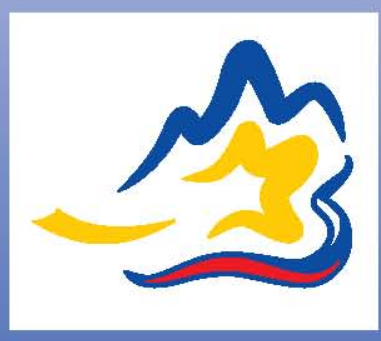

\title{
Responding to the challenge of cancer in Europe
}

Edited by

Michel P Coleman

Delia-Marina Alexe

Tit Albreht

Martin McKee
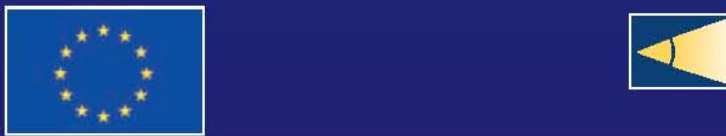
Responding to the challenge of cancer in Europe 
(C) Institute of Public Health of the Republic of Slovenia, 2008

All rights reserved. Please address requests for permission to reproduce or translate this publication to:

Institute of Public Health of the Republic of Slovenia

Trubarjeva 2

1000 Ljubljana

Slovenia

The views expressed by authors or editors do not necessarily represent the decisions or the stated policies of the Ministry of Health of the Republic of Slovenia, the European Commission, or the European

Observatory on Health Systems and Policies or any of its partners.

CIP - Cataloguing in publication

National and University Library, Ljubljana, Slovenia

$616-006(4)$

RESPONDING to the challenge of cancer in Europe / edited by

Michel P. Coleman ... [et al.]. - Ljubljana : Institute of Public Health of the Republic of Slovenia, 2008

ISBN 978-961-6659-20-8

1. Coleman, Michel P.

COBISS.SI-ID 236838144

Printed and bound in the Republic of Slovenia by Tiskarna Radovljica

Further copies of this publication are available from:

Institute of Public Health of the Republic of Slovenia

Trubarjeva 2

1000 Ljubljana

Slovenia 


\section{Responding to the challenge of cancer in Europe}

Edited by Michel P Coleman, Delia-Marina Alexe, Tit Albreht and Martin McKee

This publication arises from the project FACT - Fighting Against Cancer Today - which has received funding from the European Union, in the framework of the Public Health Programme. 



\section{Contents}

List of tables, figures and boxes

vii

Foreword

xiii

Zofija Mazej Kukovic

Acknowledgements

$X V$

About the contributors

$x v i i$

Chapter 1 Responding to the challenge of cancer in Europe

Delia-Marina Alexe, Tit Albreht, Martin McKee and Michel P Coleman

Chapter 2 The burden of cancer in Europe

Freddie Bray

Chapter 3 The causes of cancer and policies for prevention

Jose M Martin-Moreno and Gudjón Magnússon

Chapter 4 Cancer screening

Matti Hakama, Michel P Coleman, Delia-Marina Alexe and Anssi Auvinen

Chapter 5 Drugs for cancer

Karol Sikora

Chapter 6 Organizing a comprehensive framework for cancer control Robert Haward

Chapter 7 Changes in the management of cancer: the example of colorectal cancer Jean Faivre and Côme Lepage 
Chapter 8 Survival of European cancer patients

Franco Berrino and Riccardo Capocaccia

Chapter 9 Information on cancer

Andrea Micheli and Paolo Baili

Chapter 10 Cancer patients - partners for change

Hildrun Sundseth and Lynn Faulds Wood

Chapter 11 The role of psychosocial oncology in cancer care Luigi Grassi and Luzia Travado

Chapter 12 Dying with cancer, living well with advanced cancer Irene J Higginson and Massimo Costantini

Chapter 13 Closing the gap: cancer in central and eastern Europe

Witold Zatoński and Joanna Didkowska

Chapter 14 Cancer control in Slovenia: achievements, shortcomings and opportunities

Maja Primic Žakelj and Tina Žagar

Chapter 15 Researching cancer

Tanja Čufer and Richard Sullivan

Chapter 16 Making progress against cancer

Tit Albreht, Martin McKee, Delia-Marina Alexe, Michel P Coleman and Jose M Martin-Moreno 


\section{List of tables, figures and boxes}

Tables

Table 2-1

Estimated number of new cases (incidence) and deaths (mortality) in 2006 for the 17 most common cancers in the European Union of 25 Member States, and for the 5 most common cancers in Greater Europe, sorted in descending order of new cases

Table 2-2 Estimated number of new cancer cases and deaths, by country, greater Europe 2002

Table 2-3a Predicted numbers (thousands) of new cancer cases in

Table 2-3b 2020 in Europe, based on incidence rates in 2002 2020 in Europe, based on crude scenarios for annual change in the overall cancer incidence rates

Table 4-1 Components of cancer screening programmes

Table 4-2

Randomized trials evaluating mortality effects of mammography screening

Table 4-3 Randomized trials evaluating mortality effects of colorectal cancer screening based on faecal occult blood testing

Table 4-4 Summary of evidence for cancer screening

Table 4-5

Cancer screening recommendations by various organizations

Table 5-1

The challenges of cancer care

Table 5-2

Drivers of molecular therapeutics

Table 5-3

Uncertainty of novel drugs for cancer

Table 5-4

Barriers to innovation

Table 5-5

High-cost cancer drugs likely to be approved by the Food and Drug Administration (FDA) and EMEA, 2007-2010

Table 8-1 Population coverage, number of adult cancer patients diagnosed 1995-99 and included in analyses, proportion of microscopically verified tumours and proportion of patients who were followed-up for less than five years of diagnosis: EUROCARE-4, by country

Table 8-2 Relative survival (\%) at one and five years after diagnosis, and five-year survival among patients who had already survived at least 1 year, by country, selected cancers: EUROCARE-4, adults (15-99 years) diagnosed 1995-99 
Table 8-3 Five-year relative survival (\%) for selected cancers in adults:

Europe (EUROCARE data) and the USA (SEER data): period analysis for 2000-2002

Table 9-1 Relative risk between maximum and minimum levels of incidence rates, mortality rates and 5-year relative survival across Europe: for all cancers combined

Table 9-2 Indicators of progress in cancer control (EUROCHIP-1)

Table 9-3 Cancer registries in Europe

Table 11-1 Psychological morbidity and risk factors for psychosocial morbidity in cancer patients

Table 11-2 Summary of key recommendations of NICE clinical guidance on supportive and palliative care

Table 11-3 Main domains of the patient-centred model

Table 12-1 Deaths from cancer in Europe and selected other parts of the world: 2000 and predicted for 2020

Table 12-2 Cancer patients: prevalence of symptoms and estimated number of cancer patients with symptoms towards the end of life

Table 12-3 Summary of results from a meta-analysis of a multiprofessional 247 palliative care team compared with conventional care.

Description of the effect size for different patient outcomes

Table 13-1 Contribution (years of life) of selected causes of death to the difference in expectation of life at birth between central and eastern Europe and EU15, 2002

Table 13-2 Cancer incidence and mortality rates (per 100 000) in Europe, 2002

Table 13-3 Number of alcohol-related deaths and rates per 100000 population in central and eastern Europe, 2002

Table 13-4 Number of alcohol-related deaths and rates per 100000 population in EU15, 2002

Table 14-1 Burden of cancer in Slovenia, 1992-1994 and 2002-2004 
Figures

Figures marked * appear in the colour section (CS) between pages 40 and 41.

Figure 2-1 Availability of cancer mortality data by country (Source: WHO 10 mortality database)

Figure 2-2 United Nations definition of the 38 countries and 4 regions of 13 Europe (Source: WHO)

Figure 2-3 Distribution of new cases and deaths, by sex, for 23 different 16 cancers in Europe, 2002 (Source: Ferlay et al, 2004)

Figure 2-4 Distribution of age-standardized (world) rates (ASR) of cancer 17 incidence and mortality, by country and sex, in descending order of incidence - all cancers except skin (Source: Ferlay et al, 2004)

Figure 2-5 Distribution of age-standardized (world) rates (ASR) of incidence and mortality, by country and sex, in descending order of incidence - lung cancer males (a) and females (b) (Source: Ferlay et al, 2004)

Figure 2-6a Distribution of age-standardized (world) rates (ASR) of incidence and mortality by country, in descending order of incidence - breast cancer (Source: Ferlay et al, 2004)

Figure 2-6b Distribution of age-standardized (world) rates (ASR) of 25 incidence and mortality (ASR) by country, in descending order of incidence - prostate cancer (Source: Ferlay et al, 2004)

Figure 2-7 Distribution of age-standardized (world) incidence and mortality rates (ASR) by country and sex, in descending order of incidence - colorectal cancer males (a) and females (b) (Source: Ferlay et al, 2004)

Figure 2-8a* Trends in age-standardized (world) mortality rates in selected countries in the four UN-defined regions of Europe all cancers combined, males (Source: WHO mortality database)

Figure 2-8b* Trends in age-standardized (world) mortality rates in selected CS countries in the four UN-defined regions of Europe - all cancers combined, females (Source: WHO mortality database)

Figure 2-9a* Trends in age-standardized (world) mortality rates in selected CS countries in the four UN-defined regions of Europe - lung cancer, males (Source: WHO mortality database)

Figure 2-9b* Trends in age-standardized (world) mortality rates in selected CS countries in the four UN-defined regions of Europe - lung cancer, females (Source: WHO mortality database) 
Figure 2-10 Stages of worldwide tobacco epidemic (Source: Edwards, 2004; adapted from Lopez, Collishaw \& Piha, 1994)

Figure 2-11 Female lung cancer mortality, Spain 1974-2003 (solid line) and predicted (dashed line) until 2020, based on age-periodcohort model (Source: Nordpred, Møller et al, 2003)

Figure 2-12 Trends in smoking prevalence in the EU 1979-2002 (Source: Cancer Research UK, EU Factsheet, 2004)

Figure 2-13* Trends in age-standardized (world) mortality rates in selected countries in the four UN-defined regions of Europe: breast cancer, females (Source: WHO mortality database)

Figure 2-14a* Trends in age-standardized (world) mortality rates in selected countries in the four UN-defined regions of Europe colorectal cancer, males (Source: WHO mortality database)

Figure 2-14b* Trends in age-standardized (world) mortality rates in selected countries in the four UN-defined regions of Europe colorectal cancer, females (Source: WHO mortality database)

Figure 2-15* Trends in age-standardized (world) mortality rates in selected countries in the four UN-defined regions of Europe prostate cancer (Source: WHO mortality database)

Figure 5-1 Chemotherapy for advanced cancer

Figure 5-2 Predicted new drug application (NDA) dates for molecular therapies in the United States of America

Figure 5-3 Global cancer market by sector: the escalating global cost of cancer drugs

Figure 5-4 The future of cancer drug development

Figure 5-5 Six areas where diagnostics help to personalize cancer medicine

Figure 5-7 2004 (purchasing power parity adjusted) of Euros)

Figure 5-8 The four building blocks of cancer's future

Figure 8-1 Mean age-adjusted five-year relative survival, adults (15-99 years) diagnosed during 1995-99 in one of 23 European countries: EUROCARE-4 study 
Figure 8-2 Age-adjusted five-year relative survival in adults 160

(15-99 years) diagnosed during 1995-99 in one of 23

European countries, both for all patients and for those who survived to the first anniversary of diagnosis (one-year survivors), EUROCARE-4 study: (a) colorectal cancer (b) breast cancer in women (c) prostate cancer

Figure 8-3 Five-year relative survival in adults (15-99 years) diagnosed 161 during 1995-99, by geographic region and by age at diagnosis, both for all patients and for those who survived to the first anniversary of diagnosis (one-year survivors), EUROCARE-4 study: (a) colorectal cancer (b) breast cancer in women (c) prostate cancer

Figure 8-4 Trends in five-year age-adjusted relative survival in adults 163 (15-99 years) by geographic region, period estimates for 1991-2002 (see text), EUROCARE-4 study: (a) colorectal cancer (b) breast cancer in women (c) prostate cancer

Figure 8-5 Age-adjusted five-year relative survival for all cancers 164 combined, by sex and country, with area-weighted mean survival for Europe, grouped by Total National Expenditure on Health

Figure 8-6 Trends in five-year and ten-year age-adjusted relative survival in adults (15-99 years) in Europe, period estimates for 1991-2002 (see text), EUROCARE-4 study: all cancers combined, by sex

Figure 9-1 Age-standardized total prevalence, incidence and survival, both sexes, all cancers combined, 1992

Figure 11-1 Distress thermometer proposed by the NCCN Panel on Distress Management in Oncology

Figure 12-1 Hypothesized trajectories of functional decline in cancer and other conditions (which may now represent the cancer trajectory)

Figure 12-2 Preliminary data on place of death by country

Figure 12-3 Model derived from a systematic review (including 1.5 million patients) of the factors associated with home or hospital death

Figure 12-4 Results of two ecological studies in Genoa (top) and London (bottom) to analyse the relationship between the proportion of people who died at home and social indicators

Figure 12-5 Model of palliative care as an increasing part of care from diagnosis onwards 
Figure 13-1 Trends in mortality in Europe from major causes at ages 20-64 254

Figure 13-2 Time trends and predicted cancer mortality in the EU 258

Figure 13-3 Smoking-attributable death rates from all cancers in 261 EU countries

Figure 13-4 Cervical cancer mortality 265

Figure 13-5 Breast cancer mortality 266

$\begin{array}{lll}\text { Figure 13-6 Colorectal cancer mortality } & 267\end{array}$

Figure 13-7 Testicular cancer mortality 268

Figure 14-1 Trends in age-standardized cancer incidence and mortality 281 rates by sex in Slovenia, 1985-2004

Figure 14-2 Burden of cancer by site and sex, Slovenia 2004282

Figure 14-3 Distribution (\%) of cancers by age and sex, Slovenia, 2004283

Figure 14-4 Five-year relative survival (95\% Cl) for selected cancers 285 among males in Slovenia during the period 2000-2004

Figure 14-5 Five-year relative survival $(95 \%$ Cl) for selected cancers 286 among females in Slovenia during the period 2000-2004

Figure 15-1 Spend per capita for cancer research in the EU15; 304 EU25+EFTA; and United States - from funding organizations (governmental and philanthropic) and through health-care/ university systems

Figure 15-2 Outputs (publications) from top 24 pharmaceutical 308 companies in the United States of America and EU25+EFTA, 1996-2003

Figure 15-3 Comparison of global outputs (publications) in cancer 308 research 1999 and 2003

Boxes

$\begin{array}{lll}\text { Box 10-1 MAC Recommendations } & 197\end{array}$

Box 10-2 The Warsaw Declaration 202

Box 10-3 European Cancer Patient Coalition 204

Box 12-1 Balfour M Mount (originator of the term palliative care) 233 discussing quality of life at the end of life, demonstrating resilience.

Box 12-2 Palliative care services in the United Kingdom 


\section{Foreword}

Europeans today enjoy healthier, wealthier and longer lives than ever, a great achievement of our societies. However, policy-makers are still facing major health challenges, such as widening health gaps between and within Member States, ageing of the population and increasing levels of chronic disease, including cancer.

Cancer, this complex group of diseases with serious implications not just for individuals and their families, but also for society in general and health systems in particular, remains an important health challenge in Slovenia, in Europe and world-wide. At present, with more than 3 million new cases and 1.7 million deaths each year, cancer represents the second most important cause of death and morbidity in Europe. If these data are linked to the data on the projection of the burden caused by other non-communicable chronic diseases and to the data on the ageing of the European population, the problem is seen to be even greater. Without effective interventions, the cancer burden will increase dramatically, but comprehensive cancer prevention and control policies can bring significant benefits.

For all these reasons, Slovenia has decided to focus on cancer as the key public health priority during its Presidency of the Council of the European Union in the first half of 2008. This offers an important opportunity to review how Member States - and the European Union as a whole - are approaching the major public health challenge of cancer as a chronic disease, and to develop future actions.

Much has been achieved in health policy at the EU level in several domains which have a direct and positive impact on cancer prevention and control. However, both the current situation and all the predictions of the future burden of cancer call for a stronger policy response across Europe.

For comprehensive cancer prevention and control it is necessary to:

- act effectively and systematically for the improvement of health of the population, using all available measures in all policy areas, since the risk factors for several major diseases, including cancer, are mainly the same; 
- promote healthy lifestyles and reduce exposure to risk factors, in order to prevent as many cancers as possible;

- detect as early as possible those cancers which could not be prevented;

- give the best possible treatment and care to cancer patients, exchanging information on best practices for diagnosis, treatment, rehabilitation and palliative care;

- encourage research that aims to identify the causes of cancer and to develop better strategies for prevention, diagnosis, treatment and cure.

This book represents an attempt to bring together the latest information and analysis to assist in meeting the challenge of cancer. Some of the most eminent European experts in cancer control have contributed to the book. Many of them will also contribute to the EU Cancer Conference in Slovenia, in February 2008. The aim of the conference is also to strengthen links between health-care professionals, cancer patients, policy-makers and other stakeholders in the cancer community, by sharing their knowledge, experience and best practices.

With this initiative on cancer during the Slovenian Presidency of the Council of the European Union, we call for immediate and concerted action to reduce cancer incidence and mortality, to improve cancer outcomes, to reduce health gaps in the prevention and control of cancer between and within EU Member States and to increase the benefits for cancer patients.

Zofija Mazej Kukovič

Minister of Health, Republic of Slovenia 


\section{Acknowledgements}

Responding to the Challenge of Cancer in Europe has been written within the Fighting Against Cancer Today (FACT) project, funded by the EU's Public Health Programme. It brings together a large body of evidence on cancer control in Europe, a priority theme in public health during the Slovenian Presidency of the European Union in 2008.

We are extremely grateful to all the authors for the enthusiasm they have brought to this project and their hard work in meeting tight deadlines.

We very much appreciated the helpful comments and suggestions from the reviewer Jan Willem W Coebergh, Professor of Cancer Surveillance at the Department of Public Health of the Erasmus Medical Centre in Rotterdam, the Netherlands, and editor (epidemiology and prevention) of the European Journal of Cancer.

This volume is one of a series produced by the European Observatory on Health Systems and Policies and it is published with the Institute of Public Health of the Republic of Slovenia. We are especially grateful to the production team in the European Observatory, Jonathan North and Caroline White, and to Peter Powell for his work on typesetting the publication.

We would like to thank Marija Seljak, Mojca Gruntar Činč, Vesna Kerstin Petrič and Nataša Hace at the Slovenian Ministry of Health; and Ada Hočevar Grom, Rade Pribaković, Mateja Gorenc and Tatjana Pokrajac at the Institute of Public Health (IVZ) of the Republic of Slovenia, for their tireless support and enthusiasm for this project.

Finally, we would like to thank Josep Figueras, the Director of the European Observatory, and members of its secretariat in Brussels, for their endeavour in this project. 



\section{About the contributors}

Tit Albreht MD PhD is a researcher in health services at the Institute of Public Health of the Republic of Slovenia, where he holds the post of Adviser to the Director. He is involved in teaching at the Department of Public Health. He trained at the Medical Faculty, University of Ljubljana, Slovenia. After completing his training in the specialty of social medicine and his Masters and Doctorate of Science at the Netherlands Institute for Health Sciences at Erasmus University, Rotterdam (in the area of health services research), his research has focused on health systems and health policy development issues at both national and international level. He is Chair of the Section on Health Services Research of the European Public Health Association (EUPHA) and a member of the Internal Network Health Policy and Reform and the Academy of Health.

Delia-Marina Alexe MD PhD is a graduate of the University of Medicine and Pharmacy "Carol Davila" in Bucharest, Romania. She trained in trauma and cancer epidemiology at the University of Athens Medical School, Greece. She joined the Non-communicable Disease Epidemiology Unit at the London School of Hygiene and Tropical Medicine, UK, in 2006. Her research interests include cancer aetiology and trends; the role of adipose tissue hormones and growth factors in cancer risk; and early life environment and cancer risk.

Anssi Auvinen MD PhD received his MD (1989) and PhD in epidemiology (1997) from the University of Tampere, Finland. He has worked as a research scientist at the Radiation and Nuclear Safety Authority and the Finnish Cancer Registry and was a visiting scientist at the National Cancer Institute in the United States. He is Professor of Epidemiology at the University of Tampere. His main research interests include cancer screening and the health effects of radiation. He is the principal investigator of the Finnish randomized trial on prostate cancer screening and is one of the coordinators of the European Randomized Study of Prostate Cancer Screening (ERSPC). He has published about 180 scientific articles. 
Paolo Baili $\mathrm{PhD}$ is a researcher at the National Cancer Institute (Istituto Nazionale dei Tumori) in Milan, Italy. He studied demographic and social statistics at the University of Bologna, Italy (graduating in 2001) and completed postgraduate studies in health statistics in 2007. Since his graduation, Dr Baili has worked for the Descriptive Epidemiology and Public Health Planning Unit of the National Cancer Institute in Milan. He was coordinator of the public health projects - European Cancer Health Indicator Project (EUROCHIP-1, EUROCHIP-2) and Tumours in Italy (i Tumori in Italia), and has also cooperated in the CONCORD, EUROCARE-3 and EUROCARE-4 projects. Dr Baili will be the coordinator of EUROCHIP-3.

Franco Berrino MD PhD is Head of the Department of Preventive and Predictive Medicine, and Director of the Epidemiology Unit at the National Institute for the Study and Cure of Tumours (Istituto Nazionale per lo Studio e la Cura dei Tumori), Milan, Italy. His research includes case-control studies on hormones and breast cancer and the methodology of case-control studies for the evaluation of screening, as well as the ORDET study on the role of hormones and diet in the aetiology of tumours. Professor Berrino is Project Leader of the EUROCARE study, aimed at analysing and explaining variations in the survival of cancer patients in Europe. He is National Coordinator of the European Prospective Investigation into Cancer and Nutrition (EPIC) study, Italian section. He also leads several other projects COS, on gene-environment interactions in the occurrence of breast cancer in young women; DIANA on diet and androgens; and STUDIA, a randomized trial of adjuvant diet to prevent weight gain during chemotherapy for breast cancer.

Freddie Bray MSc PhD obtained his first degree in statistics in 1993 (University of Aberdeen, UK), his MSc in medical statistics in 1994 (University of Leicester, UK) and his PhD in 2005 (London School of Hygiene and Tropical Medicine, UK). He worked at the South Western Cancer Registry in England from 1994-1998, before joining the Unit of Descriptive Epidemiology, International Agency for Research on Cancer, Lyon, France from 1998-2005. He is currently at the Cancer Registry of Norway, in Oslo.

Riccardo Capocaccia MSc PhD is Director of the Cancer Epidemiology Unit at the National Health Institute (Istituto Superiore di Sanità) in Rome, Italy. $\mathrm{He}$ is a statistician with a background in mathematics and wide experience in cancer epidemiology. He is Project Leader of EUROPREVAL, the BioMedfunded project aimed at estimating cancer incidence and prevalence in European countries. He is responsible for database implementation and 
analysis in the EUROCARE project, examining cancer survival in populationbased cancer registries in Europe. He is also involved in the CONCORD project for the worldwide comparison of cancer survival.

Michel P Coleman BA BM BCh MSc FFPH has been Professor of Epidemiology and Vital Statistics at the London School of Hygiene and Tropical Medicine since 1995. He was Deputy Chief Medical Statistician at the Office for National Statistics, 1995-2004, and Head of the Cancer and Public Health Unit at the London School of Hygiene and Tropical Medicine, 1998-2003. He has worked for the World Health Organization at the International Agency for Research on Cancer in Lyon, France (1987-1991), and was Medical Director of the Thames Cancer Registry in London (19911995). His main interests include trends in cancer incidence, mortality and survival, and the application of these tools to the public health control of cancer.

Massimo Costantini MD is a physician specializing in oncology. He is responsible for the Regional Palliative Care Network, a unit of the National Cancer Research Institute in Genoa, Italy. His research interests are in the areas of palliative care, quality of life, and psycho-oncology. He was Medical Director of the Hospice G. Ghirotti of Genoa (2002-2004). Since 2007, he has been Visiting Professor in Palliative Care at the Department of Palliative Care, Policy and Rehabilitation, King's College, London.

Tanja Cufer MD PhD is Professor of Oncology at the Medical Faculty, University of Ljubljana, Slovenia. She also serves as Senior Consultant at the Institute of Oncology, Ljubljana. She is an elected Member of the General Board of the European Organisation for Research and Treatment of Cancer (EORTC) and has recently become a Member of the International Affairs Committee of the American Society of Clinical Oncology. Her main research interests are breast cancer, bladder cancer and prognostic and predictive factors, with a focus on proteases. She has a special interest in drug development and clinical trials.

Joanna Didkowska $\mathrm{PhD}$ works in the Department of Epidemiology and Cancer Prevention, National Cancer Registry at the Maria Sklodowska Curie Memorial Cancer Center and Institute of Oncology in Warsaw, Poland. Since 1999, she has been Chair of the Association of Cancer Registries in Poland. She focuses on descriptive cancer epidemiology in Poland, especially prediction models, and the determinants of the health status of Poles and inhabitants of central and eastern Europe. She works on analyses of data from the National Cancer Registry. She has authored over 50 publications (including 6 books) and is co-author and editor of the annual report Cancer in Poland. 
Jean Faivre MD is Professor at the Department of Gastroenterology, University of Burgundy, and Director of the Epidemiology Unit for Digestive Cancers (Registre Bourguignon des Cancers Digestifs) (INSERM U866) in Dijon, France. He is the French representative on the Advisory Committee of cancer experts of the European Union, and a member of the Board of the European Cancer Prevention Organization (ECP); the French National Cancer Institute (INCa); the Board of the Latin American Association of Cancer Registries and the Committee on Cancer Screening (Ministry of Health, INCa). He collaborates in the French network of cancer registries and the EUROCARE project. Professor Faivre is also Chair of the Cancerology Federation of University Hospitals and Honorary Chairman of the French Federation of Digestive Oncology (FFCD) and of the French Society of Gastroenterology.

Lynn Faulds Wood is an award-winning television presenter and journalist who survived advanced colon cancer after almost a year of medical delay. She has made many television programmes on cancer. She has helped to produce the world's first research-based guidance on symptoms of colon cancer, and helped to pioneer diagnostic training programmes in colonoscopy and formal training centres around the United Kingdom. She is currently President of the European Cancer Patient Coalition, with a membership of over 250 cancer patient organizations in 37 countries. She also runs the British charity Lynn's Bowel Cancer Campaign.

Luigi Grassi MD is Professor and Chair of Psychiatry, Department of Medical Sciences of Communication and Behaviour, University of Ferrara, Italy; and Chief of the Clinical Psychiatry Unit, Department of Neurosciences and Rehabilitation and Department of Mental Heath, S. Anna University Hospital and Local Health Agency in Ferrara, Italy. His research and teaching are mainly in clinical psychiatry, consultation-liaison psychiatry and psychooncology. Dr Grassi is the author of several scientific papers and books. He is President of the International Psycho-Oncology Society (IPOS) and the Italian Society of Psycho-Oncology (SIPO) and Chair of the Section on Psycho-Oncology of the World Psychiatric Association (WPA).

Matti Hakama PhD is Professor Emeritus at the University of Tampere, Finland, where he teaches epidemiology; and is Director of the Mass Screening Registry, based in the Finnish Cancer Registry. His main field of research is cancer epidemiology, with a special focus on bio-banks, cancer registration and screening for cancers of the cervix, breast, colorectum and prostate. He also leads other research programmes on oral health, lower urinary tract symptoms, erectile dysfunction and varicose veins. 
Robert A Haward MB ChB DPH FFPH is Emeritus Professor of Cancer Studies at Leeds University. He qualified at Bristol University in 1968 and pursued a career in public health medicine in three district authorities before being appointed Regional Director of Public Health for Yorkshire from 1986 to 1994 . He was then appointed Professor of Cancer Studies in Leeds until his retirement in 2006. He remains involved in national research activities. His academic career combined extensive national work developing cancer policies, with research interests in cancer services delivery. Much of this research used population-based data from the Northern and Yorkshire Cancer Registry and Information Service, of which he was also the Medical Director.

Irene J Higginson MD PhD is Professor of Palliative Care and Policy and Head of the Department of Palliative Care and Policy, King's College, London. She qualified in medicine from Nottingham University, and has worked in wide-ranging medical and university positions, including radiotherapy and oncology, in-patient and home hospice care, at the Department of Health (England) and various universities. Her research interests and publications are in the following areas: quality of life and outcome measurements; evaluation of palliative care (especially of new services and interventions); epidemiology; clinical audit; effectiveness; psychosocial factors and care; symptom assessment; cachexia and anorexia; and care of the elderly.

Côme Lepage $\mathrm{MD} \mathrm{PhD}$ is a hepato-gastroenterologist and consultant physician in gastroenterology and hepatology at Dijon University Hospital, France. He holds an appointment from the French Institute for Health and Medical Research (INSERM) in the Côte d'Or Digestive Cancer Registry. After undergraduate studies at François Rabelais University, Tours, he completed his doctorate at Burgundy University, Dijon, France. His main area of interest is in cancers of the digestive tract. His research interests are in cancer epidemiology and prevention, particularly colon cancer and rare cancers of the digestive tract.

Guðjón Magnússon MD PhD is Professor of Public Health, Reykjavik University, Iceland. He qualified in medicine at the University of Iceland (1971), postgraduate training in public health in Edinburgh and Stockholm followed (1974-1980) and he obtained his PhD from the Karolinska Institutet in Stockholm, Sweden (1980). He was Deputy Chief Medical Officer of Iceland (1980-90); Deputy Secretary General in the Icelandic Ministry of Health and Social Insurance (1991-95); and Dean of the Nordic School of Public Health in Gothenburg, Sweden (1996-2002). He was Director of the Division of Health Programmes at the WHO Regional Office for Europe in Copenhagen, Denmark, from 2002 until his recent retirement. He has held 
various academic positions in public health and has published numerous articles on public health and health services research.

Jose M Martin-Moreno MD PhD MPH DrPH received his medical and public health education and training from the University of Granada and the National School of Health, both in Spain; and Harvard University in the United States. His specialties are preventive medicine and public health, and occupational medicine. He is Professor of Public Health at the University of Valencia, Spain, and a specialist at the University Clinical Hospital. He also acts as an adviser to the WHO Regional Office for Europe in Copenhagen, Denmark; the International Agency for Research on Cancer; the European Institute of Oncology; and other institutions. He has previously served as Director General of Public Health and Chief Medical Officer of Spain. His main research interest is in the field of cancer epidemiology and prevention.

Andrea Micheli MD is Director of the Descriptive Epidemiology and Public Health Planning Unit (Fondazione IRCCS Istituto Nazionale dei Tumori), in Milan, Italy, and Associate Professor of Sociology at the Medical Faculty of the University of Milan. $\mathrm{He}$ is experienced at coordinating national and international projects on cancer aetiology, survival, estimation of prevalence and public health/cancer control. He has also directed major epidemiological studies in collaboration with other institutes and public health networks in Europe. He has published more than 120 scientific papers. He has been project leader, coordinator or a member of the scientific board for the following projects: ORDET; EUROCARE; ITACARE; CONCORD; ELDCARE; EUROPREVAL; ITAPREVAL; Tumours in Italy (i Tumori in Italia); and the Estimation of cancer prevalence in the United States. Dr Micheli is project leader of the European Cancer Health Indicators Project (EUROCHIP).

Martin McKee CBE MD DSc is Professor of European Public Health at the London School of Hygiene and Tropical Medicine, where he codirects the School's European Centre on Health of Societies in Transition. He is also a research director at the European Observatory on Health Systems and Policies. His main fields of research include health systems and the determinants of disease in populations and health policy, with a focus on eastern Europe and the countries of the former Soviet Union.

Maja Primic Žakelj MD DSc is a specialist in epidemiology and a doctor of cancer epidemiology at the Institute of Oncology, Ljubljana, Slovenia. She is Head of the Epidemiology and Cancer Registries of the Institute of Oncology. This comprises the Cancer Registry of Slovenia; Cancer Registry of the Institute of Oncology, Epidemiology Unit; and the Cancer Screening Registry, where the national cervical cancer screening programme is coordinated. 
Dr Primic Žakelj was elected Associate Professor at the Faculty of Medicine, University of Ljubljana in 1996. Cooperating with several NGOs, especially the Cancer League, she is also active in public education on cancer prevention. She has been involved in several national and international epidemiological studies, currently EUROCARE, CONCORD, EUROCHIP and EUROCAN+PLUS. She also serves as the national cancer control coordinator.

Karol Sikora MA MB BCh PhD FRCR FRCP FFPH is Medical Director of CancerPartnersUK, which is creating the United Kingdom's largest cancer network as a series of joint ventures with National Health Service (NHS) Trusts. He is Professor of Cancer Medicine and honorary Consultant Oncologist at Imperial College School of Medicine, Hammersmith Hospital, London. His main interests are cancer service organization, breast cancer and molecularly targeted drugs. He is Scientific Director of Medical Solutions PLC, a cancer diagnostic company. He has recently been appointed Dean of Britain's first independent medical school at the University of Buckingham.

Richard Sullivan MD PhD qualified in medicine at St. Mary's Hospital, London, and trained in urology. He undertook a $\mathrm{PhD}$ and post-doctoral research at University College London before moving to industry, where he worked in medical affairs and the R\&D divisions of radiology, interventional devices and oncology. He joined Cancer Research UK in 1999. He has served on the Cancer Research UK Executive Board, where he was responsible for the management of the clinical research portfolio and clinical policy development in a broad range of areas, from paediatric regulations to European research policy. He is Chair of the European Cancer Research Managers Forum; UK Director of the Council for Emerging National Security Affairs (CENSA), a Washington-based think-tank; and visiting Professor of International Relations at Graz, Austria. He is now at the London School of Economics and Political Sciences. He continues with his research interests in a variety of areas, including public health, biomedical research policy and the anti-cancer properties of medicinal mushrooms.

Hildrun Sundseth heads the Brussels office of the European Cancer Patient Coalition (ECPC). This is a patient-led umbrella organization bringing together some 250 cancer patient groups (ranging from common cancers such as those of the lung, colon, breast and prostate to the rarer cancers) to speak with a single voice in the European health-care debate. She is responsible for ECPC's strategy on all EU policies, legislation and measures that affect cancer patients and their care. She provides the secretariat for MEPs against Cancer (MAC), a forum of Members of the European Parliament which brings together 60 MEPs who have pledged to make the fight against cancer into a priority issue once more for EU and Member State action. 
Luzia Travado MSc DipPsych is a clinical psychologist and psychotherapist, specialized in health psychology. She completed her studies at the University of Lisbon, Portugal. She is Head of the Clinical Psychology Unit at Centro Hospitalar de Lisboa Central, where she began her career and has pioneered psychosocial programmes since 1985. She teaches psycho-oncology and has coordinated postgraduate courses on psycho-oncology and palliative care at the Independent University, Lisbon. Dr Travado collaborates in the Southern European Psycho-Oncology Study (SEPOS). She serves as adviser to the National Coordinator for Oncological Diseases, Portugal, and as Director of the International Psycho-Oncology Society.

Tina Žagar studied at the Faculty of Mathematics and Physics at the University of Ljubljana, Slovenia, and obtained her University Diploma in 2004. She is a postgraduate student in biostatistics at the University of Ljubljana and expects to obtain a DSc in Statistics in 2009. Since 2004, she has been working at the Epidemiology and Cancer Registries, Institute of Oncology, Ljubljana. Her main fields of interest are cancer epidemiology, spatial analysis with mapping, and survival of cancer patients.

Witold A Zatoński MD ScD is Professor at the Institute of Oncology, Cancer Epidemiology and Prevention and Head of the Department of Cancer Epidemiology and Prevention at the Marie Sklodowska-Curie Memorial Cancer Center, Warsaw, Poland. He is a member of the Committee on Epidemiology and Public Health at the Polish Academy of Sciences. His contribution to the national and international health arena has been recognized by many prestigious awards. Professor Zatoński has been a consultant to the World Health Organization and Regional Coordinator for Tobacco Control with the International Union Against Cancer (UICC). He has been at the forefront of public health and tobacco control in Poland. 


\section{Chapter 1 \\ Responding to the challenge of cancer in Europe}

Delia-Marina Alexe, Tit Albreht, Martin McKee and Michel P Coleman

The term "cancer" is commonly used to cover a wide range of diseases which all share a common feature, namely that cells in affected organs or tissues of the body (e.g. breast, lung, skin or bone marrow) continue to grow indefinitely, without reference to the needs of the body. Many cancers have the capacity to spread to other parts of the body and to kill the patient. With more than 3 million new cases and 1.7 million deaths each year (Ferlay et al., 2007), cancer currently represents the second most important cause of death and morbidity in Europe.

Cancers have many causes. A few are the result of faulty genes; some are a consequence of an individual's life history (e.g. how many children they have borne); some represent the long-term effects of exposure at any stage of life to cancer-causing agents such as tobacco smoke; and many involve a combination of these factors. The cause or causes of many cancers remain unknown. Most cancers become much more common with advancing age.

The total annual numbers of new cases and deaths (per 100000 population) for all cancers combined vary as much as two-fold between Member States of the European Union (EU). The range of survival rates is similarly wide. For individual cancers, the variation across Europe is even greater. This reflects a wide range of social and epidemiological factors in Member States: cancer prevention programmes; screening programmes; cancer control plans; individual lifestyles and occupational exposures; the existence and accessibility of health-care 
facilities and technological infrastructure; and the availability of human, financial and material resources for health and economic development.

The annual rates of newly diagnosed cancer patients (incidence rates) and deaths from cancer (mortality rates) are changing. Some cancers (e.g. stomach cancer) are becoming less common, but others are increasing, such as malignant melanoma - the most dangerous form of skin cancer. The time trends in cancer risk also vary between European countries and some cancers show different trends between men and women, or young and old, or poor and rich. For instance, lung cancer rates are falling in many countries among men (particularly the more affluent groups) but increasing among women, particularly the young. In other countries, lung cancer rates are still increasing in both sexes. The pattern is similarly varied for many cancers, so the public health profile of cancer in Europe is complex. Trends in the incidence and mortality rates are also influenced by successes in health promotion (e.g. tobacco control), efficient screening (e.g. breast, bowel, cervix) and better treatment. These have been reflected in lower incidence, reduced mortality, higher survival, improved life expectancy and a better quality of life for cancer survivors.

In the past, a diagnosis of cancer was often a sentence of death, but increases in medical knowledge (particularly innovations in imaging, surgery, radiotherapy and pharmaceuticals) have made it possible to offer a higher probability of cure to some cancer patients. For many other cancers, modern treatment means that a patient is more likely to die with a cancer, rather than of it - even if the cancer is not cured or eradicated, the patient may die from some other cause, not as a direct consequence of the cancer.

At the same time, a greater understanding of the causes of cancer means that primary prevention is often possible - by reducing or eliminating the risk of developing the disease. One of the most important medical discoveries of the twentieth century was the role of tobacco smoking as a cause of cancers of the lung and various other organs. The consequences of implementing antismoking measures are now becoming apparent rapid declines in the occurrence of cancers of the lung and some other organs in countries where tobacco use has declined. Mortality has fallen substantially as a direct result.

Vaccines against the types of human papilloma virus (HPV) that cause cervical cancer have recently been licensed in the EU and they may be in widespread use within a few years. 
Advances in genetics and in genetic epidemiology and the Human Genome Project (http://www.genome.gov/), in particular, now offer new perspectives for diagnosis, treatment and (soon) possibly even prevention of many diseases, including cancer.

These developments have enormous consequences for health services. The management of cancer increasingly involves a complex package of interventions, requiring careful coordination of a wide range of professionals (oncologists, surgeons, imaging specialists, pathologists, specialist nurses, psycho-oncologists) in multidisciplinary teams. This radical shift challenges the traditional role of the individual medical specialist. The complexity of cancer treatment requires specialists to keep up to date with the rapid and continuing evolution of scientific evidence on diagnosis, treatment and care in order to achieve the best possible outcomes for their patients.

The proportion of gross domestic product (GDP) spent on health care varies two-fold across EU countries, as do the number of doctors per head of population and the availability of radiotherapy equipment such as linear accelerators. These variations have major consequences for Member States' ability to deliver effective health services for their cancer patients.

Survival from some cancers has improved markedly across Europe since 1990. However, survival for many cancers still varies widely between Member States (Berrino et al. 2007). This may be due to differences in public education about cancer symptoms or the stage of disease at first contact with a health professional, as well as to variations in the accessibility, efficiency, skill and resources of the diagnostic and treatment services. The latest cancer drugs are often extremely expensive, raising difficult questions about who will benefit and whether the benefits of treatment are sufficient to justify the economic costs to a nation.

Greater understanding of the human needs of cancer patients is also focusing attention on previously neglected areas of care, in particular patients' psychosocial needs and care at the end of life. Patients who receive psychosocial services to help with the psychological impact of cancer, the consequences of treatment and (when cure is not possible) palliative care, may be enabled to reach the end of their lives with dignity and without pain.

The following challenges in cancer control can be identified:

- increase in the numbers of cases and deaths; 
- improved diagnosis and treatment with improved survival rates;

- prolonged life with the disease - requiring more prolonged monitoring and rehabilitation for younger, economically active patients and for many elderly patients;

- huge increase in the costs of diagnostic and treatment services, partly as a result of rapid advances in technology.

Yet perhaps the most important challenge in cancer control is the synchronization of plans and services for primary prevention, screening, diagnosis, treatment and rehabilitation within a country.

The overall outcome of a society's efforts at cancer control results from the interplay of all these factors. The only realistic approach to controlling some cancers remains primary prevention (e.g. for tobacco smoking and lung cancer). Other cancers (e.g. of the breast, colon, cervix uteri) require the efficient implementation of organized, population-based screening programmes essential for detection and diagnosis at an early stage of disease, thus providing better opportunities for survival and full recovery. Lastly, advances from research in diagnostics and in surgical and medical treatment now provide excellent treatment opportunities for some cancers (e.g. rectal and testicular cancers).

\section{The Slovenian initiative on cancer}

The Government of Slovenia assumes the Presidency of the EU in the first half of 2008. Its decision to focus on cancer provides an important opportunity to reassess the public health challenge of cancer, and to suggest how policy-makers in Europe should respond to it.

The goal of the Slovenian Presidency initiative is to close the gaps in cancer prevention, diagnosis, treatment, care and survival that exist between EU Member States. Many Member States experience similar gaps between different regions of the country or between rich and poor citizens.

A key step towards this goal was to review the current status of cancer control in the EU in order to produce policy recommendations for those concerned with the prevention, management and palliation of cancer. This book is the result. It has been produced as a collaboration between internationally recognized public health institutes in the EU, under the umbrella Fighting Against Cancer Today (FACT). FACT is 
co-funded by the Government of Slovenia and the European Commission's Health and Consumer Protection Directorate-General, with additional support from the European Observatory on Health Systems and Policies.

The book provides an overview of the epidemiology of cancer, including a discussion of the major risk factors, how these have changed and the policies implemented to tackle them. It also examines the burden of cancer and highlights the wide diversity in current incidence, mortality and survival rates between Member States, as well as projections of how these measures are likely to evolve in the near future.

Comprehensive cancer plans are discussed as an approach to cancer control, with emphasis on integrated strategies that span primary prevention, early diagnosis, mass screening, effective treatment, rehabilitation and palliation. Each of these elements of the cancer patient pathway is reviewed in depth in successive chapters. Contributors examine the current status and plausible future developments for cancer screening in the EU; drug discovery, evaluation and deployment; the role of psychosocial oncology; and the provision of palliative care. Current patterns of cancer survival are also reviewed.

The challenges facing cancer researchers in Europe today are examined, along with the research agenda and the crucial contribution that can be made by Europe-wide collaborative research. The information required to track changes in incidence, outcomes and responses to cancer in the $\mathrm{EU}$ is reviewed, along with the optimal indicators for assessing progress against cancer in Europe, including the benefits of research using data from cancer registries.

Three case-studies are provided. One focuses on changes in the clinical management of cancer, using the example of colorectal cancer in France. Two broader descriptions of cancer control evoke the current situation, recent achievements and continuing challenges in eastern Europe and in Slovenia. Both case-studies reveal substantial gaps in prevention and access to health care but also great potential for improvement in cancer control.

Cancer patients provide a unique and crucial contribution to the wider debate about how best to manage their disease. The experience of cancer patients is examined, including their role in the provision of self-care, and how patients and their carers can become invaluable partners with their health-care professionals in the management of their own disease. 


\section{Summary}

It is hoped that this book offers a useful review of the current status of cancer control in Europe, and that the recommendations distilled from it will provide suitable stimuli to policy-makers. If the book contributes in some small way to reducing the huge burden of cancer in Europe, it will have served its purpose.

\section{REFERENCES}

Berrino F et al. (2007). Survival for eight major cancers and all cancers combined for European adults diagnosed in 1995-99: results of the EUROCARE-4 study, Lancet Oncol, 8(9):773-783.

Ferlay J et al. (2007). Estimates of the cancer incidence and mortality in Europe in 2006, Ann Oncol, 18(3):581-592.

Human Genome Project. Bethesda, MD, USA, National Human Genome Research Institute (available online at:http://www.genome.gov/, accessed 2 November 2007). 


\section{Chapter 2 \\ The burden of cancer in Europe}

Freddie Bray

\section{Introduction}

Europe comprises only one eighth of the total world population but has around one quarter of the global total of cancer cases - some 3.2 million new patients per year. While the disproportionate cancer burden is readily apparent, the disease patterns in Europe cannot simply be generalized - overall cancer incidence and mortality rates vary at least two-fold between European countries and the differences are often far greater for specific cancers.

The cancer burden (numbers and rates of new cancers diagnosed each year and of deaths from cancer) is changing with time - both for all cancers combined and for individual types of cancer. The different countries and regions of Europe show marked differences in the speed and direction of trends in cancer incidence and mortality rates for many common forms of cancer. Sex-specific differences are readily apparent for most cancers too, whether examined by residence or over time. With some exceptions, the observed variations in incidence and mortality rates largely reflect the varying prevalence and distribution of risk factors within and between European countries, as well as disparities in the effective delivery of cancer control measures. In most European countries, the combined demographic effects of population ageing and population growth will ensure a steady and continuing increase in the number of cancer patients diagnosed each year over the next 15 years, largely irrespective of changes in the incidence rates of common cancers.

Strategies to reduce the extent of the disease burden in the EU need to be set locally, to reflect the similarities and differences in the observed cancer rates in Member States. A comparative situation analysis is required - a critical assessment of cancer incidence, mortality and survival patterns at country-bycountry level, within defined regions of Europe. 
This chapter provides an overview of cancer incidence and mortality rates and trends in greater Europe, quantifying the current burden of cancer and highlighting the dominant cancers that may therefore be considered as the main priorities for prevention. The results are interpreted in the light of current understanding of the causes of cancer and the prospects for prevention.

The chapter ends with an estimate of the cancer burden in Europe circa 2020, derived from the projection of national cancer incidence rates to the forecasted age- and sex-specific populations.

\section{Data sources and methods}

Before discussing the cancer profile in Europe, it is helpful to consider the definitions of terms such as cancer incidence and mortality, as well as the sources of such information, their availability and, where necessary, their estimation. Cancer incidence is the frequency of the occurrence of new cases of cancer in a defined population over a given period of time, usually a year. It is expressed either as the absolute number of cases or, more usually, as a rate - the annual number of new cases per unit of population per year (e.g. 250 cases per 100000 population per year). The number of cases arising in the population in a given year (the numerator of the incidence rate) is divided by the population from which the cases arose (the denominator). The use of incidence rates facilitates the comparison of incidence between populations because the rates all refer to the same base population (100000 in this example). Such comparisons may provide clues to the underlying determinants (or causes) of cancer. Incidence rates also help the planning and prioritizing of resources for primary prevention - i.e. the reduction of cancer incidence by removing the causes before cancer develops, by either individual or communal means.

Population-based cancer registries collect and classify information on all new incident cases of cancer in a defined population. They also provide statistics on incidence for the purposes of assessing and controlling the impact of cancer in the community. There are currently more than 170 cancer registries in Europe, covering national populations (e.g. the Nordic countries, the Netherlands, Slovenia) or certain regions within a country (e.g. in Italy, Spain, France). The founding of cancer registries in Europe has occurred unsystematically over the last half-century, variously dependent on official policy to support and fund such activities, or on individual initiatives by research-orientated clinicians and pathologists (Parkin et al., 2001). As a result, European cancer registries differ enormously in the size of the population covered and the number of accumulated years of complete data 
available. Where regional registries do not cover the entire national population, they may not be entirely representative of the national profile of the cancer burden or patterns of risk.

Comparable, complete and accurate registry data are essential for drawing reliable inferences about geographical and temporal variations in incidence rates. The Cancer Incidence in Five Continents series is a major reference work. It was first published in 1962. The ninth volume covers new diagnoses of cancer from 1998 to 2002 in 100 registries in 29 European countries (Curado et al., 2007). Inclusion in the series is a good marker of the quality of an individual registry's data, because the editorial process includes numerous assessments of data quality.

Cancer mortality measures the impact of cancer, expressed either as the number of deaths occurring or as a rate (number of deaths per 100000 persons per year). Mortality is a product of both the incidence and the casefatality of a given cancer. Mortality rates estimate the average risk to the population of dying from a specific cancer, while fatality represents the probability that an individual with cancer will die from it. Fatality is the inverse of cancer survival - the time between the diagnosis of cancer and death. Data are derived from vital registration systems in which (usually) a medical practitioner certifies the fact of death and the date and cause of death. The International Classification of Diseases (ICD) provides a standardized system of nomenclature and coding, and a suggested format for the death certificate.

Mortality data are affected by the degree of detail and accuracy of the recorded cause of death and by the completeness of death registration. These are known to vary considerably between countries and over time. However, the available mortality data are more comprehensive than incidence data - the WHO mortality databank contains national cancer mortality data for 35 countries in Europe, available over extended periods of time for many of those countries (Fig. 2-1). The ready availability of mortality data partly explains their common application as a surrogate for incidence, particularly in the study of time trends of cancer. Yet both geographical patterns and temporal trends of cancer mortality should be interpreted cautiously where there are marked differences in survival between European populations, or where cancer prognosis has improved markedly with time.

Since the launch in 1987 of the Europe Against Cancer programme to control cancer, a number of efforts have been made to compile and publish estimates of cancer incidence and mortality in the European community and its Member States. The first of these estimated the cancer burden in 1980 and 


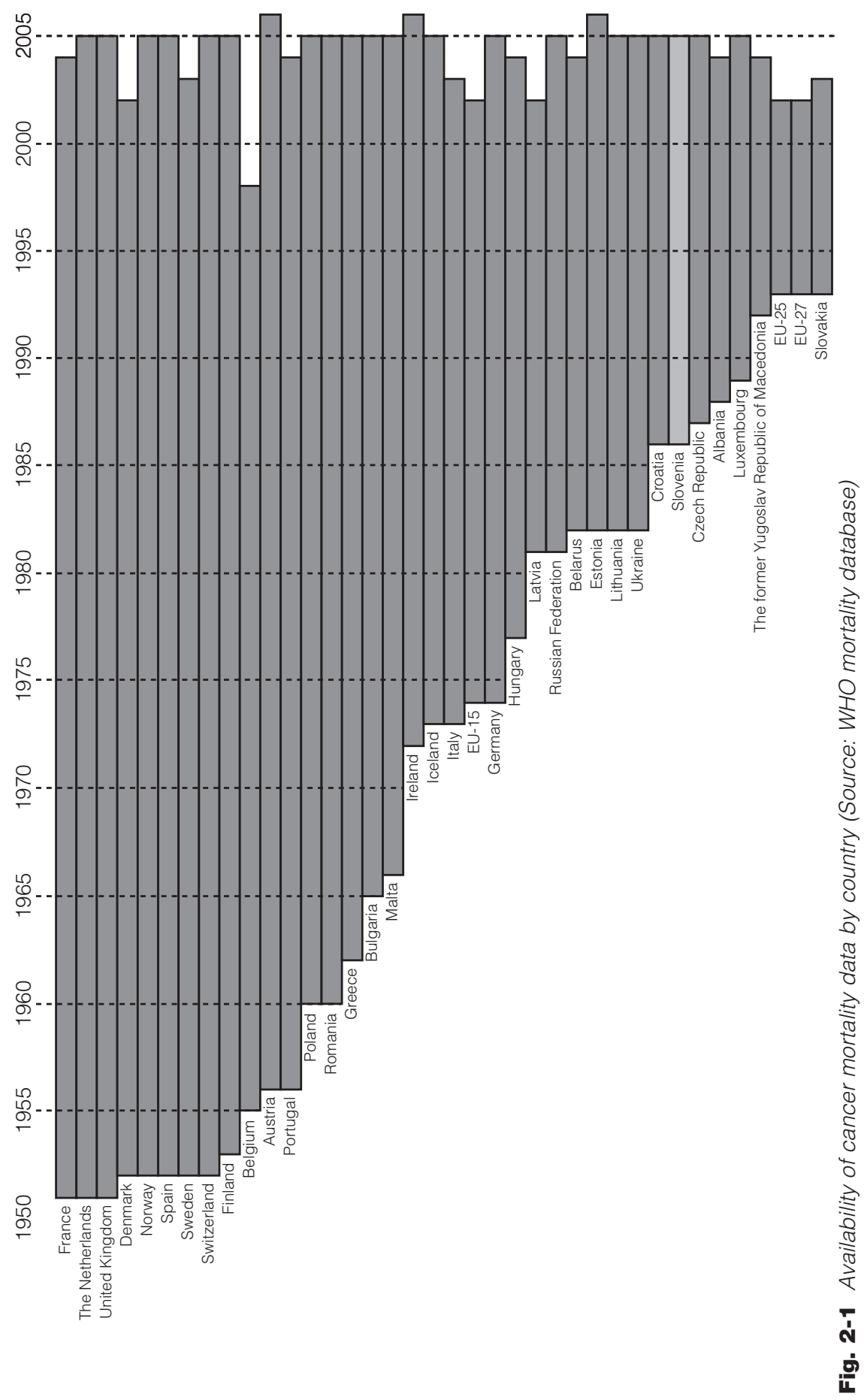


proposed a methodology for estimating national incidence by applying a ratio of the available incidence and mortality data from cancer registries to national mortality data (Jensen et al., 1990). This methodology was adopted in papers providing estimates for 1990 (Black et al., 1997) and 1995 (Bray et al., 2002). More recent methods (Ferlay et al., 2007) have incorporated a prediction model (Dyba, Hakulinen \& Paivarinta, 1997) that extrapolates recent linear trends in an attempt to improve on these estimates.

Several sources of statistical information are used to describe cancer patterns in Europe in this chapter. These include the recent article providing estimates of cancer-specific incidence and mortality in Europe for 2006 (Ferlay et al., 2007). The more detailed GLOBOCAN 2002 database (Ferlay et al., 2004; Parkin et al., 2001), a global and country-specific compilation of incidence and mortality estimates for 23 cancers in 2002, is the main source for illustrative examples of geographical and cancer-specific comparisons. Mortality data extracted from the WHO mortality databank are used to describe time trends for the most common cancers in Europe (Fig. 2-1).

Given the impact of age on cancer risk, and the phenomenon of an ageing population throughout Europe, it is vital to account for the effects of age when comparing cancer rates between populations and over time. Direct standardization procedures are used here to present age-standardized rates adjusted using the world standard population (Doll, Payne \& Waterhouse, 1966; Segi \& Kurihara, 1960) and the cumulative risk (Doll \& Smith, 1982), two summaries of the sets of age-specific rates that adjust for the effects of age. Strictly speaking, these are valid comparative indicators of risk only where the patterns of cancer risk by age are similar in the populations being compared (Day \& Charnay, 1982). Age-standardized rates may mask important variations in the direction and magnitude of age-specific trends. For example, contrasting cancer trends in younger and older populations may signal either differential effects of treatment where mortality trends are considered, or the presence of birth cohort influences (often seen more clearly for incidence). These generational effects may be related to birth itself, e.g. possible perinatal risk factors affecting the subsequent risk of testicular cancer (Garner et al., 2005). More commonly they relate to influences (risk factors) shared within the same cohort as they age, e.g. uptake and cessation of tobacco smoking and its consequences on future lung cancer rates as these cohorts age (Brown \& Kessler, 1988). Appropriate references are made to studies that have suggested important age- and cohort-specific trends.

Aggregated cancer statistics are presented at two European levels. The EU as of January 2007 and the combined territories of the 27 EU Member States is denoted by EU27; EU25 refers to the EU during the previous wave of 
enlargement. Greater Europe (or EU38) signifies the United Nations definition of Europe that contains 38 countries, and enables a useful breakdown of Europe into four designated geographical regions (Fig. 2-2).

\section{Overview of cancer burden in Europe in 2006}

According to published estimates for 2006 (Ferlay et al., 2007), there were about 2.3 million new cases of cancer and over 1 million cancer deaths in the EU25. In the continent of Europe as a whole, there were almost 3.2 million new cancer diagnoses and 1.7 million cancer deaths (Table 2-1). Men bore slightly more than half $(55 \%)$ of the total disease burden (new cases and deaths). This imbalance between the sexes is seen both in the EU25 (1.25 million cases in men and 1.04 million cases in women) and in greater Europe ( 1.7 million cases of cancer in men and 1.5 million cases in women).

Four cancers dominate the overall cancer burden profile throughout Europe. Cancers of the breast (in women), prostate, colorectum (colon and rectum combined, or large bowel) and lung accounted for over half the total cancer incidence burden in 2006. With an estimated 320000 new cases, female breast cancer was the most frequently diagnosed cancer in the EU25, closely followed by around 300000 new cases estimated for both prostate and colorectal cancer (Table 2-1). Lung cancer ranked fourth in the EU25, with an estimated 265000 new cases in 2006. In greater Europe, prostate cancer took fourth position behind breast, colorectal and lung cancer.

These four cancers in combination also explain a large proportion - around $45 \%$ - of the cancer mortality burden in the EU25. However, the ranking of cancers by frequency of death differs from the ranking for incidence, varying with the probability of death associated with each type of cancer. Lung cancer was by far the most frequent cause of cancer death in the EU25 in 2006, with an estimated 236000 deaths - one in five of all deaths from cancer (Table $2-1)$. Colorectal cancer ranked second with 140000 deaths (12\% of total cancer mortality), followed by breast cancer in women $(7.3 \%)$ and prostate cancer $(5.8 \%)$.

The ten next most frequent types of cancer account for a further $30 \%$ of the total burden of cancer incidence (and mortality). As individual cancers, they each represent some $2 \%$ to $4.5 \%$ of the total cancer burden (Table 2-1). The most frequent of these were cancers of the bladder $(4.6 \%$ of all new cancers in EU25), uterus (cervix and body of uterus combined, 3.6\%) stomach $(3.5 \%)$, oral cavity and pharynx $(3.1 \%)$, kidney $(2.8 \%)$ and nonHodgkin lymphoma (3.2\%). Stomach cancer was one of the most common cancers in the mid-twentieth century but its incidence has been declining 


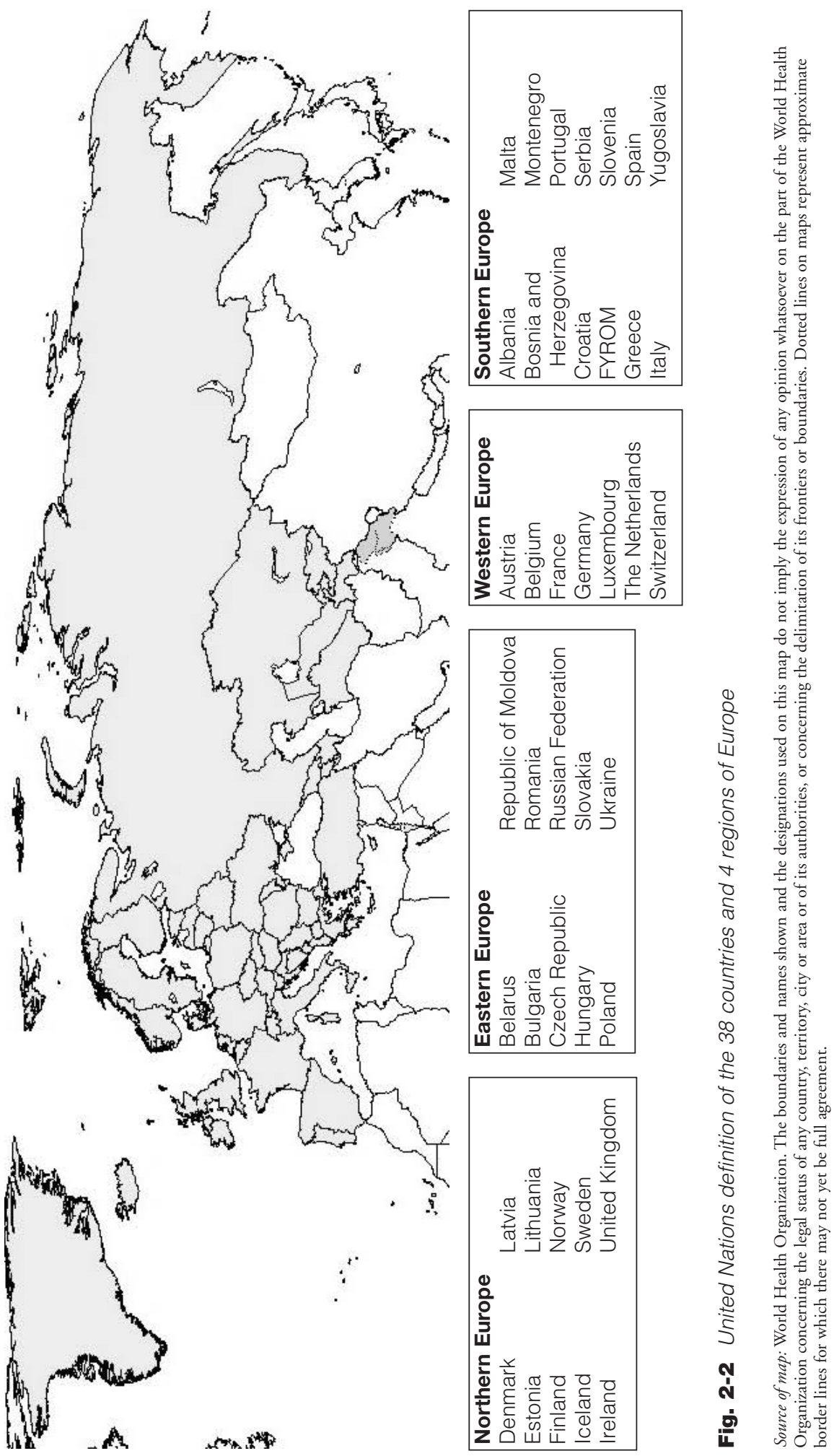




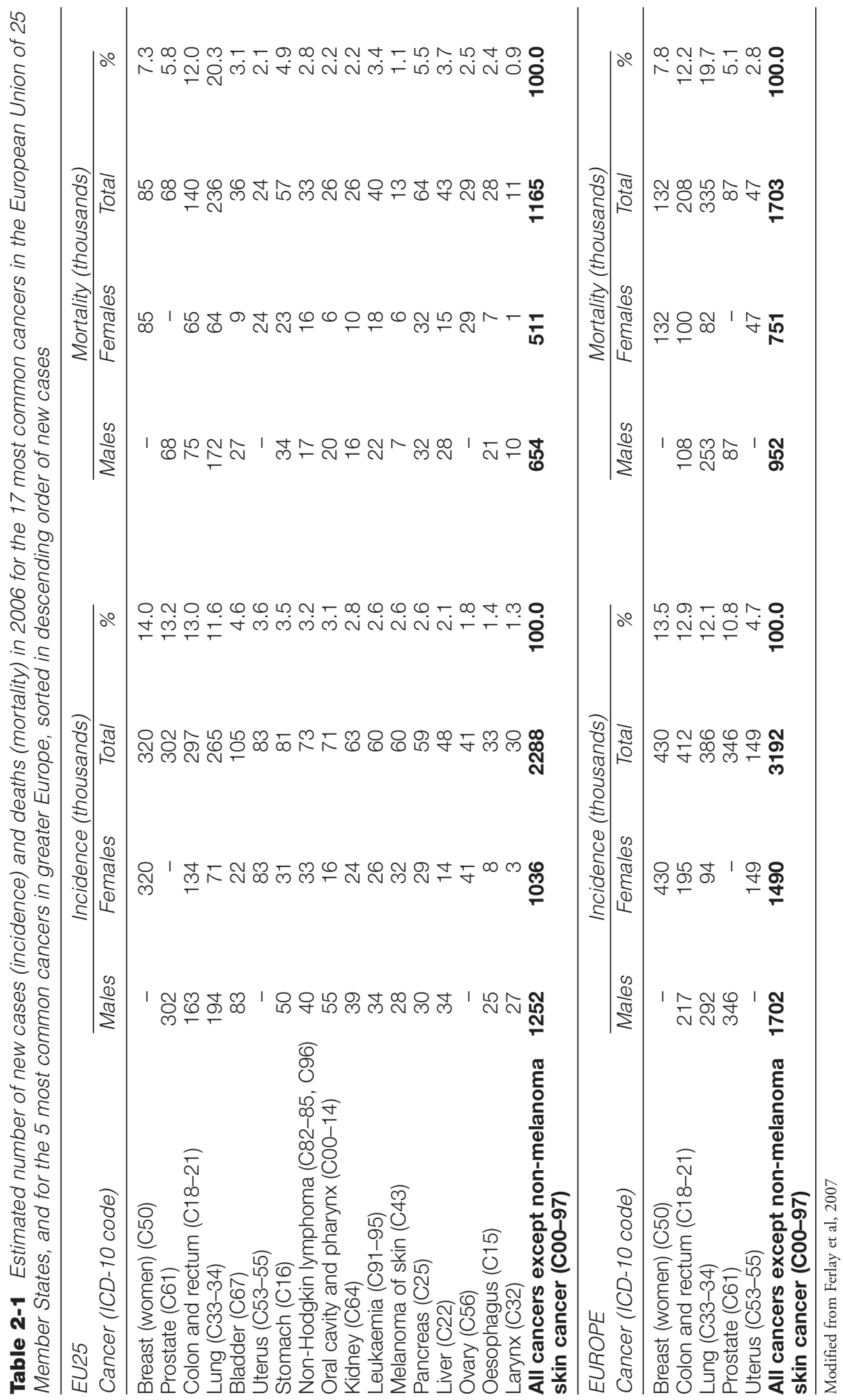


steadily for several decades and it now ranks behind bladder cancer. Pancreatic cancer is ranked thirteenth for incidence but is associated with a very poor prognosis. It is one of the leading causes of cancer death in the EU25 with an estimated 64000 deaths annually, ranking fifth behind prostate cancer.

Fig. 2-3 shows the variations in the ranking of incidence and mortality for each sex. The cancers with the highest burden among men were those of the lung, prostate, colon and rectum, stomach and bladder. In females, the principal cancers were those of the breast, colon and rectum, lung, stomach and ovary. However, the incidence of cancers of the cervix and body of the uterus equalled, or exceeded, that of ovarian cancer.

\section{Key geographical variations in cancer in Europe}

New cancer cases and cancer deaths are highest for breast, prostate, lung and colorectal cancer, but is this pattern repeated in each country and region? What are the priority cancers nationally and regionally? To what extent should cancer control policies differ between Member States and Europe as a whole? Fig. 2-4 shows the striking variations in the overall cancer rates in greater Europe around 2002. There is a two-fold range in age-adjusted incidence and mortality rates over the 38 countries of greater Europe in men, and a 1.5-fold variation in women.

Overall cancer incidence rates in men are notably higher in Hungary than in every other country in Europe. Rates are also high in several western European countries (including Belgium, France and Luxembourg) but tend to be relatively low in a number of countries in south and west Europe. Hungary has substantially higher overall cancer mortality rates in men but rates are also among the highest in other eastern European countries (including Slovakia and the Czech Republic).

In women, overall incidence is highest in Denmark and Iceland; and high in Norway, Sweden and the United Kingdom of Great Britain and Northern Ireland. As for men, the incidence rates for women are lowest in a number of southern and eastern European countries. Denmark and Hungary have the highest overall cancer mortality rates among women but mortality is also high in Ireland and the Czech Republic.

A substantial proportion of the cancer burden in Europe may be attributed to environmental causes of cancer - a vaguely defined array of dietary, social and cultural practices. The overall cancer incidence profile shown above is a composite of diagnoses of many different cancers. Assuming these estimates are reasonable descriptions of the overall cancer pattern, the variations in 


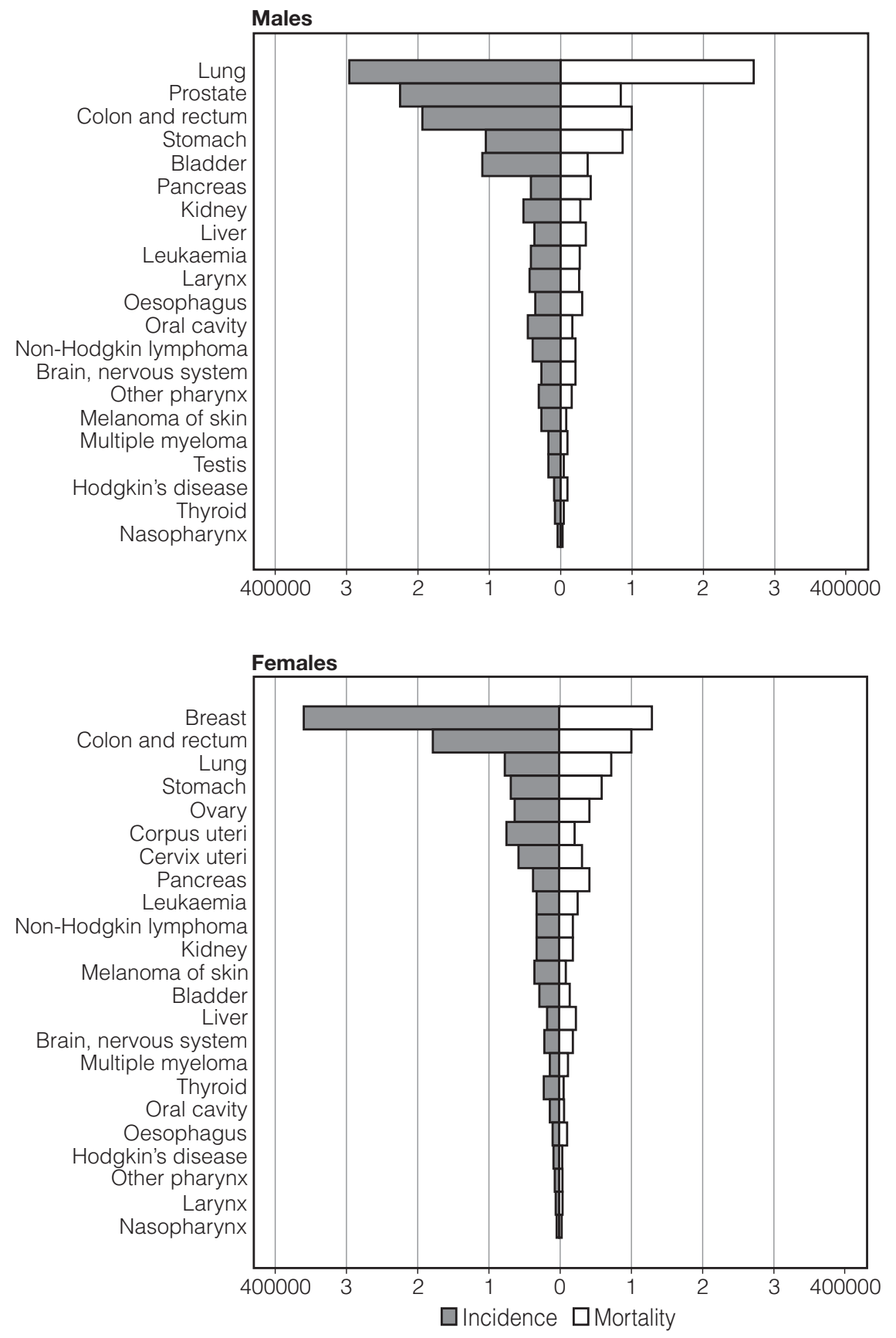

Fig. 2-3 Distribution of new cases and deaths, by sex, for 23 different cancers in Europe, 2002 (Source: Ferlay et al, 2004) 


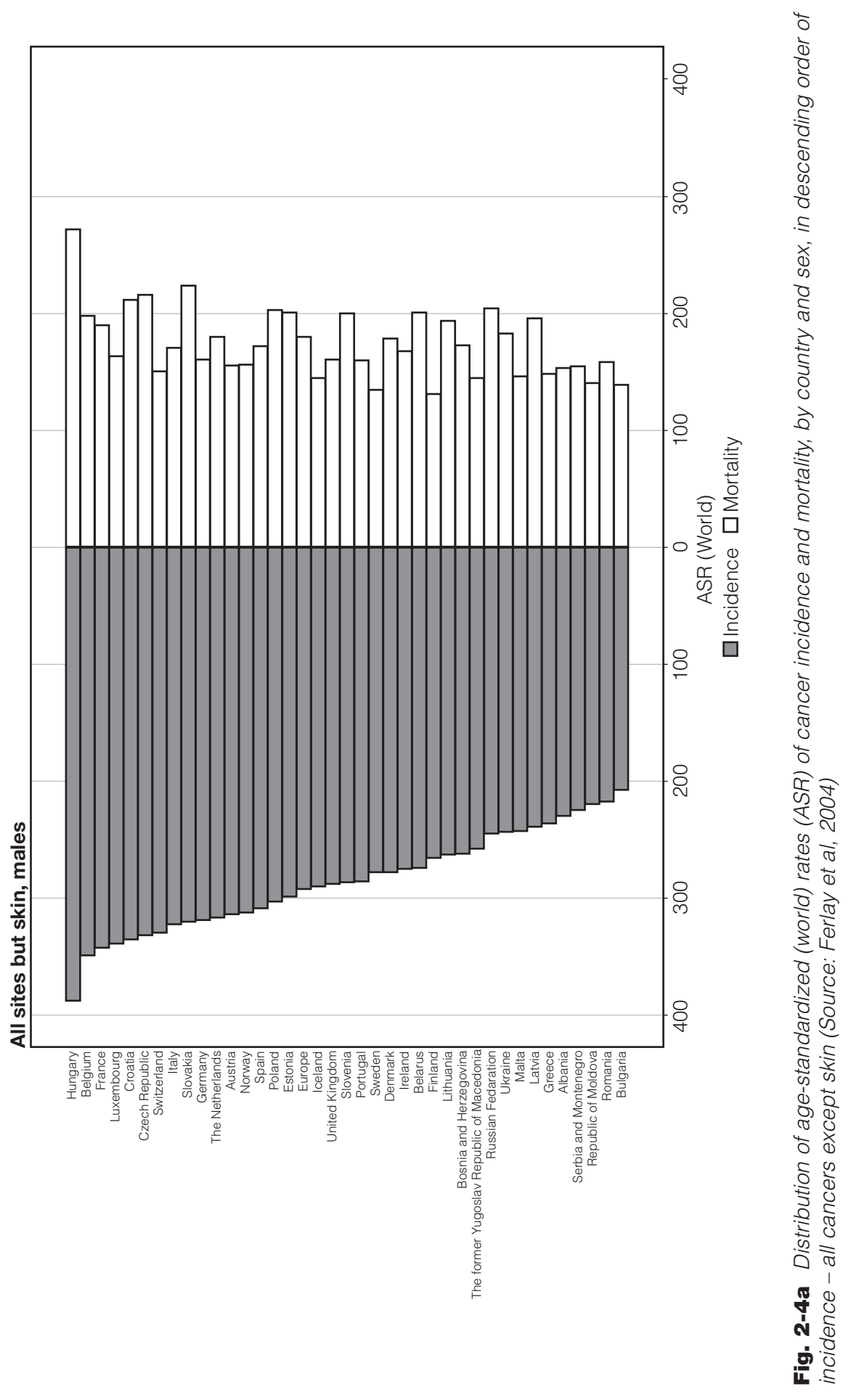




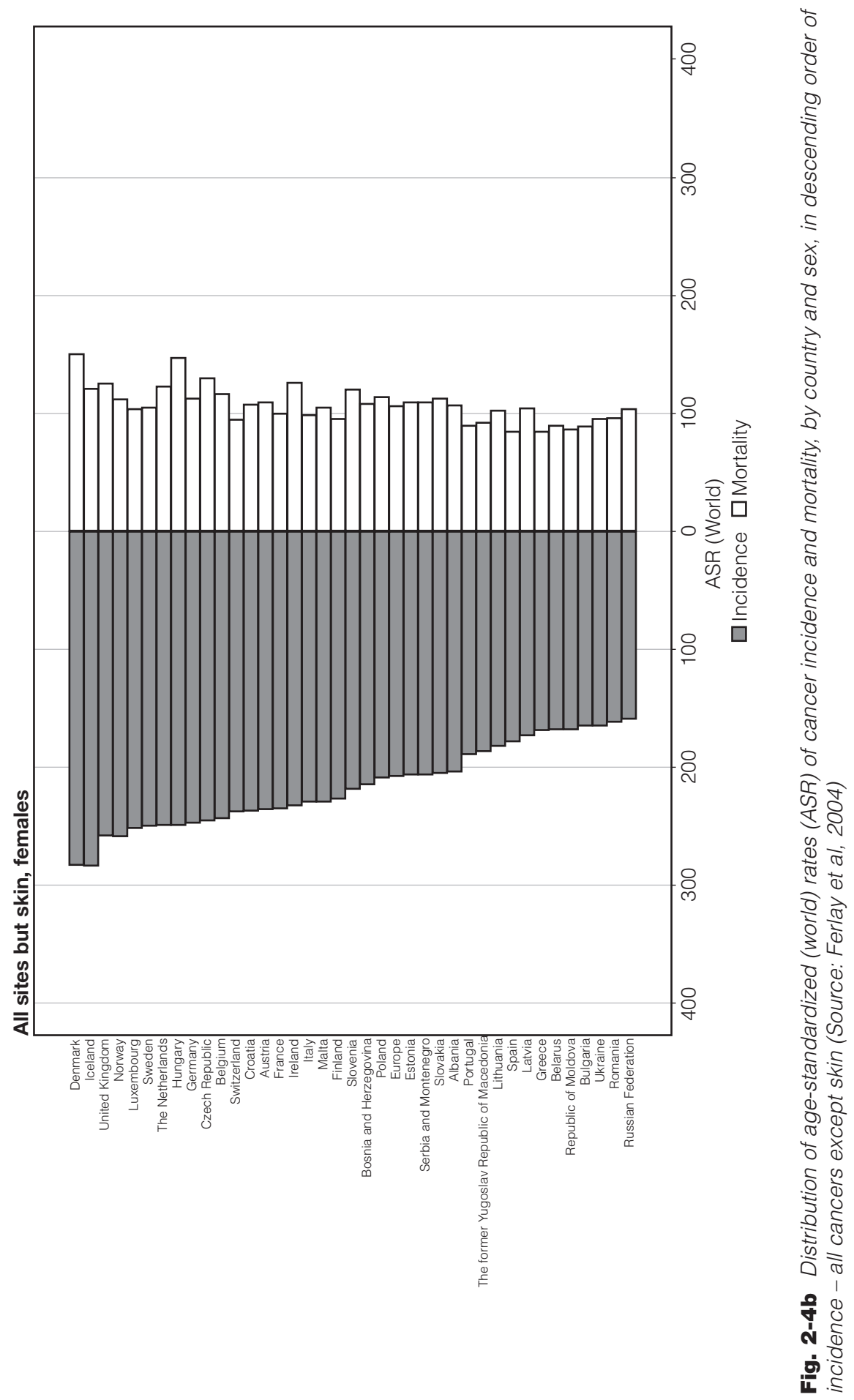


cancer incidence partially reflect underlying differences in the distribution of the determinants or risk within each country and the local effectiveness of primary prevention measures (particularly tobacco control). The rates will also reflect the availability and effectiveness of organized screening programmes aimed at reducing the occurrence of invasive cervical cancer (IARC, 2004). Rapid increases in the incidence of prostate cancer have been observed in countries that have widely adopted the prostate specific antigen (PSA) test as a diagnostic tool (Quinn \& Babb, 2002).

Cancer mortality as an indicator reflects both incidence and case fatality, and therefore depends on the availability and effectiveness of organized screening programmes aimed at early cancer diagnosis (e.g. mammography screening for breast cancer), as well as the availability and quality of cancer treatment and management at national level.

Table 2-2 provides estimates of the numbers of new cancer cases and deaths that occur in each country and their overall contribution to the European disease burden. It is noteworthy that lung cancer is the most common cancer in over half of the 38 countries in Europe (particularly eastern and southern Europe). With only one exception, it was the most common cause of cancer death in every European country around 2002. Lung cancer is responsible for at least one in five cancer deaths in the majority of European countries. Lung cancer is responsible for almost one in four cancer deaths in countries as diverse as Belgium, Poland, Greece and the United Kingdom.

The patterns of age-adjusted lung cancer incidence and mortality for males and females are depicted separately and ranked by incidence in Fig 2-5. As for all cancers combined, Hungary has the highest lung cancer rates in men, followed by Poland, Croatia and Belgium. In women, rates are high in Iceland and Denmark (seen in the rates for all cancers combined), elsewhere in northern Europe (the United Kingdom, Ireland, Norway, Sweden) and in the Netherlands, Hungary and Poland. This ranking of countries is seen in both sexes for both mortality and incidence, reflecting the generally poor and unchanging mean age-adjusted five-year relative survival from lung cancer of around $12 \%$ in Europe (Berrino et al., 2007). As the vast majority of lung cancer cases and deaths are attributable to tobacco smoking in Europe, the rates in each country reflect the current phase of the lung cancer epidemic in terms of past smoking exposure and the dose, duration and type of tobacco consumed (Gilliland \& Samet, 1994).

Breast cancer is by far the most frequent cancer in women by both incidence and mortality (Fig. 2-3). It was estimated to be the most commonly occurring cancer in 2002 for both sexes in 7 of the 38 European countries, including 
Table 2-2 Estimated number of new cancer cases and deaths, by country, greater Europe 2002

\begin{tabular}{|c|c|c|c|c|c|c|c|c|}
\hline \multirow[b]{2}{*}{ Region } & \multirow[b]{2}{*}{$\begin{array}{l}\text { Cases } \\
\text { sands) }\end{array}$} & \multirow{2}{*}{\multicolumn{2}{|c|}{$\begin{array}{r}\text { Deaths } \\
\% \text { (thousands) }\end{array}$}} & \multirow[b]{2}{*}{$\%$} & \multicolumn{2}{|c|}{ Cases } & \multicolumn{2}{|c|}{ Deaths } \\
\hline & & & & & $\begin{array}{r}\text { Most } \\
\text { common }\end{array}$ & $\begin{array}{l}\% \text { of all } \\
\text { cancers }\end{array}$ & $\begin{array}{r}\text { Most } \\
\text { common }\end{array}$ & $\begin{array}{l}\% \text { of all } \\
\text { cancers }\end{array}$ \\
\hline Europe & 2821 & 100.0 & 1701 & 100.0 & Lung & 12.4 & Lung & 17.5 \\
\hline Eastern Europe & 903 & 32.0 & 637 & 37.4 & Lung & 15.9 & Lung & 20.6 \\
\hline Belarus & 30 & 1.1 & 20 & 1.2 & Lung & 14.1 & Lung & 19.7 \\
\hline Bulgaria & 24 & 0.8 & 15 & 0.9 & Lung & 13.5 & Lung & 19.2 \\
\hline Czech Republic & 47 & 1.7 & 29 & 1.7 & Colorectal & 16.3 & Lung & 20.3 \\
\hline Hungary & 49 & 1.7 & 33 & 2.0 & Lung & 17.8 & Lung & 23.6 \\
\hline Poland & 135 & 4.8 & 85 & 5.0 & Lung & 17.8 & Lung & 23.9 \\
\hline Republic of Moldova & 9 & 0.3 & 5 & 0.3 & Breast & 14.4 & Lung & 16.3 \\
\hline Romania & 60 & 2.1 & 42 & 2.4 & Lung & 15.1 & Lung & 20.7 \\
\hline Russian Federation & 388 & 13.7 & 297 & 17.5 & Lung & 18.7 & Lung & 20.7 \\
\hline Slovakia & 19 & 0.7 & 12 & 0.7 & Colorectal & 15.9 & Lung & 18.8 \\
\hline Ukraine & 141 & 5.0 & 97 & 5.7 & Lung & 15.1 & Lung & 19.3 \\
\hline Northern Europe & 426 & 15.1 & 241 & 14.2 & Lung & 13.0 & Lung & 18.3 \\
\hline Denmark & 25 & 0.9 & 16 & 0.9 & Breast & 15.4 & Lung & 22.1 \\
\hline Estonia & 5 & 0.2 & 3 & 0.2 & Lung & 14.0 & Lung & 20.3 \\
\hline Finland & 21 & 0.7 & 10 & 0.6 & Breast & 17.1 & Lung & 18.2 \\
\hline Iceland & 1 & 0.0 & 1 & 0.0 & Breast & 15.1 & Lung & 20.3 \\
\hline Ireland & 13 & 0.5 & 8 & 0.5 & Colorectal & 14.4 & Lung & 19.3 \\
\hline Latvia & 8 & 0.3 & 6 & 0.3 & Lung & 14.3 & Lung & 19.1 \\
\hline Lithuania & 12 & 0.4 & 8 & 0.5 & Lung & 13.1 & Lung & 18.7 \\
\hline Norway & 21 & 0.7 & 11 & 0.6 & Colorectal & 15.6 & Lung & 16.6 \\
\hline Sweden & 43 & 1.5 & 22 & 1.3 & Prostate & 18.4 & Lung & 14.4 \\
\hline United Kingdom & 277 & 9.8 & 156 & 9.2 & Breast & 14.8 & Lung & 22.6 \\
\hline Southern Europe & 617 & 21.9 & 348 & 20.5 & Lung & 13.0 & Lung & 20.3 \\
\hline Albania & 6 & 0.2 & 4 & 0.2 & Lung & 16.1 & Lung & 21.9 \\
\hline Bosnia and Herzegovina & 12 & 0.4 & 7 & 0.4 & Lung & 15.5 & Lung & 21.4 \\
\hline Croatia & 21 & 0.7 & 12 & 0.7 & Lung & 15.3 & Lung & 22.3 \\
\hline Greece & 39 & 1.4 & 24 & 1.4 & Lung & 16.5 & Lung & 23.8 \\
\hline Italy & 292 & 10.4 & 156 & 9.2 & Colorectal & 12.9 & Lung & 21.0 \\
\hline Malta & 2 & 0.0 & 1 & 0.0 & Breast & 16.8 & Lung & 16.8 \\
\hline Portugal & 38 & 1.3 & 21 & 1.3 & Colorectal & 13.2 & Colorectal & 13.7 \\
\hline Serbia and Montenegro & 32 & 1.1 & 21 & 1.2 & Lung & 17.0 & Lung & 21.5 \\
\hline Slovenia & 8 & 0.3 & 5 & 0.3 & Colorectal & 14.7 & Lung & 19.1 \\
\hline Spain & 162 & 5.7 & 93 & 5.5 & Colorectal & 13.6 & Lung & 19.4 \\
\hline $\begin{array}{l}\text { The former Yugoslav } \\
\text { Republic of Macedonia }\end{array}$ & 6 & 0.2 & 3 & 0.2 & Lung & 13.3 & Lung & 20.3 \\
\hline Western Europe & 874 & 31.0 & 475 & 27.9 & Breast & 14.4 & Lung & 18.7 \\
\hline Austria & 37 & 1.3 & 19 & 1.1 & Colorectal & 14.1 & Lung & 17.5 \\
\hline Belgium & 52 & 1.8 & 30 & 1.8 & Lung & 14.9 & Lung & 24.0 \\
\hline France & 269 & 9.5 & 149 & 8.8 & Breast & 15.6 & Lung & 17.6 \\
\hline Germany & 408 & 14.5 & 218 & 12.8 & Colorectal & 15.6 & Lung & 18.1 \\
\hline Luxembourg & 2 & 0.1 & 1 & 0.1 & Colorectal & 14.0 & Lung & 21.7 \\
\hline The Netherlands & 70 & 2.5 & 40 & 2.3 & Lung & 15.0 & Lung & 23.2 \\
\hline Switzerland & 35 & 1.3 & 17 & 1.0 & Prostate & 14.5 & Lung & 17.9 \\
\hline
\end{tabular}

Source: Ferlay et al, 2004 


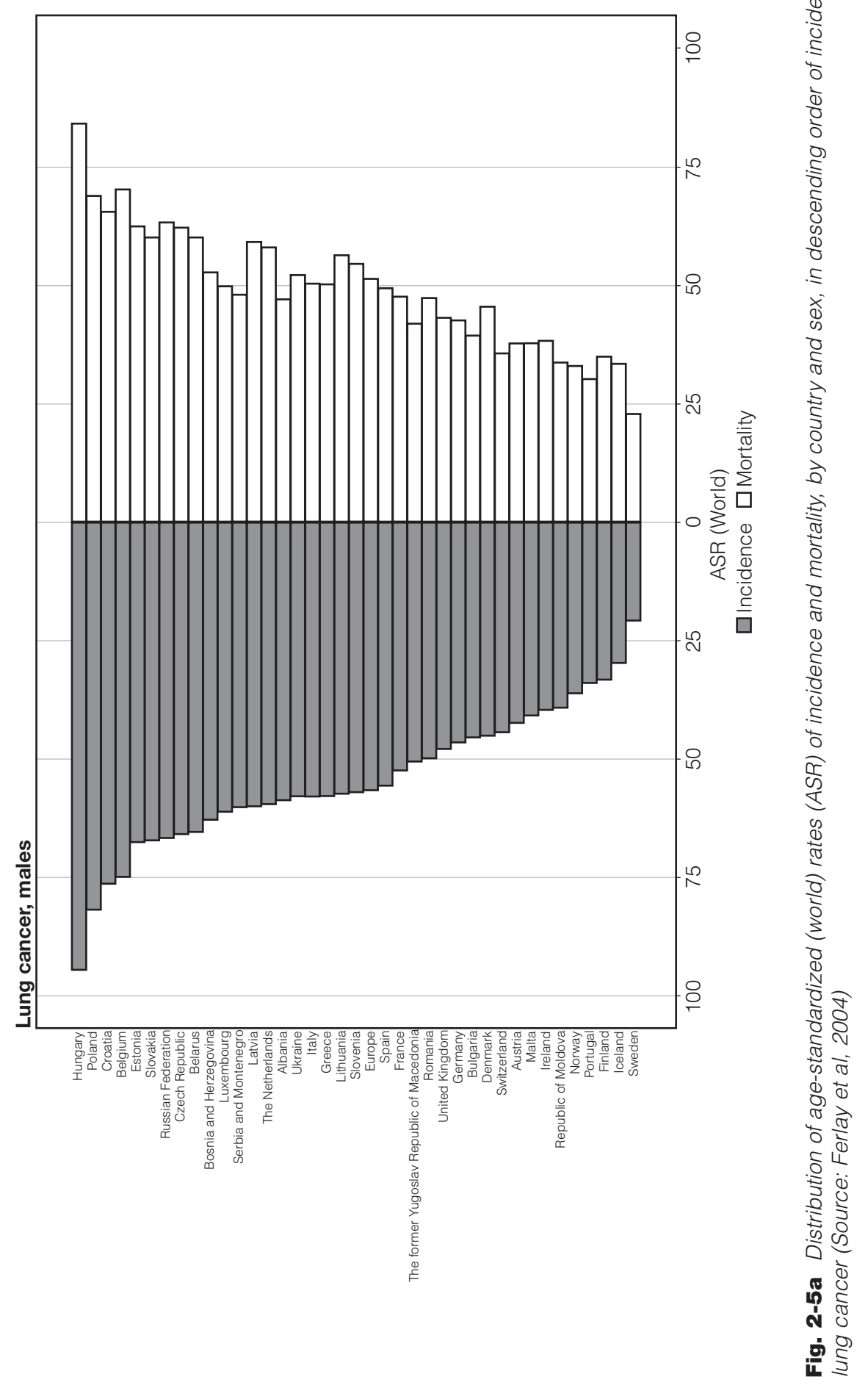




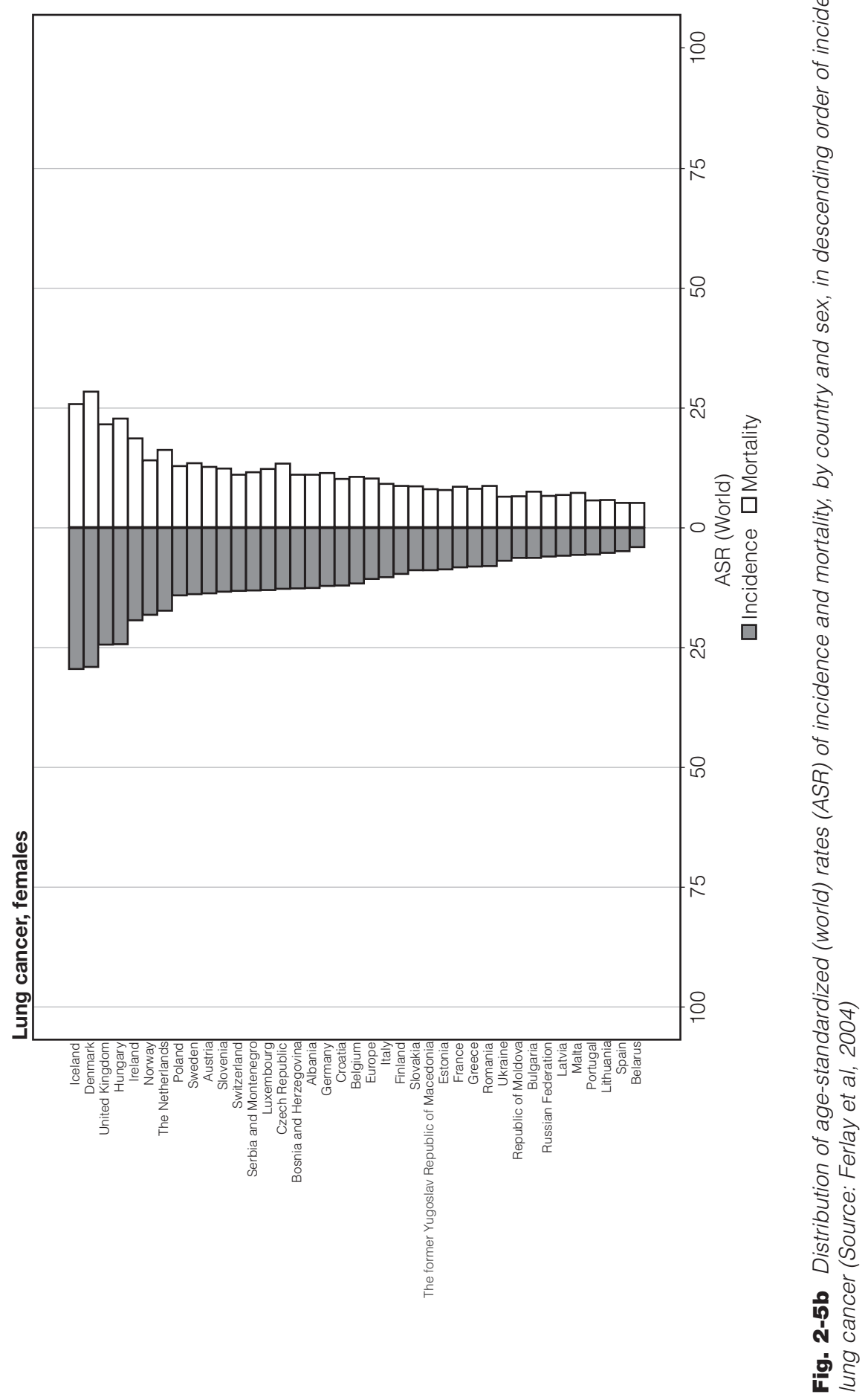


Denmark, Finland, France and the United Kingdom (Table 2-2). Incidence rates are generally higher in northern and western Europe and relatively low in most eastern European countries (Fig. 2-6a). They may partly reflect the differing national and regional prevalence of risk factors associated with affluence and socioeconomic status including parity, age at menstruation and menopause, obesity, height and alcohol consumption. Some of the excess incidence may be attributable to the time-varying implementation of mammography screening programmes in certain high-resource countries within Europe.

There is much less variation in breast cancer mortality and the death rates are not strongly correlated with incidence rates, although the incidence-tomortality ratios in eastern Europe tend to be less favourable than those in northern Europe. These variations probably reflect a combination of east-west differences in breast cancer incidence and (particularly in some higherresource countries) reductions in breast cancer mortality produced by the collective impact of the introduction of mammography screening programmes and better treatment regimens for node-positive disease (Botha et al., 2003; Bray, McCarron \& Parkin, 2004; IARC, 2002b).

Prostate cancer was the second most common cancer in European men in 2002, and the most common cause of cancer overall in Sweden and Switzerland (Table 2-2). There is at least a seven-fold variation in prostate cancer incidence in Europe (Fig. 2-6b). The high rates in many northern and western countries largely reflect the diagnosis of latent cancers in asymptomatic individuals screened by the PSA test. Prostate cancer mortality is less affected by early diagnosis of asymptomatic cancers, and a major focus of interest is the extent to which mortality has been affected by early diagnosis and improved treatment. Some correlation between prostate cancer incidence and mortality is apparent but it remains a possibility that a differing distribution of underlying risk factors could explain part of the variability. This may reflect differentials in survival linked to resource levels, as more latent cancers are detected by screening procedures. The underlying causes of this disease remain elusive (Gronberg, 2003; Signorello \& Adami, 2002).

Colorectal cancer was estimated to be the most common cancer in 11 of the 38 countries of Europe in 2002 including the Czech Republic, Slovakia, Ireland, Norway, Italy and Germany. It was the most common cause of death from cancer in Portugal. Colorectal cancer incidence and mortality rates are highest among men in the Czech Republic, Hungary and Slovakia. Among females, incidence rates are also high in Norway, Germany and Denmark (Fig. 2-7). Migrants from low- to high-risk countries acquire the higher colorectal 


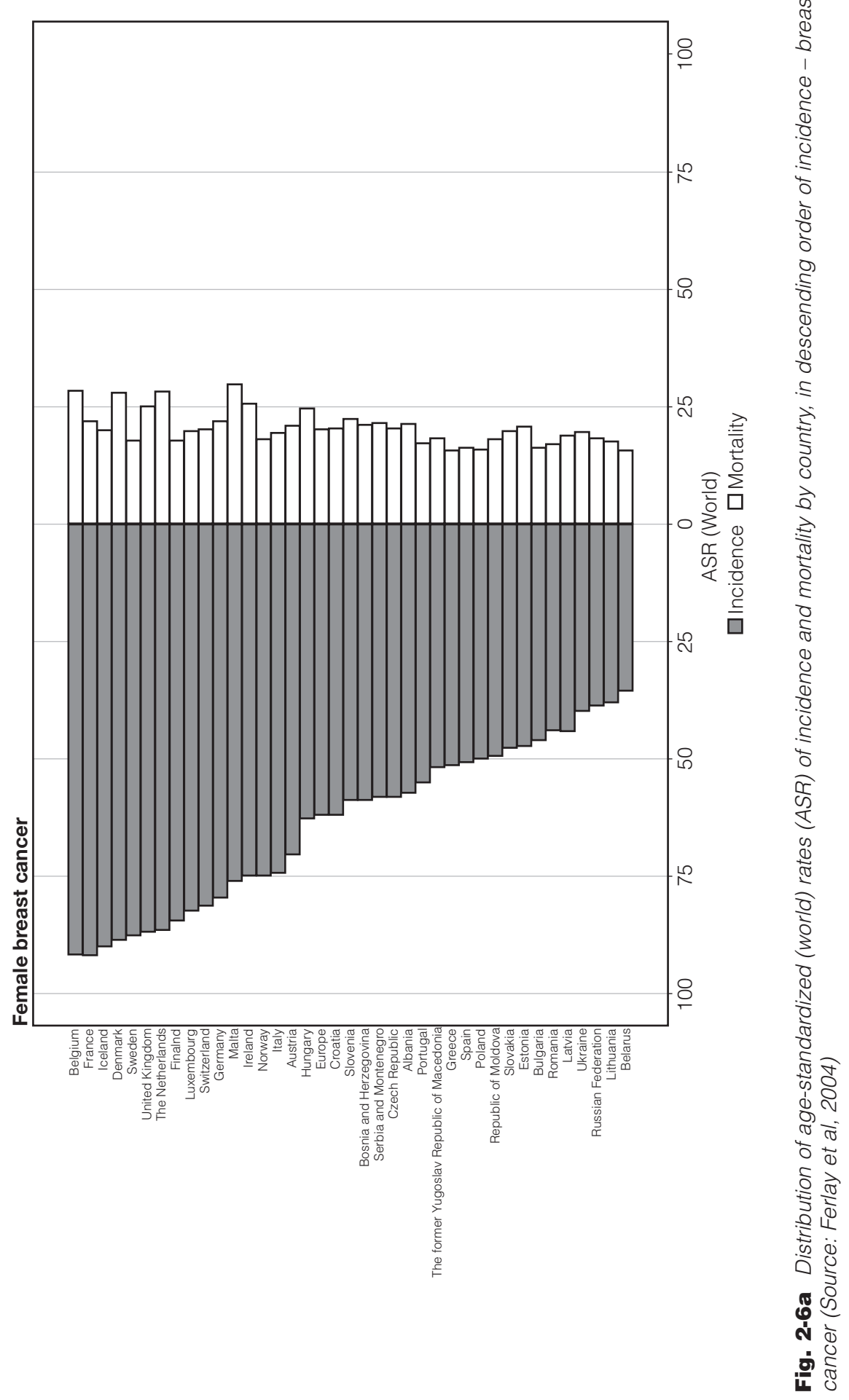




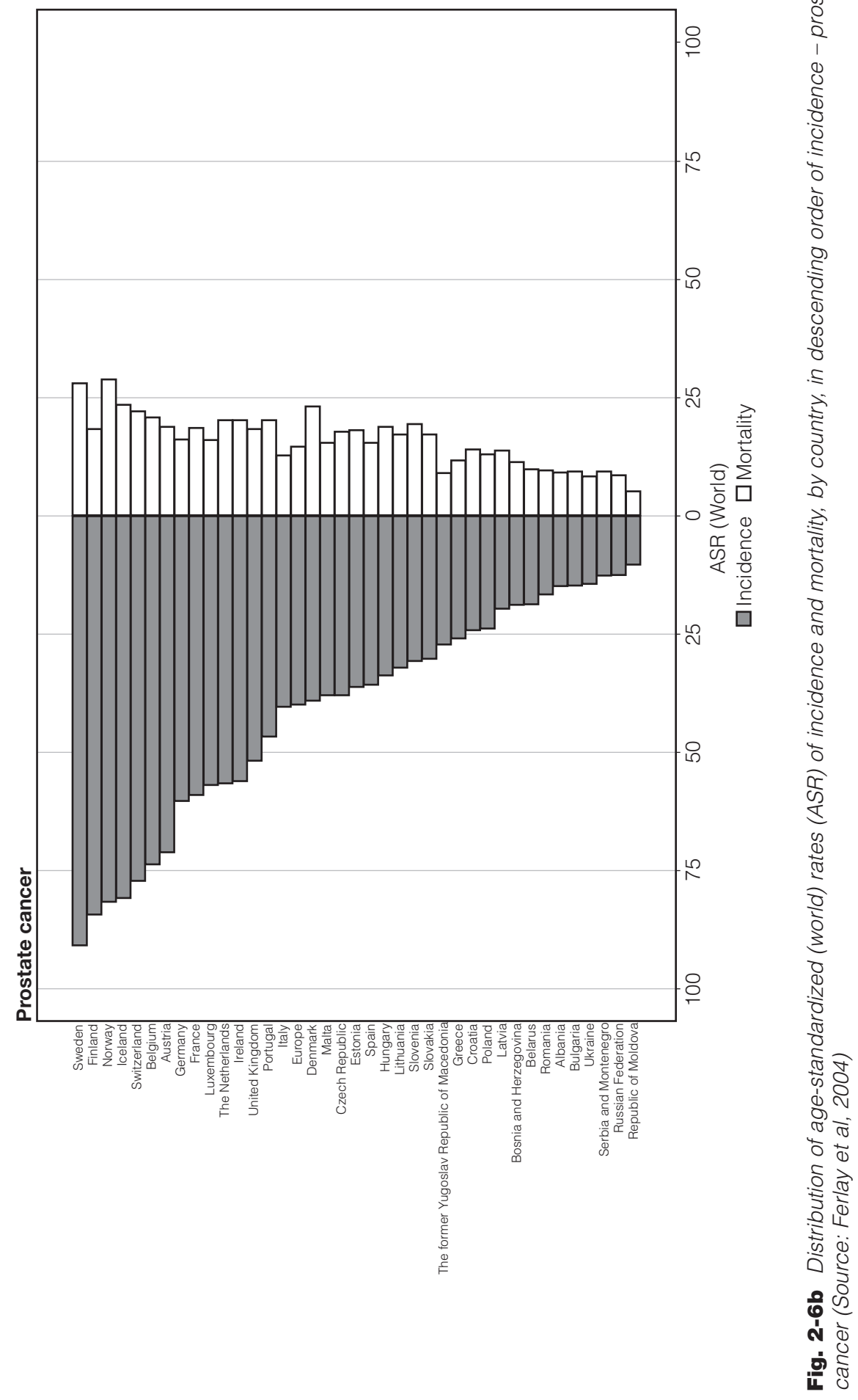




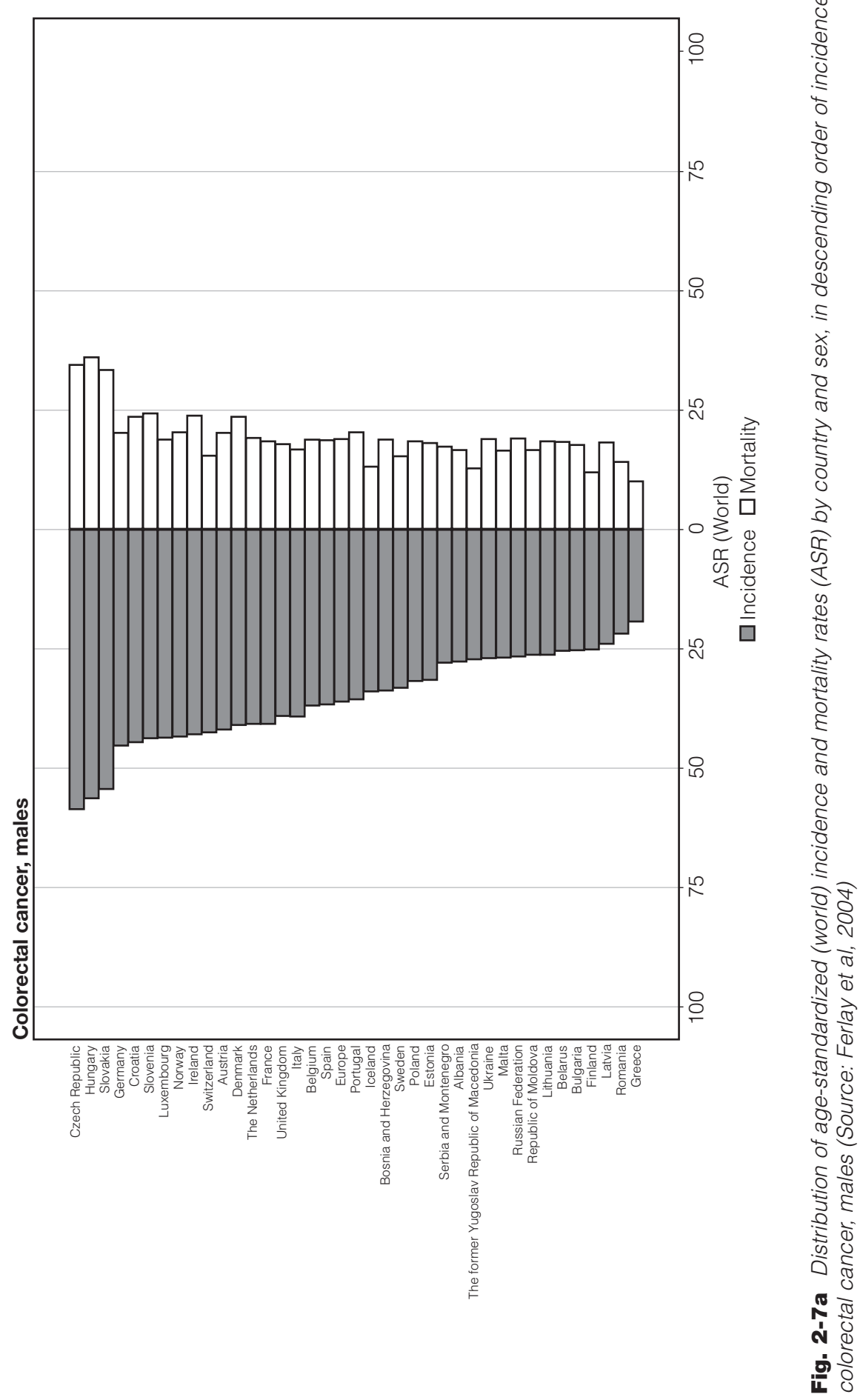




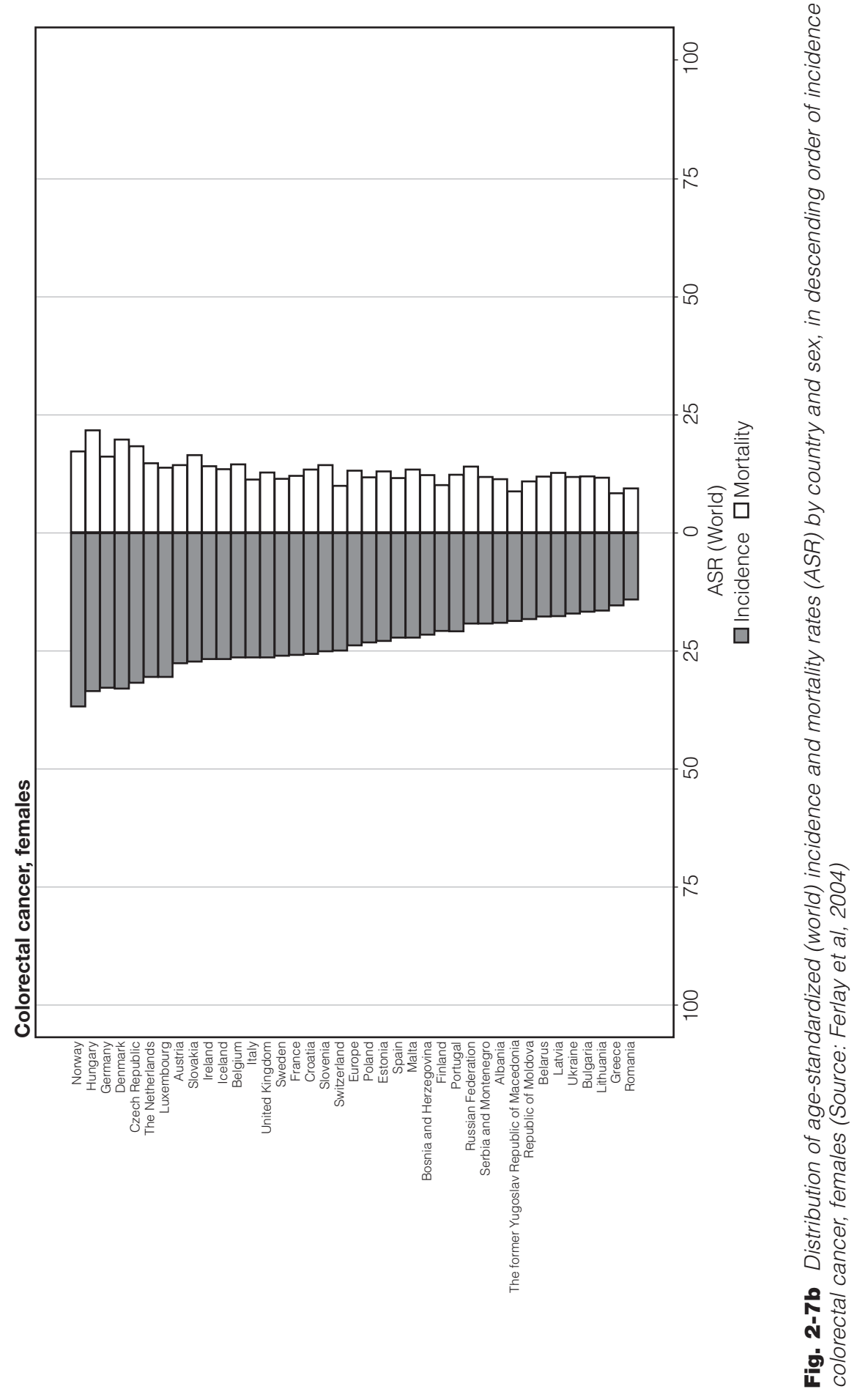


cancer incidence of the host country within a single generation. This suggests a major influence of lifestyle factors (such as diet) in the causation of colorectal cancer, including during adult life. There are consistent associations between an increased risk of colorectal cancer and a high intake of red and processed meat; a high body mass index ${ }^{1}$ (BMI) and obesity; and a sedentary lifestyle. Similarly, the protective effects of high levels of vegetable consumption are observed consistently (IARC, 2003; IARC, 2002a; Norat et al., 2005; Pischon et al., 2006; Potter \& Hunter, 2002).

The overall cancer incidence and mortality rates in Fig. 2-4 largely capture the cumulative burden imposed by the most frequently occurring and mortalitycausing cancers in Europe, as described above. The high overall cancer rates among Hungarian men partially reflect their high rates of lung and colorectal cancer. Rates in France and Belgium partly mirror the high incidence of cancers of the lung and prostate; many of the PSA-detected prostate cancers would be latent rather than overt clinical cancers. In women, the all-cancer incidence and mortality rates reflect the breast cancer rates, as well as those of other common cancers. The high overall cancer incidence rates in Denmark, Iceland and the United Kingdom reflect the high rates of breast and lung cancer in these populations.

\section{Key temporal variations in cancer in Europe}

The trends in cancer mortality are examined for each of 23 countries and 4 regions of Europe - for all cancers combined and for the four most common causes of cancer death. The relative merits and complexities of interpreting time trends in either cancer incidence or cancer mortality have been much debated, but mortality (rather than incidence) trends are used for simplicity and because national mortality data are available for more countries and over longer time spans.

Mortality trends depend on the accuracy of information about the cause of death recorded on the death certificate: this may differ between countries or change over time. Mortality trends are also rather poor surrogates for trends in cancer risk (i.e. cancer incidence) particularly where there have been improvements in outcomes (decreasing case fatality) over time. Trends in cancer mortality should therefore be interpreted and compared cautiously. It is usually considered that the joint description of cancer incidence, mortality and survival serves best to confirm and clarify understanding of the complex changes in cancer over time (Boyle, 1989; Dickman \& Adami, 2006). Informative trends and patterns in these other indicators will be mentioned below.

\footnotetext{
${ }^{1}$ Weight in kilograms divided by the square of the height in metres.
} 
It is evident that the all-cancer mortality trends in men vary considerably between populations (Fig. 2-8a, colour section). Following a sustained period of increasing mortality, declines are now being observed in almost all European countries. The year of peak cancer mortality in men varies by country and region. The declines are reasonably uniform in western Europe, starting as early as the mid-1960s in Austria but only since the early 1990s in Germany. Declines are more recent in much of eastern Europe, starting around the mid-1990s, although a continuing increase is clearly seen in Romania. Trends in the southern European countries of Greece and Portugal are stable.

The overall cancer mortality trends in men partly reflect the course of lung cancer mortality, given its heavy burden and high death toll. For example, Hungary and Finland are countries in differing phases of the smoking epidemic (Fig. 2-9a, colour section) and the all-cancer mortality rates among men are clearly heavily influenced by mortality from this neoplasm.

All-cancer mortality rates are lower in women than in men, and recent time trends tend to be favourable. This is most evident in western Europe (declines over 40 years) but similar trends are seen elsewhere, most notably in Finland. Cancer mortality rates are decreasing in northern and southern Europe, but the downturn is rather recent (Fig. 2-8b, colour section). Death rates among women in the United Kingdom began to decline rapidly from the late1980s; the smaller decrease in Denmark began in the mid-1990s. In eastern Europe, the trends in all-cancer mortality in women are more variable - stable in Poland, but (as in men) increasing in Romania. The all-cancer mortality rates are a composite - for instance, the major decline in cancer mortality in British women reflects declines in breast, lung and colorectal cancer mortality. Conversely, the increase among Romanian women reflects uniform increases in death rates from all three cancers.

The prognosis for lung cancer has been consistently poor for decades, so trends in the mortality rates in men and women provide a good indicator of the changing risk of developing the disease in each population (Fig. 2-9a, 2-9b (colour section) and 2-10). In turn, these trends relate closely to the tobacco-smoking habits of successive generations (birth cohorts) (Brown \& Kessler, 1988). Those European countries in which smoking was first established were the first to see a drop in smoking prevalence. Some decades later, in the same generations of men, this was followed by a decline in both incidence and mortality from lung cancer. Changes were first seen among younger age groups - a decline in overall rates was observed as these generations of men reached the older ages at which lung cancer is most common. The United Kingdom was the first to show a decline in rates (mid- 
1970s) followed closely by Finland and, more recently, Sweden, Denmark and most other western European countries. Recent declines or plateaux in lung cancer mortality were also observed during the 1990s. Romania is one possible exception - lung cancer mortality rates are increasing steadily among males.

European women generally acquired the smoking habit more recently. In contrast to men, lung cancer mortality is rising still in many European countries, most notably in southern and eastern Europe. Lung cancer rates in Spanish women have historically been very low but the decline during the 1970s and 1980s may have resulted from lower exposure to environmental tobacco smoke at home following a significant reduction in the prevalence of smoking among men (Brown \& Kessler, 1988). However, rising mortality among younger generations (born since the early 1940s) accords with the rise in smoking prevalence among Spanish women (Brown \& Kessler, 1988).

Lung cancer rates in Spanish women can be represented as being at an early stage of the epidemic (Lopez et al., 1994) (Fig. 2-10). On the basis of recent cohort trends and population forecasts, it is likely that the death rates will increase substantially over the next few decades, as the lung cancer epidemic matures and the population ages (Fig. 2-11).

It is more encouraging that upward trends in lung cancer rates in women are flattening in some countries, including the United Kingdom and (more recently) Denmark. In Lithuania, the Russian Federation and Ukraine, lung cancer death rates already appear to be falling. Given the substantial and lasting influence that cigarette consumption will impose on the direction and magnitude of future lung cancer incidence and mortality rates, it remains essential to monitor trends in the age- and sex-specific smoking prevalence in the countries and regions of Europe. A recent compilation of trends in the proportion of male and female smokers in ten Member States (Fig. 2-12) is encouraging (downward trends in both sexes in most countries). However, there is a clear warning of the emerging lung cancer epidemic in French and Spanish women (see box in Fig. 2-12), given the recent increases in smoking prevalence.

Time trends in breast cancer mortality reflect both the trends in incidence (and its determinants) and the impact of early diagnosis - either through screening or from increasing individual awareness of the disease and its symptoms. In the higher-resourced European countries, recent advances in breast cancer therapy have contributed greatly to improved survival and a subsequent reduction or stabilization of mortality rates. This can be seen clearly in the Netherlands, Switzerland and the United Kingdom. Less emphatic declines in breast cancer mortality can be seen in most countries in the last decade (Fig. 


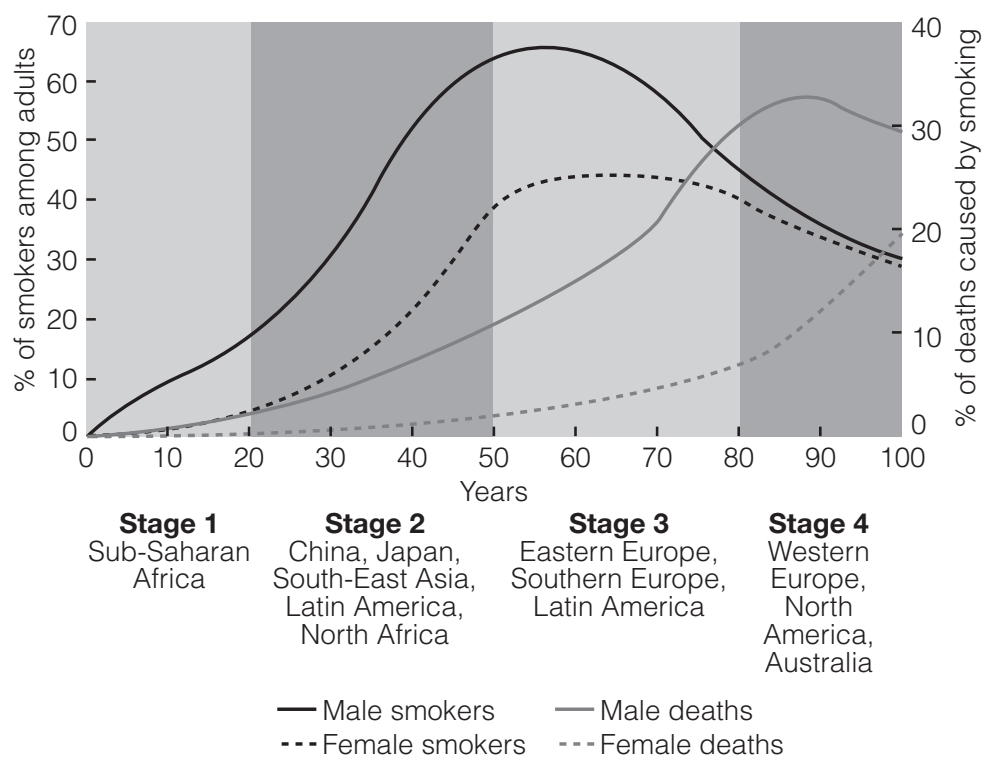

Fig. 2-10 Stages of worldwide tobacco epidemic (Source: Edwards, 2004; adapted from Lopez, Collishaw \& Piha, 1994)

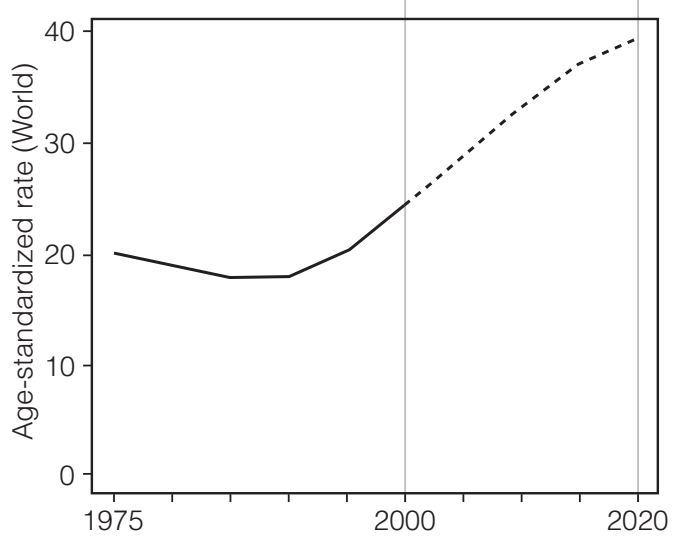

Fig. 2-11 Female lung cancer mortality, Spain 1974-2003 (solid line) and predicted (dashed line) until 2020, based on age-period-cohort model (Source: Nordpred, Møller et al, 2003)

2-13, colour section) and even in several countries without national screening programmes. Breast cancer mortality still appears to be increasing in Romania and Lithuania.

Mortality from colorectal cancer is increasing rapidly in men in most southern and eastern European countries. Rates have stabilized in the high-risk Czech Republic and intermediate-risk Italy (Fig. 2-14a, colour section). Rates have 


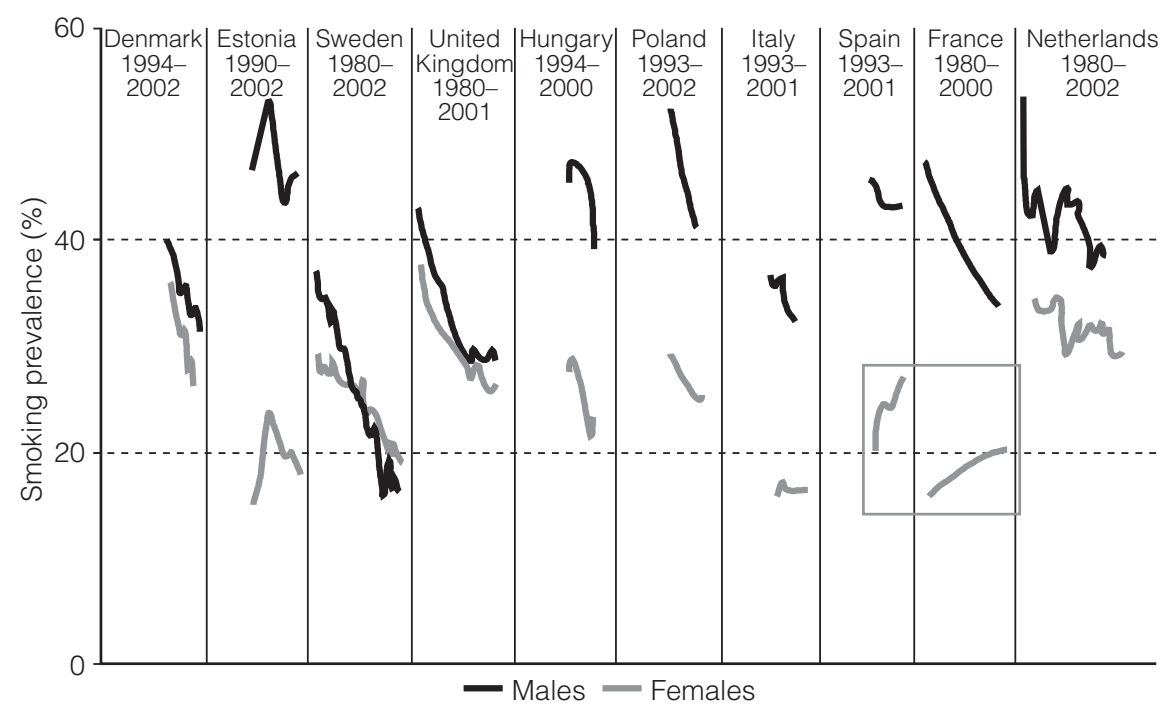

Fig. 2-12 Trends in smoking prevalence in the EU 1979-2002 (Source: Cancer Research UK, EU Factsheet, 2004)

been falling since the 1970s in most western and northern European countries, except in Lithuania and Norway where colorectal cancer mortality appears to have stabilized. The temporal patterns are quite similar in European women, although the rates tend to be slightly lower (Fig. 2-14b, colour section). These changes are due to many different factors, including changes in incidence and general improvement in the results of treatment, but they are unlikely to be due to improved early detection in screening examinations. The principal cause of the increased risk in eastern European countries may be related to the westernization of modes of life, particularly diet. Conversely, some improvements in the quality of diet in younger generations may explain the cohort effects in incidence recently observed in Sweden and the United Kingdom, with decreasing incidence rates among younger age groups (Swerdlow, dos Santos Silva \& Doll, 2001; Thorn et al., 1998).

Prostate cancer mortality rates in most European countries increased between the 1960s and the 1980s. They appear to have been falling since the 1990s in many countries, including Spain and Italy in the south; Finland, Norway and the United Kingdom in the north; and all six western European countries illustrated in Fig. 2-15 (colour section). It is not clear to what extent the increasing trends in prostate cancer mortality up to the 1980s reflect genuine increases in risk (incidence). Certainly, from the late 1980s, much of the rapid increase in incidence seen in many western and northern European countries is due to detection of latent disease through PSA testing. The burning 
question is the extent to which advances in treatment, in combination with PSA-related early detection, have been responsible for the declines in prostate cancer mortality. While some of the more substantial declines have occurred in countries where PSA testing became widespread in the early to mid-1990s (e.g. Finland and Norway), the downturns in mortality appear to have occurred earlier than would be predicted from mean estimates of lead time. Similar declines in mortality have been observed where screening activity has been less marked (Italy, Spain, United Kingdom) (Fig. 2-15, colour section).

Combined investigation of cancer- and age-specific data on incidence, mortality and survival is needed to understand better the underlying determinants of time trends (Dickman \& Adami, 2006). However, all-cancer mortality trends have been used most widely as a marker of progress against cancer in recent times. Some consider the direction of these trends to be the indicators of progress, pointing towards success or failure in controlling cancer. In the 1980s and early 1990s, several American and European commentators drew rather pessimistic conclusions (given the unfavourable trends in overall cancer mortality up to that point) and recommended that cancer control strategies should be redirected towards prevention and effective screening, with less emphasis on treatment-focused approaches (Bailar \& Smith, 1986; Becker, Smith \& Wahrendorf, 1989; Geddes, Balzi \& Tomatis, 1994).

However, the use of all-cancer mortality trends to evaluate progress in cancer control has been heavily criticized. This is partly because this measure is dominated by cancer risk in the elderly and the "prevalence of carcinogenic agents in the distant past, which are irrelevant" (Doll, 1990), rather than reflecting recent progress - including therapeutic improvements and reductions in risk among young people. Further, trends in all-cancer mortality rates are a composite of trends in many cancer types. These differ widely both in incidence and in outcome, as well as in their amenability to primary prevention, screening and treatment - in short, all the strategies that underpin cancer control.

It is interesting to compare cancer mortality trends up to the mid-1980s - a time when effective treatments had only recently been introduced - with more recent trends. Despite the caveats about using such an indicator, this indicates a more positive view of recent trends in all-cancer mortality in the 23 countries examined here. It is clear that primary prevention, secondary prevention (screening) and improvements in cancer treatment and care have all played important roles. It is also obvious that the degree of success varies widely between European countries and regions. The trends are perhaps more favourable for men - death rates for lung and other smoking-related cancers 
are falling as the tobacco epidemic reaches a mature phase in many countries. Women's tobacco habits are at an earlier phase and lung cancer rates are still increasing in most countries, though there are signs of a plateau in the rates in a few countries. Breast cancer mortality rates in women have been falling for a decade or more in countries in which screening and optimal treatment have been available. Recent declines in prostate and colorectal cancer are also linked to improving therapy and cancer management but, as for breast cancer, such breakthroughs are restricted largely to countries with sufficient resources and expertise to introduce effective cancer control measures. Undoubtedly, the trends in some European countries are of particular concern, for example, in Romania, where all-cancer mortality rates are increasing in both men and women.

In terms of the strategic prospects for prevention, these mortality trends imply major roles for primary prevention, early diagnosis and screening, as well as cancer treatment and care. A more detailed situation analysis would reveal the complexity of the cancer profile in Europe, and the likely successes and failures of cancer control in each European country. For example, cervical cancer is among the four most common cancers in many eastern European countries (Czech Republic, Estonia, Hungary, Poland, Slovakia) but a vaccine now offers real preventive promise. Stomach cancer has become less common in most countries, but remains the third most common cancer in Hungary.

The third revision of the European Code Against Cancer provides recommendations for individuals. The recommendations are designed to reduce cancer occurrence in the community and they advocate primary prevention, including the avoidance of smoking, obesity and excessive sun exposure; prudent consumption of alcohol; daily physical exercise; increased consumption of fruit and vegetables; and reduced intake of animal fat (Boyle et al., 2003). The Code also recommends that women should participate in quality-assured screening programmes for cervical cancer (from the age of 25) and breast cancer (from the age of 50), and that both men and women should participate in colorectal screening with integral quality assurance procedures from the age of 50 .

Existing knowledge and available technologies make it possible to prevent and control cervical cancer better than ever, with testing and vaccination for the HPV strains known to be major causes of the disease. Prophylactic HPV vaccines may help to reduce HPV prevalence but they will not impact on cervical cancer rates for at least a decade. If HPV vaccines were to offer lifelong immunity, implementation of high-coverage vaccination programmes would selectively impact on cases attributable to HPV16 and HPV18. In high-risk countries, parallel implementation of successful cytology screening 
programmes may therefore be a priority. Countries with existing cytology screening programmes will need to be maintained for at least the next decade and probably much longer.

\section{Cancer mortality projections for 2020}

Health-service planning is an integral component of cancer control programmes (Armstrong, 1992). Estimates of potential numbers and rates of cancer may indicate to what extent the causes of cancer and societal interventions are likely to affect its frequency. The specific objectives of predicting the future cancer burden are dependent on who requires the information (Hakulinen, 1996; Hakulinen \& Hakama, 1991). For instance, health-care providers need accurate and routinely updated estimates of numbers of cancer patients in order to allocate the finite resources available for prevention, treatment and palliative care.

Predictions are frequently obtained via a linear extrapolation of recent trends using a simple statistical model; population projections are applied to the predicted cancer incidence or mortality rates in order to estimate future numbers of cancer cases or deaths. On the European scale, however, it is not easy to predict the incidence and mortality burden in, say, 2020, even for the most common cancers. Historical patterns are not always a sound basis for future projections, and past trends clearly differ between the countries and regions of Europe.

Instead, it is assumed for simplicity that the current overall cancer incidence rates will still apply in 2020. This allows prediction of the numbers of new cases by applying the latest available incidence rates to the sex- and age-specific populations forecast for 2020. Irrespective of any future changes in risk, population growth and ageing are extremely important in determining the likely future burden of cancer. Demographic change will continue to have major consequences over the next 15 years in Europe. Scenarios of changing cancer incidence and mortality trends are also provided to indicate how the predicted numbers of cases would change if overall cancer incidence rates were to increase.

Using this approach, it is predicted that by 2020 , and in the absence of any change in risk or any intervention, there would be a total of about 3.4 million new cases of cancer each year in greater Europe. This is a $20 \%$ increase from 2002 (Table 2-3a). Much of this rise in the total cancer burden will occur among men and women aged 65 or over, the result of population ageing in each country. 
Table 2-3a Predicted numbers (thousands) of new cancer cases in 2020 in Europe, based on incidence rates in 2002

\begin{tabular}{lrrc}
\hline & $\begin{array}{r}2002 \\
\text { (thousands) }\end{array}$ & $\begin{array}{r}2020 \\
\text { (thousands) }\end{array}$ & $\begin{array}{r}\text { Increase since } \\
\text { 2002 (\%) }\end{array}$ \\
\hline Males & & & \\
Aged less than 65 & 632 & 731 & $\mathbf{1 6}$ \\
Aged 65 or over & 868 & 1133 & $\mathbf{3 1}$ \\
All ages & 1500 & 1864 & $\mathbf{2 4}$ \\
Females & & & \\
Aged less than 65 & 608 & 660 & $\mathbf{9}$ \\
Aged 65 or over & 713 & 857 & $\mathbf{2 0}$ \\
All ages & 1321 & 1517 & $\mathbf{1 5}$ \\
Persons & & & \\
Aged less than 65 & 1240 & 1391 & $\mathbf{1 2}$ \\
Aged 65 or over & 1581 & 1990 & $\mathbf{2 6}$ \\
All ages & $\mathbf{2 8 2 1}$ & $\mathbf{3 3 8 1}$ & $\mathbf{2 0}$ \\
\hline
\end{tabular}

Projections assume that incidence rates estimated for 2002 still apply in 2020; increases depend only on demographic change (see text)

Source: Ferlay et al, 2004

Of course, the risk of developing cancer is unlikely to remain constant between 2002 and 2020. To give some indication of the impact on the likely numbers of cases given future changes in risk, Table 2-3b shows the number of new cases that would occur in 2020 if the all-cancer incidence rates observed in 2002 were to stay the same, or alternatively to increase or decrease by $1 \%, 2 \%$ or $3 \%$ per annum. With constant incidence rates, a $24 \%$ increase in the number of new cancers in men is projected, and a $15 \%$ increase for women. A minor decline in cancer rates (1\% decrease per annum) will have little effect on the projected numbers of cases, because of population ageing and growth. Overall cancer rates would need to decrease by around 2\% per year to keep the overall numbers of cases below 2 million a year by 2020 . In contrast, a $1 \%$ annual increase in overall cancer incidence rates would produce more substantial net increases in the total number of new cancers diagnosed each year ( $49 \%$ for men, $37 \%$ for women) and a predicted total cancer burden of over 4 million new cancer cases by the year 2020 .

\section{Summary and concluding remarks}

It is difficult to predict the combined effect of trends in the incidence and mortality of the many types of cancer in 38 European countries, since the trends differ so widely between countries by age, sex and type of cancer. Undoubtedly, trends in some European countries raise particular concerns for example, all-cancer mortality rates in Romania are increasing in both men 
Table 2-3b Predicted numbers (thousands) of new cancer cases in 2020 in Europe, based on crude scenarios for annual change in the overall cancer incidence rates

\begin{tabular}{lrrrrr}
\hline & \multicolumn{2}{c}{ Males } & & \multicolumn{2}{c}{ Females } \\
\cline { 2 - 3 } \cline { 5 - 6 } & $\begin{array}{r}\text { Cases } \\
\text { in 2020 } \\
\text { (thousands) }\end{array}$ & $\begin{array}{r}\text { Change } \\
\text { since }\end{array}$ & & $\begin{array}{r}\text { Cases } \\
\text { in 2020 } \\
\text { 2002 (\%) }\end{array}$ & $\begin{array}{r}\text { Change } \\
\text { since }\end{array}$ \\
\hline $3 \%$ decline in rates & 1077 & -28 & & 877 & -34 \\
$2 \%$ decline in rates & 1296 & -14 & & 1054 & -20 \\
$1 \%$ decline in rates & 1555 & 4 & & 1266 & -4 \\
No change in rates & $\mathbf{1 8 6 4}$ & $\mathbf{2 4}$ & & $\mathbf{1 5 1 7}$ & $\mathbf{1 5}$ \\
1\% increase in rates & 2230 & 49 & & 1814 & 37 \\
$2 \%$ increase in rates & 2662 & 77 & & 2166 & 64 \\
$3 \%$ increase in rates & 3173 & 112 & & 2582 & 95 \\
\hline
\end{tabular}

Source: Ferlay et al, 2004

and women. Most countries are making progress in cancer control with more effective primary prevention, screening and treatment. Irrespective of future changes in cancer risk, foreseeable demographic changes will substantially increase the magnitude of cancer incidence over the next few decades. The largest increase will be in the number of cancers diagnosed in older persons in Europe.

The implementation of effective strategies may limit some of the impact of this predictable trend, particularly strategies to reduce and nullify the tobacco epidemic; effective screening for cancers of the cervix, breast and large bowel (colon and rectum); and adoption of treatment regimens that are proven to be effective and are accessible to all patients. It is vital to make adequate provision for greater numbers of cancers among the elderly.

Competing health priorities for scarce resources have had (and will continue to have) major impact on the capacity of many European nations to deliver cancer control effectively to their populations. A detailed situation analysis at national level is a key step in implementing effective cancer policies. Such an analysis should attempt to describe adequately the geographical and temporal patterns of incidence, mortality and survival for each of the common cancers, by age and sex, and to establish priority areas for cancer control. Such activities should be planned on the basis of resources within each country. A parallel analysis should consider the current status of the national cancer plan; the total national expenditure on health and the proportion of that expenditure allocated to cancer control, and finally which elements of the overall policy to combat cancer should be prioritized. 


\section{REFERENCES}

Armstrong BK (1992). The role of the cancer registry in cancer control. Cancer Causes Control, 3(6):569-579.

Bailar JC III, Smith EM (1986). Progress against cancer? N Engl J Med, 314(19):1226-1232.

Becker N, Smith EM, Wahrendorf J (1989). Time trends in cancer mortality in the Federal Republic of Germany: progress against cancer? Int J Cancer, 43(2):245-249.

Berrino F et al. (2007). Survival for eight major cancers and all cancers combined for European adults diagnosed in 1995-99: results of the EUROCARE-4 study. Lancet Oncol, 8(9):773-783.

Black RJ et al. (1997). Cancer incidence and mortality in the European Union: cancer registry data and estimates of national incidence for 1990. Eur J Cancer, 33(7):1075-1107.

Botha JL et al. (2003). Breast cancer incidence and mortality trends in 16 European countries. Eur J Cancer, 39(12):1718-1729.

Boyle P (1989). Relative value of incidence and mortality data in cancer research. Recent Results Cancer Res, 114:41-63.

Boyle P et al. (2003). European Code Against Cancer and scientific justification: third version, Ann Oncol, 14(7):973-1005.

Bray F, McCarron P, Parkin DM (2004). The changing global patterns of female breast cancer incidence and mortality. Breast Cancer Res, 6(6):229-239.

Bray F et al. (2002). Estimates of cancer incidence and mortality in Europe in 1995. Eur J Cancer, 38(1):99-166.

Brown CC, Kessler LG (1988). Projections of lung cancer mortality in the United States: 19852025. J Natl Cancer Inst, 80(1):43-51.

CRUK EU factsheet (2004). Cancer in the European Union (available at: http://info. cancerresearchuk.org/cancerstats/geographic/cancerineu/, accessed 19 December 2007).

Curado MP et al., eds (2007). Cancer incidence in five continents, Vol. IX. (IARC Scientific Publications No. 160). Lyon, IARC.

Day NE, Charnay B (1982). Time trends, cohort effects and ageing as influence on cancer incidence. In: Magnus K, ed. Trends in cancer incidence: causes and practical implications. Washington, D.C, Hemisphere Publishing Corporation pp 51-65.

Dickman PW, Adami HO (2006). Interpreting trends in cancer patient survival. J Intern Med, 260(2):103-117.

Doll R (1990). Are we winning the fight against cancer? An epidemiological assessment. EACR-Muhlbock Memorial Lecture. Eur J Cancer, 26(4):500-508.

Doll R, Payne P, Waterhouse J, eds. (1966). Cancer incidence in five continents: a technical report. New York, Springer.

Doll R, Smith P (1982). Comparison between registries age standardised rates. In: Waterhouse J, et al., eds. Cancer incidence in five continents, Vol. IV, IARC Scientific Publications No. 42. Lyon, France, IARC.

Dyba T, Hakulinen T, Paivarinta L (1997). A simple non-linear model in incidence prediction. Stat Med, 16(20):2297-2309.

Edwards R. The problem of tobacco smoking. Br Med J, 2004;328(7433):217-9.

Ferlay J et al. (2004). GLOBOCAN 2002: cancer incidence, mortality and prevalence worldwide. Lyon, France, IARC Press (IARC CancerBase No. 5. version 2.0).

Ferlay J et al. (2007). Estimates of the cancer incidence and mortality in Europe in 2006. Ann Oncol, 18(3):581-592.

Garner MJ et al. (2005). Epidemiology of testicular cancer: an overview. Int J Cancer, 116(3):331-339. 
Geddes M, Balzi D, Tomatis L (1994). Progress in the fight against cancer in EC countries: changes in mortality rates, 1970-90. Eur J Cancer Prev, 3(1):31-44.

Gilliland FD, Samet JM (1994). Lung cancer. In: Doll R, Fraumeni JF, Muir CS, eds. Trends in cancer incidence and mortality. Cancer Surv, 19-20:175-179.

Gronberg H (2003). Prostate cancer epidemiology. Lancet, 361:859-864.

Hakulinen T (1996). The future cancer burden as a study subject. Acta Oncologica, 35:665-670.

Hakulinen T, Hakama M (1991). Predictions of epidemiology and the evaluation of cancer control measures and the setting of policy priorities. Soc Sci Med, 33(12):1379-1383.

IARC (2002a). IARC handbooks of cancer prevention Vol 6. Weight control and physical activity. Lyon, IARC Press.

IARC (2002b). IARC handbooks of cancer prevention Vol 7. Breast cancer screening. Lyon, IARC Press.

IARC (2003). IARC handbooks of cancer prevention Vol 8. Fruit and vegetables. Lyon, IARC Press.

IARC (2004). IARC handbooks of cancer prevention Vol 10. Cervix cancer screening. Lyon, IARC Press.

Jensen OM et al. (1990). Cancer in the European Community and its Member States. Eur J Cancer, 26(11-12):1167-1256.

Lopez AD, Collishaw NE, Piha T (1994). A descriptive model of the cigarette epidemic in developed countries. Tobacco Control, 3:242-247.

Norat $\mathrm{T}$ et al. (2005). Meat, fish, and colorectal cancer risk: the European prospective investigation into cancer and nutrition. J Natl Cancer Inst, 97(12):906-916.

Møller B, et al. (2003) Prediction of cancer incidence in the Nordic countries: empirical comparison of different approaches. Stat Med, 22:2751-2766.

Parkin DM, Bray FI, Devesa SS (2001). Cancer burden in the year 2000. The global picture. Eur J Cancer, 37(Suppl. 8):S4-66.

Parkin DM et al. (2001). Estimating the world cancer burden: Globocan 2000. Int J Cancer, 94(2):153-156.

Pischon T et al. (2006). Body size and risk of colon and rectal cancer in the European prospective investigation into cancer and nutrition (EPIC). J Natl Cancer Inst, 98(13):920-931.

Potter J, Hunter D (2002). Colorectal cancer. In: Adami HO, Hunter D, Trichopolous D, eds. Textbook of cancer epidemiology. Oxford, UK, Oxford University Press:188-211.

Quinn M, Babb P (2002). Patterns and trends in prostate cancer incidence, survival, prevalence and mortality. Part I: international comparisons. BJU Int, 90(2):162-173.

Segi M, Kurihara M (1960). Cancer mortality for selected sites in 24 countries (1950-1957). Sendai, Japan, Tohoku University of Medicine.

Signorello L, Adami HO (2002). Prostate cancer. In: Adami HO, Hunter D, Trichopolous D, eds. Textbook of cancer epidemiology. Oxford, UK, Oxford University Press:188-211.

Swerdlow A, dos Santos Silva I, Doll R (2001). Cancer incidence and mortality in England and Wales: trends and risk factors Oxford, UK, Oxford University Press.

Thorn M et al. (1998). Trends in colorectal cancer incidence in Sweden 1959-93 by gender, localization, time period, and birth cohort. Cancer Causes Control, 9(2):145-152.

World Health Organization. WHO Statistical Information System (WHOSIS), WHO Mortality Database (available at: http://www.who.int/healthinfo/morttables/en/index.html). 

Figure 2-8a Trends in age-standardized (world) mortality rates in selected countries in the four UN-defined regions of Europe: all cancers combined, males
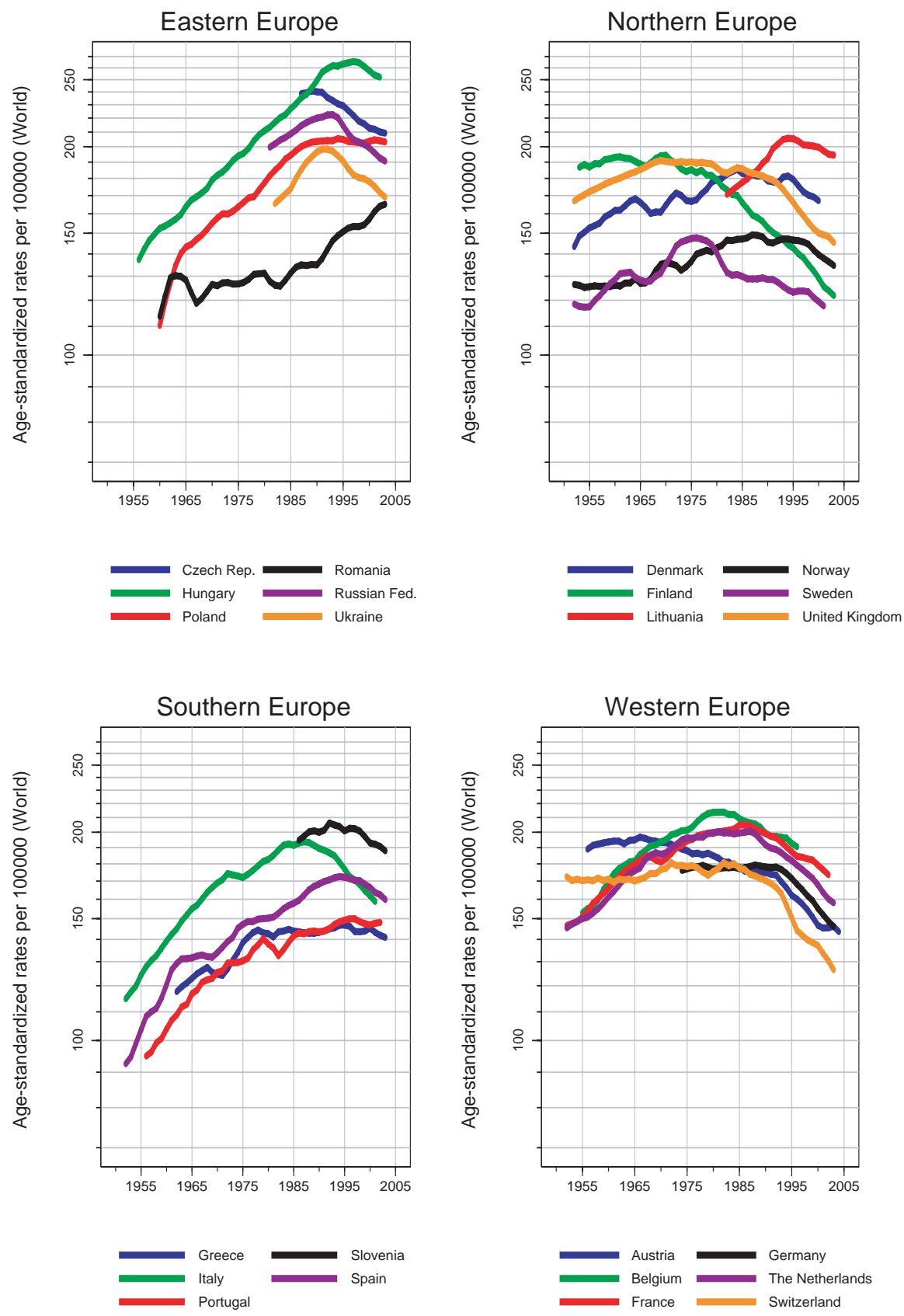
Figure 2-8b Trends in age-standardized (world) mortality rates in selected countries in the four UN-defined regions of Europe: all cancers combined, females
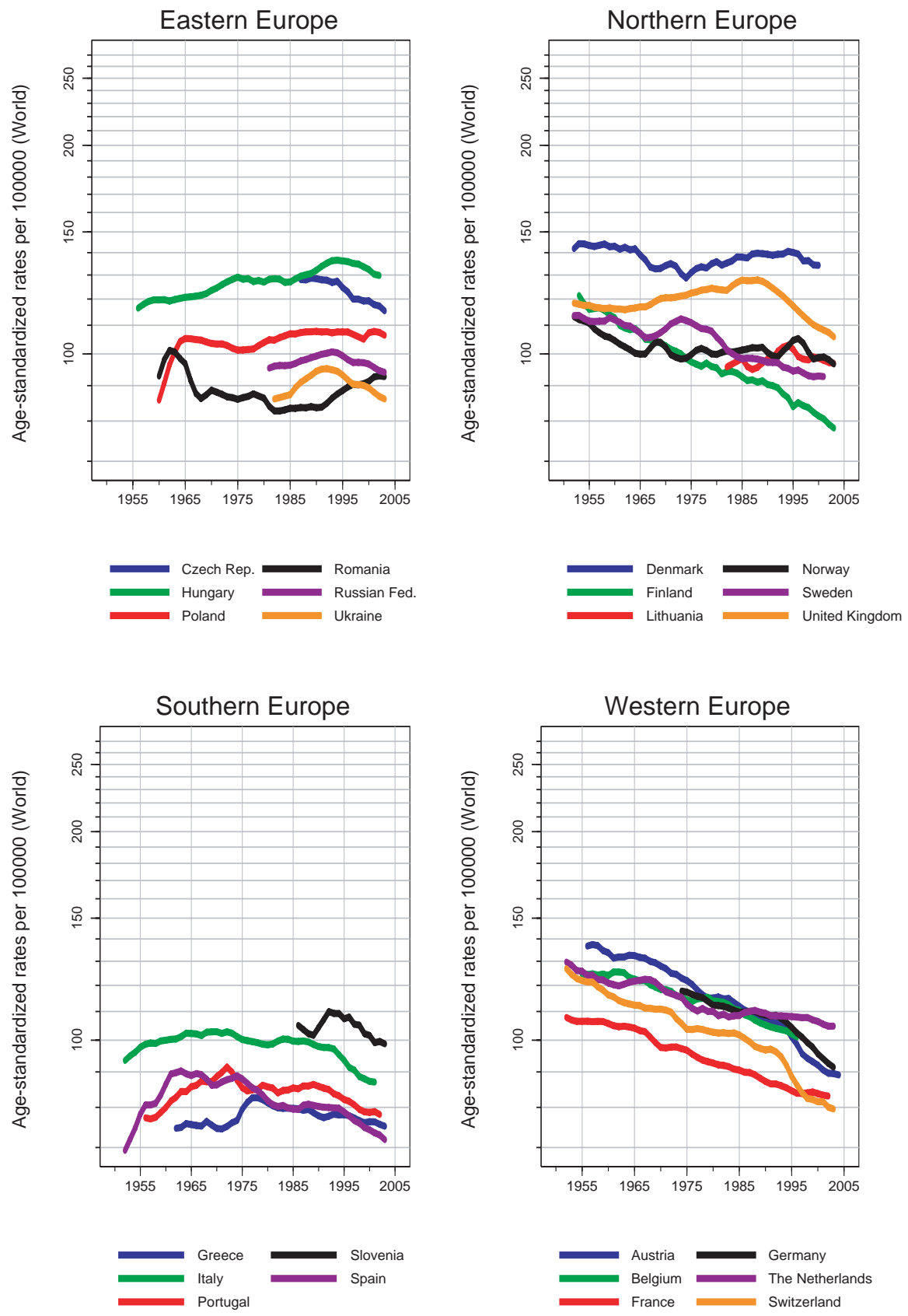
Figure 2-9a Trends in age-standardized (world) mortality rates in selected countries in the four UN-defined regions of Europe: lung cancer, males
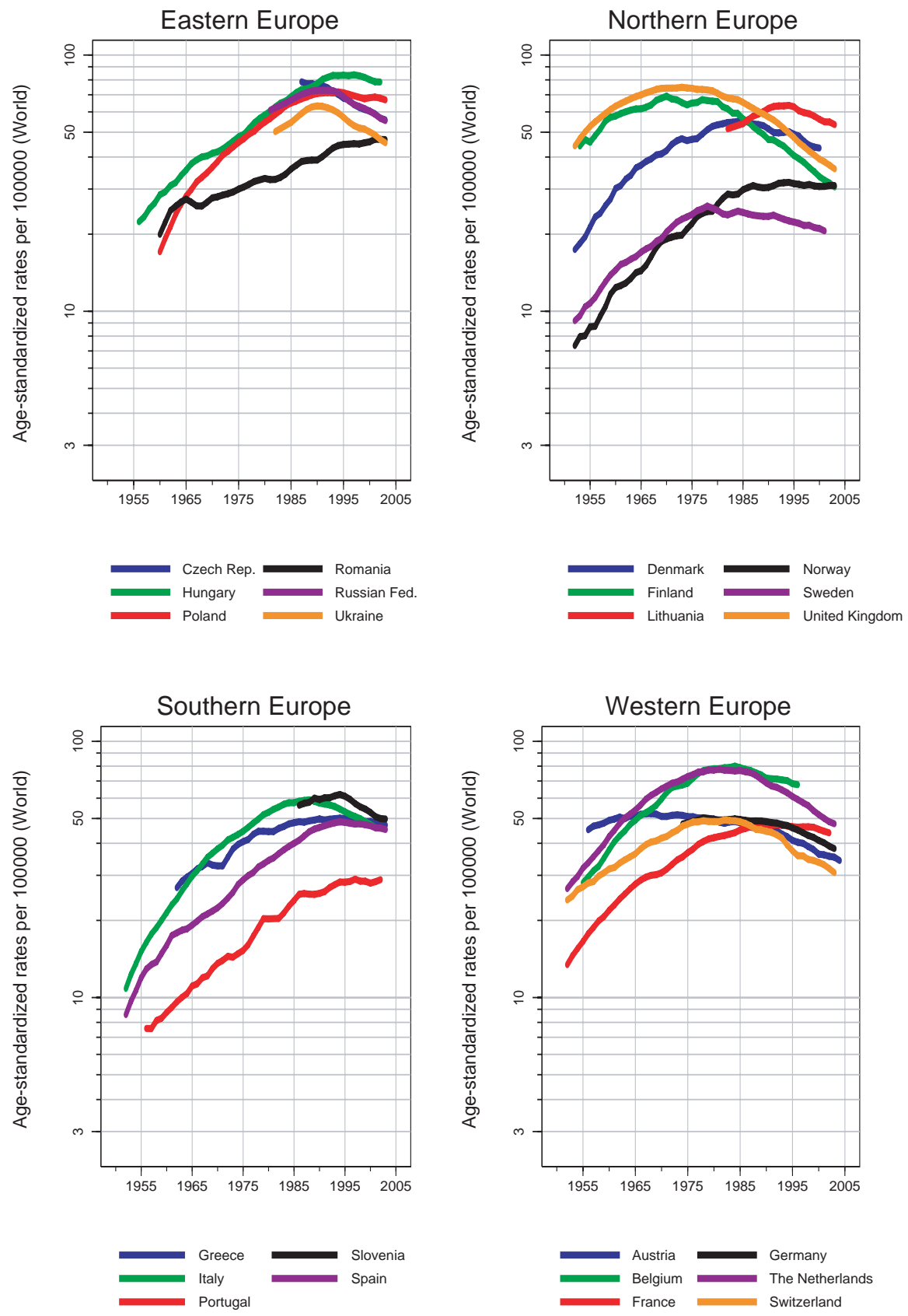
Figure 2-9b Trends in age-standardized (world) mortality rates in selected countries in the four UN-defined regions of Europe: lung cancer, females
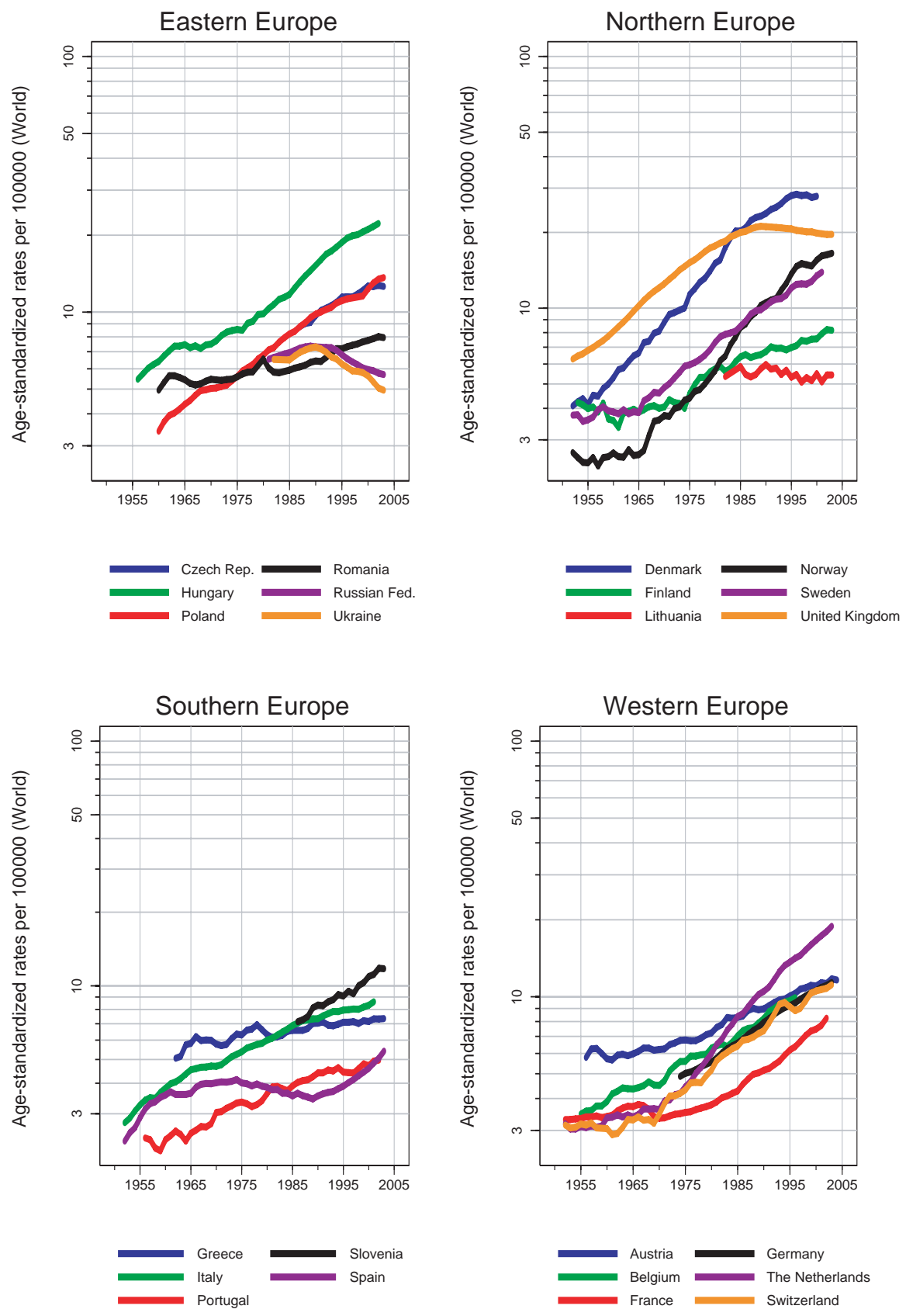
Figure 2-13 Trends in age-standardized (world) mortality rates in selected countries in the four UN-defined regions of Europe: breast cancer, females
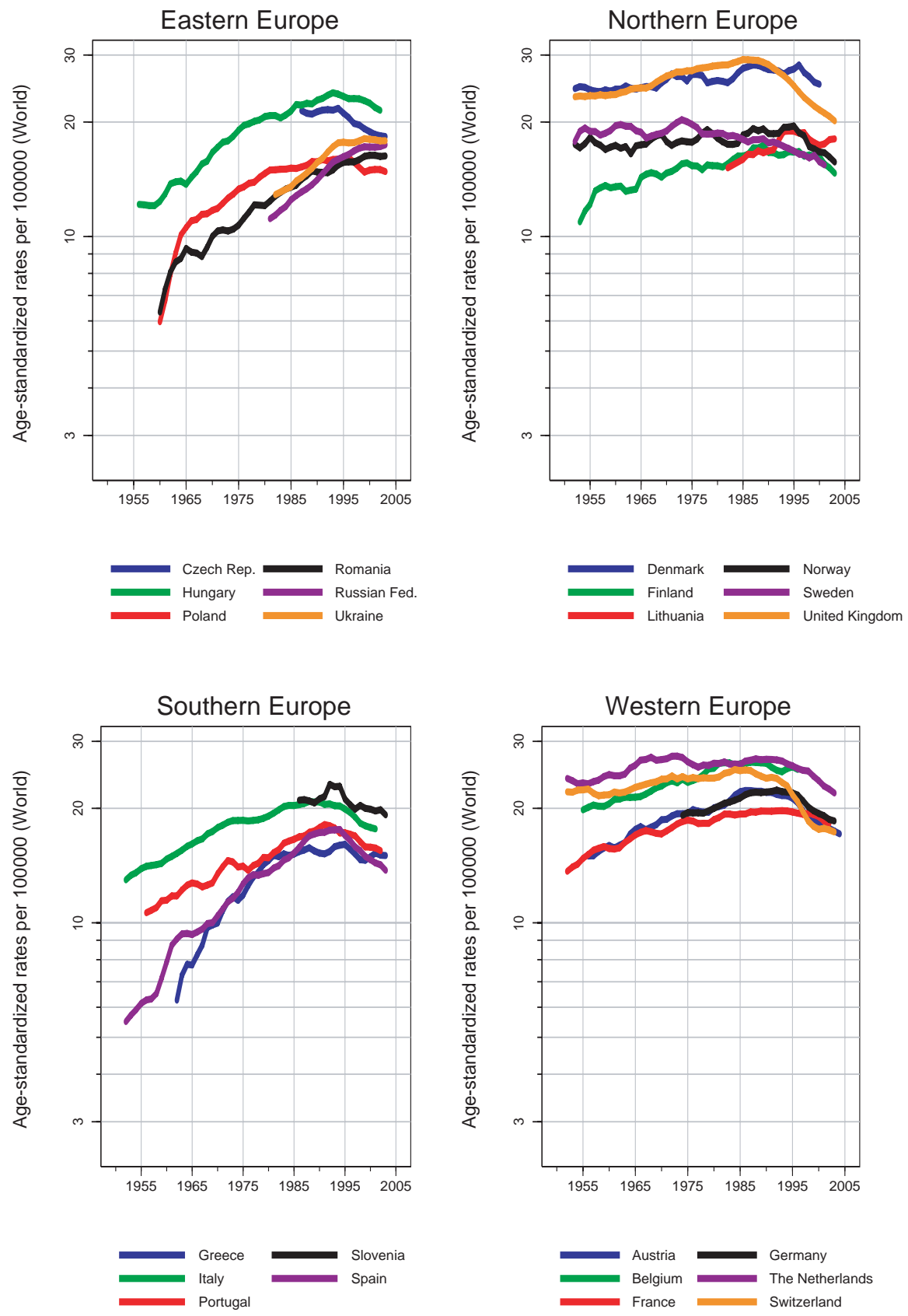
Figure 2-14a Trends in age-standardized (world) mortality rates in selected countries in the four UN-defined regions of Europe: colorectal cancer, males
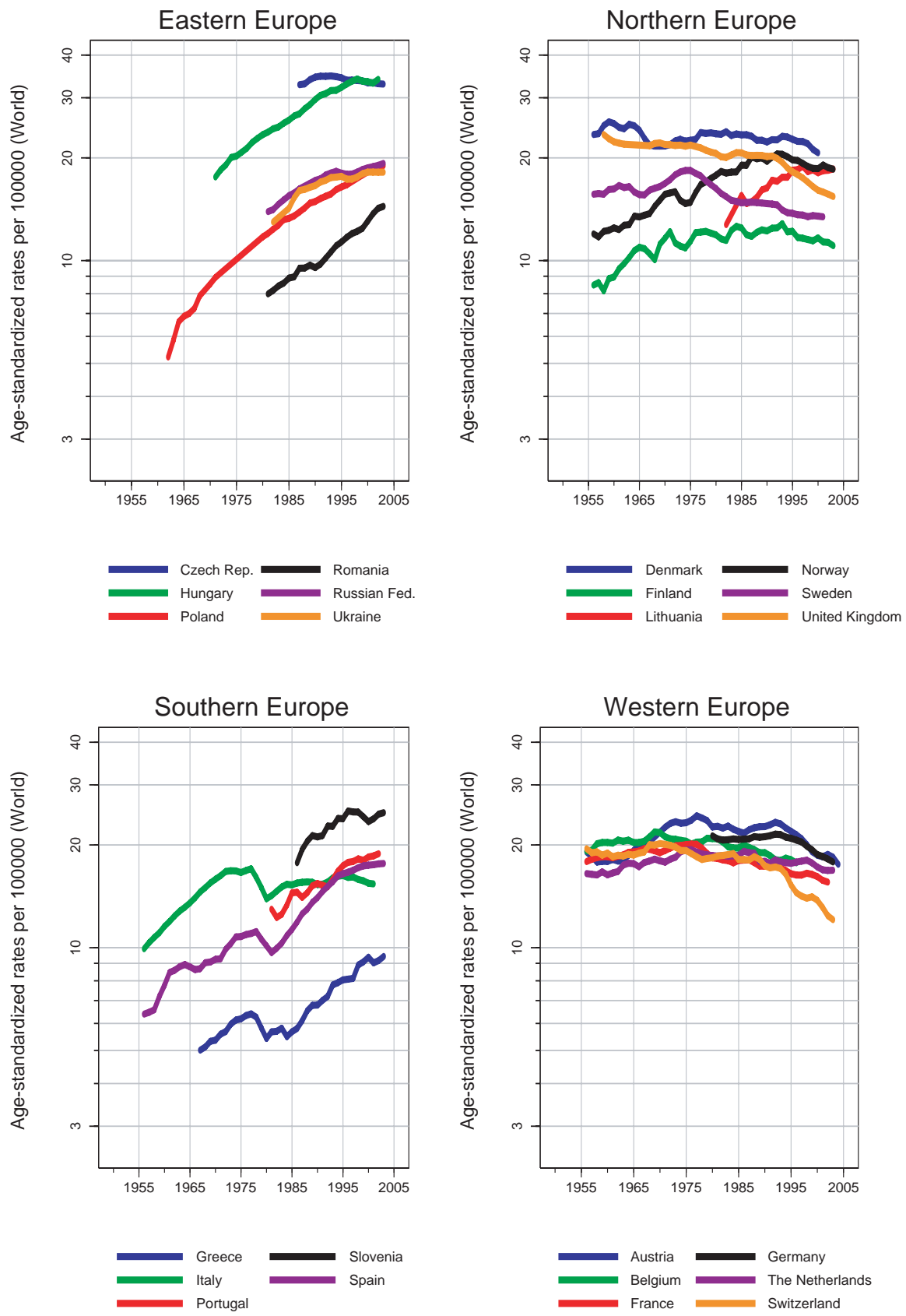
Figure 2-14b Trends in age-standardized (world) mortality rates in selected countries in the four UN-defined regions of Europe: colorectal cancer, females
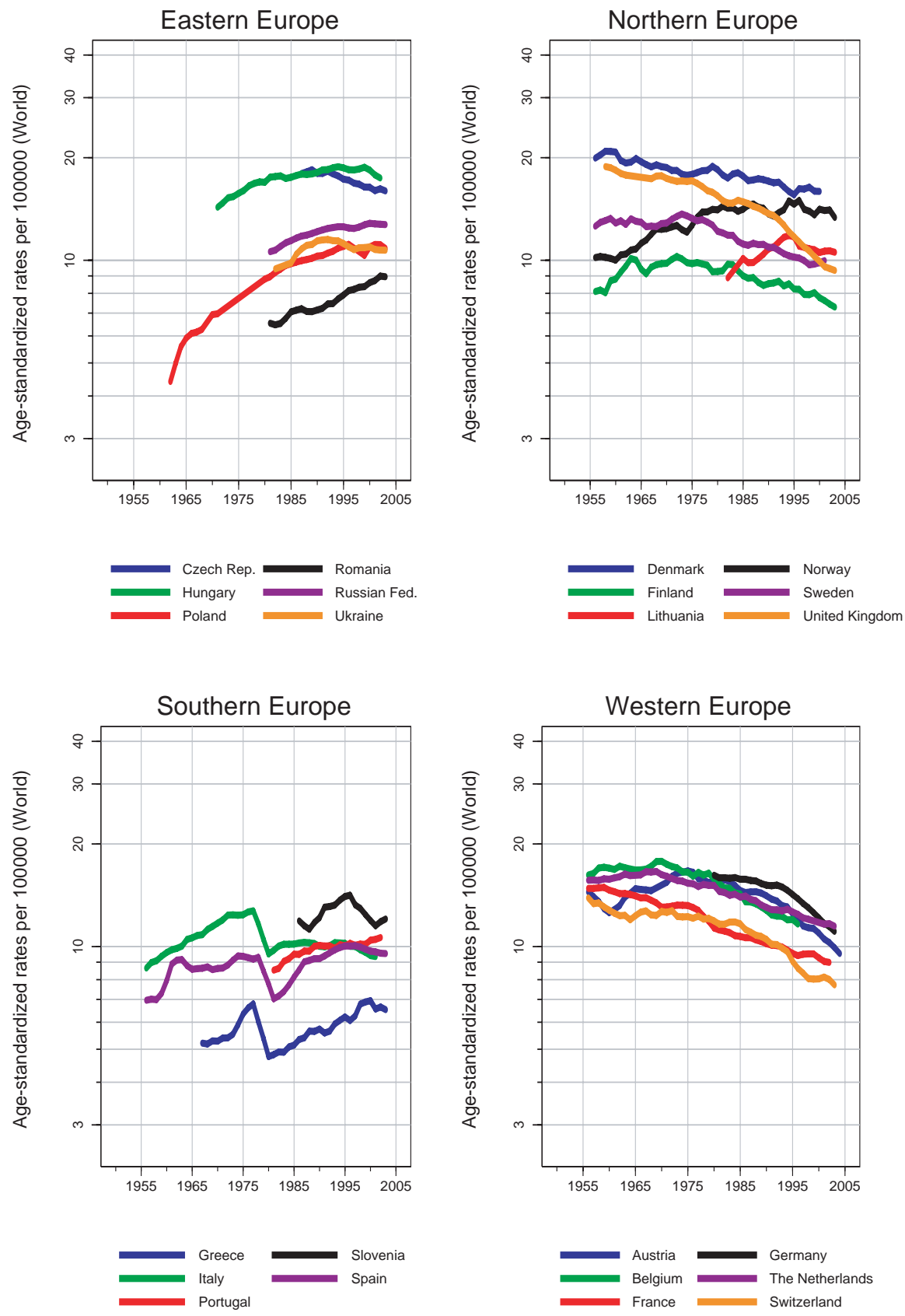
Figure 2-15 Trends in age-standardized (world) mortality rates in selected countries in the four UN-defined regions of Europe: prostate cancer
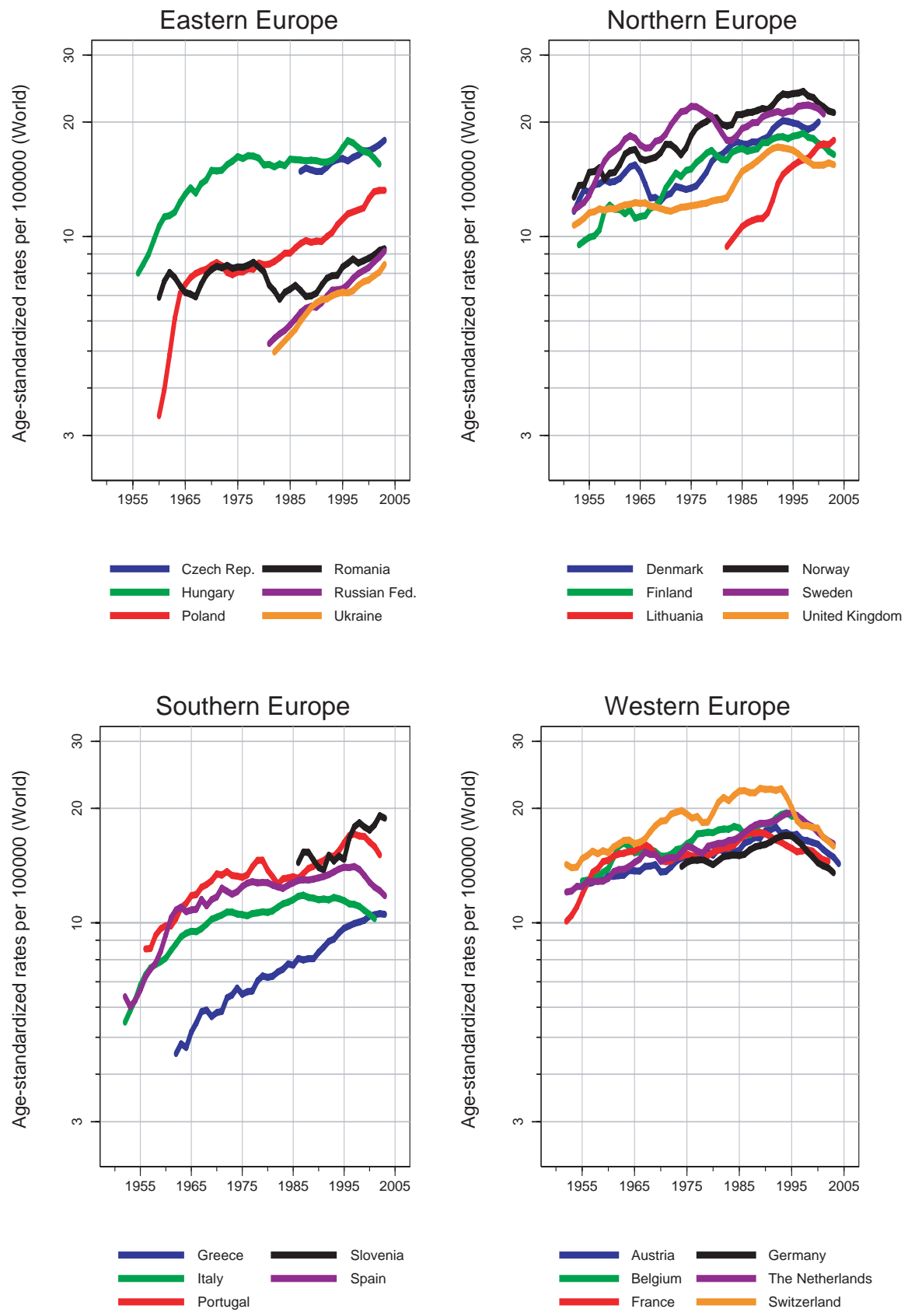


\section{Chapter 3 \\ The causes of cancer and policies for prevention}

Jose M Martin-Moreno and Gujjón Magnuisson

\section{Introduction}

This chapter presents a review of the main causes of cancer, mainly from an epidemiological viewpoint, and addresses policy-making for the prevention of cancer. The underlying idea of cancer prevention is to explore the causes (determinants) of the disease which may be acted upon or controlled. Disease prevention requires identification of those determinants that can be modified through public health actions, especially those related to the environment and to lifestyle habits.

Each of the following sections outlines the risk factors involved in the causation of cancer and the principal measures for cancer prevention, using the action plan in the European Code Against Cancer as a framework. This is followed by brief conclusions and recommendations.

\section{Key lifestyle risk factors for cancer and some prevention policies}

\section{Tobacco smoking}

Tobacco smoking is the most significant preventable cause of cancer. Among more than 4000 substances isolated from tobacco products, around 40 are known to be carcinogenic, including benzene, cadmium, chromium, 4-aminobiphenyl, 2-naphthylamine, acrylonitrile and benzo[a]pyrene (Hecht, 2005; IARC, 2004; IARC, 1986). 
Between 25\% and 30\% of all cancers diagnosed in developed countries are directly linked to tobacco smoking. In developed and developing countries combined, the proportion of cancers due to smoking is no lower than $16 \%$. Cigarette smoking is also a well-established risk factor for other diseases, harming both the (active) smoker and those exposed to the exhaled smoke (passive smokers). As an acquired behaviour pattern - a voluntary habit tobacco smoking is the largest single avoidable cause of premature death (Boyle et al., 2004).

Studies conducted in Europe, Japan and North America have shown that $87-91 \%$ of lung cancers in men $(57-86 \%$ in women) are attributable to cigarette smoking. For both sexes, 43-60\% of cancers arising in the oesophagus, larynx and oral cavity are attributable to the effect of tobacco, either alone or in combination with alcohol consumption. Lastly, a significant proportion of cancers of the urinary bladder and pancreas, a smaller proportion of cancers of the kidney, stomach, cervix and nose, and myeloid leukaemia, are also causally related to tobacco smoking (Boyle et al., 2003).

The length of the latent period means that tobacco-related cancers observed today are related to cigarette smoking patterns over several previous decades. The increased cancer risk decreases rapidly, or even ceases, on quitting or reducing smoking. The benefit of smoking cessation is clear within five years and is progressively more noticeable with the passage of time (Pisinger \& Godtfredsen, 2007).

The magnitude and distribution of exposure to tobacco smoking in Europe is particularly distressing (Costanza et al., 2006). The EU is one of the largest producers and a major exporter of cigarettes. In central and eastern Europe, there has been a significant increase in the smoking habit (Boyle et al., 2004).

The European Tobacco Control Report 2007 estimates smoking prevalence in the WHO European region to be about $40 \%$ for men and $18 \%$ for women. The prevalence of smoking among men has stabilized or is falling in most countries but there is a slight upward trend among women, especially in eastern European countries. This is worrying, especially since recent studies show that the risks associated with cigarette smoking can be particularly large for females (Costanza et al., 2006; Mucha et al., 2006). It is also of concern that the prevalence of smoking remains high among general practitioners in many parts of Europe. Doctors should set an exemplary lifestyle in terms of health. This should be a target for immediate action.

The World Health Report 2002 estimated that smoking was the second most important risk factor (after high blood pressure) in the WHO European Region in 2000 . This accounted for $12.3 \%$ of the total years of life lost due to 
premature deaths and years lived in disability (disability-adjusted life-years, DALYs) (Mucha et al., 2006). Within the EU and the European Economic Area (EEA), this ranges from $5.6 \%$ in Cyprus and $7.7 \%$ in Finland to $17.7 \%$ in Denmark and $20.9 \%$ in Hungary.

Mortality from cancer of the trachea, bronchus and lung can be used as a proxy for smoking prevalence and as a marker of past trends in exposure of the population to tobacco smoke. These markers show a clear difference in cancer risk between men and women - the age-standardized death rate in 2004 was 13.8 per 100000 population among women and 65 per 100000 among men (WHO, 2007b) .

The trend analysis offers a rather optimistic perspective. Since the early 1990s there has been a decrease in mortality rates from cancers related to smoking in the male population. This reflects decreasing smoking prevalence among males as a result of antismoking efforts implemented in many European countries since the early 1980s. However, mortality from cancers related to smoking among women is rising at different speeds in European countries. In general, this reflects the increasing prevalence of smoking among women since the early 1980s (see Chapter 2).

In all countries, the rates of premature mortality due to tobacco smoking are inversely related to education and/or income, particularly among males. Thus, smoking has been identified as a major contributing factor to the gap in mortality and healthy life expectancy between the least and the most disadvantaged in society. For instance, premature deaths from lung cancer in the United Kingdom are five times higher among men in unskilled manual work than among those in professional work (European Commission, 2003).

Tobacco smoking is also a cause of many other important diseases, including heart disease, stroke and chronic obstructive pulmonary disease (COPD or chronic bronchitis) (Boyle et al., 2004). Tobacco smoke exhaled by smokers, commonly referred to as environmental tobacco smoke, is responsible for deleterious effects on those who inhale it. This passive smoking increases the risk of lung cancer (Boyle et al., 2003) as well as heart disease and respiratory disease. Passive smoking is particularly harmful to small children.

Smoking during pregnancy has important repercussions for the offspring. It increases the risk of stillbirth, has been associated with low birth-weight and seems to impair a child's subsequent mental and physical development. After birth, smoking by either parent increases a child's risk of respiratory tract infection, severe asthma and sudden death (Boyle et al., 2004). 


\section{Alcohol}

Alcohol consumption also plays an important role in the causation of cancer. The more a person drinks, the higher the risk. There is no clear threshold (safe level of alcohol consumption) but there is evidence that men who have two or more drinks per day, and women who have one or more, have an increased risk of developing cancers of the oral cavity, pharynx, larynx and oesophagus. Higher risks for breast, colorectal and liver cancer have also been associated with alcoholic beverages (Boyle et al., 2003; Pöschl \& Seitz 2004).

The WHO European Region has the highest alcohol intake per capita of any WHO Region and twice as high as the world average. Alcohol is the third most important risk factor for the burden of disease at all ages in this Region, surpassed only by high blood pressure and tobacco smoking. Alcohol consumption is the leading risk factor among young people. As a consequence, the burden of diseases related to alcohol in Europe is twice the world average (WHO, 2006). The most recent Eurobarometer survey on attitudes towards alcohol (March 2007) reported that $75 \%$ of EU citizens claimed to have drunk alcoholic beverages during the past 12 months and an increase in alcohol consumption has been observed in the EU since 2003 (European Commission, 2007b).

Alcohol drinking increases the risk of cancers of the upper digestive and respiratory tracts, even in the absence of tobacco smoking. The cancer risk increases exponentially when these two factors are combined (Boyle et al., 2003).

Alcohol is also a major risk factor for other causes of death. These include injuries (e.g. traffic, occupational and leisure-time injuries), the leading cause of death among young people in the EU (Petridou et al., 2007).

Alcohol consumption during pregnancy has a detrimental effect on the development of the foetus and its central nervous system, often resulting in malformations, behavioural disorders and cognitive deficits in the postnatal period. It may also affect the risk of cardiovascular diseases, although this seems to depend on the dose of alcohol consumed over time (the well-known J-shaped pattern) (Boyle et al., 2003; Cook \& Reuter, 2007).

\section{Diet and nutrition}

It has been estimated that about one third (30-40\%) of all cancer mortality may be related to diet. This is not firmly established and the research domain is very dynamic; new reports about diet, nutrition and the risk of cancer appear almost weekly (Divisi et al., 2006; Willett, 2006). 
Initially, research focused on the risk of developing cancer in relation to the intake of dietary fat, particularly from animal sources. Results from ecological and experimental studies pointed strongly towards a positive association. However, findings from retrospective and prospective epidemiological studies in man have found no significant association between the consumption of dietary fat and the risk of developing breast or colorectal cancer (Boyle et al., 2003).

Whole-grain cereals and those with high fibre content have been found to reduce the risk of colorectal cancer and other digestive tract cancers in a few European studies. More research is needed to confirm this relationship because these findings have not always been replicated in large cohort and intervention studies (Willett, 2006).

The incidence and mortality of many types of cancer is lower in southern Europe (e.g. Spain, Greece, Italy) than in other European regions. This has been attributed to the Mediterranean diet, rich in olive oil, fish, vegetables and fruit but low in animal fat. Again, more research is necessary (Martin-Moreno, 2000; Willett, 2006).

A number of epidemiological studies on fruit and vegetables indicate that they are beneficial for the prevention of different chronic diseases and cancer. Specifically, a diet rich in fruit and vegetables seems to reduce the risk for a wide variety of cancers, particularly those of the oesophagus, stomach, colon, rectum and pancreas. This association was reported in several studies from Europe (most using a case-control design) but the evidence is less consistent in cohort studies from North America. Any protective effect from fruit and vegetables was apparently most marked for epithelial cancers, in particular those of the digestive and respiratory tract. The association was weak or nonexistent for hormone-related cancers.

As possible approaches to prevention, fruit and vegetables contain a large number of potentially anticarcinogenic agents. These have complementary and overlapping mechanisms of action, although the exact nutrients which confer protection are still unknown. The available evidence on cancer causation is not strong or specific enough to recommend vitamin or mineral dietary supplements at a public health level (Boyle et al., 2003; Willett, 2006).

Recent developments may shed more light because complex nutrient-gene interactions can now be investigated with new DNA chip technology and functional proteomics. Research into nutrient-gene interactions is expected to provide the understanding of pathophysiological mechanisms of cancer causation and prevention. Also, it will improve the ability to conduct cancer surveillance, which is crucial for identifying populations at risk (Go et al., 
2001; Willett; 2006). However, methods in this field still need to be improved (see Chapter 5).

\section{Physical activity, obesity and body composition}

There is consistent evidence that some form of regular physical activity is associated with a reduction in the risk of developing colon cancer. A reduction in the risk of cancers of the breast, body of the uterus (endometrium) and prostate has also been suggested. Although this effect seems to be strongly linked to the impact of physical activity on body weight, the preventive effect of regular exercise for some cancers seems to act independently of weight control for some cancers (Boyle et al., 2003; Melzer, Kayser \& Pichard, 2004).

Maintaining a healthy weight is important for reducing the risk of other chronic diseases, such as heart disease and diabetes, as well as cancer (Eyre, Kahn \& Robertson, 2004). Being overweight or obese increases the risk of several cancers, including those of the breast (among postmenopausal women), colon, endometrium, oesophagus, kidney, gallbladder and other organs. One of the main causal mechanisms is the increased production and circulation of estrogen and insulin caused by excess weight. These hormones can stimulate cancer growth (Ballard-Barbash et al., 2006; Boyle et al., 2003).

Most countries in Europe have seen rapid increases in the prevalence of obesity in recent years. Overweight and obesity have become serious public health challenges. Some $30-80 \%$ of adults in the countries of the WHO European Region are affected. About $20 \%$ of children and adolescents are overweight; a third of these are actually obese (WHO, 2007 - in press). Obesity is rising rapidly and is expected to affect 150 million adults and 15 million children by 2010. This trend is especially alarming in children and adolescents. Childhood obesity has been increasing steadily during the last decades - the current rate is 10 times higher than in the 1970s, just a generation ago.

In western Europe, it has been estimated that being overweight or obese accounts for approximately $11 \%$ of all colon cancers, $9 \%$ of breast cancers, $39 \%$ of endometrial cancers, $37 \%$ of oesophageal adenocarcinomas, $25 \%$ of kidney cancer and 24\% of gallbladder cancers (Boyle et al., 2003). Body composition (reflected in fat distribution and being lean rather than obese) may be an indicator of how the body handles calories. This may be more important for controlling cancer risks than the overall energy intake.

\section{Some prevention policies at work}

Lifestyle factors play an important role in the causation of cancer. There is a 
strong justification for cancer prevention activities focused on reversing behavioural patterns linked to tobacco smoking, alcohol drinking, unhealthy diet and physical inactivity. Tackling these risk factors has the potential to address the underlying causes of many other major diseases. A common agenda may be identifiable to prevent not only cancer but also cardiovascular disease and diabetes (Eyre, Kahn \& Robertson, 2004).

Mono-sectoral strategies that target individual behaviour by providing information and public counselling do not seem to be fully effective. Cancer prevention policies must be located in the broad context of social and economic environments, far beyond the health sector. On that basis, WHO has developed different normative strategies for addressing the major determinants of a range of chronic diseases, including cancer (Ullrich et al., 2004).

Much is known about strategies that can prevent the initiation of tobacco use among young people and promote successful cessation. Despite this, vigorous advocacy is needed to create and sustain effective tobacco-control programmes. For the first time in the history of the WHO, a legally binding international treaty has been approved by its Member States (World Health Assembly, 2003). The Framework Convention on Tobacco Control (FCTC) entered into force on 27 February 2005, the ninetieth day after the deposit of the fortieth instrument of ratification. Norway was the first country to ratify the convention (16 June 2003); 14 other Member States across the EU and EEA were among the 40 contracting parties. The European Community ratified the FCTC on 30 June 2005.

The FCTC is a unique public health tool that will facilitate international cooperation through protocols (Wipfli et al., 2004). The second meeting of the parties took place in Bangkok between 30 June and 6 July 2007. There is much hope that this will act as a solid basis to proceed with the practical development of the goals contained in the FCTC (Magnusson, 2007). The FCTC aims at the necessary systematic approach to address issues such as pricing and tax measures; protection from exposure to tobacco smoke; regulation of tobacco products; tobacco use cessation; restriction of tobacco advertising, promotion and sponsorship; strengthening the regulations on tobacco product packaging and labelling; controlling illicit trade of tobacco products; and banning sales to, and by, minors.

To be efficient and successful, a tobacco policy must be comprehensive and maintained over a long period. The importance of implementing effective interventions can be shown by their impact on the rates of lung cancer. Rates are now low in those Nordic countries which have adopted integrated policies and programmes against smoking and maintained them since the early 1970 s. 
In the United Kingdom, tobacco smoking has declined by 46\% since 1970 . Consequently, lung cancer mortality among men has been decreasing since 1980 , although the rate remains high. France saw an $11 \%$ reduction in tobacco consumption between 1993 and 1998, following the implementation of antitobacco measures (Boyle et al., 2003; Boyle et al., 2004). But there is still a lot of room for improvement. All EU Member States should consistently implement the strategies described above and identified as successful in the FCTC.

There is evidence that a daily intake of pure alcohol (ethanol) as low as $10 \mathrm{~g}$ per day (equivalent to about one can of beer, one glass of wine or one shot of spirit) is associated with some increase in breast cancer risk relative to nondrinkers. The intake associated with a significant risk of cancers of the upper digestive and respiratory tracts, liver and colorectum is probably somewhat higher (approximately 20-30 g/day). Advice on the individual recommended limits of alcohol consumption should include these points and the WHO message for limiting alcohol consumption: "less is better". Those who drink should not exceed $20 \mathrm{~g}$ of ethanol per day for men (i.e. approximately two drinks of beer, wine or spirit) and $10 \mathrm{~g}$ per day for women (Boyle et al., 2003).

In 1992, the WHO European Region was the first to launch a region-wide action plan on alcohol. Two consecutive action plans (1992-1999, 20002005) and two ministerial conferences (European Charter on Alcohol, 1995; Declaration on Young People and Alcohol, 2001) have had policy implications and offered paths for development and implementation of effective measures in European countries (WHO, 2006). The most recent instrument is the Framework for Alcohol Policy in the WHO European Region (adopted as resolution EUR/RC55/R by the Regional Committee 2005 in Bucharest). This focuses on alcohol-free settings within a range of environments (Cook \& Reuter, 2007; WHO, 2006) for:

- young people, including sports and leisure;

- transport, both at land and sea;

- the workplace - promoting the public health view that alcohol should not be a part of normal working life;

- pregnancy - in the absence of demonstrated safe limits, abstinence from alcohol during pregnancy is recommended and actively encouraged.

Finally, the European Commission's October 2006 communication concerning the strategy to support Member States in reducing alcohol-related harm at the EU level - is a very important policy instrument (European Commission, 2006). 
Following dietary recommendations may have the potential to reduce cancer incidence in Europe by as much as $30-40 \%$. The proposed changes aim to reduce the levels of saturated fats, added sugar and salt; remove trans fatty acids from the diet; increase consumption of fruit and vegetables; and increase physical activity. In line with $\mathrm{WHO}$ and American FDA recommendations, the five-a-day regime of fruit and vegetables is advocated (minimum $400 \mathrm{~g} /$ day, i.e. 2 pieces of fruit and $200 \mathrm{~g}$ of vegetables). Further research is needed to clarify the complex relationship between some dietary factors and cancer, in particular the protective role of fruit and vegetables (Boyle et al., 2003; Willett, 2006).

Nutritional practices in central and eastern European countries are changing rapidly towards a more westernized type of diet, with adverse effects on death rates from chronic disease (WHO, 2007 - in press). These regions require particular attention to promoting a healthy diet.

The development of affordable, safe and healthy choices for consumers and responsible marketing of food products, especially to children, would provide key complementary messages. This requires simple, clear, non-misleading and consistent food labels that provide consumers with information on the composition of food (Ullrich et al. 2004).

Another general public-health message is to undertake some brisk, physical activity every day, and maintain a BMI in the range of $18.5-25 \mathrm{~kg} / \mathrm{m}^{2}$ (Boyle et al., 2003). Those who are already overweight or obese should reduce their BMI to below $25 \mathrm{~kg} / \mathrm{m}^{2}$. A lifestyle that incorporates a healthy diet, exercise and weight control reduces cancer risk and the risk of other chronic diseases. A balance between calorific intake and energy expenditure is the critical factor in maintaining a healthy BMI (Ballard-Barbash et al., 2006).

Following the rationale explained for tobacco prevention and control, WHO developed non-binding recommendations for the promotion of healthy diet and physical activity. The Global Strategy on Diet, Physical Activity and Health was adopted by Member States in May 2003 at the 57 $7^{\text {th }}$ World Health Assembly (World Health Assembly, 2004). In contrast to the FCTC, this does not contain any legal obligation to implement its recommendations. However, it does provide an important template for developing national plans of action and approaches for dealing with this important public health problem (Waxman, 2004). 


\section{Occupational and environmental factors - risks and opportunities for prevention}

\section{Occupational factors}

Approximately $5 \%$ of cancers have been attributed to occupational environments. However, like most work-related ill-health, it is probable that the importance of exposure to carcinogenic risk factors in work settings is underestimated (Boyle et al., 2003; Siemiatycki et al., 2004). In fact, occupation-related cancers must be considered a central public health issue.

Occupational exposures have been linked most frequently to malignant neoplasms of the lung, urinary bladder, larynx and nasopharynx, liver, nose and nasal cavity and mesothelioma, leukaemia, and non-melanoma skin cancer. Several other malignant tumours have also been associated with occupational exposures, but less evidence exists. These include cancers of the oral cavity, oesophagus, stomach, colon and rectum, pancreas, breast, testis, kidney, prostate, brain and bones; soft tissue sarcoma, lymphomas and multiple myeloma (Boyle et al., 2003; Siemiatycki et al., 2004).

Some 35 occupational agents are classified as probably carcinogenic in humans (Group 2A of the International Agency for Research on Cancer, IARC) and many are still widely used, e.g. 1,3-butadiene and formaldehyde. Over 200 agents, groups of agents or exposure circumstances are classified as possibly carcinogenic to humans (Group 2B), based on carcinogenicity data derived from animal experiments.

In the early 1990s, around 23\% (approximately 32 million) of those employed in the EU were thought to be exposed to carcinogenic agents at levels above the natural background. Although exposure to these agents remains widespread, it occurs mostly at low levels. The most common occupational exposures concern solar radiation, passive smoking, crystalline silica, diesel exhausts, radon, wood dust, benzene, asbestos, formaldehyde, polycyclic aromatic hydrocarbons, chromium (VI), cadmium and nickel compounds (Boyle et al., 2003; Siemiatycki et al., 2004).

Most of the well-known or suspected occupational-related carcinogens have been evaluated by IARC in Lyon, France, (Boyle et al., 2003; Siemiatycki, Richardson \& Boffetta, 2006; Siemiatycki et al., 2004). Of 102 agents, groups of agents or exposure circumstances classified as human carcinogens (Group 1 of the IARC classification), 29 are chemical or physical agents, groups of agents or mixtures that occur predominantly in the workplace. IARC has also classified 16 industrial processes or occupations as carcinogenic to humans, including the rubber industry, painters, etc. 
The production or use of certain chemicals in EU Member States has been limited under the REACH provisions of the EU Chemicals Policy Review (EuroWorksafe, 2007). For example, dichlordiethylene sulphide (mustard gas) and 2-naphthylamine have been banned; mining associated with exposure to ionizing radiation and some other high-risk industries have recently been stopped. However, there is still widespread exposure to other carcinogens, such as metals and dioxins. Cancers caused by occupational exposures remain a problem that needs to be tackled appropriately (EuroWorksafe, 2007; Lamontagne \& Christiani, 2002).

\section{Air pollution and water contaminants}

Key risk factors in air pollution include: residential proximity to industrial point sources; combustion products such as polycyclic organic matter (POM) particulate matter, radionuclides, 1,3-butadiene and aldehydes; organic fibres (mainly asbestos); and radon (Samet \& Cohen, 2006). Carcinogens can be measured in indoor and outdoor environments; toxicological and epidemiological data indicate the potential for human carcinogenicity.

Air pollutants may be widespread as fine particles. Several studies have associated these particles with a slightly increased risk of lung cancer, even at low urban exposure levels. A large number of subjects may be exposed to such agents in the general environment for lengthy periods. In the EU the increased cancer risk due to this exposure is relatively modest, but important (Nawrot et al., 2007).

Particulate matter (PM) is a pollutant comprising a complex mixture of solid and/or liquid particles of organic and inorganic substances suspended in the air. Studies of the short-term effects of PM on health, based on the association between daily changes in PM concentrations and various health outcomes, have been conducted in many cities in the WHO European Region during the last decade. The findings indicate some short-term, acute health effects and a significant increase in the risk of death from cardiovascular disease and lung cancer (Anderson et al., 2004; WHO, 2005). More research is needed on lung cancer risk from air pollution in order to guide public health policies on these exposures (Boyle et al., 2003; Samet \& Cohen, 2006).

There has been no quantification of the impact of several environmental carcinogenic exposures (including arsenic) via contaminated drinking water. Exposure to arsenic probably affects only some limited population groups. There is inconclusive evidence for other widespread exposures to disinfection by-products in drinking water (Cantor et al., 2006), such as nitrate, organic chemicals from human commerce (e.g. agricultural pesticides), asbestiform fibres present in water, other inorganic solutes and fluoride. 
There is evidence that drinking water contains a mixture of known or suspected carcinogenic substances, typically found at trace level concentrations (less than 100 parts per billion [ppb]). The following substances have a proven or suggested carcinogenic risk:

- inorganic arsenic (and possibly other trace metals) - involved in the causation of several cancers, including non-melanoma skin cancer and cancers of the bladder, lung and kidney;

- synthetic organic chemicals (especially disinfection by-products) - linked with cancers of the urinary bladder and (possibly) the large bowel;

- radium - increases the risk of osteosarcoma;

- radon in water - linked with lung cancer through contribution to airborne radon levels in the home;

- nitrate - increases the risk of gastrointestinal and other cancers.

On the other hand, water hardness, magnesium and calcium confer protection against cancers at several sites.

The Water Framework Directive (2000/60/EC of the European Parliament and of the Council establishing a framework for the Community action in the field of water policy) was adopted in 2000 and is still being improved (Anderson et al., 2004). In March 2007, the European Commission organized the European Water Conference at which more than 400 participants discussed the first implementation report and the launch of the Water Information System for Europe (WISE). Material produced in this conference should facilitate appropriate development of this field of public health (WHO, 2005).

\section{lonizing radiation}

There is comprehensive evidence of the association between high doses of ionizing radiation and cancer in humans (Boice, 2006). Recently, IARC classified X-rays, gamma rays and neutrons as carcinogenic to humans (Group 1). This is irrespective of the pattern of energy release and the penetrating power of the various types of ionizing radiation (Boyle et al., 2003).

Natural terrestrial and cosmic background radiation is the principal source of ionizing radiation for humans. However, man-made sources give much greater public concern, e.g. nuclear power production, nuclear accidents (e.g. Chernobyl), atmospheric nuclear testing and other similar exposures. The consequences of exposure to high doses of ionizing radiation have been well documented through studies of the atomic bomb survivors of Hiroshima 
and Nagasaki. High-dose ionizing radiation is used in medicine for therapeutic reasons (mainly radiotherapy for treating cancer).

In 1955, a UN General Assembly resolution established the The United Nations Scientific Committee on the Effects of Atomic Radiation (UNSCEAR) in response to widespread concerns about the effects of radiation on human health and the environment. Over the decades, UNSCEAR has evolved to become the world authority on the effects of ionizing radiation (UNSCEAR, 2007).

UNSCEAR has estimated that the population risk of dying from cancer after exposure to an acute dose of $1000 \mathrm{mSv}$ (millisieverts) of ionizing radiation would be around $13 \%$ for women and $9 \%$ for men. These estimates might be $50 \%$ lower for chronic exposures. The average annual effective dose is $2.4 \mathrm{mSv}$ worldwide; the population lifetime exposure to all sources of ionizing radiation has been estimated to account for $1 \%$ of all fatal cancers. Statistical models are now available to gauge precisely the health effects of ionizing radiation (Akushevich et al., 2006).

Radiation for diagnostic purposes is a matter of public concern for the population groups undergoing examinations e.g. mass screening programmes for healthy individuals (e.g. mammography for breast cancer, or computed tomography (CT) scans for lung cancer) or when thyroid disease is suspected. There is evidence that mammography screening programmes substantially reduce breast cancer mortality. This benefit greatly exceeds the potential cancer risk induced by radiation exposure during mammograms. Still, unnecessary exposure to ionizing radiation should be avoided, even though the collective exposure from diagnostic tests is small compared to natural radiation (Boice, 2006; Boyle et al., 2003).

\section{Solar radiation}

Solar ultraviolet (UV) radiation is part of the electromagnetic radiation spectrum arising from the sun (Green \& Whiteman, 2006). Sunlight exposure is the main environmental cause of skin cancer and UV light is the solar spectrum component involved. Those affected are mainly fair-skinned, particularly people with red hair, freckles and a tendency to burn in the sun (Boyle et al., 2003).

Three main types of skin cancer are related to sun exposure. Squamous cell carcinoma shows the clearest relationship with cumulative sun exposure and is the most common form of skin cancer among people who work outdoors. Recipients of transplanted organs are also at high risk of developing these 
tumours because of the combined effects of the unchecked growth of HPV in their skin (caused by immunosuppression) and sun exposure.

Basal cell carcinoma is the most common type of skin cancer but its severity is limited since this tumour is localized at skin level. This type of skin cancer apparently shares an aetiological relationship to sun exposure with melanoma.

Lastly, cutaneous melanoma seems to be related to intermittent sun exposure such as sunbathing and outdoor sports. A history of sunburn has been repeatedly described as a risk factor. Worldwide, incidence rates for cutaneous melanoma have risen faster than those for any other malignancy in Caucasian populations over the last 30 years. Mortality rates have continued to climb despite improving survival rates over this period (Giblin \& Thomas, 2007).

In Europe, the incidence of melanoma doubled between the 1960s and the 1990s. This is attributed to an increase in intense sun exposure in the past century. The incidence of squamous cell and basal cell cancers has also increased during this period in all European countries. These tumours are significantly less life-threatening than melanoma but they account for $95 \%$ of all skin cancers. Treatment is a considerable financial burden for individuals and health-care systems. Skin cancer remains an important challenge for cancer prevention and control (Giblin \& Thomas, 2007; Green \& Whiteman, 2006).

\section{Electromagnetic fields and other non-ionizing radiation}

The possible carcinogenic effects of non-ionizing radiation - from sources such as power lines, electrical equipment and mobile phones - are a matter of public concern (Boyle et al., 2003; Savitz \& Ahlbom, 2006). While current exposure levels have shown little evidence of an associated risk of cancer, the International Commission on Non-Ionizing Radiation Protection (ICNIRP) issues guidelines for limiting exposure (ICNIRP, 2007).

Power lines produce extremely low frequency (ELF) electromagnetic fields $(50-60 \mathrm{~Hz})$. Electromagnetic fields are characterized by their frequency (inversely correlated with wavelength) (Savitz \& Ahlbom, 2006) and their intensity. Electric fields do not penetrate the body. Power-line magnetic fields penetrate most materials and cause additional human exposure that exceeds the typical background field (roughly 0.1 microtesla $[\mu \mathrm{T}]$ ) up to a distance of about $50 \mathrm{~m}$ from the power line, depending on the voltage and the wiring configuration (Boyle et al., 2003).

Since 1979, at least 24 studies on childhood cancer and power line exposure have been published, including two recent meta-analyses. However, the 
association is still not clear (Savitz \& Ahlbom, 2006). Studies that enrolled large sample sizes of cancer cases found no excess risk of cancer among adults living in the vicinity of power lines. Nonetheless, an association between some cancers and exposure to ELF magnetic fields has been suggested in various occupational studies. Epidemiological studies suggest that the carcinogenic effects of magnetic fields (if any) are concentrated among people with high exposures, which are uncommon in Europe (Boyle et al., 2003).

\section{Opportunities for environmental prevention}

Some risk factors related to lifestyle are potentially controllable through behaviour modification. Occupational and environmental carcinogens are also amenable to preventive strategies - cancers arising from these are highly preventable.

Primary responsibility for the prevention of occupational cancer rests with the manufacturers and distributors of carcinogenic substances and the companies who use them, rather than the workers affected by cancers (Lamontagne \& Christiani, 2002). In recent decades, extensive preventive measures have averted many cancers related to workplace exposures (Boyle et al., 2003). For example, the ban on the use of beta-naphthylamine in the chemical and rubber industries has resulted in a lower incidence of urinary bladder cancer related to occupational exposure. But there is still a long way to go. The Global Plan of Action for Workers' Health 2008-2017 was endorsed at the 60 $0^{\text {th }}$ World Health Assembly in May 2007 as an update to the WHO Global Strategy on Occupational Health for All. The WHO Regional Office for Europe will work with governments, trade unions, employers, professional associations and other stakeholders to implement this in European countries (WHO, 2007c).

Air pollution should always be minimized and controlled appropriately. This requires more research on the relationship between air pollution and lung cancer to guide policies for the protection of public health (Boyle et al., 2003; Samet \& Cohen, 2006).

Strategies to minimize contamination of drinking water and exposure to water contaminants include various watershed protection programmes and water treatment options, some of which are expensive and technologically complex. Strategies to reduce exposure to chemical factors by water contamination include the application of alternative disinfection procedures (or more selective use of existing methods) to reduce disinfectant by-products; and the use of more advanced water-treatment technologies to remove organic, inorganic and particulate contaminants (European Commission 2007a). 
The International Commission on Radiological Protection (ICRP) issues recommendations for radiological protection based on existing scientific literature. It is vital to apply the regulations strictly and to follow the advice provided by the national radiation protection offices, including the avoidance of unnecessary exposure to radiation (Boyle et al., 2003; Samet \& Cohen, 2006).

Exposure to solar radiation should be limited - to reduce total lifetime exposure in general and to avoid extreme sun exposure and sunburn in particular (Green \& Whiteman, 2006), especially for those Europeans who are more prone to skin cancer, e.g. fair-skinned people. A significant fall in mortality rates is anticipated following the improvements in early detection, but changes resulting from primary prevention should also be fostered. A change in behaviour is required to limit sun exposure, accomplished through public education campaigns and targeted health policies. The best preventive message would be to keep out of the sun (especially in summer), to use high sunprotection factor (SPF) sunscreens when this is not possible and to follow the advice detailed in the European Code Against Cancer (Boyle et al., 2003).

Lastly, more research is needed about the health effects of electromagnetic fields. Current scientific knowledge is substantial but cannot be easily translated into preventive measures (Savitz \& Ahlbom, 2006; WHO, 2007a).

\section{Other cancer determinants and related preventive strategies}

Infectious agents

Infectious agents cause some cancers. At least 15\% (and up to 20\%) of human malignancies worldwide are attributable to persistent infections with bacteria, viruses or parasites. The EU percentage is lower (10\%), affecting mainly cancers of the cervix uteri, liver and stomach, and certain malignancies of the blood-forming or lymphatic systems (haemolymphopoietic malignancies).

During the last decade, research has elucidated the role of HPV as a cause of cervical cancer. A dozen types of HPV have been identified in 99\% of biopsy specimens from cervical cancer worldwide. Five (HPV 16, 18, 31, 33, 45) account for over $85 \%$ of cervical cancer specimens in Europe (WHO, 2007).

There is no effective medical treatment against HPV but new vaccines have been developed and very sensitive and specific tests to detect HPV DNA in cervical cells are now available. This new HPV testing could be recommended among women who present with borderline or low-grade cytological abnormalities. Moreover, HPV DNA testing may offer a more sensitive alternative to cytology (Pap smear) in primary cervical cancer screening and 
could improve the follow-up of women who have been treated for cervical intraepithelial neoplasia (CIN). Regarding HPV vaccines, they have been licensed in more than 30 countries of the WHO European Region, and although there is already sufficient evidence that they are safe, highly immunogenic and effective specifically against the most common oncogenic HPV types (HPV 16 and 18), their overall effectiveness in our population is still being studied. It is expected that, in the near future, enough experience will have been acquired to confirm the real impact of these vaccines; and about their interaction with comprehensive, population based, organized cervical cancer prevention programmes (Chan \& Berek, 2007; Davies et al., 2007).

Chronic infections with the hepatitis B and $\mathrm{C}$ viruses (HBV and $\mathrm{HCV}$ ) have been associated with an increased risk of liver cancer. HBV is the main cause of liver cancer in sub-Saharan Africa and south-east Asia, but is less important in Europe. Nevertheless, over $70 \%$ of 503 liver cancer patients in a large case series from six European liver centres had markers of either HBV or HCV infection (Mueller et al., 2006). An effective HBV vaccine has been available for 20 years but is not yet used systematically in national immunization programmes in Europe. National policies of universal vaccination against HBV should be reconsidered because selective vaccination of high-risk groups rarely works. Travelling and migration help to mix the high- and low-risk populations. HBV infection in young adulthood (through sexual intercourse or contaminated needles) carries a much lower risk of chronic hepatitis and liver cancer than infection at birth or during childhood, but it frequently leads to acute hepatitis.

$\mathrm{HCV}$ is becoming an increasing problem in certain areas of the EU (especially Italy, Greece, Spain) and among certain population groups, mainly intravenous drug users. No vaccine is available and treatment of all infected individuals with pegylated interferon-2a (with or without ribavirin) is still being assessed for effectiveness. Currently, prevention of HCV infection relies on strict control of blood and blood derivatives and avoiding exposure from needles re-used in medical and non-medical procedures (e.g. acupuncture, tattooing, etc) (Boyle et al., 2003; Mueller et al., 2006).

The bacterium Helicobacter pylori (HP) is associated with an approximate sixfold increased risk of cancer of the stomach, particularly the lower part. Of approximately 78000 new cases each year in the EU, 65\% may be attributable to HP (assuming 35\% HP prevalence in the general population). The current treatment of HP infection is effective, based on the use of proton pump inhibitors and antibiotics (Wang, Yuan \& Hunt, 2007). However, it can be complicated by poor patient compliance, antibiotic resistance and recurrence of infection. A recent meta-analysis indicates that HP infection is 
strongly associated with early gastric cancer. Treatment of HP infection can induce regression of gastric lymphoma. Future studies will reveal more.

In summary, major improvements in the ability to identify markers of chronic infection have raised awareness of the role of infectious agents in the causation of various types of cancer in the last three decades. Contrary to conventional wisdom, antibacterial and antiviral treatments and vaccination programmes may offer important tools for cancer control. In order to reduce the impact of infectious agents it is important to strengthen surveillance, prevention and control of communicable diseases, and particularly to use safe and effective vaccines, when they are available. Pending further knowledge about the population impact of HPV vaccines (Chan \& Berek, 2007; Davies et al., 2007; WHO, 2007), the best advice is to introduce systematic vaccination against HBV (Boyle et al., 2003). Vaccines against cancer caused by infectious agents are among the most promising approaches to prevention.

\section{Exogenous hormones}

It is well-known that the mutagenic effects of sex steroid hormones can contribute to the causation of cancers of the reproductive organs. Genotoxic effects of the metabolites of sex steroid hormones have also been shown (Lacey, Colditz \& Schottenfeld, 2006). Oral contraceptives (OC) and postmenopausal hormone replacement therapy (HRT) are also associated with an increased risk of some of these cancers.

Many studies have shown that breast cancer risk increases among current or recent $\mathrm{OC}$ users but tends to level off in the first few years after cessation of use. Their use is also associated with a higher risk of cervical cancer in HPVpositive women. An increased risk of liver cancer in OC users has been reported (Giannitrapani et al., 2006) but the public health importance of this association in developed countries is unclear. Conversely, OC use substantially reduces the risk of ovarian cancer for up to 20 years after cessation of use, and may reduce the risk of endometrial cancer. Several studies have suggested a reduced risk of colorectal cancer (La Vecchia et al., 2001) but this is open to further research.

Hormone replacement therapy (HRT) may increase the risk of breast cancer. A combined estrogen-progestogen HRT is associated with an excess risk of breast cancer after a few years of use but this increased risk appears to be restricted to current users. It is important to note that treatment with unopposed estrogens (given without progestogens) is strongly related to an excess risk of cancer of the uterus but combined (estrogen-progestogen) HRT is not. HRT has also been reported to be associated positively with the risk of 
ovarian cancer, and inversely with the risk of colorectal cancer, although its relationship with duration and other time-related factors remains unclear (Boyle et al., 2003; Lacey, Colditz \& Schottenfeld, 2006). Given its adverse effects on cardiovascular diseases, HRT should not be recommended for disease prevention. It remains more useful for the short-term relief of postmenopausal symptoms; other treatments should be considered for osteoporosis (Dull, 2006).

Immunological factors, hereditary risk of cancer and genetic modifiers of cancer risk

A greater understanding of basic immunological principles has advanced the knowledge of cancer aetiology, prevention and treatment (Arlen, Dahut \& Gulley, 2006). Immunodeficiency disorders (either inherited or acquired after birth) can have profound effects on the risk of specific cancers. The primary inherited immunodeficiency syndromes are rare disorders that increase the risk of recurrent and persistent infections, and may eventually lead to a higher risk of lymphoproliferative malignancies. Severe acquired immunodeficiency may also have important consequences. The increasing frequency of immune impairment (whether due to immunosuppressive drugs given after an organ transplant or from the spread of HIV infection) has had marked effects on cancer incidence in the affected groups. These include an increase of skin cancers, non-Hodgkin lymphoma and Kaposi's sarcoma; and many other cancers to a lesser extent. In some cases this may be due to releasing the immunological control of incipient malignancies related to infection (Morgan, Linet \& Rabkin, 2006). More research on immunological factors is necessary to identify new approaches for prevention and immunotherapy.

To address the importance of genetic factors in the risk of malignant neoplasms, it is necessary to underline that cancer is a result of a breakdown in the genetic control of cell growth and behaviour. In nearly all cases, a cell becomes capable of uncontrolled growth and of spreading to other sites following a succession of genetic errors. A genetic error in the germ-line in certain individuals predisposes them to cancer in practically every cell in the body. These changes may be inherited - whole families are affected. Comprehensive study of the family history of cancer and the development of population-based data on familial cancers (following up members of cancerprone families) has shown an ever-expanding list of clinical syndromes (Lindor, Lindor \& Greene, 2006).

Increasing discoveries in the last decade have shown the genes that underlie hereditary forms of cancer. This research has the main advantage of offering an approach to more accurate diagnoses (in some cases before symptoms 
emerge) and thus has potential as a screening tool. Any defective gene that is predisposed to malignancy is usually a key element of an important pathway. Consequently, the discovery of these genes has led to a better understanding of the causes of common cancers.

The next phase of genetic discovery will be the identification of genes that contribute to the heritable component of the cause of cancer but do not have enough individual influence to account for families with a classic pattern of inheritance of cancer. Association studies of cases of familial breast cancer have identified mutations in this gene, showing it to be a significant risk factor in predisposition. In most cases, a defective function of at least one other unidentified gene is required to precipitate disease (Caporaso, 2006). Such genes are likely to interact with environmental triggers to cancer in a proportion of people who inherit them, since they confer a mild-to-moderate increase in predisposition to cancer. Such advances will increase the number of genetic variations known to carry an increased risk of malignancy. The main challenge is to quantify the risk associated with such genetic variations in different environmental settings, and to intensify research on geneenvironment interactions in relation to cancer risk. Multidisciplinary research is essential; biobanks and large-scale population-based studies will be required (Boyle et al., 2003; Preston, 2007).

\section{The European Code Against Cancer}

In 1987, the EU set up Europe Against Cancer, an ambitious programme to meet the public health challenge of cancer. Among other activities, a committee of experts was commissioned to create a series of prevention messages and guidelines targeting all EU citizens. The first European Code Against Cancer (ECAC) was developed in 1987 and formally approved in 1988. It comprises ten recommendations (six on cancer prevention, four on early diagnosis) which could reduce both cancer incidence and mortality.

In 1994, six years after the implementation of the ECAC, the European Commission asked the European Institute of Oncology to form a group of international experts to review the recommendations. A second version of the ECAC included new features. A third update began in 2002 (Boyle et al., 2003), directed by an executive board comprising specialists in public health and oncology; cancer associations; and cancer prevention units of European ministries of health. A scientific committee of independent experts carried out an exhaustive study of each ECAC recommendation. Over 100 scientists participated in this process, which was completed in 2003. 
The new ECAC highlighted that many aspects of public health could be improved and many deaths from cancer could be prevented if people made appropriate choices about certain health and lifestyle habits.

The recommendations to prevent cancer are summarized below.

\section{A. Many aspects of general health can be improved, and certain} cancers avoided, if you adopt a healthier lifestyle.

\section{1) Do not smoke; if you do smoke, stop doing so as soon as possible.}

If you cannot stop smoking, never smoke in the presence of non-smokers.

Between $25 \%$ and $30 \%$ of all cancers diagnosed in European countries are related to tobacco smoking. Not only does smoking cause cancer, it also causes other serious diseases such as chronic obstructive pulmonary disease and heart disease. Tobacco smoking harms the user, but second-hand smoke also affects the non-user (passive smoker). Therefore, promoting the benefits gained from smoking cessation is well worthwhile.

\section{2) Avoid obesity.}

Obesity (defined as a Body Mass Index equal to, or more than, $30 \mathrm{~kg} / \mathrm{m}^{2}$ ) is one of the major, and one of the most preventable, causes of morbidity and mortality. It is a significant risk factor for many chronic diseases, including cancer. It leads to an increased risk of diabetes and cardiovascular diseases.

\section{3) Do brisk but moderate physical exercise every day.}

Numerous studies have shown the protective effect of physical activity on the risk of cancer, particularly cancers of the colon, breast, uterus and prostate. If there are no medical counter-indications, it is advisable to do 30 minutes physical exercise per day, at least three times a week. More vigorous activities may offer additional benefits for cancer prevention.

\section{4) Increase your daily intake and variety of fruit and vegetables: eat at least 5 servings a day. Cut back on foods containing animal fats.}

Sufficient evidence exists that eating fruit and vegetables is beneficial because it reduces the risk of a whole range of tumours, especially those of the oesophagus, stomach, colon, rectum and pancreas. WHO advocates the 5-aday plan as the recommended daily amount to reduce the risk of developing cancer. Ideally, this plan consists of 2 fruit and 3 vegetable portions each day.

\section{5) If you drink alcohol (wine, beer or spirits) do so in moderation, a} maximum of two drinks a day for men, and one for women is recommended. 
Compelling evidence exists that alcohol consumption increases the risk of developing cancer of the oral cavity, pharynx, larynx, oesophagus, liver, colon and rectum, and breast. This risk tends to increase with the amount of ethanol consumed. A marked increase in the risk of cancers of the respiratory and upper digestive tracts is associated with smoking and drinking alcohol simultaneously, since one factor multiplies the effect of the other.

In any case, alcohol consumption must not exceed $20 \mathrm{~g}$ of alcohol a day (two drinks) in healthy adult men and $10 \mathrm{~g}$ a day (one drink) in healthy adult women. No safe limits are recommended for alcohol consumption in minors. Women should not drink at all while pregnant.

6) Care must be taken to avoid excessive sun exposure. It is particularly important to protect children and adolescents. Those who are prone to sunburn must take precautions when sunbathing throughout life.

Ultraviolet light is the main component involved in skin cancer, which is more frequent in white-skinned people who live in places where solar radiation is high. The best recommendation is to moderate time spent in the sun. People should reduce their total lifetime sun exposure, avoid prolonged exposure to the sun in general and sunburn in particular.

\section{7) Strictly apply the legislation designed to prevent any exposure to} carcinogenic substances. Follow all health and safety instructions about the use of such substances. Follow the radiation protection regulations.

Approximately $5 \%$ of all cancers are related to occupational carcinogenic exposures. The identification of numerous carcinogenic substances of a natural and artificial nature has enabled the prevention of occupational and environmental exposures. The ECAC message addresses all those responsible for laws and their observation and exhorts citizens to protect their own health, and that of others, by following the instructions and regulations on carcinogenic pollutants.

Individual protection systems in the workplace are essential where hazardous substances may reach levels that exceed those of the environment in general. Knowledge about carcinogenic substances and how to reduce exposure to them is essential in cancer prevention. The carcinogenic effects of ionizing radiation from both natural and artificial sources are well established therefore unnecessary exposures should be avoided.

\section{B. Public health programmes exist which can prevent cancer from developing or increase the probability that a cancer is cured.}


Early detection is important for reducing cancer mortality. It is well established that cancer survival is better for patients diagnosed at an early, localized stage of disease than for those detected at later, more advanced stages. The earlier cancer is detected, the more effectively it can be treated. It is important to alert the population to the different symptoms of cancer-related diseases so that medical advice can be sought immediately any of these appear. Great efforts have been made to organize early-detection programmes (screening) and to search for new diagnostic methods to enable early diagnoses and increase the prospects of cure. Cancer screening is covered in detail in Chapter 4, but its role in the ECAC is covered briefly here.

\section{8) Women over 25 should participate in cervical screening programmes.}

HPV vaccination does not yet replace the need for an organized mass population screening programme for cervical cancer, based on cytology. Most recommendations about the age at which women should participate in a screening programme are based on studies of the prevalence of lesions at different ages and the association between sexual activity and cervical cancer. Participation in screenings between the ages of 20 and 30 is compatible with this theory. It is advisable to continue participation until the age of 60 , with tests at intervals of 3-5 years. HPV infection, usually transmitted sexually, is the most important risk factor for cervical cancer and early detection through screening programmes has proved very effective. New vaccines may be introduced into the immunization schedule of various Member States of the EU imminently.

\section{9) Women over 50 should participate in breast screening programmes.}

Breast screening by mammography at two-yearly intervals can detect breast tumours too small to cause symptoms or to be detected by ordinary clinical examination. Treatment is more effective at this early stage. Mammogram programmes have effectively reduced breast cancer mortality but efforts are required to ensure that women from more disadvantaged and less informed social groups participate fully in these programmes. Well-organized programmes with participation rates of $70 \%$ or more should lead to an average reduction of $20 \%$ in breast cancer mortality in women over the age of 50 . There is a consensus that participation in a breast cancer screening programme should be recommended for all women aged 50-69.

\section{0) Men and women over 50 should participate in colon cancer screening programmes.}

Colorectal cancer can also be detected before symptoms occur, and treated more effectively as a result. Premalignant lesions (adenomatous polyps) enable 
this tumour to be detected early from traces of blood in the faeces. Faecal occult blood (FOB) testing is effective as part of a mass screening strategy if positive tests can be followed up by flexible sigmoidoscopy or colonoscopy. Other novel techniques, like the virtual colonoscopy (a 3-D CAT scan), may also prove beneficial. Screening of men and women aged 50-75 at 3-5 year intervals may prove highly effective, providing all the necessary arrangements are managed coherently within a comprehensive cancer plan.

\section{1) Participate in vaccination programmes against hepatitis $B$.}

Each year 30000 liver cancers are diagnosed in the EU; most of them are caused by HBV and HCV. The European Code recommends vaccination against HBV because there is clear evidence that it is highly effective; universal (whole population) vaccination may be one way of preventing this particular cancer. Vaccines against cancer caused by infectious agents are one of the most promising tools for cancer prevention.

\section{Getting the message across and assessing the impact of ECAC}

The ECAC must not be limited to a small group of specialists. Health-care professionals and health-education organizations must take its message to the general population through educational interventions. Their efficacy can be assessed by accurate measurement of the impacts on individual behaviour and the long-term community results (in health indicators such as cancer incidence or mortality). Furthermore, these assessments may improve understanding of the psychosocial determinants that are key to success. These activities should be seen as an investment in improving the effectiveness of educational interventions. Cancer prevention may benefit from such actions and the effort is well worthwhile.

\section{Conclusions}

There is a large body of knowledge about the causes of cancer and associated preventive strategies but further research will increase understanding. A set of lifestyle and environmental factors involved in the causation of cancer is already well defined - these include tobacco smoking; alcohol consumption; dietary and nutritional factors; lack of physical activity; occupational and other environmental risks; and infectious agents.

Many of these unhealthy lifestyle and environmental determinants also contribute to increases in other non-communicable diseases such as diabetes mellitus, cardiovascular disease and chronic obstructive pulmonary disease. 
The European Code Against Cancer provides a practical framework for health promotion and cancer prevention, as well as alternative strategies to target the main causes of cancer. These have proved effective when implemented properly.

Knowledge about primary prevention has not always been translated into effective prevention in EU Member States. Cancer prevention is a complex undertaking that must involve stakeholders from many sectors of society and target the social and economic dimensions responsible for the cancer burden. In an ideal world, all stakeholders would recognize the primacy of health, and health policies would apply effective cancer control strategies. However, vested interests in society conflict with these goals and they hinder both the development and application of cancer prevention strategies.

Communication is vital. Consistent messages, effective strategies and multiple channels of communication must take account of customs, norms, values and leadership patterns (in single communities or society as a whole) in order to alter human behaviour by promoting healthy environments and lifestyles. It should be possible to advocate these policies for pragmatic and economic reasons - cancer has substantial direct and indirect impacts on national economies and places a tremendous economic burden on all countries.

\section{Recommendations}

It is essential to develop a comprehensive framework to control cancer and other chronic diseases in each Member State and at EU level. This should incorporate the policies and experience of $\mathrm{WHO}$ and other international organizations.

A response to the global burden of chronic disease (including cancer) requires a strategic assessment of the global processes likely to be most effective in generating country-level commitments to policy change and influencing industry. A number of $\mathrm{EU}$ partnerships, economic incentives and international legal instruments could contribute to a more effective global response to cancer prevention.

A broader European frame of reference for lifestyle-related chronic diseases would be particularly useful. This might bring together the European Code Against Cancer, the Framework Convention on Tobacco Control and the Global Strategy on Diet, Physical Activity and Health. The use of a wide range of public health instruments would promote a broad preventive strategy, e.g. EU legal obligations and non-binding recommendations, advocacy and policy advice. Real success will depend on the ability of interest groups (within and 
between Member States) to influence the political process in order to develop cancer prevention policies and programmes across the EU. This will be demanding, but the effort is certainly worthwhile.

\section{REFERENCES}

Akushevich I et al. (2006). Population models for the health effects of ionizing radiation. Radiats Biol Radioecol, 46(6):663-674.

Anderson HR et al. (2004). Meta-analysis of time series studies and panel studies of particulate matter (PM) and ozone (O3). Report of a WHO task group. (Available on-line at: http://www. euro.who.int/document/e82792.pdf, accessed 7 November 2007). Copenhagen, WHO.

Arlen PM, Dahut WL, Gulley J L (2006). Immunotherapy for prostate cancer: what's the future? Hematol Oncol Clin North Am, 20(4):965-983,xi.

Ballard-Barbash R et al. (2006). Obesity and body composition New York. In: Schottenfeld D, Fraumeni J. eds. Cancer epidemiology and prevention, 3rd edition. New York, Oxford University Press: 422-448.

Boice JD (2006). Ionizing radiation. In: Schottenfeld D, Fraumeni J. eds. Cancer epidemiology and prevention. New York, Oxford University Press: 259-293.

Boyle P et al. (2003). European Code Against Cancer and scientific justification: third version (2003). Ann Oncol, 14(7):973-1005.

Boyle P et al. (2004). Tobacco and public health: science and policy. Oxford, Oxford University Press.

Cantor KP et al. (2006). Water contaminants. In: Schottenfeld D, Fraumeni J. eds. Cancer epidemiology and prevention, 3rd edition. New York, Oxford University Press:382-404.

Caporaso NE (2006). Genetic modifiers of cancer risk. In: Schottenfeld D, Fraumeni J. eds. Cancer epidemiology and prevention. New York, Oxford University Press:577-600.

Chan JK, Berek JS (2007). Impact of the human papilloma vaccine on cervical cancer. J Clin Oncol, 25(20):2975-2982.

Cook P, Reuter P ( 2007). When is alcohol just another drug? Some thoughts on research and policy. Addiction, 102:1183-1188.

Costanza MC et al. (2006). Gender differentials in the evolution of cigarette smoking habits in a general European adult population from 1993-2003. BMC Public Health, 6:130.

Davies P et al. (2007). The future of cervical cancer prevention in Europe. Coll Antropol, 31(Suppl. 2):11-16.

Divisi D et al. (2006). Diet and cancer. Acta Biomed, 77:118-123.

Dull P (2006). Hormone replacement therapy. Prim Care, 33:953-963.

European Commission (2003). Smoking and the environment: actions and attitudes. Brussels, European Commission.

European Commission (2006). Communication from the Commission to the Council, the European Parliament and the Committee of the Regions. An EU strategy to support Member States in reducing alcohol related harm. Brussels 24.10.2006 Com (2006) 625. Brussels, European Commission.

European Commission (2007a) European Water Conference. 22-23 March 2007, Brussels (available at:http://ec.europa.eu/environment/water/water-framework/2007conference/index_ en.htm, accessed 21 July 2007). Brussels, European Commission.

European Commission (2007b). Special EUROBAROMETER 272. Attitudes towards alcohol (available at:http://ec.europa.eu/health/ph_determinants/life_style/alcohol/documents/ebs272_ en.pdf, accessed 20 July 2007). Brussels, European Commission. 
EuroWorksafe (2007). European semantic portal on occupational cancer risks and prevention (available at: http://www.euroworksafe.org/portal/media-type/html/user/anon/page/default.psml; jsessionid=D3CBF1DA2B2B48388008D934BBF4FAD6?js_language $=e n$, accessed 20 July 2007).

Eyre H, Kahn R, Robertson RM (2004). Preventing cancer, cardiovascular disease, and diabetes: a common agenda for the American Cancer Society, the American Diabetes Association, and the American Heart Association. Diabetes Care, 27(7):1812-1824.

Giannitrapani L et al. (2006). Sex hormones and risk of liver tumor. Ann N Y Acad Sci, 1089:228-236.

Giblin AV, Thomas JM (2007). Incidence, mortality and survival in cutaneous melanoma. $J$ Plast Reconstr Aesthet Surg, 60(1):32-40.

Go VL, Wong DA, Butrum R (2001). Diet, nutrition and cancer prevention: where are we going from here? J Nutr, 131(Suppl. 11):3121S-3126S.

Green AC, Whiteman DC (2006). Solar radiation. In: Schottenfeld D, Fraumeni J eds. Cancer epidemiology and prevention. New York, Oxford University Press:294-305.

Hecht SS (2005). Carcinogenicity studies of inhaled cigarette smoke in laboratory animals: old and new. Carcinogenesis, 26(9):1488-1492.

IARC (1986). Tobacco smoking. IARC monographs on the evaluation of the carcinogenic risk of chemicals to humans. Vol. 38. Lyon, International Agency for Research on Cancer.

IARC (2004). Tobacco smoke and involuntary smoking. (IARC monographs on the evaluation of carcinogenic risks to humans. Vol. 83). Lyon, International Agency for Research on Cancer.

ICNIRP (2007). International Commission on Non-Ionizing Radiation Protection: aim \& roots (available at:http://www.icnirp.de/aim.htm, accessed 21 July 2007). Oberschleissheim, Germany, International Commission on Non-Ionizing Radiation Protection.

Lacey JV, Colditz GA, Schottenfeld D (2006). Exogenous hormones. In: Schottenfeld D, Fraumeni J. eds. Cancer epidemiology and prevention. New York, Oxford University Press:468488.

Lamontagne AD, Christiani DC (2002). Prevention of work-related cancers. New Solut, 12(2):137-156.

La Vecchia C et al. (2001). Oral contraceptives and cancer: an update. Drug Saf, 24:741-754.

Lindor NM, Lindor CJ, Greene MH (2006). Hereditary neoplastic syndromes. In: Schottenfeld D, Fraumeni J. eds. Cancer epidemiology and prevention, 3rd edition. New York, Oxford University Press:562-576.

Magnusson RS (2007). Non-communicable diseases and global health governance: enhancing global processes to improve health development. Global Health, 3:2.

Martin-Moreno J M (2000). The role of olive oil in lowering cancer risk: is this real gold or simply pinchbeck? J Epidemiol Community Health, 54:726-727.

Melzer K, Kayser B, Pichard C (2004). Physical activity: the health benefits outweigh the risks. Curr Opin Clin Nutr Metab Care, 7(6):641-647.

Morgan GJ, Linet MS, Rabkin CS (2006). Immunologic factors. In: Schottenfeld D, Fraumeni J. eds. Cancer epidemiology and prevention. New York, Oxford University Press:541-561.

Mucha L et al. (2006). Meta-analysis of disease risk associated with smoking, by gender and intensity of smoking. Gend Med, 3(4):279-291.

Mueller NE et al. (2006). Infectious agents. In: Schottenfeld D, Fraumeni J, eds. Cancer epidemiology and prevention. New York, Oxford University Press:507-548.

Nawrot TS et al. (2007). Lung cancer mortality and fine particulate air pollution in Europe. Int J Cancer, 120(8):1825-1826; authors' reply:1827.

Petridou ET et al. (2007). Unintentional injury mortality in the European Union: how many more lives could be saved? Scand J Public Health, 35:278-287. 
Pisinger C, Godtfredsen NS (2007). Is there a health benefit of reduced tobacco consumption? A systematic review. Nicotine Tob Res, 9:631-646.

Pöschl G, Seitz HK (2004). Alcohol and cancer. Alcohol and Alcoholism, 39:155-165.

Preston RJ (2007). Epigenetic processes and cancer risk assessment. Mutat Res, 616(1-2):7-10.

Samet JM, Cohen AJ (2006). Air pollution. In: Schottenfeld D, Fraumeni J. eds. Cancer epidemiology and prevention, 3rd edition. New York, Oxford University Press:355-381.

Savitz DA, Ahlbom A (2006). Electromagnetic fields and radiofrequency radiation. In: Schottenfeld D, Fraumeni J. eds. Cancer epidemiology and prevention. New York, Oxford University Press:306-321

Siemiatycki J, Richardson L, Boffetta P (2006). Occupation. In: Schottenfeld D, Fraumeni J. eds. Cancer epidemiology and prevention, 3rd edition. New York, Oxford University Press:322-354.

Siemiatycki J et al. (2004). Listing occupational carcinogens. Environ Health Perspect, 112:1447-1459.

Ullrich A et al. (2004). Cancer prevention in the political arena: the WHO perspective. Ann Oncol, 15(Suppl. 4):249-256.

UNSCEAR (2007). Mandate of the Committee: UNSCEAR Secretariat - United Nations (available at: http://www.unscear.org/unscear/en/about_us/mandate.html, accessed 21 July 2007). Vienna, The United Nations Scientific Committee on the Effects of Atomic Radiation.

Wang C, Yuan Y, Hunt RH (2007). The association between helicobacter pylori infection and early gastric cancer: a meta-analysis. Am J Gastroenterol, 102:1-10.

Waxman A (2004). WHO's global strategy on diet, physical activity and health. Response to a worldwide epidemic of non-communicable diseases. Scand J Nutrition, 48:58-60.

WHO (2005). Particulate matter air pollution: how it harms health (available at: http://www.euro.who.int/document/mediacentre/fs0405e.pdf, accessed 20 July 2007). Berlin, Copenhagen \& Rome, World Health Organization (Fact sheet EURO/04/05).

WHO (2006). Framework for alcohol policy in the WHO European Region. Copenhagen, World Health Organization.

WHO (2007). Can we prevent cervical cancer? Entre nous (available at: http://www.euro.who.int/ document/ens/en64.pdf, accessed 24 July 2007). Copenhagen, World Health Organization.

WHO (2007a). Environmental health policy (available at: http://www.euro.who.int/envhealthpolicy/ 20030405_3, accessed 23 July 2007). Copenhagen \& Rome, World Health Organization.

WHO (2007b). The European tobacco control report 2007. Copenhagen, World Health Organization.

WHO (2007c). Occupational health (available at: http://www.euro.who.int/occhealth, accessed 20 July 2007). Berlin, Copenhagen \& Rome, World Health Organization.

WHO (2007 - in press). The challenge of obesity in the WHO European Region and the strategies for response. Copenhagen, World Health Organization.

Willett WC (2006). Diet and nutrition. In: Schottenfeld D, Fraumeni J. eds. Cancer epidemiology and prevention, 3rd edition. New York, Oxford University Press: 405-421.

Wipfli H et al. (2004). Achieving the Framework Convention on Tobacco Control's potential by investing in national capacity. Tob Control, 13(4):433-437.

World Health Assembly (2003). Resolution 56.1: WHO Framework Convention on Tobacco Control (FCTC) (available at: http://www.who.int/tobacco/areas/framework/final_text/en/, accessed 20 July 2007).

World Health Assembly (2004). Resolution 57.17: Global strategy on diet, physical activity and health (available at: http://www.who.int/gb/ebwha/pdf_files/WHA57/A57_R17-en.pdf, accessed 20 July 2007). 


\section{Chapter 4 Cancer screening}

Matti Hakama, Michel P Coleman, Delia-Marina Alexe and Anssi Auvinen

\section{Introduction}

This chapter begins with an examination of the theoretical basis of cancer screening. This is followed by an evaluation of screening initiatives from a population health perspective and a discussion of the organization of mass screening programmes. The status of cancer screening in the $\mathrm{EU}$ is summarized, along with evidence for the effectiveness of existing screening programmes. There is also a brief review of the evidence on screening for cancers for which no screening programme is currently in place, such as lung cancer and melanoma.

\section{Theoretical basis of screening}

Screening involves testing for disease in people without symptoms, with the primary purpose of reducing mortality from the target disease, in this case cancer. In addition to its effect on length of life, screening also has other important consequences, including the use of economic resources (usually an increase in health expenditure) and implications for the quality of life, both positive and negative.

Cancer is always a potentially lethal disease, therefore the primary goal of screening and treating patients is to save lives. Public health policies related to cancer screening are invariably initiated, managed and evaluated with the aim of reducing mortality. Mortality is therefore the most important indicator of effectiveness.

Screening is appropriate when a cancer has a detectable preclinical phase during which it can be treated to prevent progression to overt, clinically detactable disease, (Cole \& Morrison, 1978). The detectable preclinical phase is known as the sojourn time (Day \& Walter, 1984). Its duration varies 
according to the natural progression of the disease, uptake of screening, access to diagnosis and the characteristics of the screening test.

An ideal screening programme should reduce the burden of disease in terms of death and morbidity, and/or improve the quality of life. Screen-detected cancer cases should have a better prognosis than those detected clinically, because the disease will have been treated at an earlier stage. Yet screening will always have some adverse effects. Screen-detected cases often include borderline abnormalities; some of these fulfil the histological criteria for malignancy, but would not progress even if left untreated, and would remain clinically indolent (Furihata \& Maruchi, 1969; Hugosson et al., 2000; IARC, 2002; IARC, 2005). Any screening programme will disclose such abnormalities so one of the adverse effects of screening is overdiagnosis, i.e. detection of indolent disease, and unnecessary treatment (overtreatment).

If a disease can be successfully treated after it has become clinically diagnosed, there is no need for screening.

Screening should not be applied to untreatable diseases.

\section{Evaluating the effectiveness of screening}

A screening programme should have high sensitivity and specificity. Sensitivity is the probability that the programme will detect all cases in the detectable preclinical phase among those screened. Specificity is the probability that the test will correctly identify those who do not have the disease. This is important because it is essential to minimize the number of false positives - healthy persons incorrectly identified as having the disease. These and other measures of performance depend on factors such as the accuracy of the screening test; the processes used to confirm positive results; attendance rates; the interval between successive screening tests; and the success of referral for diagnostic confirmation of screen-positive cases.

Screening can be described in terms of process and outcome measures. For instance, the process indicators in a mammography-based programme for breast cancer include coverage of the target population, identification of preclinical breast cancer and achieving a more favourable stage distribution than that seen without screening. However, an evaluation cannot be based on process indicators alone. These are necessary but not sufficient requirements for effectiveness.

Case detection by screening and a favourable stage distribution may simply indicate overdiagnosis or length-biased sampling, that is the tendency of 
screening to detect preferentially the slower-growing tumours (Feinleib \& Zelen, 1969).

Overdiagnosis is common when screening for preinvasive lesions of the cervix uteri or for prostate cancer. This is because of the high prevalence of preinvasive or indolent lesions during the detectable preclinical phase. Initial results from spiral computed tomography (CT) to screen for lung cancer suggest that it also leads to overdiagnosis.

Screening detects a disproportionate number of slow-growing cancers compared with normal clinical diagnosis. Thus, screen-detected cancers tend to have more favourable survival than clinically detected disease (Feinleib \& Zelen, 1969). As far as possible, evaluations should be designed to eliminate the consequences of length bias.

Lead time (Hutchison \& Shapiro, 1968) is the amount of time by which the diagnosis of disease is brought forward compared with diagnosis in the absence of screening. By definition, an effective screening programme gives some lead time, because earlier diagnosis is a requirement for achieving the goals of screening. Therefore, even if screening does not postpone death, survival from the time of diagnosis is, on average, longer for a screen-detected case than for one that is detected clinically. Comparison of survival between screen-detected and symptom-detected patients is therefore biased unless it is corrected for lead time. The methods available for correction are crude (at best) and, in general, survival remains an invalid indicator of the effectiveness of screening.

Process indicators cannot be used to estimate effectiveness, and evaluation should focus on the outcome - mortality from cancer. However, screening programmes also affect morbidity and (more broadly) the quality of life. Such effects should be examined in screening decisions but considered separately from process indicators.

Process measures, such as the proportion of all surgical procedures that are tissue-conserving (breast-conserving surgery and conization of the cervix uteri), are also invalid indicators of effect because they do not capture the main objective of screening - mortality reduction.

A randomized controlled trial (RCT), with mortality as its end-point, is the optimal and often the only valid means of evaluating the effectiveness of a screening programme. Cohort and case-control studies are often used as substitutes for RCTs when evaluating screening programmes. Most evidence on the effectiveness of screening programmes stems from comparisons of time trends and geographical differences between populations that were subjected to screening of variable intensity. However, the non-experimental approaches 
remain quite crude and insensitive and do not provide a solid basis for decision-making.

Effectiveness trials provide efficacy estimates when corrected for non-response and selection by attendance (Cuzick, Edwards \& Segnan, 1997). For instance, screening for colorectal cancer with the faecal occult blood (FOB) test was evaluated in randomized trials in Denmark and England. The estimates for effectiveness were 18\% in Denmark (Jörgensen, Kronborg \& Fenger, 2002) and $12 \%$ in England (Hardcastle, Chamberlain \& Robinson, 1996). After correction for attendance in the first round and selection (mortality difference between non-attenders and controls), the efficacy estimates were $24 \%$ in Denmark and $32 \%$ in England.

Intervention studies without control groups (also called demonstration projects or single-arm trials) and other non-experimental designs (cohort and case-control studies) have been proposed for the evaluation of mass screening programmes, but inherent biases are involved in all of these approaches. A randomized approach must always be considered the gold standard.

Screening programmes can be introduced as a public health policy in an experimental fashion, with comparison of screened and unscreened groups allocated at random. A newly introduced programme is unlikely to cover the total population immediately because resources may limit availability to the entire target population. Under such circumstances, screening may be limited to a randomly allocated sample of the population, rather than a self-selected or haphazardly selected fraction. As long as the resources available cover only a proportion of the population, it is ethically acceptable to carry out a randomized trial. The trial does not withhold screening from anybody, but gives a priori an equal chance to everyone in the target population. In this context, the equipoise (lack of firm evidence for or against an intervention), an ethical requirement for conducting a randomized trial, gradually disappears as evidence is accrued within the programme. For those planning public health services, this will provide the most reliable basis for providing or withholding new screening activities.

\section{Organizing a screening programme}

Screening is an umbrella term covering a range of activities that starts with defining the target population and extends to the treatment and follow-up of screen-detected patients. A screening programme links all these activities into a coherent sequence. 
Table 4-1 Components of cancer screening programmes

1. Definition of target population

2. Identification of individuals

3. Measures to achieve sufficient coverage and attendance, e.g. personal invitation

4. Test facilities for collection and analysis of screen material

5. Organized quality-control programme for obtaining screen material and its analysis

6. Adequate facilities for diagnosis, treatment and follow-up of patients with screen-detected disease

7. Referral system linking the persons screened with laboratories (providing information about normal screening tests) and clinical facilities (responsible for diagnostic examinations following abnormal screening tests and management of screen-detected abnormalities) Coordination

8. Monitoring, quality control and evaluation of the programme: availability of incidence and mortality rates for the entire target population, and for attenders and non-attenders respectively.

Different cancer screening programmes consist of different components (Table 4-1).

Screening can be opportunistic (spontaneous, unorganized) or organized (mass screening, screening programmes). The major differences lie in the level of organization and planning, and the systematic nature and scope of the activity. The components described in Table 4-1 are characteristics of organized rather than opportunistic screening.

The age range to be covered and the screening interval are major organizational considerations in any screening programme. For example, in western populations with a similar risk of disease and available resources, cervical cancer screening policies range from annual smears from the start of sexual activity to a cervical smear every five years in the age range $30-55$ years. Hence there is a ten-fold difference in the cumulative number of tests over a lifetime.

Selective screening involves applying the screening test to the proportion of the population that is known to be at above-average risk for disease. The purpose of screening only high-risk groups is to reduce the resources required and to limit any adverse effects of the test. A selective screening programme should detect a substantial proportion of the disease in the entire target population, i.e. the majority of the entire disease burden should appear in the high-risk group. Of course, all screening programmes are selective to some degree, according to age and sex, but usually the term is applied to selection by other parameters, e.g. screening for liver cancer in those infected with hepatitis B. 
So far, selective screening based on high-risk populations defined by aetiological risk factors has failed in cervical and breast cancer screening. Programme sensitivity has been low and a substantial proportion of the disease in the total target population has occurred in the low-risk group outside the screening programme. Existing methods of selective screening, based on risk factors, are not likely to be sufficiently valid to be incorporated into public health policy, except in countries with very few resources, where the alternative is not to screen at all. The following section examines the evidence in relation to specific cancers.

\section{Screening for cervical cancer}

Cervical cancer: disease burden and natural history

Cervical cancer is the second most common cancer among women worldwide. The great majority of the disease burden occurs in developing countries (Sankaranarayanan \& Ferlay, 2006). In 2004, approximately 31000 women in the EU developed cervical cancer and almost 14000 died from it (Arbyn, Autier \& Ferlay, 2007).

Virtually all cases of cervical cancer are a consequence of infection with human papilloma virus (HPV), but most infections clear spontaneously within twelve months or less. Persistence of infection is a feature common to oncogenic HPV types. Cervical cancer develops gradually, progressing through a series of precursor lesions from mild abnormality (atypia) into more aberrant lesions (dysplasia) and eventually malignant changes (initially in situ, then microinvasive and, finally, frankly invasive carcinoma). The detectable preclinical phase of cervical cancer has been estimated to be as long as 12 to 16 years.

Prognosis for local disease is good (relative survival around $90 \%$ at five years); advanced disease generally has a poor outcome (relative survival for stage IV disease is about $10 \%$ at five years). Overall, the five-year survival rate has been $67 \%$ in Europe (Berrino et al., 2007).

\section{Screening for cervical cancer}

The objective of cervical cancer screening is to reduce both incidence and mortality. A successful screening programme detects early, preinvasive lesions during the preclinical detectable phase and is able to reduce deaths by preventing the occurrence of invasive cancer. Diagnostic assessment requires colposcopy examination, with assessment of morphological features of the cervix as well as histological evaluation. 
The value of the Papanicolaou (Pap) screening test in reducing the risk of invasive cancer and mortality has been firmly established. It is estimated that regular screening reduces the risk of cancer by $80 \%$ to $98 \%$ (Olesen, 1988 ; WHO 1986). Organized screening programmes for cervical cancer using Pap smears have been shown to be more effective than opportunistic or nonorganized screening. Opportunistic screening typically misses the women at greatest risk (Anttila et al., 2004).

The effectiveness of cytological smears in cervical cancer screening has never been established with current, methodologically stringent evaluation criteria. However, there is extensive and consistent evidence showing reductions in both incidence of, and mortality from, invasive carcinoma. This is dependent on a well-organized screening programme.

In Finland, the population-based cervical cancer screening programme which began in 1963 achieved a 60\% reduction in the incidence of cancer at 10 years (Nieminen, Kallio \& Hakama, 1995). In Norway, a population-based nationwide cervical cancer screening programme was introduced in 1995. Two years later the incidence of invasive cancer was 22\% lower (Nygard, Skare \& Thoresen, 2002). In the United Kingdom the incidence of cervical cancer in women aged 20-69 years fell by 33\% between 1991-1993 and 1998-2000; mortality fell by $36 \%$ over the same period (Canfell, Sitas \& Beral, 2006). Conversely, the incidence of invasive cancer increased in an area of Denmark where organized screening had been discontinued (Lynge, 1998).

Most screening programmes start with women aged between 18 and 30 years and are discontinued after age 60 to 70 . In some programmes, the frequency of screening varies according to the individual's initial result, either starting with annual screening and increasing the interval after negative results or, conversely, initially offering a longer, three-year to five-year interval that is shortened if there is any abnormality (Olesen, 1999).

Other screening methods include direct visualization of the cervix, liquidbased cytology and HPV screening. Visual inspection was shown to be an effective method for reducing the risk of invasive disease and death in developing countries (Sankaranarayanan et al., 2007).

\section{Adverse effects of screening for cervical cancer}

Overdiagnosis of preinvasive lesions (i.e. detection and treatment of changes that would not have progressed into malignancy) is common in cervical cancer screening because only a small proportion of preinvasive lesions will develop into a cancer, even if left untreated. The cumulative risk of an abnormal screening test is relatively high compared with the lifetime risk of cancer in the 
absence of screening (10-15\% or higher versus approximately 3\%). Even the probability that preinvasive lesions such as cervical intraepithelial neoplasia will require treatment may be twice as high as the risk of cervical cancer. Treatment also has several adverse effects, such as a predisposition to complications of pregnancy following surgical treatment of the cervix, and infertility following hysterectomy.

\section{Status of cervical screening in the EU}

Almost all EU countries have a screening policy for cervical cancer. However, there are major variations in how the screening is organized, the type of screening activities, the targeted age range and the recommended screening interval, as well as payment strategies. A review in 2004 (Mackay et al, 2006) showed that national screening programmes were in place in the Nordic countries, the United Kingdom, Latvia, Slovenia, the Netherlands and Hungary. Regional screening programmes were operational in Spain, Portugal, Italy, Romania, Czech Republic, Austria and Belgium. Pilot programmes existed in France, Greece, Ireland and Estonia. No population-based screening programme was in place in Germany, although there was a screening policy.

In many regions or countries there are inadequacies in the population targeted, the registration of subjects, the evaluation or monitoring of the programme and the choice of screening interval. The recommended screening interval ranges between three and five years in most EU countries for which information is available. Some countries or regions recommend an excessive number of smears, with consequent potential for overdiagnosis and overtreatment. Similarly, the population covered by the screening programmes varied between 30\% in Slovenia and 100\% in the Nordic countries and Italy (Anttila et al., 2004).

EU recommendations state that cervical cancer screening should be offered on a population basis in organized screening programmes. Pap smear screening for cervical abnormalities should start by the age of 30 (at the latest) and definitely not before the age of 20 (Council of the European Union, 2003).

Detailed European guidelines on quality assurance screening programmes have been developed (European Cancer Network). Centralized data systems are essential for monitoring and evaluating the effectiveness of such programmes.

\section{HPV and cervical cancer}

As noted above, HPV is the principal cause of cervical cancer. At least 20 of the many types of HPV are regarded as oncogenic (cancer-causing). 
Commercially available tests based on nucleic acid hybridization can identify more than 10 different types. No trials comparing the effectiveness of HPV testing with cytological smears have been completed, but preliminary findings indicate that while HPV screening is likely to be at least as effective as screening based on Pap smears, it is also likely to have more adverse effects, including lower specificity.

The development of a vaccine against HPV infection is likely to have major public health implications. It has the potential to influence the conditions in which screening operates, possibly reducing the demand for cervical cancer screening by reducing the risk of disease. This may take at least one generation to achieve.

\section{Screening for breast cancer}

\section{Breast cancer: disease burden and natural history}

Breast cancer is the most common cancer among women, with increasing incidence in most populations. In 2006, there were about 430000 newly diagnosed cases in Europe (about 14\% of the total cancer burden) and almost 132000 breast cancer deaths (Ferlay et al., 2007).

Autopsy studies indicate that carcinoma in situ (CIS) of the female breast occurs frequently. About $20 \%$ of women will develop CIS during their lifetime but only a small fraction of these are diagnosed (Ottesen, 2003). The mean sojourn time has been estimated at two to eight years but this tends to be longer at older ages and may depend on the histological type.

The prognosis of breast cancer is relatively favourable. Five-year relative survival of more than $80 \%$ has been reported for women diagnosed during 1995-99 in Europe (Berrino et al., 2007).

\section{Screening for breast cancer}

In screening, the primary target lesion is early invasive cancer. However, ductal carcinoma in situ is also detected with up to a fifth of the frequency of invasive cancer.

Mammography involves radiological imaging of the breast (either one or two views) read by one or two radiologists. A screen-positive finding is a lesion that is suspicious for breast cancer. Two views are likely to increase the sensitivity by approximately $20 \%$, with the greatest incremental benefit for detection of small cancers among women with dense breast tissue. Some screening programmes use two views at the first screening and only one (mediolateral 
Table 4-2 Randomized trials evaluating mortality effects of mammography screening

\begin{tabular}{llrlrl}
\hline Reference & Setting & $\begin{array}{r}\text { Sample } \\
\text { size }\end{array}$ & $\begin{array}{l}\text { Age } \\
\text { range }\end{array}$ & $\begin{array}{r}\text { Follow-up } \\
\text { (years) }\end{array}$ & $\begin{array}{r}\text { Mortality }^{*} \\
\left(10^{-5}\right)\end{array}$ \\
\hline Shapiro, 1994 & Greater New York & 60995 & $40-64$ & 18 & $23 / 29$ \\
Andersson \& Janzon, 1997 & Malmö & 42283 & $45-70$ & 19 & $45 / 55$ \\
Andersson \& Janzon, 1997 & Malmö & 17793 & $43-49$ & 9 & $26 / 38$ \\
Tabar et al., 2000 & Kopparberg & 56448 & $40-74$ & 20 & $27 / 33$ \\
Nystrom et al., 2002 & Östergötland & 76617 & $40-74$ & 17 & $30 / 33$ \\
Alexander et al., 1999 & Edinburgh & 52654 & $45-64$ & 13 & $34 / 42$ \\
Miller et al., 2002 & Canada & 50430 & $40-49$ & 13 & $37 / 38$ \\
Miller et al., 2000 & Canada & 39405 & $50-59$ & 13 & $50 / 49$ \\
Nystrom et al., 2002 & Stockholm & 60117 & $40-64$ & 15 & $15 / 17$ \\
Bjurstam, 2003 & Gothenburg & 51611 & $39-59$ & 13 & $23 / 30$ \\
Hakama et al., 1997 & Finland & 158755 & $50-64$ & 4 & $16 / 21$ \\
\hline
\end{tabular}

* Mortality rate per 100000 in screened/unscreened groups

oblique) in subsequent screens. Double reading appears to increase both the recall rate and the detection of breast cancer by about 10\%. Diagnostic assessment requires an initial needle biopsy or excision (open surgical) biopsy. The effectiveness of mammography screening has been documented in a number of randomized trials (Table 4-2). These have shown consistent mortality reductions of 20-35\% among women in the 50-69 age range.

In Sweden, for example, the reduction in breast cancer mortality after 15 to 20 years of follow-up, ranged from 12\% (Stockholm trial) to $18 \%$ (Kopparberg and Malmö trials). The trial in Edinburgh, Scotland reported a $21 \%$ difference in breast cancer mortality between intervention and control groups after 14 years of follow-up.

Existing randomized trials have been criticized for methodological weaknesses (Gotzsche \& Olsen, 2000; Olsen \& Gotzsche, 2001), on the grounds that inadequate randomization and exclusions after randomization produced a lack of comparability between the trial arms. A systematic review that excluded studies with possible shortcomings finally evaluated only two trials; these showed no benefit from breast cancer screening (Olsen \& Gotzsche, 2001). It was also argued that breast cancer mortality is not a valid end-point for screening trials. These criticisms would be a serious challenge to the scientific basis of mammography screening if they were generally accepted as accurate, but their validity has been firmly rebutted by several investigators, both in open debates and in peer-reviewed publications. The critics' dismissal of all the positive randomized trials is generally considered to be inappropriate because, essentially, it is based on a mechanistic evaluation of technical criteria that are of questionable relevance to the results. 
Other screening tests include: digital mammography, which has been adopted recently; magnetic resonance imaging; clinical breast examination, and breast self-examination.

No study has evaluated the effect of digital mammography on breast cancer mortality, and no randomized trial has compared the performance of magnetic resonance imaging and mammography. Likewise, no randomized trial has evaluated the effectiveness of clinical breast examination alone, but it was included in the intervention arm of some trials. It may increase the sensitivity of screening if used as an ancillary test in a mammography screening programme. Lastly, no reduction in breast cancer mortality has been reported in the two trials that estimated the effectiveness of breast self-examination (Gao et al., 2006).

\section{Adverse effects of breast cancer screening}

Overdiagnosis, and subsequent unnecessary treatment of lesions that would not have progressed, may not be as common in breast cancer screening as for several other cancer types. Estimates of overdiagnosis have ranged from 3\% to $5 \%$.

Mammography delivers a small dose of ionizing radiation (1-2 mGy) to the breast. This can be expected to increase the risk of breast cancer but the excess risk is likely to remain very small (1-3\% or smaller increase in relative risk), well below the advantage gained by the reduction in breast cancer mortality. On the other hand, early diagnosis can improve the quality of life by allowing a wider range of treatment options and the possibility of avoiding radical surgery (and possibly adjuvant chemotherapy).

\section{Status of mammography screening in the EU}

The Council of Europe recommends population-based, organized mammography screening for breast cancer in women aged 50-69 using screening programmes that comply with European guidelines on quality assurance (Council of the European Union, 2003).

Screening programmes are organized either regionally or nationally, incorporating quality-assurance mechanisms for both radiology and pathology services. Most programmes target women in the 50-69 age group, with a twoyear interval between tests. Several northern European countries have achieved $80 \%$ participation and recall rates of $1 \%$ to $8 \%$.

In addition to the randomized trials described, screening programmes have been evaluated in a few notable studies. In Finland, breast cancer mortality 
was compared between women aged 50-59 who had been invited to attend a breast cancer screening programme and those who had not. This yielded a $24 \%$ reduction in breast cancer mortality but this was not statistically significant. In the Netherlands, a statistically significant reduction in breast cancer mortality was reported following the introduction of mammography screening among women aged 50-69. A 19\% reduction was found between the period before the introduction of screening and the end of follow-up (Fracheboud et al., 2004).

An evaluation of mammography screening in seven Swedish counties, begun between 1978 and 1990, targeted women mostly aged 40 to 69. This found a significant $32 \%$ reduction in breast cancer mortality in counties with a 10 -year history of screening, and an $18 \%$ reduction in counties with shorter screening histories (Duffy et al., 2002).

The effectiveness of the breast screening programme in England and Wales was assessed by comparing mortality from breast cancer after the introduction of the programme with that expected in the absence of screening, predicted using an age cohort model. Women aged 50 to 69 were invited with a threeyear screening interval. Breast cancer mortality fell by $21 \%$ after the introduction of screening, but most of the decline was attributed to improvements in treatment. The estimated reduction in breast cancer mortality gained from screening was $6 \%$.

These assessments are based on non-randomized studies, which are more prone to bias than randomized trials. However, the results are generally consistent with the mortality reduction observed in screening trials, suggesting that the results of the trials are not atypical.

\section{Screening for colorectal cancer}

\section{Colorectal cancer: disease burden and natural history}

Colorectal cancer ranks as the second most common cause of cancer death in Europe. It accounted for 13\% (413 000 incident cases) of all newly diagnosed cancers and for about 20\% (335 000 deaths) of cancer deaths in 2006 (Ferlay et al., 2007).

The majority of colorectal carcinomas are thought to arise from adenoma, either flat or polypoid. Adenomas (particularly those with a diameter of $1 \mathrm{~cm}$ or more, exhibiting dysplasia) and early carcinoma comprise the principal targets of screening. Screening can either reduce incidence by removal of premalignant lesions or increase it by earlier detection of invasive cancer, with 
possible overdiagnosis. The detectable preclinical phase has been estimated as two to six years.

Diagnostic examination always involves colonoscopy, which also allows removal of polyps. The prognosis of colorectal cancer is moderately good. A five-year relative survival rate of $54 \%$ has been reported in Europe (Berrino et al., 2007).

\section{Screening for colorectal cancer}

Several screening methods are available for colorectal cancer screening, including faecal occult blood (FOB) testing, sigmoidoscopy, colonoscopy and double-contrast barium enema.

FOB testing is based on detection of haemoglobin in stools using guaiacimpregnated patches. An oxidative reaction (pseudoperoxidase activity) results in a colour change that is detectable on inspection. Hemoccult II® is the most commonly used test but is not specific for human blood and may yield false positives in those who have eaten undercooked meat recently. Other tests detect human haemoglobin immunologically but they are more expensive. Rehydration (adding water to the specimen) can be used to increase the detection rate, but this also increases the number of false positive results. For screening, usually two specimens are obtained on three consecutive days.

Several randomized trials have evaluated the effectiveness of FOB screening. Both one- and two-year screening intervals have been used and most studies have targeted the 45-75 age group. Three randomized trials evaluating incidence and mortality have been completed (Table 4-3).

A $6-18 \%$ reduction in mortality is found consistently with biennial screening. A meta-analysis based on the trials available in 1998 estimated the pooled reduction in mortality to be $16 \%$ (Towler et al., 1998).

The Nottingham trial used biennial FOB tests (Hemoccult II®) and three to six rounds of screening. This found $2.1 \%$ of individuals to be screen-positive at the first round and $2.7 \%$ at subsequent rounds. Adenomas were identified in $0.8-1 \%$ of those screened; cancer was identified in $0.2-0.5 \%$. However, there was no reduction in colorectal cancer incidence in the screened group (151 vs. 153 per 100 000). In contrast, those screened experienced a significant $19 \%$ reduction in mortality (70 vs. 81 per 100000$)$ after a median 11 years of follow-up.

The Danish Funen study used a biennial FOB test (Hemoccult II@) protocol over a total of nine screening rounds $-1 \%$ of subjects were screen-positive at the first screen and, on average, $1.2 \%$ in subsequent screens. The mortality 
Table 4-3 Randomized trials evaluating mortality effects of colorectal cancer screening based on faecal occult blood (FOB) testing

\begin{tabular}{|c|c|c|c|c|}
\hline Reference (setting) & $\begin{array}{r}\text { Sample } \\
\text { size }\end{array}$ & $\begin{array}{l}\text { Age } \\
\text { range }\end{array}$ & $\begin{array}{l}\text { Length of } \\
\text { follow-up } \\
\text { (years) }\end{array}$ & Mortality $(R R)^{\star *}$ \\
\hline $\begin{array}{l}\text { Mandel et al., } 1999 \\
\text { (Minnesota, USA) }\end{array}$ & $46551^{*}$ & $50-80$ & 15 & $0.67(0.51-0.87)$ \\
\hline $\begin{array}{l}\text { Scholefield et al., } 2002 \\
\text { (Nottingham, UK) }\end{array}$ & 152850 & $45-74$ & 11 & $0.87(0.78-0.97)$ \\
\hline $\begin{array}{l}\text { Kronborg et al., } 2004 \\
\text { (Fynen, Denmark) }\end{array}$ & 61933 & $45-75$ & $\begin{array}{l}11 \\
14\end{array}$ & $\begin{array}{l}0.89(0.78-1.01) \\
0.82(0.69-0.97)\end{array}$ \\
\hline
\end{tabular}

* Three arms: annual and biennial screening with control

** Ratio of the mortality rates in the screening and control arms of each trial. Value less than 1.0 indicates a beneficial effect

reduction was $11 \%$ after a mean follow-up of 14 years (99 vs. 110 per 100000 , including deaths from screening-related interventions). No decrease in colorectal cancer incidence was observed (206 vs. 202 per 100 000).

Other screening tests include flexible sigmoidoscopy, screening colonoscopy and the recently introduced faecal DNA analysis. Compliance with screening sigmoidoscopy has been $50 \%$ or lower but the detection rate is higher than in FOB testing, suggesting higher sensitivity. Several case-control studies have found that sigmoidoscopy significantly reduces mortality from colorectal cancer by between $60 \%$ and $80 \%$. However, the reduction in mortality achievable with sigmoidoscopy remains unclear - selection bias and other systematic errors may affect the results so the evidence is not as strong as that from randomized trials. A population-based randomized trial is under way in Norway and should provide important new information. This is comparing one sigmoidoscopy with no intervention in 20000 subjects aged 50-64.

Screening colonoscopy has the advantage of visualizing the entire colon but the procedure is expensive, involves substantial discomfort and has a risk of complications such as perforation of the bowel (reported in 1-2 patients per 10 000). No trials have evaluated the effectiveness of screening colonoscopy but demonstration projects, which lacked control groups, have reported detection rates of $5-10 \%$ for advanced neoplasia (carcinoma or large, dysplastic or villous adenoma). This is approximately one-third higher than for examinations covering only the distal colon.

Faecal DNA analysis has been introduced as a new option for colorectal cancer screening. Early results have shown good sensitivity and acceptable specificity, but no studies have been conducted to assess its effectiveness in reducing mortality. 


\section{Adverse effects of colorectal cancer screening}

The FOB test is safe, but false positive results require follow-up diagnostic examinations that cause inconvenience and some risk for the patient and incur costs for the health-care system. Some degree of overdiagnosis is likely, because not all precursor lesions will advance to cancer. However, the morbidity associated with the removal of polyps is low.

\section{Status of screening for colorectal cancer in the EU}

The Council of Europe recommends FOB screening for colorectal cancer in men and women aged 50-74 (Council of the European Union, 2003). Quality-assurance guidelines for screening are being developed by a consortium of experts supported by the European Commission, using methods similar to those employed for breast and cervical cancer.

The existing national screening programme in Finland is expanding gradually and by randomization. In 2007, about one third of the Finnish population was covered. Regional initiatives have been implemented in several other EU countries including France, Italy, the Netherlands, Poland and the United Kingdom.

In conclusion, FOB testing has been shown to reduce mortality from colorectal cancer in several randomized trials. It appears to be an underutilized opportunity for cancer control. Other screening modalities are also available, but there is very limited evidence of their effectiveness.

\section{Screening for prostate cancer}

\section{Prostate cancer: disease burden and natural history}

Prostate cancer has increased rapidly in the past 10 to 15 years in most industrialized countries. Currently it is the most common cancer among men in several countries. In Europe, there were about 346000 newly diagnosed cases of prostate cancer in 2004 ( $20 \%$ of all incident cases of cancer) and about 87000 deaths (9\% of all cancer deaths) (Ferlay et al., 2007).

The target lesion for screening is early invasive prostate cancer; the diagnosis is formally confirmed by prostate biopsy. The natural course of prostate cancer is highly variable, with some very indolent, slow-growing tumours and some that are highly aggressive. Premalignant lesions such as prostatic intraepithelial neoplasia (PIN) exist, but are not strongly predictive of prostate cancer. They are not considered indications for treatment. 
Latent prostate cancer is a common autopsy finding: it has been detected in more than $10 \%$ of men dying before the age of 50 years, and much more frequently than that in older men. The common occurrence of indolent prostate cancer is a clear indication of the scope for overdiagnosis.

The mean lead time in prostate cancer has been estimated at 6 to12 years. Prognosis is favourable, with five-year relative survival rates of about $77 \%$ in Europe (Berrino et al., 2007).

\section{Screening for prostate cancer}

Prostate specific antigen (PSA) is a serine protease (enzyme) secreted by the prostate gland. It is usually found in low concentrations in serum, with levels increased by prostate diseases such as benign prostatic hyperplasia, prostatitis or prostate cancer.

Two large randomized trials are being carried out - one in Europe, the other in the United States. The European Randomized Study of Screening for Prostate Cancer (ERSPC, http://www.erspc.org/) includes eight centres in the Netherlands, Finland, Sweden, Italy, Belgium, Spain, Switzerland and France. More than 200000 men aged 50-74 years have been recruited so far. The first analyses of the effect on mortality are planned for 2010 .

In the United States, the Prostate, Lung, Colorectal and Ovarian Cancer Screening Trial (PLCO) recruited 76705 men aged 55-74 for its prostate component between 1993 and 2001. Both serum PSA and digital rectal examination are used as screening tests. No mortality results are available yet.

Several published ecological studies and time-series analyses have correlated the frequency of PSA testing (or the incidence of prostate cancer as a surrogate for PSA testing) with prostate cancer mortality. The results have been inconsistent. The shortcomings inherent in these approaches preclude firm conclusions.

Digital rectal examination is the other main screening test. Its impact on death from prostate cancer has been evaluated in five case-control studies. These have not yielded consistent results: two indicated a 30-50\% reduction in risk; the other three failed to show a benefit. The lack of a clear effect is thought to be due to the fact that digital rectal examination detects only cancers that are large enough to be palpable. In many cases the cancer has spread beyond the prostate capsule by this stage and is no longer curable. Hence, the chief limitation of digital rectal examination is its low sensitivity for the detection of early disease. 


\section{Adverse effects of prostate cancer screening}

Overdiagnosis is potentially a major problem in prostate cancer screening. It has been estimated that $30-45 \%$ of cancers detected by screening would not have been diagnosed during the individual's lifetime in the absence of screening. Treatment of prostate cancer has several major adverse effects, including high rates of erectile dysfunction and urinary incontinence following surgery and irritation of the rectum and bladder (chronic radiation cystitis and radiation proctitis) following radiotherapy.

In summary, prostate cancer screening by serum PSA or any other test has not yet been shown to reduce mortality. The use of serum PSA levels as a screening test should be restricted to randomized trials. Such trials are ongoing and should provide important evidence.

\section{Screening for other cancers}

\section{Lung cancer}

The target lesion for lung cancer screening is early, resectable (stage 1) carcinoma. Conclusive diagnosis of early lung cancer is based on biopsy, usually obtained by bronchoscopy for central tumours and excision biopsy for more peripheral tumours. Survival is among the worst for any cancer type, with five-year relative survival of approximately $12 \%$ in Europe (Berrino et al., 2007).

The screening protocols available for lung cancer include screening with chest $\mathrm{X}$-rays with or without sputum cytology and spiral low-dose CT. Chest X-rays and sputum cytology are ineffective in reducing mortality from lung cancer but the effectiveness of screening based on spiral CT remains unclear.

\section{Ovarian cancer}

The natural history of ovarian carcinoma is not well understood, particularly the relative frequency of cancers developing from benign or borderline lesions or de novo. The duration of any detectable preclinical phase is also unknown. The relatively poor average five-year survival rate (42\%) in Europe reflects the often advanced stage at diagnosis (Berrino et al., 2007).

Screening tests include transvaginal or transabdominal ultrasound for imaging, and serum CA-125 as a biochemical marker. There is no evidence that ovarian cancer screening can reduce mortality. Preliminary results from non-randomized studies are not encouraging - the sensitivity is low (too many 
cases missed) and false positive findings are common (too many healthy women identified as having disease).

\section{Oral cancer}

Oral cancer is one of the leading cancers in some areas of the world, largely due to tobacco chewing. A recent cluster-randomized trial of visual inspection for oral cancer achieved a $20 \%$ reduction in mortality among more than 190000 subjects (Sankaranarayanan et al., 2005).

\section{Cutaneous melanoma}

Incidence has been increasing rapidly in most industrialized countries for several decades. It now ranks among the ten most common cancers in several European countries. Some of this increase could be due to more active casefinding and changes in diagnostic criteria, but this does not seem a likely explanation (van der Esch et al., 1991). Mortality has not shown a similar increase.

Survival is favourable if cutaneous melanoma is detected at an early stage. A substantial proportion of melanomas (approximately one fifth) arise from atypical naevi. Visual inspection can be used to identify early melanoma (or premalignant lesions); diagnostic assessment requires a skin biopsy. No randomized trial has been conducted to evaluate the effect of screening on melanoma mortality.

\section{Neuroblastoma}

Neuroblastoma is an uncommon childhood tumour. Screening can be performed via a urine test for the catecholamine metabolites HMA and VMA (homovanillic acid and vanillylmandelic acid) secreted by most (60-80\%) of these tumours. The effects of screening have been evaluated by comparing screened and unscreened cohorts in Germany, Canada and Japan. Screening has been associated with a two- to six-fold increase in the incidence rate, with cases being diagnosed at earlier ages. Unfortunately, this has not been counterbalanced by a reduction in incidence at older ages. No reduction in mortality or in the occurrence of advanced disease has been demonstrated. Neuroblastoma screening in Japan has been stopped as a result.

\section{Liver cancer}

Serum alpha-fetoprotein (AFP) levels and ultrasound have been used as a combined screening test for hepatocellular cancer. Two randomized trials in 
high-risk subjects have been carried out in China among chronic carriers of hepatitis B virus, who are at greatly increased risk of liver cancer. The smaller study, among 5500 men in Qidong county, found a non-significant 20\% reduction with six-monthly AFP tests. The larger trial (18 000 people) using twice-yearly AFP tests and ultrasonography, achieved a one-third reduction in five-year mortality from liver cancer.

\section{Gastric carcinoma}

Fluoroscopic imaging (photofluorography) and endoscopy have been used to screen for stomach cancer. Several case-control studies and two cohort studies have evaluated the effect of gastroscopy, but have not provided consistent results. No randomized trials have been reported so there is insufficient evidence of effectiveness.

\section{Cancer screening guidelines}

A summary of the current evidence for cancer screening is provided in Table 4-4. Several international and national organizations have made recommendations for cancer screening (Table 4-5). These have been based on a variety of approaches from expert opinion and consensus-development conferences to more objective methods of evidence synthesis. In the EU, detailed quality-assurance guidelines are available for breast cancer and cervical cancer screening; guidelines for colorectal cancer screening are under development.

The different guidelines have some degree of consistency but also some variations. Some organizations have taken a stricter approach to evidence. For example, the American Cancer Society tends to adopt a low threshold for advocating screening. Similarly, medical specialty societies tend to be relatively eager to adopt screening recommendations (not shown in the table). The role of the organizations and the task of the working groups also affect the outcome - those with more responsibility for planning health-care services tend to apply more stringent evaluation criteria. Also, countries with publicly financed health care tend to be more conservative than those with fee-for-service financing systems.

\section{Conclusions}

Evidence on mortality effects from large randomized trials is required in order to establish the benefits of screening. Screening tests are available for many types of cancer, but either their effectiveness has not been evaluated adequately 
Table 4-4 Summary of evidence for cancer screening

\begin{tabular}{|c|c|c|c|c|c|}
\hline \multirow[b]{2}{*}{ Primary site } & \multirow[b]{2}{*}{$\begin{array}{l}\text { Screening } \\
\text { method }\end{array}$} & \multicolumn{2}{|c|}{ Efficacy } & \multicolumn{2}{|c|}{ Effectiveness } \\
\hline & & $\begin{array}{l}\text { Non- } \\
\text { randomized }\end{array}$ & Randomized & $\begin{array}{l}\text { Randomized } \\
\text { trial }\end{array}$ & $\begin{array}{l}\text { Service } \\
\text { screening }\end{array}$ \\
\hline \multirow[t]{3}{*}{ Cervical cancer } & Pap smear & & NA & NA & $0-80 \%$ \\
\hline & $\begin{array}{l}\text { Visual } \\
\text { inspection }\end{array}$ & & & $35 \%$ & NA \\
\hline & HPV testing & & NA & NA & NA \\
\hline Breast cancer & Mammography & & $35 \%$ & $15-25 \%$ & $6-20 \%$ \\
\hline \multirow[t]{3}{*}{ Colorectal cancer } & $\begin{array}{l}\text { Faecal occult } \\
\text { blood (FOB) }\end{array}$ & & $24 \%$ & $15 \%$ & NA \\
\hline & Sigmoidoscopy & & NA & NA & NA \\
\hline & Colonoscopy & & NA & NA & NA \\
\hline \multirow[t]{2}{*}{ Lung cancer } & $\begin{array}{l}\text { Chest X-ray } \pm \\
\text { sputum cytology }\end{array}$ & & None & & NA \\
\hline & Low-dose CT & & NA & NA & NA \\
\hline \multirow[t]{2}{*}{ Prostate cancer } & Serum PSA & & NA & NA & NA \\
\hline & $\begin{array}{l}\text { Digital rectal } \\
\text { examination }\end{array}$ & None & NA & NA & NA \\
\hline Oral cancer & $\begin{array}{l}\text { Visual } \\
\text { inspection }\end{array}$ & & NA & $20 \%$ & NA \\
\hline Liver cancer & $\begin{array}{l}\text { Serum AFP } \pm \\
\text { ultrasound }\end{array}$ & & NA & $20-33 \%$ & NA \\
\hline Ovarian cancer & $\begin{array}{l}\text { Ultrasound+ } \\
\text { CA } 125\end{array}$ & & NA & NA & NA \\
\hline
\end{tabular}

or a lack of effectiveness has been demonstrated. Even when efficacy trials (typically conducted in specialist centres with volunteer subjects) have been successful, pilot studies are still required to demonstrate the feasibility of mass screening. Also, an organized screening programme requires continual evaluation to ensure that the benefits are maintained. Ideally, this is achieved by incorporating a randomized design, comparing outcomes for subjects allocated randomly to early entry to the screening programme with the outcomes for those included later.

For two primary cancers, sufficient knowledge exists that a public health policy of screening is effective in reducing risk. The Pap test and visual inspection both reduce the incidence of invasive cervical cancer, while mammography reduces mortality from breast cancer.

Large randomized trials have shown the efficacy of screening - reducing mortality from colorectal cancer with the FOB test; from liver cancer with the AFP test and ultrasound; and from oral cancer with visual inspection. 


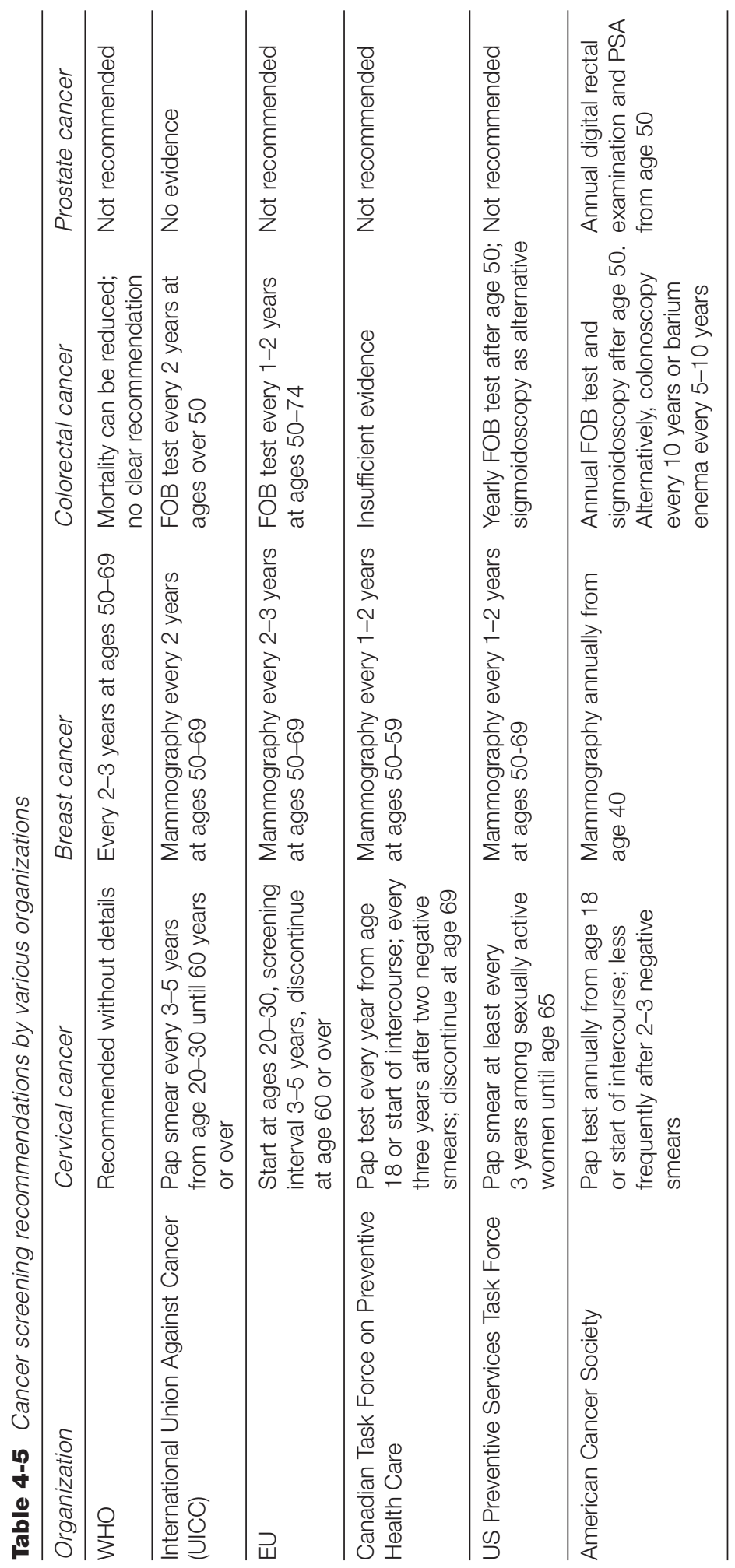


Currently, there is no evidence that these results are applicable in an effective mass screening programme.

Randomized trials have provided sufficient evidence that sputum cytology and X-ray screening for lung cancer will not reduce mortality from the disease. Evaluation of screened and unscreened cohorts for neuroblastoma deaths showed no reduction of mortality.

No randomized trials have been conducted for any other type of cancer, or other screening tests.

\section{REFERENCES}

Alexander FE et al. (1999). 14 years of follow-up from the Edinburgh randomised trial of breast cancer screening. Lancet, 353(9168):1903-1908.

Andersson I, Janzon L (1997). Reduced breast cancer mortality in women under age 50: updated results from the Malmo Mammographic Screening Program. J Natl Cancer Inst Monogr, (22):63-67.

Anttila A et al. (2004). Cervical cancer screening programmes and policies in 18 European countries. Br J Cancer, 91(5):935-941.

Arbyn M, Autier P, Ferlay J (2007). Burden of cervical cancer in the 27 Member States of the European Union: estimates for 2004. Ann Oncol, 18(8):1423-1425.

Berrino F et al. (2007). Survival for eight major cancers and all cancers combined for European adults diagnosed in 1995-99: results of the EUROCARE-4 study. Lancet Oncol, 8(9):773-783.

Bjurstam N et al. (2003). The Gothenburg breast screening trial. Cancer, 15;97(10):2387-2396.

Canfell K, Sitas F, Beral V (2006). Cervical cancer in Australia and the United Kingdom: comparison of screening policy and uptake, and cancer incidence and mortality. Med J Aust, 185(9):482-486.

Cole P, Morrison AS (1978). Basic issues in cancer screening. In: Miller AB ed. Screening in cancer. (UICC Technical Report Series, Vol. 40). Geneva, UICC:7-39.

Council of the European Union (2003). Council recommendation of 2 December 2003 on cancer screening, Official Journal of the European Union (2003/878/EC, L327/34- 38).

Cuzick J, Edwards R, Segnan N (1997). Adjusting for non-compliance and contamination in randomised clinical trials. Stat Med, 16:1017-1029.

Day NE, Walter SD (1984). Simplified models of screening for chronic disease: estimation procedures from mass screening programmes. Biometrics, 40(1):1-14.

Duffy SW et al. (2002). The impact of organized mammography service screening on breast carcinoma mortality in seven Swedish counties. Cancer, 95(3):458-469.

European Cancer Network. European Guidelines for Quality Assurance in Cervical Cancer Screening. (available at:http://www.cancernetwork.de/cervical/cerv_guidelines.htm, accessed 28 November 2007).

European Randomized Study of Screening for Prostate Cancer (ERSPC) web site (available at:http://www.erspc.org/, accessed 19 December 2007).

Feinleib M, Zelen M (1969). Some pitfalls in the evaluation of screening programs. Archives of Environmental Health, 19:412-415.

Ferlay J et al. (2007). Estimates of the cancer incidence and mortality in Europe in 2006. Ann Oncol, 18(3):581-592. 
Fracheboud J et al. (2004). Decreased rates of advanced breast cancer due to mammography screening in the Netherlands. Br J Cancer, 91:861-867.

Furihata R, Maruchi N (1969). Epidemiological studies on thyroid cancer in Nagano prefecture, Japan. In: Hedinger CE ed. Thyroid cancer. (UICC Monograph Series, Vol. 12). Berlin, Springer-Verlag:79.

Gao DL et al. (2006). Evaluation on the effect of intervention regarding breast self-examination for decreasing breast cancer mortality. Zhonghua Liu Xing Bing Xue Za Zhi, 27:985-990.

Gotzsche PC, Olsen O (2000). Is screening for breast cancer with mammography justifiable? Lancet, 355(9198):129-134.

Hakama M et al. (1997). Effectiveness of the public health policy for breast cancer screening in Finland: population based cohort study. Br Med J, 314(7084):864-867.

Hardcastle JD, Chamberlain JO, Robinson MHE (1996). Randomized controlled trial of faecal-occult-blood screening for colorectal cancer. Lancet, 348:1472-1477.

Hugosson J et al. (2000). Would prostate cancer detected by screening with prostate specific antigen develop intoclinical cancer if left undiagnosed? BJU Int, 85:1978-1984.

Hutchison GB, Shapiro S (1968). Lead time gained by diagnostic screening for breast cancer. J Natl Cancer Inst, 41(3):665-681.

IARC (2002). Breast cancer screening. IARC handbooks of cancer prevention, Volume 7. Lyon, IARC Press.

IARC (2005). Cervix cancer screening. IARC handbooks of cancer prevention, Volume 10. Lyon, IARC Press.

Jörgensen O, Kronborg O, Fenger C (2002). A randomised study on screening for colorectal cancer using faecal occult blood testing: results after 13 years and seven biennial screening rounds. Gut, 50:29-39.

Kronborg O, et al. (2004). Randomized study of biennial screening with a faecal occult blood test: results after nine screening rounds. Scand J Gastroenterol, 39(9):846-851.

Lynge E (1998). Mammography screening for breast cancer in Copenhagen April 1991-March 1997. Mammography Screening Evaluation Group, APMIS Suppl, 83:1-44.

Mackay J, Jemal A, Lee NC, Parkin DM (eds) (2006). The Cancer Atlas. Atlanta, Ga. American Cancer Society, 2006:70-71 (available at: http://www.cancer.org/downloads/AA/CancerAtlas22. pdf, accessed on 28 November 2007)

Mandel JS et al. (1999). Colorectal cancer mortality: effectiveness of biennial screening for fecal occult blood. J Natl Cancer Inst, 91(5):434-437.

Miller AB et al. (2000). Canadian national breast screening study-2: 13-year results of a randomized trial in women aged 50-59 years. J Natl Cancer Inst, 92(18):1490-1499.

Miller AB et al. (2002). Canadian national breast screening study-1: breast cancer mortality after 11 to 16 years of follow-up. Ann Intern Med 137: E-305?E-315.

Nieminen P, Kallio M, Hakama M (1995). The effect of mass screening on incidence and mortality of squamous and adenocarcinoma of cervix uteri. Obstet Gynecol, 85(6):1017-1021.

Nygard JF, Skare GB, Thoresen SO (2002). The cervical cancer screening programme in Norway, 1992-2000: changes in Pap smear coverage and incidence of cervical cancer. J Med Screen, 9(2):86-91.

Nystrom L et al. (2002). Long-term effects of mammography screening: updated overview of the Swedish randomised trials. Lancet, 359(9310): 909-919.

Olesen F (1988). A case-control study of cervical cytology before diagnosis of cervical cancer in Denmark. Int J Epidemiol 17:501-508.

Olesen F (1999). Detecting cervical cancer: the European experience. Hong Kong Med J, 5(3):272-274. 
Olsen O, Gotzsche PC (2001). Screening for breast cancer with mammography. Cochrane Database Syst Rev: CD001877.

Ottesen GL (2003). Carcinoma in situ of the female breast. A clinico-pathological, immunohistological and DNA ploidy study. APMIS Suppl:1-67.

Sankaranarayanan R, Ferlay J. (2006). Worldwide burden of gynaecological cancer: the size of the problem. Best Pract Res Clin Obstet Gynaecol, 20(2):207-225.

Sankaranarayanan R et al. (2005). Effect of screening on oral cancer mortality in Kerala, India: a cluster-randomised controlled trial. Lancet, 365(9475):1927-1933.

Sankaranarayanan R et al. (2007). Effect of visual screening on cervical cancer incidence and mortality in Tamil Nadu, India: a cluster-randomised trial. Lancet, 370:398-406.

Scholefield JH et al. (2002). Effect of faecal occult blood screening on mortality from colorectal cancer: results from a randomised controlled trial. Gut, 50(6):840-844.

Shapiro S (1994). Screening: assessment of current studies. Cancer, 74(Suppl. 1):231-238.

Tabar L et al. (2000). The Swedish two-county trial twenty years later. Updated mortality results and new insights from long-term follow-up. Radiol Clin North Am, 38(4):625-651.

Towler B et al. (1998). A systematic review of the effects of screening for colorectal cancer using the faecal occult blood test, hemoccult. Br Med J, 317(7158):559-565.

van der Esch EP et al. (1991). Temporal change in diagnostic criteria as a cause of the increase of malignant melanoma over time is unlikely. Int J Cancer, 47(4):483-489.

WHO (1986). Screening for cancer of the uterine cervix, 1ARC. Geneva, World Health Organization. 


\section{Chapter 5 \\ Drugs for cancer}

Karol Sikora

\section{Introduction}

Over the last twenty years a huge amount of fine detail has been amassed about the basic biological processes that become disturbed in cancer. These include the key elements of the disturbances in growth factor binding, signal transduction, gene transcription control, cell cycle checkpoints, apoptosis and angiogenesis related to the disorganized growth of cancer cells (Sikora, 2002). These have proved to be fertile areas to hunt for rationally based anticancer drugs and have produced a record number of novel compounds, currently in cancer treatment trials. A number of such targeted drugs are now licensed for routine clinical use, including rituximab, trastuzumab, imatinib, gefitinib, bevacizumab, lapatinib and cetuximab. Clearly there will be a marked shift in the types of agents used in the systemic treatment of cancer over the next decade. This will impose huge financial pressures on all health-care systems.

Currently, drugs are defined for use empirically and relatively ineffectively for different types of cancer. The new agents have precise targets and will revolutionize cancer-therapy prescribing. In future, a series of molecular lesions will be identifed in tumour biopsies and patients will receive.drugs that target these directly. The Human Genome Project provides a vast repository of comparative information about normal and malignant cells. The new therapies will be more selective, less toxic and given for prolonged periods sometimes for the rest of the patient's life. This radical overhaul of the provision of cancer care (Sikora, 2004) will make it more like the delivery of diabetes care. Community nursing and patient education will be as important as the chemotherapy protocols devised by the cancer centres.

Investment in more sophisticated diagnostics is required. Systems which examine multiple factors using complex bioinformatics such as genomics, proteomics, metabolomics and methylomics provide fascinating clues about disturbed growth. The development of simple, reproducible and cheap assays 
for specific biomarkers will produce a battery of tests to guide treatment choice and monitor its effectiveness. These companion diagnostics, or "theranostics", will be as important to innovative care as the new drugs themselves (Philip et al., 2007). Over the next decade it is likely that these new tests will be rooted firmly in tissue pathology; histopathologists are essential to move this exciting field forward. Ultimately, the fusion of tissue analysis and imaging technologies will offer the possibility for virtual biopsies of any part of the body (Del Vecchio et al., 2007).

Individual cancer risk assessment enables tailored prevention messages and a specific screening programme to detect early cancer, with far-reaching public health consequences. Cancer prevention drugs will be developed to reduce the risk of further genetic deterioration. The discovery of low-penetrance risk polymophisms detected by fast and cheap technologies will make it possible to band populations by their cancer risk (Velasquez \& Lipkin, 2007).

By the end of this decade the cost of sequencing an individual's total genome will fall to less than US\$1000. This will allow for sophisticated analyses of the relevant importance of genetics and lifestyle in an individual's development of cancer. The use of gene arrays to monitor serum for fragments of DNA containing defined mutations could ultimately develop into an implanted gene chip. Having detected a significant mutation, the chip would signal the holder's home computer to instigate a series of investigations based on the most likely type and site of the primary tumour.

The prevalence of cancer will increase as a result of improved survival and a shift to types of cancer with longer survival, such as prostate cancer. Some estimates suggest a three-fold increase in the number of people living with cancer in the developing world. This will create new challenges for assessing risks of recurrence, designing care pathways, the use of information technology (IT) and improving access to services. There will be new opportunities for further targeting and development of existing therapies as experience of risk factors grows over the longer term; careful monitoring of patient experiences could help to improve results. Soon, cancer could become a long-term management issue for many patients - they will enjoy a high quality of life despite a degree of chronic illness and morbidity (Nuffield Trust et al., 2003).

The funding of cancer care will become a significant problem (Bosanquet \& Sikora, 2006). Already within Europe there is inequitable access to the taxanes for breast and ovarian cancer and gemcitabine for lung and pancreatic cancer. Even countries that spend similar total amounts on health care and cancer have enormous variations in access to the new molecular-targeted agents. 
Many of these drugs are only palliative, adding just a few months to life; the compounds emerging are likely to be far more successful and their long-term administration will be considerably more expensive. Increased consumerism in medicine will lead to increasingly informed and assertive patients who use global information networks to seek out novel therapies and bypass traditional referral pathways. It is likely that far greater inequity will result from the integrated molecular solutions for cancer that may develop. Cost-effectiveness analyses will be used to scrutinize novel diagnostic technology, as well as cancer therapies.

Major innovations in the following six areas are likely to have the greatest impact on cancer:

- molecularly targeted drugs with associated sophisticated diagnostic systems to personalize care;

- biosensors to detect, monitor and correct abnormal physiology and provide surrogate measurements of cancer risk;

- ability to modify the human genome through systemically administered novel targeted vectors;

- continued miniaturization of surgical intervention through robotics, nanotechnology and more precise imaging;

- computer-driven interactive devices to help with everyday living;

- use of virtual-reality systems together with novel mood-control drugs to create an illusion of wellness and contentment.

\section{The future delivery of cancer care}

By 2030, cancer will be considered a chronic disease like diabetes, heart disease, hypertension and asthma. These conditions impact on the way people live but will not lead inexorably to death. The model of prostate cancer will be more usual - many men die with it rather than from it. There will be progress in preventing cancers and even greater progress in understanding the myriad causes. The new ways in which cancer will be detected, diagnosed and treated are crucial to understanding the future (Fig. 5-1).

When a cancer does develop, it will be made controllable through refinements of current technologies and techniques in imaging, radiotherapy and surgery, together with the availability of targeted drugs. Patients will be monitored closely after treatment; cure will be sought but will not be the only satisfactory outcome. Fear that cancer will definitely kill (still prevalent in the early years 


\begin{tabular}{|c|c|c|}
\hline High complete response & High complete response & Low complete response \\
\hline High cure & Low cure & Low cure \\
\hline $5 \%$ & $40 \%$ & $55 \%$ \\
\hline Hodgkin's disease & Acute myeloid leukaemia & Non-small cell lung cancer \\
\hline $\begin{array}{l}\text { Acute lymphoblastic } \\
\text { leukaemia }\end{array}$ & Breast & Colon \\
\hline Testis & Ovary & Stomach \\
\hline Choriocarcinoma & Small-cell lung cancer & Prostate \\
\hline Childhood & Sarcoma & Pancreas \\
\hline Burkitt lymphoma & Myeloma & Glioma \\
\hline
\end{tabular}

Fig. 5-1 Chemotherapy for advanced cancer

of the $21^{\text {st }}$ century) will be replaced by an acceptance that many forms of cancer are a consequence of old age.

Looking into the future is fraught with difficulties. In the 1980s, who could have imagined the impacts on global communication resulting from mobile phones, the internet and low-cost airlines? Medicine will be overtaken by similarly unexpected innovations.

For these reasons, it is difficult to produce economic analyses of the likely impact of future developments in cancer care. Technologies are developing fast, particularly in imaging and the exploitation of the human genome. The greatest benefit will be achieved simply by assuring that the best care possible is available to the most patients; irrespective of socioeconomic circumstances and of any scientific developments. But this is unrealistic. Well-informed patients (with adequate funds) will ensure that they have rapid access to the newest and the best - whatever the location. More patients will benefit from better diagnosis and newer treatments, with greater emphasis on quality of life. Inevitably, innovation will bring more inequality to health and health care.

The outcome of the same quality of care differs between socioeconomic groups today and will continue to do so. It is the role of governments to ensure health equity for all their constituents. Table 5-1 shows the challenges that need to be addressed in order to deliver most health benefit. 
Table 5-1 The challenges of cancer care

- Increasing the focus on prevention.

- Improving screening and diagnosis and their impact on treatment.

- New targeted treatments - how effective and affordable will they be?

- How will patients' and carers' expectations translate into care delivery?

- Reconfiguring health services to deliver optimal care.

- How will reconfiguration impact on professional territories?

- Will society accept the financial burden of these opportunities?

- Developing processes to bring equitable access to care within individual countries.

\section{New treatment approaches}

Future cancer care will be driven by the least invasive therapy consistent with long-term survival. Although still desirable, eradication of the disease will no longer be the primary aim of treatment. Cancers will be identified earlier and the disease process regulated similarly to chronic diseases such as diabetes. The roles of surgery and radiotherapy will depend on the type of cancer, the stage at which it is identified and the performance of drugs being developed now.

Cancer treatment will be shaped by a new generation of drugs that depend critically on the relative success of agents currently in development. Fuller understanding of the benefits of new compounds such as kinase inhibitors is likely over the next three to five years. It is estimated that about 500 drugs are currently being tested in clinical trials on cancer patients. Around 300 of these inhibit specific molecular targets (Dietel, 2007). This number is set to rise dramatically - some 2000 compounds will be available to enter clinical trials by the end of 2007 , and some 5000 by 2010 . Many of these candidate drugs will be directed at the same molecular targets and the industry is racing to screen those most likely to succeed in the development process.

Tremendous commercial pressures can be anticipated, because most of the existing high-cost cytotoxic chemotherapy drugs will lose patent protection by 2009. Without new premium-priced innovative drugs, the normal economic drivers of the pharmaceutical industry will simply disappear.

Small molecules and monoclonal antibodies are the main focus of current research. Most of these are designed to target specific gene products that control the biological processes associated with cancer such as signal transduction, angiogenesis, cell cycle control, apoptosis, inflammation, invasion and differentiation. Treatment strategies involving cancer vaccines and gene therapy are also being explored (Table 5-2). Although it is not known 
Base case launch year in the US

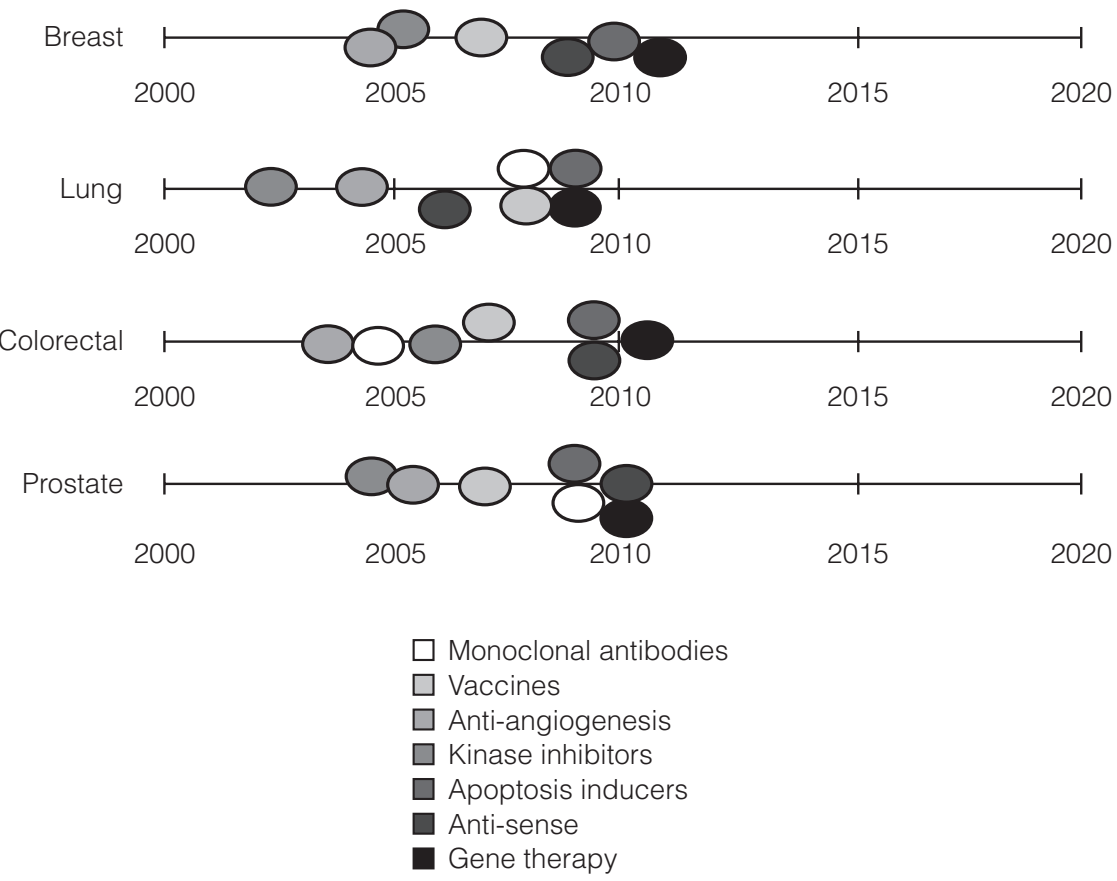

Source: Bosanquet \& Sikora, 2006.

Note: Huge numbers of novel therapies will come into clinical use outside the research setting between 2005 and 2010.

Fig. 5-2 Predicted new drug application (NDA) dates for molecular therapies in the United States of America

exactly what these targeted agents will look like, there is growing confidence that they will work. Their overall efficacy at prolonging survival is more uncertain - many could be just expensive palliatives. In future, advances will be driven by a better biological understanding of the disease process.

Already drugs targeted at a molecular level are emerging - trastuzumab, directed at the HER2 protein; imatinib, which targets the BCR-ABL tyrosine kinase; and gefitinib and erlotinib, directed at EGFR tyrosine kinase (Fig. 5-2). These therapies will be used across a range of cancers. In future it will be important to know whether a patient's cancer has particular biological or genetic characteristics. Traditional categories will continue to be broken down and genetic profiling will enable targeted treatment. Patients will understand that treatment options are dependent on their genetic profile. The risks and benefits of treatment will be much more predictable. 
Table 5-2 Drivers of molecular therapeutics

- Human Genome Project and bioinformatics

- Expression vectors for novel protein target production

- Computer-aided drug design

- Robotic high-throughput screening

- Combinatorial chemistry

- Platform approach to drug discovery

- Huge increase in number of molecular targets.

Therapies will emerge from knowledge of the human genome and the use of sophisticated bioinformatics (Simon, 2006). Targeted imaging agents will be used to deliver therapy at screening or diagnosis and treatment strategies for individual patients will change as technology allows the disease process in that patient to be tracked much more closely. Drug resistance will become much more predictable. Biomarkers will allow assessment of whether a drug is working on its target or if an alternative treatment strategy should be sought. Tumour regression will become less important as clinicians look for molecular patterns of disease and its response.

There will be a greater focus on therapies designed to prevent cancer. A tangible risk indicator and risk-reducing therapy (similar to cholesterol and statins for heart disease) would allow people to monitor, and intervene to reduce, their own risk. Subtle changes in cellular activity will be detectable and these will enable treatment to be delivered early in the disease process. This will lead to less aggressive treatment. The role of industry in the development of new therapies will continue to change. Increasingly, smaller and more specialized companies linked to universities will deliver drug candidates and innovative diagnostics to the established pharmaceutical industry for commercialization and marketing. Table 5-3 lists the uncertainties facing developers of cancer drugs.

People will become used to living with risk and will have much more knowledge about their propensity for disease. Computer programs will enable people to determine their own predisposition to cancer. In turn, this will encourage health-changing behaviour and efforts to seek information about the treatment options available. Patients will be more involved in decisionmaking as medicine becomes more personalized. Indeed, doctors may find themselves directed by well-informed patients. In combination with an environment in which patients are able to exercise choice, this will help to drive innovation towards those who will benefit. However, inequity based on education, wealth and access is likely to continue. 
Table 5-3 Uncertainty of novel drugs for cancer

- Will the new generation of small-molecule kinase inhibitors really make a difference or provide only expensive palliation?

- How will industry cope as most high-value cytotoxics become generic (out of patent) by 2009?

- Can expensive late-stage attrition (removal of a drug because of problems identified at the end of its development) really be avoided?

- How will sophisticated molecular diagnostic services be provided?

- Will effective surrogates for cancer-preventive agents emerge?

- Will patient choice involve cost considerations in guiding therapy?

\section{Barriers to innovation}

Innovation in cancer treatment is inevitable but there are certain prerequisites for the introduction of new therapies. First, innovation has to be translated into usable therapies. These must be deliverable to the right biological target and to the right patient in a way that is acceptable to patient, health-care professional and society. Innovation must also be marketed successfully so that the potential benefits are understood by professionals, patients and fundholders. Investments in research will inevitably create a market for innovation even if the benefits achieved are minimal.

The explosion in new therapies for cancer care continues. Prices will remain high. In 2007, the global cost of cancer drugs was estimated to be US\$ 31 billion - US\$ 18 billion (more than 50\%) of this is spent in the United States alone, with only $4.8 \%$ of the world's population. The global cancer drug market could reach US $\$ 300$ billion by 2027 if effective drugs emerge from the research and development pipeline. This cost will spread more widely around the world.

A number of confounding factors will reduce markets as therapies and costs multiply. Blockbuster drugs will become redundant as technology identifies patients who will not respond to therapy. Doctors will know the precise stage of the disease process at which treatment is necessary. As cancer gradually becomes a chronic disease like any other, cancer patients will also have more comorbidity at the time of diagnosis; this will bring associated drug-drug interactions and an increase in care requirements.

How to balance this equation? Pharmaceutical companies are unlikely to undertake studies that may fragment their market - their interest is selling drugs, and this would reduce their market penetration. Fundholders will need to drive research that leads to rational prescribing. There is a risk that pharmaceutical companies will stop developing drugs for cancer, preferring to 


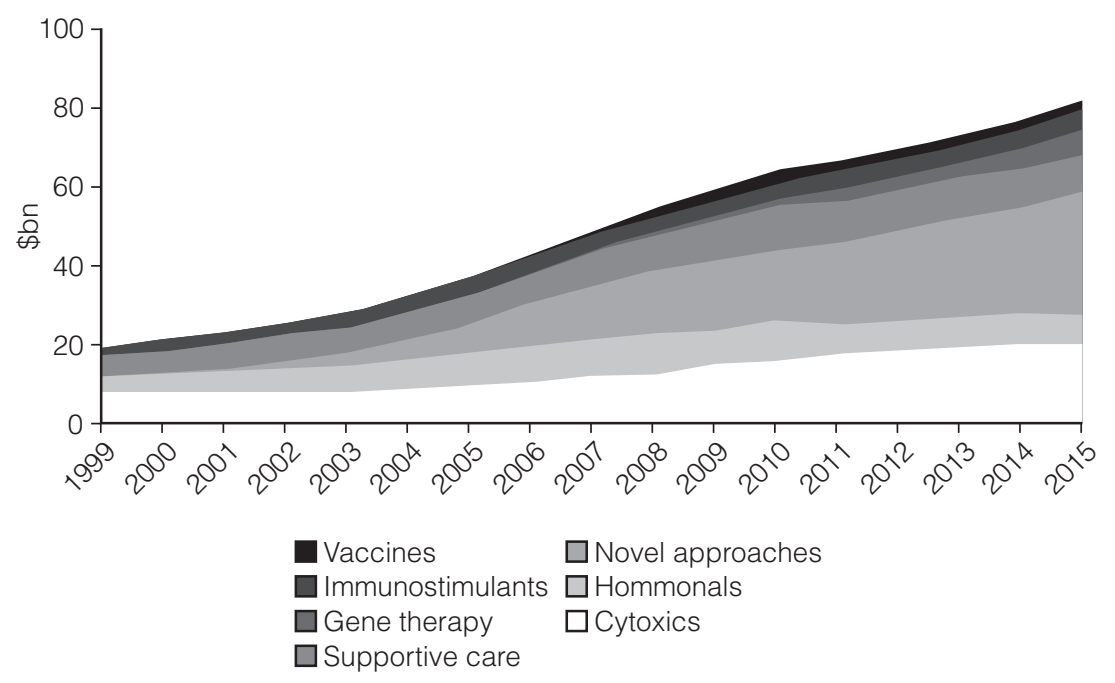

- 2010 sales $\$ 64 \mathrm{bn}$, compound annual growth rate (CAGR) $12 \%$ driven by

- new technology - particularly biologically targeted therapies

- earlier intervention

- patient numbers (ageing population - other diseases controlled)

Note: By 2015, it is estimated that global consumption will exceed US\$ 80 billion. Over 55\% of this will be used in the United States of America - containing only $4.8 \%$ of the world population.

Fig. 5-3 Global cancer market by sector: the escalating global cost of cancer drugs

focus on therapeutic areas with less individual variation and therefore more scope for profit. Furthermore, development costs are rising. Ten years ago, the average cost of developing a new cancer drug was around US\$ 400 million; now it is US\$ 1 bilion. At this rate of growth the cost of developing a new drug could soon reach US\$ 2 billion - unsustainable in a shrinking market. The process of developing drugs needs to be made faster.

The European Commission plans to increase clinical research in Europe by allocating $€ 1$ billion over the next seven years. The Innovative Medicines Initiative (IMI) is modelled on the US Federal Drug Administration's Critical Path Initiative, launched in 2004 (Sinha, 2007). It will allocate funds for joint academic-industrial research to address bottlenecks in cancer drug development. It remains to be seen whether this will lead to a useful realignment. Cynics suggest that the money will be absorbed by the large companies without producing much real gain for patients.

Research should be made simpler but it is being hampered by changes in legislation concerned with privacy and prior consent. The EU Clinical Trials Directive will make quick, hypothesis-testing drug trials impossible (see Chapter 15). Other challenges (such as obtaining consent for new uses of existing human tissue) result from political anxiety caused by failures to obtain 


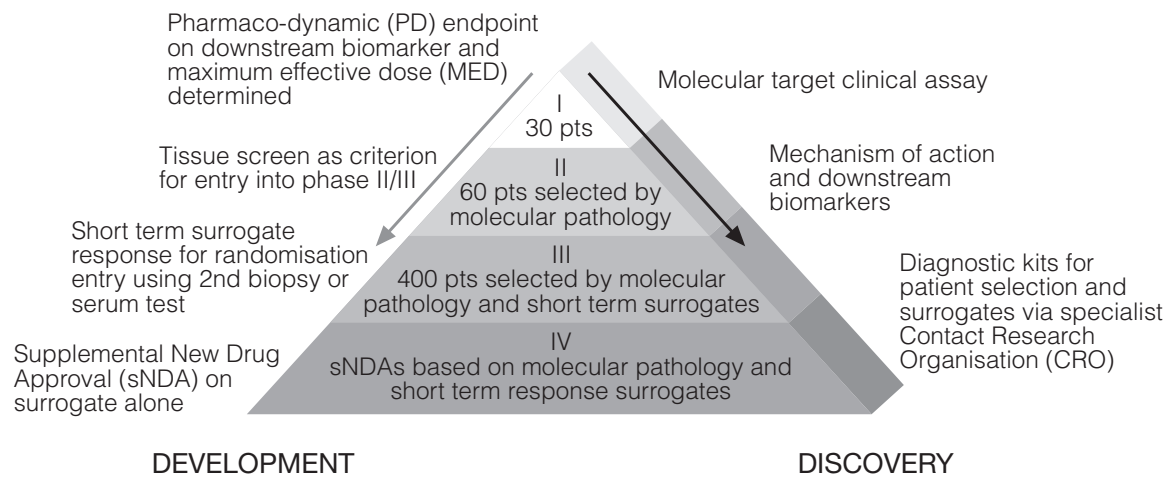

Note: Drugs will enter patients for the first time accompanied by effective biomarkers. These will be used to identify surrogate markers of response, selecting patients early in pivotal studies either to continue or to stop a specific trial. In addition, continued laboratory research will be used to create diagnostic kits to identify signatures of response.

Fig. 5-4 The future of cancer drug development

\begin{tabular}{|ll|}
\hline Diagnostic & Value \\
\hline Predisposition screen & Identify patients for chemo-prevention \\
Screen for presence of cancer & Increase in patients - earlier disease \\
Pharmacodynamic biomarker & Establishes pharmacological dose \\
Surrogate marker of clinical efficacy & Early indication of proof of concept \\
Predictive reclassification of disease & Targets therapy to those likely to respond \\
Patient-specific toxicity prediction & Avoid adverse events, adjust dose \\
\hline
\end{tabular}

Fig. 5-5 Six areas where diagnostics help to personalize cancer medicine

consent for removing and storing tissues in the early years of the $21^{\text {st }}$ century. However, surveys have shown that patients who consented to tissue being used for one purpose were happy for it to be used for another. They do not wish to be reminded of their cancer years later. To overcome these constraints, regulators will have to accept surrogate markers rather than clinical outcomes when approving new therapies. Outcome studies may well move to postregistration surveillance of a drug's efficacy, similar to arrangements for cholesterol-lowering agents today (Fig. 5-4).

The rise of personalized medicine will remove the temptation to overtreat. Doctors and patients will know whether a particular treatment is justified (Fig. 5-5). 
Table 5-4 Barriers to innovation

- The drug industry will continue to compete for investment in a competitive, capitalist environment.

- Blockbuster drugs drive profit - niche products are unattractive in today's market.

- Personalized therapies pose difficulties for the current industrial environment.

- Use of surrogate endpoints will be essential in the procedure to approve and license new drugs.

- Novel providers will emerge, for both diagnostic and therapeutic services.

- Payers will seek robust justification for the use of high-cost agents.

With evidence available to support decisions, treatment failure (with all its associated costs) will be less common (Table 5-4).

Cancer care costs are spiralling out of control in every health-care environment. Ageing populations with a wide range of medical problems require vastly increasing amounts of care. New technology (drugs, devices, procedures) is a powerful inflationary driver in an information-rich, consumer-oriented world (Jonsson \& Wilking, 2007). Different health-care systems use a variety of approaches to dampen demand, including copayments, top-up payments and deductibles (patient pays first tranche of costs for any treatment).

Whether overt or covert, rationing inevitably leads to inequity. A study in 2007 showed clearly a wide variation in cancer drug use in different EU countries (Fig. 5-6). A comparative study on the availability of radiotherapy showed considerable differences across Europe (Department of Health, 2007).

There is evidence of a growing use of co-payments to break through the access barriers to health care (Charlson, Lees \& Sikora, 2007). This applies to areas as diverse as implanted hearing-aid devices; access to diagnostics such as MRI scans; and home nursing care. Politicians of all persuasions appear to be unaware of their existence and reluctant to become involved in debate.

Cancer patients are developing sophisticated approaches to buy extra clinical services. These may be obtained either directly from their health providers (through selective use of the private sector to upgrade basic care) or simply through what are euphemistically called 'brown-envelope' payments to doctors and pharmacists; such payments have become common practice in several European countries. It may result in a more detailed explanation of their disease and the treatment options; jumping long queues for radiotherapy; or access to drugs generally denied because of funding problems. 


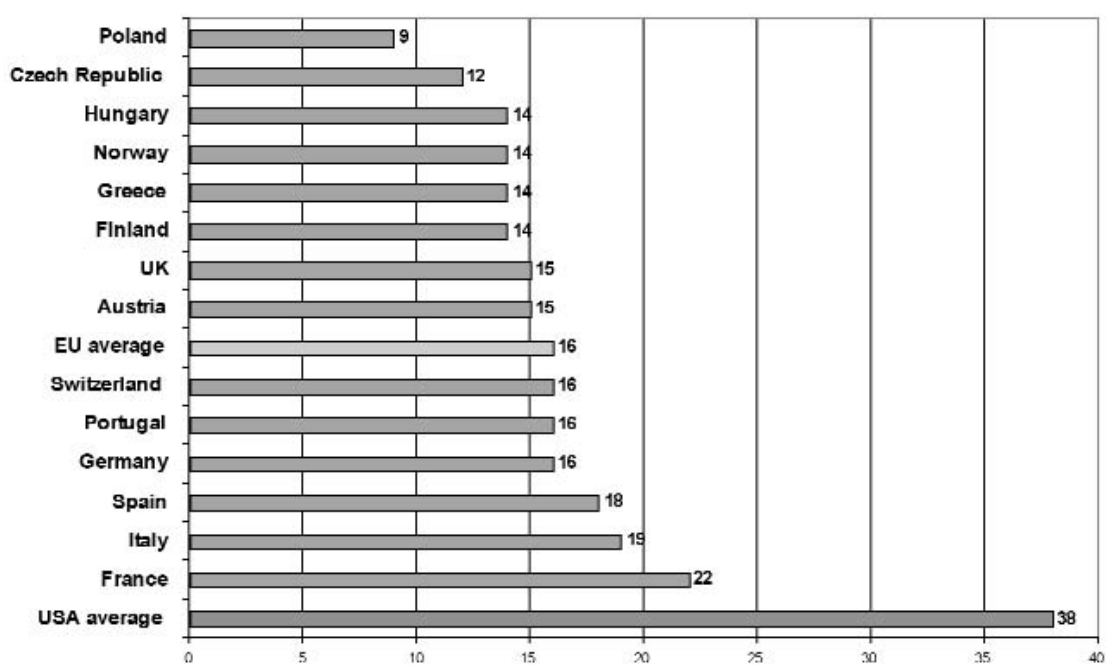

Source: Jonsson \& Wilking, 2007

Fig. 5-6 Annual spend per person ( $€$ ) on cancer drugs in the EU in 2004 (purchasing power parity adjusted)

\section{Options for paying for cancer treatments}

\section{Denial}

A doctor may deny the existence of a drug, device or service and refuse to discuss any possible benefits during a consultation. This method of dealing with the situation is no longer viable in an age of patient empowerment and free availability of information via the Internet. A recent report shows that patients want all the information even if they cannot access all the treatments (Cancerbackup, 2007). This approach is the ultimate of social solidarity - if a drug is not available for all, then none will receive it. It is a difficult policy to enforce in a democracy (Sikora, 2007).

\section{Transfer entire care to the private sector}

After a full discussion of the available options, the patient is referred for entirely private care either by the same consultant or by a colleague at a private hospital. Without insurance, this amounts to signing a blank cheque, because unforeseen medical complications may require expensive treatment. It is also hugely inequitable - only patients of substantial means can afford this approach, yet everyone has paid for basic health care, through taxation or social insurance. 


\section{Obtain upgrade only in the private sector}

After a full discussion of the available options, the patient is referred (to the same consultant or a colleague at a private hospital) for private provision of the component of care that is not available in the public sector. This may result in treatment from two oncologists and potential difficulty in dealing with treatment-related complications. Should these be treated free by the state, by the insurer or by further payment in the private sector? While this approach is fairer than making patients pay for all their care, it is more inconvenient. Treatment is received in two places; two separate sets of records could lead to confusion and errors in treatment.

\section{Provision of upgraded drug invoiced to the patient}

After full discussion of the available options, the patient receives the relevant upgrade within the same provider unit. The patient is invoiced for this extra treatment, including a charge to cover hospital costs and improve the overall quality of care for all. Clinical care and information flow is seamless and any complications can be dealt with expeditiously. This strategy does mean that patients in the same day ward can receive different drugs based solely on their ability to pay but private and public patients are treated at the same units across Europe.

\section{Leaving the patient to their own devices}

A patient dissatisfied with the clinical response obtains the relevant upgrade from an internet pharmacy, without informing their consultant. These markets could be driven underground if edicts ban the use of co-payments to obtain drugs; and patients would not inform their health professionals. This is clearly the most dangerous option. Certain complementary and alternative therapies already follow this pattern. Undoubtedly, unscrupulous suppliers and service providers would emerge to meet this new market.

\section{Immediate cost pressures}

Huge changes are taking place in cancer medicine, and they will have a significant impact on the costs of optimal care. The biggest financial impact will come from the registration of several high-cost molecularly targeted drugs in adjuvant settings for common diseases such as lung, breast and colorectal cancer. The precedent set by trastuzumab (Herceptin) is likely to be repeated for several other agents over the next 12 months - bevacizumab (Avastin), erlotinib (Tarceva), lapatinib (Tykerb) and cetuximab (Erbitux) (Fig. 5-7). 


\begin{tabular}{|lllc|}
\hline Drug & Generic & Manufacturer & Cost per annum \\
\hline Herceptin & trastuzumab & Roche & 88.2 \\
Mabthera & rituximab & Roche & 58.8 \\
Glivec & imatinib & Novartis & 73.5 \\
Erbitux & cetuximab & BMS/ Merck Serono & 88.2 \\
Avastin & bevacizumab & Genentech/Roche & 102.9 \\
Tarceva & erlotinib & Roche & 95.6 \\
Sutent & sunitinib & Pfizer & 73.5 \\
\hline
\end{tabular}

Fig. 5-7 Marketed targeted therapies and their costs (in thousands of Euros)

The strategy will be the same - initial licensing for metastatic cancer will be sought in the United States, but this is likely to be followed rapidly in the EU and Japan as industry seeks global markets. There are huge variations in per capita drug spend across Europe (Fig. 5-6) but the average spend is half that of the United States.

Adjuvant studies, where drugs are given routinely after successful removal of the primary tumour to prevent recurrence, will be pursued aggressively to bring these new drugs into the earlier phase of cancer management and to increase sales. Big pharmaceutical companies have incurred huge research and development expenditures for molecularly targeted therapies that are expected to be future blockbusters. These include both small molecules (taken as daily oral medication) and monoclonal antibodies (intravenous infusions every two or three weeks).

The American Society of Clinical Oncology (ASCO) holds the industry's most important clinical meeting in June each year (ASCO, 2007); attended by over 35000 delegates from all over the world. At the 2007 meeting in Chicago, it was estimated that 22 small molecule tyrosine kinase inhibitors and 18 monoclonal antibodies are likely to be licensed for sale in the United States within the next two to three years (Table 5-5). Regulatory packages are now so efficient that near-simultaneous registration will occur at all three major global regulators in Washington, London and Tokyo. The European Medicines Agency (EMEA) controls the entry of a drug to all EU markets.

In 2007 there will be at least six powerful new molecularly targeted anticancer drugs administered as simple tablets. It is unlikely that many EU countries will be able to afford them all. The dynamic is bound to change as powerful tyrosine kinase inhibitors become available as simple tablets, without the need for complex administration systems. Monoclonal antibodies are predicted to double the number of intravenous infusions required by 2011, stretching 
Table 5-5 High-cost cancer drugs likely to be approved by the Food and Drug Administration (FDA) and the European Medicines Agency for the Evaluation of Medicinal Products (EMEA), 2007-2010

\begin{tabular}{ll}
\hline Small molecules & Monoclonal antibodies \\
Sorafenib & Bevacizumab \\
Sunitinib & Pertuzumab \\
Axitinib & Nimotuzomab \\
Lapatinib & Galiximab \\
Tipifarnib & Catumaxomab \\
Cediranib & Eculizamab \\
Erlotinib & Tositumomab \\
Gefitinib & Nimotuzumab \\
Imatinib & Alemtuzumab \\
Ipsinemib & Apomab \\
Motesanib & Volociximab \\
Vandetanib & Panitumomab \\
Bosutinib & Adecatumumab \\
Lestaurtinib & Lexatumumab \\
Nilotinib & Lumiliximab \\
Fulotinib & Ipilimumab \\
Brivanib & \\
Dasatinib & \\
Pazopanib & \\
Everolimus & \\
Selicilib & \\
\hline
\end{tabular}

delivery capacity to the limit. Emerging independent providers are likely to provide over-the-counter diagnostic services for personalized medicine by using genomics and proteomics to choose the right therapy. Almost certainly, these companies will target a vast self-pay market as well as marketing their diagnostics to state and private insurers.

Increasingly, patients will order drugs through global internet pharmacies, thereby bypassing drug companies' traditional strategies of selling to doctors, other health professionals and state health systems. In a consumerist world patient information flow will become a far more powerful marketing tool. The pharmaceutical and medical-device industry makes increasing use of direct-to-consumer marketing, using advertisements and subtle public relations activities to generate positive press stories.

Recently, Roche was widely criticized for creating Cancer United (Cancer United, 2007). This cancer charity, purporting to lobby for better cancer care in Europe, was funded exclusively by Roche and run by its own PR agency. Pfizer recently hired the agency responsible for Coca Cola branding to advise 
on its cancer portfolio. Some time ago AstraZeneca bought Salick Health Care (now Aptium Oncology) - a series of cancer centres in the United States partly to gain better understanding of doctors' prescribing habits. A new ethical framework of operations is needed urgently.

\section{The longer-term future}

Cancer will become incidental to day-to-day living, not necessarily eradicated but causing patients less anxiety. People will have far greater control over their medical destinies and patients in all socioeconomic groups will be better informed. Surgery and chemotherapy will not be rationed on grounds of age, since all interventions will be less damaging - psychologically and physically.

This picture is dependent on the emergence of the requisite technological innovations. For example, will people really live in "smart" houses in which televisions play a critical role in monitoring their health and well-being? It is also dependent on health-care professionals working with each other and valuing the input of carers. These will provide even more voluntary support because of the increasing number of people in older age groups compared with those of working age.

The reality for cancer care may be rather different. The ideal will exist for a minority of patients but the majority may not have access to the full range of services. Older people, having been relatively poor all their lives, may suffer from cancer and a huge range of comorbidities that will limit their quality of life. Will there be enough younger people to provide care for them all - rich and poor?

As with all health issues, access will be determined by cost and political will. In 2007 a cancer patient consumes direct medical care costs of about $£ 25000$ ( $€ 36750$ ) in the United Kingdom, 70\% of which is spent in the last six months of life (Bosanquet \& Sikora, 2006). At a conservative estimate, this could increase four-fold to $£ 100000$ ( $€ 147$ 000) per patient per year by 2027 as patients live with (rather than die from) cancer and have access to new technologies. The current annual cost of targeted therapies is shown in Fig. 5-7. In theory, cancer care could absorb an ever-increasing proportion of the health-care budget. This is likely to reflect patients' wishes as surveys reveal that the majority believe that cancer care should be the highest priority; far beyond any other disease.

Assuming that part of the health service will still be funded from taxation this expenditure might require the tax rate to rise to $60 \%$ in the United Kingdom. Inevitably, there will be conflicting demands on resources: the choice may be 
drugs or care costs. However, although expensive, the technology will be used more judiciously as it will be better targeted. Another argument suggests that empowered patients use fewer and less expensive medicines, in effect lowering overall costs. Although costs for treating individuals will increase, overall costs will decrease as more care is delivered at home. However, people will live longer so the lifetime costs of cancer care will rise, together with the cost of managing comorbidities.

Politicians will be faced with new dilemmas. Increases in cancer prevalence could cause massive increases in the cost of delivering innovative care. Will cancer care need to be rationed in a draconian way?

The political power of older people will increase as more people live longer and their chronic problems do not necessarily cause physical or mental incapacities. This educated elderly population will have high expectations, sharpened through the first two decades of the $21^{\text {st }}$ century, and will not tolerate the standards of care offered now. They will wield considerable influence. Will a tax-based health system be able to fund their expectations? Politicians will have to consider the alignment between patients' requirements and the wishes of tax-payers and voters. Currently, fewer than $50 \%$ of voters pay tax - this is set to fall further as the population ages. Will younger taxpayers tolerate the expensive wishes of mainly elderly non-tax-payers? The interests of voters may be very different to the interests of tax-payers. An exclusively tax-funded health service may be impossible; co-payments and deductibles will be an inevitable part of the new financial vocabulary. Social solidarity and consumerism are uneasy companions.

Fig. 5-8 shows the four components of cancer's future - technology, delivery, finances and society.

Whichever system is put in place, there is the prospect of a major socioeconomic division in cancer care. A small percentage of the elderly population will have made suitable provision for their retirement, in terms of health and welfare, but the vast majority will not be prepared.

Policy-makers need to start planning now, as they are doing for the looming pension crisis. Cancer patient and health-advocacy groups should be involved in the debate, to ensure that difficult decisions are reached by consensus. Societal change will create new challenges in the provision of care. A decline in hierarchical religious structures, a reduction in family integrity through increasing divorce, greater international mobility and even the increased selfishness of a consumer-driven culture will leave many people lonely, with no social or psychological support to lean on at the onset of serious illness. There will be a global shortage of carers - the unskilled, low-paid but essential 


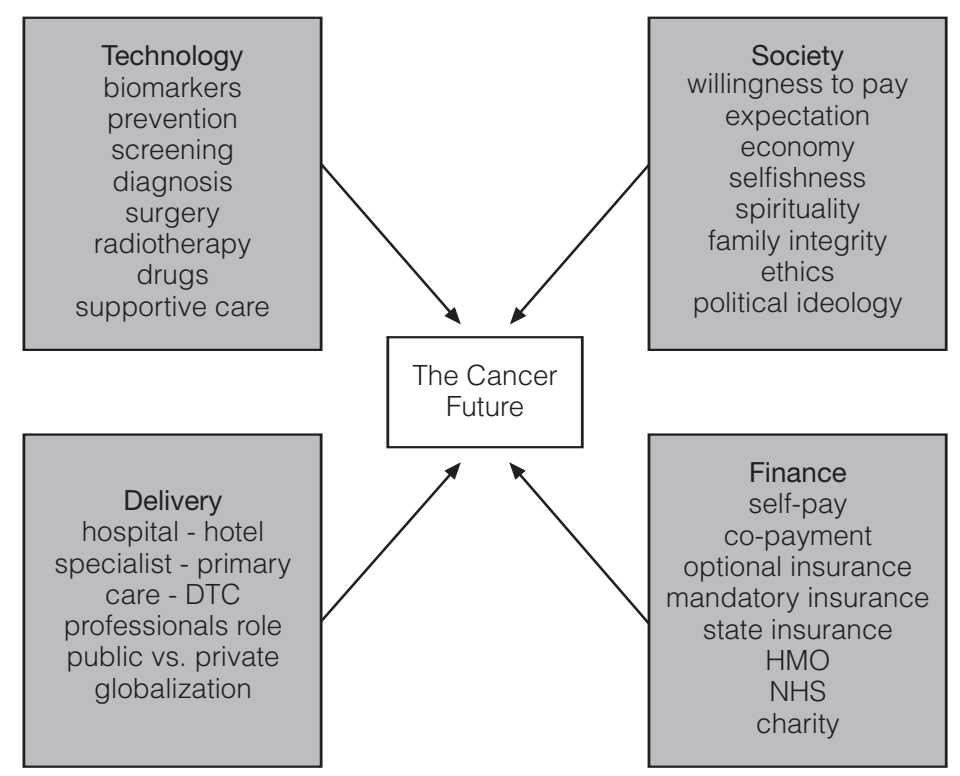

Fig. 5-8 The four building blocks of cancer's future

component of any health-delivery system. The richer parts of the world are recruiting carers from poorer countries, but the supply of this precious human capital is limited.

New financial structures will emerge as novel consortia from the pharmaceutical, financial and health-care sectors enable people to buy into the level of care they wish to pay for. Cancer, cardiovascular disease and dementia will be controlled, joining today's list of chronic diseases such as diabetes, asthma and hypertension. Competing private-sector providers will run hospitals as attractive health hotels in which global franchises provide speciality therapies, similar to the internationally branded shops in today's malls. Governments will have long ceased to deliver care. The United Kingdom's NHS may well be one of the last centralized systems to disappear, converting to UK Health - a regulator and safety-net insurer - by the end of this decade.

The ability of technology to improve cancer care is assured. But this will come at a price - not only direct costs but also those incurred from looking after the increasingly elderly population it will produce. Eventually, humans may simply run out of things to die from. New ethical and moral dilemmas will arise as living long and dying quickly become the mantras of $21^{\text {st }}$ century medicine. The cancer future will emerge from the interaction of four factors: the success of new technology, society's willingness to pay, future health-care delivery systems and the financial mechanisms that underpin them (Fig. 5-8). 


\section{Conclusions}

- Drugs are increasingly important in modern cancer care but they are only one component. They must be integrated into sophisticated care pathways from education, prevention and diagnosis to palliative care.

- Compared with other modalities of treatment and care, such as early detection, radiotherapy and palliative care, the prioritization of drugs is exaggerated by high-cost public relations activities from the pharmaceutical industry.

- Increased use of sophisticated molecular diagnostics will provide personalized medicine. This will reduce drug wastage and costs.

- Within Europe it will become increasingly difficult to achieve equity for whole populations in terms of access to drugs. Different countries will choose different thresholds to trigger the availability of drugs for defined groups of patients.

- Global, specialized private providers of cancer care are emerging. These may provide higher-quality services more efficiently and cheaply than state-run services. They are likely to drive increased consumerism amongst patients and their relatives.

- Mechanisms that allow patients to top up their care need to be fair, transparent and free from corruption. Brown-envelope payments (bribes) to doctors for extra services such as new cancer drugs must be avoided across Europe.

- Politicians must understand the importance of good cancer care for their voters. This needs to be translated into an effective structure for cancer diagnosis, referral and treatment in each country.

- The pharmaceutical industry will continue to overemphasize the benefits of drug-based treatments and will use increasingly sophisticated public relations techniques to promote its products. This activity needs to be transparent and not achieved by covert funding of patient-advocacy groups.

- Health-care fundholders in Europe need to examine the cost-effectiveness of new technologies as closely as the efficacy of the drugs themselves.

- Governments must ensure that all their constituents have access to clinically proven interventions that maximize the length and quality of life. Robust health technology assessment is essential, as is equitable distribution of treatment resources. 


\section{REFERENCES}

ASCO (2007). ASCO annual meeting proceedings. J Clin Oncology, 25(18 S):1-960.

Bosanquet N, Sikora K (2006). The economics of cancer care. Cambridge, Cambridge University Press.

Cancerbackup (2007). Cancer - a public priority. Attitudes towards cancer treatment in Britain. August 2006 (available at http://www.cancerbackup.org.uk/News/Campaigns/CancerValues/ CancerAPublicPriorityAug2006.pdf)

Cancer United (2007). Web site available at: http://www.cancerunited org/, accessed 28 November 2007.

Charlson P, Lees C, Sikora K (2007). Free at the point of delivery - reality or political mirage? Doctors for reform. London (available at: http://www.doctorsforreform.com/filedata/ CopyofFreeatthepointofdelivery-realityorpoliticalmirage.pdf, accessed 19 December 2007).

Del Vecchio S et al. (2007). Nuclear imaging in cancer theranostics. QJ Nucl Med Mol Imaging, 51:152-163.

Department of Health (2007). Report to ministers from the National Radiotherapy Advisory Group. London, Department of Health.

Dietel M (2007). Predictive pathology of cytostatic drug resistance and new anti-cancer targets. Recent Results Cancer Res, 176:25-32.

Jonsson B, Wilking N (2007). A global comparison regarding patient access to cancer drugs. Ann Oncol, 18(Suppl. 3):1-74.

Nuffield Trust et al. (2003). 2020 vision: our future healthcare environments. Norwich, The Stationery Office.

Philip R et al. (2007). Shared immunoproteome for ovarian cancer diagnostics and immunotherapy: potential theranostic approach to cancer. J Proteome Res, 6:2509-2517.

Sikora K (2002). The impact of future technology on cancer care. Clin Med, 2:560-568.

Sikora K (2004). Cancer 2025: the future of cancer care. Expert Review of Anticancer Therapy Supplement, 4(3s1):1-78.

Sikora K (2007). Paying for cancer care - a new dilemma. J R Soc Med, 100:166-169.

Simon R (2006). Validation of pharmacogenomic biomarker classifiers for treatment selection. Cancer Biomark, 2(3-4):89-96.

Sinha G (2007). European Union creates its own "critical path". J Natl Cancer Inst, 99(11):832833.

Velasquez JL, Lipkin SM (2007). Genetic testing to identify high-risk populations for chemoprevention studies. Cancer Biomark, 3(3):163-168. 


\section{Chapter 6 Organizing a comprehensive framework for cancer control}

Robert Haward

\section{Introduction}

Comprehensive national policies or plans specifically directed at improving the organization of services for people with cancer are relatively new. Cancer care has always been part of the health care provided within national health systems across the EU. It is only in the last 10 to 15 years, however, that more systematic approaches to formulating and applying improvements in the structures and processes used for delivering these services, including treatment services, have been adopted in some countries. The rationale is that outcomes can be greatly improved by more effective clinical organization and better operational delivery of cancer services (Ludwig, 2006; Marwick, 1999; Micheli et al., 2003).

The traditional concept of cancer control placed most stress on classic public health measures such as prevention, early diagnosis and the role of primary care. The importance of improving the clinical organization and operation of secondary and tertiary services has become a key issue only recently. The principles behind cancer control policies remain valid, but modern cancer strategies and plans have extended their scope, ambition and detail, breaking new ground in the methods of defining and addressing clinical care.

Cancer outcomes can be influenced by interventions of all kinds - from primary prevention to end-of-life care. Inevitably, the organization and delivery of public health programmes designed to improve cancer outcomes 
and reduce cancer mortality (including clinical services for patients diagnosed with cancer) is complex. It involves an unusually wide range of professional expertise, and contributions from organizations at all levels of the health system. For maximum benefit, planned activity is required across the full spectrum of interventions that can improve population and individual outcomes. This may be summarised as follows:

- Populations need effective programmes for cancer prevention, screening and early diagnosis in order to achieve long-term reductions in cancer mortality.

- People who may have cancer need prompt access to appropriate specialists for accurate diagnosis and subsequent clinical management.

- Cancer specialists come from a range of medical disciplines and clinical professions. They need to work effectively together within a multidisciplinary service if the best decisions are to be made about each patient's diagnosis, treatment and support, and treatments are to be delivered safely and effectively.

- Diagnosis and treatment services are available at primary, secondary and tertiary care levels - locally and at a distance. Most cancer patients interact with services from more than one part of the health-care system and with different providers. It is imperative that the roles and operational practices of the entire system for delivering cancer services develop logically and fit together well.

- Health-care professionals at every level must communicate effectively and coordinate the delivery of services, in order to ensure that each patient's pathway of care functions well.

- Complex interventions for diagnosis (e.g. for lymphoma) or treatment (e.g. surgery or chemo-radiation for oesophageal cancer) should not normally be offered in centres with low volumes of such interventions. They should be concentrated where all the necessary expertise can be assembled costeffectively and the results audited consistently.

- Patients' needs must be central to the organization and delivery of services. Patients' views on different therapeutic options and expected outcomes should be part of the clinical decision-making process. Quality of life and psychosocial issues are important, and care plans should always take them into account.

The first explicit attempt to prepare a comprehensive national cancer policy was published by the Chief Medical Officers of England and Wales in 1995 (Expert Advisory Group on Cancer, 1995). The Calman-Hine report, named 
after the Chief Medical Officers involved, was accepted by government as the basis for the future provision of cancer services in the United Kingdom. It played a crucial role in raising awareness within the United Kingdom of the shortcomings in cancer care, and it had far-reaching consequences. For the first time, it offered a clear and radical strategy to address longstanding weaknesses and improve the organization of cancer services. It was a policy framework rather than a detailed plan, but the policy goal was ambitious:

All patients should have access to a uniformly high quality of care in the community or hospital wherever they may live to ensure the maximum possible cure rates and best quality of life. Care should be provided as close to the patient's home as is compatible with high-quality, safe and effective treatment.

The first comprehensive national cancer plans followed a few years later in Denmark (National Board of Health, 2000), England (Department of Health, 2000) and France (French National Cancer Institute, 2003). All began around 2000. Although each of these plans reflected their national context, they had important similarities. All three went beyond the shaping of policy goals to address practical issues of implementation. Their scope and detail were far more comprehensive than either the initial United Kingdom framework or any other strategic plans available at that time. All adopted a five-year timeframe to initiate the required changes, but it was anticipated that implementation would extend beyond that period and that further steps would be necessary.

The Danish National Board of Health published its National Cancer Plan status and proposals for initiatives in relation to cancer treatment under the auspices of an advisory Cancer Steering Committee. This was a comprehensive plan covering the full range of cancer control (from prevention to rehabilitation and palliative care) with the aim of improving cancer treatment and reducing cancer mortality. This became known as National Cancer Plan I when the second-stage plan was published in 2005 (National Board of Health, 2005). Denmark was the first country to produce a follow-up plan in order to maintain the momentum of the first plan and to deliver further changes in the structure and operation of national cancer services.

The first National Cancer Plan in England (Department of Health, 2000), built on the 1995 policy. It had a particular focus on improving access to diagnosis and care; developing and implementing service guidance; and addressing shortfalls in key manpower and equipment. The plan included new prevention and research initiatives as well as specific targets. It was supported by substantial new resources, phased in over three years. An updated plan the Cancer Reform Strategy - was published in late 2007. 
The French cancer plan was similar to those of Denmark and the United Kingdom, but it was developed and presented very differently. It began in 2000 at the highest political level, with a call for action from President Jacques Chirac, who made cancer one of three top political priorities for his second term of office. In a characteristically bold call for global action, he described cancer as "one of the greatest challenges of our century".

The French cancer plan was published in March 2002 and dealt with all the key themes of prevention, screening and treatment. It included social and domiciliary support for patients, reflecting the particular context of these issues in France. The plan also addressed teaching and research in some detail. The Institut National du Cancer (INCa) was established in May 2005 as a major new national structure to oversee the implementation of the plan. Substantial resources were made available to support the programme, and funds for additional facilities and manpower were clearly identified.

Other EU Member States have implemented cancer plans to make progress in cancer control, or are actively developing such plans. The scale and scope of such cancer plans varies between countries - for example, Finland and Sweden have successfully addressed many of the important cancer policy issues within their normal political and management systems for organizing health care, rather than through comprehensive cancer plans. Both countries have wellorganized cancer services and good survival outcomes in the EUROCARE data (Coleman et al., 2003). Other EU countries, such as Germany and Spain, devolve most of the responsibilities for health-care planning to sub-national levels. These regional cancer plans may be considered more practical than national initiatives.

The case for explicit cancer plans is that they generate greater governmental and political commitment with a much higher profile for cancer control and often an explicit decision to accord a high priority to improving cancer care. National cancer plans generate clear policy goals. They can help to overcome difficult problems such as unequal access to health-care resources or resistance to key changes from vested interests. Such issues can be much harder to resolve without the overriding political commitment provided by a cancer plan.

\section{Drivers for change}

It seems likely that many influences have operated (independently and jointly) to raise the importance of the organization of cancer services as an issue requiring action in so many countries. Politicians and health policy-makers have been faced by a combination of hard evidence about the variability of cancer services and outcomes, backed by expert opinion from cancer clinicians 
and other health-care professionals. Individual patients and patient groups have been exerting growing pressure for governments to do more for cancer patients and give higher priority to improving services. Many cancer professionals have been openly supportive of these patient voices. It is likely that this synergy between professionals and patients has been politically effective.

When the first results of the EUROCARE study became available in 1995, they provided the first systematic and credible international comparisons of cancer survival (Berrino et al., 1995). At the same time, they demonstrated the vital role of effective and complete cancer registration as a prerequisite for effective cancer control. The existence of statistically significant differences in cancer survival across Europe invited the judgement that the differences might be regarded in part as an indicator of the relative performance of national cancer care systems. Whilst interpretation posed difficulties, in particular whether the differences were real, or due to artefacts arising from variations in the completeness or accuracy of the recording of cancer cases by clinicians and cancer registries, there is no doubting the importance of those first results. Many types of cancer showed consistent international patterns of survival that gave some countries particular cause for concern. Denmark and the United Kingdom have complete population coverage from their cancer registries. Their survival rates for many cancers were lower than in other western European countries, with which they naturally compared themselves. Both countries concluded that there were flaws in their cancer services that needed to be addressed. There were similarities in the underlying weaknesses in service delivery including variability of care; fragmentation of complex cancer treatments between too many hospitals and clinicians (Kehlet \& Laurberg 2006; Lauritsen et al., 2005; Marx et al., 2006); and shortcomings in nonsurgical oncology services.

France performed well in the EUROCARE results, although cancer registration data were not comprehensive. Internationally, the French healthcare system is regarded as one of the best. There were significant regional and social inequalities in cancer incidence, however, and problems with achieving uniform access to high-quality cancer care, with evidence that care varied significantly. There was a particular need to redress a historical neglect of cancer prevention. Despite past successes in the delivery of good health care, economic pressures in France were difficult.

All health systems and even strong economies struggle with the pressures imposed by the need for cancer services to meet more exacting clinical standards and respond to rising patient expectations. The rising costs of cancer treatment (including rapid increases in the cost of anticancer drugs - see 
Chapter 5) also create tension. Countries may find it easier to cope with such pressures if they adopt systematic health technology assessment; set public health and clinical priorities; and clarify what treatments can be provided and under what circumstances. Such approaches provide a context for rational decision-making about the use of inevitably scarce resources.

There is an influential trend of growing pressure from patients and their carers for greater involvement in decisions about their care, with more information and better communication (see Chapter 10). An increasing willingness to talk openly about cancer has been most apparent for breast cancer - patients and patient groups publicly challenge weaknesses in the care offered. The profile of other cancers (e.g. bowel and prostate) has lagged behind, but this is changing.

Descriptive evidence showed considerable variability in access, diagnosis, treatment and subsequent care (Blais et al., 2006; Chouillet, Bell \& Hiscox, 1994; Eaker et al., 2005; Harries et al., 1996; Morris, 1992; Pitchforth, Russell \& Van der Pol, 2002; Richards et al., 1996; Sainsbury et al., 1995a). These disparities can reflect unacceptable variations in the quality of cancer services. Prognosis is highly dependent on the diagnostic process and primary management being right first time - failings at this stage are often irreversible.

Advances in knowledge challenge clinicians and health systems to refine the management of patients. The best research defines the standard of care, and the relationship between research and the quality of patient care is increasingly important. There is some evidence that care provided in a unit that takes an active part in clinical trials may produce better outcomes (Fayter et al., 2006). Countries such as France and the United Kingdom have responded by expanding the links between cancer treatment systems and clinical research, particularly randomized clinical trials. It is anticipated that this synergy will also help to improve service delivery.

\section{The goals of effective cancer control}

The overriding aim of national policies for cancer services is to improve outcomes for patients and reduce cancer mortality. The guiding principle is that the structures and processes for delivering cancer services should be those most likely to produce good outcomes and to use available resources effectively and efficiently.

The key outcome measure is survival but this is neither the only one nor, for many patients, the most important. Other outcomes include quality of life combining well-being, psychosocial factors and the impact of specific forms of morbidity; and the patient's experience of cancer and the care they receive. 
Morbidity arises both from the cancer itself and from its treatment but this can be minimized, or even avoided, by optimal clinical management.

Cancer patients often require prolonged health care and their experiences of the health-system are important. It is extremely important that cancer patients' experience of health care is as good as it can be. Too many patients face more problems than they should during an inevitably difficult time. These difficulties can be reduced greatly by good communication from health-care professionals and useful information about their condition and the available treatment options.

A comprehensive cancer plan should address the full spectrum of interventions. The traditional epidemiological perspective of primary, secondary and tertiary prevention is often expressed through the more personal concept of the patient or care pathway. This describes the logical sequence from primary prevention (to reduce cancer incidence); screening and early diagnosis; and access to services for symptomatic diagnosis, staging and primary treatment (to improve survival and reduce mortality). For many patients, the care pathway will also include the management of progressive or recurrent disease; palliative and end-of-life care; and appropriate psychosocial support.

The implementation of measures to improve the clinical organization and operational policies of cancer services takes time and effort (Haward, 2006). Changes may be required in clinical services - how and where they are created; how they function; how they relate to other services; and the staff and facilities required. Any health system must address the challenge of narrowing discrepancies between what has been done and what ought to be done. Some changes may be controversial because they have significant consequences for health-care providers and clinical professionals, particularly if an aspect of service needs to be moved to another location. Centralization of a service or mode of treatment affects the roles of both "losing" and "receiving" hospitals and their staff, and is likely to increase travel time and costs for some patients. The crucial test should be "What arrangement is most likely to improve patient outcomes?", and not "What difficulties may be encountered in promoting change?"

\section{Key issues in improving cancer services}

\section{Access}

People need information about how best to manage their health and when to seek professional advice. Fear of cancer can be reduced by more openness and 
greater emphasis on the benefits of early diagnosis. For patients in whom cancer may be suspected, or excluded as a possible diagnosis, adequate professional awareness is required of the indications for further action, including prompt access to blood tests, X-rays, ultrasound or endoscopy. Clear procedures, supported by local guidelines, should be in place to ensure that patients who may have cancer have prompt access to appropriate specialists. Efficient organization of rapid access is complex - many people for whom clinical suspicion of cancer is appropriate will not have the disease.

Evidence from the EUROCARE studies indicates that the differences in cancer survival between EU Member States, and between Europe and the United States, are explained partly by differences in the stage at presentation (Ciccolallo et al., 2005; Sant, 2001; Sant et al., 2004; Sant et al., 2003). It is much harder to determine why these differences arise but better understanding of the reasons for delay in presentation or diagnosis should lead to improved access to cancer services. Socioeconomic factors play a part, as does the operation of health systems, including the expertise of the doctors who first see the patient; ease of access to key investigations such as endoscopy; and the availability of specialist cancer services. Clinical policies and guidelines play a key role in addressing these issues effectively within each health system.

\section{Specialization and multidisciplinary clinical practice}

The United Kingdom's 1995 policy framework sought to achieve two fundamental changes in order to transform the delivery of cancer services. The first was to ensure that all patients had early access to a specialist appropriate for their type of cancer, e.g. a breast or colorectal surgeon rather than a general surgeon for whom cancer is only a small part of their clinical caseload. The second was to ensure that the different medical specialists and other relevant professions for each type of cancer worked closely together in multidisciplinary teams with defined membership and working arrangements. Before the introduction of this policy, many cancer patients never saw a cancer specialist and properly established multidisciplinary teams for treating cancer were the exception not the rule.

The evidence base for this policy relies on several strands. There is evidence of a gradation of practice or outcome, with generalists performing less well or achieving poorer outcomes than specialists. One early paper reported the magnitude of the observed differences in breast cancer (Gillis \& Hole, 1996). Five-year survival was $9 \%$ higher for patients cared for by specialist surgeons. Ten-year survival was $8 \%$ higher and the risk of death was $16 \%$ lower (95\% CI: 6-25\%). The authors concluded that survival differences of this 
magnitude have significant implications for women with breast cancer. Studies of this type now cover many different cancer types and usually show better outcomes for patients treated by specialists (Bachmann et al., 2003; Grilli et al., 1998; Junor, Hole \& Gillis, 1994; Sainsbury et al., 1995b; Selby, Gillis \& Haward, 1996).

Diagnostic issues have been studied too. For lymphoma there is consistent evidence of greater accuracy in specialist diagnostic services, and that accuracy is improved by specialist pathological review of diagnostic reports on haematological malignancies (Lester et al., 2003). The level of discrepancies between diagnoses made by local clinicians and specialists is similar in most studies, generally around $25 \%$.

Expert opinion supports specialization on the grounds that it is more probable that specialists will have wider experience of the diagnosis and staging of disease; be more aware of the full range of treatment options; and have a fuller understanding of their indications and potential adverse effects. They will be more experienced and proficient in appropriate technical aspects of treatment. Because cancer is a significant part of their work, they can participate more readily in multidisciplinary working and should find it easier to remain up to date and participate in audit, teaching and research.

Evidence of the benefits from multidisciplinary working is currently weak, Limited literature analyses the impact of teamwork in medical settings, including cancer (Amir, Scully \& Borrill, 2004; Baldwin et al., 2004; Haward et al., 2003; Ruhstaller et al., 2006; Whelan, Griffith \& Archer, 2006). The evidence does suggest that teams that work well together achieve better results than those that do not, or are incomplete. Despite the limited evidence, expert opinion overwhelmingly supports the principle of multidisciplinary practice. Important contributions from different disciplines and professions combine to ensure the best decisions for individual patients. Good teams take account of all important information within a collective process, although their methods vary. For example, the United Kingdom model defines the membership and operational arrangements for multidisciplinary teams managing each type of cancer. The arrangements are peer-reviewed and regularly validated against explicit standards.

Evidence of the impact of specialization overlaps with evidence of the importance of caseload (see below), since specialists normally have higher cancer caseloads than generalists.

\section{Clinical caseload (volume) at clinician and hospital level}

There are now many studies on this topic and several systematic reviews 
(Davoli et al., 2005; Halm, Lee \& Chassin, 2002; Hewitt \& Petitti, 2001; Hillner, Smith \& Desch, 2000; Teisberg et al., 2001).

One review of the relationship between hospital volume and mortality concluded that there is compelling support for the hypothesis that a higher hospital caseload equates with better outcome (Hillner, Smith \& Desch, 2000). A further review (Hewitt \& Petitti, 2001) for the Institute of Medicine in the United States suggested a significant relationship between volume and outcomes, again highlighting complex cancer surgery. In the largest systematic review to date (Halm, Lee \& Chassin, 2002), 135 of 272 studies reviewed met the inclusion criteria. These studies covered 27 procedures and clinical conditions. Among these, $71 \%$ of the studies of hospital volume and 69\% of the studies of physician volume reported statistically significant associations between higher volume and better outcomes, strongest for surgery on pancreatic and oesophageal cancer. The authors concluded that high volume is associated with better outcomes across a wide range of procedures and conditions, with the caveats that the magnitude of the association varies greatly and the clinical and policy significance of the findings is complicated by the methodological shortcomings of many studies. Differences in case mix and the processes of care between high- and low-volume providers may explain part of the observed relationship between volume and outcome. A review of the same issues in Norway reached similar conclusions (Teisberg et al., 2001).

The main scientific conclusion from these reviews is that higher caseloads for complex procedures are better for patient outcomes. Low caseloads should generally be avoided. Therefore, although many common surgical procedures can be performed safely and effectively in well-organized local services, complex surgery should be restricted to specialists in higher-volume centres that serve bigger populations. The striking and important similarity in results from the studies of different cancers suggests that, with higher caseloads:

- patients are more likely to be actively managed

- important complications will be reduced

- more patients will survive the perioperative period

- longer-term survival and mortality rates improve

- lengths of stay are shorter, reducing costs.

Together, these studies provide a scientific basis for concentrating some services in facilities serving large populations (typically 1-5 million persons) rather than providing them in local settings. The literature is consistent in both specific and general conclusions. It deserves to have an important bearing on the way services are structured and operated. The evidence is strongest for 
complex surgical procedures for cancers of the upper gastrointestinal tract pancreas, stomach and oesophagus. For pancreatic resections the evidence is dramatic.

There is legitimate concern about the quality of this evidence because necessarily it comes from observational studies, rather than randomized clinical trials. Also, low caseloads are impossible to audit reliably because the numbers of patients and events are too small for statistically robust conclusions, so policy conclusions must be based on an overall assessment of the evidence. There is therefore scope for controversy. The issue has been forcefully expressed in an editorial, entitled "Taking action on the volumequality relationship: how long can we hide our heads in the colostomy bag?" (Smith, Hillner \& Bear, 2003):

The data for much of surgical oncology are compelling enough to demand changes in practice, referral patterns, or both. Complication or mortality rates that are unexplainably high for similar patients are simply not acceptable. If these decisions did not involve livelihood, prestige, and power, we would have demanded action long ago.

\section{Cancer centres and cancer geography}

Cancer centres emerged in most countries before the evidence linking specialization and caseload to better outcomes. The case for cancer centres was founded on a belief that they could offer the fullest range of expertise to treat almost all types of cancer to high standards and would make efficient use of scarce skills and expensive equipment.

There are several reasons why cancer centres have been adopted widely. Many cancers are uncommon and some are rare; some subtypes of common cancers (such as breast cancer) are also rare and complex to treat. No single clinician would have sufficient experience to manage such diseases adequately, unless their practice covered a substantial population. Cancer centres provide the means to bring together enough patients with particular types of rare or uncommon forms of cancer to ensure that they can be managed correctly. Cancer centres also provide a logical base in which to maintain expertise in the delivery of complex treatments for more common cancers, such as radical surgery for cancers of the oesophagus or pancreas.

Some facilities fit naturally into the cancer centre model. For example, radiotherapy tends to be used only for cancer patients. Safe and effective operation of large and expensive radiotherapy installations requires substantial investment in scarce skills such as medical physics, radiation oncology and therapeutic radiography. The growing sophistication of clinical practice, and 
of the computerized equipment in radiotherapy, adds force to these arguments. Modern cancer care needs staff from many different medical disciplines and professions. The complexity of delivering efficient cancer services to a wide range of patients explains the attraction of the cancer centre. It also provides a common identity for staff treating cancer, generating an ethos that supports teaching and research, which in turn adds value to the enterprise.

These arguments do not imply that all cancer patients should be treated in cancer centres. Common cancers occur often enough for most clinical management (except radiotherapy) to be performed safely and efficiently at hospitals much nearer to the patient's home. Even when a particular treatment needs to be provided in a cancer centre, it may be better for other components of care (diagnostic procedures, chemotherapy, follow-up, palliative care) to be provided at district hospitals or other local facilities. For example, links with primary care and community support are easier to manage from district hospitals, where the necessary relationships are well established.

The consequence of a rational case for both local and centralized service components demonstrates a natural cancer geography based on a continuum from the provision of local services in primary care and the community; management of common tumours at district hospitals; and the use of cancer centres for uncommon tumours and complex therapies, plus radiotherapy and chemoradiation. This model requires an explicit commitment to coordination at both operational and policy levels in order to work well. All the organizations involved need a shared basis for making decisions about the development and operation of the whole cancer service. This must include arrangements for sharing information, communications and medical records. Such processes may be relatively informal, or they may be created more formally within organizational entities such as cancer networks.

\section{Case study - the English National Cancer Plan 2000}

A brief account of the implementation of the English cancer plan may serve as a useful example. The NHS Cancer Plan (Department of Health, 2000) built on an earlier policy framework (Expert Advisory Group on Cancer, 1995) that had established clear goals and laid down the essential structures for a specialist, multidisciplinary service. The cancer plan had strong political support from the government of the day. It had four main aims:

1. To save more lives.

2. To ensure that people with cancer get the right professional support as well as the best treatments. 
3. To tackle inequalities in health that mean unskilled workers are twice as likely to die from cancer as professionals.

4. To build for the future through investment in the cancer workforce, through strong research and through preparation for the genetics revolution.

The plan made new commitments in several areas.

On prevention, the plan concentrated on smoking and diet, with new initiatives and resources. There were specific targets to reduce the prevalence of smoking and to narrow the socioeconomic gap between prevalence rates in the least and most affluent populations.

A new target on speed of access required that no cancer patient should wait longer than one month between an urgent referral for suspected cancer and the beginning of treatment (except for good clinical reasons or patient choice).

Substantial new money was provided to enable further development of hospices and specialist palliative care. Population screening in breast cancer was extended to new age groups; cervical screening was enhanced; and there was a commitment to move towards the implementation of bowel cancer screening. Informed access to PSA testing for prostate cancer was made available, but population screening was not.

An important theme of the plan was to improve services for cancer patients. Emphasis was placed on improving access to cancer services, with new guidelines on referral from primary care and a cancer-specific timetable for the implementation of referral targets, starting with breast cancer. Expanding staffing in key disciplines was central to progress, as were increases in the associated infrastructure. An extra $£ 570$ million per year was allocated to support these developments, an inflation-adjusted increase of more than $30 \%$ over three years. By the end of the plan, it was expected that the number of cancer specialists would have risen by one third (around 1000). The impact on related services such as gastroenterology and urology was recognized and numbers of cancer nurses and therapy radiographers were increased.

The structure and organization of services was improved in two main ways: firstly, through the development and implementation of national service guidance on how services for each type of cancer should be delivered. This dealt with local and centralized aspects of service. For the first time in the United Kingdom, some complex work was restricted to larger centres only. The plan emphasized the need for services at all levels to work closely together. The cancer network was selected as the model, and networks were given resources to facilitate their role in leading and supporting implementation. 
Secondly, a quality improvement programme was launched to empower staff to resolve operational problems (such as access to scans) in innovative ways. This became known as the Cancer Services Collaborative.

The government appointed a cancer clinician to prepare the plan and lead the process. This new role of National Cancer Director has been a highly successful innovation. The independent Healthcare Commission was given specific responsibility to oversee the implementation of the national cancer plan. This introduced a radical means of monitoring progress by establishing a programme for national peer review of cancer services, based on specific published standards. This process examines how well cancer services meet the new requirements.

Research was encouraged through the establishment of a new National Cancer Research Institute, comprising all the main bodies involved in cancer research. A specific sum of $£ 20$ million was provided to support the recruitment of cancer patients into randomized trials and other well-designed studies. Within three years, this programme met its target of doubling the number of cancer patients recruited into clinical studies (to $7.5 \%$ of incident cases).

Independent evaluations of the cancer plan (Commission for Health Improvement \& The Audit Commission, 2001; Doll \& Boreham, 2005; Haward \& Amir, 2000; Morris, 2004; Morris, Forman \& Haward, 2007; National Audit Office, 2005a; National Audit Office, 2004; National Audit Office, 2005) have been positive. These show measurable progress on improving patient experiences, meeting targets for access to cancer services and reconfiguring services. Multidisciplinary care of cancer patients is now the norm. New staff and facilities have been delivered. Survival is improving but it is impossible to attribute this to any particular change because so many changes have been made across the entire spectrum of cancer care.

\section{Improving cancer services - some ways and means}

\section{Implementation}

It is easier to prepare policies, strategies or plans than to implement them. Cosmetic changes such as redesignating existing structures may provide an illusion of progress but improving the quality and effectiveness of clinical services always takes time, effort and a combination of many different approaches. It is crucial to establish specific structures and mechanisms designed expressly for the purpose of driving the implementation process, over and above normal management arrangements within a given country. 


\section{Leadership}

It is important to gain the active support of the professional and lay communities involved. Leadership and support for the desired changes from within these communities are powerful aids to progress. Experience in both the United Kingdom and France shows the importance of designating national leadership for the cancer plan. The issues are complex, and cancerspecific leadership can be more effective than relying on managers who may lack the necessary insights into the nature of the changes required and the problems that must be resolved if the plan is to be implemented successfully.

\section{Resources}

Specific resources help to make the right things happen. The French cancer plan (French National Cancer Institute, 2003) specified in detail the intended expansion in staff and facilities. By March 2005, it had created the cancer networks known as canceropôles - an association of about 50 teams and 500 staff including medical and non-medical posts, e.g. psychologists, radiotherapists, nurses, secretarial and administrative staff, physiotherapists and dietitians. There was substantial investment in major equipment $€ 8$ million for PET or MRI scanners and $€ 4.5$ million for radiotherapy equipment. Within two years (by the end of 2004), 294 of the 345 authorized linear accelerators were in place.

\section{Targets}

Targets should be used sparingly and backed by efficient monitoring and performance management. The impact of targets to reduce waiting times for cancer treatment in the United Kingdom provides an example of the value of selective target-setting to address longstanding problems.

\section{Benchmarking}

This term applies to the evaluation of treatment facilities or staffing arrangements (usually in relation to population or workload) on a basis that is specifically designed to be comparable within a country, or even internationally. It improves understanding of the need for investment in expensive skills and facilities and exposes the need for remedial action where staff or equipment levels appear unacceptably low (or high) relative to workload. It has been particularly valuable for radiotherapy services (Bentzen et al., 2005; Slotman et al., 2005), the provision of scanners (PET, MRI and CT) and the recruitment of key oncology staff. 


\section{Service guidance}

In England and Wales, a rigorous evidence-based methodology (Bentzen et al., 2005; Haward, 1998; Haward, 2003; Slotman et al., 2005) was used to develop national service guidance for each type of cancer and for supportive and palliative care. The guidance was published from 1996 to 2007. The national cancer plan included a specific commitment to implement the guidance and this is monitored closely. The guidance has enabled fundamental changes in the configuration of the United Kingdom's cancer services. Each guidance document defines the multidisciplinary teams necessary to deliver services, the roles and responsibilities of all parts of the service, and how they fit together. It explicitly addresses whether any services should be concentrated in facilities serving large populations.

\section{Clinical guidelines}

Clinical guidelines (Grimshaw, Eccles \& Russell, 1995; Grimshaw \& Russell, 1993; Woolf, 1992) are more widely used (and better understood) than service guidance. They can be valuable in improving care and should be developed using reputable evidence-based methods. Local processes are required to agree (or modify) the application of clinical guidelines, and for clinical audit.

\section{Engaging staff}

Plans and policies can appear too remote to many staff. It is highly desirable to involve health-care staff at all levels and for them to engage with these issues. Cancer collaboratives were used in the United Kingdom (Kerr et al., 2002) to encourage and empower staff to identify local problems and to think afresh how to solve them, outside the normal constraints of departmental boundaries and policies. The results were startling - staff successfully resolved many longstanding constraints such as waiting times for scans, or the ways in which patients moved through their care pathways.

\section{Systematic training}

Systematic training programmes for specific new techniques can involve clinicians and improve their effectiveness. For example, rectal cancer outcomes are greatly improved by best surgical practice, especially the technique of total mesorectal excision. This procedure was adopted systematically through a specific training programme in Holland (Kapiteijn, Putter \& van de Velde, 2002), Sweden (Martling et al., 2005) and Norway (Wibe et al., 2002), the first countries to adopt the technique nationally. The programmes included all surgeons designated to perform colorectal cancer surgery; pathologists and 
radiologists were also crucial to success. Colorectal teams in the United Kingdom later adopted this strategy, which has led to better management of rectal cancer. A similar strategy is now being used for sentinel nodes in breast cancer.

\section{Peer review and accreditation}

The use of peer review and formal accreditation has been advocated for breast cancer services across the EU (Blamey \& Cataliotti, 2006). These have already been used in some specialist areas like pathology and on a large scale in the United Kingdom (Scrivens et al., 2001) to examine all services. The latter has proved a powerful learning mechanism for both the reviewers and the reviewed - exposing service weaknesses and enabling unsafe practices to be addressed urgently. Follow-up of problems, including repeat visits, combine to make the process a potent and constructive force for better services.

\section{Monitoring}

It is crucial to know whether desired and planned changes have actually happened. Several mechanisms are important. Cancer registries provide essential population-based information on incidence, demography, trends and survival outcomes. Improved clinical information systems aid understanding of the operation of cancer services. Clinical audit allows actual performance to be assessed against the expected standard.

\section{Research}

This is of vital importance in its own right. Enabling the conduct of research is a key component of all strategies to improve the quality of services. The French plan set a goal of raising the level of cancer research activity more generally and has established seven new network structures (canceropôles) across the country to promote clinical research and increase recruitment to randomized trials. The United Kingdom's plan used new research networks to provide the infrastructure required to support patients' entry into studies throughout the country, with considerable success (Sinha, 2007).

\section{Conclusions}

There is considerable variability in the delivery of cancer services and the outcomes achieved both within and between countries. Understanding this variability and identifying how and where services fall short of current standards can lead to significant improvements in outcomes for people with 
cancer. Improvements in the quality and effectiveness of services are achievable with existing knowledge. A systematic approach to these issues is likely to improve population and individual outcomes.

Comprehensive national cancer plans have been shown to be effective in improving the performance of cancer services and the outcomes they achieve. Their potential as a means of promoting better cancer care should be carefully considered. It is vital that there are effective arrangements to ensure the implementation of these plans. Evidence-based strategies should be used to improve patients' access to multidisciplinary management teams that can deploy the full range of appropriate therapies. Effective coordination of service delivery must apply both to the individual patient and to the overall operation of services. The diagnosis and treatment of uncommon cancers and complex procedures should be concentrated in high-volume centres, with regular audit of results.

Cancer control policies should be developed for the full spectrum of relevant interventions from primary prevention to end-of-life care. Performance and outcomes should be monitored and evaluated. Cancer registries are essential for the long-term evaluation of trends in incidence and survival in populations, and for comparison of these measures within and between countries.

\section{Recommendations}

EU Member States should develop or continue to improve their cancer planning, using an integrated approach and evidence-based strategies for each of the following domains:

- Primary prevention and screening.

- Providing rapid access to diagnosis and multidisciplinary clinical care, using the full range of appropriate therapies and taking account of patients' preferences.

- Coordinating cancer care throughout the process - from diagnosis to therapy, including palliative care.

- Concentrating uncommon or highly complex diagnostic and therapeutic procedures in clinical services that have caseloads sufficient to maintain quality, with regular audit of results.

- Ensuring adequate management of patients' quality of life and psychosocial care.

- Evaluating cancer outcomes. 


\section{REFERENCES}

Amir Z, Scully J, Borrill C (2004). The professional role of breast cancer nurses in multidisciplinary breast cancer care teams. Eur J Oncol Nurs, 8(4):306-314.

Bachmann MO et al. (2003). Influence of specialization on the management and outcome of patients with pancreatic cancer. Br J Surg, 90(2):171-177.

Baldwin LM et al. (2004). Access to multidisciplinary cancer care: is it linked to the use of breast-conserving surgery with radiation for early-stage breast carcinoma? Cancer, 100(4):701709.

Bentzen SM et al. (2005). Towards evidence-based guidelines for radiotherapy infrastructure and staffing needs in Europe: the ESTRO QUARTS project. Radiother Oncol, 75(3):355-365.

Berrino F et al. (1995). Survival of cancer patients in Europe: the EUROCARE study (IARC Scientific Publications No. 132). Lyon, International Agency for Research on Cancer.

Blais $S$ et al. (2006). Social determinants of access to reference care centres for patients with colorectal cancer - a multilevel analysis. Eur J Cancer, 42:3041-3048.

Blamey RW, Cataliotti L (2006). EUSOMA accreditation of breast units. Eur J Cancer, 42(10):1331-1337.

Chouillet AM, Bell CM, Hiscox JG (1994). Management of breast cancer in southeast England. Br Med J, 308:168-171.

Ciccolallo L et al. (2005). Survival differences between European and US patients with colorectal cancer: role of stage at diagnosis and surgery. Gut, 54:268-273.

Coleman MP et al. (2003). EUROCARE-3 summary: cancer survival in Europe at the end of the 20th century. Ann Oncol, 14(Suppl. 5):128-149.

Commission for Health Improvement and the Audit Commission (2001). NHS cancer care in England and Wales (National Service Framework Assessments No. 1). London, UK, Commission for Health Improvement and the Audit Commission.

Davoli M et al. (2005). Volume and health outcomes: an overview of systematic reviews. Epidemiol Prev, 29(Suppl. 3-4):3-63.

Department of Health (2000). The NHS cancer plan. London, Department of Health.

Doll R, Boreham J (2005). Recent trends in cancer mortality in the UK. Br J Cancer, 92(7):1329-1335.

Eaker $S$ et al. (2005). Regional differences in breast cancer survival despite common guidelines. Cancer Epidemiol Biomarkers Prev, 14:2914-2918.

Expert Advisory Group on Cancer (1995). A policy framework for commissioning cancer services. London, Department of Health.

Fayter D et al. (2006). Systematic review of barriers, modifiers and benefits involved in participation in cancer clinical trials (CRD Report 31). York, UK, Centre for Reviews and Dissemination, University of York.

French National Cancer Institute (2003). Mission interministérielle pour la lutte contre le cancer. Plan Cancer 2003-2007 (French) (available at: http://www.e-cancer.fr/)

Gillis CR, Hole DJ (1996). Survival outcome of care by specialist surgeons in breast cancer: a study of 3786 patients in the west of Scotland. Br Med J, 312(7024):145-148.

Grilli R et al. (1998). Do specialists do it better? The impact of specialization on the processes and outcomes of care for cancer patients. Ann Oncol, 9(4):365-374.

Grimshaw J, Eccles M, Russell I (1995). Developing clinically valid practice guidelines. J Eval Clin Pract, 1(1):37-48.

Grimshaw JM, Russell IT (1993). Effect of clinical guidelines on medical practice: a systematic review of rigorous evaluations. Lancet, 342(8883):1317-1322. 
Halm EA, Lee C, Chassin MR (2002). Is volume related to outcome in health care? A systematic review and methodologic critique of the literature. Ann Intern Med, 137(6):511-520.

Harries $S$ et al. (1996). A survey of the management of breast cancer in England and Wales. Ann $R$ Coll Surg Engl, 78:197-202.

Haward RA (1998). Preparing guidelines and documented clinical policies. Ann Oncol, 9(10):1073-1078.

Haward RA (2003). Using service guidance to shape the delivery of cancer services: experience in the UK. Br J Cancer, 89(Suppl. 1):S12-S14.

Haward RA (2006). The Calman-Hine report: a personal retrospective on the UK's first comprehensive policy on cancer services. Lancet Oncol, 7(4):336-346.

Haward RA, Amir Z (2000). Progress in establishing non-surgical oncology within English cancer units. Br J Cancer, 83(3):284-286.

Haward R et al. (2003). Breast cancer teams: the impact of constitution, new cancer workload, and methods of operation on their effectiveness. Br J Cancer, 89(1):15-22.

Hewitt M, Petitti D (2001). Interpreting the volume-outcome relationship in the context of cancer care. Washington DC, National Cancer Policy Board, Institute of Medicine and National Research Council, National Academy Press.

Hillner BE, Smith TJ, Desch CE (2000). Hospital and physician volume or specialization and outcomes in cancer treatment: importance in quality of cancer care. J Clin Oncol, 18(11):23272340 .

Junor EJ, Hole DJ, Gillis CR (1994). Management of ovarian cancer: referral to a multidisciplinary team matters. Br J Cancer, 70(2):363-370.

Kapiteijn E, Putter H, van de Velde CJ (2002). Impact of the introduction and training of total mesorectal excision on recurrence and survival in rectal cancer in the Netherlands. Br J Surg, 89(9):1142-1149.

Kehlet H, Laurberg S (2006). Gastrointestinal surgery - why specialisation? Ugeskr Laeger, 168:1519-1521.

Kerr D et al. (2002). Redesigning cancer care. Br Med J, 324(7330):164-166.

Lauritsen M et al. (2005). Gastric resection for cancer in Denmark, 1999-2004. Ugeskr Laeger, 167:3048-3051.

Lester JF et al. (2003). The clinical impact of expert pathological review on lymphoma management: a regional experience. Br J Haematol, 123(3):463-468.

Ludwig H (2006). Keynote comment: inequalities and shortcomings in European cancer care. Lancet Oncol, 7:276-277.

Martling A et al. (2005). Impact of a surgical training programme on rectal cancer outcomes in Stockholm. Br J Surg, 92(2):225-229.

Marwick C (1999). Better cancer patient care requires systemic changes. J Am Med Assoc, 281:1575-1576.

Marx CI et al. (2006). Ovarian cancer in Denmark. Status of the surgical intervention. Ugeskr Laeger, 168:1537-1540.

Micheli A et al. (2003). European health systems and cancer care. Ann Oncol 14(Suppl. 5):v41v60.

Morris E (2004). The impact of the Calman-Hine report on the processes and outcomes of care for Yorkshire's breast, colorectal and lung cancer patients. Leeds, UK, University of Leeds.

Morris E, Forman D, Haward B (2007). The impact of the Calman-Hine report: analysis of breast and colorectal cancer surgical workloads and the degree of surgical site specialization in the Yorkshire region of the UK, 1990-2000. Eur J Cancer Care (Engl), 16(2):150-155. 
Morris J (1992). Regional variation in the surgical treatment of early breast cancer. Br J Surg, 79(12):1312-1313.

National Audit Office (2004). Tackling cancer in England: saving more lives (3242. HC 364 Session 2003-2004, 1-64). London, The Stationery Office.

National Audit Office (2005). Tackling cancer: improving the patient journey (1-69). London, The Stationery Office.

National Audit Office (2005a). The NHS Cancer Plan: a progress report (1-37). London, The Stationery Office.

National Board of Health (2000). National Cancer Plan - status and proposals for initiatives in relation to cancer treatment. Copenhagen, Denmark, National Board of Health.

National Board of Health (2005). National Cancer Plan II. Copenhagen, Denmark, National Board of Health.

Pitchforth E, Russell E, Van der Pol M (2002). Access to specialist cancer care: is it equitable? Br J Cancer, 87(11):1221-1226.

Richards MA et al. (1996). Variations in the management and survival of women under 50 years with breast cancer in the south east Thames region. Br J Cancer, 73(6):751-757.

Ruhstaller $\mathrm{T}$ et al. (2006). The multidisciplinary meeting: an indispensable aid to communication between different specialities. Eur J Cancer, 42(15):2459-2462.

Sainsbury R et al. (1995a). Does it matter where you live? Treatment variation for breast cancer in Yorkshire. The Yorkshire Breast Cancer Group. Br J Cancer, 71(6):1275-1278.

Sainsbury R et al. (1995b). Influence of clinician workload and patterns of treatment on survival from breast cancer. Lancet, 345(8960):1265-1270.

Sant M (2001). Differences in stage and therapy for breast cancer across Europe. Int J Cancer, 93(6):894-901.

Sant $\mathrm{M}$ et al. (2003). Stage at diagnosis is a key explanation of differences in breast cancer survival across Europe. Int J Cancer, 106(3):416-422.

Sant $M$ et al. (2004). Breast carcinoma survival in Europe and the United States. Cancer, 100(4):715-722.

Scrivens E et al. (2001). Evaluation of national cancer peer review 2001 (CASU Working Paper 1, Controls Assurance Support Unit). Keele, Health Care Standards Unit, Keele University.

Selby P, Gillis C, Haward R (1996). Benefits from specialised cancer care. Lancet, 348(9023):313-318.

Sinha G (2007). United Kingdom becomes the cancer clinical trials recruitment capital of the world. J Natl Cancer Inst, 99(6):420-422.

Slotman BJ et al. (2005). Overview of national guidelines for infrastructure and staffing of radiotherapy. ESTRO-QUARTS: work package 1. Radiother Oncol, 75(3):349-354.

Smith TJ, Hillner BE, Bear HD (2003). Taking action on the volume-quality relationship: how 



\section{Chapter 7 \\ Changes in the management of cancer: the example of colorectal cancer}

Jean Faivre and Côme Lepage

\section{Introduction}

Whatever the nature of the health-care facility in which cancer patients are treated or otherwise cared for, population-based data on their management represent the only viable approach for overall assessment. Most publications on the management of cancer patients come from specialized centres. Population-based studies are rare because they require accurate, detailed and comprehensive data on diagnosis and treatment (seldom available from cancer registries) over a long period.

Over the past few decades, many developments have taken place in the management of cancer. Advances initiated in specialized centres have gradually spread and been incorporated into routine clinical practice, but their impact at population level is not well known.

This short review aims to provide a population-level evaluation of how cancer is managed today and to review the impact of recent trends, e.g. the emphasis on evidence-based medicine and the use of clinical or consensus guidelines and recommendations. Space precludes a comprehensive overview of the changes for each major type of cancer. Colorectal cancer will be used as an example, since many of the changes in its management mirror those that are recommended or are being implemented for other cancers, in principle if not in detail. 
Colorectal cancer was chosen because it represents a major health problem. It has been estimated that in 2006, 413000 people in Europe were newly diagnosed with colorectal cancer and 207000 Europeans died from it (Ferlay et al., 2007). Cancers of the colon and rectum combined comprise the second most common malignant tumour in Europe, both in the number of new cases and the number of deaths.

Early diagnosis is the key to good prognosis. Clinical diagnosis is not difficult, but lack of public awareness of the symptoms, and fear or lack of responsiveness to those symptoms, appears to lead to delay in diagnosis. The prognosis for colorectal cancer is still only moderate, and there are wide international variations within Europe. The five-year relative survival ranges from 30\% to 58\% for patients diagnosed between 1990 and 1994 (Sant et al., 2003). There is also evidence of significant socioeconomic variations in outcome within countries (Coleman et al., 2004; Dejardin et al., 2006).

Many recent changes have altered the management of colorectal cancer (Mitry et al., 2005). These include increased use of surgical resection of the cancer; improved surgical techniques (total mesorectal excision); reduced postoperative mortality; implementation of effective adjuvant chemotherapy in stage III colon cancer and neo-adjuvant radio-chemotherapy in rectal cancer $^{2}$; and the development of multidisciplinary consulting meetings.

A number of EU countries are gradually implementing mass screening using faecal occult blood (FOB) tests. These initiatives follow several large randomized trials that have shown a clear reduction in mortality from colorectal cancer (Faivre et al., 2004; Hardcastle et al., 1996; Kronborg et al., 1996). The EU Council has issued recommendations (Council of the European Union, 2003). Flexible sigmoidoscopy is also being used for screening in some settings. Colorectal cancer can be cured, or even effectively prevented, by detection and resection of a cancer when diagnosed at an early stage, or by removal of adenomas from the bowel lining.

\section{Resection of colorectal cancer}

Colorectal cancer is managed by surgical resection of the primary tumour whenever possible. Radical surgery (i.e. surgery of curative intent) offers the only approach to obtaining a definitive cure although endoscopic removal of a malignant adenoma (polyp) may suffice as a radical treatment.

\footnotetext{
2 'Adjuvant' chemotherapy or radiotherapy is usually used to describe treatments given after primary surgical treatment (e.g. to destroy tumour cells that may remain after surgery). 'Neo-adjuvant' treatments are given before surgery (e.g. to shrink a tumour before surgical removal).
} 
The rate of surgical resection for colorectal cancer varies widely within Europe. A EUROCARE high-resolution study ${ }^{3}$ provides data for patients registered in 1990 by 10 European cancer registries (Gatta et al., 2000). The proportion of resected colorectal cancers varied between $77 \%$ and 93\% It was higher in France, Italy or the Netherlands than in Spain or the United Kingdom. For the same year, American cancer registries included in the Surveillance, Epidemiology and End Results (SEER) programme reported a resection rate of $92 \%$ (Ciccolallo et al., 2005).

A survey of colon cancer management was conducted by eight populationbased cancer registries in France in 1995 (Phelip et al., 2005). There was no significant variation: resection of the primary tumour was performed on average in $90 \%$ of cases, with a very narrow range from $88 \%$ to $93 \%$ among the eight départements (counties) involved. A resection rate around $90 \%$ is not far from the optimum, but the data suggest that improvement is still possible in some countries. Several reports show that in the best-performing countries, a major improvement in the proportion of resected cases was seen between 1975 and 1990, after which it levelled out (Bouvier et al., 2004; Faivre-Finn et al., 2002a; Iversen et al., 2005). This trend was not the result of a planned health policy. On the contrary, it arose from gradual changes in the habits and opinions of clinicians, particularly anaesthetists and surgeons. Such changes in clinical opinion, and especially in clinical practice, have not yet been observed in all European countries.

It is important to underline that the increase in the proportion of patients whose cancer could be resected has been associated with an improvement in the stage at diagnosis (shift toward earlier stage), which is the most important influence on the eventual prognosis (Mitry et al., 2005). Several explanations can be offered: earlier consultation; more frequent and more rapid referral for investigation by general practitioners; more forceful attitudes among surgeons; and also because patients considered too advanced for resection in the past included some patients who are now correctly identified as having early-stage disease and are resected accordingly.

A review of 28 independent studies showed a lower surgical resection rate in elderly patients than in younger patients (Colorectal Cancer Collaborative Group, 2000). The difference between age groups may be due to later presentation, poor performance status, a higher level of comorbidity or simply that clinicians expect a poorer outcome in elderly patients. However, the gap in resection rates between these age groups is closing. Recent data suggest that

\footnotetext{
${ }^{3}$ In this context, a "high-resolution" study implies one in which detailed clinical data on diagnosis, treatment and other variables are specially re-abstracted from the clinical records for a large random sample of patients. These data are more extensive than the usual (low-resolution) data abstracted for routine cancer registration.
} 
for colon cancer, the resection rate is now similar in all age groups up to 85 years and is lower only in the oldest age group (Bouvier et al., 2005). For rectal cancer, the decline in resection rate appears slightly earlier, after the age of 80 . This may be explained by the fact that rectal surgery is more complex.

Some patients may be unsuitable for surgery because of pre-existing comorbidity; this is particularly true for elderly patients. In a population-based study in the Netherlands, the proportion of patients with one or more comorbid conditions varied from about $40 \%$ in patients aged 50-64 years to more than $70 \%$ in those aged 80 or over (Lemmens et al., 2005a). Postoperative morbidity also increases progressively with age, as does the duration of hospital stay (Gross et al., 2006; Lemmens et al., 2005a). An increasing frequency of thromboembolic, respiratory and cardiovascular complications has been reported in relation to age. Some comorbid conditions at the time of diagnosis are predictive of complications after surgery, especially chronic obstructive pulmonary disease and deep vein thrombosis (Lemmens et al., 2007).

It is worth underlining that the few studies available on this topic suggest that elderly patients who are selected for surgery have a subsequent quality of life that is comparable in most respects to that of younger patients. A Canadian study compared the quality of life among patients aged 80 years and over who had undergone surgery for colorectal cancer with that in a group aged less than 70 (Mastracci et al., 2006). The two groups scored similarly on the European Organisation for Research and Treatment of Cancer (EORTC) scales for the quality of life, except for physical functioning and stoma-related problems. Most patients did not require special assistance or alternative living arrangements after discharge from hospital and were able to return to their preoperative level of functioning.

It has been shown that the annual volume of a given procedure in American hospitals is predictive of both short- and long-term survival after surgical resection for cancers of the colon and rectum (Mastracci et al., 2006; Schrag et al., 2002). There was no evidence that underlying differences in the characteristics of the patients (age, sex, race, cancer stage, comorbid illness, socioeconomic status) accounted for these results. In the United States, colorectal cancer surgery is currently performed at many hospitals with very low annual case volumes. This is associated with unfavourable outcomes that are not attributable to differences in case mix.

Available population-based data indicate that the outcomes of surgery can be good for even the oldest age groups. An elderly patient who is believed to be fit for surgery can tolerate a standard surgical procedure without excessive risk 
of postoperative complications. A comprehensive age-specific assessment for determining operative risk should assist in a more rational selection of patients who appear unlikely to benefit from surgery.

One Swedish county has centralized rectal cancer surgery in one hospital and reduced the number of surgeons operating on this condition (from 26 to 4). This reorganization has reduced postoperative mortality and overall morbidity rates by more than half (Smedh et al., 2001). Surgery must be restricted to centres performing an acceptable minimum number of cases each year in order to maintain competence and expertise.

In France, minimum volume thresholds for colorectal cancer surgery have been implemented under the national cancer plan. It has been calculated that $36 \%$ of public hospitals and private institutions performing colorectal surgery would have to discontinue this surgery. Only $8 \%$ of colorectal cancer patients would need to be referred elsewhere, however, because these centres perform only a few procedures each year.

\section{Implementation of therapeutic improvements}

\section{Chemotherapy for stage III colon cancer}

In 1989 and 1990, two large randomized trials demonstrated the efficacy of adjuvant chemotherapy for stage III colon cancer (Laurie et al., 1989; Moertel et al., 1990). These trials compared chemotherapy based on 5-fluorouracil (5-FU) with no chemotherapy. On the evidence of these results, the US National Institutes of Health Consensus Development Conference recommended the use of adjuvant chemotherapy in 1990. This produced an immediate and dramatic increase in the use of adjuvant chemotherapy for stage III colon cancer in the United States (Cronin et al., 2006; Dobie et al., 2006; Jessup et al., 2005; Neugut, Fleischauer \& Sundararajan, 2002; Potosky et al., 2002).

The change has been much more gradual in Europe (Bouchardy et al., 2001; Faivre-Finn et al., 2002b; Lemmens et al., 2005b). In Burgundy, France, it was four years before the proportion of patients under the age of 65 who were treated with adjuvant chemotherapy for colorectal cancer rose to nearly optimal values; it took six years for those aged 65 to 74 (Faivre-Finn et al., 2002b). Treatment for patients aged 75 and over has still not reached this level and the differences between the United States and Europe remain.

Available data for the year 2000 suggest that only $20-25 \%$ of elderly patients with stage III colorectal cancer received adjuvant chemotherapy in Europe (Bouchardy et al. 2001; Faivre-Finn et al. 2002b; Lemmens et al. 2005b) 
compared with 40-50\% in the USA (Cronin et al., 2006; Dobie et al., 2006; Jessup et al., 2005; Neugut, Fleischauer \& Sundararajan, 2002; Potosky et al., 2002). Data from the population-based SEER programme ${ }^{4}$ suggest that 5 FU-based chemotherapy for patients aged 65 or over was associated with a $34 \%$ reduction in mortality. This is similar to the difference described in the randomized studies (Sundararajan et al., 2002).

More frequent use of adjuvant chemotherapy in elderly patients would reduce the number of deaths from colorectal cancer. A review of seven randomized trials has indicated only a small increase in toxicity with 5-FU-based chemotherapy in elderly people (Kohne et al., 2001). While pre-existing comorbidity makes some elderly patients unsuitable for chemotherapy, the risk of toxicity is not sufficient to justify withholding chemotherapy from elderly patients with bowel cancer. In one American study, 53\% of patients aged 75 to 84 received adjuvant chemotherapy in the absence of comorbidity, $47 \%$ had one comorbid condition and 37\% had two (Ayanian et al., 2003). Physicians' attitudes may explain the low utilization of chemotherapy.

Another American study suggested that elderly patients were just as likely as younger patients to accept chemotherapy. However, having chosen to receive treatment, they were less likely to accept major toxicity in exchange for added survival (Yellen, Cella \& Leslie, 1994). The primary determinant of the elderly patient's decision to accept or decline chemotherapy was their physician's advice (Newcomb \& Carbone, 1993). Physicians' awareness of chemotherapy for the elderly must be improved. Treatment decisions for elderly patients should be taken in the context of multidisciplinary consultancy meetings that include advice from a geriatrician.

\section{Palliative chemotherapy}

Palliative chemotherapy has been used in the treatment of advanced colon cancer for many years. However, it was not until 1993 that a randomized study showed that it increased the quality of care and survival compared with best supportive care alone (Kohne et al., 2001). The 1995 survey of colon cancer management in eight population-based registries in France indicated that palliative chemotherapy was used in $62 \%$ of patients aged under 75 (Phelip et al., 2005). In direct contrast with the situation for surgical resection of the primary, however, there was a wide range between the eight départements (49-85\%). This suggests equally wide differences in practitioners' awareness of the value of palliative chemotherapy. Among patients aged 75 or

\footnotetext{
${ }^{4}$ Part of the Natonal Cancer Institute, this operates population-based cancer registration in a number of states and territories of the United States, currently covering some $26 \%$ of the national population.
} 
older, only $9 \%$ received palliative chemotherapy, but without significant regional differences.

These patterns may be explained by clinicians who consider that these patients may be too old to be treated effectively and that chemotherapy is more prone to produce unwanted side-effects in the elderly. However, the treatment for an individual patient should be decided on the basis of known benefits, rather than possible side-effects and the expected impact on the quality of life. A comprehensive geriatric assessment is particularly important in this context.

\section{Adjuvant radiotherapy}

Radiotherapy is now known to be an effective adjuvant treatment to surgery (Glimelius et al., 2003), but this was not always so. In Burgundy, France, trends in the practice of adjuvant radiotherapy were analysed in relation to contemporary scientific knowledge (Faivre-Finn et al., 2000). Although radiotherapists in France have been recommending this treatment for nearly 40 years, the evidence for it was weak before 1995. Some experts favoured postoperative radiotherapy, others preoperative. This explains why only $27 \%$ of patients who underwent radical surgery (with curative intent) for colorectal cancer received radiotherapy during the period 1976-1987.

Between 1985 and 1990, seven trials were published comparing preoperative or postoperative adjuvant pelvic irradiation with surgery alone (Glimelius et al., 2003). For postoperative radiotherapy, two studies reported a nonsignificant reduction in local recurrence, a third was negative. In contrast, three studies of preoperative radiotherapy reported a significant reduction in local recurrence and the fourth reported a non-significant decrease.

It became clear that preoperative radiotherapy was more effective, although postoperative radiotherapy had a moderate effect on local recurrence. Further, the only trial comparing pre- and post-operative radiotherapy demonstrated that local recurrence was significantly reduced in the preoperative arm (Pahlman \& Glimelius, 1990). These publications appear to have had an impact on clinicians' practice. The proportion of patients treated with adjuvant radiotherapy rose to $53 \%$ during $1998-2000$, with a shift towards preoperative radiotherapy.

Other population-based studies in the United States (Ayanian et al., 2003; Mastracci et al., 2006) and the Netherlands (Martijn et al., 2003) show that $50-60 \%$ of patients receive adjuvant radiotherapy. These results suggest that substantial improvement has occurred, largely following the publication of the results of clinical trials, but the use of adjuvant radiotherapy has not yet reached its full potential. This is particularly true for elderly people. Again, 
population-based data indicate that about two thirds of patients under the age of 75 receive radiotherapy, but only $40 \%$ of those aged 75 and over. This may be limited by the need for transportation between home and the few specialized centres. It has also been shown that elderly patients who undergo surgery after preoperative radiotherapy develop more complications (especially pneumonia and cardiac complications) than patients who have surgery alone (Shahir et al., 2006).

\section{Total mesorectal excision}

It is well established that the quality of surgery is particularly important in rectal cancer. Although no randomized trial is available, there is strong evidence that local recurrence rates are lower with total mesorectal excision than with conventional resection (Heald \& Ryall, 1986; MacFarlane, Ryall \& Heald, 1993; Quirke et al., 1986). This finding is related to the large difference in local recurrence between the two techniques.

A community-based study reported a $22.7 \%$ cumulative local recurrence rate at five years following conventional resection over the period 1976-2000 (Manfredi et al., 2006). Similar results were suggested in a review of recurrence rates reported in the reference arm of trials comparing surgery with surgery plus radiotherapy (Pahlman \& Glimelius, 1990). In striking contrast, there is evidence that the local recurrence rate with total mesorectal excision was less than 10\% (Dahlberg, Glimelius \& Pahlman, 1999). Studies from Sweden and the Netherlands have underlined the improved outcomes for rectal cancer following training programmes for total mesorectal excision (Dahlberg et al., 1998; Kronborg et al., 1996). It seems vital that such a strategy should be implemented in other countries.

\section{Continence-preserving operations}

Continence-preserving operations for rectal cancer are recommended whenever possible. A substantial increase in the rate of these operations was reported in France between 1976 and 1990 (Faivre-Finn et al., 2002a). This trend is due partly to reduction of the recommended distal margins ${ }^{5}-\mathrm{a}$ distal clearance of just $1 \mathrm{~cm}$ beyond the visible tumour, measured in an unpinned specimen, has been shown to be adequate (Heald \& Karanjia, 1992; Vernava et al., 1992). The number of patients with a distal margin less than $2 \mathrm{~cm}$ increased from 50\% (1976-1995) to 69\% (1986-1995) (Manfredi et al., 2006). This study also indicates that the increasing proportion of tumours

\footnotetext{
${ }^{5}$ The length of large bowel, beyond the part visibly affected by tumour, which the surgeon is recommended to remove at radical surgery. "Unpinned" refers to the natural length of the excised bowel, before it is prepared for pathological examination.
} 
excised with small distal margins was not associated with an increase in local recurrence.

\section{Evaluation of recommendations on the management of colorectal cancer}

\section{Clinical guidelines}

Various expert groups have prepared recommendations, or guidelines, to assist clinicians in planning each patient's treatment. Clinical practice guidelines can be defined as consensus statements of expert opinion that have been systematically prepared from the latest evidence to assist the practitioner's decision about appropriate management for specific clinical circumstances (Audet, Greenfield \& Field, 1990). Guidelines are also considered to improve the effectiveness of health-care services and to reduce unnecessary costs.

Consensus conferences are increasingly used to disseminate new medical evidence. In practice, adherence to clinical guidelines depends on the physician's awareness of them. Some studies have shown that the implementation of guidelines based on rigorously evaluated facts does improve clinical practice (Grilli \& Lomas, 1994; Grimshaw \& Russell, 1993; Ray-Coquard et al., 1997). Other data suggest that they may have little effect (Kosecoff et al., 1987). Their impact on clinical practice may vary from one guideline to another (Grimshaw \& Russell, 1993).

Community-based studies represent the best approach for checking the implementation of guidelines and their impact on the outcome of treatment. A study in Burgundy, France, was conducted in 2000, two years after the consensus conference on colon cancer (Lepage et al., 2006) to determine if patients were being treated in accordance with the recommendations. Pretreatment work-up was classified as in conformity with the guidelines in $48 \%$ of cases, incomplete in $22 \%$ and excessive in $30 \%$. Incomplete work-up referred to either incomplete exploration of the colon or the absence of an abdominal ultrasound examination. Excessive work-up was related to measurement of CEA (carcino-embryonic antigen), no longer recommended as a preoperative measure because it does not influence the diagnosis or the strategy for treatment. This probably reflects the difficulty that physicians experience in changing long-standing clinical practice. Surgical resection was recommended as the main treatment where possible, and this appears to have been closely followed - it is difficult to envisage a major improvement on a $90 \%$ resection rate. 
The number of lymph nodes removed surgically for pathological examination, to detect spread of the tumour, is important for reliable staging of the disease. The recommended number of at least eight lymph nodes had been harvested in only $69 \%$ of cases. Pathologists must be made more aware that sufficient numbers of lymph nodes need to be examined in order to provide optimal treatment for patients with colon cancer.

Nearly $70 \%$ of the patients received chemotherapy treatment according to the recommendations in the guidelines. Patients with stage II colon cancer were overtreated most often - despite the current recommendations, one quarter of those under 75 years of age were given chemotherapy but should not have been. This may be explained by the fact that the survival difference between treated and untreated patients is statistically significant when patients with stage II and III disease are pooled, because of the important survival benefit in stage III. The subject has been controversial; certain experts and representatives of the pharmaceutical industry have suggested that chemotherapy may be effective in some situations. In contrast, three-quarters of patients aged 75 years or more with stage III colon cancer were not being treated, whereas they should have been.

It may be concluded that adherence to official recommendations varies from one recommendation to another. For some guidelines, clinical adherence is good. The main reasons for divergence from the consensus standard appear to be inertia (CEA measurement), difficulty in carrying out the recommended action (examination of sufficient number of lymph nodes), and lack of familiarity with the clinical background (chemotherapy in the elderly).

\section{Multidisciplinary consulting meetings}

The 1998 consensus conference on the management of colon cancer in France and the National Cancer Plan (2003) both underlined the need for multidisciplinary meetings and for including as many patients as possible in therapeutic trials. French cancer registries conducted a special survey in 2000 (Bouvier et al., 2007). Globally, multidisciplinary meetings were held for only one third of patients, a proportion that varied ten-fold between different residential districts. The place of diagnosis also affected the practice $-52 \%$ of university hospitals, $31 \%$ of non-university hospitals and $29 \%$ of private clinics held multidisciplinary meetings. Although a multidisciplinary meeting is of particular importance in deciding the best treatment for patients over 75 , fewer elderly patients were the subjects of multidisciplinary review.

It can be safely concluded that the proportion of patients whose management was decided by a multidisciplinary approach is much too low. Such cross- 
sectional surveys will need to be repeated in order to aid understanding of how the measures included in the Cancer Plan are being implemented in practice. Regular review of performance in each hospital might also be helpful.

\section{Clinical trials}

It is well known that therapeutic trials should include as many patients as possible. A survey conducted by 12 cancer registries in France in 2000 indicated that only $4.3 \%$ of patients with colorectal cancer were included in a therapeutic trial (Bouvier et al., 2007). Considering only those patients who met the eligibility criteria for available randomized trials, the effective overall proportion included was $7.3 \%$. This ranged widely between geographical districts - from $0.7 \%$ to $16.4 \%$.

This geographical variability emphasizes the importance of measures to develop clinical research, announced under the Cancer Plan. In particular, the development of mobile teams with clinical research assistants should improve the dissemination of information and help to reduce geographical variation. Presentation of a patient's clinical dossier at a multidisciplinary meeting doubled the chance of inclusion in a trial $-10.3 \%$ compared to $5.3 \%$ with no multidisciplinary meeting. Interestingly, trial inclusion was not influenced significantly by the health-care facility responsible for diagnosis - whether public or private.

In 2000, no trial was available for patients over the age of 75 . On the request of investigators, $1 \%$ of elderly patients were included in trials. This may help to explain current undertreatment of older subjects. Either elderly patients must be considered eligible for clinical trials or trials devoted to subjects over 75 should be proposed.

\section{Development of mass screening}

Over the past 20 years, considerable research efforts have been launched to evaluate the capacity of various screening tests to reduce colorectal cancer mortality and incidence (see Chapter 4). Available studies indicate that biennial screening with faecal occult blood (FOB) tests is effective in reducing mortality. The three European studies, performed in general populations of average risk, provided very similar results. The overall reduction in colorectal cancer mortality in the population as a whole was in the narrow range of 15$18 \%$, but $33-39 \%$ among those who actually participated in the screening studies (Faivre et al., 2004; Hardcastle et al., 1996; Kronborg et al., 1996). Comparable results were obtained in a study among volunteers in the United States (Mandel et al., 1999). 
High compliance with the screening test is essential in these programmes. Compliance must be at least $50 \%$ in the first round of screening and remain high in successive rounds in order to reduce mortality significantly. Cancer screening must be coordinated at the national level and organized regionally. Maximum effectiveness is achieved by rigorous organization - central invitation procedures, a call-and-recall system and evaluation of quality assurance. The active participation of primary-care physicians is crucial to obtain a high participation rate. They must be able to motivate patients to comply with the screening programme and the requirements of the primary screening test, and to ensure that all subsequent investigations are carried out.

Taking account of these trials, the European Commission's Advisory Committee on Cancer Prevention recommended that colorectal cancer screening should be organized across the EU for those aged 50 to 74 (Advisory Committee on Cancer Prevention, 2000). This resulted in the inclusion of colorectal cancer screening in the European Code Against Cancer (Boyle et al., 2003) and a statement by the Council of the European Union (Council of the European Union, 2003).

So far, this recommendation has only been followed by some Member States. National programmes have been launched or announced in Finland, France, Germany, the Netherlands and the United Kingdom. Pilot studies are ongoing in Denmark, Ireland, Italy and the Czech Republic. Member States that have so far shown no interest in a colorectal cancer screening programme (opting for different health-policy priorities) have failed to recognize the huge importance and potential impact of this disease on their populations. It is possible that several of these countries have concluded (incorrectly) that the effectiveness of colorectal cancer screening has not yet been sufficiently firmly established, or that it can be difficult to reproduce the benefits reported from the trials in the general population.

Some subjects at particularly high risk of colorectal cancer require regular diagnostic surveillance with colonoscopy. Persons with a history of colorectal cancer or adenoma are one such group; others include those with longstanding inflammatory bowel disease; and first-degree relatives of an index case who developed colorectal cancer before the age of 60. People with a suspected inherited susceptibility to colorectal cancer must be referred for genetic counselling and adequate follow-up.

\section{Conclusions and recommendations}

Population-based cancer registries play an important role in the improvement of cancer control. In particular, they contribute to the evaluation of how 
cancer is managed, and quantify the impact of new treatments at a population level, by providing data on population-based survival. They are also essential for evaluating the effect of clinical guidelines on cancer management and screening. Adequate manpower is required for regular collection of the necessary data. Adequate funding is needed to achieve this crucial public health function.

Cancer registry data indicate that the quality of care has improved. In particular, surgical resection rates for colorectal cancer and many other solid tumours are not far from the optimum in many European countries. Equally, the available data indicate that improvements are still possible in some countries. Thus, treatment of curative intent is performed less often in the elderly, partly because of poor performance status or the presence of comorbidity, but also because clinicians have lower expectations (which may not be justified) of a successful outcome. The available population-based data indicate that surgery can have good outcomes in even the oldest age groups. A comprehensive geriatric assessment to determine operative risk should assist in the selection of patients who may otherwise appear unlikely to benefit from surgery. Advanced age alone must not be a contraindication to surgery.

It has been clearly demonstrated that hospital volume predicts both short- and long-term survival following surgical resection. Minimum thresholds must be implemented for the number of procedures of a given type that a centre must perform each year. Complex treatments and rare procedures must be concentrated in centres where all the necessary expertise is available.

Adjuvant, neo-adjuvant and palliative treatments of proven clinical effectiveness are sometimes implemented too slowly. A multidisciplinary approach to cancer care is required to make the best decisions about each patient's diagnosis, treatment and support. This is particularly the case for elderly patients. Available data suggest that substantial changes have occurred but these treatments have not yet reached their full potential for the elderly, anywhere in Europe. Again, the importance of multidisciplinary consultations for such patients must be stressed, including the advice of a geriatrician.

Cancer screening has become a political priority in Europe. A Council recommendation was adopted in December 2005, endorsed by the health ministers of all Member States. So far, fewer than half of the Member States have followed the recommendation for FOB screening for colorectal cancer in men and women aged 50 to 74 , either by introducing a national screening programme or by conducting pilot studies.

Other EU recommendations concern mammography screening for breast cancer and cervical smear screening for cervical abnormalities. The scientific 
evidence to recommend screening for other cancers is inadequate. It is clear that many EU Member States have not implemented the Council recommendations on cancer screening. Public health specialists, clinicians and cancer patient groups must apply sustained pressure to encourage their national governments to act.

\section{REFERENCES}

Advisory Committee on Cancer Prevention (2000). Recommendations on cancer screening in the European Union. Eur J Cancer, 36(12):1473-1478.

Audet AM, Greenfield S, Field M (1990). Medical practice guidelines: current activities and future directions. Ann Intern Med, 113(9):709-714.

Ayanian JZ et al. (2003). Use of adjuvant chemotherapy and radiation therapy for colorectal cancer in a population-based cohort. J Clin Oncol, 21(7):1293-1300.

Bouchardy $\mathrm{C}$ et al. (2001). Adjuvant chemotherapy for colon carcinoma with positive lymph nodes: use and benefit in routine health care practice. Br J Cancer, 85(9):1251-1257.

Bouhier $\mathrm{K}$ et al. (2004). Changing practices for diagnosis and treatment of colorectal cancer in Calvados:1990-1999. Gastroenterol Clin Biol, 28(4):371-376.

Bouvier AM et al. (2005). Trends in the management and survival of digestive tract cancers among patients aged over 80 years. Aliment Pharmacol Ther, 22(3):233-241.

Bouvier AM et al. (2007). Place of multidisciplinary consulting meetings and clinical trials in the management of colorectal cancer in France in 2000. Gastroenterol Clin Biol, 31(3):286-291.

Boyle et al. (2003). European Code Against Cancer and scientific justification: third version (2003). Ann Oncol, 14(7):973-1005.

Ciccolallo et al. (2005). Survival differences between European and US patients with colorectal cancer: role of stage at diagnosis and surgery. Gut, 54:268-273.

Coleman MP et al. (2004). Trends and socioeconomic inequalities in cancer survival in England and Wales up to 2001. Br J Cancer, 90(7):1367-1373.

Colorectal Cancer Collaborative Group (2000). Surgery for colorectal cancer in elderly patients: a systematic review. Lancet, 356(9234):968-974.

Council of the European Union (2003). Council recommendation of 2 December 2003 on cancer screening. Official Journal of the European Union (2003/878/EC, L327/34- 38).

Cronin DP et al. (2006). Patterns of care for adjuvant therapy in a random population-based sample of patients diagnosed with colorectal cancer. Am J Gastroenterol, 101(10):2308-2318.

Dahlberg M, Glimelius B, Pahlman L (1999). Changing strategy for rectal cancer is associated with improved outcome. Br J Surg, 86(3):379-384.

Dahlberg M et al. (1998). Improved survival in patients with rectal cancer: a population-based register study. Br J Surg, 85(4):515-520.

Dejardin O, Remontet L, Bouvier AM (2006). Socioeconomic and geographic determinants of survival of patients with digestive cancers in France. Br J Cancer, 95:944-949.

Dobie SA et al. (2006). Completion of therapy by Medicare patients with stage III colon cancer. J Natl Cancer Inst, 98(9):610-619.

Faivre-Finn C et al. (2000). Changes in the practice of adjuvant radiotherapy in resectable rectal cancer within a French well-defined population. Radiother Oncol, 57(2):137-142.

Faivre-Finn C et al. (2002a). Colon cancer in France: evidence for improvement in management and survival. Gut, 51(1):60-64. 
Faivre-Finn C et al. (2002b). Chemotherapy for colon cancer in a well-defined French population: is it under- or over-prescribed? Aliment Pharmacol Ther, 16(3): 353-359.

Faivre J et al. (2004). Reduction in colorectal cancer mortality by fecal occult blood screening in a French controlled study. Gastroenterology, 126(7):1674-1680.

Ferlay J et al. (2007). Estimates of the cancer incidence and mortality in Europe in 2006. Ann Oncol, 18(3):581-592.

Gatta G et al. (2000). Understanding variations in survival for colorectal cancer in Europe: a EUROCARE high resolution study. Gut, 47(4):533-538.

Glimelius B et al. (2003). A systematic overview of radiation therapy effects in rectal cancer. Acta Oncol, 42(5-6):476-492.

Grilli R, Lomas J (1994). Evaluating the message: the relationship between compliance rate and the subject of a practice guideline. Med Care, 32(3):202-213.

Grimshaw JM, Russell IT (1993). Effect of clinical guidelines on medical practice: a systematic review of rigorous evaluations. Lancet, 342(8883):1317-1322.

Gross CP et al. (2006). Multimorbidity and survival in older persons with colorectal cancer. J Am Geriatr Soc, 54(12):1898-1904.

Hardcastle JD, Chamberlain JO, Robinson MHE (1996). Randomized controlled trial of faecal-occult-blood screening for colorectal cancer. Lancet, 348:1472-1477.

Heald RJ, Karanjia ND (1992). Results of radical surgery for rectal cancer. World J Surg, 16(5):848-857.

Heald RJ, Ryall RD (1986). Recurrence and survival after total mesorectal excision for rectal cancer. Lancet, 1(8496):1479-1482.

Iversen LH et al. (2005). Age and colorectal cancer with focus on the elderly: trends in relative survival and initial treatment from a Danish population-based study. Dis Colon Rectum, 48(9):1755-1763.

Jessup JM et al. (2005). Adjuvant chemotherapy for stage III colon cancer: implications of race/ethnicity, age, and differentiation. J Am Med Assoc, 294(21):2703-2711.

Kohne CH et al. (2001). Chemotherapy in elderly patients with colorectal cancer. Ann Oncol, $12(4): 435-442$.

Kosecoff J et al. (1987). Effects of the National Institutes of Health Consensus Development Program on physician practice. J Am Med Assoc, 258(19):2708-2713.

Kronborg O et al. (1996). Randomised study of screening for colorectal cancer with faecaloccult-blood test. Lancet, 348(9040):1467-1471.

Laurie JA et al. (1989). Surgical adjuvant therapy of large-bowel carcinoma: an evaluation of levamisole and the combination of levamisole and fluorouracil. The North Central Cancer Treatment Group and the Mayo Clinic. J Clin Oncol, 7(10):1447-1456.

Lemmens VE et al. (2005a). Co-morbidity leads to altered treatment and worse survival of elderly patients with colorectal cancer. Br J Surg, 92(5):615-623.

Lemmens VE et al. (2005b). Adjuvant treatment for elderly patients with stage III colon cancer in the southern Netherlands is affected by socioeconomic status, gender, and comorbidity. Ann Oncol, 16(5):767-772.

Lemmens VE et al. (2007). Which comorbid conditions predict complications after surgery for colorectal cancer? World J Surg, 31(1):192-199.

Lepage C et al. (2006). Are the recommendations of the French consensus conference on the management of colon cancer followed up? Eur J Cancer Prev, 15(4):295-300.

MacFarlane JK, Ryall RD, Heald RJ (1993). Mesorectal excision for rectal cancer. Lancet, 341(8843):457-460. 
Mandel JS et al. (1999). Colorectal cancer mortality: effectiveness of biennial screening for fecal occult blood. J Natl Cancer Inst, 91(5):434-437.

Manfredi $S$ et al. (2006). Incidence and patterns of recurrence after resection for cure of colonic cancer in a well defined population. Br J Surg, 93(9):1115-1122.

Martijn H et al. (2003). Improved survival of patients with rectal cancer since 1980: a population-based study. Eur J Cancer, 39(14):2073-2079.

Mastracci TM et al. (2006). The impact of surgery for colorectal cancer on quality of life and functional status in the elderly. Dis Colon Rectum, 49(12):1878-1884.

Mitry E et al. (2005). Improvement in colorectal cancer survival: a population-based study. Eur J Cancer, 41(15):2297-2303.

Moertel CG et al. (1990). Levamisole and fluorouracil for adjuvant therapy of resected colon carcinoma. N Engl J Med, 322(6):352-358.

Neugut AI, Fleischauer AT, Sundararajan V (2002). Use of adjuvant chemotherapy and radiation therapy for rectal cancer among the elderly: a population-based study. J Clin Oncol, 20:2643-2650.

Newcomb PA, Carbone PP (1993). Cancer treatment and age: patient perspectives. J Natl Cancer Inst, 85(19):1580-1584.

Pahlman L, Glimelius B (1990). Pre- or postoperative radiotherapy in rectal and rectosigmoid carcinoma. Report from a randomized multicenter trial. Ann Surg, 211(2):187-195.

Phelip JM et al. (2005). Are there regional differences in the management of colon cancer in France? Eur J Cancer Prev, 14(1):31-37.

Potosky AL et al. (2002). Age, sex, and racial differences in the use of standard adjuvant therapy for colorectal cancer. J Clin Oncol, 20(5):1192-1202.

Quirke P et al. (1986). Local recurrence of rectal adenocarcinoma due to inadequate surgical resection. Histopathological study of lateral tumour spread and surgical excision. Lancet, 2(8514):996-999.

Ray-Coquard I et al. (1997). Impact of a clinical guidelines program for breast and colon cancer in a French cancer center. J Am Med Assoc, 278(19):1591-1595.

Sant M et al. (2003). EUROCARE-3: survival of cancer patients diagnosed 1990-94 - results and commentary. Ann Oncol, 14(Suppl. 5):v61-v118.

Schrag D et al. (2002). Hospital and surgeon procedure volume as predictors of outcome following rectal cancer resection. Ann Surg, 236(5):583-592.

Shahir MA et al. (2006). Elderly patients with rectal cancer have a higher risk of treatmentrelated complications and a poorer prognosis than younger patients: a population-based study. Eur J Cancer, 42(17):3015-3021.

Smedh K et al. (2001). Reduction of postoperative morbidity and mortality in patients with rectal cancer following the introduction of a colorectal unit. Br J Surg, 88(2):273-277.

Sundararajan V et al. (2002). Survival associated with 5-fluorouracil-based adjuvant chemotherapy among elderly patients with node-positive colon cancer. Ann Intern Med, 136(5):349-357.

Vernava AM III et al. (1992). A prospective evaluation of distal margins in carcinoma of the rectum. Surg Gynecol Obstet, 175(4):333-336.

Yellen SB, Cella DF, Leslie WT (1994). Age and clinical decision making in oncology patients. J Natl Cancer Inst, 86(23):1766-1770. 


\section{Chapter 8 \\ Survival of European cancer patients}

Franco Berrino and Riccardo Capocaccia

\section{Introduction}

Since the earliest days of scientific medicine, the proportion of patients who are cured of a disease has been considered the basic parameter by which to assess the effectiveness of medical treatment for it (Louis, 1835). For chronic diseases, the proportion of patients who are cured is usually estimated as the probability of survival, in the absence of other causes of death, at various intervals of time after the diagnosis. In cancer research, the survival of cancer patients in clinical trials is a straightforward indicator - the greater the proportion of survivors, the better the treatment.

By contrast, in studies of the effectiveness of cancer control, which require population-based data from cancer registries, survival is a more complex indicator. The interpretation of survival differences between populations and over time requires careful insight. A longer interval between the dates of diagnosis and death may simply be the result of earlier diagnosis, but it may of course also be due to postponement of the eventual date of death, or even both. The distinction is important, because inexperienced readers usually interpret differences in survival as being attributable solely to differences in the quality of cancer treatment.

Disentangling the contribution of these two components of variation or trends in survival is far from straightforward. Later death may indeed reflect the effectiveness of better treatment, but it may also be the result of conventional treatment being more effective precisely because the diagnosis was made earlier. Earlier diagnosis and earlier treatment do not necessarily delay the eventual date of death, however, and in such circumstances they are not necessarily advantageous for the patient. This is sometimes referred to as "useless early diagnosis", even if it may confer the advantage of a less mutilating treatment. 
There is growing evidence that the increasing availability of highly sensitive screening and other diagnostic techniques may cause overdiagnosis (and thus overtreatment) of cancer, i.e. the diagnosis of tumours that are formally malignant, but very slow-growing, and which would not otherwise be detected in the patient's lifetime. Overdiagnosis has been reported, or suggested, for cancers of the breast (Zahl, Strand \& Maehlen, 2004), kidney, prostate (Fleshner \& Klotz, 2002; Telesca, Etzioni \& Gulati R, 2007), and lung (Bach et al., 2007; Sone et al., 1998), as well as for melanoma (Welch, Woloshin \& Schwartz, 2005) and childhood neuroblastoma (Schilling, Spix \& Berthold, 2002; Woods, Gao \& Shuster, 2002). Therefore, when evaluating differences in cancer survival between populations, or trends over time, one must always consider that part of the difference may be due to lead-time bias (i.e. the length of time by which diagnosis has been brought forward) in one or other of the populations or time periods, or, alternatively, to overdiagnosis.

Usually, earlier diagnosis is associated with postponement of death. Thus, screening programmes have proved effective in reducing death rates for cancers of the cervix uteri, breast and large bowel (colon and rectum). It is also likely that earlier treatment is important in reducing mortality rates for several other cancers. Longer survival reflects greater or more efficient investment in cancer control, regardless of whether it is due to earlier diagnosis or to better treatment.

The detection of large differences in cancer survival between populations should stimulate health-care planners and politicians to determine the cause whether it be late diagnosis, lack of provision (or inaccessibility) of modern treatment, or different pathological characteristics of the tumours - and to implement strategies to reduce the differences. When survival in one country is conspicuously lower than in other countries of similar wealth, the health system may not be functioning as it should. But the problem is not restricted to countries with low survival: very high survival may also suggest that there is substantial overdiagnosis.

Population-based cancer registration in Europe began in the Nordic countries, the United Kingdom and Slovenia, where national registration started from the 1950s and 1960s. From the 1970s and 1980s onwards, regional cancer registration has also been implemented in many central, southern and western European countries. Cancer registries have provided population-based survival statistics since the 1960s (Cutler, 1964). However, the largest coordinated initiative has been the EUROCARE project, a very large European cancer registry-based study of the survival and care of cancer patients (Berrino, Capocaccia \& Estève, 1999; Berrino, Capocaccia \& Gatta, 2003; Berrino et al., 2007; Berrino et al., 1995; Verdecchia et al., 2007). The aims of 
EUROCARE are to monitor, analyse and explain between-country differences and trends in cancer survival. EUROCARE provides the most systematic data available on the patterns of cancer survival in Europe, and some of the latest results are presented in this chapter.

\section{The EUROCARE project}

The EUROCARE project started in 1989 with financial support from the European Community. It was later funded by the Italian foundation Compagnia di San Paolo. A first monograph was published in 1995, with data on cancer patients diagnosed between 1978 and 1984 among 30 populations in 12 countries. The second monograph (1999) included data on cancer patients diagnosed between 1985 and 1989 among 45 populations in 17 European countries, while the third (2003) covered patients diagnosed between 1990 and 1994 among 56 populations in 22 countries. More detailed information is available on the EUROCARE web site (http://www. eurocare.it/).

The most recent study (EUROCARE-4) concerns cancer patients diagnosed between 1995 and 1999, and is based on data from 23 European countries. More than half (47) of the 83 participating cancer registries also provided data on the survival of cancer patients diagnosed between 2000 and 2002, and 30 registries had sufficient data to study five-year and ten-year cancer survival trends from the early 1990s to the early 2000s (Verdecchia et al., 2007).

For 13 of the 23 countries that contributed data to EUROCARE-4, the entire population is covered by cancer registration (Austria, Denmark, England, Finland, Iceland, Ireland, Malta, Northern Ireland, Norway, Scotland, Slovenia, Sweden and Wales) (Table 8-1). The other ten countries were represented by one or more regional cancer registries covering part of the national population $(58 \%$ in Belgium, $43 \%$ in Portugal, $34 \%$ in the Netherlands, 28\% in Italy, 17\% in France and in Switzerland, 16\% in Spain, $9 \%$ in Poland, $8 \%$ in the Czech Republic and slightly more than $1 \%$ in Germany). In order to obtain an estimate of the mean survival in Europe, we extrapolated the data for countries with partial coverage to their whole population. Regional estimates were also derived, according to the geographical areas defined by the United Nations (GLOBOCAN 2002 database http://www.dep.iarc.fr/globocan/database, accessed 5 May 2007).

- Southern Europe (represented in EUROCARE by cancer registries from Italy, Malta, Portugal, Slovenia, Spain). 
Table 8-1 Population coverage, number of adult cancer patients diagnosed 1995-99 and included in analyses, proportion of microscopically verified tumours and proportion of patients who were followed-up for less than five years: EUROCARE-4, by country

\begin{tabular}{|c|c|c|c|c|}
\hline & $\begin{array}{l}\text { ation } \\
1 \%)\end{array}$ & $\begin{array}{r}\text { Number of } \\
\text { patients }\end{array}$ & $\begin{array}{r}\text { Microscopically } \\
\text { verified }^{2}(\%)\end{array}$ & $\begin{array}{l}\text { Censored within } \\
\text { five years (\%) }\end{array}$ \\
\hline Austria & 100 & 146217 & 93 & 19 \\
\hline Belgium & 58 & 79622 & 87 & 18 \\
\hline Czech Republic & 8 & 16651 & 87 & 17 \\
\hline Denmark & 100 & 101349 & 91 & 9 \\
\hline Finland & 100 & 91135 & 95 & 11 \\
\hline France & 17 & 80016 & 96 & 1 \\
\hline Germany & 1 & 24658 & 95 & 20 \\
\hline Iceland & 100 & 4541 & 96 & 10 \\
\hline Ireland & 100 & 59259 & 86 & 9 \\
\hline Italy & 28 & 365832 & 85 & 10 \\
\hline Malta & 100 & 5757 & 89 & 10 \\
\hline Netherlands & 34 & 107444 & 95 & 13 \\
\hline Norway & 100 & 84125 & 92 & 9 \\
\hline Poland & 9 & 56131 & 79 & 9 \\
\hline Portugal & 43 & 31276 & 94 & 23 \\
\hline Slovenia & 100 & 31655 & 92 & 8 \\
\hline Spain & 16 & 94306 & 91 & 9 \\
\hline Sweden & 100 & 185485 & 98 & 11 \\
\hline Switzerland & 17 & 37758 & 95 & 10 \\
\hline UK England ${ }^{3}$ & 100 & 900115 & 83 & 9 \\
\hline UK Northern Irelanc & 100 & 28687 & 81 & 8 \\
\hline UK Scotland & 100 & 110905 & 85 & 8 \\
\hline UK Wales & 100 & 56162 & na & 8 \\
\hline All cases & & 2699086 & 87 & 10 \\
\hline
\end{tabular}

- Central Europe (Austria, Belgium, Germany, France, the Netherlands and Switzerland).

- Eastern Europe (Czech Republic and Poland).

- Northern Europe (Denmark, Finland, Iceland, Ireland, Norway, Sweden and the United Kingdom, all with 100\% coverage).

These countries include a large proportion of the population of Europe, and they will be referred to below as "Europe". The important issue of whether average survival estimates for the pool of these countries are representative of cancer survival in Europe as a whole has been addressed extensively elsewhere (Coleman et al., 2003).

The main survival indicator used in the EUROCARE study is relative survival. This is the ratio of the observed survival among the cancer patients and the survival that would have been expected if the cancer patients had simply had the same mortality as people in the general population of the same age and sex 
(expected survival). For cancer patients, relative survival is an estimate of their survival from cancer, in the absence of any other cause of death. In children and young adults, observed and relative survival are often similar, because other causes of death are not common. Among elderly patients, however, the difference becomes substantial, because other causes of death are very common. For example, the average risk of death within five years among people aged 75 and over in Europe is about 60\%; if the observed survival of cancer patients in this age group at five years were $20 \%$, the relative survival would be $33 \%(0.20 / 0.60=0.33)$. The difference between $20 \%$ and $33 \%$ represents the impact of other causes of death (background mortality), which we wish to eliminate in the comparisons of cancer survival between different countries. That is why the use of relative survival facilitates the comparison of cancer survival between countries with widely different background mortality.

In the EUROCARE project, relative survival was estimated using the Hakulinen method (Hakulinen 1982; US Department of Human Services, 2001), with estimates of background mortality derived from population life tables for each cancer registry area.

Age is a major determinant of relative survival (Berrino et al., 1995; Berrino et al., 2003; Berrino et al., 1999). To account for differences in the age profile of cancer patients in each country or region, relative survival was adjusted for age by the direct method, using a set of age-specific weights specially designed for international cancer survival comparison - the International Cancer Survival Standards (Corazziari, Quinn \& Capocaccia, 2004). These standards reflect three different age distributions: one for the majority of cancers, which mainly affect the elderly, another for those which affect mainly young adults (e.g. testicular cancer, non-Hodgkin lymphoma, acute lymphatic leukaemia); and a third for cancers for which the risk does not vary greatly with age (e.g. cervix uteri, thyroid, brain).

One limitation of conventional survival estimates is that they refer to patients diagnosed some years before the analyses are carried out, and they may be less relevant for patients diagnosed more recently. To address this limitation, we also used data from those cancer registries that could provide data for cancer patients diagnosed during 2000-2002 (and followed up to 31 December 2003), and the analysis was restricted to the survival experience within this more recent time period (period analysis). Experience has shown that these "period" survival estimates usually provide a very good prediction of the longer-term survival that will eventually be observed for those patients, some time in the future (Brenner \& Hakulinen, 2002; Brenner, Soderman \& Hakulinen, 2002; Ellison, 2006; Talback, Stenbeck \& Rosen, 2004). 
This chapter presents conventional five-year survival estimates, derived from the data for 2.7 million adults (aged 15 years or over) in Europe who were diagnosed with cancer during 1995-99. It also includes "period" survival estimates, to predict survival at five and ten years after diagnosis for patients diagnosed during the period 2000 to 2002 . We do this both for all cancers combined, and for selected individual types of cancer.

\section{Survival of cancer patients in Europe}

Overall, the 83 cancer registries in the EUROCARE- 4 study provided data for about 3 million adult cancer patients diagnosed during the period 1995 to1999. After the exclusion of cases where the cancer was not the patient's first cancer $(6 \%)$, or was reported only from a death certificate (4\%) or at autopsy $(0.5 \%)$, or with faulty data that the cancer registries were not able to correct (0.1\%), 2699086 cancer patients were included in the analyses.

Overall, about $90 \%$ of these patients were followed up for at least five years (Table $8-1$ ). The $10 \%$ followed up for less than five years were mainly diagnosed in 1999, and could only be followed up for about four years by the end of the study on 31 December 2003. Only 1\% were lost to follow-up (censored from the analysis) within four years of diagnosis. For the great majority of cases, there was microscopic verification of the cancer diagnosis, ranging from more than 95\% in France, Iceland, the Netherlands and Sweden to below $80 \%$ in Poland and the United Kingdom.

The average five-year relative survival in Europe, weighted by geographic region, ranges from $94 \%$ for men with testicular cancer to $6 \%$ for patients with pancreatic cancer (Fig. 8-1). For all cancers and both sexes combined, relative survival at five years was $52 \%$. The figure was $46 \%$ for men and $58 \%$ for women.

For breast cancer (women), skin melanoma, Hodgkin's disease, and cancers of the thyroid, lip and testis, five-year relative survival was $80 \%$ or higher. These cancers with a good prognosis represent about $20 \%$ of all cancers, with breast cancer alone accounting for $15 \%$.

Five-year relative survival is fairly good (60-79\%) for another one fifth (20\%) of cancers, including those of the larynx, uterus (corpus and cervix), prostate and bladder; and chronic lymphatic leukaemia, accounting for another fifth (20\%) of all cancers. Five-year relative survival is only moderate (in the range 20-60\%), however, for most cancers, including frequent cancers such as those of the stomach $(24 \%)$, colon and rectum $(54 \%)$, ovary $(42 \%)$; and nonHodgkin lymphoma (55\%). 


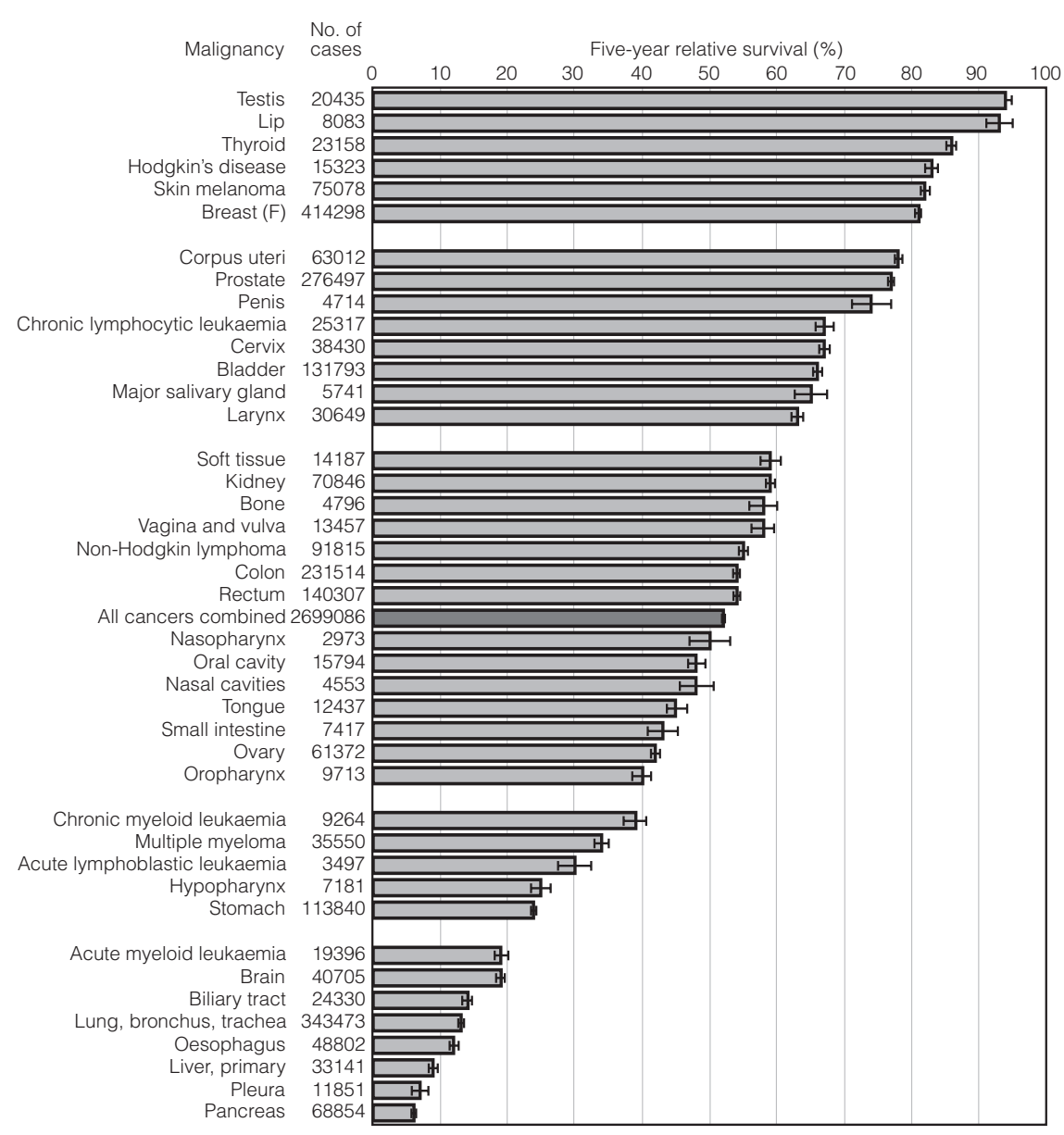

Fig. 8-1 Mean age-adjusted five-year relative survival, adults (15-99 years) diagnosed during 1995-99 in one of 23 European countries: EUROCARE-4 study

Cancers with a poor prognosis (less than 20\% survival at five years) include those of the oesophagus, liver and biliary tract, pancreas, lung and pleura, and acute myeloid leukaemia. These account for about $20 \%$ of all cancers. They are usually diagnosed at an advanced stage or have no effective treatments. The main cause of most of these cancers is known, however, so primary prevention should become the public health priority.

For most cancers, survival is higher in the Nordic countries (except Denmark) and in central European populations; slightly lower in southern European populations; lower in the United Kingdom and Ireland; and lowest in eastern European populations. The notable exceptions are testicular cancer and Hodgkin's disease, for which survival is remarkably similar all over Europe. 


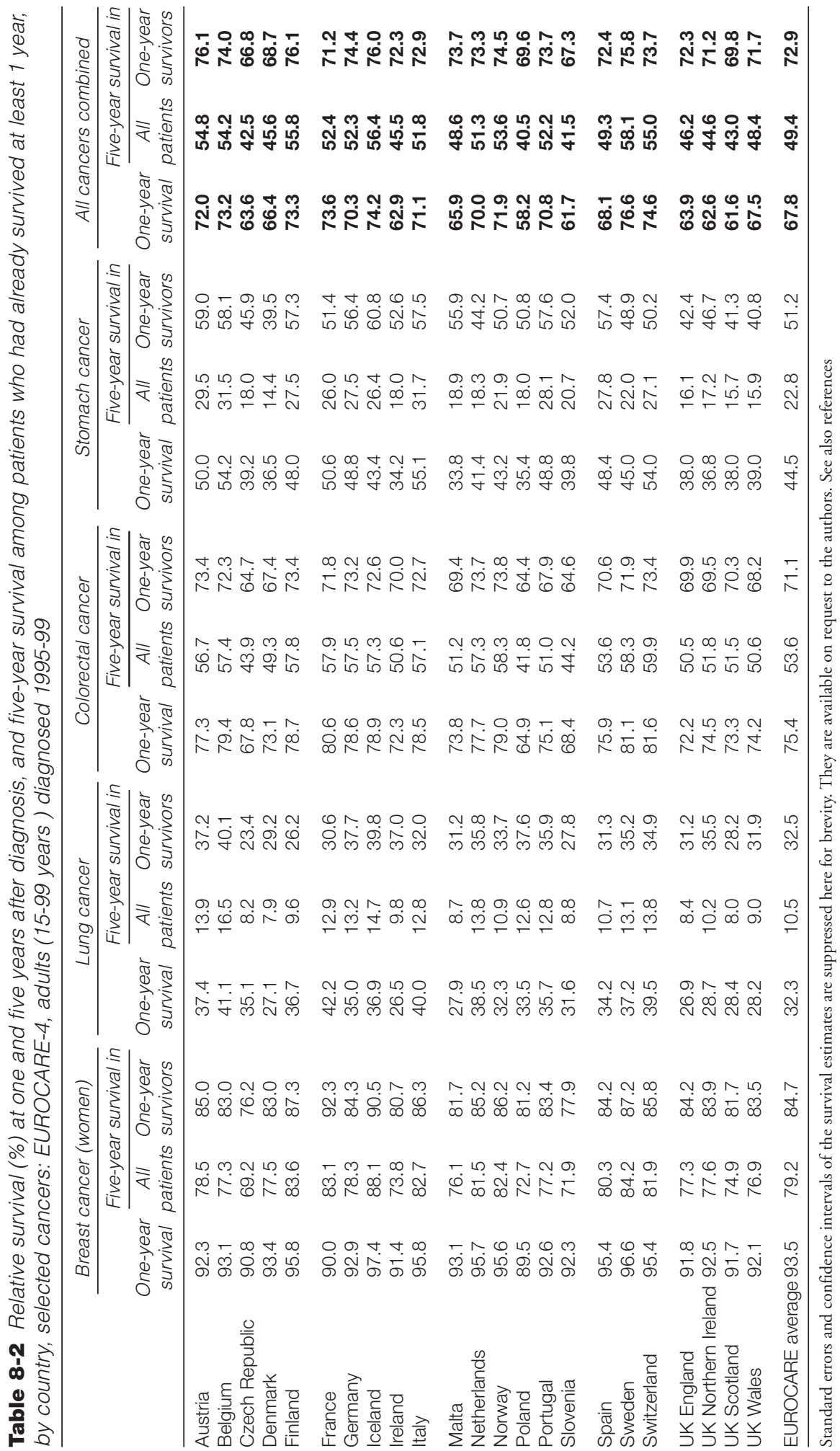


Survival varies widely between the countries and regions of Europe, both for all cancers combined and for individual cancers. Table 8-2 includes agestandardized relative survival estimates for selected common cancers at one year and five years after diagnosis. It is also helpful to examine the survival of patients who survived to the first anniversary after diagnosis. This "conditional survival" to the fifth anniversary among one-year survivors reflects the chances of survival to five years after the patient has survived the immediate effects of the stage of disease at diagnosis and its treatment in the first year. It is calculated as the ratio of the relative survival estimates at five years and at one year. International variation in these conditional survival estimates is less marked than for overall five-year survival, suggesting that the main reason for international differences in survival differences is mortality in the first year after diagnosis.

Thus for colorectal cancer, the international differences in survival among patients who survived the first year are much smaller than for overall five-year survival (Fig. 8-2a). For women with breast cancer, the differences in survival for women who survived the first year were smaller, but still evident (Fig. 8-2b). For prostate cancer, by contrast, the same pattern and a similar degree of variability is observed for both overall and conditional five-year survival, suggesting that international variations in mortality in the first year after diagnosis can only explain a small fraction of the international variation in five-year survival (Fig. 8-2c).

We can examine these differences in five-year survival - both overall, and conditional on survival to the first anniversary of diagnosis - separately in each age group and region of Europe. For colorectal cancer, overall survival declines with increasing age at diagnosis, but this pattern disappears with conditional survival, indicating that the effect of age on survival is largely due to differences in mortality in the first year after diagnosis, in all regions of Europe (Fig. 8-3a). For breast and prostate cancer (Figs. 8-3b \& 8-3c), survival is somewhat worse in the youngest and oldest age groups, but again, conditional survival shows smaller differences among the oldest patients. Interestingly, the difference between overall and conditional survival is larger in the populations of eastern Europe, intermediate for the United Kingdom and Ireland, and smaller for other European populations. This suggests that international differences in overall five-year survival are largely due to differences in mortality during the first year after diagnosis. EUROCARE does not have systematic information on the stage of disease at diagnosis, but these differences between total and conditional survival suggest that cancer is generally diagnosed at a more advanced stage in eastern Europe, the United Kingdom and Ireland than in northern and western Europe. 

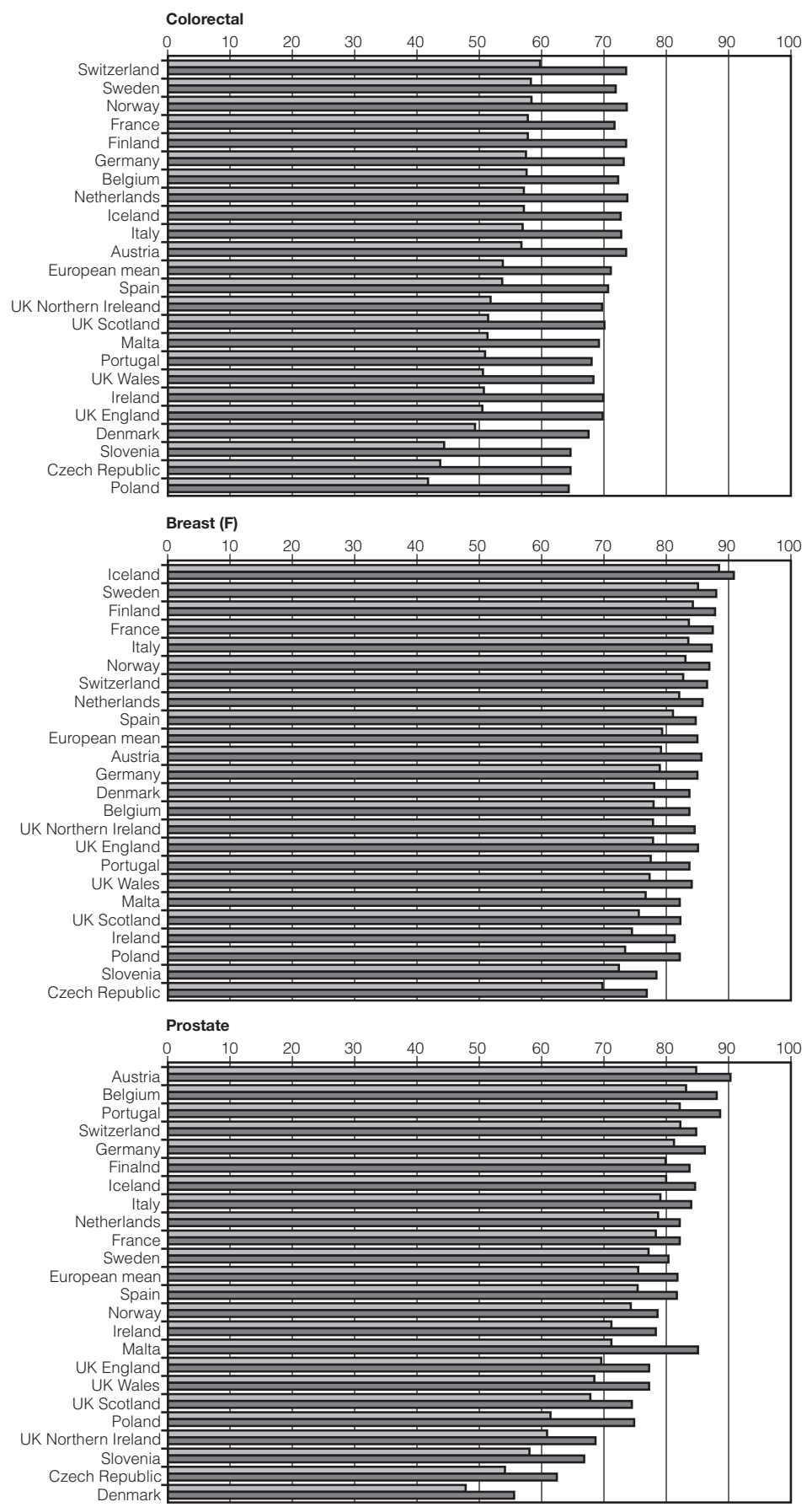

$\square$ All patients $\square$ One-year survivors

Figure 8-2 Age-adjusted five-year relative survival in adults (15-99 years) diagnosed during 1995-99 in one of 23 European countries, both for all patients and for those who survived to the first anniversary of diagnosis (one-year survivors), EUROCARE-4 study: (a) colorectal cancer (b) breast cancer in women (c) prostate cancer 


\section{Colorectal}
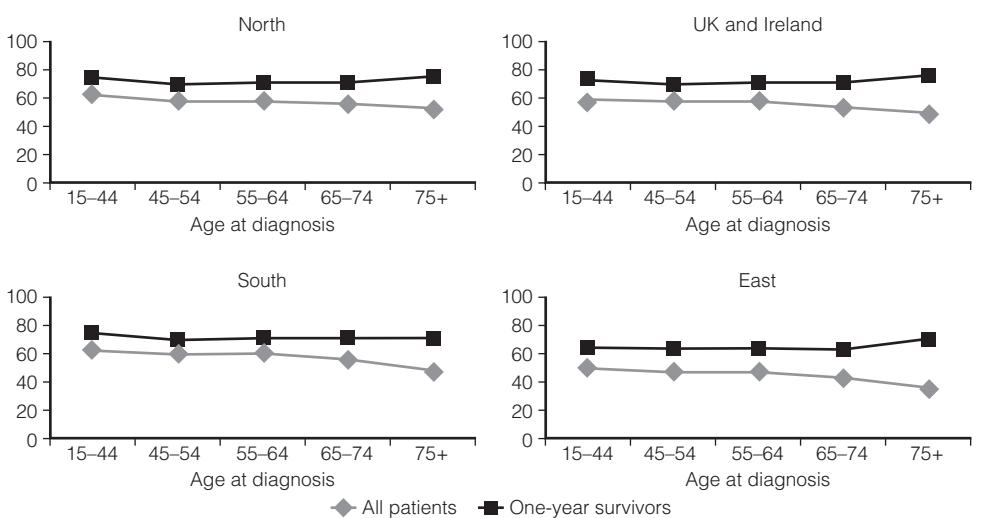

\section{Breast (F)}
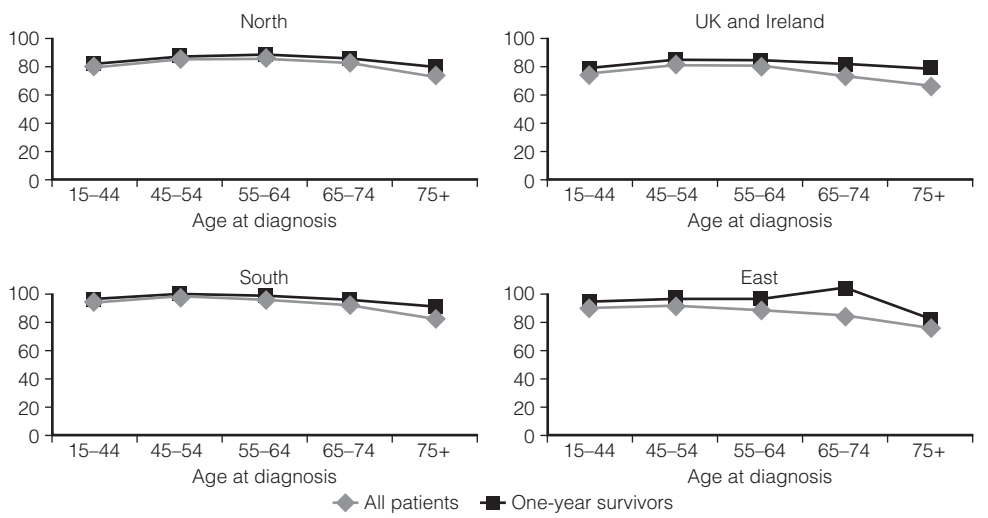

\section{Prostate}
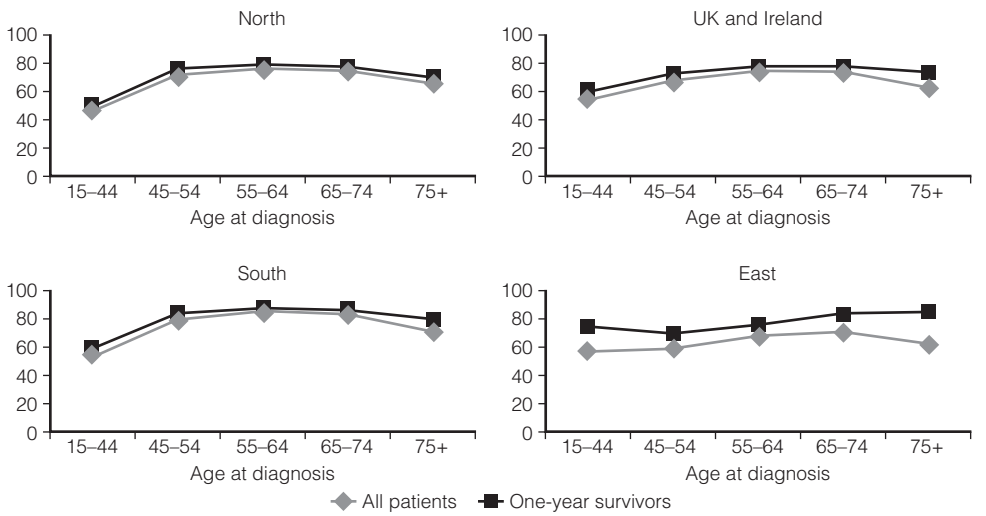

Figure 8-3 Five-year relative survival in adults (15-99 years) diagnosed during 1995-99, by geographic region and by age at diagnosis, both for all patients and for those who survived to the first anniversary of diagnosis (one-year survivors), EUROCARE-4 study: (a) colorectal cancer (b) breast cancer in women (c) prostate cancer 
Short-term predictions of survival for patients diagnosed between 2000 and 2002, from period analysis, suggest that these geographical gaps in cancer survival in Europe may well diminish in the near future. Thus, five-year survival for cancers of the colon and rectum, breast and prostate cancer has increased in all European areas (Figs. 8-4a, 8-4b \& 8-4c), but the increase in survival has been more marked for eastern European populations (data available for Poland and the Czech Republic only); intermediate for the United Kingdom and Ireland; and lower for the Nordic countries and southern Europe. In eastern European populations, survival increased from $30 \%$ to $47 \%$ for colorectal cancer, from $60 \%$ to $74 \%$ for breast cancer and from $40 \%$ to $68 \%$ for prostate cancer.

We also examined an all-cancers survival index that combines the estimates of relative survival for all cancers, separately for men and women, and for each country. The index is adjusted for age and for "case-mix" - the proportion of cancers of each type among the total number of cancers. Since case-mix varies markedly with sex, this was done for men and women separately, using the proportions of each type of cancer diagnosed between 1995 and 1999. This all-cancers survival index can be seen as a simple and directly comparable measure of the cancer survival that would be seen in each region or country if the age distribution of cancer patients were the same in each region or country and the proportion of cancers of each type were also the same. For Europe as a whole, the regionally-weighted mean cancer survival index was $49.6 \%$ for all adults $-44.8 \%$ for men, $54.6 \%$ for women (Fig. 8-5 - these estimates differ slightly from those given above, because they are adjusted for age).

We examined the all-cancers survival index in each country in relation to the total national expenditure on health, measured in US dollars per person, adjusted for purchasing power parity (US\$ PPP) (OECD, 2004). We also ranked the countries on this measure and grouped them into four classes of total national health expenditure, from under US\$1000 to over US\$3000 per person per year (Fig. 8-5: data for United Kingdom available only as a single country). There was a moderate correlation between total national expenditure on health and the five-year relative survival index for all cancers combined, adjusted for age and case-mix $\left(r^{2}=0.56\right.$ for women and 0.43 for men). There were notable exceptions, however: Denmark and the United Kingdom had lower survival than countries with similar national expenditure on health. Finland had better survival than expected, given its moderate health expenditure. Spain, Italy and Portugal had better survival than countries with comparable health expenditure, but they are only partially covered by cancer registration. Survival in the regions included in the analyses may not be representative of that in the whole country, from which the total health 

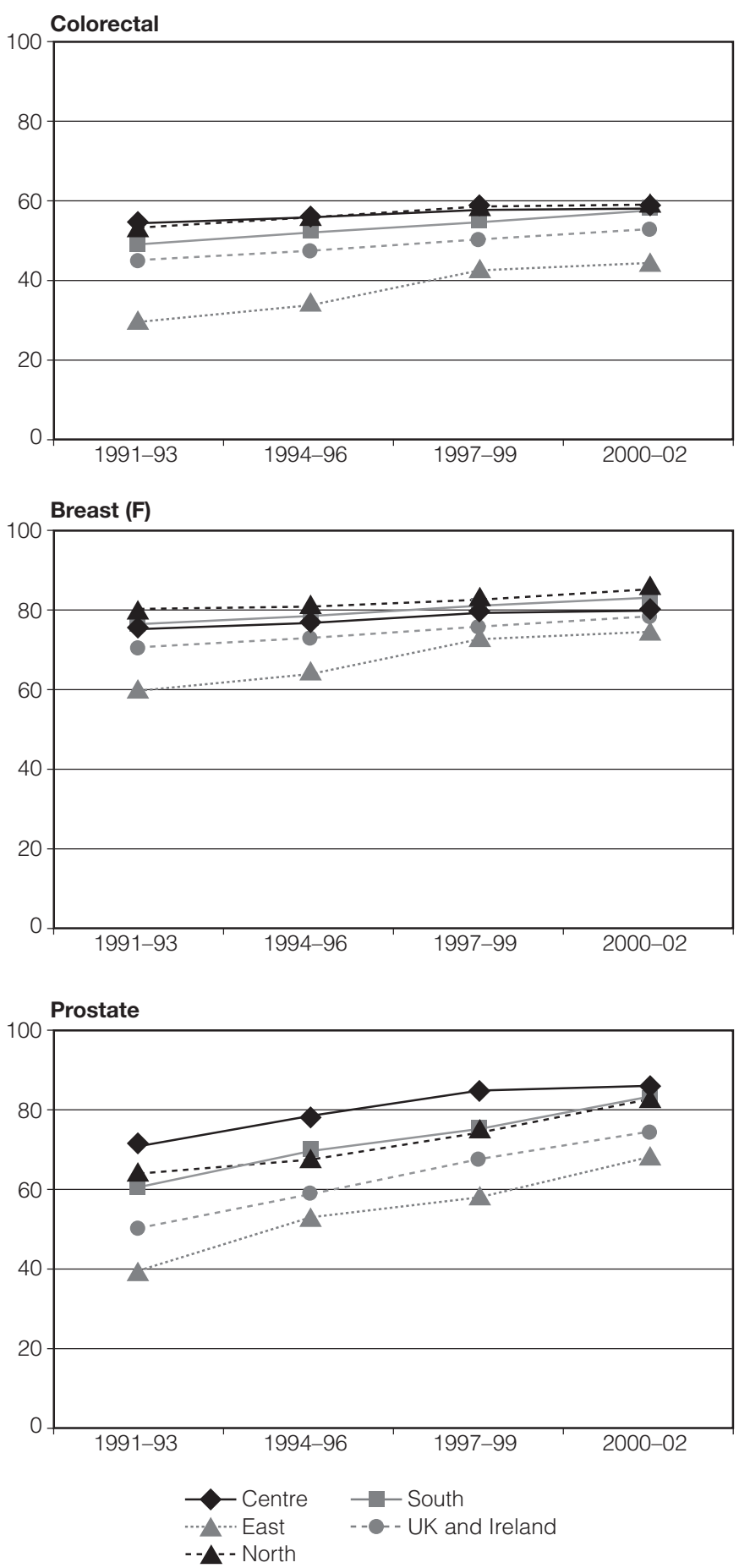

Figure 8-4 Trends in five-year age-adjusted relative survival in adults (15-99 years) by geographic region, period estimates for 1991-2002 (see text), EUROCARE-4 study: (a) colorectal cancer (b) breast cancer in women (c) prostate cancer 

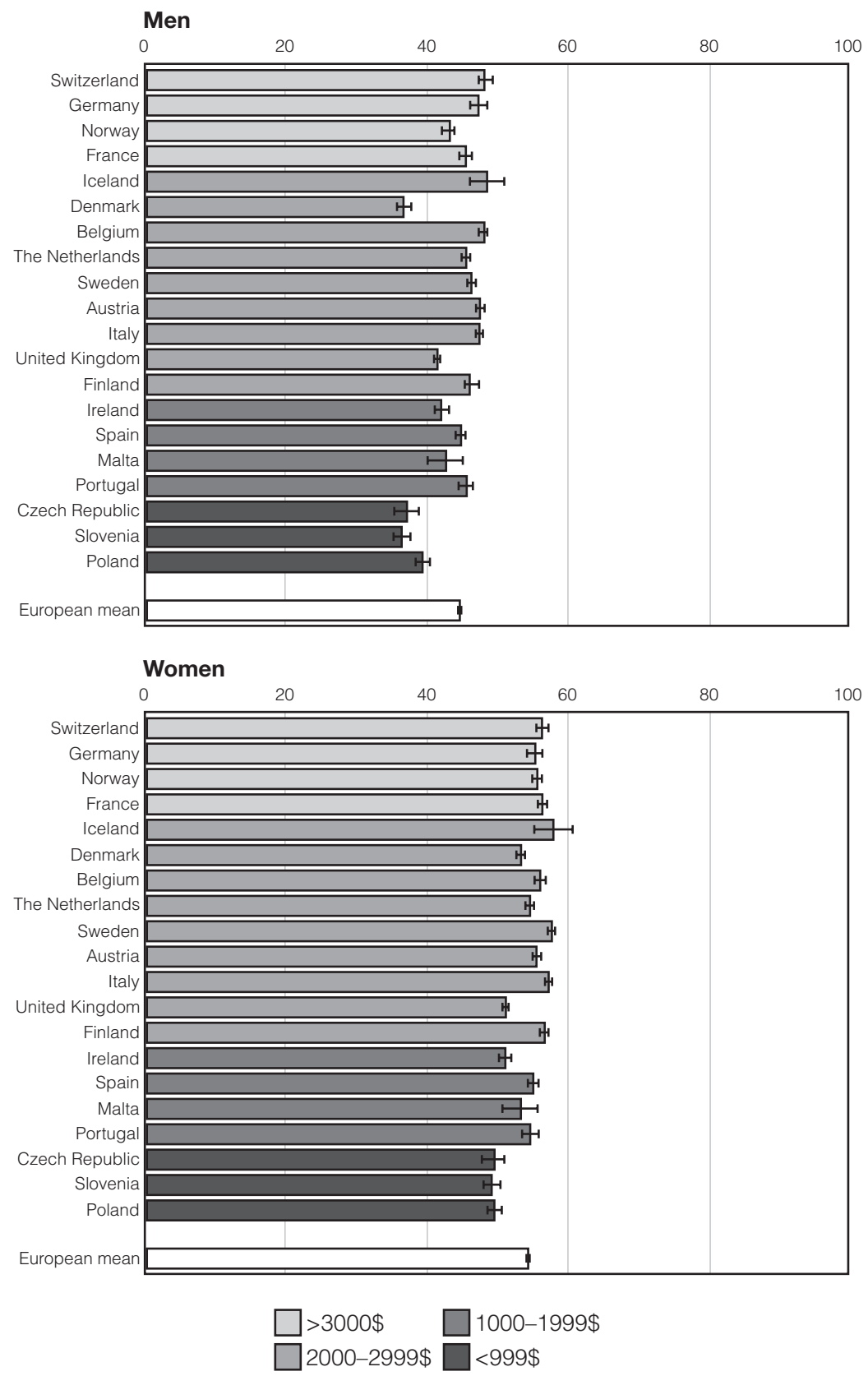

Figure 8-5 Age-adjusted five-year relative survival for all cancers combined by sex and country, with area-weighted mean survival for Europe, grouped by Total National Expenditure on Health (US\$PPP per person) 
expenditure is derived. In Italy, for instance, the cancer registries are mainly in the wealthier, northern part of the country (AIRTUM, 2007).

It should also be noted that total national expenditure on cancer treatment is only a small fraction of total national expenditure on health, recently estimated at 6.4\% (Jonsson \& Wilking, 2007) even though it is fairly well correlated with the overall figure. The direct cost of cancer management per person in 2004, for instance, was estimated to be €PPP 146 in Sweden, ЄPPP 147 in Germany, €PPP 124 in France, €PPP 92 in the Netherlands, €PPP 50 in the Czech Republic and €PPP 30 in Poland.

We compared cancer survival in Europe with that in the United States by contrasting the EUROCARE-4 results for selected cancers with corresponding data from the SEER programme (Table 8-3). Europe is represented by 47 cancer registries that provided data on cancer patients who were diagnosed during 2000-2002 and followed up to the end of 2003. The United States is represented by the 14 SEER cancer registries. Table 8-3 shows period estimates of five-year relative survival in the United States and Europe, as well as the range of survival figures in Europe.

Average cancer survival is higher in the United States, with a few exceptions: cancers of the stomach and testis, Hodgkin's disease and acute myeloid leukaemia (AML). However, the average cancer survival estimates for the United States are usually within the range covered by the European countries contributing to EUROCARE-4. For all but two of the selected cancers (prostate and colorectal cancer) survival in at least one European population was higher than average survival in the areas covered by the SEER programme of the United States, including cancers of the stomach, corpus uteri, testis and kidney, Hodgkin's disease and myeloid leukaemia.

Considering all cancers together, five-year survival estimates were much higher for American cancer patients (66.3\% for men, 62.9\% for women) than for European patients ( $47.3 \%$ for men, $55.8 \%$ for women). The large USEuropean difference in cancer survival for men, however, is largely due to the lower incidence in the United States of highly lethal cancers, such as lung and stomach cancer, and to the exceptionally high incidence and survival of prostate cancer. This is also why the overall survival index for men in the USA is higher than that for women - the reverse of the situation in Europe. If we exclude prostate cancer, the US-European difference in cancer survival for men falls by about half - to $46.9 \%$ for the United States, $38.1 \%$ for Europe. It should also be noted that survival in SEER areas may well be higher than in other areas of the United States. 


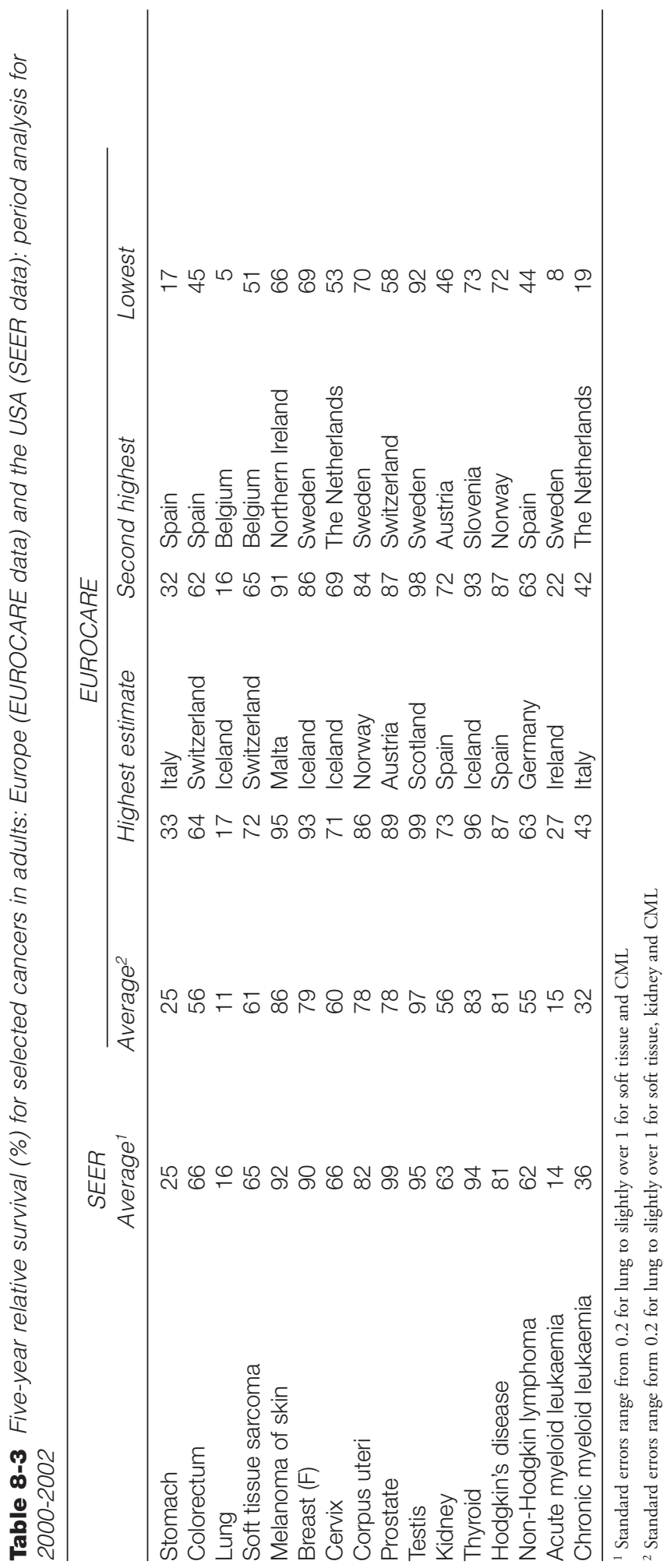



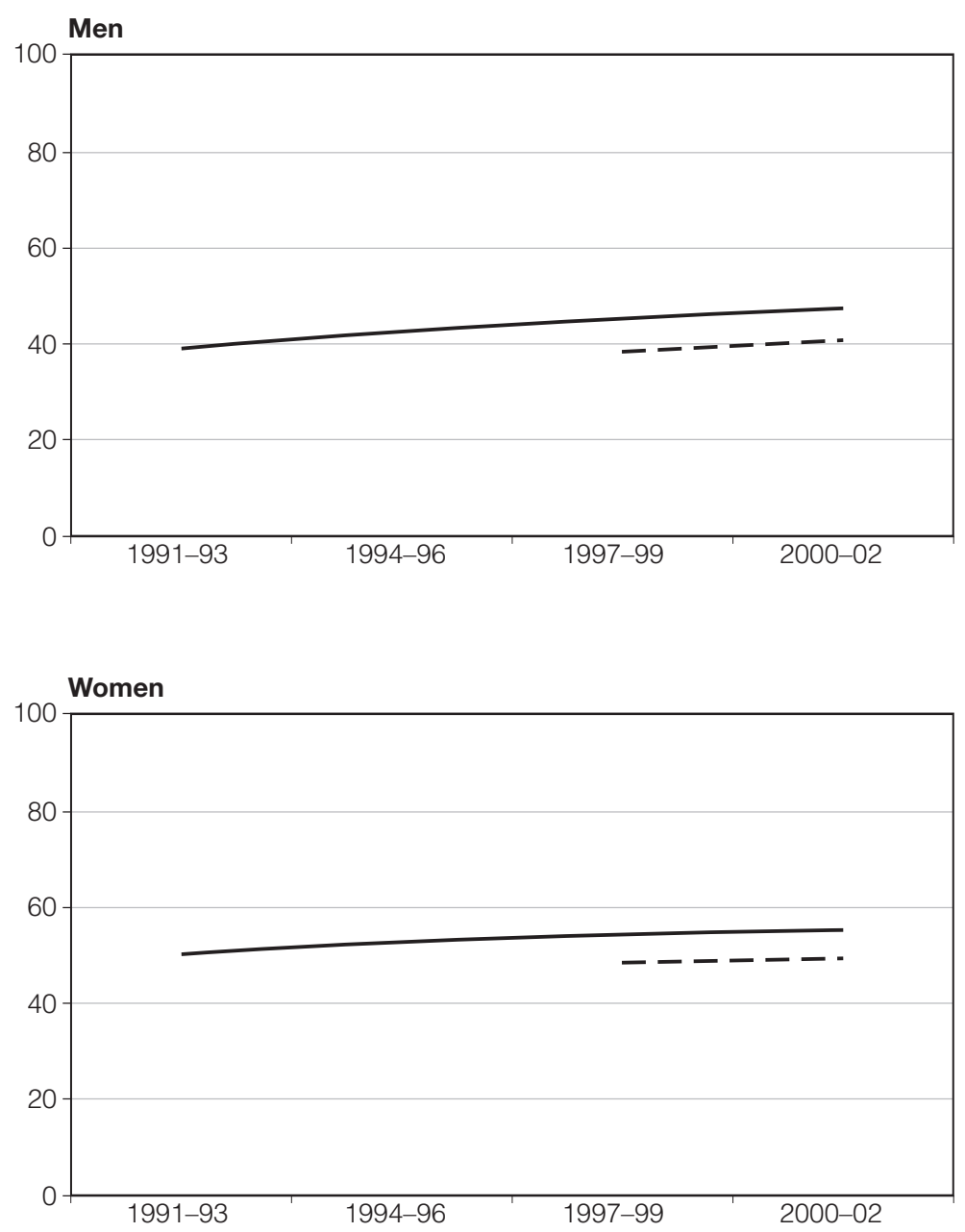

Figure 8-6 Trends in five-year and ten-year age-adjusted relative survival in adults (1599 years) in Europe, period estimates for 1991-2002 (see text), EUROCARE-4 study: all cancers combined, by sex

We examined European trends in overall cancer survival at five and ten years, using period analysis of data from 16 countries (30 cancer registries) that were able to provide data for patients diagnosed during the entire period 19892002. Overall survival has increased steadily, but short-term predictions of relative survival up to 10 years for patients diagnosed as recently as 2000-02 do not yet reach $50 \%-49 \%$ for women and only about $41 \%$ for men (Fig. 8-6).

\section{Discussion}

The EUROCARE study of the survival of cancer patients diagnosed in Europe during the second half of the 1990s and followed up to the end of 2003 shows 
that, after correcting for non-cancer mortality and age at diagnosis, survival up to five years after diagnosis for all cancers combined was $44.8 \%$ for men, $54.6 \%$ for women and $49.6 \%$ overall. Predictions of five-year survival for patients diagnosed during 2000-02, using period analysis of data from more than half the cancer registries contributing to EUROCARE-4, were $47.3 \%$ for men, $55.8 \%$ for women.

An important finding is that all-cancer survival increased markedly in countries where it was previously low. This has led to some reduction in international differences in cancer survival. Previous EUROCARE projects found marked inter-country and inter-regional differences in survival (Sant et al., 2003a). Although survival increased almost everywhere with time, survival differences persisted and sometimes increased (Sant et al., 2001). The new EUROCARE-4 finding, that the European cancer survival gap is narrowing, is highly encouraging, and it suggests marked improvement in cancer care in countries with poor survival from cancer.

The underlying causes of the observed increases in survival are unlikely to be the same for all sites. For example, the increase in breast cancer survival in the United Kingdom (Quinn \& Allen, 1995) and in many other European countries (Sant, Francisci \& Capocaccia, 2006) has been accompanied by reduced mortality. This probably reflects the establishment of organized screening programmes and some real improvements in cancer care. The increase in survival for prostate cancer may be largely due to the widespread introduction of prostate-specific antigen (PSA) testing, with consequent early diagnosis and strong lead-time bias (Brenner $\&$ Arndt, 2005). The decrease in mortality for prostate cancer, evident in several European countries, may be largely due to improved treatment (Baade, Coory \& Aitken, 2004).

Survival remains much lower in eastern Europe than in Western Europe. Within western Europe, survival in the United Kingdom and Denmark is still lower than in other countries for several cancers. Five-year breast cancer survival was around 83\% in Finland and Sweden; high in the French, Italian and Dutch populations covered by cancer registration (81-83\%); and about $77 \%$ in the United Kingdom and Denmark. Five-year colorectal cancer survival was above $57 \%$ in the Nordic countries and several central and southern European populations, but only 51\% in the United Kingdom and $49 \%$ in Denmark.

It is important to consider whether this difference of about $6 \%$ in relative survival at five years between the United Kingdom and Denmark and other western countries could be due to bias or artefacts in cancer registration. Two major sources of artefact can bias comparative survival estimates: 
incomplete ascertainment (registration) of incident cases and incomplete ascertainment of death in registered cancer patients. Several EUROCARE cancer registries used active follow-up techniques, specifically to check the vital status (dead, alive or unknown) of patients who appeared from cancer registry records to be alive, and found only rare instances of such errors (Sant \& Gatta, 1999). If we suppose, in the extreme case, that as many as $5 \%$ of patients who had died were misclassified as alive in the cancer registry records, then breast cancer and colorectal cancer survival would increase by $1 \%$ and $2 \%$ respectively (Sant \& Gatta, 1999). Under-ascertainment of the death of registered cancer patients cannot plausibly account for the difference in survival between the United Kingdom and Denmark and other western European countries.

Incomplete registration of newly diagnosed cancer patients could explain some of the observed differences in cancer survival, if it affected particularly those who would survive long periods and if this selective under-ascertainment were more marked in registries with artefactually low survival. However, if the under-ascertainment of long-term survivors were to account for a $6 \%$ difference in survival for breast or colorectal cancer, then cancer registries in countries with artefactually low survival would have to have failed to register some $20 \%$ to $30 \%$ of five-year survivors, without missing any patients who died. That would also imply that the true incidence of cancer in those countries was seriously underestimated. These circumstances are highly implausible, and we can reasonably conclude that the survival differences reported by EUROCARE-4 are likely to correspond fairly closely to reality.

Increasing age was confirmed as having a major adverse effect on relative survival from cancer (Berrino et al., 1995; Berrino et al., 1999; Berrino et al., 2003; Sant \& Gatta, 1999). Most of the effect of age was due to differences in short-term survival, suggesting either that older people may have more advanced disease at diagnosis, or else that they do not receive treatments intended to cure them or prolong their survival as often as younger patients.

It is a consistent finding of all EUROCARE studies that between-country survival differences are small for relatively uncommon treatable cancers such as testicular cancer and Hodgkin's disease, and for cancers with very poor prognosis. By contrast, for common cancers with an intermediate prognosis (for which early diagnosis is typically a major determinant of outcome) survival rates still differ markedly, and probably unacceptably, between countries of the EU. The survival differences between the best and the rest still represented about 150000 cancer deaths between 1995 and 1999, or about $12 \%$ of the 1.2 million cancer deaths within five years of diagnosis. Understanding the reasons for these persistent differences - even if we may 
expect them to diminish somewhat in the near future - is important for the public health response to cancer in Europe.

As we highlighted in the introduction, survival is a complex indicator of a country's performance in cancer control. Longer survival may be due to better treatment, or more effective treatment because of earlier diagnosis, or may be simply due to earlier diagnosis of the cancer (lead-time bias), without affecting the time it takes for cancer to kill the patient. Alternatively, a cancer may not be lethal at all, because the patient will die of some other cause (Berrino, 2003).

The EUROCARE approach to disentangling these possible determinants of survival, and of international differences in survival, includes high-resolution studies. These are population-based inter-country comparisons of survival that make use of detailed information from clinical records. Adjustment can then be made for disease stage at diagnosis, which also requires careful evaluation of the investigations used to determine the stage of disease (Gatta et al., 2000; Sant et al., 2003b). Stage-specific comparisons may be biased by so-called "stage migration". Where extensive diagnostic procedures are common practice, many cancers that would otherwise be classified as localized are then accurately classified to a more advanced stage category. This shift leads to an increase in the survival of both localized and advanced cases, even though the overall survival estimate is unaffected (Feinstein, Sosin \& Wells, 1985).

EUROCARE studies have adjusted for stage at diagnosis and a few major diagnostic determinants of stage (e.g. number of lymph nodes examined for tumour spread in the surgical specimens). These studies have shown that the differences in survival from breast and colorectal cancer among western European populations, for example (and the corresponding differences between Europe and the United States) are largely due to differences in the timing of diagnosis, rather than differences in treatment (Gatta et al., 2000; Gatta et al., 2003; Sant et al., 2004; Sant et al., 2003b). Survival differences for testicular cancer, on the other hand, seem to be largely due to differences in access to appropriate treatment (Sant et al., 2007).

Variation in the distribution of cancer by subsite, histological type and some other biological features within the categories of the standard International Classification of Diseases (ICD), could provide other reasons for geographical variation in survival, as well as differences in treatment (Gatta et al., 2003). These should be explored further in future studies at population level.

The proportion of patients who appear to be "cured" of their cancer has been estimated, as has the average survival of patients who die from their cancer (Verdecchia, De Angelis \& Capocaccia, 1998). The observation that the 
proportion of patients who are "cured" of their disease has increased over time suggests that the increases in survival that we have observed are not only due to lead time, but may also in many cases reflect more effective treatment.

That conclusion can be challenged if substantial overdiagnosis is suspected. Overdiagnosis (i.e. of patients who would never have been diagnosed without a test such as PSA) may artificially increase the proportion of patients who appear to be "cured". To overcome this difficulty, the EUROCARE approach would be to study the trend in the proportion of cured patients by stage of disease at diagnosis. If the proportion of cured patients increases only among patients who were diagnosed at a very localized stage of disease, the assumption of overdiagnosis would be strengthened. If, however, the proportion of cured patients also increases among those who were diagnosed at a more advanced stage, this would imply a real improvement in outcome. That type of information about cancer control cannot be obtained from clinical trials, only from observational studies in entire populations. Such studies require the collection of standardized information on stage and other diagnostic variables for all newly diagnosed cancer patients, or a very large sample of them. That will require a major investment, but nothing approaching the scale of investment in new cancer treatments.

Population-based relative survival reflects the impact of both early diagnosis and effective care on the outcome of cancer. It is of critical importance for rational planning of national cancer services (UK Department of Health, 2000).

EUROCARE-4 included most EU countries, as well as Iceland, Norway and Switzerland. It should be noted that Greece and Luxembourg currently have no population-based cancer registration. Bulgaria, Cyprus, Hungary, Latvia, Lithuania and Romania did not contribute to EUROCARE-4. Estonia and Slovakia contributed population-based cancer survival data to EUROCARE3, but were unable to contribute to EUROCARE-4 for legal or administrative reasons related to concerns about confidentiality. This retreat from public health norms represents a serious loss to the understanding of cancer control in the EU. Expanded coverage of eastern European populations will be a priority for future EUROCARE studies. In order to achieve that, however, the EU will need to take action to strengthen cancer registration, to extend registration and facilitate participation in cancer survival analysis in all Member States.

We conclude with the following observation: among cancer patients diagnosed between 1995 and 1999, the overall average relative survival at five years was $52 \%$ and the total number of cancer deaths in the first five years after 
diagnosis was 1309 836. If all countries had attained the mean survival (57\%) of Norway, Sweden and Finland (countries with high survival and medium-tohigh total national expenditure on health), there would have been 150693 (approximately 12\%) fewer cancer deaths within five years of diagnosis.

\section{APPENDIX: EUROCARE-4 WORKING GROUP}

Austria: M Hackl (Austrian National Cancer Registry); W Oberaigner (Tyrol Cancer Registry); Belgium: E Van Eycken (Flemish Cancer Registry), Denmark: HH Storm (Danish Cancer Society, Dept. Cancer Prevention \& Documentation); Czech Republic: J Holub (West Bohemia Cancer Registry); Finland: T Hakulinen (Finnish Cancer Registry); France: G Hédelin, M Velten (Bas-Rhin Cancer Registry); AV Guizard (Calvados General Cancer Registry); G Launoy (Calvados Digestive Cancer Registry); J Faivre (Côte d'Or Digestive Cancer Registry); PM Carli, M Maynadié (Côte d'Or Haematological Malignancies Registry); A Danzon (Doubs Cancer Registry); A Buémi (HautRhin Cancer Registry); B Tretarre (Hérault Cancer Registry); M Colonna (Isère Cancer Registry), F Molinié (Loire Atlantique Breast and Colon Cancer Registry); S Bara (Manche Cancer Registry); C Schvartz (Marne-Ardennes Thyroid Cancer Registry); N Raverdy (Somme Cancer Registry); P Grosclaude (Tarn Cancer Registry); Germany: H Brenner (Abt. Klinische Epidemiologie und Alternsforschung Deutsches Krebsforschungszentrum); H Ziegler (Saarland Cancer Registry); Iceland: L Tryggvadottir (Icelandic Cancer Registry); Ireland: H Comber (National Cancer Registry of Ireland); Italy: F Berrino (Project Leader), C Allemani, P Baili, L Ciccolallo, G Gatta, F Lucca, A Micheli, M Sant, S Sowe, G Zigon (Fondazione IRCCS, Istituto Nazionale dei Tumori); P Crosignani, P Contiero (Cancer Registry and Environmental Epidemiology Unit, Fondazione IRCCS, Istituto Nazionale dei Tumori); F Bellù (Registro Tumori A. Adige/Tumorregister Südtirol); A Giacomin (Biella Cancer Registry); S Ferretti (Ferrara Cancer Registry); E Paci, E Crocetti (Firenze Cancer Registry); D Serraino, L Dal Maso, M De Dottori, M Lise (Friuli Venezia Giulia Cancer Registry); M Vercelli, C Casella (Liguria Cancer Registry, IST University; Genova); F Pannelli (Macerata Cancer Registry, Marche Childhood Cancer Registry); M Federico, I Rashid (Modena Cancer Registry); M Fusco (Napoli Cancer Registry); A Traina (Palermo Breast Cancer Registry); V De Lisi, L Serventi (Parma Cancer Registry), R Tumino (Ragusa Cancer Registry); L Mangone (Reggio Emilia Cancer Registry); F Falcini (Romagna Cancer Registry); G Senatore, A Iannelli (Salerno Cancer Registry); M Budroni (Sassari Cancer Registry); S Piffer (Trento Cancer Registry); R Zanetti, S Patriarca, S Rosso (Torino Cancer Registry); F La Rossa (Umbria Cancer Registry); P Zambon, S Guzzinati 
(Venetian Cancer Registry); R Capocaccia, E Carrani, R De Angelis, P Roazzi, M Santaquilani, E Grande, R Inghelmann, A Simonetti, A. Tavilla, S Francisci, A Verdecchia (Centro Nazionale di Epidemiologia, Istituto Superiore di Sanità, Rome); Malta: M Dalmas (Malta National Cancer Registry); Norway: F Langmark, (Cancer Registry of Norway, Institute of Population-based Cancer Research); Poland: J Rachtan (Cracow Cancer Registry), S Góźdź, U Siudowska, R Mężyk (Holycross Cancer Centre); M Bielska-Lasota (Independent Unit of Oncological Education, M. Sklodowska-Curie Cancer Centre, Warsaw); M Zwierko (Warsaw Cancer Registry); Slovenia: M PrimicŽakelj (Cancer Registry of Slovenia); Portugal: PS Pinheiro (Southern Portugal Cancer Registry), Spain: A Mateos (Albacete Cancer Registry); I Izarzugaza (Basque Country Cancer Registry); A Torrella-Ramos (Castellón Cancer Registry); R Marcos-Gragera (Girona Cancer Registry); C Martinez-Garcia (Granada Cancer Registry); C Navarro, MD Chirlaque (Murcia Cancer Registry); E Ardanaz, C Moreno (Navarra Cancer Registry); J Galceran (Tarragona Cancer Registry); Sweden: M Talbäck (Centre for Epidemiology, The National Board of Health and Welfare); Switzerland: G Jundt (Basel Cancer Registry); M Usel (Geneva Cancer Registry); H Frick (Grisons Cancer Registry); S Ess (St. Gall-Appenzell Cancer Registry); A Bordoni (Ticino Cancer Registry); JC Luthi, I Konzelmann (Valais Cancer Registry); N Probst (Cancer Registry Zurich); JM Lutz, P. Pury (Coordinating Centre); The Netherlands: O Visser (Amsterdam Cancer Registry); JWW Coebergh (Eindhoven Cancer Registry); R Otter (Comprehensive Cancer Centre, Groningen); United Kingdom - England: DC Greenberg (Eastern Cancer Registration and Information Centre); MP Coleman (London School of Hygiene and Tropical Medicine); M Quinn, N Cooper (Office for National Statistics); T Moran (North West Cancer Intelligence Service); D Forman (Northern and Yorkshire Cancer Registry and Information Service); M Roche, $S$ Edwards (Oxford Cancer Intelligence Unit); J Verne (South West Cancer Intelligence Service); H Møller (Thames Cancer Registry); D Meechan, J Poole (Trent Cancer Registry); G Lawrence (West Midlands Cancer Intelligence Unit); United Kingdom - Northern Ireland: A Gavin (Northern Ireland Cancer Registry,) United Kingdom - Scotland: RJ Black, DH Brewster (Scottish Cancer Registry); United Kingdom - Wales: JA Steward (Welsh Cancer Intelligence and Surveillance Unit).

\section{REFERENCES}

AIRTUM (2007). Italian cancer figures, report 2007: survival. Rome, AIRTUM.

Baade PD, Coory MD, Aitken JF (2004). International trends in prostate cancer mortality: the decrease is continuing and spreading. Cancer Causes Control, 15(3):237-241. 
Bach PB et al. (2007). Computed tomography screening and lung cancer outcomes. J Am Med Assoc, 297(9):953-961.

Berrino F (2003). The EUROCARE study: strengths, limitations and perspectives of population-based, comparative survival studies. Ann Oncol, 14(Suppl 5):v9-v13.

Berrino F et al. (1995). Survival of cancer patients in Europe: the EUROCARE study (IARC Scientific Publications No. 132). Lyon, International Agency for Research on Cancer.

Berrino F et al. (1999). Survival of cancer patients in Europe: the EUROCARE-2 study (IARC Scientific Publications No. 151). Lyon, International Agency for Research on Cancer.

Berrino F et al. (2003). Survival of cancer patients in Europe: the EUROCARE-3 study. Ann Oncol, 14(Suppl. 5):1-155.

Berrino F et al. (2007). Survival for eight major cancers and all cancers combined for European adults diagnosed in 1995-99: results of the EUROCARE-4 study. Lancet Oncol, 8(9):773-783.

Brenner H, Arndt V (2005). Long-term survival rates of patients with prostate cancer in the prostate-specific antigen screening era: population-based estimates for the year 2000 by period analysis. J Clin Oncol, 23(3):441-447.

Brenner H, Hakulinen T. (2002). Up-to-date long-term survival curves of patients with cancer by period analysis. J Clin Oncol, 20(3):826-832.

Brenner H, Soderman B, Hakulinen T (2002). Use of period analysis for providing more upto-date estimates of long-term survival rates: empirical evaluation among 370,000 cancer patients in Finland. Int J Epidemiol, 31:456-462.

Coleman MP et al. (2003). EUROCARE-3 summary: cancer survival in Europe at the end of the 20th century. Ann Oncol, 14(Suppl. 5):128-149.

Corazziari I, Quinn M, Capocaccia R. (2004). Standard cancer patient population for age standardising survival ratios. Eur J Cancer, 40(15):2307-2316.

Cutler SJ (1964). International symposium on end results of cancer therapy. (National Cancer Institute Monograph Number 15). Bethesda, MD, US Government Printing Office.

Ellison LF (2006). An empirical evaluation of period survival analysis using data from the Canadian Cancer Registry. Ann Epidemiol, 16:191-196.

EUROCARE web site, Italy (available at:http://www.eurocare.it/, accessed 2 December 2007).

Feinstein AR, Sosin DM, Wells CK (1985). The Will Rogers phenomenon. Stage migration and new diagnostic techniques as a source of misleading statistics for survival in cancer. $N$ Engl J Med, 312:1604-1608.

Fleshner N, Klotz L (2002). Role of "saturation biopsy" in the detection of prostate cancer among difficult diagnostic cases. Urology, 60(1):93-97.

Gatta G et al. (2000). Understanding variations in survival for colorectal cancer in Europe: a EUROCARE high-resolution study. Gut, 47(4):533-538.

Gatta G et al. (2003). Differences in colorectal cancer survival between European and US populations: the importance of sub-site and morphology. Eur J Cancer, 39:2214-2222.

GLOBOCAN 2002 [online database]. International Agency for Research on Cancer (IARC), Lyon (available at:http://www.dep.iarc.fr/globocan/database, accessed 5 May 2007).

Hakulinen T (1982). Cancer survival corrected for heterogeneity in patient withdrawal. Biometrics, 38(4):933-942.

Jonsson B, Wilking N (2007). A global comparison regarding patient access to cancer drugs. Ann Oncol, 18(Suppl. 3): 1-74.

Louis PCA (1835). Recherches sur les effets de la saignée dans quelques maladies inflammatoires et sur l'action de l'émétique et des vésicatoires dans la pneumonie. Paris, Baillère.

OECD (2004). OECD health data 2004. 1st edition. Paris, Organisation for Economic Cooperation and Development. 
Quinn M, Allen E (1995). Changes in incidence of and mortality from breast cancer in England and Wales since introduction of screening. United Kingdom Association of Cancer Registries. Br Med J, 311:1391-1395.

Sant M, Francisci S, Capocaccia R et al. (2006). Time trends of breast cancer survival in Europe in relation to incidence and mortality. Int J Cancer, 119(10):2417-2422.

Sant M, Gatta G (1999). The EUROCARE database. In: Berrino F, Capocaccia R, Estève J, eds. Survival of cancer patients in Europe: the EUROCARE-2 study. (IARC Scientific Publications No. 151). Lyon, IARC Scientific Publications:15-31.

Sant $\mathrm{M}$ et al. (2001). Cancer survival increases in Europe, but international differences remain wide. Eur J Cancer, 37(13):1659-1667.

Sant M et al. (2003a). EUROCARE-3: survival of cancer patients diagnosed 1990-94 - results and commentary. Ann Oncol, 14(Suppl. 5):61-118.

Sant $M$ et al. (2003b). Stage at diagnosis is a key explanation of differences in breast cancer survival across Europe. Int J Cancer, 106(3):416-422.

Sant $M$ et al. (2004). Breast carcinoma survival in Europe and the United States. Cancer, 100(4):715-22.

Sant $M$ et al. (2007). Ten-year survival and risk of relapse for testicular cancer: a EUROCARE high-resolution study. Eur J Cancer, 43(3):585-592.

Schilling FH, Spix C, Berthold F (2002). Neuroblastoma screening at one year of age. $N$ Engl $J$ Med. 346(14):1047-1053.

Sone $S$ et al. (1998). Mass screening for lung cancer with mobile spiral computed tomography scanner. Lancet, 351(9111):1242-1245.

Talback M, Stenbeck M, Rosen M (2004). Up-to-date long-term survival of cancer patients: an evaluation of period analysis on Swedish Cancer Registry data. Eur J Cancer, 40(9):1361-1372.

Telesca D, Etzioni R, Gulati R (2007). Estimating lead time and overdiagnosis associated with PSA screening from prostate cancer incidence trends. Biometrics, doi:10.1111/j.15410420.2007.00825.x

UK Department of Health (2000). NHS cancer plan (available at:http://www.doh.gov.uk, accessed 26 June 2007) London, Department of Health.

US Department of Human Services (2001). National Cancer Institute, Surveillance, Epidemiology and Results program. SEER cancer statistics review, 1973-1999. Bethesda, MD, US Department of Health and Human Services.

Verdecchia A, De Angelis R, Capocaccia R (1998). The cure for colon cancer: results from the EUROCARE study. Int J Cancer, 77:322-329.

Verdecchia A et al. (2007). Recent cancer survival in Europe: a 2000-02 period analysis of EUROCARE-4 data. Lancet Oncol, 8(9):784-796.

Welch HG, Woloshin S, Schwartz LM (2005). Skin biopsy rates and incidence of melanoma: population-based ecological study. Br Med J, 331(7515):481.

Woods WG, Gao RN, Shuster JJ (2002). Screening of infants and mortality due to neuroblastoma. N Engl J Med, 346(14):1041-1046.

Zahl PH, Strand BH, Maehlen J (2004). Incidence of breast cancer in Norway and Sweden during introduction of nationwide screening: prospective cohort study. $\mathrm{Br} \mathrm{Med} J$, 328(7445):921-924. 



\title{
Chapter 9
}

\section{Information on cancer}

\author{
Andrea Micheli and Paolo Baili
}

\section{Introduction}

Earlier chapters highlight the differences between indicators of cancer outcomes in Europe (incidence, mortality and survival). Table 9-1 shows the gap between the countries with the highest and lowest levels of selected outcome indicators (for all cancers) expressed as relative risks, in accordance with the most recent data available on the web.

To understand, at least in part, the reasons for these inequalities in cancer incidence, mortality and survival, it is necessary to have access to knowledge about a range of social and epidemiological parameters. These include lifestyles; socioeconomic status; implementation of preventive actions, cancer registration and the organization of screening programmes; the distribution of health-care facilities and technological infrastructure; the resources available

Table 9-1 Relative risk between maximum and minimum levels of incidence rates, mortality rates and 5-year relative survival across Europe: for all cancers combined

\begin{tabular}{|c|c|c|c|c|}
\hline \multirow[b]{2}{*}{ Indicator } & \multirow[b]{2}{*}{ Reference year } & \multirow{2}{*}{$\begin{array}{c}\text { No. of countries* } \\
(E \cup 27+E E A)\end{array}$} & \multicolumn{2}{|c|}{ Relative risk } \\
\hline & & & Men & Women \\
\hline Mortality rate ${ }^{1}$ & 1996-2001 & $27+3$ & 2.1 & 1.8 \\
\hline Incidence rate ${ }^{2}$ & 2002 & $22+3$ & 1.7 & 1.7 \\
\hline 5 -yr relative survival ${ }^{3}$ & $\begin{array}{l}\text { Diagnosis: } 1990-94 \\
\text { Followed up to } 1999\end{array}$ & $17+3$ & 1.7 & 1.6 \\
\hline
\end{tabular}

* Countries for which the indicator is available from national or regional cancer registries and through estimation procedures (incidence).

${ }^{1}$ Age-standardized cancer mortality rate (world standard). Source: WHO, http://epicancer.iss.it/. Maximum levels in Hungary (men 396 deaths per 100 000, women 202 deaths per 100 000). Minimum levels in Sweden (men: 192 deaths per 100000$)$ and Spain (women: 113 deaths per 100 000).

${ }^{2}$ Age-standardized cancer incidence rate (world standard). Source: GLOBOCAN, http://www-dep.iarc.fr/. Maximum levels in Belgium (men: 347 new cases per 100 000) and Denmark (women: 286 new cases per 100 000). Minimum levels in Bulgaria (men: 207 new cases per 100 000; women: 168 new cases per 100 000).

${ }^{3}$ The risk is calculated as the cumulative mortality excess after 5 years since diagnosis. Source: EUROCARE-3, www.eurocare.it. Age-adjusted 5-year relative survival: minimum levels in Poland (men: 22\%; women: 35\%); maximum levels in Austria (men: 55\%) and France (women: 59\%). 
for health; and economic development. Clearly, the systematic collection and exploitation of such data, in the form of indicators at national level, would facilitate meaningful comparison between countries.

In 1997, the European Commission launched a health monitoring programme (HMP). One element of this was to define health indicators for the EU. The HMP programme was followed by two more wide-ranging public health programmes (PHP) covering the periods 2003-2008 and 2008-2013 (http://ec.europa.eu/health/ph_overview/overview_en.htm). The Commission is promoting the standardized collection of a list of public health indicators in all Member States. Since 2001, this has been undertaken by the European Community Health Indicators projects (ECHI-1, ECHI-2, ECHIM) (http://ec.europa.eu/health/ph_information/documents/ev20040705_rd09_ en.pdf).

The first European Cancer Health Indicator Project (EUROCHIP-1) was initiated under the auspices of the HMP, with the goal of providing the European Community Health Indicators programme with a list of indicators to monitor trends in cancer in Europe. In 2001, more than 130 experts from various fields of cancer research and policy (physicians, epidemiologists, health planners, etc.) and from all of the then 15 Member States, participated in the development of a list of indicators. These were based on criteria such as reliability, comparability, ease of collection and national representativeness. The outcome of the project was a comprehensive list of indicators relevant to cancer control, describing cancer in terms of burden, prevention measures, standards of care and cure rates. This list is presented in Table 9-2 (Eurochip web site; Micheli, Capocaccia \& Martinez, 2003). The indicators lie within the following domains.

- Prevention: indicators referring to exposure to risk factors or protective factors for cancer and other chronic diseases (e.g. tobacco smoking, level of physical activity, obesity, consumption of fruit and vegetables, etc).

- Epidemiology and cancer registration: indicators of cancer occurrence and care (incidence, mortality, prevalence, survival, stage at diagnosis).

- Screening: indicators covering programmes of early diagnosis of cancer (breast, cervical and colorectal cancer).

- Cancer treatment and clinical aspects: indicators pertaining to the performance of cancer diagnosis and treatment resources, as well as palliative care.

- Macro-social and economic variables related to cancer outcomes. 
This chapter describes the development of these indicators, the sources of data used to generate them and the process of creating standardized mechanisms to collect information that is, at present, difficult to obtain (indicators marked with an asterisk in Table 9-2) (http://ec.europa.eu/health/ph_information/ indicators/docs/shortlist_en.pdf).

\section{The Cancer Information System}

Table 9-1 highlights the unacceptable inequalities in the burden of cancer in Europe. One reason for these inequalities is the non-uniform application of scientific knowledge about cancer control. The main purpose of the Cancer Information System in the EU is to bridge the gap between cancer research and cancer control activities. The prerequisites for achieving this goal are:

- availability of population-based data;

- completeness of data collection in all European countries;

- standardization of data collection methods, to allow comparison across Europe;

- ability to adapt information systems to changing circumstances.

The indicators were chosen to enable standardized comparisons within Europe that would facilitate learning from best practice about how to lower cancer risk; provide optimal cancer treatment; prolong survival; optimize cancer patients' quality of life; and reduce inequalities in the burden of cancer.

Given that data collection systems reflect the specific organizational characteristics of national health systems it is inevitable that these indicators are not totally comparable across the EU. The sources of information may be cancer registries, health surveys, cancer screening programmes and a range of other databases.

\section{Cancer registries}

Information on cancer is much more widely available than for other diseases, reflecting the long tradition, in most European countries, of "populationbased cancer registries". Cancer registries should hold records for all patients with cancer diagnosed in the population they cover.

Epidemiological indicators such as incidence (indicator 2.2 in Table 9-2), survival (2.3) and prevalence (2.4) are routinely collected by cancer registries. Data on mortality (2.5) are collected by national statistical offices in each country and supplied to cancer registries for linkage with registration data. In some cases the only record is the death certificate - death certificate only 


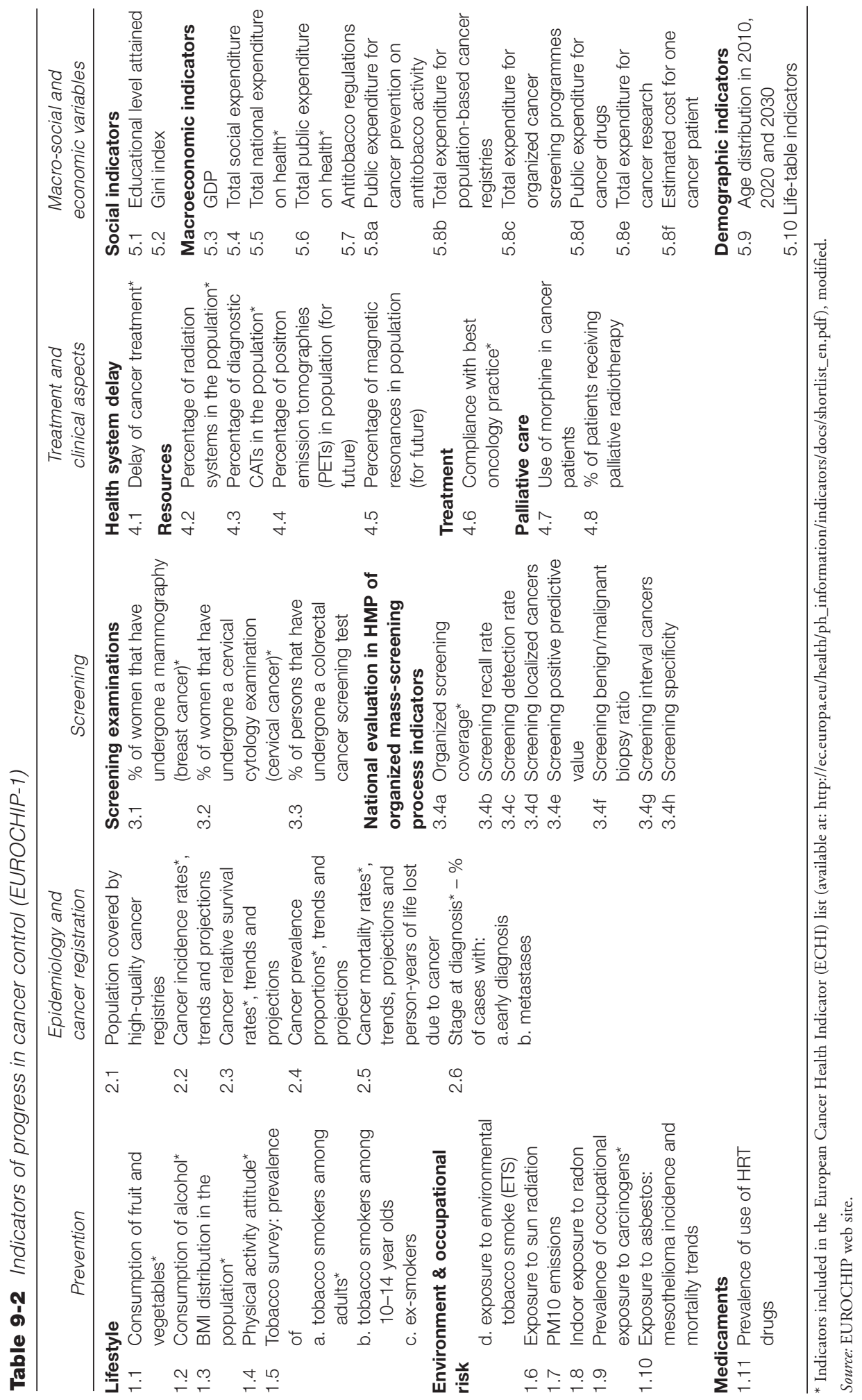


(DCO) records. In some countries, cancer registries cover the entire population, while in others coverage only extends to limited geographical areas. The European Network of Cancer Registries (ENCR) and the IARC produce cancer incidence estimates at national level. When the information is incomplete, they use statistical and mathematical models to impute the missing values and provide estimates of cancer incidence (Ferlay et al., 2004). Another EU-funded project, EUROCARE, provides cancer survival estimates (Capocaccia, Gatta \& Roazzi, 2003)., while the EUROPREVAL project focuses on cancer prevalence (Micheli, Mugno \& Krogh, 2002).

Cancer registries use established quality criteria in collecting the data used to estimate cancer incidence. The IARC monograph "Cancer incidence in five continents" (Curado et al., 2007) reports the attainment of these quality criteria (i.e. internal consistency; histological verification of cancer diagnosis; percentage of cases registered by DCO, or with unspecified anatomic site or of unknown age). At the time of writing, Greece, Hungary, Luxembourg and Romania are not included in the book, because relevant cancer registries had not yet been established or the epidemiological data that was available was not easily comparable with data from other countries (Table 9-3).

Cancer registries can collect information on patients' cancer history from existing routine health-service data systems.

The EUROCHIP pilot studies (http://www.tumori.net/eurochip/ dom_c\&t. php) investigated the feasibility of obtaining indicators that could be associated with international variations in cancer survival, as reported by the EUROCARE project (Capocaccia, Gatta \& Roazzi, 2003).

- Stage at diagnosis (indicator 2.6 in Table 9-2): percentage of cases with early diagnosis and percentage of cases with metastases at presentation, among all incident cases. These indicators are proxies for diagnostic awareness and the intensity of investigation in cases of suspected cancer.

- Delay in cancer treatment (4.1): average time between the date of cancer diagnosis and the date of first treatment.

- Compliance with guidelines for best practice (4.6): indicator aimed at reflecting compliance with best practice in oncology.

\section{Health surveys}

Indicators of behaviours and lifestyles and of screening examinations (Table 9-2) can be collected via health surveys. Indicators from national surveys are available in many countries; however, many are not readily comparable. 
Table 9-3 Cancer registries in Europe

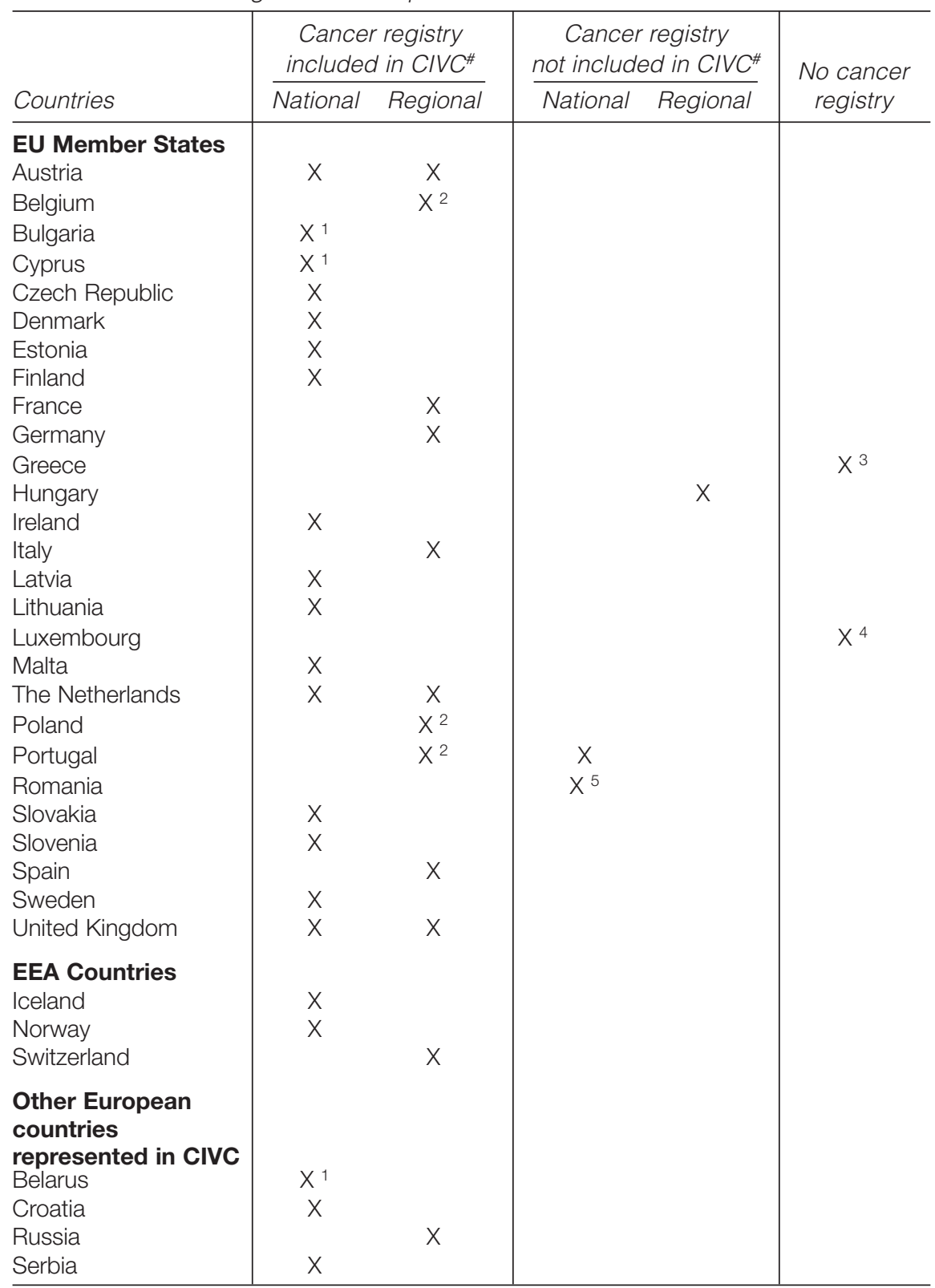

\# CIVC: Cancer incidence in five continents - volume IX (Curado et al., 2007)

${ }^{1}$ Some care is required in the analysis of all the data for some or all cancer sites (Curado et al., 2007)

${ }^{2}$ Some care is required in the analysis of some data for some or all cancer sites (Curado et al., 2007)

${ }^{3}$ Greece: a proposal for the urgent initiation of a pilot study was submitted to the Ministry of Health in June 2007

${ }^{4}$ Luxembourg: a national steering committee was charged by the Minister of Health to organize a cancer registry in July 2007

${ }^{5}$ Romania: national registration required by law since 2002 
The Statistical Office of the European Communities (EUROSTAT) and the EC Directorate General for Health and Consumer Affairs (DG-SANCO) are currently developing a European Health Survey System (EHSS) that would facilitate standardized health-survey modules (http://ec.europa.eu/health/ ph_information/dissemination/reporting/ehss_en.htm). The EHSS involves a combination of existing national or international survey tools with specifically designed modules of common questions. It comprises a core health interview survey (managed by the Community Statistical Programme) and a set of special health interview survey modules (managed by the Public Health Programme).

The goals of the EHSS can be summarized as:

- identification of health problems

- description of the health status and health needs of a population

- estimation of prevalence and distribution of health indicators

- analysis of social (in)equality in health and access to health services

- study of health-care consumption and its determinants, as well as preventive care

- study of trends in health status, lifestyle and health-care service consumption in the population.

During 2007-2008, all EU Member States will implement the European Health Interview Survey (EHIS). The great advantage of this survey is that, for the first time, European and national statistics offices in all Member States will use a standardized questionnaire (current version available at: http://circa. europa.eu/Public/irc/dsis/health/library, accessed 19 November 2007). The data obtained will be comparable across Europe. The EHIS will be performed every five years, in a manner determined by each country. The various modules are set out below.

- Health status (EHSM): includes questions on self-perceived health. Module will be used to estimate the "self-perceived prevalence" of various chronic diseases (including cancer).

- Health care (EHCM): includes questions on the use of care, including screening examinations. With this module it is possible to estimate indicators such as the percentage of women who have undergone mammography (indicator 3.1), the percentage of women who have undergone cervical cytology (3.2), or the percentage of people who have undergone a colorectal cancer screening test (3.3). 
- Health determinants (EHDM): includes questions about lifestyles. With this module, indicators on fruit and vegetable consumption (indicator 1.1), alcohol consumption (1.2), body mass index (1.3), attitude to physical activity (1.4), and smoking prevalence (1.5), will be estimated.

\section{Organized screening registries}

Indicators of the implementation of cancer screening examinations in the population (indicators 3.1-3.3 in Table 9-2) include attendance at either organized or opportunistic screening. Currently, they can only be collected for many Member States by means of health surveys, because organized screening programmes are not available in all countries (see Chapter 4).

Nevertheless, it is important to define the geographical coverage of organized screening programmes ${ }^{6}$ (indicator 3.4a) as it has been shown that wellorganized, population-based, integrated, quality-controlled screening programmes for cervical cancer and breast cancer reduce mortality. Opportunistic or non-organized screening initiatives will miss many people (in the case of cervical cancer, typically those at greatest risk) and cannot be monitored and systematically evaluated for clinical and diagnostic quality. Lastly, they cannot be monitored for potential adverse effects of screening, such as excessive doses of radiation and unnecessary subsequent investigation.

The EU currently recommends that cancer screening should be offered on a population basis in organized screening programmes for three cancer sites: breast, cervix and colorectal (http://eur-lex.europa.eu/LexUriServ/site/en/oj/ 2003/1_327/1_32720031216en00340038.pdf). Such screening programmes cannot be implemented without a population registry.

Indicators 3.4a-3.4h (Table 9-2) refer to organized screening programmes in terms of clinical and diagnostic quality. Surveys to measure these indicators have been performed (Anttila et al., 2004) and are now included in the EUNICE project (http://ec.europa.eu/health/ph_projects/2004/action1/ action1_2004_33_en.htm), with screening registries being used as sources of data. These indicators vary, however, between cancer types - e.g. for cervical cancer, the majority of screen-detected lesions are treated at a precancerous stage; for breast cancer, the disease is usually detected and treated in the invasive stage.

\footnotetext{
${ }^{6}$ Population resident in areas where organized screening is implemented and to which screening is offered, as a proportion of the national population in the corresponding age-sex group
} 


\section{Other databases}

EUROCHIP-1 and ECHI suggested the continuation of the collection of a list of indicators covered by the Organisation for Economic Co-operation and Development (OECD, 2005) - radiotherapy provision (indicator 4.2 in Table 9-2); computed tomography (indicators 4.2 and 4.3); and expenditure on health care (5.5 and 5.6).

Measurement of the prevalence of occupational exposure to carcinogens (indicator 1.9) was under the responsibility of the International Information System on Occupational Exposure to Carcinogens (CAREX) (http://www.ttl. fi/Internet/English/Organization/Collaboration/Carex/). This database, subsidized by the Europe Against Cancer programme, estimated the burden of occupational exposure to carcinogens in various countries by specific agent and by industries for the period 1990 to 1993 (Austria, Belgium, Denmark, Finland, France, Germany, Greece, Ireland, Italy, Luxembourg, the Netherlands, Portugal, Spain and the United Kingdom) and for 1997 (Czech Republic, Estonia, Latvia and Lithuania). By updating the database using the same methodology, it is possible to study changes in occupational exposure to carcinogens over the past 10 years in the EU.

\section{Cancer information and cancer control}

Cancer control includes prevention and treatment of cancer, and improvements in the survival and quality of life of cancer patients, by transferring knowledge obtained from research, surveillance and outcomes evaluation into policies and actions.

A well-functioning cancer information system is vital to study the efficacy of cancer control actions and interventions. Comparison of population-based outcomes allows a better understanding of which policies may be transferable to other countries. Such a system also sheds light on the performance of broad areas of cancer control. For example:

- successes in primary prevention can be monitored using indicators such as the reduction in the number of persons exposed to risk factors (short term) and the decrease in cancer incidence (long term);

- a measurable outcome of successful early diagnosis activities is the reduction in cancer mortality for screen-detectable cancers;

- the modernization of diagnostic and therapeutic infrastructure can improve cancer survival; 
- rehabilitation and palliative care contribute to a better quality of life for cancer patients.

Fig. 9-1 shows the correlation between cancer incidence and prevalence in various European countries (Micheli, Mugno \& Krogh, 2002). The area of each circle, each representing a country, is proportional to the five-year relative survival of cancer patients in that country. An increase in the total prevalence is seen as incidence increases. Western European countries (clustered mainly on the right of the figure) tend to show higher incidence and prevalence compared with central and eastern European countries (on the left). This figure also captures the net results of cancer control activities. Primary prevention reduces cancer incidence while effective treatment and early diagnosis increase survival and thus prevalence. For example, Sweden stands out as having a low incidence and high prevalence of cancer. It has longstanding experience in cancer prevention that is supported by a high-level information system. This simple graphic illustrates the power of indicators to capture important aspects of cancer control in Europe.

\section{Conclusions}

One key role for a cancer information system is to facilitate linkages between researchers and public-health decision-makers, providing the latter with highquality research and data to support their decisions. Thus, the development of the cancer information system is a fundamental tool for the reduction of inequalities in the burden of cancer across Europe. It facilitates the decisionmaking process at strategic and operational level, leading to concrete actions in the field of cancer control, including the monitoring of their implementation and the evaluation of their impact.

\section{Recommendations}

An optimal cancer information system that can meet the needs of Europe's population in the $21^{\text {st }}$ century requires that each Member State facilitate:

- population-based cancer registries, to enable access to population-based, comparable indicators of the cancer burden and cancer outcomes and, ultimately, detailed information on diagnostic and clinical procedures. This implies both adequate funding and availability of data, especially where overzealous application of data protection laws either prevent cancer registries from functioning or prevent their data being used for public health purposes; 

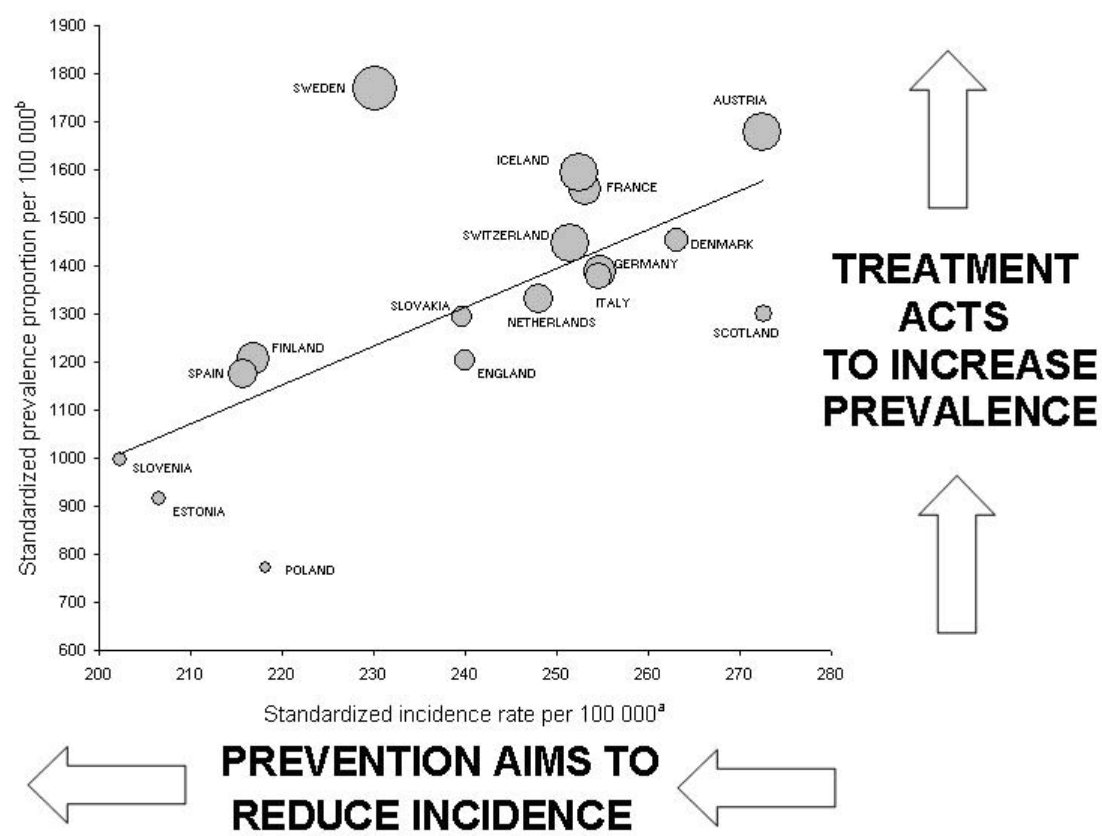

Notes: Circle areas are proportional to 5-year relative survival. Total prevalence refers to all persons in a given population diagnosed in the past with cancer and alive on the reference day. Circle diameters are proportional to EUROCARE ageadjusted 5-year relative survival (\%), 1988-1992 incidence period. Source: Berrino et al., 1995.

a World standard age-adjusted rate per 100000 people, incidence period 1988-1992. Source: Parkin et al., 1997.

b Total prevalence at 31st December 1992, world standard age-adjusted proportion per 100000 people. Source: Micheli, Mugno \& Krogh, 2002.

Source: Micheli, Mugno \& Krogh, 2002 (modified).

Fig. 9-1 Age-standardized total prevalence, incidence and survival, both sexes, all cancers combined, 1992

- best coverage of the national population by population-based cancer registries;

- implementation of the European Health Survey by the national statistical office in each Member State, to permit the collection of comparable indicators on lifestyle and screening examinations;

- organization of population-based screening programmes and registries.

\section{Acknowledgments}

This chapter was supported by EUROCHIP-2: European Cancer Health Indicator Project (Phase 2) (European Commission, Health and Consumer Protection Directorate-General). 
EUROCHIP-2 Working Group - Austria: W Oberaigner (Cancer Registry of Tirol); S De Sabata (International Atomic Energy Agency); Belgium: E Van Eycken (Belgian Cancer Registry); H Sundseth (European Cancer Patient Coalition); Bulgaria: Z Valerianova (Bulgarian National Cancer Registry); V Zlatkov (Sofia Medical University); P Kostova (National Oncology Hospital, Sofia); Czech Republic: Z Kamberska (Institute of Health Information \& Statistics); Cyprus: C Papageorgiou (Ministry of Health); Denmark: M Von Euler (University of Copenhagen); Estonia: P Veerus (National Institute for Health Development); Finland: R Sankila, A Anttila (Finnish Cancer Registry); France: J Bloch (Direction Générale de la Santé); P Grosclaude, A Danzon (FRANCIM); Greece: L Tzala (Centre for Disease Control \& Prevention); KV Kamposioras (University Hospital of Ioannina); G Ferentinos (General Hospital of Halkida); D Mauri (Papageorgiou Hospital); Ireland: H Comber (Irish National Cancer Registry); Italy: R Capocaccia (EUROPREVAL); A Verdecchia (Istituto Superiore di Sanità); F Berrino (EUROCARE); C Amati, I Casella, L Ciccolallo, C Allemani (Fondazione IRCCS, Istituto Nazionale dei Tumori); G Gatta (RARECARE); M Sant (HAEMACARE); F Merzagora (Osservatorio Salute Donna); Latvia: I Viberga (Riga Stradins University); L Engele (Riga East Hospital Cancer Center); Lithuania: J Kurtinaitis (Lithuanian Cancer Registry); Luxembourg: S Couffignal (Centre de Recherche Public Santé); Malta: M Dalmas, R Busuttil (Malta National Cancer Registry); The Netherlands: R Otter, M Schaapveld (Comprehensive Cancer Centre North Netherlands); Poland: M BielskaLasota (M Sklodowska-Curie Memorial Cancer Center and Institute of Oncology); Portugal: A Miranda (South-Regional Cancer Registry); Romania: I Apostol (Dr Victor Babes Foundation); Slovakia: I Pleško, M Ondrusova (National Cancer Registry of Slovakia); Slovenia: M Primic-Žakelj, V Zadnik (Cancer Registry of Slovenia); Spain: C Martinez (Escuela Andaluza de Salud Publica); C Navarro (Consejería de Sanidad de Murcia); Switzerland: J-M Lutz (Association of Swiss Cancer Registries); C Quinto (ISPM Basel); I Mortara (UICC); United Kingdom: MP Coleman (London School of Hygiene \& Tropical Medicine); I Kunkler, A Gregor (Western General Hospital, Edinburgh); A Gavin, F Bannon (Northern Ireland Cancer Registry).

\section{REFERENCES}

Anttila A et al. (2004). Cervical cancer screening programmes and policies in 18 European countries. Br J Cancer, 91(5):935-941.

Berrino F et al. eds. (1995). Survival of cancer patients in Europe: the EUROCARE study (IARC Scientific Publications No. 132). Lyon, International Agency for Research on Cancer.

Capocaccia R, Gatta G, Roazzi P (2003). The EUROCARE-3 database: methodology of data collection, standardisation, quality control and statistical analysis. Ann Oncol, 14:14-27. 
Curado MP et al. (2007). Cancer incidence in five continents, Vol. IX. (IARC Scientific Publications No. 160). Lyon: International Agency for Research on Cancer.

EUROCHIP web site (available at: http://www.tumori.net/eurochip, accessed 19 November 2007). Milan, Italy, National Institute of Cancer.

Ferlay J et al. (2004). GLOBOCAN 2002: Cancer incidence, mortality and prevalence worldwide (IARC CancerBase No. 5. version 2.0). Lyon, IARC Press.

(available at: http://ec.europa.eu/health/ph_overview/overview_en.htm, accessed 2 December 2007).

(available at: http://ec.europa.eu/health/ph_information/documents/ev20040705_rd09_en.pdf, accessed 2 December 2007)

(available at:http://circa.europa.eu/Public/irc/dsis/health/library, accessed 2 December 2007).

(available at: http://ec.europa.eu/health/ph_information/indicators/docs/shortlist_en.pdf, accessed 2 December 2007)

(available at: http://ec.europa.eu/health/ph_information/dissemination/reporting/ehss_en.htm, accessed 2 December 2007).

(available at: http://ec.europa.eu/health/ph_projects/2004/action1/action1_2004_33_en.htm, accessed 2 December 2007).

(available at: http://eur-lex.europ(a.eu/LexUriServ/site/en/oj/2003/1_327/1_32720031216en00340038. pdf, accessed 2 December 2007).

(available at: http://www.ttl.fi/Internet/English/Organization/Collaboration/Carex/, accessed 2 December 2007).

(available at: http://www.tumori.net/eurochip/dom_c\&t.php, accessed 2 December 2007).

Micheli A, Mugno E, Krogh V (2002). Cancer prevalence in European registry areas. Annals of Oncology, 13:840-865.

Micheli A, Capocaccia R, Martinez C (2003). Cancer control in Europe: a proposed set of European cancer health indicators. Eur J Public Health, 13(Suppl. 3):116-118.

OECD (2005). Health data 2004, 3rd ed [CD-ROM]. Paris, OECD, Health Policy Unit.

Parkin DM, Whelan SL, Ferlay J (2002). Cancer incidence in five continents, Vol. VIII (IARC Scientific Publications No. 155). Lyon, International Agency for Research on Cancer. 



\section{Chapter 10 Cancer patients - partners for change}

Hildrun Sundseth and Lynn Faulds Wood

\section{Introduction}

Cancer affects us all. A cancer diagnosis has a profound impact on a person's life. Often it feels like a death sentence, plunging us into despair and turning our life and the lives of our family and friends upside down. This happens to over 5000 people in the EU every day (Boyle \& Ferlay, 2005). We may get used to these shocking cancer statistics, but when they wear the familiar faces of our family members, friends and neighbours, it shatters our world.

One in three Europeans will be diagnosed with cancer at some time in their life, so this devastating disease, which comes in over 200 different forms, will touch most of us and hardly any family will be spared. While every person will have to cope with the disease in their own way, all that we patient groups, family and friends can hope for is that the person who has suddenly become a "patient" gets the best possible, up-to-date, patient-friendly and evidencebased treatment and supportive care. At such times, it is vital that nobody is left alone to struggle and that every cancer patient is supported by family and friends, and a responsive medical system.

For many of us, the survivors, nothing will ever be the same again. And some of us feel compelled to use our personal experience for a wider societal and political cause. Yes - for lobbying for change: for a more robust, responsive and patient-centred approach to cancer control by our governments, encompassing prevention, early diagnosis, screening and the highest quality of treatment and care, including psychosocial and palliative care. We also wish to encourage further research, without which some cancers will remain untreatable and unbeatable. 
Until recently, too many people diagnosed with cancer died of their disease. Today, more and more people can or could survive, and yet - across Europe thousands die unnecessarily (Coleman et al., 2003). Experts tell us, the patients, that we now know how to prevent about $50 \%$ of all cancers (Boyle et al., 2003). All the more shocking then, that every year so many people still develop cancer and die unnecessarily. Why have we not learned the lessons of controlling cancer more effectively? Of course, there are some cancers for which we do not yet possess the knowledge to prevent, treat or cure. But, sadly for others, the chance of surviving cancer is often affected by where you live, where you are treated, whether you have vital information and whether you are able and strong enough to fend for yourself in what often seems an impersonal system.

Astonishingly, within the EU, there seems to be little political will to share and apply evenly the knowledge we do have on how to prevent, diagnose and treat cancer, and how to care for patients. Europe seems to have no legal basis for this. This is comprehensible only if we consider that countries in the EU came together first of all to form a common market, but it remains incomprehensible, all the same, if one considers that good health is what people treasure most. Small wonder that most EU citizens feel far removed from their EU.

The European Cancer Patient Coalition (ECPC) would argue that if Europe wants to keep in touch with its citizens and benefit from the free movement of people and services, and indeed to implement the Lisbon Strategy of becoming the most advanced and competitive information society in the world, there seems to be a need for fresh thinking.

We have known about the dangers of tobacco use for over 50 years, but only now has Europe mustered the political will to act accordingly. ECPC congratulates all those EU Member States that have implemented the WHO Framework Convention on Tobacco Control.

Why, when so many of us are affected by cancer, has Europe been so slow to react to this scourge that haunts us and - given the data and projections we have from important studies such as EUROCARE (Coleman et al., 2003) will continue to do so, even more in the future?

We are fortunate that European societies treasure health as a common good. At a time when illness strikes, it is reassuring that the individual person can count on a safety-net based on the principle of solidarity and health protection for all citizens, including the weakest members of society. But it is imperative for all of us to do all that we can to stem the cancer tide. Given our ageing populations, there is simply no time to lose. Europe must act now. 
Cancer creates such a heavy and complex burden of disease for society that no single organization, institution or government can cope with it adequately in isolation. We, cancer patients, see an urgent need to mobilize all the available energy and expertise to bring about change for the better. The ECPC stands ready to play its part. Only if we have managed to achieve such change will ECPC feel that we have done ourselves out of a job. But until that time, we are ready to follow our motto: "Nothing about us, without us" and to argue forcefully the case of all those who find it more difficult to fend for themselves.

The change from passive to empowered patient is a recent phenomenon. The HIV/AIDS community has shown us how. Led by the desire to improve the situation for ourselves and others, cancer patients have organized themselves in groups. No longer satisfied with the status quo, we have thrown off the fatalistic attitude that has often surrounded cancer. We have become strong advocates for our own cause.

Our advocacy work has become more professional. Our voice is clear, forceful and persistent. In turn, patient groups are increasingly recognized as having an important role to play in responding to the cancer patient's need for support. At a more strategic level, we are also increasingly seen as having a constructive contribution to make in arguing for more efficient, responsive and effective health-care systems, leading to improved cancer control.

Like that of most European patient groups, ECPC's advocacy work is focused on two broad categories:

- raising awareness and advocacy

- patient support and capacity building.

In the cancer field, this spans the whole range of what is commonly understood as cancer control. We are committed to creating cancer awareness in the general public and advocating the need for improved primary prevention, screening, early diagnosis and the best treatment and care. To that end, we are working to increase cancer patients' representation and influence at the highest level of decision-making, both nationally and Europe-wide, in all areas that affect patients' health, and wherever they live in Europe. This also means pointing out the disparities and inequalities that exist within countries and across the EU.

\section{ECPC and our objectives}

The urgent need for Europe-wide action is one of the reasons why ECPC came into existence three years ago. We are a European umbrella organization 
bringing together cancer groups representing both the common and the rarer cancers. As a patient-led organization, we want all cancer patients across the EU to have their say in influencing the way cancer is controlled.

Health is a common good, yet everything surrounding it is political. Our governments and health ministries are committed to protecting the health of their citizens, but we realize that it is a real challenge to allocate the finite resources available for health fairly and effectively. That is where ECPC's interest lies; and that is why the many patient groups that belong to our member organizations have decided to join forces. We currently represent over 250 patient groups from across the EU Member States, determined to give cancer patients a strong, unified voice.

Our aim is first and foremost political - to ensure that policy-makers, politicians, health professionals, the media and the general public recognize the serious burden of cancer and the need for concerted action to reduce unnecessary death and suffering.

So how is ECPC setting about this tough agenda, and why are we concentrating on Europe when health care is still largely a national concern, and patients want their health taken care of as close to home as possible?

Health care is national, but we live in a world that is increasingly interconnected and where information can be shared rapidly. We are all subject to outside influences. Knowledge is global and it is on this sharing of knowledge and best practice that much of our hope for improvement is based. Unless we look over the fence to our neighbours, we cannot compare performance, and know if we should be doing better than we are. For this reason, ECPC supports the robust evidence provided by the data from cancer registries in studies like EUROCARE (Coleman et al, 2003). Cancer registries provide a detailed picture of the current situation on cancer control - telling us who does better in prevention or survival, and who could improve. Only if we have this evidence can we explore the reasons why, and lobby for change. So in our view, tackling cancer is not only a medical and scientific task but also an economic and a social challenge - it is hugely political. Twenty-seven countries have decided to share their destiny in an EU, and we need to concentrate at all levels.

ECPC realized that in order to improve cancer outcomes for all citizens and cancer patients, we first needed to focus on creating more political will to act against cancer. This, in turn, would provide our member organizations with the necessary leverage and tools for their efforts to improve outcomes for their citizens and cancer patients, at the level where they affect the individual. Realizing that our health-care systems are facing increasing budget pressures 
due to an ageing population and the possibility of many sophisticated and expensive new treatments, we decided on a more constructive partnership approach: how can we do more and do it better, more efficiently and effectively? And how can patients themselves contribute?

\section{Investing in prevention}

While our national health-care systems are mostly struggling with the acute care of their patients, ECPC feels that a shift towards investing in the health of citizens and that of future generations is urgently needed. We must place a greater emphasis on primary prevention and screening. In the 1990s, Europe was leading in cancer policy through the Europe Against Cancer programme, which addressed prevention, research, information and regulation for cancer control, and mounted a strong campaign against tobacco addiction. It gave us the European Code Against Cancer, with 10 recommendations for preventing the most common cancers.

At the Slovenian cancer summit (Ljubljana, November 2006), EU Commissioner Potočnik reported that the European Commission estimates that about 92000 cancer deaths are averted each year (United against Cancer Summit, 2006). More than ever, the European Code Against Cancer is an excellent public health tool that tackles key lifestyle issues and is relevant for other diseases, not just cancer (Boyle et al, 2003). ECPC's earnest wish is that the Code continues to be promoted so that it becomes known by every child and adult.

The Europe Against Cancer programme also provided comparative data that enabled many governments to see that their measures to control cancer were not as effective as those of other countries.

Under the European Treaty, health care may remain a national rather than a European issue for some time to come. However, the European Commission and Parliament have both recognized the importance of cancer prevention and have forcefully communicated this to all Member States. In addition, the EU has passed legislation and many rules, set up public health and research programmes, a European medicines agency and a disease prevention and control centre, all of which have an impact on national health-care delivery and on cancer patients. 


\section{Our policy and advocacy work - marshalling political will}

MEPs Against Cancer (MAC)

Anxious that cancer should remain firmly on the EU's political agenda despite closure of the Europe Against Cancer programme in 2002, ECPC encouraged Members of the European Parliament to set up an informal all-party forum MEPs Against Cancer, or MAC.

Now comprising over 60 members from all the major political groups, the parliamentary interest group has grown into a strong political force within a very short time. Having pledged to strengthen Europe's fight against cancer, as a first step, its members signed the MAC Statement (http://www. mepsagainstcancer.org/index.php?option $=$ com_content\&task=view\&id=18\& Itemid=32). This includes seven policy recommendations and calls on all European health ministers to take urgent steps to combat the major inequalities in cancer survival rates within countries and across the EU.

The MAC Statement has focused the cancer community on specific actions. One key recommendation is to set up a European cancer task force to share best practices and eradicate the gaps in cancer control. The all-party group holds regular meetings and conferences to inform colleagues in the European and national parliaments, as well as health attachés (civil servants from national health ministries, based in Brussels) and Commission officials about cancer issues that need urgent attention. For example, last year MAC discussed the benefits of a national cancer plan and its role in ensuring fair, equitable and improved outcomes for citizens and cancer patients.

By providing the MAC secretariat, ECPC helps politicians to stay in close touch with the concerns of their constituents. For this reason, volunteers from the cancer patient community have developed the web site (http://www. mepsagainstcancer.org) where all papers issued by MAC, such as meeting reports, the MAC Statement, press releases and position statements can be found.

The first two MAC meetings of 2007 concentrated on strategic issues. Investing in Future Generations: Cancer Prevention in Children was held in March; Making Cancer a Priority for the EU Presidencies was held in June. For the EU Presidencies meeting, MAC brought together high-level officials from Portugal and Slovenia and European and national politicians to collaborate and strengthen the cancer initiatives.

Cancer patients can now count on politicians arguing their cause. Already MAC is sending a strong signal to national capitals that immediate action is needed both to reduce cancer rates and to improve outcomes (Box 10-1). 


\section{Box 10-1 MAC Recommendations}

1. Urgently develop and, where existing, improve national cancer plans, setting priorities and effectively allocating resources for improving cancer control and research across the EU.

2. Firmly tackle the socioeconomic and geographic divide, which leads to inequalities in cancer control.

3. Make high-quality and up-to-date prevention, treatment and care attainable for all cancer patients in each European Member State.

4. Vigorously promote cancer awareness in the general public through the existing Europe against Cancer code, making a special effort in new Member States.

5. Invest in cancer prevention in Member States through implementing the Council Recommendation on Cancer Screening of December 2003 and setting up national high-quality screening programmes

6. Oppose discrimination because of age, race, gender and domicile in respect of the latest cancer treatments.

7. Set up a cancer task force at European level, to exchange best practice and to highlight once again that tackling cancer is a priority and sending a strong political signal that immediate and concerted action is needed now.

\section{Signed by}

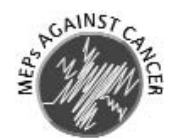

MAC

Members of the European Parliament against Cancer

\section{EU Presidencies: Slovenia and Portugal take up the challenge}

The United Against Cancer Summit - a wake-up call

In anticipation of the Slovenian EU Presidency in the first six months of 2008, 
ECPC organized the Central and Eastern Europe (CEE) Patient Summit (Making Cancer a Priority) in Ljubljana in November 2006, to buttress Europe's flagging fight against cancer. More than just lengthy declaration and communiqués, the summit illustrated how politicians and patient groups can work together to compel new thinking.

It was shown that the cancer survival gap between European countries was widening - this was the wake-up call sounded by the summit. Experts produced glaring evidence gleaned from cancer registries and the EUROCARE study showing that inequality in cancer control and outcomes is actually increasing in the new Member States. Unless we took urgent action now to forestall a looming cancer divide in new Member States and future accession countries, even more people would be losing their life to cancer in the coming years.

This landmark summit meeting on cancer set off a chain of events which ECPC sincerely hopes will bring about real-life change for cancer patients. For instance, the Slovenian Minister of Health announced that his country would highlight the gaps in health and cancer care as an EU priority issue. So cancer patients can take heart that MAC's call to action has already found a welcome resonance with governments. Slovenia pledged to examine the whole range of weapons that we have to fight cancer, from prevention to screening; and a robust integrated approach to cancer management. Importantly, this would include the need for continued research, which is badly needed for all those cancers that are currently still untreatable.

Cancer patients now have their hopes firmly pinned on Slovenia, which will be the first of the 10 new Member States that joined the EU in 2004 to take up the EU Presidency. Slovenia will have the difficult task of rallying EU health ministers' support to step up measures for cancer control (covering prevention, early diagnosis, treatment and care) and to argue for a more coordinated application of present knowledge to tobacco control. Without such a drive, Europe risks abandoning the health of many future generations to cancer.

In turn, ECPC will do all it can to muster its national member groups behind this opportunity for change.

\section{Portuguese Presidency Round Table on Health Strategies in Europe}

Working with the Slovenian Presidency, Portugal has already started work on the Council of Health Ministers' action against cancer. At a time when the 
Commission is reflecting on its new health strategy, Portugal emphasized the need for solid cancer registries, comprehensive cancer plans and screening, in their Presidency Political Roundtable in July 2007.

Linking up the work of MAC and the cancer summit with the Portuguese and Slovenian Presidencies enabled our Portuguese member to be involved in organizing the round table's parallel session on cancer, thus ensuring that cancer patients' needs were truly in the centre of the high-level deliberations. Once more, proof of how patient groups with a European and national remit can work together to break down barriers for the good of all.

The recommendations from the Portuguese Presidency Round Table on Health Strategies in Europe will provide vigorous input to the Commission's forthcoming White Paper: "Together for health" and the Council's conclusions.

\section{Adding value to the work of our national members}

In addition to the efforts that we make, on behalf of our members, to break down political and territorial barriers that hamper Europe from sharing best practice, ECPC is actively working with other institutions and bodies in the regulatory, scientific and medical fields. ECPC contributes the perspective of the cancer patient to all European legislation and other initiatives that could affect the treatment of cancer patients. Recent examples include the EU Paediatrics and Advanced Therapies Regulations and the debate on whether or not to legislate on improving patient information. ECPC is involved in two European consortia: one that will produce quality screening guidelines for colon cancer, and another that will establish a European surveillance system for rare cancers. We then channel the information back to our patient organizations at grass-roots level.

A prominent example of how European legislation can go wrong if the voice of cancer patients is not heard can be found in the Clinical Trials Directive, which was adopted before ECPC came into existence. Rather than streamlining European trials and making them more effective, the EU legislation actually had the opposite effect: it has significantly delayed and increased the cost of cancer trials. Fortunately, there is now general agreement that the Directive must be reviewed. This time, cancer patients and researchers can rest assured that ECPC will be keeping a close watch. 


\section{Clinical trials information can be life-saving for cancer patients}

Having the possibility to enrol in early clinical trials is often a matter of life and death for cancer patients. There is some evidence that being cared for in a unit that takes an active part in clinical trials may lead to better outcomes (Fayter et al., 2006). That is why ECPC has made it a priority to lobby for transparency and better public access to clinical trial information. We wish to see clinical trials information made available in a public register which patients across the EU can access in their own language. To that end, ECPC has been working with WHO's Clinical Trials Registry Platform to ensure the interconnectivity of such registers, so that cancer patients will have access to essential information about how and where to enrol in ongoing trials of new cancer treatments - whatever their language skills, and wherever they live in Europe.

\section{EMEA - Patient and Consumer Working Party (PCWP)}

EMEA has recognized the contribution that patient groups can make and has opened its door to a fruitful dialogue with patient groups including the ECPC. This innovative step allows the cancer patient's voice to be heard by the regulator.

Since November 2005, all cancer medicines are approved and issued with a single European licence by EMEA. The ECPC sits on the official EMEA Patient and Consumer Working Party, a permanent forum for dialogue between the agency and patients' and consumer organizations as representatives of the end-users of medicines. ECPC is specifically consulted about the user-friendliness of the patient information leaflets in drug packages, as well as on the European Public Assessment Report and issues of pharmaco-vigilance and risk management. ECPC is also involved in the preparation of guidelines, and is regularly consulted by EMEA scientific committees, working parties and scientific advisory groups.

The European regulator thus provides welcome recognition of the unique contribution that patient groups can make. ECPC's members will lobby for such worthwhile interaction between regulator and patient groups to become standard practice for national medicines agencies.

More details about the work of the EMEA's Patient and Consumer Working Party are given on the EMEA web site (http://www.emea.europa.eu/). 


\section{Patient support - taking heart from the examples of best practice}

MAC meeting on prostate health - encouraging men to speak up

Although many well-known personalities (including famous politicians) have had prostate cancer, men have generally been reluctant to talk about "their cancer". Unlike women, who publicly confronted breast cancer and thereby became pioneers in creating awareness and influencing policy and care along the way, men have mostly kept silent.

As the MAC secretariat, ECPC was in a unique position to help Europa Uomo, the prostate cancer coalition and ECPC member, to tell MEPs about prostate health. Men tend to think that prostate cancer does not match the seriousness of breast cancer, but they are wrong and cancer statistics bear this out. It is estimated that one in ten men will develop symptomatic prostate cancer in their lifetime, making this the most common cancer in men and, after lung and colorectal cancer, the third biggest cancer killer. In the EU each year there are over 300000 new cases and more than 68000 men will die from prostate cancer.

Prostate cancer is generally slow-growing and affects mainly older men. In fact, more than $75 \%$ of all prostate cancers are found in men over the age of 65 . There are over 2 million men followed up or treated for prostate cancer in the EU. There is much overdetection and subsequent overtreatment, which can rob men of their quality of life in later years.

MAC agreed that men must follow the example of women and speak out. Given that all across Europe our populations are ageing, a concerted effort was needed to raise awareness about prostate cancer. MAC has pledged to help men to overcome this information deficit.

The Warsaw Declaration: the policy tool for closing the east-west gap

This declaration was developed for the Warsaw CEE Cancer Patients Advocacy Groups Summit by the ECPC and partners from the Polish Foundation for Humanitarian Aid "Res Humanae" and the Institute for Patients' Rights and Health Information. Patient groups have been spurred to join forces and promote the declaration in a unique show of solidarity. It provides a useful example of how patient groups can use statistics to build a broad political campaign.

Recently, ECPC's Romanian member (Federaţia Asociaţiilor Bolnavilor de Cancer - FABC, the Romanian cancer patient association), used the Warsaw Declaration in its demonstration against the Romanian Government's 
Box 10-2 The Warsaw Declaration

\section{WARSAW DECLARATION \\ Five years after the adoption of the Paris Charter Against Cancer adopted at the First World Cancer Summit, the situation of cancer patients is still dramatic.}

\section{Whereas}

Total health expenditure as proportion of GDP in CEE countries is less than in the European Union.

Prevalence and survival rates for every type of cancer are lower than in the rest of Europe.

Inadequate health prevention measures such as opportunistic screening, delay in early diagnosis and innovative treatment, lack of best quality care account for the poor cancer control in CEE countries.

Public awareness and understanding of cancers and their prevention, robust anti-smoking campaigns, promotion of healthier lifestyles are low or fragmented.

According to $\mathrm{WHO}$ predictions, cancer is expected to increase to epidemic proportions due to the ageing population all across Europe, including CEE and Accession Countries.

\section{Recommendations}

Because good health is a basic human aspiration;

Because all European Governments share the goal of protecting and improving their citizens' health and well-being;

Because the right to health protection is enshrined in the European Treaties;

\section{We, the signatories call on policy makers, politicians and key stakeholders urgently to:}

1. Develop national cancer plans, setting priorities and allocating resources, for improving cancer control and research in all CEE countries and assure patients' groups monitoring over the implementation of these plans

2. Invest in cancer prevention by promoting awareness, information and education campaigns about the risk factors of cancer, building on the European Code against Cancer.

3. Invest in national screening programmes as recommended by the European Union; and implement high quality EU standards to support early diagnosis.

4. Make high-quality up-to-date treatment, rehabilitation and care attainable for all cancer patients throughout Europe.

5. Encourage and ensure patient participation in all decisions on health policy and health care affecting cancer.

6. Advance cancer control as a priority for action where necessary to qualify for grants from the EU Structural Funds.

7. Oppose discrimination because of age, race, gender, domicile and economic status in respect of the latest cancer treatment.

8. Encourage and adopt national Charters of Patients' Rights according to European guidelines. 
constant changes to the provision of cancer care which threatened the lives of cancer patients. Marching to their Ministry of Health under the ECPC banner "Nothing about us, without us", the FABC succeeded in attracting media attention and obtaining consultative status on committees dealing with cancer care.

\section{An example of good practice from Germany - expert patients}

Everyone who has been given a diagnosis of cancer knows only too well the black hole of despair and the feeling of helplessness this brings. This is where Mamazone (Frauen und Forschung gegen Brustkrebs - women and research against cancer), ECPC's German member, has found an innovative way to bring support and confidence to women with breast cancer. Based on the concept that knowledge is power, Mamazone women have developed a series of intensive courses and lectures that enable them to understand their disease better and to regain control over their lives.

Women with breast cancer become expert patients (Projekt Diplompatientin) by participating in training seminars organized by Mamazone and a circle of specialist lecturers. The quality of the seminars given by high-ranking experts in oncology would be the envy of many a university programme: Mamazone persuaded renowned specialists to share their knowledge with patients. The course arms women with vital knowledge and improved understanding about their breast cancer, enabling them to become experts in their own disease. In many instances, this newly acquired knowledge helps to chase away the sense of helplessness that is attached to cancer, when the body seems to turn against itself. Women regain control over their lives and their bodies.

Such expert patients become true partners with their medical specialists. Participation in the course helps many women to endure and cope better with their illness. They become patients who work with their doctors, asking searching questions about their treatment and the latest research and development. The passive patient is replaced by one who is uniquely informed, determined to get through the disease and lead as normal a life as is possible.

Not only does the Mamazone course provide a most beneficial learning experience for women, it also serves as an excellent communication platform for doctors and their patients. The two-way dialogue offers useful insights for everyone - patients, their specialists and their general practitioners. Often, this brings a new dimension to the latest breast cancer treatment. Expert patients understand their disease and are uniquely able to articulate what it feels like to live with it. They can also improve their doctor's knowledge. 


\section{Box 10-3 European Cancer Patient Coalition}

ECPC now has over 250 member organizations in 37 countries, representing both the common (colon, lung, prostate and breast) and rarer cancers, for which the patient's voice is seldom heard. ECPC maintains links with a number of EU institutions: the Commission, the Parliament, the Council and and European Medicines Agency. ECPC is campaigning to make the fight against cancer an EU priority once again, filling seats on important EU committees, running the MAC secretariat and organizing cancer patient summits (Slovenia November 2007) and masterclasses in advocacy across Europe.

During the last six years, the reputation of this seminar has grown, as has the number of participants. Organized by patients for patients, the course has become a model of best practice. Its professional value is now acknowledged and recognized because participating doctors receive official continuing education credits for taking part.

\section{Colon cancer - how ECPC is helping improve survival}

Colon cancer ${ }^{7}$ is the second biggest cancer killer in most European countries. Survival in Europe is much lower than in the United States, yet this cancer is largely preventable and curable (Gatta et al., 2003).

The EUROCARE research in the late 1990s showed that most countries in Europe could do better, especially the United Kingdom and CEE countries. Since its inception, ECPC has been heavily involved in helping to prevent the unnecessary toll of deaths from this common cancer across Europe.

ECPC President Lynn Faulds Wood was diagnosed with advanced colon cancer in 1991. She had never previously heard of the disease, she was misdiagnosed for nearly a year and attributes her survival to luck. She is leading ECPC's efforts to combat colon cancer. For example, ECPC is a partner in the European Commission's project to develop Colorectal Cancer Quality Screening Guidelines. Lynn Faulds Wood has been helping to set up screening programmes in countries where they do not exist - or do not function adequately - such as Slovenia and Cyprus. She has also been helping to encourage prevention and symptom awareness campaigns to fill the vacuum between the announcement of screening programmes and their introduction.

When I was diagnosed, I discovered that - worldwide - there was no evidence-based guidance on the symptoms of this common cancer. We have

${ }^{7}$ Colon cancer is the term most widely known to the public. Bowel (or colorectal) cancer includes both cancer of the colon and cancer of the rectum, the last part of the bowel. In this chapter, the term colon cancer covers all these cancers. 
helped to develop a major symptoms database in the UK. From that, we have produced new advice on symptoms, different from what doctors learn in medical school, and that is what we are helping to spread across Europe.

This new advice has been translated into several languages and work is proceeding on "people-friendly" language in the different countries to describe the symptoms. ECPC is sharing materials freely rather than re-inventing the wheel.

ECPC is now setting up a pan-European colon cancer patient network, to disseminate the official screening guidance as soon as the European Commission development project is completed.

ECPC is also involved in raising awareness of the need for more effective colorectal cancer surgery across Europe. We are looking into why some surgeons have vastly better results than the average, and encouraging improved training.

\section{Conclusions}

Good health is one of our most precious possessions. It is the ECPC's dearest wish that our society and our governments - both national and European - do everything possible to protect their citizens' health and encourage them to become actively engaged in preserving it. Good health for the more fortunate among us may be a gift, an accident of our genetic heritage. But for others it can become a struggle which cannot be tackled by the individual alone. It requires that all the energies and resources of our societies are mobilized.

Health must also be integrated into all government policies, not just left solely in the hands of the Ministry of Health. WHO has defined health as a total state of physical, mental and social well-being - a laudable ambition. More than ever, health is a highly political issue, spanning not only medicine and science, but also the strength of a country's economic and social position. Health is wealth, measured in both monetary and humanitarian terms.

The ECPC and cancer patients across Europe have our hopes pinned on Slovenia's Presidency of the EU (January to July 2008). Slovenia must build the political will required to force through the level of change and investment in health needed to close the gaps in cancer control, both within European countries and between the countries of east and west Europe.

Slovenia will have the difficult task of rallying EU health ministers' support to step up measures for cancer control; to argue for a more coordinated application of present knowledge on tobacco control and, more generally, on 
cancer prevention, early diagnosis, treatment and care. We, the cancer patients, stand ready to give our full support and we are eager to work in partnership with governments and other important players to bring about change.

\section{Cancer patients' recommendations for improving cancer control in Europe}

Here are our recommendations for improving cancer control in the EU and its Member States, and for breaking down the political and territorial barriers that deter Europe from sharing best practice.

- Step up cancer control initiatives for all cancers, to include robust measures for prevention, screening, early diagnosis and an integrated approach to cancer management that provides equal access to best-quality treatment and care, including psychosocial and palliative care.

- In addition to acute care, governments and health-care systems must shift towards investing in the health of citizens by focusing on prevention, to stem the increasing cancer tide.

- Promote the European Code Against Cancer to every man, woman and child.

- Institute tough tobacco control measures.

- Set up population-based screening programmes according to agreed EU quality guidelines.

- Encourage further research into all those cancers that are currently untreatable.

- Ensure that data protection legislation does not hamper the collection of data by cancer registries, or restrict unnecessarily the research that is done with their data, or prevent the development of electronic record systems (ehealth).

- Based on the evidence provided by cancer registries, produce a new European cancer strategy to improve primary prevention, screening, early diagnosis and best-quality treatment and care.

- Modern technology tools must be harnessed to make health-care systems safer, more efficient and effective, and to improve their quality.

- Health policy-makers, health systems and health professionals must become more responsive to patients' needs by working in true partnership with them. 
- Patients must be empowered by having access to the vital information, in their own language, which enables them to make informed decisions about their care. This should include an EU registry for interconnected information on clinical trials to encourage participation of more cancer patients in such trials.

- Taking the European Medicines Agency as a model, institute an official interaction between the national medicines regulator and national patient groups.

- Include the voice of cancer patients in everything that affects their health and care.

- Set up a European cancer task force to provide fresh impetus and a European framework for tackling inequalities and sharing best practice, such as European cancer treatment guidelines and centres of excellence for rare cancers across the EU.

\section{REFERENCES}

Boyle P et al. (2003). European Code Against Cancer and scientific justification: third version (2003). Ann Oncol, 14(7):973-1005.

Boyle P, Ferlay J (2005). Cancer incidence and mortality in Europe, 2004. Ann Oncol, 16(3):481-488.

Coleman MP et al. (2003). EUROCARE-3 summary: cancer survival in Europe at the end of the 20th century. Ann Oncol, 14(Suppl. 5):128-149.

EMEA web site. London (available at:http://www.emea.europa.eu/, accessed 2 December 2007).

Fayter D et al. (2006). Systematic review of barriers, modifiers and benefits involved in participation in cancer clinical trials (CRD Report 31). York, UK, Centre for Reviews and Dissemination, University of York.

Ferlay J et al. (2007). Estimates of the cancer incidence and mortality in Europe in 2006. Ann Oncol, 18(3):581-592.

Gatta G et al. (2003). Differences in colorectal cancer survival between European and US populations: the importance of sub-site and morphology. Eur J Cancer, 39:2214-2222.

MEPs Against Cancer statement. Brussels (available at: http://www.mepsagainstcancer.org/ index.php?option=com_content\&task=view\&id=18\&Itemid=32, accessed 15 December 2007).

MEPs Against Cancer web site. Brussels (available at: http://www.mepsagainstcancer.org, accessed 2 December 2007).

United against Cancer Summit (2006). Cancer is everybody's business. Ljubljana, Slovenia. 



\section{Chapter 11 \\ The role of psychosocial oncology in cancer care}

Luigi Grassi and Luzia Travado

\section{Introduction}

Cancer is one of the most common diseases worldwide and the second leading cause of death. According to the World Health Organization's International Agency for Research on Cancer (IARC), there were 3.2 million new cases and 1.7 million deaths from cancer in Europe in 2006. The EU25 countries accounted for nearly 2.3 million new cases and over 1 million cancer deaths.

The incidence of cancer increased between 1950 and 2005, partly as a result of population growth and ageing. Survival from cancer is also improving, with several million people being cured or living with cancer for many years in Europe (Coleman et al., 2003).

In this context, evaluating and understanding the psychosocial aspects of cancer and its treatment has become a mandatory part of cancer care. Although the psychosocial dimensions of cancer have been explored in the literature since the 1950s, it is only over the past 25 years that this area has developed into a specific discipline and subspecialty, known as psychooncology (Holland, 2002).

Psycho-oncology addresses a range of psychosocial, behavioural, spiritual and existential dimensions along the entire journey travelled by the cancer patient and his or her family. Its goal is that all cancer patients and their families receive optimal psychosocial care at all stages of disease and survivorship (Holland, 2003).

In this chapter, we will discuss the main psychosocial and social concomitants of cancer, their consequences for cancer patients and their families, and the development and application of guidelines for screening and evaluation of the psychosocial needs of cancer patients. We will also describe the contribution 
that psychosocial interventions can make to the process of education and training of cancer physicians, and the evidence-based psychosocial treatment of cancer patients that can be pursued by means of a multidisciplinary approach.

\section{Psychosocial issues in cancer}

\section{The psychosocial response to cancer}

The diagnosis and treatment of cancer involves a series of dramatic changes that affect both the patient and his or her family, as well as their social milieu. The impact of cancer has four main dimensions: physical, emotional, spiritual and interpersonal.

At the physical level, cancer and its treatment have significant effects on the body and its functions, with important repercussions for the individual's body image. The anatomic site of cancer may influence the patient's emotional response to the disease: patients may cope differently with "visible cancers", like breast cancer or head and neck cancers, than with "less visible" cancers, such as leukaemia or lung cancer.

The type of cancer therapy, including surgery, chemotherapy, radiotherapy and hormone therapy, may also affect the patient differently at the physical level, involving possible physical mutilation (e.g. amputations, stomas, hair loss) and symptoms (e.g. pain, nausea and vomiting, fatigue). Lastly, the stage of cancer at diagnosis and its biological characteristics also play a role in this process, with different implications in terms of physical integrity and body image arising from earlier cancers than from more advanced ones. Overall, cancer alters the performance status and functional activity of patients. It can lead to problems in carrying out their daily activities and have other effects such as poor concentration, memory impairment and altered sexuality.

From the psychological point of view, the loss of certainties, the instability of one's own emotional status (e.g. fears, anxiety, worries, sadness), the need to depend on others, the reduction of self-esteem, the change of perspective in the future, and the threat of possible death, are some examples of the numerous experiences that cancer patients have to deal with during the trajectory of their illness. Psychological disorders (e.g. phobia, depression, sleep disorders) may also emerge (see next section) with further suffering for the patient and his/her family.

The impact of cancer at the spiritual level should also be taken into account. Religious affiliation and faith, the whole set of personal values, the meaning given to one's own life and existence, the change in the perception of time and 
being, are important concomitants of cancer and other life-threatening diseases in general.

The social and interpersonal levels are also altered by cancer and its treatment. The sense of belonging ("to be with") and communicating ("to put in common with someone") in the patient's family, the microcosm of their close relationships and the macrocosm of society as a whole, are also threatened or impaired by cancer. Feelings of loneliness or abandonment, problems in returning to work, marginalization or even stigmatization are common issues reported by cancer patients.

These factors may fluctuate throughout the various phases of cancer management (diagnosis, treatment, discharge from hospital, follow-up, survivorship, palliative care) and the biological course of cancer (remission, recurrence, progression, death). In turn, these factors may affect the psychosocial implications and dimensions of cancer. For these reasons, the capacity of patients to cope with the multiple losses caused by cancer should be constantly monitored in clinical practice.

In the early 1960s, Kübler-Ross indicated that psychological adjustment of patients with advanced cancer may be reached by passing through a series of phases: namely shock, denial, anger, bargaining, depression and acceptance (Kübler-Ross, 1969). Subsequent research has demonstrated that this model remains useful in understanding the psychological response of cancer patients at every stage of their illness.

These phases do not occur in a rigid sequence. They may overlap or shift with the patient's emotional condition, which may also vary. More specific coping styles may also emerge, depending on the patient's personality, previous experience and social support. Thus, cancer patients may adopt several styles to respond emotionally, cognitively and behaviourally to the disease. Some of these styles, such as a fighting spirit (i.e. the tendency to confront and actively face the illness), seem to favour the adjustment to illness. Others may indicate a poorer adjustment to illness and a higher risk of psychological morbidity. These less favourable strategies include hopelessness (tendency to adopt a pessimistic attitude to the illness) and anxious preoccupation (the tendency to perceive the impact of illness in one's own life at all times).

\section{Psychosocial disorders and psychosocial morbidity}

The psychological adjustment to cancer is therefore a complex process, in which "normal" psychological responses such as sadness or preoccupation can be transformed into clinically significant states, with symptoms of distress or more definite psychopathological conditions. 
Table 11-1 Psychological morbidity and risk factors for psychosocial morbidity in cancer patients

\section{Psychological morbidity}

- Mood disorders: adjustment disorders with depressed mood, major depression.

- Anxiety disorders: adjustment disorders with anxious mood, post-traumatic stress syndrome, phobia.

- Somatoform disorders: hypochondriasis.

- Sexual disorders: loss of libido, inability to achieve orgasm.

\section{Risk factors for psychosocial morbidity}

Medical factors

- Stage of disease.

- Type of treatment: surgery, chemotherapy, radiotherapy, hormone-therapy, immunotherapy.

- Physical symptoms (e.g. loss of mobility, pain, fatigue, nausea and vomiting, hot flushes).

\section{Individual factors}

- Psychological and psychiatric issues (e.g. family or personal history of psychiatric disorders).

- Personality (e.g. pessimism; maladaptive coping styles such as hopelessness, anxious preoccupation, emotional repression, external locus of control).

\section{Interpersonal and social factors}

- Poor social support from interpersonal ties: close social support (e.g. family, spouse, close friends) and wider social support (e.g. church, association, work, neighbours).

- Stressful events.

It has been reported that in a number of patients, the "crisis of cancer" is accompanied by a sense of personal growth and a radical change in one's own perception of life, with new and more positive meanings given to life. The impact on many cancer patients is different, however, with dramatic consequences for the patient's psychosocial equilibrium and quality of life (van't Spijker et al., 1997).

Psycho-oncology studies have shown that $30-40 \%$ of cancer patients are also diagnosed with psychopathological disorders, according to the International Classification of Disease $\left(10^{\text {th }}\right.$ ed.) or the Diagnostic and Statistical Manual of Mental Disorders ( $4^{\text {th }}$ ed.). Among these disorders, the most common are mood, anxiety, somatoform and sexual disorders (Grassi et al., 2005a). The main types of psychosocial disorder and the risk factors for developing them are listed in Table 11-1.

The Southern European Psycho-Oncology Study (SEPOS) undertook a multicentre study in Italy, Portugal and Spain. This showed that about 17\% of cancer patients had developed some clinically relevant anxiety disorders and 
9-14\% had depressive disorders, irrespective of the type of cancer diagnosis and its treatment (Grassi et al., 2004).

Current psychiatric disease classifications and diagnostic criteria cannot be readily transferred to oncology. Important psychosocial conditions that are not correctly evaluated by these tools may affect a further $25-30 \%$ of cancer patients. Health anxiety, irritable mood, demoralization, interpersonal problems or abnormal illness behaviour are some of the disorders that should be routinely taken into consideration when assessing the status of cancer patients because they may severely affect patients' quality of life, as well as the doctor-patient relationship (Grassi et al., 2007).

Emotional distress can also be triggered by other events during the course of the disease, such as the perception of a new suspicious symptom; hearing the cancer diagnosis from a health-care professional; awaiting treatment, a change in treatment or the end of treatment; discharge from hospital; failure of treatment, recurrence or progression of disease; and the approaching end of life.

\section{Consequences of psychosocial disorders}

Distress symptoms and psychosocial morbidity secondary to cancer have significant consequences. The difficulty in adjusting to cancer and the psychopathological conditions associated with it, especially depression, may cause (Grassi et al., 2005a):

- maladaptive coping and abnormal illness behaviour, with negative effects on compliance with treatment, health behaviour and quality of life;

- increased length of stay in hospital or impaired rehabilitation;

- higher perception of pain and reduced ability to respond to physical symptoms;

- less effective response to primary chemotherapy;

- increased risk of suicide;

- higher risk of recurrence and decreased survival from cancer.

Psychosocial problems also reverberate within the family, increasing the emotional distress among the patient's caregivers and, after his/her death, a greater risk of complicated or traumatic grief among relatives (Grassi et al., 2007).

Despite the major implications of psychosocial morbidity for clinical care, psychosocial issues in cancer are still all too often dismissed or underestimated. 
Cancer care professionals tend to confuse clinical depression or anxiety disorders with sadness and preoccupation, based on the incorrect belief that "it is normal for the patient to feel sadness because of his/her cancer". Thus, 30$40 \%$ of all cancer patients suffering from psychological problems are not recognized as such by their doctors (Grassi et al., 2005a). As a result, they are not referred to psycho-oncology services for a more specific assessment and proper treatment (Grassi et al., 2000). Nevertheless, unless there is a major step-change in the cultural attitude towards psychiatry and clinical psychology applied to medicine, especially in oncology, cancer patients with maladjustment disorders will continue to be denied assistance and treatment for their psychological conditions.

Some signs of improvement are apparent. These include the development and dissemination of guidelines for the management of psychological distress and training courses to improve doctors' communication skills, and thus to help them recognize and treat psychosocial problems and psychiatric disorders in cancer patients.

\section{The evaluation and management of psychosocial dimensions in cancer}

\section{Assessment of psychosocial dimensions}

Cancer patients need their preoccupations and personal values to be listened to and taken into consideration by their doctors. They need to be informed honestly about their disease and supported in a humanistic way. Assessment of the emotional problems of patients and their families should become part of every clinical intervention by oncologists and cancer care professionals, and in that sense a mandatory component of clinical care.

Several instruments have been proposed as clinical tools for routine use in cancer settings, to improve the detection and referral for treatment of maladjustment and psychosocial morbidity. These include psychometric scales and inventories such as the Hospital Anxiety and Depression Scale (HADS), Brief Symptom Inventory (BSI), Profile of Mood States (POMS), Mental Adjustment to Cancer Scale (MAC) and multidimensional quality of life instruments. Most of these have been validated, are available in many languages and have been shown to have good sensitivity (low number of false negative cases) and specificity (low number of false positive cases).

However, physicians involved in cancer care have indicated that lack of selfconfidence in exploring the psychosocial dimensions of cancer and in using psychometric instruments, as well as lack of time, are important reasons for 
not detecting and referring patients for psychological care. The presence of psycho-oncologists in cancer settings and the implementation of psychooncology services could create a real network, promote multidisciplinary work and facilitate education in the use of these instruments.

Standards and clinical practice guidelines for measuring distress in cancer patients

Instruments for diagnosing psychosocial conditions in cancer patients are not yet used routinely in all cancer settings. One approach would be to encourage nurses and doctors to become familiar with simpler instruments that can at least enable them to assess the level of distress of their cancer patients.

In 1997, the National Comprehensive Cancer Network (NCCN) (www.nccn.org) developed the first set of clinical practice standards and guidelines for the management of psychosocial distress. Updates have been made almost every year (NCCN, 2007). A panel of 23 professionals in psychiatry, oncology, psychology, social work, nursing and the clergy worked with a patient representative to create an instrument for rapid, routine assessment of psychosocial morbidity. The panel also developed practical guidelines for psychosocial care, to be used by clinicians (NCCN, 1999; Holland, 2000; Holland et al., 2007; NCCN, 2007).

According to the NCCN, distress has been very broadly defined as:

... a multifactorial, unpleasant, emotional experience of a psychological (cognitive, behavioural, emotional), social andlor spiritual nature that may interfere with the patient's ability to cope effectively with cancer, its physical symptoms and its treatment. Distress extends along a continuum, ranging from common normal feelings of vulnerability, sadness and fears to problems that can become disabling, such as depression, anxiety, panic, social isolation and existential and spiritual crisis.

The NCCN panel developed a screening instrument for distress - the distress thermometer, which allows the patient to indicate his/her level of distress on a scale from 0 to 10 . An accompanying 'problem list' helps the patient to identify the causes of distress (psychological, social, physical, spiritual) (Fig. 11-1). This concept parallels the approach used to inquire about the patient's level of pain. A score of 5 or more on the distress scale should alert the nurse or oncologist to refer the patient for mental health, social work or pastoral counselling, depending on the problem. The distress thermometer has been shown to be a valid tool when compared with other psychometric instruments, such as the Hospital Anxiety and Depression Scale and the Brief Symptom Inventory. 


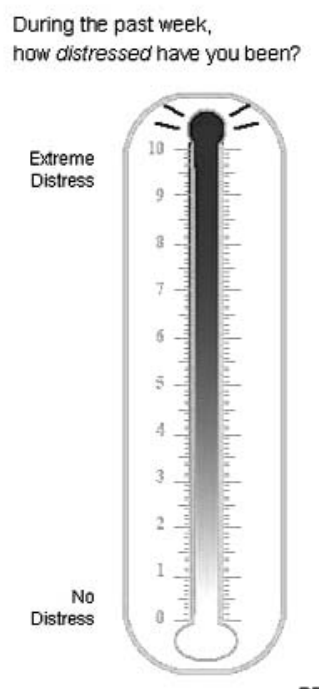

Please indicate your level of distress on the thermometer and check the causes of your distress.

how distressed have you been?

Practical problems

_ Housing

- Insurance

Work/school

- Transportation

_ Child care

Family problems

_ Partner

- Children

Emotional problems

_ Worry

— Sadness

— Depression

_ Nervousness

Spiritual/religious concerns

Relating to God

_ Loss of faith

— Other problems
Physical problems

_Pain

_ Nausea

Fatigue

_ Sleep

_ Getting around

_ Bathing/dressing

_ Breathing

— Mouth sores

_Eating

_ Indigestion

_ Constipation/diarrhea

_Bowel changes

- Changes in urination

_ Fevers

_ Skin drynitchy

- Nose dryicongested

_ Tingling in hands/teet

_Feeling swollen

_ Sexual problems

BREIF SCREENING TOOL AND PROBLEM LIST

Reproduced with permission from The NCCN 1.2007 Distress Management Clinical Practice Guidelines in Oncology. (CNational Comprehensive Cancer Network, 2007 (available at: http://www.nccn.org, accessed 30 August 2007). View the most recent and complete version of the guideline online at: www.nccn.org.

Fig. 11-1 Distress thermometer proposed by the NCCN Panel on Distress Management in Oncology

Clinical practice guidelines for the management of psychosocial distress are currently under development in Australia, Germany, the United Kingdom, Hungary, Italy, Israel, Spain, the United States and Canada. In these countries, it has been suggested that distress should be added as the sixth vital sign (after pain) in order to raise the level of attention to this need during clinic visits (Holland \& Bultz, 2007).

Simple instruments like the distress thermometer can be used repeatedly at each visit of the patient, or whenever considered necessary, thus providing a regular assessment of psychosocial problems as well as indicating possible approaches to manage them. In the SEPOS study, the distress and the mood thermometers (a similar 0 to 10 scale examining the level of mood) have been shown to be valid against the HADS in identifying cancer patients who need psychosocial intervention to help them adjust to their illness (Gil et al., 2005).

It is taking a long time for clinical practice guidelines to be disseminated and to produce a significant change in practice patterns, but they do serve as benchmarks against which the quality of psychosocial care can be measured. The National Psychosocial Oncology Standards for Canada have been used since 1999 to guide the provincial and federal governments in planning and budgeting for psychosocial care in cancer (Canadian Association of 
Psychosocial Oncology, 1999; www.capo.ca). More comprehensive clinical guidelines, such as the Clinical Practice Guidelines for the Psychosocial Care of Adults with Cancer developed by the Australian National Breast Cancer Centre and National Cancer Control Initiative (http://www.nhmrc.gov.au/ publications/synopses/cp90syn.htm) are also available.

\section{Intervention}

Both the diagnosis of cancer and cancer treatment can cause stress, emotional turmoil and psychosocial disorders. It is essential to consider both how to manage these conditions clinically and, more broadly, how to organize cancer services to provide optimal and comprehensive care to cancer patients and their families.

Two levels of intervention can be considered. The first concerns the need for cancer care professionals to be trained in biopsychosocial approaches. The second concerns the specific role of psychosocial interventions carried out by either clinical psychologists or psychiatrists trained in psycho-oncology.

\section{Training health-care professionals to manage the psychosocial aspects of cancer}

Cancer care professionals' awareness about the various psychosocial aspects of oncology can be raised by dissemination of information. Almost all oncology textbooks now include a section covering psycho-oncology. Cancer websites also detail the psychosocial dimensions of cancer.

The literature on this topic has increased exponentially over the last 20 years. The process has been facilitated by the cancer patient advocacy movement, which has emphasized that good clinical care cannot be based solely on a technical approach, and that a more comprehensive approach is needed, taking both clinical and psychological needs into account.

An interesting initiative was launched recently by The International PsychoOncology Society (IPOS), in cooperation with the European School of Oncology. They developed the first on-line core curriculum on the psychosocial aspects of cancer care, available in eight languages - English, French, German, Hungarian, Italian, Japanese, Portuguese and Spanish (International Psycho-Oncology Society, 2006). A series of lectures on the main topics in psychosocial oncology is already available on the following topics: communication and interpersonal skills in cancer care; anxiety and adjustment disorders in cancer patients; distress management in cancer; depression and depressive disorders in cancer patients; and psychosocial 
assessment in cancer patients. Further lectures will be released by 2008: family issues in cancer care; palliative care; grief and bereavement; psychological intervention; psychopharmacology intervention; ethical issues in psychooncology; and multidisciplinary approaches in cancer care. The web site broadcast (web cast) is rapidly becoming part of the teaching curricula in medical schools, residency programmes and courses in psychosocial oncology in some European countries. These lectures represent a good way to sensitize health-care professionals to the psychosocial aspects of cancer care and to prompt them to contact their national societies for information on specific psycho-oncology courses.

A further aspect of psycho-oncology concerns hands-on training courses in communication. Research has shown that communication skills can be taught and that training oncologists through peer-led workshops has a strong positive impact on their clinical care.

Well-conducted trials in communication skills training have shown that doctors who acquired new competences in communicating with their patients have also had an increased awareness and appreciation of psychosocial issues in cancer, and an improved relationship with their patients. There is also evidence from a European study that cancer patients need to perceive that their doctors are interested in their concerns and are emotionally present (Bredart et al., 2003). This view is reinforced by the fact that improved communication skills are associated with positive outcomes in the cancer patient, such as compliance with medical treatment, symptom resolution, pain control, adjustment to illness and patient satisfaction (Fallowfield \& Jenkins, 2004; Maguire, 1999).

The characteristics and aims of training courses are critically important. It has been shown that if the main purpose of communication skills training is to raise awareness about a specific disorder, such as depression, this can facilitate early detection and referral to psycho-oncology services and may help in preventing psychological complications (Maguire, 2002). On the other hand, if the purpose of the training is more general, this is not necessarily followed by better recognition of cancer patients' emotional problems (Merckaert et al., 2005).

It has also been shown that training in communication can reduce cancer physicians' own psychosocial morbidity and burn-out (Armstrong \& Holland, 2004). Some new models have been developed by examining doctors' own difficulties in relating to cancer patients (Travado et al., 2005), thus facilitating the doctors' evaluation of the most common psychological problems of their patients (Grassi et al., 2005b). 
In conclusion, communication skills and training in the psychosocial consequences of cancer should become a mandatory part of the curricula for all cancer professionals.

\section{Evidence-based psychosocial interventions}

The liaison between cancer care and psychosocial-care professionals is an important component of multidisciplinary treatment in oncology. A wide range of psychosocial approaches and treatments have been developed to provide cancer patients with a specific intervention for conditions in which psychological disorders and maladjustment to cancer and its treatment might emerge.

Thirty years ago, psychotherapy was not considered to be as scientific as other medical interventions but new data have provided evidence for the positive impact of psychological and psychosocial approaches in cancer care (Fawzy et al., 1995). Psychosocial interventions such as counselling, education, coping and psychological support, as well as psychotherapy sensu stricto (e.g. individual, group and family psychotherapy) have been shown to be effective in several studies (Fawzy, 1999).

The choice of psychosocial intervention depends on the psychological condition (e.g. phobia, major depression, adjustment disorder); type and phase of illness (e.g. diagnosis, initial treatment, follow-up, recurrence and retreatment, palliative care); context (e.g. outpatient clinic, hospital, hospice, home); and, obviously, the availability of psycho-oncology services with trained professionals. Sociodemographic variables (e.g. age, gender, social support) should also be considered.

Another important consideration is that patients with more intense emotional symptoms seek psychosocial support more often than those who have sufficient social support in their personal lives (Plass \& Koch, 2001). Furthermore, specific psychosocial interventions are more efficient for patients who develop psychosocial morbidity. Thus, the use of specialized psychosocial interventions in cancer patients who show only minor symptoms of maladaptation is not useful, but it may be important for them to benefit from self-help groups or supportive and educational interventions by social services. This underscores the usefulness of suitable screening and evaluation guidelines as a way to guide patients who need help to the appropriate psycho-oncology services.

Specialized psychotherapeutic interventions in oncology such as group, individual and family therapy can provide benefit in reducing symptoms; improving the quality of life, well-being and return to work; and better illness behaviour (Fawzy \& Fawzy, 1998). 
Psychopharmacological interventions can be effective in treating some psychological disorders, but they should only be recommended by clinicians (usually psychiatrists) trained in the use of these drugs, their side-effects and their interaction with other cancer treatments. More research is needed in this area (Andrykowski \& Manne, 2006; Lepore \& Coyne, 2006; Newell, SansonFisher \& Savolainen, 2002).

\section{The need for an integrated multidisciplinary patient- centred approach to cancer care}

\section{Quality of life assessments as endpoints in cancer care}

There is evidence that psychosocial interventions reduce psychological morbidity and improve patients' adjustment to illness, quality of life and wellbeing, thus making such interventions an important component of cancer treatment. The quality of life of cancer patients is as important as the duration of their survival, and this should be an essential consideration in cancer care. However, it is only recently that this concept has gained more attention (Rowland, 1997; Gunnars, Nygren \& Glimelius, 2001; Jacobsen, Davis \& Cella , 2002), since better cancer treatment has resulted in longer survival or even cure, thus allowing more focus on quality of life issues.

The side-effects of cancer treatment have traditionally been seen as a way of monitoring the impact and efficacy of cancer therapy. This over-simplified concept of the quality of life - disease-focused, based on physical symptoms and functional ability - has broadened to include the assessment of other important dimensions such as psychological well-being, social functioning and spirituality (Aaronson et al., 1993; Cella et al., 1993; Power \& Kuyken, 1998; Stewart, Hays \& Ware, 1988).

Several reliable cancer-specific instruments are now available to assess multidimensional quality of life, e.g. EORTC QLQ-C30 and FACT (Sloan et al., 2006) in monitoring the progress of cancer care.

The patient's own perceptions of their quality of life are important, because medicine is shifting from a disease-focused model to a more patient-centred approach. Patients are now better informed about their illnesses and their treatment options, and more often want to become partners in the clinical decision-making process, striving to balance the various demands of the therapeutic options proposed by the clinician with their personal preferences in choosing a specific treatment. There is a shift towards a more patientcentred model of clinical practice and demands from patients and consumer advocacy organizations to address the psychosocial needs of cancer patients as 
an integral part of their care. These have converged to make the assessment of cancer patients' quality of life a key component of oncological practice and research (Sloan et al., 2006; Travado, 2006).

For cancer patients, the incorporation of quality-of-life assessments as an endpoint in their care can help address their perceptions of well-being and bring their own perspective into play, and may assist in focusing treatment and health care appropriately on their personal sense of well-being. Assessment of the patient's quality of life may also serve to foster patients' awareness of their own quality of life and promote more informed participation in their care. For professionals, the use of such instruments provides data on outcomes which can be used in making treatment decisions. It also increases their awareness of patients' well-being and facilitates the discussion of quality-of-life issues (Detmar et al., 2002).

Quality-of-life tools may also be relevant to screening for distress, providing clinicians with a means for systematic recognition of psychosocial morbidity associated with cancer and its treatment, and the opportunity to refer patients for evaluation or support, thereby helping to improve their patients' quality of life.

Addressing these often neglected aspects of patients' functioning may have positive implications for their overall well-being and satisfaction with the health-care system (Travado, 2006). Mental-health interventions have been shown to reduce health-care utilization, so routinely addressing the quality of life of cancer patients may reduce health-care costs. Lastly, patients' quality of life can also be considered a useful indicator of the quality of performance in cancer care (Moher et al., 2004).

Quality-of-life assessments still need to be included in routine clinical practice, research and clinical trials (Davis \& Cella, 2002; Sloan et al., 2006). The FDA and EMEA have recognized quality of life as an important endpoint in clinical research and have helped promote its value, but assessment of the quality of life is still not mandatory (Kiebert et al., 2000). The same situation is seen in clinical practice, where the assessment of patients' quality of life is not a routine requirement.

To conclude, regular assessment of cancer patients' quality of life improves both understanding of their needs and monitoring of the quality of care they receive. Improvement in the organization of cancer care should include routine assessment of cancer patients' quality of life in order to meet patients' needs and to prevent the development of more complex problems. 
The biopsychosocial model and the patient-centred care approach

It is now widely acknowledged that comprehensive cancer care requires a significant shift from the traditional biomedical, paternalistic perspective to a more patient-centred, biopsychosocial approach.

Traditionally, the focus in treating cancer has been mostly biological, while patients were usually expected to comply with prescriptions and to play a quite passive role. But cancer patients need to be considered as whole persons. Their psychological and social dimensions, and their participation in decisionmaking, should be given as much prominence as the biological aspects of their illness in the process of cancer care (Reis, 1998).

The European recommendations for best practice refer to the need for a multidisciplinary team approach in order to attain the best survival rates and quality of life for cancer patients. Organizations such as the European Society of Mastology (EUSOMA), in collaboration with the European Organisation for Research and Treatment of Cancer - Breast Cancer Cooperative Group (EORTC-BCCG) and the European Breast Cancer Coalition (Europa Donna), have worked together to summarize the basic requirements for a specialist breast unit (Blamey \& Cataliotti, 2006a; Blamey \& Cataliotti, 2006b). These requirements emphasize the need for the core team to include both specialists (e.g. in surgery, radiology and oncology) and also patientsupport staff who specialize in psychological care, including breast-care nurses and psychologists.

This initiative is a good example of how attitudes to the provision of optimal care are changing. Although psycho-oncology services are already available in many cancer centres across Europe (http://www.psycho-oncology.net/ index.html), there is still much work to be done to integrate psycho-oncology into fully standard cancer care. In some countries where comprehensive and high-quality care for cancer patients has become a priority, the provision of psychosocial care has been considered vital, and regulatory procedures and recommendations have been proposed. For example, the National Institute for Health and Clinical Excellence (www.nice.org.uk) offers clinical guidance for improving supportive and palliative care and services for cancer patients (http://guidance.nice.org.uk/csgsp and Table 11-2).

Despite psychosocial care being widely recognized as an important part of cancer care, it is still far from being offered as a routine standard of care, from the onset of disease and in all phases of diagnosis, treatment and survivorship. Referral to supportive care or patient-support groups in Europe has been reported as inconsistent, and the availability of these services is scarce or even completely absent in some countries (Reuben, 2004). Where available, they 
Table 11-2 Summary of key recommendations of NICE clinical guidance on supportive and palliative care

Key recommendations:

- People affected by cancer should be involved in developing cancer services.

- There should be good communication, and people affected by cancer should be involved in decision-making.

- Information should be available free of charge.

- People affected by cancer should be offered a range of physical, emotional, spiritual and social support.

- There should be services to help people living with the after-effects of cancer manage these for themselves.

- People with advanced cancer should have access to a range of services to improve their quality of life.

- There should be support for people dying from cancer.

- The needs of family and other carers of people with cancer should be met.

- There should be a trained workforce to provide services.

Source: National Institute for Health and Clinical Excellence (NICE), 2004. Supportive and palliative care services for adults with cancer (Understanding NICE guidance - information for adults with cancer, their families and carers, and the public). London: NICE. Available from www.nice.org.uk/guidance. Reproduced with permission.

are in many cases provided through cancer charities or advocacy groups. Thus, an important part of cancer care is either completely neglected or assigned to third parties. Community resources, although very important, are only complementary in cancer care: they cannot replace psycho-oncology services and the efficiency and high standard of an integrated multidisciplinary (biopsychosocial) and patient-centred care approach.

In 2001, patient-centred care was defined as "respectful of and responsive to individual patients' preferences, needs and values, and ensures that patient values guide all clinical decisions" (Radwin, 2003). Therefore, the focus of optimal care should not be just the treatment of the disease but also understanding the perceptions and expectations of the cancer patient. Such an approach has priority over the provider's agenda (signs and symptoms), and includes patient participation, shared decision-making, patient choice and support tailored to the patient's needs. Patients themselves strongly recommended this model of cancer care (Little et al., 2001) (Table 11-3) which should include good communication, partnership and discussion of health enhancement and risk reduction.

Patient-centred care is associated with higher patient satisfaction and compliance with care (Mallinger, Griggs \& Shields, 2005), and with better health outcomes (Stewart, 2001). However, the provision of such care is far from complete and of very variable quality (Stewart, 2001). Doctors remain ambivalent about the psychosocial aspects of patient care (Travado et al., 2005) and current arrangements are far from optimal. 
Table 11-3 Main domains of the patient-centred model

- Exploring the experience of disease and illness: patients' ideas about the problem, feelings, expectations for the visit, and effects on function.

- Understanding the whole person: personal and developmental issues (for example, feeling emotionally understood) and the context (the family and how life has been affected).

- Finding common ground (partnership): problems, priorities, goals of treatment, and roles of doctor and patient.

- Health promotion: health enhancement, risk reduction, early detection of disease.

- Enhancing the doctor-patient relationship: sharing power, the caring and healing relationship.

Source: Little et al., 2001. Reproduced with permission of the BMJ Publishing Group Ltd.

The development of a curriculum in medical education covering communication skills and psychosocial areas would help to close this gap, as would the implementation of programmes for patient education (Parker et al., 2005; Stewart, 1995).

\section{Conclusions}

Cancer and its treatment have a tremendous psychological and social impact, alongside its physical impact. It is accompanied by a series of dramatic changes that involve the physical, emotional, spiritual, interpersonal and social dimensions of the person affected by cancer. At least $50 \%$ of cancer patients suffer from stress-related symptoms, including anxiety, irritable mood, and demoralization. Many of them develop more serious psychopathological conditions, such as major depression or post-traumatic stress disorder.

Psychosocial morbidity has significant clinical consequences, including poor compliance with treatment, reduced quality of life, higher perception of pain and other physical symptoms, higher risk of suicide, recurrence of cancer and decreased survival.

Psycho-oncology services provide interventions aimed at preventing or reducing the emotional impact of cancer and improving patients' skills to cope with the demands of treatment and the uncertainty of the disease. There is evidence that providing these services to patients and their families as part of standard regular care reduces the distress and psychosocial morbidity associated with cancer and improves quality of life during and after cancer treatment.

Each cancer patient's needs are unique. Patients have become better informed. They demand more information about their medical condition and a share in 
decision-making with their doctors. Thus, a multi-professional and patientcentred approach is the best way to provide cancer care to patients.

Quality of life is an important patient-centred measure and an indicator of the quality of care. It informs clinicians about treatment outcomes and patients' needs, enables better planning of care and addresses areas of need that might otherwise be missed. Cancer is a chronic disease for which survival is improving, so patients' quality of life and their well-being are of the utmost importance.

A biopsychosocial approach improves doctor-patient relationships and patient satisfaction; reduces physician burn-out, and facilitates the detection of psychosocial problems secondary to cancer. It promotes patient-centred care by focusing on patients' needs and preferences, which produces better results. Communication skills are a basic professional component of psychosocial care that can be taught and improved. Peer-led workshops for health-care professionals (particularly oncologists) seem to have a strong positive impact on clinical care.

Having better-adjusted patients will result in significant benefits for the individual, the family, the social context and, presumably, for the national health economy. In spite of the evidence of psychosocial care benefits, however, psycho-oncology services are not yet offered regularly as standard care to all European citizens suffering from cancer. As an important element of high-quality care, psycho-oncology services should be mandatory and included in all comprehensive cancer care programmes and national cancer plans in Europe.

The reduction of avoidable inequalities in life expectancy, health status and access to high-quality health services across the recently enlarged EU will contribute to achieving the goal of a more cohesive Europe.

Solidarity and equity requires all Europeans to have the same opportunities to access optimal cancer care. This will enable them to attain a better quality of life, well-being, satisfaction with their care, social adjustment and inclusion in wider society.

\section{Recommendations}

The following recommendations aim to inform cancer policies in Europe in achieving optimal cancer care for European citizens.

- Psychosocial care should be provided as a standard component of treatment for all cancer patients. It should be included in all comprehensive cancer care programmes and European national cancer plans, and made available in every cancer centre. 
- All cancer patients and their families should receive optimal psychosocial care at all stages of the disease and survivorship, as part of regular standard care.

- Assessment of the psychosocial concomitants of cancer should be mandatory in clinical care. Nurses and doctors should become familiar with simple instruments for screening for psychosocial morbidity and the psychosocial consequences of cancer.

- Specific psycho-oncology interventions (e.g. psychotherapy and psychopharmacology) should become part of the standard treatment offered to cancer patients and their families, when needed.

- Communication skills training for health professionals, in particular oncologists and cancer physicians, should be a mandatory part of all curricula for clinical practice.

- Quality-of-life assessments should be routinely included across cancer care settings, as a standardized way to estimate patients' needs, to inform treatment options and to monitor the quality of care.

- A biopsychosocial and patient-centred approach should be implemented in routine cancer clinical care in order to attain optimal results.

\section{REFERENCES}

Aaronson NK et al. (1993). The European Organization for Research and Treatment of Cancer QLQ-C30: a quality-of-life instrument for use in international clinical trials in oncology. J Natl Cancer Inst, 85(5):365-76.

American Psychiatric Association (2000). Diagnostic and statistical manual of mental disorders, $4^{\text {th }}$ edition - revised. Washington, American Psychiatric Press.

Andrykowski MA, Manne SL (2006). Are psychological interventions effective and accepted by cancer patients? I. Standards and levels of evidence. Ann Behav Med, 32(2):93-97.

Armstrong J, Holland J (2004). Surviving the stresses of clinical oncology by improving communication. Oncology (Williston Park), 18(3):363-368; discussion:373-375.

Blamey RW, Cataliotti L (2006a). EUSOMA accreditation of breast units. Eur J Cancer, 42(10):1331-1337.

Blamey RW, Cataliotti L (2006b). Requirements of a specialist breast unit: first revision. European guidelines for quality assuring in breast screening and diagnosis, $4^{\text {th }}$ ed. European Commission, Belgium, European Community:343-354.

Bredart A et al. (2003). Patients' satisfaction ratings and their desire for care improvement across oncology settings from France, Italy, Poland and Sweden. Psychooncology, 12:68-77.

Canadian Association of Psychosocial Oncology web site (available at: www.capo.ca, accessed 18 December 2007)

Cella DF et al. (1993). The functional assessment of cancer therapy scale: development and validation of the general measure. J Clin Oncol, 11(3):570-579. 
Coleman MP et al. (2003). EUROCARE-3 summary: cancer survival in Europe at the end of the 20th century. Ann Oncol 14(Suppl. 5):128-149.

Davis K, Cella D (2002). Assessing quality of life in oncology clinical practice: a review of barriers and critical success factors. J Clin Outcomes Manage, 9:327-332.

Detmar SB et al. (2002). Health-related quality-of-life assessments and patient-physician communication: a randomized controlled trial. J Am Med Assoc, 288(23):3027-3034.

Fallowfield L, Jenkins V (2004). Communicating sad, bad, and difficult news in medicine. Lancet, 363(9405):312-319.

Fawzy FI (1999). Psychosocial interventions for patients with cancer: what works and what doesn't. Eur J Cancer, 35(11):1559-1564.

Fawzy FI, Fawzy NW (1998). Group therapy in the cancer setting. J Psychosom Res, 45(3):191200.

Fawzy FI et al. (1995). Critical review of psychosocial interventions in cancer care. Arch Gen Psychiatry, 52(2):100-113.

Gil F et al. (2005). Use of distress and depression thermometers to measure psychosocial morbidity among southern European cancer patients. Support Care Cancer, 13: 600-606.

Grassi L et al. (2000). Psychosocial problems secondary to cancer: an Italian multicentre survey of consultation-liaison psychiatry in oncology. Italian Consultation-Liaison Group. Eur J Cancer, 36(5):579-585.

Grassi L et al. (2004). Psychosocial morbidity and its correlates in cancer patients of the Mediterranean area: findings from the Southern European Psycho-Oncology Study. J Affect Disord, 83(2-3):243-248.

Grassi L et al. (2005a). Psychiatric concomitants of cancer, screening procedures, and training of health-care professionals in oncology: the paradigms of psycho-oncology in the psychiatry field. In: Christodoulou GN, ed. Advances in psychiatry, Vol II. Athens, World Psychiatric Association:59-66.

Grassi L et al. (2005b). A communication intervention for training southern European oncologists to recognize psychosocial morbidity in cancer. I - development of the model and preliminary results on physicians' satisfaction. J Cancer Educ, 20(2):79-84.

Grassi L et al. (2007). Psychological factors affecting oncology conditions. In: Porcelli P, Sonino N, eds. Psychological factors affecting medical conditions. A proposal for a new classification for $D S M-V$. Basel, Karger Press:57-71.

Gunnars B, Nygren P, Glimelius B (2001). Assessment of quality of life during chemotherapy. Acta Oncol, 40(2-3):175-184.

Holland J. (2000). An algorithm for rapid assessment and referral of distressed patients. In: Perry CM ed. American Society of Clinical Oncology educational book. Alexandria, ASCO:129138.

Holland JC (2002). History of psycho-oncology: overcoming attitudinal and conceptual barriers. Psychosom Med, 64(2):206-221.

Holland JC (2003). American Cancer Society Award lecture. Psychological care of patients: psycho-oncology's contribution. J Clin Oncol, 21(Suppl. 23):253s-265s.

Holland JC, Bultz BD (2007). The NCCN guideline for distress management: a case for making distress the sixth vital sign. J Natl Compr Canc Netw, 5(1):3-7.

Holland JC et al. (2007). Distress management. J Natl Compr Canc Netw, 5:66-98.

International Psycho-Oncology Society (2006). Multilingual core curriculum in psychosocial aspects of cancer care. IPOS (available at: http://www.ipos-society.org/professionals/meetingsed/core-curriculum/core-curriculum-pres.htm., accessed 16 December 2007)

Jacobsen PB, Davis K, Cella D (2002). Assessing quality of life in research and clinical practice. Oncology (Williston Park), 16(Suppl.10):133-139. 
Kiebert G et al. (2000). Practice and policy of measuring quality of life and health economics in cancer clinical trials: a survey among co-operative trial groups. Qual Life Res, 9(10):10731080 .

Kubler Ross E. (1969). On death and dying. London, Tavistock.

Lepore SJ, Coyne JC (2006). Psychological interventions for distress in cancer patients: a review of reviews. Ann Behav Med, 32(2):85-92.

Little P et al. (2001). Preferences of patients for patient centred approach to consultation in primary care: observational study. $\mathrm{Br}$ Med J, 322(7284):468-472.

Maguire P (1999). Improving communication with cancer patients. Eur J Cancer, 35(10):14151422 .

Maguire P. (2002). Improving the recognition of concerns and affective disorders in cancer patients. Ann Oncol, 13(Suppl. 4):177-181.

Mallinger JB, Griggs JJ, Shields CG (2005). Patient-centered care and breast cancer survivors' satisfaction with information. Patient Educ Couns, 57(3):342-349.

Merckaert I et al. (2005). Factors that influence physicians' detection of distress in patients with cancer: can a communication skills training program improve physicians' detection? Cancer, $104(2): 411-421$.

Moher D et al. (2004). Measuring the quality of breast cancer care in women. Evid Rep Technol Assess (Summ), 105:1-8.

National Breast Cancer Centre and National Cancer Control Initiative. Clinical practice guidelines for the psychosocial care of adults with cancer (available at: http://www. nhmrc.gov.au/ publications/synopses/cp90syn.htm, accessed 19 December 2007).

National Comprehensive Cancer Network (1999). NCCN practice guidelines for the management of psychosocial distress. National Comprehensive Cancer Network. Oncology (Williston Park), 13(5A):113-147.

National Comprehensive Cancer Network (2007). Clinical practice guidelines in oncology. ${ }^{\mathrm{TM}}$ Distress management V.I.2007/2008. Jenkintown, PA, NCCN.

National Comprehensive Cancer Network web site (available at: http://www.nccn.org, accessed 16 December 2007).

National Comprehensive Cancer Network. NCCN clinical practice guidelines in Oncology. (available at: http://www.nccn.org/professionals/physician_gls/default.asp, accessed 16 December 2007).

National Institute for Health and Clinical Excellence web site (available at: http://www. nice.org.uk/, accessed 2 December 2007).

National Institute for Health and Clinical Excellence. Clinical guidance for improving supportive and palliative care and services for cancer patients (available at: http://www.nice. org.uk/csgsp, accessed 2 December 2007).

Newell SA, Sanson-Fisher RW, Savolainen NJ (2002). Systematic review of psychological therapies for cancer patients: overview and recommendations for future research.J Natl Cancer Inst, 94(8):558-584.

Parker PA et al. (2005). What do we know about facilitating patient communication in the cancer care setting? Psychooncology, 14(10):848-858; discussion:859-860.

Plass A, Koch U. (2001). Participation of oncological outpatients in psychosocial support. Psychooncology, 10(6):511-520.

Power M, Kuyken W (1998). The World Health Organization Quality of Life Assessment (WHOQOL): development and general psychometric properties. Soc Sci Med, 46(12):15691585 .

Radwin LE (2003). Cancer patients' demographic characteristics and ratings of patient-centered nursing care. J Nurs Scholarsh, 35(4):365-370. 
Reis JC (1998). O sorriso de Hipócrates: a integração biopsicossocial dos processos de saúde e doença (Hippocrates' smile: a biopsycho-social integration of health and disease processes). Lisbon, Vega.

Reuben S (2004). Living beyond cancer: a European dialogue. President's Cancer Panel, 20032004 Annual Report Supplement. National Institutes of Health, US Department of Health and Human Services.

Rowland J (1997). Outcomes assessment: cancer-specific quality-of-life measures: beyond the research setting (American Society of Clinical Oncology (ASCO) Educational Book) $33^{\text {rd }}$ Annual Meeting, May 1997.

Sloan JA et al. (2006). The clinical significance of quality of life assessments in oncology: a summary for clinicians. Support Care Cancer, 14(10):988-998.

Stewart AL, Hays RD, Ware JE Jr. (1988). The MOS short-form general health survey. Reliability and validity in a patient population. Med Care, 26(7):724-735.

Stewart MA (1995). Effective physician-patient communication and health outcomes: a review. Cam Med Ass J, 152(9):1423-1433.

Stewart M (2001). Towards a global definition of patient centred care. Br Med J, 322(7284):444-445.

Travado L (2006). Placing quality of life assessments on oncologists' agenda. Support Care Cancer, 14(10):979-981.

Travado L et al. (2005). Physician-patient communication among southern European cancer physicians: the influence of psychosocial orientation and burnout. Psychooncology, 14(8):661670.

van't Spijker A, Trijsburg RW, Duivenvoorden HJ (1997). Psychological sequelae of cancer diagnosis: a meta-analytical review of 58 studies after 1980. Psychosom Med, 59(3):280-293.

World Health Organization (1992) International classification of disease, 10th edition. Geneva, WHO. 



\section{Chapter 12 \\ Dying with cancer, living well with advanced cancer}

Irene J Higginson and Massimo Costantini

\section{Introduction}

Why is it important to devote attention to people with advanced cancer and those who die from it in a book on cancer in Europe? There are four epidemiological reasons. First, it affects very many people - at present there are more than 1.7 million deaths from cancer in Europe each year (Ferlay et al., 2007). By 2020, WHO estimates that globally more than 15 million people will experience cancer and 10 million will die from it each year (WHO, 2003), a 60\% increase in deaths. In western Europe, the number of people who die from cancer will increase by $31 \%$ to over 630000 each year (Table 12-1).

Second, despite extensive efforts to prevent and cure cancer, overall five-year survival from cancer is only between $50 \%$ and $60 \%$ in high-income countries.

Table 12-1 Deaths from cancer in Europe and selected other parts of the world: 2000 and predicted for 2020

\begin{tabular}{lccc}
\hline & Number of deaths 2000 & Predicted deaths 2020 & \% increase \\
\hline Northern Europe & 245000 & 310000 & 27 \\
Southern Europe & 342400 & 419600 & 23 \\
Eastern Europe & 638200 & 765500 & 20 \\
Western Europe & 483700 & 631300 & 31 \\
TOTAL Europe & $\mathbf{1 7 0 9 3 0 0}$ & $\mathbf{2 1 2 6 4 0 0}$ & $\mathbf{2 4}$ \\
North America & 636000 & 962100 & 51 \\
South America & 458700 & 840900 & 83 \\
Sub-Saharan Africa & 316300 & 523600 & 66 \\
TOTAL WORLD & 6200000 & 10000000 & 61 \\
\hline
\end{tabular}

Source: WHO, 2003 
Optimal treatment combined with early detection leads to a high rate of cure or remission for some cancers (e.g. of the cervix, breast, oral cavity and colon; and lymphomas). Yet, survival remains poor for some cancers (e.g. of the oesophagus, pancreas and lung).

Third, these improvements in the early detection and management of cancer have meant that cancer patients live with their illness for longer than in the past. The transition from radical, intensive treatment to a phase of less active life-prolonging treatment and palliative care remains poorly understood.

Fourth, the general ageing of the population will mean that people who have cancer are older. Other chronic and progressive diseases will also become more common, among all people affected by cancer, both patients and their families or caregivers. This brings with it problems of comorbidity for those people with cancer, so that their health needs become much more complex, as well as leading to changes in the structure of society, with more and more older people living alone or at a distance from their family.

However, there is also a fifth reason - not epidemiological, but perhaps the most important. Patients with advanced cancer can experience profound symptoms, which, unless alleviated, result in great suffering for them and their family (Davies \& Higginson, 2004; Solano, Gomes \& Higginson, 2006). There are emotional, social and spiritual consequences associated with cancer, disability and facing the end of life, for patients, their families and those close to them (Davies \& Higginson, 2004; Saunders \& Sykes, 1993). All these require effective support, communication and care. The effects on the family can continue long into bereavement, affecting their subsequent health and well-being.

In response to these needs, and the demands of patients and families for effective and coordinated support at this phase of the illness, most European countries have developed palliative care services. As Dame Cicely Saunders, the founder of the modern palliative care movement, explained:

Palliative care begins from the understanding that every patient has his or her own story, relationships and culture, and is worthy of respect as a unique individual. This respect includes giving the best available medical care and making the advances of recent decades fully available, so that all have the best chance of using their time well. (Davies \& Higginson, 2004)

\section{Living with, and dying from, advanced cancer in Europe}

Advanced cancer brings with it a complex web of problems, all of which interact with each other. Not only are there functional changes and the 
Box 12-1 Balfour M Mount (originator of the term palliative care) discussing quality of life at the end of life, demonstrating resilience

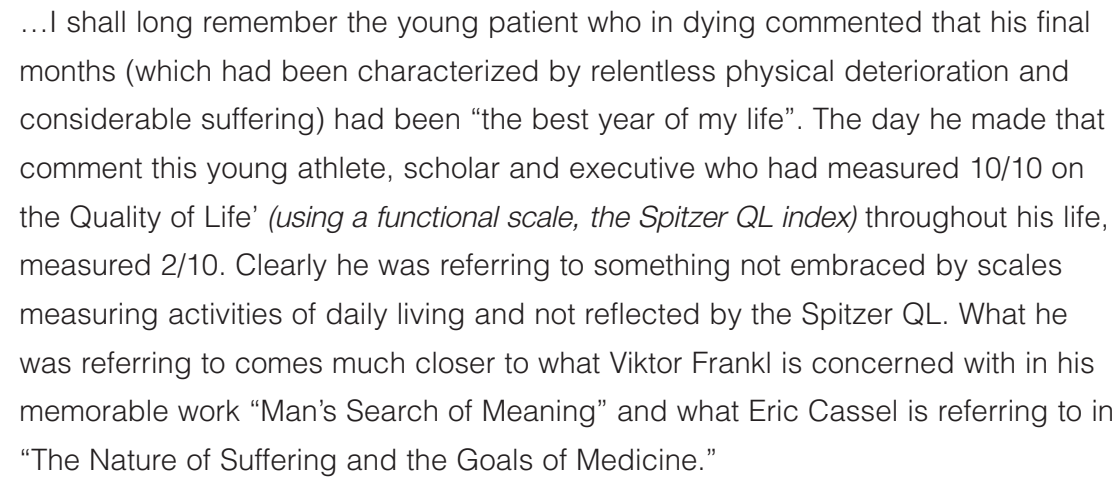

Source: Mount \& Scott, 1983.

emergence of symptoms, but emotional, social and spiritual effects on the individual, including coping with the impact of loss and the challenge of facing impending death (Davies \& Higginson, 2004). There are profound effects on the family, who are often involved in care of the patient, as well as coping with their own grief. There is also the increasing need to interact with health and social care services, as the individual and their family require help to manage the illness. All this changes, often rapidly, and requires continuous and often challenging adaptation for the patient, the family and health- and social-care professionals (Davies \& Higginson, 2004).

On the other hand, we should not forget that many individuals, families and communities demonstrate great resilience and grow during this period, providing their symptoms and difficulties are adequately managed and they have time to do so. Indeed some patients rate their quality of life very highly during advanced cancer, even higher than before they were ill (Box 12-1). Improving our ability to deal with the problems of patients and providing effective services to allow for such growth is a key goal for cancer care.

Research into factors that promote resilience suggests that paying attention to spiritual and psychological well-being, and creating meaningful narratives of living and dying is important (Nakashima \& Canda, 2005). Similarly, Chochinov has developed programmes to improve dignity, which include involving patients in preparing a narrative account of their lives (Chochinov et al., 2005). A key issue is to ensure that patients and families are given the means - if they wish - to live well until they die, as well as to die well. 

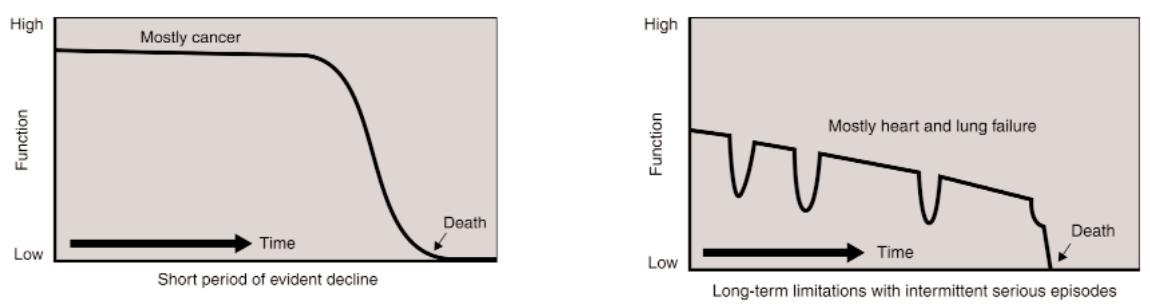

Source: Lynn \& Adamson, 2003

Fig. 12-1 Hypothesized trajectories of functional decline in cancer and other conditions (which may now represent the cancer trajectory)

\section{Functional trajectories}

Much attention has been given to the functional decline that accompanies cancer. This is probably because chemotherapy trials often study functional status as a proxy for quality of life. In the United States, Lynn and colleagues modelled the trajectory of functional decline in cancer and other conditions (Lynn \& Adamson, 2003). As Fig. 12-1 suggests, they propose a period of stable (good) function, followed by a period of rapid decline. However, the data on which this model was validated are based on cross-sectional data collected on different patients at different times before death. Therefore we cannot be sure that these trajectories reflect the longitudinal trajectory of individual patients. Further, as the cancer population has aged, with concomitant comorbidity, and treatments have evolved, giving second-, thirdand even fourth-line chemotherapies and improved surgery and radiotherapy for palliative effect, it is likely that the trajectory of cancer patients is less smooth than that in the Figure, and may even be more like that of organ failure or frailty.

\section{Cancer symptoms - how many people in Europe are affected?}

Symptoms are very common in advanced cancer, with patients having a median of 11 symptoms on admission to palliative care services (Tsai et al., 2006). Pain, breathlessness, fatigue, anorexia, constipation and insomnia are especially common; occurring in some combination in virtually all patients (Solano, Gomes \& Higginson, 2006). Using the methods of Higginson (Higginson, 1997) to develop an epidemiological assessment of need for palliative and terminal care, we can estimate the likely annual numbers of people in Europe with symptoms and problems. Applying symptom prevalence data to the numbers of cancer deaths in Europe suggests that there are, among patients who eventually die of their cancer, up to 1.6 million 
Table 12-2 Cancer patients: prevalence of symptoms and estimated number of cancer patients with symptoms towards the end of life

\begin{tabular}{|c|c|c|c|}
\hline $\begin{array}{r}\text { Sym } \\
( \\
\text { car } \\
\text { syst } \\
(1\end{array}$ & $\begin{array}{l}\text { ymptom prevalence } \\
\text { (\%) in advanced } \\
\text { ancer according to } \\
\text { stematic review (A) } \\
\text { (no. of patients) }\end{array}$ & $\begin{array}{l}\text { Symptom prevalence } \\
(\%) \text { in a random } \\
\text { sample of people in } \\
\text { the last year of life }(B)\end{array}$ & $\begin{array}{c}\text { Number of cancer } \\
\text { patients in Europe } \\
\text { experiencing the } \\
\text { symptom each year } \\
\text { (C) (lowest possible } \\
\text { - highest) }\end{array}$ \\
\hline Pain & $\begin{array}{r}35-96 \\
10379\end{array}$ & 84 & $595000-1632000$ \\
\hline Depression & $\begin{array}{r}3-77 \\
4378\end{array}$ & 38 & $51000-1309000$ \\
\hline Anxiety & $\begin{array}{l}13-79 \\
3274\end{array}$ & $\mathrm{~N} / \mathrm{A}$ & $221000-1343000$ \\
\hline Confusion & $\begin{array}{r}6-93 \\
9154\end{array}$ & 33 & $102000-1581000$ \\
\hline Fatigue & $\begin{array}{r}32-90 \\
2888\end{array}$ & $\mathrm{~N} / \mathrm{A}$ & $544000-1530000$ \\
\hline Breathlessness & $\begin{array}{r}10-70 \\
10029\end{array}$ & 47 & $170000-1190000$ \\
\hline Insomnia & $\begin{array}{r}9-69 \\
5606\end{array}$ & 51 & $153000-1173000$ \\
\hline Nausea & $\begin{array}{r}6-68 \\
9140\end{array}$ & 51 & 102 000-1 171810 \\
\hline Constipation & $\begin{array}{r}23-65 \\
7602\end{array}$ & 47 & $391000-1105000$ \\
\hline Diarrhoea & $\begin{array}{r}3-29 \\
3392\end{array}$ & $\mathrm{~N} / \mathrm{A}$ & $51000-493000$ \\
\hline Anorexia & $\begin{array}{r}30-92 \\
9113\end{array}$ & 71 & $510000-1564000$ \\
\hline Loss of bladder control & $\mathrm{N} / \mathrm{A}$ & 37 & 629000 \\
\hline Loss of bowel control & $\mathrm{N} / \mathrm{A}$ & 25 & 425000 \\
\hline Unpleasant smell & $\mathrm{N} / \mathrm{A}$ & 19 & 323000 \\
\hline Bedsores & N/A & 28 & 476000 \\
\hline
\end{tabular}

Notes:

(A) Symptoms as per Solano, Gomes and Higginson (2006), based on a systematic review of symptoms in advanced cancer. Range is the range of prevalence found in the study, no. patients is the number of patients included in the studies. (B) Symptoms as per Cartwright and Seale studies (Cartwright, 1991; Seale, 1991), based on a random sample of deaths and using the reports of bereaved carers about the last year of life.

(C) Estimated number of patients with symptom in Europe based on prevalence and 1.7 million cancer deaths. Range is lowest to highest, prevalence is higher when patients are near the end of life. The true figure is likely to lie between the ranges, probably nearer the top end, as the prevalence in the last year of life suggests. Note: patients are most likely to have multiple symptoms.

Source: Method of calculation based on Higginson, 1997

patients with pain (Table 12-2) each year. In around one third of patients this pain will be severe, requiring complex treatment (Higginson, 1997; Tsai et al., 2006).

There have been many advances in the management of symptoms. It is beyond the scope of this chapter to review these, and major textbooks, e.g. Palliative 
Medicine (Bruera et al., 2006), and The Oxford Textbook of Palliative Medicine (Doyle et al., 2004), deal with these developments. Needless to say, pain and many other symptoms can often be treated effectively. Treatment requires impeccable assessment of cause (there may be overlapping symptoms caused by the cancer, treatment or comorbid conditions) and effects, and skill in using analgesics to balance effect on pain against potential side effects, including constipation (which should always be anticipated and pre-empted), nausea and confusion. There is a need to balance these with the individual's psychological, social and spiritual problems, as it is important to treat the total person and not fragments of them. For example, sleep quality is related to pain, feelings of hopelessness and pain treatment in advanced cancer patients (Mystakidou et al., 2007a).

In 1986, WHO published simple but effective guidance on the management of pain. This "analgesic ladder" proposed non-opioid analgesics as the first step, followed by a mild opioid (step 2) or strong opioids (step 3) in patients with persistent pain. Non-opioid drugs (including non-steroidal antiinflammatory drugs - NSAIDs) are effective analgesics for patients with mild cancer pain and can be combined with opioids in patients with moderate to severe pain. Drugs should be given orally (wherever possible) and regularly, rather than waiting for pain to break through (Higginson \& Bruera, 2002).

Pain management has advanced considerably in recent decades, although the WHO approach remains at the heart of practice. There are new analgesics, different routes of administration (e.g. sublingual, subcutaneous, transdermal, intra-nasal), long- and short-acting medications for different pain durations, techniques to manage neuropathic pain (which involves the nerves and is most difficult to treat) and research into methods to "switch" opioids, which may be useful if side effects are problematic, in instances of renal failure and, in some cases, to improve effectiveness of pain relief (Higginson \& Bruera, 2002).

Study of other symptoms and interactions between symptoms lags behind that on pain, but nevertheless shows promising progress for many symptoms, including constipation, nausea, vomiting and breathlessness, although fatigue and anorexia remain problematic for many.

\section{Emotional concerns}

Psychological and emotional concerns are common in advanced cancer but may be dismissed by professionals and, sometimes, by the patient's family. In-depth discussion is often needed to identify the causes of the problems and these may be quite different from what one might expect. There are many 
interwoven factors that may include the family, finances, spiritual needs, guilt, anger, fear of dying and unrelieved physical symptoms. The patient may employ different defence mechanisms and coping strategies, some of which may be unexpected or difficult to interpret, including:

- regression - becoming more childlike;

- denial - blotting out or ignoring some realities;

- rationalization - providing an alternative everyday explanation (rather than the true one) for symptoms or feelings;

- intellectualization - becoming theoretical (often used by doctors and nurses in painful situations);

- projection - pushing problems onto others;

- displacement - displacing emotional energy into other thoughts and activities;

- introjection - looking within oneself to find solutions;

- repression - unconscious suppression of painful memories;

- withdrawal and avoidance - withdrawing from and avoiding painful situations.

Understanding these mechanisms can help carers to explain and empathize with a patient's behaviour. It is only when the mechanisms are excessive that problems occur - for example, excessive introjection can result in self-blame, isolation and depression; excessive projection can result in alienation of friends and family members or paranoid states; excessive displacement can lead to complete exhaustion followed by severe depression or anxiety.

The stress of prolonged illness or the shock of a recent diagnosis of advanced cancer can predispose a person to psychiatric and psychological problems, particularly if they have few external supports (for example, if they live alone, have few friends, or are very poor), have limited communication skills (such as in learning difficulties or impaired vision or hearing), or if they have a history of mental health problems. Assessment and management requires skill and a multiprofessional multimodal approach (Higginson \& Costantini, 2002; Higginson et al., 2003). Assessing psychological problems can be complex, but there are now simple screening methods that work well in advanced cancer (Hoffman \& Weiner, 2007; Mystakidou et al., 2007b). 


\section{Socioeconomic impacts}

First in the United States and then in Europe (Giorgi Rossi et al., 2007), surveys have revealed the socioeconomic impact of terminal disease on both patient and caregiver. Of 1271 caregivers interviewed in the Italian Survey of Dying of Cancer - ISDOC (Giorgi Rossi et al., 2007), 44\% reported difficulties in their regular employment during the last three months of the patient's life. Of the $68 \%$ of families who had to pay for some of the care, $37 \%$ had to pay for drugs; $36 \%$ for nursing and assistance; and $22 \%$ for physicians. In order to cover the costs of patient care, $26 \%$ of families in this Italian survey and $23 \%$ in a similar American survey used all or most of their savings. In the Italian survey, the duration of time the patient was completely dependent strongly determined the effect that caregiving had on the caregiver's regular employment and on the family's financial situation.

There are also differences between ethnic groups. A mortality followback survey in the United Kingdom found that a comparable proportion of caregivers of 50 black Caribbean and 50 white British patients reported experiencing restrictions in activities of daily living during their last year of life which included needing help toileting, washing and shaving; and requiring help at night. However, significantly more $(70 \%, \mathrm{n}=26)$ black Caribbean respondents stated they needed more help assisting their dependants with these tasks compared to $33 \%(n=14)$ of those caring for white dependants. Significantly more respondents caring for black Caribbean dependants also reported that they gave up visiting friends and relatives; going out for social occasions; and entertaining people at home, although similar numbers from both ethnic groups reported that they found the overall experience of providing care was rewarding (Koffman \& Higginson, 2003).

\section{Relationships and communication}

In all European countries, communication about cancer can be very problematic. Training programmes have been developed and evaluated for many of the critical points in cancer care, including breaking bad news, truthtelling, explaining treatment options and giving information about clinical trials. However, end-of-life care is least studied. Studies of patients with very advanced and progressive illness, or of their bereaved carers, identify three main areas of communication that are important:

1. information giving, with an empathetic approach from professionals to patients;

2. communication between the patient and their family regarding end-of-life issues; and 
3. communication between the different professionals involved in care, such as between doctors and nurses and between community and inpatient settings, to avoid contradictory or redundant information and ambiguity (Higginson \& Costantini 2002).

In a study across three European countries, data were collected on 1326 patients: 416 in the United Kingdom, 411 in Ireland and 499 in Italy. Severe communication problems at the end of life were found among up to $40 \%$ of patients (Higginson \& Costantini, 2002). Problematic communication was more common where the patient had respiratory or breast cancers and a shorter time in palliative care.

Talking to patients about the end of life is especially difficult. In a study of bereaved family members whose relatives had died from cancer (50 from the black Caribbean community and 50 from the white British community), in both groups over two thirds of patients knew they might die, although in over half of these cases no one had actually told them this (Higginson \& Koffman, 2003). In an Italian study only $12 \%$ of patients dying from cancer had received information about the bad prognosis of the disease, although according to the caregivers half of the patients had realized their poor prognosis (Costantini et al., 2006).

\section{The family and lay caregivers}

Concern for the patient and family, as the unit of care, is an essential element in the care of people who are dying. The word "family" is meant in its broadest sense and encompasses close relatives (often a spouse, children or siblings), a partner and close friend(s) who are significant for the patient. The end of life places a particular strain on families and on relationships (McLean \& Jones, 2007). Families should have every available option to meet their choices, and expert recognition of their cultural and individual needs. Not everyone will have the time to embark on long family discussions, but everyone can recognize the family or carers by name and accept them as an integral part of the team caring for the patient. This includes acknowledging the concerns of the family or friends and finding mechanisms for these to be heard and discussed, whether care is at home, in hospital or in a hospice.

The evidence supporting this approach is two-fold. Firstly, descriptive studies provide evidence of dissatisfaction and problems when involvement of the family does not occur (Hearn \& Higginson, 1998). Secondly, there is evidence from comparative and descriptive studies in Europe and the United States of higher satisfaction among carers when services do provide these family support systems. A systematic review of both comparative studies and randomized 
trials affirmed this, although many of the studies were small, retrospective and used matched (rather than randomly allocated) groups (Higginson et al., 2003).

\section{Place of death}

For many people, home is more than a physical space - it represents familiarity, the presence of loved ones and the possibility of enjoying "normal" life. These are the reasons why systematic reviews of studies of preferences have found that more than $50 \%$ of people with a progressive illness want to die at home (Higginson \& Sen-Gupta, 2000). Several countries are making substantial efforts to enhance home care, prompted by increased commitments to users' choice; rapidly increasing elderly populations; the quest to maximize scarce resources; and the experience of nearly four decades of palliative care practice.

Despite these efforts, preliminary data reported in the $\mathrm{WHO}$ analysis of palliative care (Davies \& Higginson, 2004) and more recent publications (Beccaro et al., 2006; Cohen et al., 2007) showed that most patients in England and Wales, the United States, Germany, Switzerland, France, Italy and Belgium die in hospitals (Fig. 12-2).

A conceptual model derived from the results of a systematic review, including 58 studies referring to over 1.5 million patients from 13 countries, shows the complex interaction of factors - related to illness, the individual and environment - which influence place of death (Fig.12-3) (Gomes \& Higginson, 2006).

Even though people often wish to die in their own homes, the probability of doing so is often lowest in areas of high socioeconomic deprivation (Fig. 12-4) (Costantini, Fusco \& Bruzzi, 1996; Higginson, Webb \& Lessof, 1994; Higginson et al., 1999). This is an important finding because those in poor areas tend to require more resources to achieve the same level of care. A study in London compared the activity of home palliative care nurses in deprived and affluent areas. It found that to achieve similar levels of home death rates, at least twice as many visits were needed in the deprived areas (Clark, 1997).

The inverse care law often operates in relation to hospice care, with provision inversely related to need. This is because hospice services are often supported by voluntary contributions, which are easier to raise in more affluent areas. In a comparison of home cancer deaths in London and New York, Decker and Higginson also found that in both cities, in all age groups, home deaths were less common in the lowest socioeconomic groups (Decker \& Higginson, 2007). 


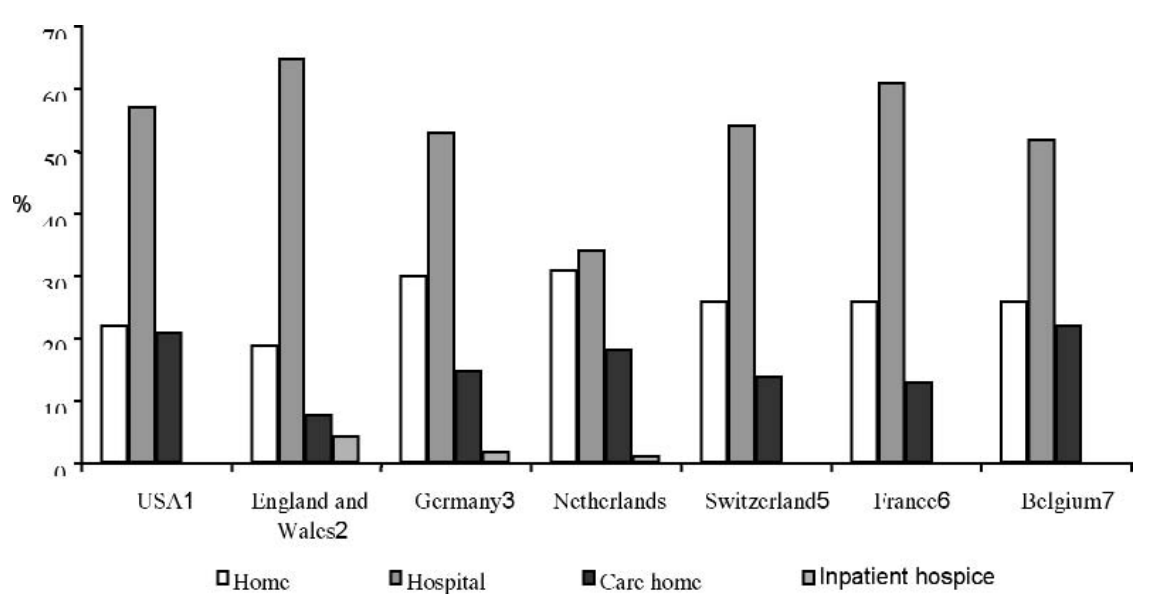

Sources: Davies \& Higginson, 2004; ${ }^{1 .}$ Weitzen et al., 2003 ; $^{2 .}$ Office for National Statistics, 2000; ${ }^{3 .}$ Thomas Schindler, personal communication; ${ }^{4}$. Central Buro of Statistics in the Netherlands, 2000, www.cbs.nl (personal communication Ribbe M). Updated with data from Cohen et al., 2007; 5. Extrapolations from Federal Statistics, 1985; ${ }^{6}$ INSERM, 1999.

Note: Data from different countries are collected in different ways and sometimes not at all. This has limited the comparisons that can be drawn, but highlights the need for health-care systems across Europe to begin to collect this information routinely. The $15 \%$ of deaths that occur in residential homes for the elderly in the Netherlands are not included in the graph.

Fig. 12-2 Preliminary data on place of death by country

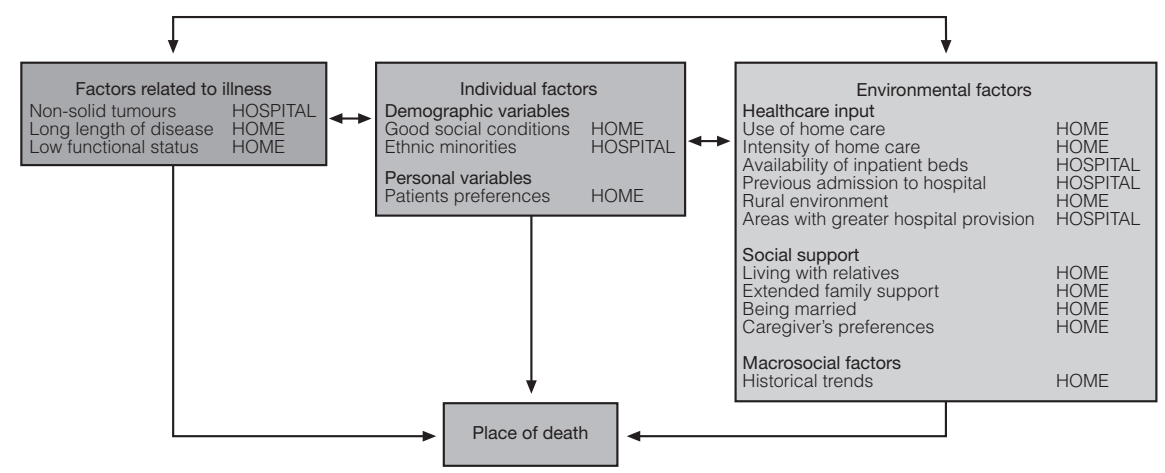

Source: Gomes \& Higginson, 2006.

Fig. 12-3 Model derived from a systematic review (including 1.5 million patients) of the factors associated with home or hospital death

\section{Bereavement}

Bereavement refers to a loss through death of someone significant. A universal occurrence, bereavement is also an especially potent and stressful life event. It predisposes people to physical and mental illness, can precipitate illness and death and aggravate existing illness (Raveis, 2006). Although there is 

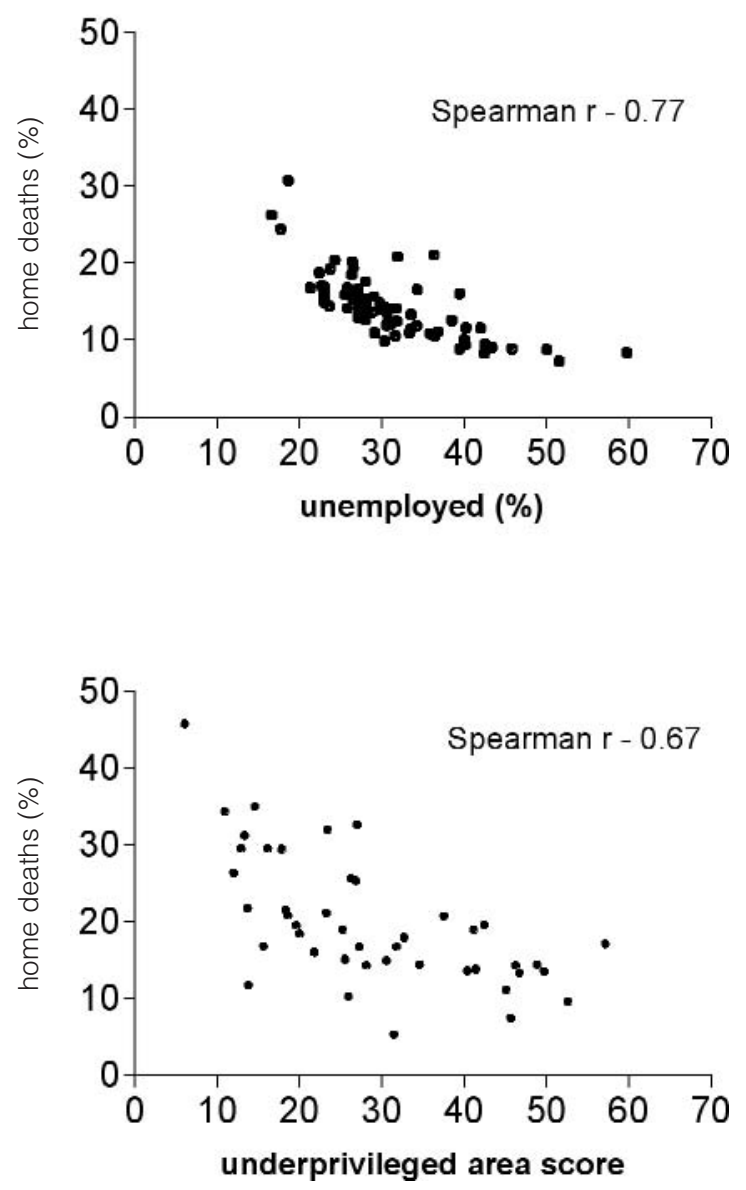

Source: Costantini, Fusco \& Bruzzi, 1996; Higginson, Webb \& Lessof, 1994

Fig. 12-4 Results of two ecological studies in Genoa (top) and London (bottom) to analyse the relationship between the proportion of people who died at home and social indicators

considerable variability in the rate at which individuals adjust to bereavement, most literature indicates that in western societies most bereaved people return to normal activities one to two years after their loss. Grief-related distress is normally highest in the first year. According to classical definitions a chronic, persistent, intensive, inhibited or delayed grief is regarded as pathological or complicated. However, any distinction between normal and pathological grief must take account of cultural differences. Grief is universally experienced, but response to loss is culturally bound. Unrestrained crying, wailing, selfmutilation or prostration may be a normal expression of grief in some societies (Raveis, 2006). 
Recognizing the needs of the family and caregivers both before and after bereavement is important in cancer care. Before the death occurs, family members may begin to express "anticipatory grief" of the death and may grieve the loss of previous relationships with the person before their death. Individual and situational factors can affect the outcome of the grieving process. These include: protracted illness; difficult or uncontrolled symptoms; a stigmatized death; the nature of the relationship; life circumstances; a history of physical and/or mental health problems; and isolation.

Principles of support in advanced cancer and after death should include viewing the patient and family as the unit of care; enabling open discussion of illness and death-related concerns; providing emotional support; facilitating practical systems; and respecting cultural, ethnic and spiritual practices.

Much attention has been given to adult grief, but bereaved children raise particular issues. A review of factors affecting children's reactions to bereavement found that truancy and behaviour problems were more common among bereaved children and adolescents. Social factors were important mediators of the response. More affluent and better supported children appeared to be buffered against major difficulties, whereas those from socially deprived circumstances or lacking supportive family environments experienced more severe and complicated grief (Lowton \& Higginson, 2002). These social circumstances were more important in influencing outcomes than were service factors, such as support offered to children. Schools may play an important role in supporting children among their peers, but rarely are teachers equipped or trained to facilitate open discussions of children's concerns.

For the minority of individuals who experience pathological grief, there is a need for rapid referral to appropriate services. However, many questions regarding bereavement remain unanswered. How common is complicated grief? Which interventions support what proportion of bereaved people most cost-effectively? And when is the right time to identify individuals and commence an intervention?

\section{Palliative care services}

Palliative care services have developed in culturally appropriate ways across Europe. While this has meant that services have responded to regional variations in health- and social-care structures it has also lead to variations in definitions, models and processes of care, within as well as between countries. 
Hospices and specialist palliative care services have increased rapidly in number worldwide. In 2007 there were over 8000 hospice or palliative care services in 100 countries of the world. In 2006, there were well over 1500 hospice and palliative care services in Europe. The number of services per million population ranged from 0 to over 20. These estimates are based on a survey by the European Association for Palliative Care Task Force on the Development of Palliative Care in Europe (a map of these services is available at: http://www.eapcnet.org/download/forTaskforces/developTF-Map.pdf). These data are difficult to interpret because of: (1) variations in the definition and size of responding services, (2) the use of self-assessments and (3) missing data and differences in the interpretation of the questions.

The main types of services within Europe include those listed below.

Inpatient hospice and specialist palliative care units with dedicated beds. The terms hospice and palliative care are often used interchangeably across Europe. The term palliative care is more appropriate in some countries, because the term hospice means hospital in some Latin-based languages and so confuses both the public and policy-makers. Some inpatient units are freestanding, being managed independently from and geographically separated from hospitals. Many of these are charitable units, a very few are private and some are part of community services. There are also inpatient units within acute hospitals, and in some instances part of a hospital ward is dedicated to palliative care.

Hospital-based palliative care teams. Usually these are advisory peripatetic teams that provide support, advice and education in the management of dying patients within the hospital. They are sometimes called mobile teams. These services rarely "take over" the management of patients and more usually advise the clinicians in charge of care on symptom management, as well as supporting the family and facilitating communication, care planning and discharge to the community or an in-patient hospice. They are often involved in multidisciplinary team meetings and may do joint ward rounds with oncologists or other specialists.

Home care teams provide support for patients and their families in the community. They usually work in partnership with existing community services, especially the family doctor or general practitioner and district nurses. They are most often advisory and do not provide "hands-on" nursing care, such as washing, dressing etc. However, this varies from country to country. For example, in Italy the community palliative care team takes over all aspects of community care, including direct care and prescribing; in the United Kingdom the services negotiate their level of support and usually prescribing 
Box 12-2 Palliative care services in the United Kingdom

In 2007, in the United Kingdom there were over 210 inpatient hospices, over 400 home care teams and over 260 palliative care teams working in hospitals. Palliative services and hospices usually offer a shared model of care. In the United Kingdom just over $50 \%$ of cancer patients who die receive care from some kind of palliative care team or nurse and $18 \%$ of people with cancer die in an in-patient hospice or palliative care unit.

and direct physical care is provided by the primary care team and social services. These teams may be based in the community, in hospital or in freestanding hospices or palliative care units.

Day care services are offered by inpatient units and home care teams. This model of care has developed primarily in the United Kingdom and is found much less often in other European countries. Patients usually attend day care centres one day per week, for the whole day, and are offered individualized services ranging from therapies (including complementary therapies), to mutual support, social and personal care activities such as bathing and hairdressing, and artistic and musical activities.

Outpatient services are sometimes offered by all the above services, offering clinic attendance for patients in the community.

Bereavement services are offered by many inpatient palliative care and hospice services, and by some home care, day care and hospital teams. Often, volunteers are involved in visiting families and sometimes group support is offered.

One feature common to all the above models is that of the multidisciplinary team. This is a key component of the approach to palliative care. The specialists that may be included in a multiprofessional palliative care team include doctors, clinical nurse specialists, social workers, chaplains, therapists and psychologists or psychiatrists. The team should aim to:

- achieve accurate and speedy assessment and diagnosis of the problems;

- plan and implement effective integrated treatment and care;

- communicate effectively with the patient, family and all other professionals and agencies involved in the care of the patient; and within the team;

- audit activities and outcomes.

A systematic review of the effectiveness of palliative care teams identified 44 relevant studies (Higginson et al., 2003). Of these, 26 included data suitable for meta-analysis, including randomized controlled trials and comparative or 
observational studies. Evidence was strongest for the effectiveness of home care studies, but there was also evidence of the effectiveness of hospital teams and inpatient hospices. Comparison of specialist multidisciplinary care and conventional care revealed a small quantitative benefit from multiprofessional teams. The main outcomes to benefit were pain, symptom control and changing the nature of therapies provided (from intensive treatments to human approaches to care) (Table 12-3). No studies conducted full costeffectiveness analyses, but those studies that examined costs showed a tendency for a reduction in hospital inpatient days resulting in lower hospital costs, although this was at the expense of greater costs in the community.

\section{New frontiers and dilemmas}

\section{Extending palliative care to earlier in the illness}

Cancer in Europe is changing, with longer periods of illness and treatments continuing later in the course of the disease. It is becoming more and more like other chronic illnesses. One important question is when palliative care should start? Patients often have symptoms and psychological and social concerns earlier in their illness. Communication is important too. WHO has recommended that palliative care should begin early in the course of illness. There is a need to move from the idea of palliative care as a rectangle which begins at a specific point close to death, to the concept of an interaction between palliative, life extending and curative treatment, each forming triangles that change in response to patient and family needs over time. Bereavement follow-up is part of this model. The use of shared clinics and ward rounds involving oncologists and palliative care specialists might be one step towards this (Fig 12-5).

\section{Assessment and outcome measurement}

Assessment of the patient is a critical aspect of palliative care, just as at other times of care. However, the standard measurements (of pulse, blood pressure, temperature and functional status) may be inappropriate at this stage if they do not contribute to planning of care. Appropriate assessment should emphasize pain and symptom control, the quality of life for the patient, fears and anxiety, psychological, social and spiritual concerns, any future wishes and the needs of family members and carers. This approach is equally relevant in clinical care and in measuring outcomes in research studies (Carr \& Higginson, 2001; Hearn \& Higginson, 1999; Higginson et al., 2006). 


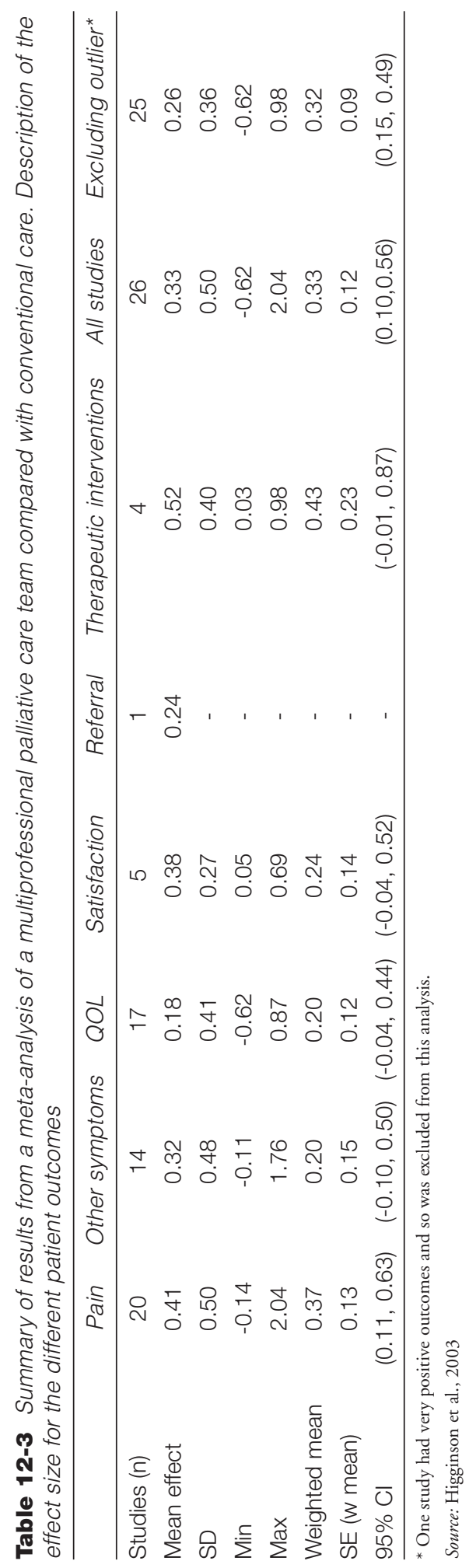


Previous situation

\section{Curative treatment}

\section{Terminal}

Care

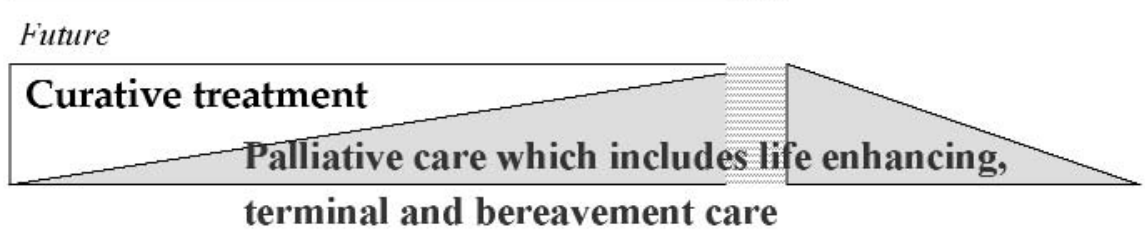

\section{Diagnosis}

Death -> Bercavement

Fig. 12-5 Model of palliative care as an increasing part of care from diagnosis onwards

There is now a range of assessment tools that have been developed and validated in the palliative care setting. The Palliative Care Outcome Scale POS (and its precursor measure the Support Team Assessment Schedule) has been validated in community, hospital and inpatient hospice settings, and featured user involvement during its design (Hearn \& Higginson, 1999; Higginson \& Donaldson, 2004; Higginson et al., 2006). The POS has been independently validated and translated into several European and African languages (Bausewein et al., 2005; Brandt et al., 2005; Serra-Prat et al., 2004; Sherry \& McAuley, 2004; Stevens et al., 2005). There are also adaptations to well-known measures such as the FACT and EORTC QLQ-C30 for palliative care (Groenvold et al., 2006; Mularski et al., 2007).

However, several challenges remain. Measures must be short and simple enough for use in patients with severe illness. They can be completed by proxy informants where patients have cognitive impairment, although more research is needed on the validity of this approach (Neumann, Araki \& Gutterman, 2000). There is a crucial need to standardize the measures used, in both research and clinical practice. Currently, a group involving King's College London (in partnership with European networks) is attempting to establish a Europe-wide project to test the use of POS and related measures, understand what measures are used and which are needed, and provide web-based resources. In addition, a systematic review is being undertaken by a collaborative group in the United Kingdom, supported by the National Cancer Research Institute. This is using Delphi exercises and statistical analysis to develop common measures and methods to map from one measure to another. 


\section{Ageing of the population with cancer}

People with cancer are growing older, and increasingly, may experience not just one cancer, but may be cured from an initial one and then experience another in later life. Further, older people have multiple comorbidities that can influence their treatment options, increase the risk of side effects from drugs and exacerbate symptoms and problems. The cancer specialist of the future will be dealing more and more with older people. This will require a strong understanding of general medical issues, unless patients are to be seen by multiple specialists, which may be time-consuming for them and impractical if they are more frail.

This is also an issue for palliative care. Palliative care services and treatments will have to find ways to support and manage patients not only in hospitals and specialist units, but also in nursing and residential homes, and to deal with patients who live alone, far from their families. They will need a better understanding of the management of diseases, symptoms and emotional concerns in diseases other than cancer.

\section{Recommendations for the future}

Looking ahead, it is possible to identify four areas that require attention.

1. Needs assessment. Hospices and palliative care services have developed in a haphazard way, often in response to local initiatives and support by champions. Although this type of development often reflects local wishes and circumstances, it means that hospices and palliative care services may not be in the places where they are most needed and may be disconnected from effective services. Epidemiologically-based needs assessment should drive future developments, coupled with statutory support, to reduce the inverse care law that applies to the provision of palliative care services.

Although many types of palliative care services have been evaluated, there is a need to compare the costs and benefits of variations in the mix of care (home versus hospital versus hospice) along with full evaluations of some of the new models of care, such as palliative care beds within hospitals, day care services and earlier integration with other professionals.

2. Investment in research. In the United Kingdom, less than $0.2 \%$ of the research spend in cancer addresses palliative and end-of-life care. The figure is not known for many other European countries, but is likely to be similar. Research in palliative care therapies and services needs to be prioritized, along with methodological development to refine further the study designs, measures and methods. 
3. Education and training in palliative care. A curriculum for palliative care doctors exists in the United Kingdom, and to some extent in other European countries. A curriculum for nurses in palliative care does not exist. There is a need for curricula relevant to different types of clinicians, such as oncologists, as well as for specialists in palliative care.

4. Assessment. This is a strategic and clinical necessity. European statistics about place of care, symptom levels and family concerns are not available. Such information is needed to understand and contrast provision and outcomes across Europe to aid policy-makers and public health physicians in the development of palliative care in the future. At the clinical level, assessment and outcome tools exist and have been translated and validated in several countries. European collaborative efforts are required to develop these tools further and to make them more widely available.

\section{Conclusions}

Palliative care is an essential component of cancer care. Its importance is becoming increasingly recognized, but it requires investment in research, education and services if it is to achieve its potential to improve the quality of care for cancer patients and their families, and to help them live well until they die, as well as to die well.

\section{REFERENCES}

Bausewein C et al. (2005). Validation and clinical application of the German version of the palliative care outcome scale. J Pain Symptom Manage, 30(1):51-62.

Beccaro M et al. (2006). Actual and preferred place of death of cancer patients. Results from the Italian Survey of Dying of Cancer (ISDOC). J Epidemiol Community Health, 60(5):412-416.

Brandt HE et al. (2005). The last days of life of nursing home patients with and without dementia assessed with the palliative care outcome scale. Palliat Med, 19(4):334-342.

Bruera E et al. (2006). Textbook of palliative medicine. London, Hodder Arnold.

Carr AJ, Higginson IJ (2001). Measuring quality of life: are quality of life measures patient centred? $\mathrm{Br}$ Med J, 322:1357-1360.

Cartwright A (1991). Changes in life and care in the year before death 1969-1987. J Public Health Med, 13(2):81-87.

Chochinov HM et al. (2005). Dignity therapy: a novel psychotherapeutic intervention for patients near the end of life. J Clin Oncol, 23(24):5520-5525.

Clark CR (1997). Social deprivation increases workload in palliative care of terminally ill patients. Br Med J, 314(7088):1202.

Cohen J et al. (2007). Hospital deaths in six European countries: population-based cross national study of factors associated with dying in hospital. In: Cohen J, ed. End-of-life decisions and place of death in Belgium and Europe. Brussels, VUB Press:149-166. 
Costantini M, Fusco F, Bruzzi P (1996). Uno studio epidemiologico a Genova dal 1986 al 1990 sul luogo di decesso per neoplasia. (An epidemiolgical study in Genova 1986-1990 on the place of death from cancer) Informatore Medico Oncologico, 5:21-24.

Costantini $\mathrm{M}$ et al. (2006). Diagnosis and prognosis disclosure among cancer patients. Results from an Italian mortality follow-back survey. Ann Oncol, 17:853-859.

Davies E, Higginson IJ (2004). Palliative care: the solid facts (available at:http://www.euro.who. int/document/E82931.pdf, accessed 22 November 2007). Copenhagen, Denmark, World Health Organization.

Decker SL, Higginson IJ (2007). A tale of two cities: factors affecting place of cancer death in London and New York. Eur J Public Health, 17(3):285-290.

Doyle D et al. (2004). Oxford textbook of palliative medicine. Oxford, Oxford University Press.

European Association for Palliative Care Task Force on the Development of Palliative Care in Europe. A map of palliative care specific resources in Europe (available at:http://www.eapcnet. org/download/forTaskforces/developTF-Map.pdf, accessed 19 December 2007.

Ferlay J et al. (2007). Estimates of the cancer incidence and mortality in Europe in 2006. Ann Oncol, 18(3):581-592.

Giorgi Rossi P et al. (2007). Dying of cancer in Italy: impact on family and caregiver. The Italian Survey of Dying of Cancer. J Epidemiol Community Health, 61(6):547-554.

Gomes B, Higginson IJ (2006). Factors influencing death at home in terminally ill patients with cancer: systematic review. Br Med J, 332(7540):515-521.

Groenvold $\mathrm{M}$ et al. (2006). The development of the EORTC QLQ-C15-PAL: a shortened questionnaire for cancer patients in palliative care. Eur J Cancer, 42(1):55-64.

Hearn J, Higginson IJ (1998). Do specialist palliative care teams improve outcomes for cancer patients? A systematic literature review. Palliat Med, 12:317-332.

Hearn J, Higginson IJ (1999). Development and validation of a core outcome measure for palliative care: the palliative care outcome scale. Palliative Care Core Audit Project Advisory Group. Qual Health Care, 8(4):219-227.

Higginson I, Webb D, Lessof L (1994). Reducing hospital beds for patients with advanced cancer. Lancet, 344(8919):409.

Higginson IJ (1997). Health care needs assessment: palliative and terminal care. Oxford, Radcliffe Medical Press.

Higginson IJ, Bruera E (2002). Care of patients who are dying, and their families. In: Souhami RL et al. eds. Oxford textbook of oncology. Oxford, Oxford University Press:1103-1120.

Higginson IJ, Costantini M (2002). Communication in end-of-life cancer care: a comparison of team assessments in three European countries. J Clin Oncol, 20(17):3674-3682.

Higginson IJ, Donaldson N (2004). Relationship between three palliative care outcome scales. Health and Quality of Life Outcomes, 2:68-75.

Higginson IJ, Koffman J (2003). Attitudes to timeliness of death and euthanasia among black Caribbean and white populations. J Palliat Med, 6: 45-249.

Higginson IJ, Sen-Gupta GJ (2000). Place of care in advanced cancer: a qualitative systematic literature review of patient preferences. J Palliat Med, 3(3):287-300.

Higginson IJ et al. (1999). Do social factors affect where patients die: an analysis of 10 years of cancer deaths in England. J Public Health Med, 21(1):22-28.

Higginson IJ et al. (2003). Is there evidence that palliative care teams alter end-of-life experiences of patients and their caregivers? Journal of Pain \& Symptom Management, 25:150168.

Higginson IJ et al. (2006). Symptom prevalence and severity in people severely affected by multiple sclerosis. J Palliat Care, 22(3):158-165. 
Hoffman MA, Weiner JS (2007). Is Mrs S depressed? Diagnosing depression in the cancer patient. J Clin Oncol, 25:2853-2856.

Koffman JS, Higginson IJ (2003). Fit to care? A comparison of informal carers of first generation black Caribbeans and white dependants with advanced progressive disease in the UK. Health and Social Care in the Community, 11:528-536.

Lowton K, Higginson IJ (2002). Early bereavement: what factors influence children's responses to death? London, King's College London and National Council for Hospice and Specialist Palliative Care Services.

Lynn J, Adamson DM (2003). Living well at the end of life: adapting health care to serious chronic illness in old age. Arlington, VA, Rand Health.

McLean LM, Jones JM (2007). A review of distress and its management in couples facing endof-life cancer. Psychooncology, 16(7):603-616.

Mount BM, Scott JF (1983). Whither hospice evaluation? J Chronic Dis, 36(11):731-736.

Mularski RA et al. (2007). Measuring outcomes in randomized prospective trials in palliative care. J Pain Symptom Manage, 34(Suppl. 1):S7-S19.

Mystakidou K et al. (2007a). Sleep quality in advanced cancer patients. J Psychosom Res, 62(5):527-533.

Mystakidou K et al. (2007b). Beck depression inventory: exploring its psychometric properties in a palliative care population of advanced cancer patients. Eur J Cancer Care (Engl), 16(3):244250.

Nakashima M, Canda ER (2005). Positive dying and resiliency in later life: a qualitative study. Journal of Aging Studies, 19:109-125.

Neumann P, Araki SS, Gutterman EM (2000). The use of proxy respondents in studies of older adults: lessons, challenges, and opportunities. Journal of the American Geriatrics Society, 48(12):1646-1654.

Raveis VH (2006). Bereavement. In: Bruera E et al. eds. Textbook of palliative medicine. London, Hodder Arnold:1044-1050.

Saunders CMD, Sykes N (1993). The management of terminal malignant disease. London, E. Arnold.

Seale C (1991). A comparison of hospice and conventional care. Soc Sci Med, 32(2):147-52.

Serra-Prat M et al. (2004). Grupo Catalan de estudio de la efectividad de los cuidados paliativos [Validation of the Spanish version of the palliative care outcome scale]. Med Clin (Barc), 123:419-420.

Sherry KL, McAuley G (2004). Symptom prevalence and the use of systematic symptom assessment. Palliat Med, 18(1):75-76.

Solano JP, Gomes B, Higginson IJ (2006). A comparison of symptom prevalence in far advanced cancer, AIDS, heart disease, chronic obstructive pulmonary disease and renal disease. J Pain Symptom Manage, 31(1):58-69.

Stevens A-M et al. (2005). Experience in the use of the palliative care outcome scale. Support Care Cancer, 13:1027-1034.

Tsai JS et al. (2006). Symptom patterns of advanced cancer patients in a palliative care unit. Palliat Med, 20(6):617-622.

Weitzen $S$ et al. Factors associated with site of death: a national study of where people die. Med Care, 2003;41(2):323-335.

WHO (2003). Global action against cancer. Geneva, World Health Organization/UICC. 


\section{Chapter 13 \\ Closing the gap: cancer in Central and Eastern Europe}

Witold Zaton'ski and Joanna Didkowska

\section{Background}

The epidemiological transformation that took place after the Second World War in Europe was significantly delayed in the central and eastern European countries (CEE) (with the exception of Czechoslovakia), compared to the countries of northern Europe and the United Kindgom (where it had already started at the beginning of the twentieth century). Communicable diseases declined rapidly, allowing many children to survive into adulthood, only to be afflicted by a rising tide of noncommunicable dísease (Zatonski, McMichael \& Powles, 1998).

From the 1960s onwards, cancer and cardiovascular diseases emerged as the leading causes of death among adults in CEE (Zatoński \& Boyle, 1996; Zatoński \& Jha, 2000; Zatoński \& Willett, 2005). Since the 1990s, as death rates from cardiovascular disease have begun to fall, cancer has emerged as the most common cause of death among young and middle-aged women (20-64 years old) in these countries. In the coming decade it seems likely to be the leading cause of death among young and middle-aged men (cardiovascular diseases still dominate in this group) (Fig. 13-1) (Boyle et al., 2003).

It is accepted that behavioural factors play a crucial role in the development of cancer. These include cigarette smoking; alcohol consumption; exposure to occupational and environmental carcinogens; sexual behaviour; obesity; diet; and physical activity. Demographic changes, particularly population ageing ${ }^{8}$,

${ }^{8}$ Cancer risk increases exponentially with age. 

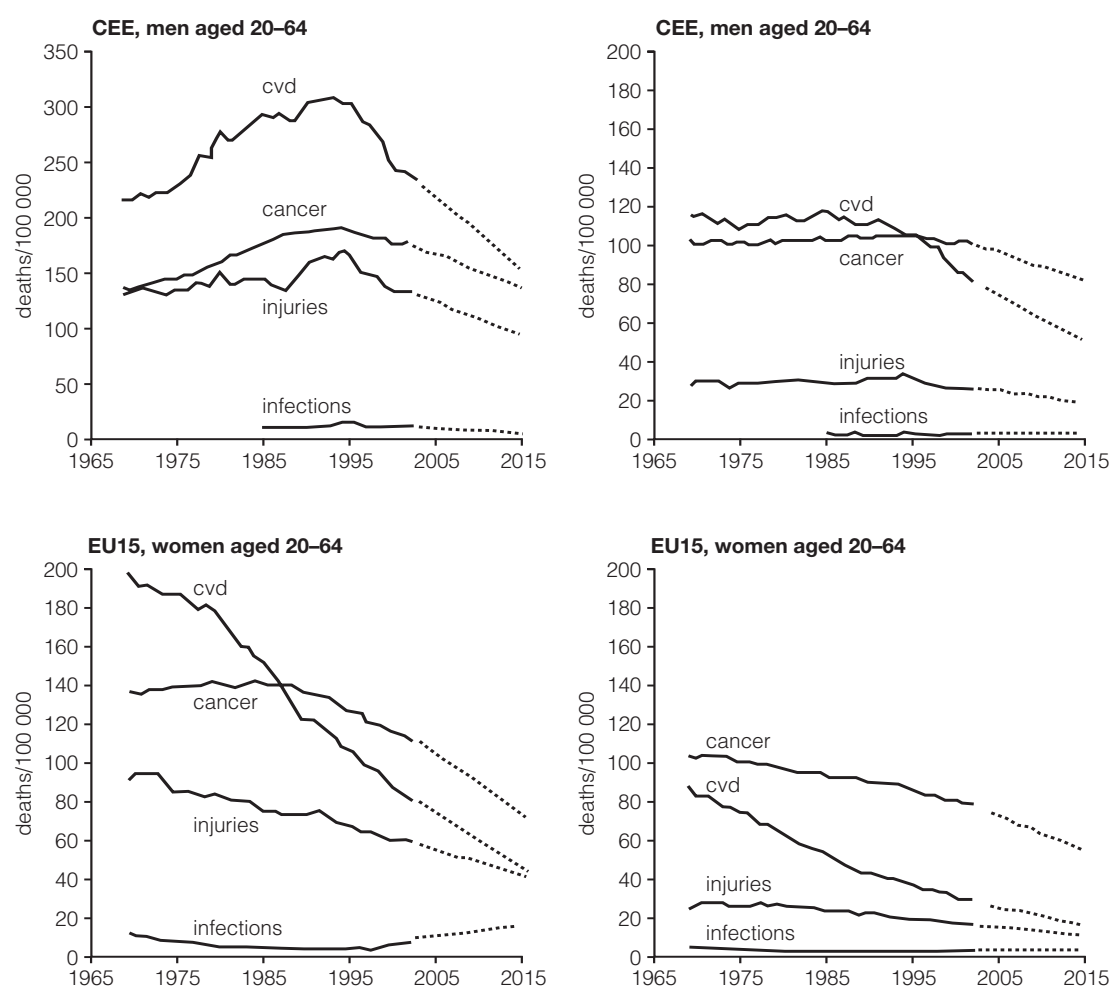

Fig. 13-1 Trends in mortality in Europe from major causes at ages 20-64

also impact on cancer incidence. These factors have continued to change in all parts of Europe, often following similar pathways but from different initial levels and at different rates. As a consequence, each country must confront a different burden of cancer - both in nature and magnitude.

Diversity is also apparent in the control of cancer in Europe. While there is considerable scope for improvement throughout the region, the past decade has seen significant progress. Much of this can be attributed to the introduction of comprehensive approaches to cancer control (including increasingly successful interventions to treat cancer) but fundamentally it stems from achievements in prevention (primary and secondary) (Boyle et al., 2003a).

Some countries have achieved much more than others. This chapter addresses the diversity in both disease burden and outcomes in Europe by contrasting the old Member States (EU15) with the newer Member States, which are largely in central and eastern Europe. The EU15 comprises Austria, Belgium, Denmark, Finland, France, Germany, Greece, Ireland, Italy, Luxembourg, the Netherlands, Portugal, Spain, Sweden and the United Kingdom. The CEE 
comprises 10 Member States: Bulgaria, the Czech Republic, Estonia, Hungary, Latvia, Lithuania, Poland, Romania, Slovakia and Slovenia. Two of the 12 newer Member States, Cyprus and Malta, are excluded for the purposes of this chapter, because they have quite different patterns of disease.

\section{Material and methods}

Data on deaths in each country have been extracted from the WHO database and cover the period 1959-2002 (WHO database). Data are not available for all countries for all years (see Chapter 2). Population data are drawn from the Department of Economic and Social Affairs Population Division of the United Nations (United Nations, 2001).

Throughout this chapter, direct standardization has been undertaken using the world standard population (Parkin et al., 1997). The share of cancer mortality attributable to tobacco was calculated following the method developed by Peto (Peto, 1994). The fraction attributable to alcohol (Rehm et al., 2007) was calculated using relative risks from existing literature combined with survey data on alcohol prevalence. A standard methodology of decomposing life expectancy was used (Preston, Heuveline \& Guillot, 2001). Predictions employed the method described by Dyba \& Hakulinen (Dyba \& Hakulinen, 2000) using STATA version 8 (European Network of Cancer Registries) and 2015 was chosen as the time horizon for predictions.

\section{Results}

\section{Life expectancy}

In the CEE countries, average life expectancy for men in 2002 was 6.84 years lower than in the EU15 countries. For the female population this difference was smaller, at 4.64 years. For all age groups the difference in life expectancy attributable to cancer is 0.84 of a year (12\% of the total gap) among men and 0.4 of a year ( $9 \%$ of the total) among women. Among the more restricted group of women of working age (20-64 years) nearly one quarter of the difference in life expectancy between the CEE and EU15 is attributable to cancer (among men it is only around 12\%) (Table 13-1). Only cardiovascular mortality (53\% of the difference for men; $79 \%$ for women) is more important than cancer. Infectious diseases make a negligible contribution to life expectancy differences between the two parts of Europe. On the other hand, injury does make a significant contribution to the life expectancy gap in the male population of working age. 


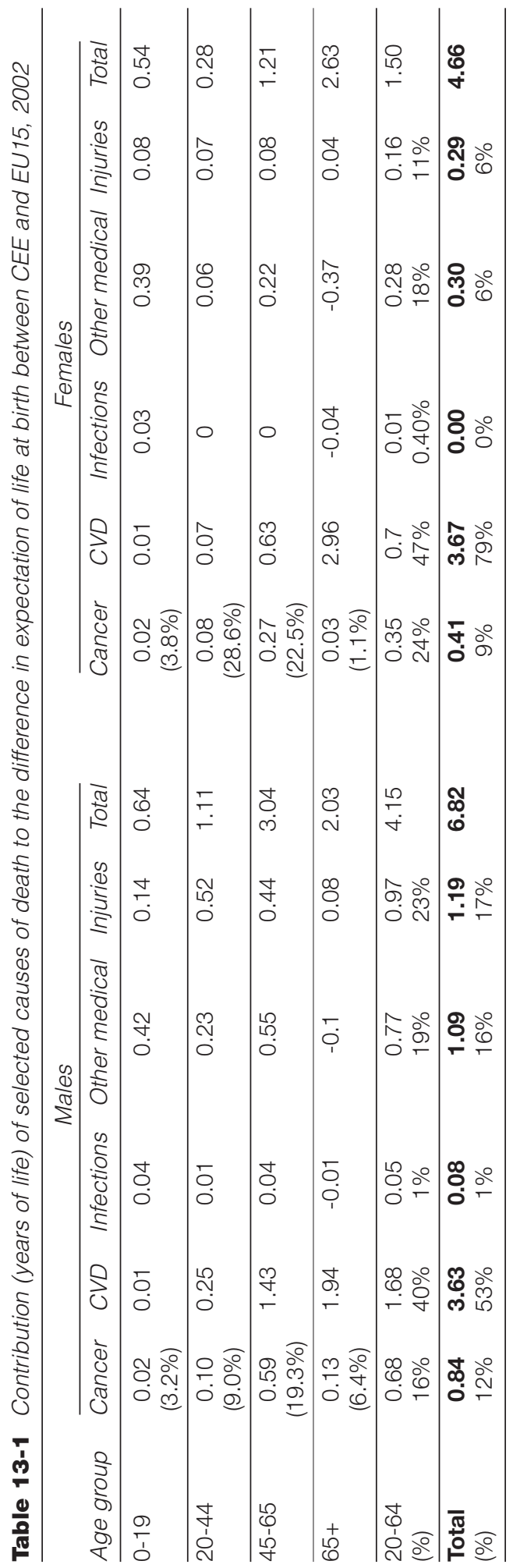


Table 13-2 Cancer incidence and mortality rates (per 100 000) in Europe, 2002

\begin{tabular}{lcccc}
\hline & \multicolumn{3}{c}{ Male } & \multicolumn{2}{c}{ Female } \\
\cline { 2 - 5 } & EU15 & CEE & EU15 & CEE \\
\hline Incidence & 300 & 284 & 234 & 203 \\
Mortality & 155 & 194 & 104 & 104 \\
\hline
\end{tabular}

Source: Ferlay et al., 2004

\section{Numbers, rates}

In 2002, 2111000 new cancer cases were diagnosed in Europe (EU15: 1746000 ; CEE: 365 000). In the same year, 1191500 people died from cancer (EU15: 950 000; CEE: 241 500). The incidence and mortality rates for men and women in the two parts of Europe are shown in Table 13-2. Although the incidence of cancer is higher in EU15 countries, the mortality rate is lower for men but equal for women.

\section{Trends}

Trends in cancer over time differ significantly by gender, age group and time period in eastern and western Europe. Only in children and youth (up to age 20 ), in both sexes and from both parts of Europe, can a decreasing mortality trend be seen over the period in question. Nevertheless, the decline was steeper in the EU15 countries (Fig. 13-2) so that, by 2002, their mortality level was significantly lower than in the CEE countries.

A constant decrease in mortality rates can be seen in young men (20-44) in the EU15 throughout the period. In contrast, the CEE countries experienced an increase in mortality rates until the 1990s, after which they declined. By 2002 there was a substantial mortality gap between the two parts of Europe.

At the beginning of the observation period, cancer mortality rates among middle-aged men (45-64) in both regions were increasing. These trends started to diverge towards the end of the 1970s when the EU15 experienced a plateau followed by a decline. In CEE a continuing increase occurred until the 1990 s, only then giving way to a small decline. As a result, in 2002, a very significant difference in mortality rates was apparent.

Historically, mortality rates in the oldest age group were higher in the EU15. After initial stagnation, mortality rates in CEE have increased in the last two decades. In the EU15, after decades during which there was a steady increase, the beginning of the 1990s saw a reversal of this trend (Fig. 13-2). In 2002, mortality rates in both regions are at comparable levels, albeit slightly higher in CEE. 

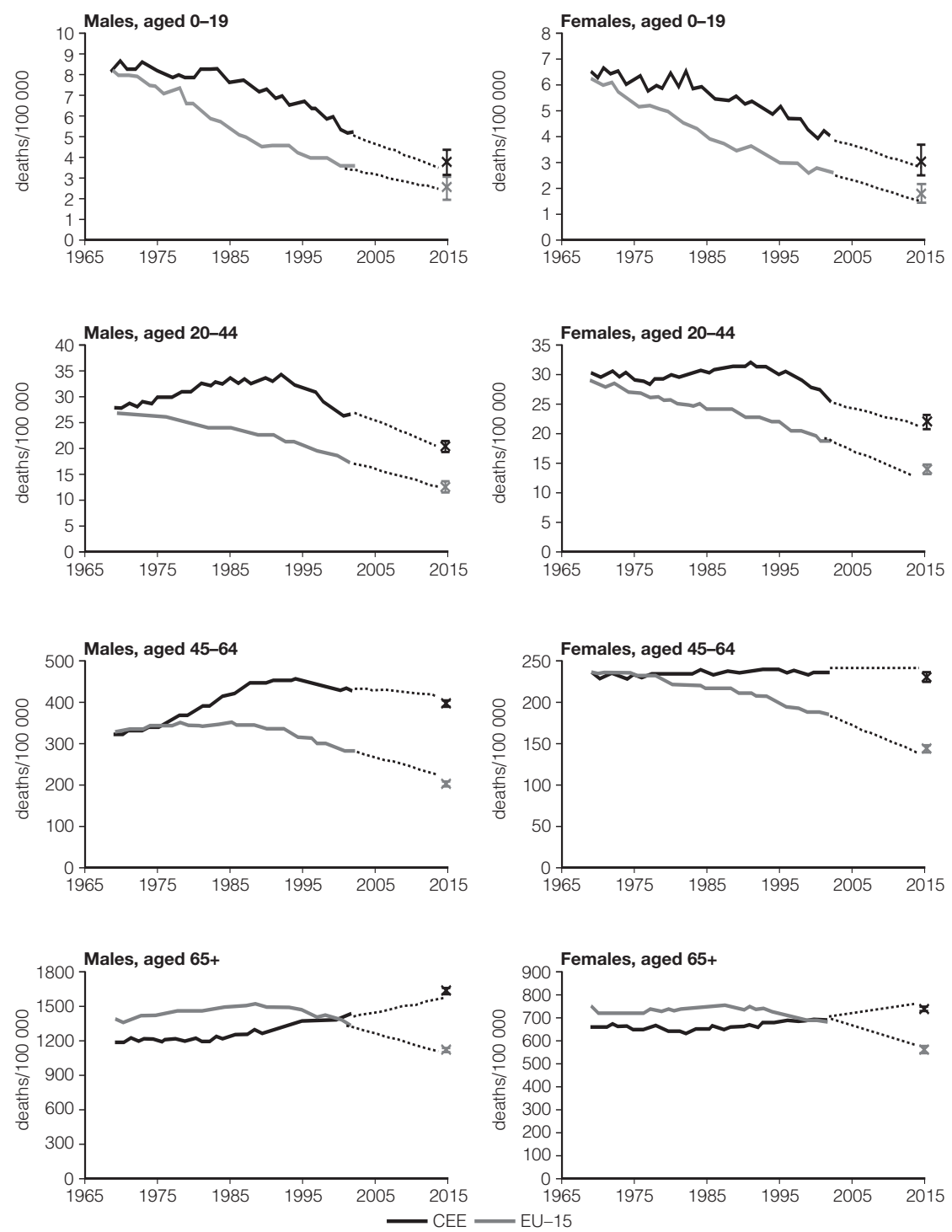

Fig. 13-2 Time trends and predicted cancer mortality in the EU

Mortality rates from cancer among adult women in the EU15 have been decreasing throughout the observation period in all age groups. The rate of decline is fastest in the youngest age group and slowest in the oldest. In CEE, on the other hand, among young women (age 20-44) there was a small increase until the end of 1980s, after which a significant decline became apparent. In 2002, a clear gap was noticeable between the EU15 and CEE. In CEE, among women in middle- and old-age, a plateau and a slight increase, respectively, were observed. However, in 2002, a mortality gap was observed 
in middle-aged women, while in the $65+$ age group this rate was almost equal in CEE and the EU15.

\section{Prediction}

On current predictions, it is estimated that there will be 300000 deaths from cancer in CEE in 2015 (170 000 men; 130000 women). Thus, in slightly over a decade, it is anticipated that there will be an increase of about 60000 in the annual number of deaths. The predicted mortality rate in CEE in 2015 thus equates to 201 per 100000 (95\% CI 198.9-204.0) for men and 105.6 per 100000 (95\% CI 104.1-107.0) for women (Fig. 13-2). On these trends, future mortality rates in CEE in the future will still be considerably higher than in the EU15 countries, by about 1.6 times for men and 1.4 times for women (Quinn et al., 2003).

The phenomenon of widening gaps in mortality is predicted to affect all age groups. In the youngest age group (0-19 years), the cancer mortality rate is predicted to fall to 3.7 per 100000 (95\% CI 3.08-4.30) for men and 3.1 per 100000 (95\% CI 2.42-3.60) for women (the actual number of deaths in this age group for both sexes is estimated to be about 540 in 2015). In younger adults (20-44 years), it is predicted that the mortality rate will fall to 19.9 per 100000 (95\% CI 18.8-21.0) for men and 21.8 per 100000 (95\% CI 20.623.0) for women. The number of deaths for both sexes is predicted to be about 8900 per year in 2015. Among older adults (45-64 years) no substantial changes in mortality rates are expected, with the current level being maintained at 406 per 100000 (95\% CI 398-414) for men and 231 per 100000 (95\% CI 225-237) for women. The number of deaths in this age group in both sexes in 2015 is estimated to be 93 000. Among the oldest adults the predicted mortality rate is 658 per 100000 (95\% CI 1629-1687) for men and 758 per 100000 (95\% CI 746-770) for women, with the deaths in this age group among females and males estimated at 191000.

The following sections examine the need for different approaches to address the higher burden of cancer in CEE. These draw on trends in mortality from different cancers to illustrate the importance of a comprehensive strategy embracing primary, secondary and tertiary prevention.

\section{Primary prevention}

Tobacco-attributable fraction of mortality

Tobacco is the leading preventable cause of cancer in Europe. It causes cancers 
of the lung and many other organs, and contributes to many other diseases. Peto et al. have developed a method to ascertain the total burden of mortality attributable to smoking (Peto et al.,1994), allowing comparison of the impact of tobacco in the EU15 and CEE countries. The trends in smokingattributable cancer deaths are shown in Fig. 13-3. The two country groupings differ in absolute levels and, in some age groups, direction of travel. Among men, there was an increase in mortality in CEE until 1995, since when it has begun to increase at ages below 65, and especially so in those aged 35-44. In contrast, tobacco-attributable deaths among older men have continuously increased.

In the EU15 countries, there was little change between 1965 and 1990, apart from among the oldest group, where it was increasing. Since then, all age groups have seen a steady decline. By 2002, rates were substantially higher in CEE at all ages under 65, although even at older ages, where the rates are now similar, the direction of travel suggests that a gap will soon open up, to the disadvantage of CEE.

Among the female population, the picture is more varied. In young women, there was a rapid acceleration in the increase in death rates during the 1980s, with rates in CEE consistently higher, by at least a factor of two. Thereafter, the levels reached a plateau, falling slightly in CEE. In middle-aged and older women, tobacco-attributable death rates have steadily risen throughout the period of observation, in both parts of Europe. However, among middle-aged women, the rate is now higher in CEE, while in women aged 65+, the rate is higher in the EU-15, reflecting the different timing of the growth of smoking among women.

\section{Alcohol-attributable fraction}

The International Agency for Research on Cancer (IARC, 1988) recognizes alcohol as a carcinogen and one that is responsible for an increasing number of cancers, especially in countries with a high alcohol consumption level (which includes all European countries). The contribution of alcohol to cancer mortality has been estimated by combining survey data on the prevalence of alcohol consumption (in 2002) with data on mortality (Rehm et al., 2007). Tables 13-3 and 13-4 present an estimation of alcohol-attributed cancer mortality in the adult population, for all regions, both sexes and all age groups, showing both mortality rates and numbers of deaths.

In the male population, alcohol-attributed cancer mortality is significantly higher in younger age groups (20-64) in CEE. The converse situation applies in the age group 65+, where mortality is higher in the EU15. 

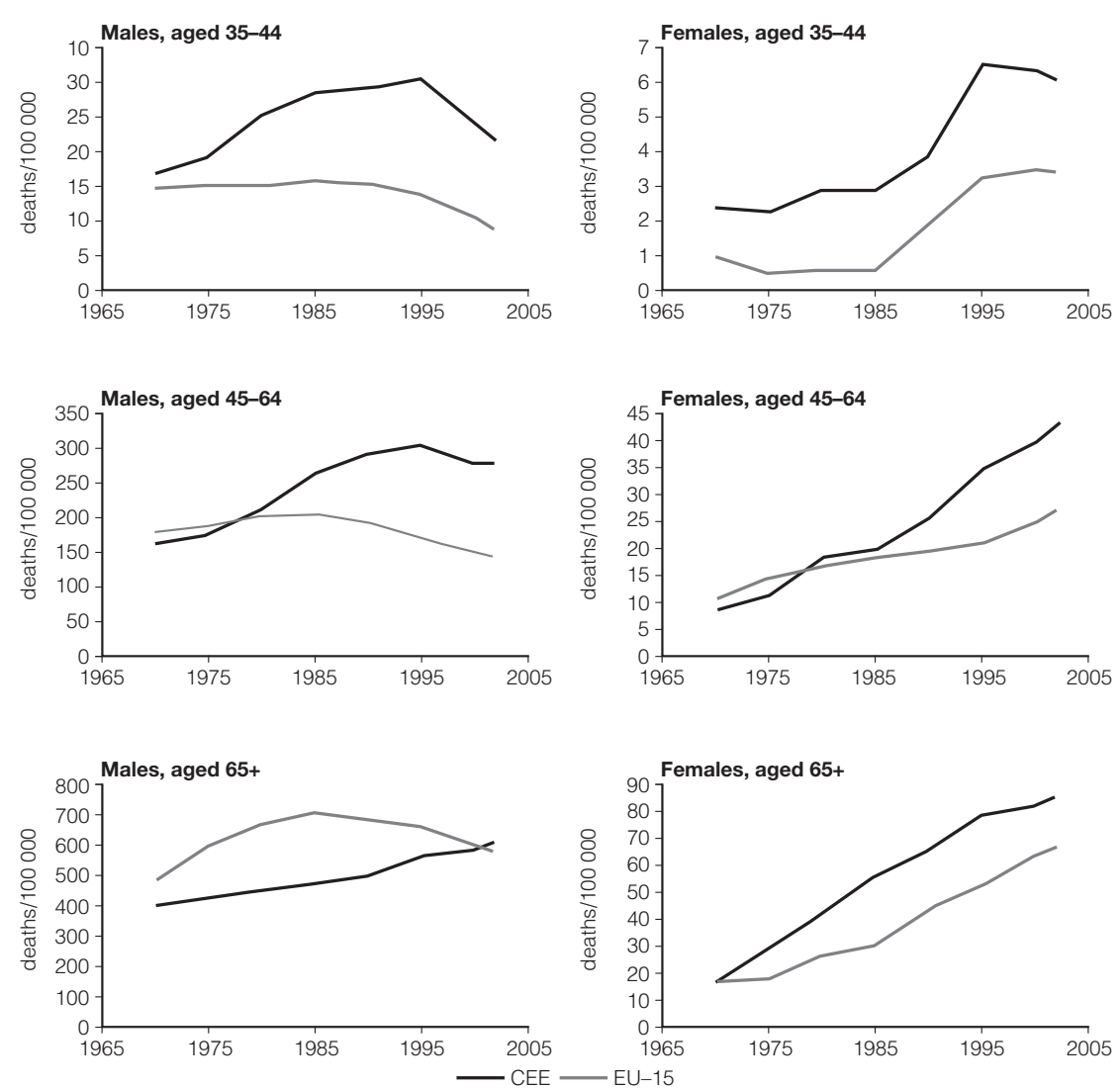

Note: CEE 1970-1980: Bulgaria, Czech Republic, Hungary, Poland, Romania, Slovakia, Slovenia; CEE 1985-2002: as before, plus Estonia, Latvia, Lithuania.

Fig. 13-3 Smoking-attributable death rates from all cancers in EU countries

In contrast, in the female population, the level of alcohol-attributed death rates is higher in the EU15 and this difference is widening with increasing age. In middle-aged women (45-64) the difference is minor (7/100 000 compared to $8.5 / 100000$ while in the oldest age group the rate is more than twice as high in the EU15 as in CEE (22/100 000 vs 10/100 000).

\section{Secondary screening}

Population-based screening programmes have been shown to be effective in reducing mortality from a number of cancers, including cervical, breast and colorectal cancer. These programmes were introduced from the 1950s and 1960s onwards in many parts of western Europe (e.g. cervical cancer screening in Finland) (Anttila et al., 1999). 


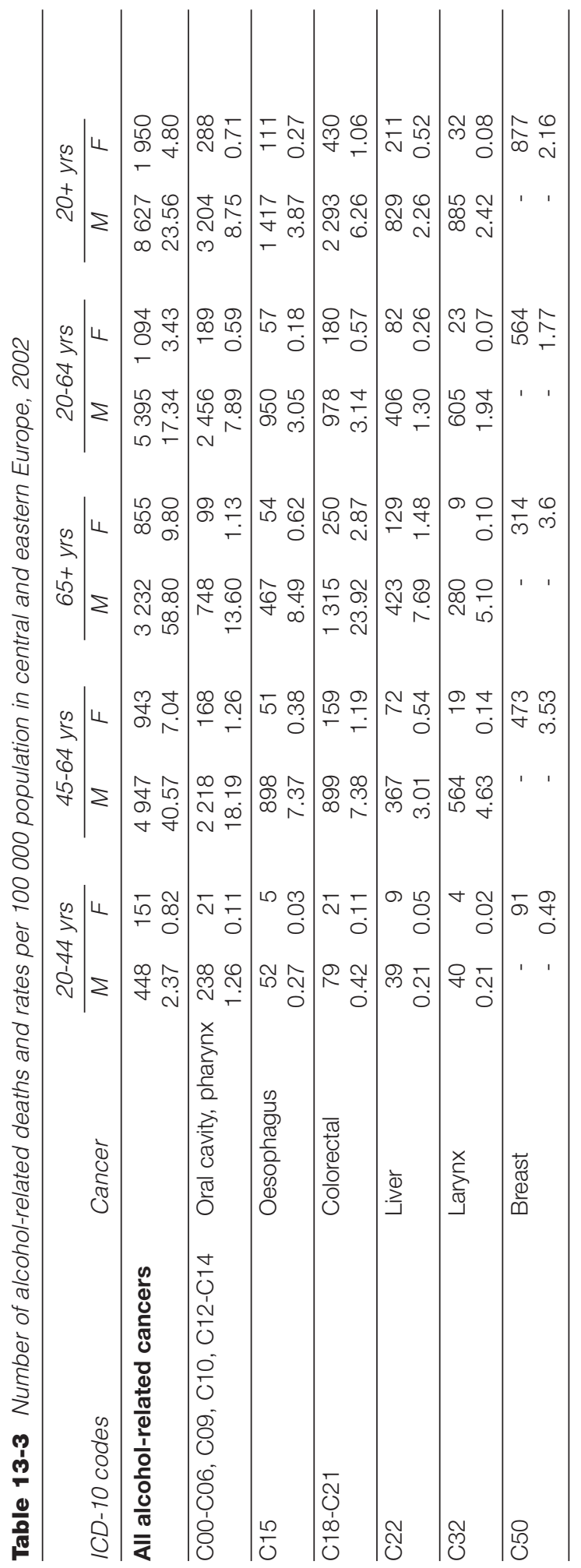




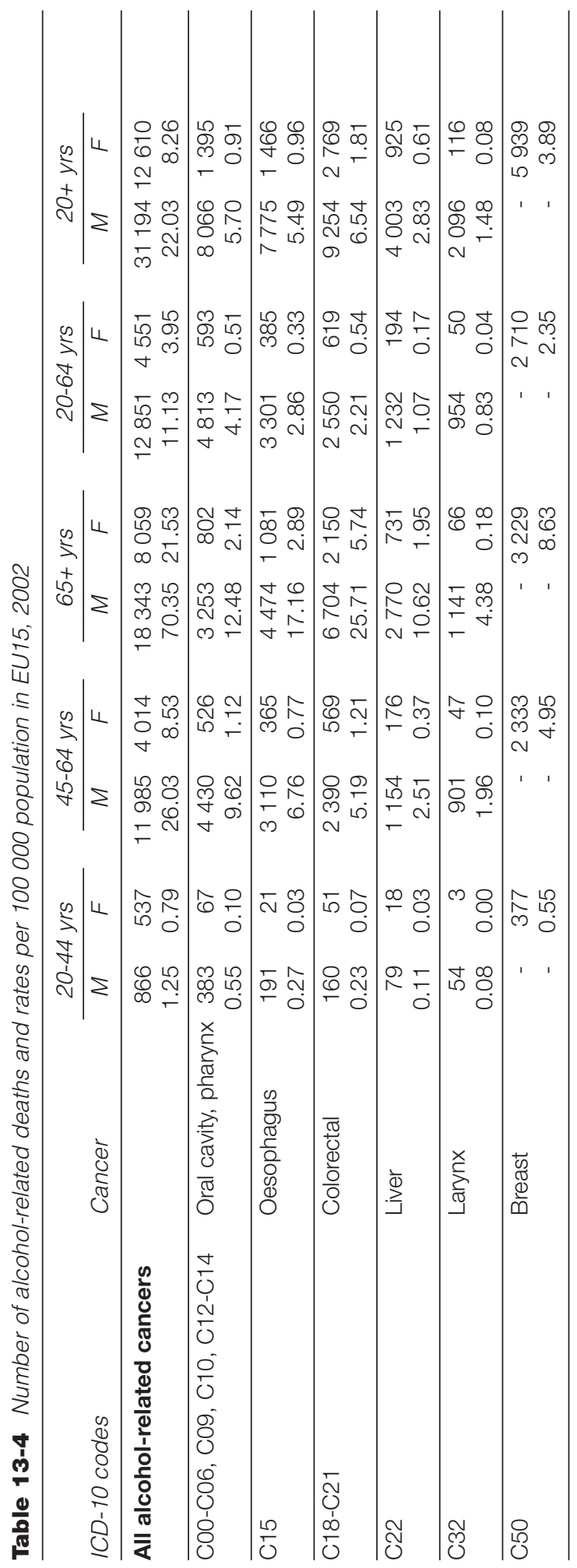




\section{Cancer of cervix uteri}

At the beginning of the $21^{\text {st }}$ century, the annual number of new cases of cervical cancer in CEE was estimated to be 13000 (in the EU15 the corresponding figure was 24000) (Ferlay et al., 2004). In 2002, in CEE almost 5700 deaths from cervical cancer were recorded (in the EU-15 the figure was about 5800). In the same year, the mortality rate in CEE (7.1/ 100 000) was more than four times higher than in the EU15 (1.7/100 000).

In western Europe, there has been a continuing decrease in mortality from cervical cancer at all age groups throughout the last half of the twentieth century (Fig. 13-4). Over that period, death from cervical cancer, especially in some north European countries, such as Finland (Hakama, Luostarinen \& Hakulinen, 2004), has become very rare.

This continuous decline is in sharp contrast with the situation in CEE. Cervical cancer mortality is significantly higher in each of the CEE countries than the average for the entire EU15. A decline observed in some CEE states (Slovenia, Czech Republic, Slovakia, Hungary, Estonia and Poland) began much later (in the 1970s and 1980s) and has been much slower than in the EU15. However, in the four remaining CEE countries (Latvia, Bulgaria, Lithuania and Romania) there is a continuing increase in cervical cancer mortality. The most dramatic case is Romania, where mortality rates have reached levels never before observed in Europe. In 2002, the difference in cervical cancer mortality between the EU15 average (1.7/100 000) and Romania (11/100 000) was six-fold.

\section{Breast cancer}

Breast cancer is another disease where screening and early treatment have been successful in diminishing mortality substantially. A 20-30\% decrease in breast cancer mortality has been observed in England and Wales (Quinn \& Allen, 1995) and the United States in the last decade (American Cancer Society, 2007). Annually, 41000 women in CEE are diagnosed with breast cancer (238653 new cases in the EU15) (Ferlay et al., 2004).

Breast cancer mortality rates are higher amongst all age groups in the EU-15 than in CEE. Earlier increases in mortality rates have, however, been arrested in almost all countries of the EU and, since the beginning of the 1990s, stagnation or even a decline in mortality levels have been observed, both in EU-15 and some CEE countries. This decline appeared earlier in EU15 countries and has been much faster than in CEE. In Romania and the Baltic States, no decline in mortality has yet been observed (Fig. 13-5). 

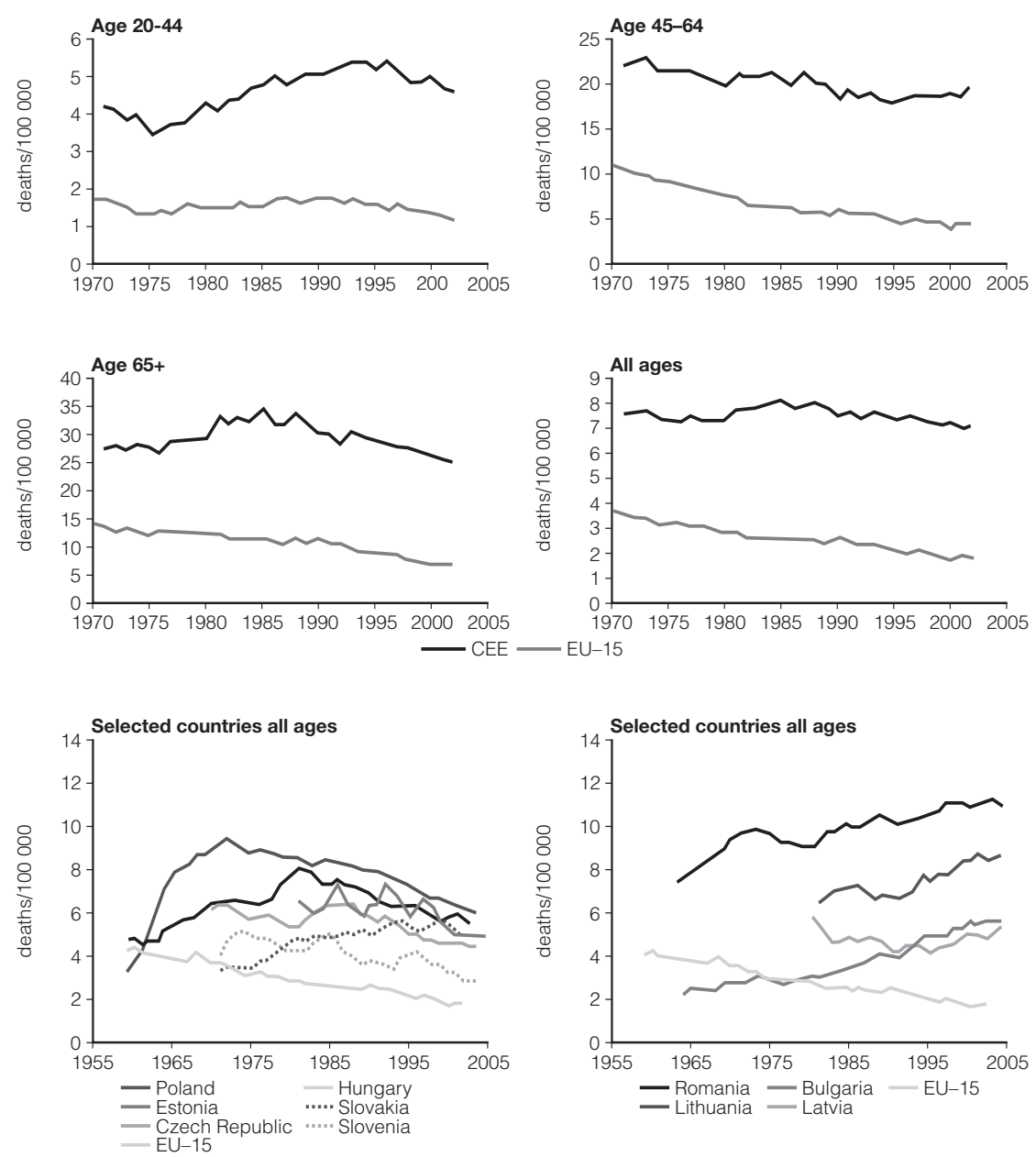

Fig. 13-4 Cervical cancer mortality

\section{Colorectal cancer}

In 2002, the number of incident cases of colorectal cancer was estimated at about 47000 in CEE countries and 238000 in the EU15 (Ferlay et al., 2004). There were 29000 deaths in CEE and 112000 in the EU15. Mortality rates in CEE were 21.4 per 100000 for males (16.5/100 000 in EU15) and 11.9 per 100000 for females (10/100 000 in EU15).

Colorectal cancer is a more frequent cause of death in CEE than in EU15 countries among both women and men and in all age groups. However, the mortality rate in the Czech Republic, Hungary and Slovakia is almost twice that in other CEE countries. For both sexes the lowest mortality is seen in Romania. In CEE countries, trends in colorectal cancers are characterized by 

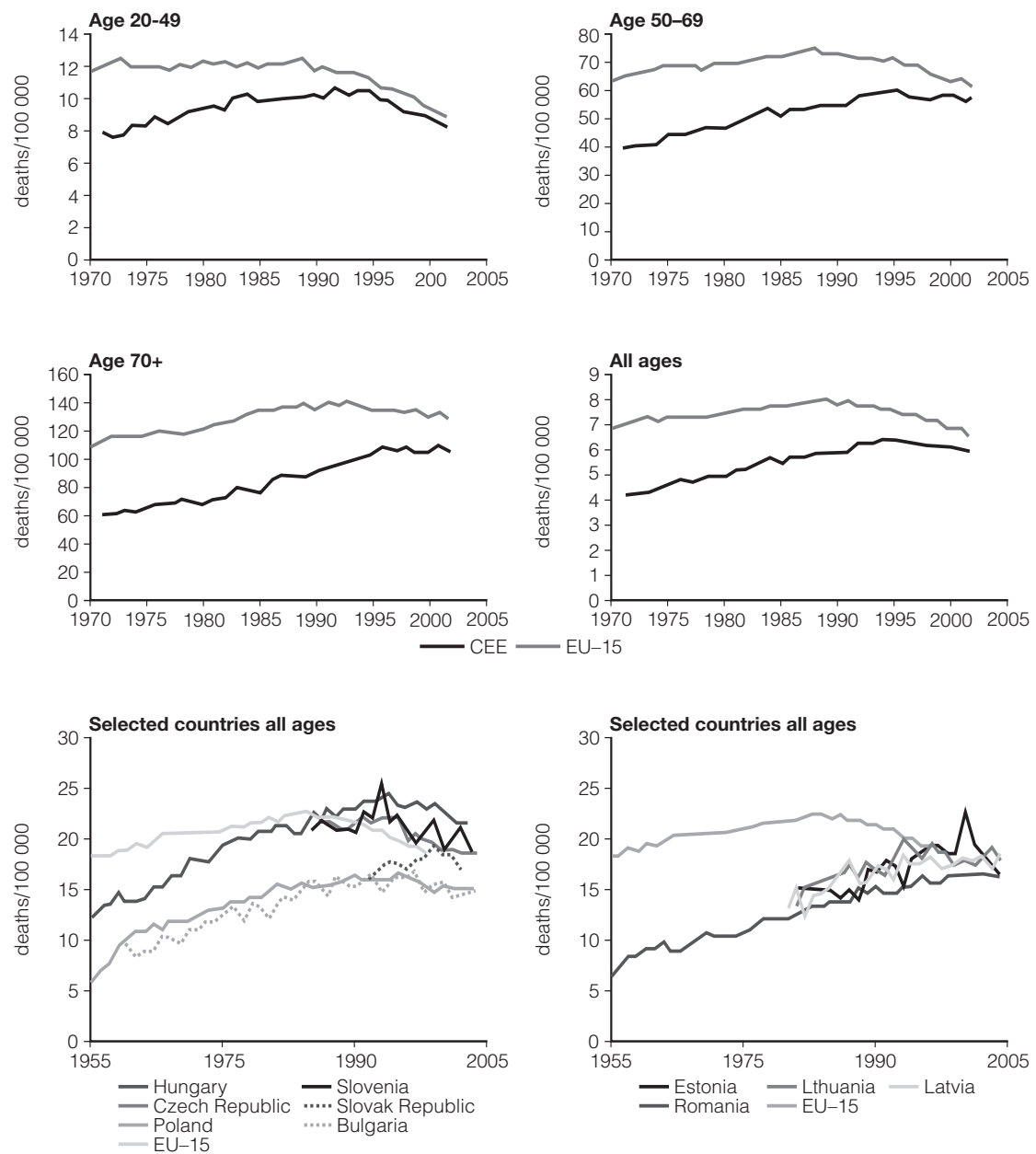

Note: 1971-1985: Poland, Hungary, Bulgaria and Romania only.

Fig. 13-5 Breast cancer mortality

a steady increase in almost all age groups, with the exception of young adults (20-44 years) (Fig. 13-6).

This contrasts with the situation in the EU15 where, in both sexes and all age groups, colorectal cancer mortality remains at the same level or is falling, with the latter especially pronounced amongst women. It should be noted that deaths from colorectal cancers are twice as frequent among men as among women, in CEE and EU15 countries.

\section{Tertiary prevention (treatment)}

Testicular cancer is one of the few cancers to have seen a dramatic improvement in the outcome of treatment in many countries. The 

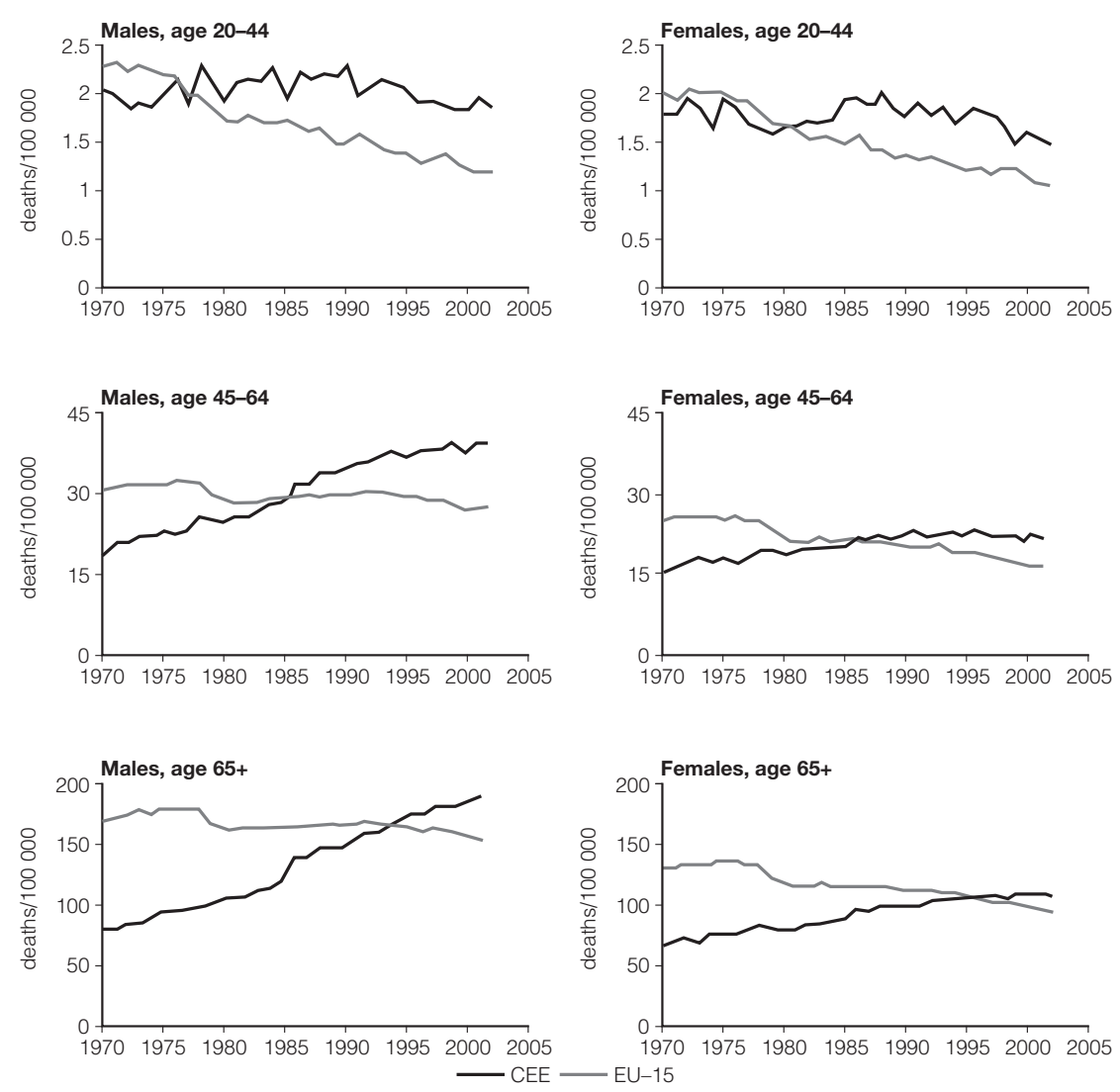

Note: 1971-1985: Poland, Hungary, Bulgaria and Romania only.

Fig. 13-6 Colorectal cancer mortality rates

combination of new treatments and evidence-based packages of care have led to reductions in mortality of up to $90 \%$ in some countries, making this cancer a candidate for an indicator of the diffusion of evidence-based medicine in CEE countries (Fig. 13-7).

Testicular cancer is now a rare cause of death (316 deaths in the entire male population of CEE and 2637 deaths in the EU15 in 2002). The mortality rate in CEE was 0.52 per 100000 (0.27/100 000 in the EU15).

Testicular cancer mortality rates have decreased in all age groups in both regions. However, the decrease in mortality began earlier in the EU15 countries - in the mid 1970s - and the pace of decline is more rapid. In CEE the decline began only around 1990, when access to modern treatment became possible. By 2002, mortality rates were still around twice as high in $\mathrm{CEE}$ as in the EU15. 

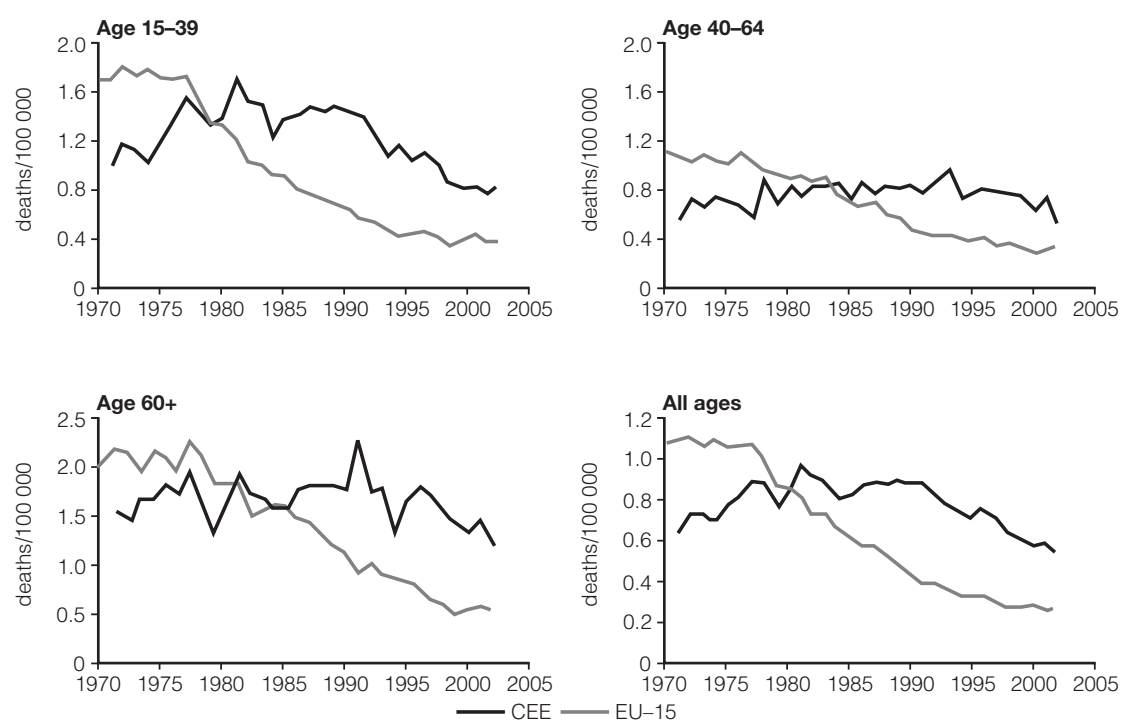

Note: 1971-1985: Poland, Hungary, Bulgaria and Romania only.

Fig. 13-7 Testicular cancer mortality

\section{Discussion}

In many regards, the pattern of health in the majority of CEE countries has now returned to the normal European pattern. A rapid decline in morbidity and mortality from cardiovascular diseases in the post-transition period will progressively close the gap with western Europe. This will leave cancer as by far the most important public health problem (Zatoński, 2007). There is a clear need for the creation of well-resourced, comprehensive, multifaceted programmes of cancer control in the eastern countries of the EU. These must combine research with the design, implementation and monitoring of cancer control programmes, ensuring that their activities are brought up to the standards already in place in many EU15 countries (Boyle et al., 2003a).

Our analysis seems to indicate that the greatest possibilities, but also the greatest unmet need, lie in secondary prevention (well-organized populationbased cancer screening). The clearest illustration of this need, over half a century in some countries of eastern and central Europe, is the dramatic increase in deaths from cervical cancer - deaths that are now almost entirely preventable.

There is also significant scope for gains from primary prevention especially, as can be seen in countries such as the United Kingdom and Finland, in tobacco control. Despite a dramatic improvement in knowledge and attitudes on 
tobacco smoking in the countries of the "new" EU, there is still much to be done. In some CEE countries, cigarettes are still cheaper than bread, even though pricing is widely accepted as among the most effective forms of tobacco (and thus cancer) control. The scale of the mountain to climb is illustrated by the fact that, in some eastern European countries, there is a higher proportion of smokers among medical doctors (who should be health role-models) than there is in the general population (Zatoński, 2004).

Finally, smoking amongst women poses a particular problem in CEE. This is apparent from the extraordinarily high level of lung cancer amongst young and middle-aged (20-64) women in Hungary. This is even higher than the rate in the male population in the EU15. In some other CEE countries (Romania, Lithuania), the prevalence of smoking and lung cancer in the female population is still amongst the lowest in Europe. This will be difficult to maintain, given the intensity of activity by tobacco companies seeking new markets.

In the last decade the CEE countries have achieved significant success in fighting against cancer through tobacco control (as in many EU-15 countries). The observed decline in the risk of smoking-attributable cancer mortality in young adults is particularly noteworthy (although it is still higher than in the EU15) (Didkowska et al., 2005; Zatoński et al., 2007). However, achievements have been much less in the population over the age of 45 . This fact emphasizes the need for wide-ranging effective interventions in the field of smoking cessation (Zatoński, 2000). Engaging health providers in tobacco control ("medicalization" of tobacco control) is a challenging task, especially in CEE countries. The need to address nicotine dependency is still inadequately recognised (Zatoński, 2000).

Among younger generations of EU citizens, the rates of smoking among men and women are converging. In a few countries (Sweden, United Kingdom, Ireland, the Netherlands, Denmark) they are reaching higher levels among women. A similar trend is now emerging with lung cancer, as is shown by the example of Sweden (Zatoński, 2007; Zatoński, 2003).

Another unsolved problem in the EU is the price of cigarettes, which remains at a very low level in CEE countries. A failure to increase taxes in CEE, at a time when taxes are increasing in many EU15 countries, is leading to a widening price gap (Joossens \& Raw, 2006).

To conclude, more than half a century after the discovery of the causal relation between smoking and lung cancer (Doll \& Bradford Hill, 1950), it is important to reflect on why the problem of tobacco control in the EU remains unresolved. It remains the most important issue, not just for cancer control 
but also for public health as a whole. Smoking, which remains the greatest single preventable cause of cancer (and ill health) in the EU, kills almost 350000 Europeans every year.

Europe is the region with the highest level of alcohol consumption in the world; this is reflected in the highest level of alcohol-attributable cancer mortality rates (Popova et al., 2007; Rehm et al., 2007). Europe is also characterized by a variety of styles of drinking alcohol. Northern Europe is dominated by binge drinking ${ }^{9}$, while southern countries are characterized by the Mediterranean style of drinking ${ }^{10}$ (La Vecchia, 2001). Unfortunately, binge drinking is rapidly becoming more popular amongst youths throughout the entire continent. Moreover, alcohol abstinence is extremely rare, especially amongst young Europeans.

Another factor connected with alcohol-attributable cancer risk, especially in $\mathrm{CEE}$, is the nature of the products being drunk. Unfortunately there are very few scientific studies on this subject. However, it is known that the phenomenon of drinking alcohol surrogates, such as aftershaves containing high concentrations of ethanol, is not uncommon (Lang et al., 2006). In south-eastern European countries, a significant portion of consumed alcohol is poorly distilled home-made fruit brandy, which contains significant levels of toxic-long-chain alcohols (Szucs et al., 2005). In Poland, a significant segment of the population (especially the least educated and poorest) consumes considerable amounts of very cheap alcohol in the form of "fruit wine". This is not made from grapes, but information about the chemical consistency and biological consequences of these types of alcohol is very fragmentary. As this brief review shows, alcohol, as a cause of cancer but also from a wider public health perspective, is still far from being controlled in the EU (McKee, 2006).

The problem is further complicated by the lack of a common pricing policy for alcohol in the EU. In many CEE countries, access to the EU was linked with "price harmonization" to the level of Mediterranean countries. In 2002, the price of vodka fell by $30 \%$ in Poland as part of the preparation for EU accession (National Statistics Office, 2006). This immediately led to an increase in vodka consumption and negative health effects, especially amongst the least well-educated and poorest males (National Statistics Office, 2006). Future progress to control alcohol-attributable cancers, particularly oral cavity

\footnotetext{
${ }^{9}$ A pattern of drinking alcohol that corresponds to males consuming five or more drinks (four or more for females) in about two hours. A drink in this context is half an ounce of alcohol (e.g. in a 12 oz. beer, a 5 oz. glass of wine, or a $1.5 \mathrm{oz}$. shot of distilled spirits). For some individuals (e.g. older people; those taking other drugs or certain medications), the number of drinks needed to reach a binge-level blood alcohol content is lower than for the 'typical adult' (Plant \& Plant, 2006)

${ }^{10}$ Daily drinking of alcohol with meals, usually wine, in moderate quantity.
} 
and laryngeal cancers, requires a coherent EU social policy on alcohol, especially in CEE countries.

An obvious way of avoiding death from cancer, even at an already advanced stage, is to cure cancers that develop. However, there have been few major breakthroughs in cancer treatment, in the sense of turning a fatal tumour into a curable one. Significant successes have been accomplished in the treatment of testicular teratoma, Hodgkin's disease, childhood leukaemia, Wilms' tumour and choriocarcinoma. However, the increase in survival of patients suffering from the most common types of cancer is still far from satisfactory.

For many cancers, the chance of successful treatment increases significantly if the disease is diagnosed at an early stage in its development. Awareness of the signs and symptoms of early disease is very important. Nevertheless, it has to be emphasized that often the first symptoms of cancer appear only after it has already developed to an advanced stage. In order to diagnose cancer during the phase when no symptoms are present, population-based screening may be done if the evidence warrants it (see Chapter 4). Cancer detection at an early, symptom-free phase can often reduce mortality. This is especially true in the case of those cancers for which early diagnosis and treatment can reduce metastasis. Thus, early detection is an important factor influencing the mortality rates (Boyle et al., 2003a).

Population-based secondary prevention (screening) was underemphasized in CEE countries for many years. leading to a significant delay in implementing effective cancer control in this part of Europe. The benefits of evidence-based screening were recognized much earlier in the EU15, especially in the case of cervical cancer and later breast cancer among women (Zatoński et al., 1996).

In CEE countries, a particularly unfavourable situation exists in relation to cervical cancer. Mortality is four times higher than in EU15 countries and this gap is not diminishing over time. Cervical cancer mortality in most European countries has decreased dramatically since the beginning of the 1960s. Finland became a model of a successful fight against the disease using a comprehensive population screening programme (Anttila et al., 1999).

In CEE it is possible to identify two developments.Firstly, there are countries where the decrease in mortality began in the 1970s and 1980s (Poland, Hungary, Czech Republic, Slovakia, Slovenia and Estonia), but these changes were much slower than in western European countries and, by the beginning of the $21^{\text {st }}$ century, there remained a very significant gap with the EU15 average. In CEE, cervical screening is typically opportunistic and far from complete, especially amongst the least educated women in rural areas. Secondly, there is another group of countries (Romania, Bulgaria, Latvia and 
Lithuania) exhibiting increasing cervical cancer mortality to levels unprecedented in Europe (Zatoński, Jeziorski \& Tyczynski, 1990; Zatoński et al., 1996). This situation, which contrasts dramatically with international experience, arose during the socioeconomic transition, perhaps reflecting a collapse in collective health programmes.

In all the countries that form CEE, there is an urgent need for a swift introduction of effective, population-based screening programmes supplemented with intensive health information campaigns. Education is a major priority, especially that targeted at less educated, less wealthy women living in rural areas. Well-planned and organized screening programmes could bring mortality in CEE countries down to the level in the EU15. However, this will require several decades (Didkowska et al., 2006).

Another very important consideration in the implementation of screening programmes in CEE countries is their cost and effectiveness. The cost depends on the frequency of screening (Mandelblatt et al., 2002; van Ballegooijen et al., 2000). It is important to draw on international experience; this shows (assuming that screening is available for women aged 20-64) that there is little difference in the reduction in mortality rates achieved with different screening intervals between three and five years: 97\% reduction in mortality rates with three-year intervals; 90\% reduction with five-year intervals (Mandelblatt et al., 2002; Miller, 1999).

The next important factor influencing the effectiveness of screening is its completeness. In Finland, after a few decades of cervical cancer screening, attendance by invited women rose to 75\% (Cancer Society of Finland, 2005). Polish experience shows how low attendance rates can lead to programme failure - only $42 \%$ attendance was achieved in a pilot study (Chosia, Bedner $\&$ Domagala, 2001). In addition, according to a study from 2006, Poland has a substantial percentage of women who have never had a cervical smear $-30 \%$ among the least educated (National Statistics Office, 2006). There is a clear need for enhanced educational programmes (e.g. based on popularization of the European Code Against Cancer) and implementation of a personal invitation system (Boyle et al., 2003a).

Historically, the incidence and mortality of breast cancer has been significantly higher in western than in eastern Europe. However, in the last decade, dramatic changes in reproductive behaviour in eastern Europe have been accompanied by a dramatic increase in breast cancer morbidity (Smallwood \& Chamberlain, 2005; Zatoński et al., 1996). The case of Poland illustrates this phenomenon well. In the last two decades a considerable increase in incidence has been observed. Between 1985 and 2004 an accelerating increase in 
incidence rates (from 26 per 100000 in 1985 to 40 per 100000 in 2004) was observed; at the same time the number of incident cases grew from 6000 to 12000 (Wojciechowska et al., 2006).

In the next few decades we can expect an increase in breast cancer incidence in CEE. Prevention can be achieved only by implementation of widespread screening, with as high a level of coverage as possible, linked to rapid access to treatment by women in whom cancer is diagnosed. Both these activities need sufficient financial support.

Colorectal cancer also requires special attention in CEE countries. The highest mortality rates in Europe are observed in the Czech Republic, Slovakia and Hungary. In addition, this cancer is characterized, as the case of Poland shows, by one of the most rapid rates of increase of all cancers. The existing epidemiological situation seems to indicate that the gap in colorectal cancer mortality between the "old" and the "new" parts of the EU is still growing. This development requires an immediate response (Zatoński et al., 1996).

The problem is complicated by the options for colorectal cancer screening. Colonoscopy screening is recommended due to its high specificity and sensitivity. This examination, conducted once or twice in a lifetime, is emerging as the best method to reduce individual risk of death from colorectal cancer. The first positive observations from a pilot programme in Poland seem to support this thesis (Regula et al., 2006).

However, colorectal cancer screening needs to overcome a few difficulties, such as the very large cost of this type of screening (training of staff, purchase of equipment, preparation of the colon) (van Ballegooijen et al., 2000). Another problem appearing in all screening programmes in CEE is widespread "cancer illiteracy". Overcoming this problem requires spreading knowledge about the benefits that result from participating in screening programmes, with the aim of changing attitudes among the general public and encouraging active participation in existing programmes.

The last two decades have seen appreciable progress in the treatment of some cancers. Introduction of new methods of chemotherapy and improved technologies allowing more precise diagnosis meant that more and more patients can receive treatment adjusted to their needs. The effectiveness of new treatments is apparent in the cases of Hodgkin's disease and testicular, breast and colorectal cancers.

Differences in cancer mortality between CEE and EU15 countries arise for several reasons. One is delay in the introduction of new clinical interventions, apparent from the comparison of trends in mortality from testicular cancer. 
The decrease in mortality seen initially in western Europe is the result of the introduction of cisplatinum to the treatment regime in the 1970s. In CEE countries, this treatment only became available over a decade later, which explains the 10-15 year delay in the decline in mortality (Zatoński et al., 1996). However, new methods of treatment are effective when introduced into CEE countries - Poland has experienced continued decreases in mortality despite a dramatically increasing trend in incidence (Zatoński, Jeziorski \& Tyczynski, 1990).

Another reason is, once again, a lack of knowledge and awareness: patients do not recognize very early symptoms of cancer or they neglect the symptoms and visit doctors too late (where they sometimes do not receive specialist help). Limited budgets for health care in CEE countries are insufficient to ensure adequate treatment and care for all patients. This is a major factor in limited access to the newest treatment methods for breast cancer among women over 70 and testicular cancer among elderly men (Zatoński et al., 1996).

\section{Conclusions}

The pattern and time trends in cancer incidence and cancer mortality are, in many respects, similar in the eastern and western parts of the EU. However, for each cancer site, in CEE there is a variable degree of delay in the emergence of trends. One consequence is that, at last, there are some favourable changes reflecting those seen a decade or more earlier in the EU15 (e.g. the levelling off in the increase of lung cancer among men).

The best prospects for progress, given current levels of knowledge, are to be found in prevention. Both primary and secondary prevention are especially neglected in CEE, as the epidemiological analysis shows. Cervical cancer is the best example. In almost half of the countries of eastern Europe, cervical cancer mortality is still increasing.

Cancer policy in CEE has a persisting problem with the low level of awareness in society (cancer illiteracy). The least educated and less wealthy in the population, in particular, have little knowledge of the importance of prevention (especially in the case of smoking) as a means of cancer control. It is essential to implement national education programmes designed to change attitudes towards cancer (building on initiatives such as the European Code Against Cancer).

Citizens in the EU15 have profited most from the introduction of cheap and effective programmes of primary prevention, exemplified by the success of 
those aimed at smoking. It can therefore be predicted with confidence that similar gains can be made in CEE.

Secondary prevention of cancer in CEE is especially neglected. For instance, relatively cheap cervical cancer screening programmes are not in place, again reflecting cancer illiteracy. In this part of the EU there is a need to create national, evidence-based, well-organized screening programmes.

The benefits of modern approaches to treatment only reach CEE countries after some delay (e.g. for testicular cancer). This is perhaps less surprising, given the high cost of medication in settings where resources are constrained and the benefits of investment in health are still not adequately recognized (Suhrcke, Rocco \& McKee, 2007).

Education of societies about the responsibility of the individual for one's own health is a clear priority, but this should be complemented by acceptance of responsibility by the state to create national cancer control programmes.

Improvements are possible only if cancer control interventions, based on the modern concept of public health (primary prevention, secondary prevention, and modern medical treatment), can be integrated. In many European countries, especially in the EU15, the burden of cancer has been tackled to varying degrees. This success was achieved not only by increasing expenditure on medical treatment, but also (and especially) by changing attitudes within society linked to well-organized primary prevention programmes (in particular reductions in smoking) and secondary prevention (screening).

\section{Acknowledgements}

The work on this paper was conducted partly within the European Commission's public heath project: HEM - Closing the Gap - Reducing Premature Mortality. Baseline Monitoring Health Evolution Following Enlargement (grant agreement no 2003121) and a collaborative grant from IARC.

The authors would like to thank their collaborators in the Division of Cancer Epidemiology and Prevention: Urszula Wojciechowska, Marta Mańczuk, and Urszula Sułkowska for help in epidemiological analysis; Łucja Didkowska, Mateusz Zatoński and Aleksandra Herbeć for help in creating the English version of the text; and Joasia Szwechowicz and Ewa Tarnowska for help in editing the text and illustrations. 


\section{REFERENCES}

American Cancer Society (2007). 2007 cancer statistics. Washington, American Cancer Society. Anttila A et al. (1999). Effect of organised screening on cervical cancer incidence and mortality in Finland, 1963-1995: recent increase in cervical cancer incidence. Int J Cancer, 83(1):59-65.

Boyle P et al. (2003). European Code Against Cancer and scientific justification: third version (2003). Ann Oncol, 14(7):973-1005.

Boyle P et al. (2003a). Measuring progress against cancer in Europe: has the 15\% decline targeted for 2000 come about? Ann Oncol, 14(8):1312-1325.

Cancer Society of Finland (2005). Cancer in Finland 2002 and 2005. Helsinki, Cancer Society of Finland.

Chosia M, Bedner E, Domagala W (2001). Czy cytologiczne badania przesiewowe moga obnizyc w Polsce wspólczynnik umieralnosci z powodu raka szyjki macicy? (Can cytology screening reduce mortality due to cervical cáncer in Poland?) Nowotwory, 51:523-529.

Didkowska J, Wojciechowska U, Zatonski W (2006). Nowotwory szyjki macicy w Polsce epidemiologiczny bilans otwarcia i'perspektywy. (Cervix uteri cancer in Poland epidemiological opening balance and perspectives). Gin. Pol, 77(9):660-666.

Didkowska J et al. (2005). Lung cancer mortality at ages 35-54 in the European Union: ecological study of evolving tobacco epidemics. Br Med J, 331(7510):189-191.

Doll R, Hill AB (1950). Smoking and carcinoma of the lung. Preliminary report. Br Med J, ii:739-748.

Dyba T, Hakulinen T (2000). Comparison of different approaches to incidence prediction based on simple interpolation techniques. Stat Med, 19(13):1741-1752.

European Network of Cancer Registries. Stata macros for short-term predictions (available at: http://www.encr.com.fr/stata-macros.htm, accessed 22 November 2007)

Ferlay J et al. (2004). GLOBOCAN 2002: Cancer incidence, mortality and prevalence worldwide. IARC CancerBase No. 5. version 2.0. Lyon, France, IARC Press.

Hakama M, Luostarinen T, Hakulinen T (2004). Survival of in situ carcinoma of cervix uteri: a 50-year follow-up in Finland. Int J Cancer, 112(6):1072-1074.

IARC (1988). Alcohol drinking. IARC Monograph 1988:44. Lyon, International Agency for Research on Cancer.

Joossens L, Raw M (2006). The tobacco control scale: a new scale to measure country activity. Tob Control, 15(3):247-253.

La Vecchia C (2001). Alcohol in the Mediterranean diet: benefits and risks. Int J Vitam Nutr Res, 71(3):210-213.

Lang $\mathrm{K}$ et al. (2006). The composition of surrogate and illegal alcohol products in Estonia. Alcohol Alcohol, 41(4):446-450.

Mandelblatt JS et al. (2002). Costs and benefits of different strategies to screen for cervical cancer in less-developed countries. J Natl Cancer Inst, 94(19):1469-1483.

McKee M (2006). A European alcohol strategy. Br Med J, 333(7574):871-872.

Miller AB (1999). The brave new world - what can we realistically expect to achieve through cancer control early in the new millennium? Chronic Dis Can, 20(4):139-150.

National Statistics Office (2006). GUS, Warsaw, National Statistics Office.

Parkin DM et al. (1997). Cancer incidence in five continents. Vol. VII. (IARC scientific Publications No. 143). Lyon, IARC.

Peto R (1994). Mortality from smoking in developed countries, 1950-2000: indirect estimates from national vital statistics. Oxford, Oxford University Press.

Plant M, Plant M (2006). Binge Britain. Oxford, Oxford University Press. 
Popova $S$ et al. (2007). Comparing alcohol consumption in central and eastern Europe to other European countries.Alcohol and Alcoholism, 2007 42(5):465-473; doi:10.1093/alcalc/agl124

Preston SH, Heuveline P, Guillot M (2001). Demography: measuring and modeling population processes. Oxford, Blackwell Publishers.

Quinn MJ et al. (2003). Cancer mortality trends in the EU and acceding countries up to 2015. Ann Oncol, 14(7):1148-1152.

Quinn M, Allen E (1995). Changes in incidence of and mortality from breast cancer in England and Wales since introduction of screening. Br Med J, 311:1391-5.

Regula J et al. (2006). Colonoscopy in colorectal cancer screening for detection of advanced neoplasia. N Engl J Med, 355(18):1863-1872.

Rehm J et al. (2007). Alcohol accounts for a high proportion of premature mortality in central and eastern Europe. Int J Epidemiol, 36(2):458-467.

Smallwood S, Chamberlain J (2005). Replacement fertility: what has it been and what does it mean? Pop Trends, 119 (Spring):16-27.

Suhrcke M, Rocco L, McKee M (2007). Health: a vital investment for economic development in eastern Europe and central Asia. Copenhagen, European Observatory on Health Systems and Policies.

Szucs $S$ et al. (2005). Could the high level of cirrhosis in central and eastern Europe be due partly to the quality of alcohol consumed? An exploratory investigation. Addiction, 100(4):536542.

United Nations (2001). World population prospects: the 2000 revision medium variant, 20012050. Department of Economic and Social Affairs of United Nations.

van Ballegooijen M et al. (2000). Overview of important cervical cancer screening process values in European Union (EU) countries, and tentative predictions of the corresponding effectiveness and cost-effectiveness. Eur J Cancer, 36(17):2177-2188.

WHO database. Geneva, Switzerland (available at:http://www.who.int/whosis/en/, accessed 1 December 2007)

Wojciechowska U et al. (2006). Nowotwory ztosliwe w Polsce w 2004 roku (Cancer in Poland in 2004). Warsaw, Centrum Onkologii - Instytut.,

Zatoński W (2000). Leczenie zespołu uzależnienia od tytoniu jest obowiązkiem lekarza, (Treatment of tobacco dependence syndrome is a physicians duty) Medipress, Supp.7:3-5.

Zatoński W (2003). Lung cancer trends in selected European Countries: what we can learn from the Swedish experience with oral tobacco (snus). In: European status report 2003 on oral tobacco. Brussels:37 54 .

Zatoński W (2004). Tobacco smoking in central European countries: Poland. In: Boyle P et al. eds. Tobacco and public health: science and policy. Oxford, Oxford University Press:235-252.

Zatoński W (2007). The east-west health gap in Europe - what are the causes? The Europ J Public Heath, 17(2):121.

Zatoński W, Boyle P (1996). Commentary. Health transformations in Poland after 1988. J Epid. and Biostatistics, 1(4):183-197.

Zatoński W, Jeziorski K, Tyczynski J (1990). Testicular cancer in Poland. Lancet, 336(8708):183.

Zatoński W, Jha P (2000). The health transformation in Eastern Europe after 1990: a second look (available at: http://www.hem.waw.pl/?idm=58,59\&cmd=1, accessed 1 December 2007) Warsaw, Cancer Center and Institute of Oncology.

Zatoński WA, McMichael AJ, Powles JW (1998). Ecological study of reasons for sharp decline in mortality from ischaemic heart disease in Poland since 1991. Br Med J, 316(7137):10471051. 
Zatoński WA, Willett W (2005). Changes in dietary fat and declining coronary heart disease in Poland: population-based study. Br Med J, 331(7510):187-188.

Zatoński W et al. (1996). Atlas of cancer mortality in central Europe. (IARC Scientific Publications No.134). Lyon, France, IARC.

Zatoński WA et al. (2007). Convergence of male and female lung cancer mortality at younger ages in the European Union and Russia. Eur J Public Health, 17(5): 450-454. 


\section{Chapter 14 \\ Cancer control in Slovenia: achievements, shortcomings and opportunities}

Maja Primic Žakelj and Tina Žagar

\section{Introduction}

As in other European countries, cancer is the second most frequent cause of death in Slovenia. In 2006, cancer incidence in Slovenia was lower than the estimated EU average for both sexes; mortality from cancer was slightly higher among males but lower among females (Ferlay et al., 2007).

The political transformation that Slovenia underwent in the previous decade had an impact on the lifestyle and health status of its population, as well as the organization of health care. Given the latent periods involved in cancer epidemiology, the consequences of these changes will continue to be seen in future years.

Interest in cancer control has a long history and one of the oldest populationbased cancer registries in Europe is based in Slovenia. Founded in 1950 at the Institute of Oncology Ljubljana, it covers the entire Slovenian population and publishes data on cancer incidence, prevalence and survival (Institute of Oncology).

\section{The burden of cancer in Slovenia}

During the last fifteen years, there has been a significant increase in both incidence of, and mortality from, cancer in Slovenia. In 1992-1994, there were (on average) 7334 new cases of cancer and 4454 cancer deaths; while in 


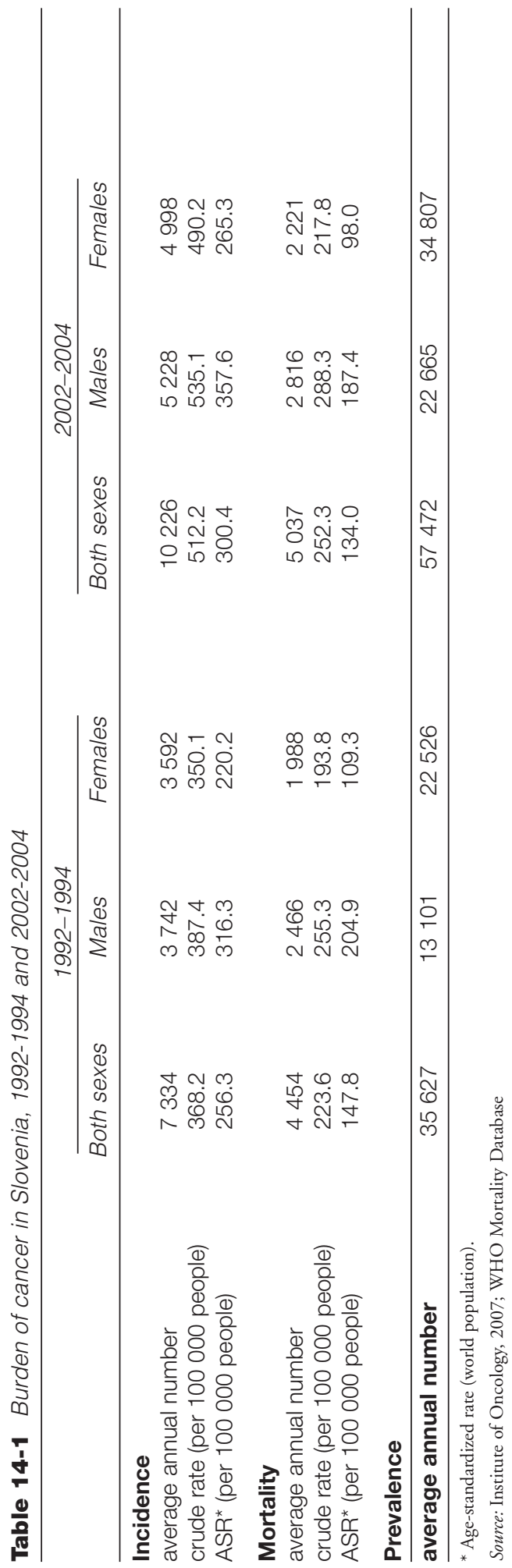




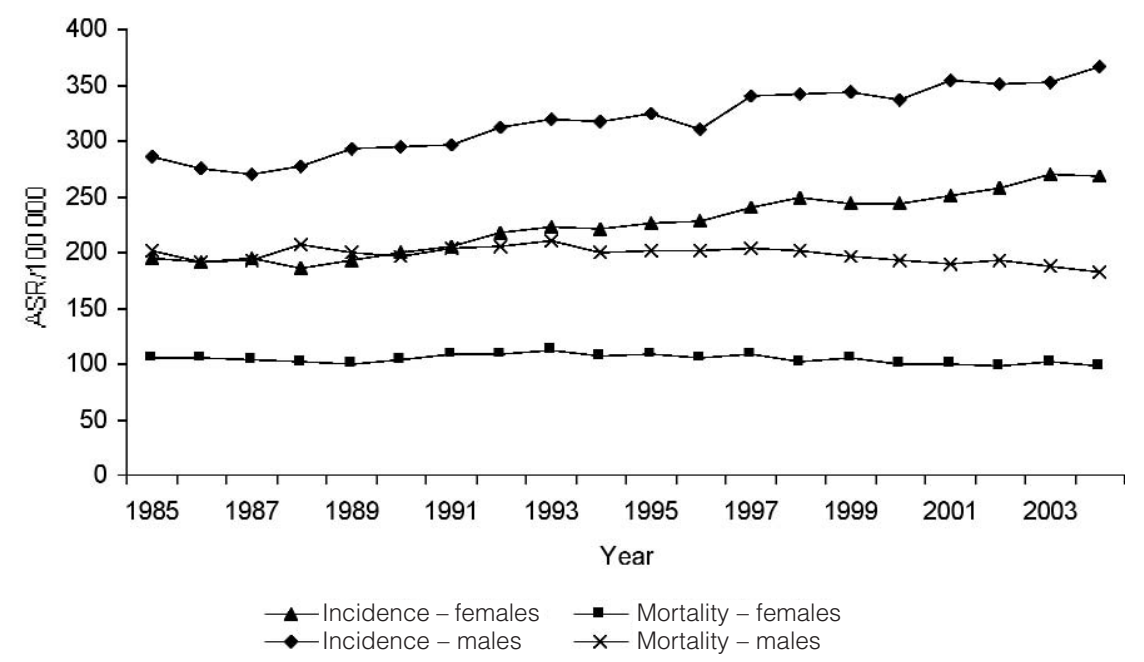

Source: Institute of Oncology; WHO Mortality Database.

Fig. 14-1 Trends in age-standardized cancer incidence and mortality rates by sex in Slovenia, 1985-2004

2002-2004, there were 10226 new cases of cancer and 5037 cancer deaths per year (Institute of Oncology, 2007). This corresponds to a 39\% increase in the crude incidence rate for both sexes; the crude mortality rate rose by $14 \%$ in males and $12 \%$ in females.

Trends in age-standardized cancer incidence and mortality rates during the period 1985-2004, for males and females, are presented in Fig. 14-1. Both incidence and mortality from cancer are higher in males than in females. While the cancer incidence rate is still increasing, mortality in males has been declining by $1.5 \%$ per year since 1997 ; in females there has been an annual decrease of $1.1 \%$ since 1993 .

The total burden of cancer is dominated by five types: non-melanoma skin, colorectal, lung, breast and prostate cancer, which represent about $56 \%$ of the total number of all new cases of cancer. Cancers of other sites are quite rare, accounting for fewer than 25 cases per 100000 people per year (Fig. 14-2).

The lung has been the most frequent cancer site among males since 1967, when it replaced stomach cancer. As the prevalence of adult male smokers has decreased over the last 30 years (from 48\% of smokers among men in 1975 to $20 \%$ in 2006), the age-standardized lung cancer mortality rate in males has started to decline in the last 10 years (from 62.6 deaths per 100000 in 1995 to 50.7 in 2004). However, the lung cancer mortality rate in females has been increasing slowly (from 9.8 per 100000 in 1995 to 11.0 in 2004), reflecting their similar and sustainable smoking habits (16\% of smokers among women 


\section{MALES}

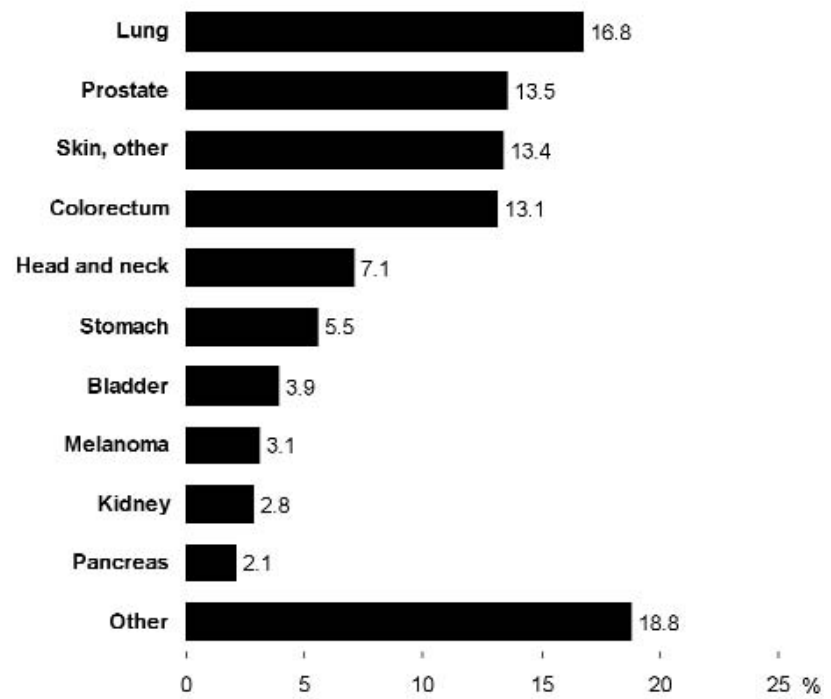

\section{FEMALES}

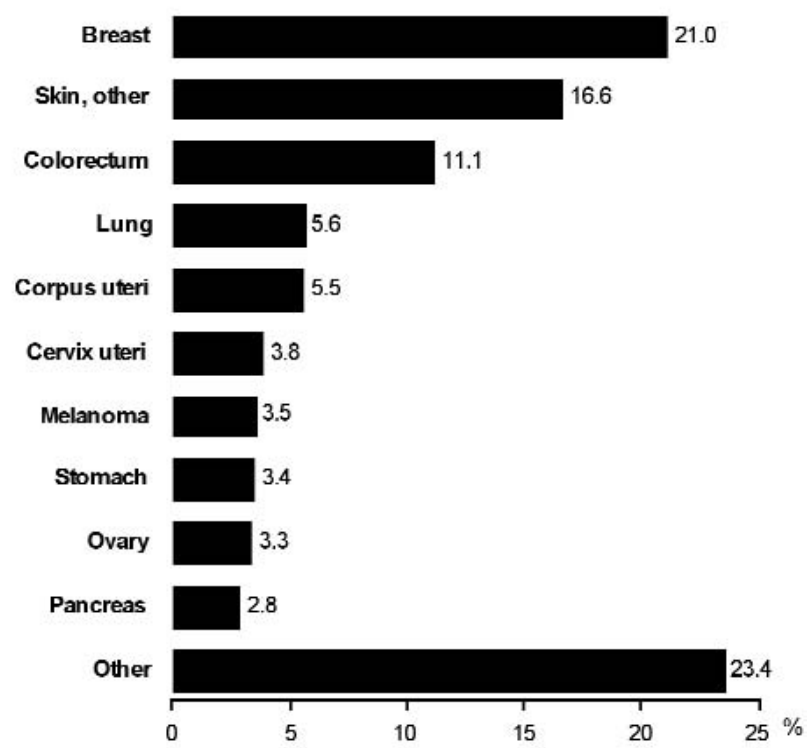

Source: Institute of Oncology

Fig. 14-2 Burden of cancer by site and sex, Slovenia 2004 

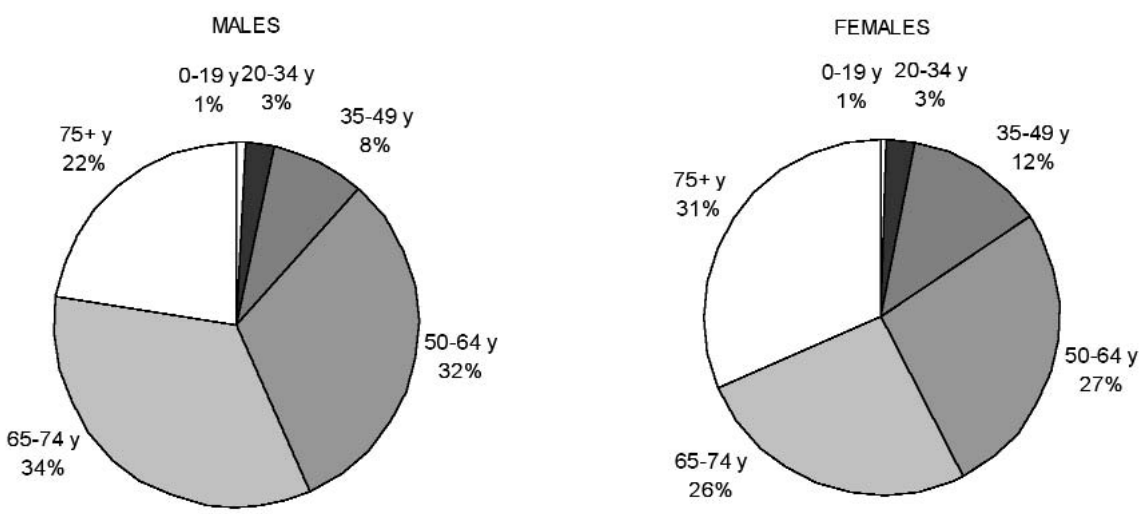

Source: Institute of Oncology

Fig. 14-3 Distribution (\%) of cancers by age and sex, Slovenia, 2004

in 1975; 20\% in 2006) (Toš, 1997; Toš, 1999; Toš, 2004; WHO Mortality Database).

The most frequent cancer site among women is the breast, accounting for $21 \%$ of all female cancers. An increasing trend in breast cancer incidence has been observed during recent years, followed by a slowly decreasing mortality rate for women aged 40-59 years, but not for older women (Tyczynski et al., 2004).

There has been an increasing trend in the incidence of colorectal cancer, nonmelanoma skin cancer, pancreatic cancer and non-Hodgkin lymphoma in both sexes.

The burden of cancer varies with age (Fig. 14-3). Cancer is a rare disease until the age of 19 years. In childhood and adolescence, the most frequent type of cancer is leukaemia.

Less than 3\% of all cancers occur among people aged 20-34, where the most frequent malignancies are testicular cancer in males and cervical cancer in females. Cancer is more common among women in the 35-49 age group, and more common among men at older ages. Between the ages of 35 and 49, the most frequent types of malignancies are oral and lung cancers in males; breast and cervical cancers in females. Colorectal cancer becomes the predominant form of cancer for both sexes for the 50-74 age group, while among older males $(75+$ years), prostate cancer is most frequently diagnosed. After the age of 75, there is a higher proportion of cancers among females, partially because of their longer life expectancy, with breast, colorectal and stomach cancers among the most common at this age.

Cancer survival, as calculated by population-based cancer registries, is a complex indicator of early diagnosis and effective care for all cancer patients. 
This reflects both the overall performance of the health system and the specific effects of cancer control measures. Data on survival of cancer patients in Slovenia have been published in two books (Pompe-Kirn et al., 1995; PompeKirn, Zakotnik \& Zadnik, 2003) and in annual reports of the Registry (Institute of Oncology), and have also been analysed in the framework of EUROCARE studies (Berrino et al., 1999; Berrino et al., 2003; Berrino et al., 2007; Verdecchia et al., 2007).

Cancer survival has improved steadily overall and for most types of cancer in both sexes between the periods 1963-1967 and 2000-2004. Compared with the first period, when the overall relative survival was $26 \%$ in males and $43 \%$ in females (Pompe-Kirn et al., 1995), cancer survival has increased to 50\% for male and 64\% for female patients diagnosed in 2000-2004 (Institute of Oncology). The better survival of women with cancer is at least in part a consequence of the different nature of cancer, with a greater number of sites where the prognosis is better, compared to what is happening with men. Specifically, in 2000-2004, about 44\% of new cancer cases among men were at sites where the 5-year survival was more than 50\%, while the corresponding proportion among females was nearly 50\% (Fig. 14-4 and Fig. 14-5).

Survival of Slovenian cancer patients is somewhere between western and eastern European countries, especially for the most common cancers (breast, colorectal, prostate) (Berrino et al., 2003; Berrino et al., 2007; Verdecchia et al., 2007). This is mainly a consequence of cancer diagnosis being made at a later stage of the disease in Slovenia and, except for cervical cancer, of the absence of population-based screening programmes. Furthermore, outcomes vary among hospitals. While rare cancers are treated mostly at the Institute of Oncology Ljubljana and survival of these patients is comparable to that in western European countries, common cancers are treated in other hospitals, where implementation of multidisciplinary care and adherence to national guidelines for treatment are patchy.

\section{Cancer control in Slovenia}

As defined by WHO, a national cancer control programme is:

... a public health programme designed to reduce the incidence and mortality of cancer and to improve the quality of life of cancer patients in a particular country or state, through the systematic and equitable implementation of evidence-based strategies for prevention, early detection, treatment, and palliation, making the best use of available resources (WHO, 2006). 
MALES, 2000-2004 (N=24 424)

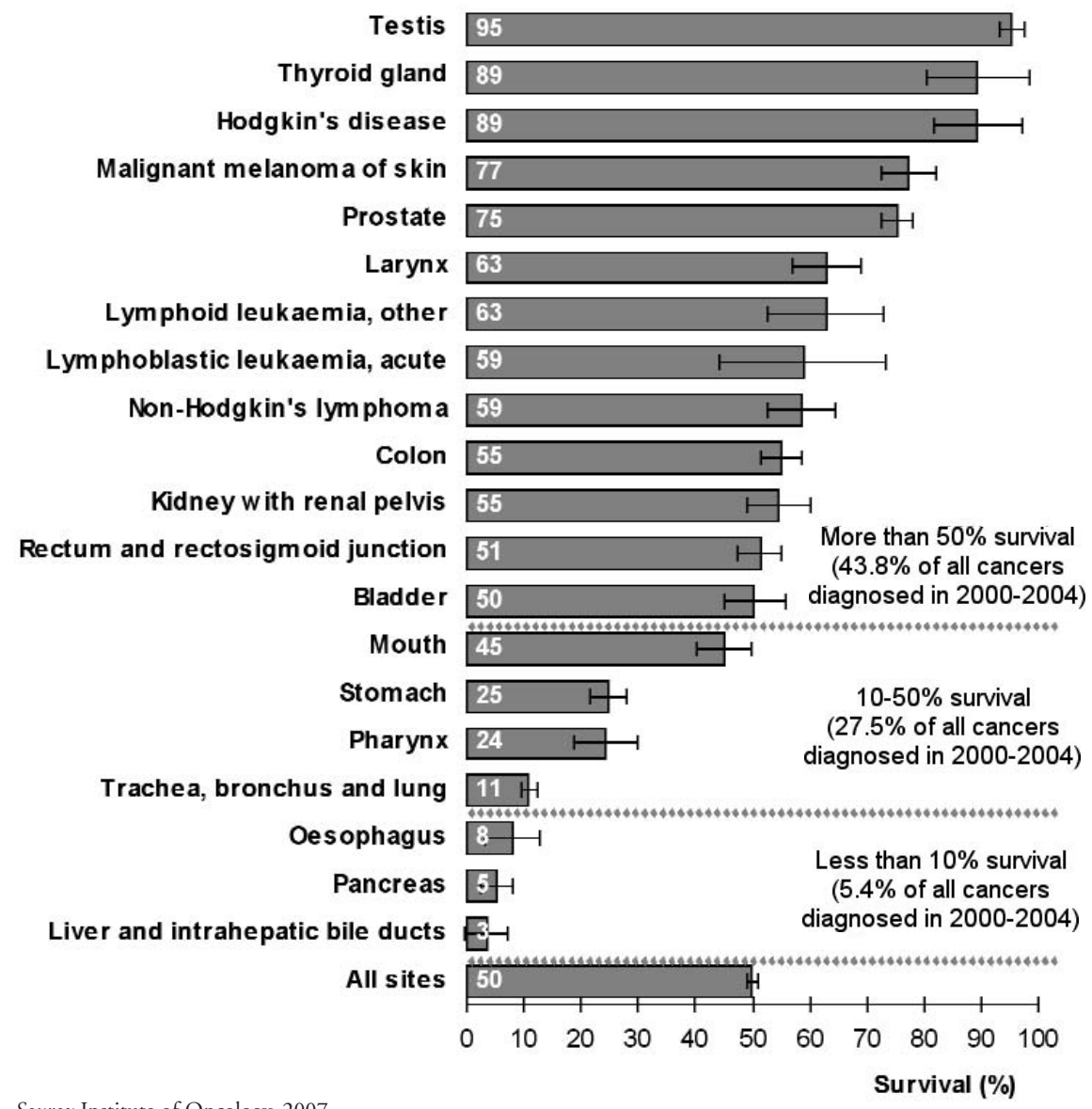

Source: Institute of Oncology, 2007

Fig. 14-4 Five-year relative survival (95\% Cl) for selected cancers among males in Slovenia, 2000-2004

In Slovenia, there is no explicit, integrated cancer control programme, although several components of one are in place. These will be reviewed in the following paragraphs.

\section{Primary prevention}

Activities in the areas of health promotion and prevention of cancer seek to reduce exposure to major behavioural risk factors such as tobacco smoking, excessive alcohol consumption, unhealthy diet, obesity and lack of physical activity (Stewart \& Kleihues, 2003). These activities are largely integrated into a wider set of activities aimed at preventing all chronic diseases. The Ministry 
FEMALES, 2000-2004 ( $\mathrm{N}=23$ 393)

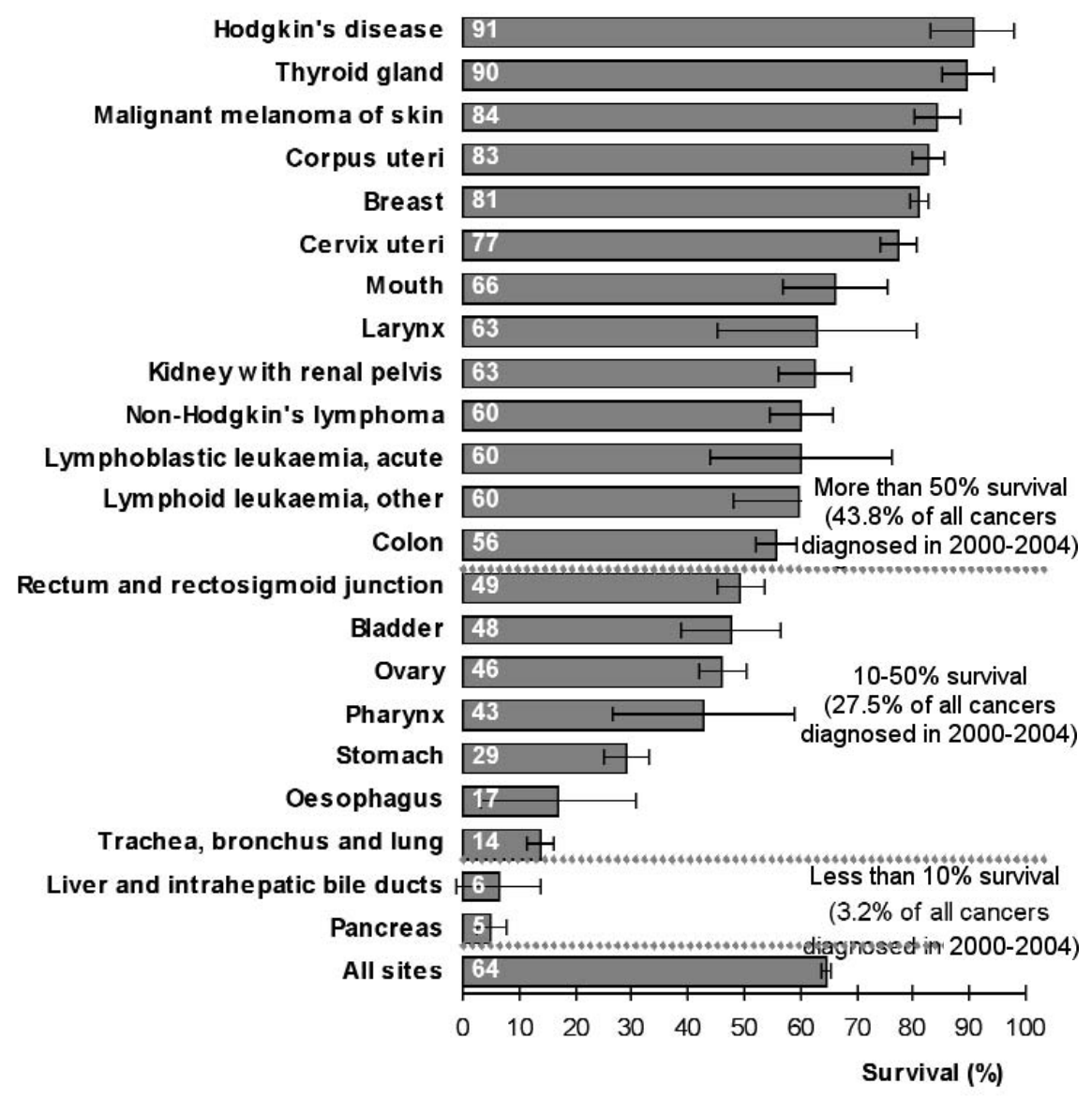

Source: Institute of Oncology, 2007

Fig. 14-5 Five-year relative survival $(95 \%$ Cl) for selected cancers among females in Slovenia, 2000-2004

of Health and its Directorate for Public Health are responsible for the formulation of national policies and strategies to enable and promote healthy lifestyles. They work closely with other ministries, as many cancer risk factors lie outside the health sector's direct influence. Health promotion and prevention programmes are implemented in primary health-care settings; schools and nursery schools; workplaces and the local community; and by several governmental and nongovernmental organizations.

There is also a legislative framework to control environmental pollution (http://www.mop.gov.si/en/legislation/environment/) and exposure to chemical carcinogens in the environment (http://www.mz.gov.si/si/ zakonodaja_in_ 
dokumenti/veljavni_predpisi/kemikalije/; http://www.mz.gov.si/si/zakonodaja_in_ dokumenti/veljavni_predpisi/pitna_voda/); exposure to occupational carcinogens, with a special act on asbestos (although the burden of occupational cancer is not explicitly monitored) (http://www.uradni-list.si/1/objava.jsp?urlid= 2005101\&stevilka=4409; http://www.uradni-list.si/1/ulonline.jsp?urlid= 200593\&dhid=78487); and exposure to ionizing radiation (http://www.mz. gov.si/si/zakonodaja_in_dokumenti/veljavni_predpisi/ionizirajoce_sevanje/).

Hepatitis B vaccine, as a means of preventing liver cancer, has been included in the national immunization programme, while human papilloma virus vaccine, as a contribution to cervical cancer prevention, has also been made available, although it is not yet reimbursed by the health insurance fund (http://www.mz.gov.si/si/zakonodaja_in_dokumenti/veljavni_predpisi/varstv o_pred_nalezljivimi_boleznimi/).

\section{Antismoking policy}

A long-term commitment to combat smoking (http://www.mz.gov.si/si/ zakonodaja_in_dokumenti/veljavni_predpisi/tobak_kajenje/) was strengthened by the Act Amending the Restriction of the Use of Tobacco Products, which was implemented 5 August 2007 (http://www.mz.gov.si/fileadmin/mz.gov.si/ pageuploads/mz_dokumenti/vprasanja_in_odgovori/ZOUTI_velja_050807/ ZOUTI_english_version.pdf). With this act, Slovenia joined Ireland, Italy, Norway, the United Kingdom and some other European countries in prohibiting smoking in all closed public areas and work places. Smoking will only be permitted in special smoking rooms; these must meet strict conditions that the Minister of Health laid down in a set of rules (http://www.uradnilist.si/1/ulonline.jsp?urlid=200780\&dhid=91444).

The amended Act also prohibits selling of tobacco products to persons younger than 18 years; they are also not allowed to sell tobacco products. Within eight months of the implementation of this Act, cigarette manufacturers will have to print the telephone number of the "stop smoking" helpline on the packaging of tobacco products along with existing warning labels. It is hoped that this Act, together with more intensive programmes aimed at reducing smoking, will help to reduce further the prevalence of smoking in Slovenia.

\section{National nutrition strategy}

Several studies among children, adolescents and adults in Slovenia have shown that their dietary habits are inadequate, in terms of number of daily meals (breakfast usually omitted), food items consumed (not enough fruit and 
vegetables, too much red and processed meat) and nutritional composition of diet (high intake of total and saturated fat, salt and total energy; low intake of dietary fibre, calcium, vitamin $\mathrm{C}$ and folic acid) (http://www.mz.gov.si/ fileadmin/mz.gov.si/pageuploads/mz_dokumenti/delovna_podrocja/javno_zd ravje/national_programme_of_food_and_nutrition.pdf). This is important given the role of diet in the aetiology of several common cancers.

The Resolution on the National Programme of Food and Nutrition Policy 2005-2010 was adopted in 2005, with the goals of ensuring safety throughout the food chain; to establish, preserve and strengthen healthy nutritional habits among the Slovenian population; and to ensure an adequate sustainable supply of high-quality, healthy food (http://www.mz.gov.si/fileadmin/ mz.gov.si/pageuploads/mz_dokumenti/delovna_podrocja/javno_zdravje/ national_programme_of_food_and_nutrition.pdf).

\section{National strategy on encouraging physical activity for better health}

Several studies have found that a high proportion of the Slovenian population, at all ages, do not undertake recommended levels of physical activity. Two cross-sectional studies undertaken in Ljubljana (within the framework of the WHO CINDI Programme) showed that only one third of adults are sufficiently active to protect their health (http://www.mz.gov.si/fileadmin/ mz.gov.si/pageuploads/mz_dokumenti/delovna_podrocja/javno_zdravje/natio nal_health_enhancing_physical_activity_programme.pdf). Moreover, the proportion of the population that is totally physically inactive has increased from $15 \%$ to $25 \%$ during the period 1990-1997. This finding has been replicated in other studies (http://www.mz.gov.si/fileadmin/mz.gov.si/ pageuploads/mz_dokumenti/delovna_podrocja/javno_zdravje/national_ health_enhancing_physical_activity_programme.pdf).

There are gender disparities in both physical activity and diet: while males tend to have worse diets, females are less physically active. Inappropriate diet, obesity and lack of physical activity have been shown to increase the risk of several cancers, including colon cancer. As colon cancer is one of the most common types of cancer in Slovenia, there is a clear need for greater effort to achieve more healthy behaviours in the Slovenian population. The National Health Enhancing Physical Activity Programme 2007-2012 encourages all forms of regular physical activity and exercise, with the aim of enhancing and maintaining health throughout life (http://www.mz.gov.si/fileadmin/mz.gov.si/ pageuploads/mz_dokumenti/delovna_podrocja/javno_zdravje/national_health_ enhancing_physical_activity_programme.pdf). 


\section{Alcohol policy}

The average per capita alcohol consumption in Slovenia in 2003 was approximately 10 litres of pure alcohol, high above the corresponding figures in the EU as a whole (WHO Regional Office for Europe, 2007). Cancer is only one of the numerous consequences of alcohol abuse. The law to restrict alcohol consumption, published in 2003, introduced several measures to reduce alcohol consumption, among them a limit on the times when alcoholic beverages can be sold in shops, and the prohibition of sales to people younger than 18 (http://www.uradni-list.si/1/ulonline.jsp?urlid=200315\&dhid= 59912-).

The major deficiency in primary prevention of cancer in Slovenia is the gap between the formal strategies and programmes and their implementation by governmental and nongovernmental organizations. This reflects a lack of coordination that would enable the best use of available resources. A very specific concern is the removal of health education from primary and secondary school curricula; thus, schools are free to implement a variety of programmes that are on the market, not all of which are best suited to the particular age group.

There are, however, some successes. Tobacco control measures, beginning in the last decades of the twentieth century, have successfully reduced the prevalence of cigarette smoking among adult males. This is reflected in the slowly decreasing lung cancer mortality rate. There is, however, still much to do to tackle smoking among females and adolescents - recognizing the need for a long-term investment in effective coordinated policies, ensuring that these include monitoring, audit and evaluation.

\section{Secondary prevention}

Secondary prevention of cancer includes early diagnosis as well as increased awareness of early signs and symptoms, at a time when treatment is most likely to be successful, enabling those affected to seek help from their physicians and receive rapid diagnosis and treatment. Thus, the focus of activities in Slovenia is on raising public awareness about the warning signs and symptoms of cancer, using a number of specific campaigns, as well as continuing training and the production of information for primary care doctors so as to help them recognize these early warnings. Despite these programmes, more than $25 \%$ of all new cancer cases (except non-melanoma skin cancer) are still diagnosed with distant metastases and 36\% with localized disease (not extending to lymph nodes or other organs) (Institute of Oncology). 
Screening refers to early detection of the disease that is not yet clinically manifest, in asymptomatic and apparently healthy individuals (see Chapter 4). Screening has been shown to reduce cancer mortality for cervical, breast and colorectal cancer (European Union, 2003). To be effective, screening programmes should be population-based, with integrated quality assurance and control, not simply opportunistic and uncoordinated. Based on the experience of several European countries, the European Council of Ministers recommended that Member States should introduce organized screening programmes for cervical, breast and colorectal cancer, providing guidance on the measures that should be incorporated in such programmes (European Union, 2003).

\section{Cervical cancer screening}

In Slovenia, opportunistic cervical cancer screening was introduced in regular gynaecological practice in 1960. The proportion of the population screened was unknown, and there were no standards for quality assurance and control. Despite a great number of smears being read, no major changes were recorded in the incidence of invasive cervical cancer in the period from 1979 until 1993. Moreover, the incidence rate started to increase again, to reach a peak in 1997 (23 new cases per 100000 females, corresponding to 241 new cases of cervical cancer).

Inspired by, and based on, the experience of countries with effectively organized screening programmes, the Slovenian Minister of Health made a decision in 1996 to nominate a group of experts to prepare a proposal for an organized cervical cancer screening programme at national level, and to test its feasibility in a pilot study.

In this pilot study, a centralized computerized information system (screening registry) has been established progressively, to record the results of all cervical smears at country level, and to enable the identification of women aged 20-64 who did not have a cervical smear taken in the last three years, in order to send them an invitation for cervical screening. This registry also continuously monitors the quality of the screening programme.

A key element of the pilot study was the development of guidelines for quality assurance and control of all procedures involved in cervical cancer screening and treatment of intraepithelial lesions. In the three years since the beginning of the national programme, nearly $70 \%$ of women in the target age group (2064 years) have had at least one cervical smear taken. Although there is still room for further improvement of the programme, the incidence of cervical cancer has already started to decline, especially among younger women, whose 
participation in screening programmes is higher than that among women aged 50 years and over (Primic-Žakelj \& Repše-Fokter, 2007).

\section{Screening for breast cancer}

No population-based breast cancer screening has been implemented in Slovenia, but women can have "preventive" mammography in diagnostic settings. Furthermore, preventive clinical breast examination is offered to women who attend gynaecology clinics in primary health care. This opportunistic screening, lacking quality control, has not achieved satisfactory results. Specifically, basic epidemiological parameters such as the proportion of localized cancers at diagnosis, breast cancer survival and mortality, have not shown any significant improvement over the last ten years (Institute of Oncology). The number of screened women is not known, but from the findings of several studies, it seems that the proportion of women in the age group 50-69, where screening is most effective, is low, that screening intervals are too short, and that many women who attend for opportunistic screening are too young to derive benefit (Primic Žakelj et al., 2006).

In response to this situation, a national breast cancer screening programme has been developed that is consistent with European guidelines, and is due to start in December 2007. When linked to effective diagnostic procedures and optimal treatment, it is hoped that this screening programme will contribute to the reduction of mortality from breast cancer in the Slovenian population.

The breast cancer screening programme is aimed at the 240000 women aged between 50 and 69 years; therefore, it is expected that each year, about 120000 women will receive an invitation for a screening test. The screening programme is based on the screening unit, which cooperates closely with the diagnostic unit, both professionally and functionally. Digital mammography machines will be used, which will be placed in two stationary screening units in Ljubljana and Maribor, and in six mobile units across the country. All women with suspicious mammography findings will be invited for further assessment at a diagnostic centre, where the treatment of all screen-detected and subsequently confirmed breast cancers will take place (Primic Žakelj, Krajc \& Maučec Zakotnik, 2006).

\section{Colorectal cancer screening}

Screening for colorectal cancer has not been yet introduced in Slovenia, although this has been planned along with the development of national clinical guidelines for the management of colorectal cancer. Colonoscopy 
screening is offered to patients in some diagnostic settings, but the volume of this activity is unknown.

\section{Other secondary prevention measures}

The Institute of Oncology Ljubljana has recently established a team for genetic counselling of families with a history of breast, colorectal and some other cancers. There is no explicit policy on prostate cancer screening using prostate specific antigen test (PSA), but this is becoming more popular on an opportunistic basis, despite the continuing controversy about its value.

\section{Diagnosis and treatment of cancer}

According to the Law on Health Care and Health Insurance, treatment of cancer is covered fully by the health insurance. Health-care centres and private practitioners provide primary and outpatient secondary health care; most of them are included in the public health-care network, which have a contract with the Health Insurance Fund. There are 12 general hospitals across the country as well as a clinical centre in Ljubljana, acting as a tertiary health-care facility.

The majority of cancer patients receive their initial diagnosis from specialists in one of the 12 Slovenian general hospitals or the clinical centre in Ljubljana, with only about $15 \%$ being diagnosed at the Institute of Oncology Ljubljana. However, about $50 \%$ of patients diagnosed elsewhere are subsequently referred to the Institute of Oncology Ljubljana. In $80 \%$ of cases, the diagnosis is confirmed by pathology and a further $10 \%$ by cytopathology (Institute of Oncology).

The Institute of Oncology, founded in Ljubljana in 1938, is the only national cancer centre in Slovenia. Besides patient care, it is involved in research and education, working under the auspices of the Medical Faculty of the University of Ljubljana. By working closely with specialists from several clinical departments treating cancer patients in the Clinical Centre in Ljubljana and through joint clinics for several cancer sites, it is seeking to develop a comprehensive response to cancer in Slovenia. While common cancers are also treated in general hospitals, the Institute of Oncology Ljubljana is the only referral centre for rare cancers. Moreover, radiotherapy is administered only at this institute.

The National Advisory Board for Oncology provides a forum that seeks to formulate guidelines for treatment of several types of cancer. Unfortunately, there is currently no adequate mechanism to monitor and audit the implementation of these guidelines in clinical practice. 
The Health Council is an advisory body to the Minister of Health, formulating contents of health programmes, including those against cancer, and acting as the highest authority on health care in Slovenia. Unfortunately, there is no specialist in oncology on this council.

\section{Palliative care}

Palliative care is an important part of the management of cancer patients. This is provided in hospital settings or by general practitioners. The Institute of Oncology Ljubljana has recently established a special team for palliative care but, at present, palliative care and specialist rehabilitation of cancer patients are the least well-developed components of cancer control in Slovenia.

\section{Conclusions}

Slovenia is faced with an increase in the cancer burden and in the prevalence of cancer risk factors. These observations argue for making cancer control a priority in the health agenda. This will require the development of new managerial systems for cancer control that would facilitate policy and programmatic decisions that take full account of the cost-effectiveness and affordability of interventions.

Slovenia can build on a number of strengths, including the well-established National Institute of Oncology and the population-based Cancer Registry, covering the whole country. Yet, while many cancer control activities have been in place for many years, there is still much room for improvement, and in particular a need to develop a comprehensive national cancer control programme that would incorporate clearly defined goals and objectives, responsibilities, financial support, adequate training and ongoing audit and quality control.

\section{Recommendations}

A comprehensive national cancer control programme should be instituted to improve cancer control, based on a systematic assessment of the strengths and weaknesses of existing services for cancer prevention and the clinical management of cancer patients.

The following issues should specifically be addressed:

- In primary prevention and health promotion there is a need to close the gap between official policies and their implementation by governmental 
and nongovernmental organizations, based on better coordination to achieve the best use of available resources.

- Given the importance of behaviours adopted in childhood and adolescence, effective and appropriate health promotion programmes should be a core part of the school curriculum.

- Evidence-based mass screening programmes should replace opportunistic screening.

- Equal access to timely and high-quality diagnosis and multidisciplinary treatment and clinical care should be available to all Slovenian people. Minimum caseloads, levels of clinical expertise and treatment guidelines should be defined and regularly monitored along with an ongoing and mandatory audit of results.

- Better communication and rapid flow of patients between primary, secondary and tertiary health levels should be promoted.

- Diagnostic and treatment facilities that are highly complex and/or used infrequently should remain concentrated at the Institute of Oncology Ljubljana. This will ensure an adequate caseload to maintain high quality of care.

- Good physical and psychological rehabilitation should enable those patients cured of cancer to return to their normal life as soon as possible.

- Palliative care should be provided to cancer patients throughout the cancer journey, linked to adequate support for their families.

- Training, education and research in oncology should become a high priority.

- The new cancer plan should incorporate clearly defined goals and objectives, responsibilities, financial support, adequate training and ongoing audit and quality control of all interventions at all levels.

- Additional managerial systems for cancer control should be developed, which would facilitate policy and programmatic decisions, taking full account of cost-effectiveness and affordability of interventions. The general public should also be involved in these decisions, given the public health perspectives of efficiency and effectiveness of all interventions.

\section{REFERENCES}

Berrino F, et al., eds. (1999). Survival of cancer patients in Europe: the EUROCARE-2 study. (IARC Scientific Publications No. 151). Lyon, International Agency for Research on Cancer. 
Berrino F, et al., eds. (2003). Survival of cancer patients in Europe: the EUROCARE-3 study. Ann Oncol, 14 (Suppl. 5): 1-155.

Berrino F et al. (2007). Survival for eight major cancers and all cancers combined for European adults diagnosed in 1995-99: results of the EUROCARE-4 study. Lancet Oncol, 8(9):773-783.

European Union (2003). Council Recommendation of 2 December 2003 on cancer screening (2003/878/EC) Official Journal of the European Union, L327:34-38.

Ferlay J et al. (2007). Estimates of the cancer incidence and mortality in Europe in 2006. Ann Oncol, 18(3):581-592.

http://www.mop.gov.si/en/legislation/environment/, accessed 1 December 2007.

http://www.mz.gov.si/fileadmin/mz.gov.si/pageuploads/mz_dokumenti/delovna_podrocja/javn o_zdravje/national_health_enhancing_physical_activity_programme.pdf, accessed 1 December 2007.

http://www.mz.gov.si/fileadmin/mz.gov.si/pageuploads/mz_dokumenti/delovna_podrocja/javn o_zdravje/national_programme_of_food_and_nutrition.pdf, accessed 1 December 2007.

http://www.mz.gov.si/fileadmin/mz.gov.si/pageuploads/mz_dokumenti/vprasanja_in_odgovori IZOUTI_velja_050807/ZOUTI_english_version.pdf, accessed 1 December 2007).

http://www.mz.gov.si/si/zakonodaja_in_dokumenti/veljavni_predpisi/ionizirajoce_sevanje/ accessed 1 December 2007.

http://www.mz.gov.si/si/zakonodaja_in_dokumenti/veljavni_predpisi/kemikalije/, accessed 1 December 2007.

http://www.mz.gov.si/si/zakonodaja_in_dokumenti/veljavni_predpisi/pitna_voda/, accessed 1 December 2007.

http://www.mz.gov.si/si/zakonodaja_in_dokumenti/veljavni_predpisi/tobak_kajenje/, accessed 1 December 2007.

http://www.mz.gov.si/si/zakonodaja_in_dokumenti/veljavni_predpisi/varstvo_pred_nalezljivim i_boleznimi/, accessed 1 December 2007.

Institute of Oncology. Cancer Incidence in Slovenia (1985-2007). Ljubljana: Institute of Oncology, Cancer Registry of Slovenia 1987-2004.

Pompe-Kirn V, Zakotnik B, Zadnik V (2003). Preživetje bolnikov z rakom v Sloveniji 1983-1997 (Cancer patients's survival in Slovenia 1983-1997). Ljubljana, Onkološki Inštitut, Register raka za Slovenijo.

Pompe-Kirn V et al. (1995). Preživetje bolnikov z rakom v Sloveniji 1963-1990 (Cancer patients' survival in Slovenia 1963-1990). Ljubljana, Onkološki Inštitut, Register raka za Slovenijo.

Primic Žakelj M, Krajc M, Maučec Zakotnik J (2006). Presejanje za raka dojk - kje smo danes. (Breast cancer screening - where are we today?) Radiol Oncol 40(1):25-30.

Primic Žakelj M, Repše-Fokter A (2007). Cervical cancer screening: a Slovenian experience. Coll Antropol, 31(Suppl. 2):23-26.

Stewart BW, Kleihues P (2003). World cancer report. Lyon, IARC Press.

Toš N (1997). Vrednote v prehodu I. Slovensko javno mnenje 1968-1990 (Values in transition I. Slovenian public opinion surveys 1968-1990. Ljubljana, CJMMK (Public Opinion and Mass Communication Research Centre).

Toš N (1999). Vrednote v prehodu II. Slovensko javno mnenje 1990-1998 (Values in transition II. Slovenian public opinion surveys 1968-1990. Ljubljana, CJMMK (Public Opinion and Mass Communication Research Centre).

Toš N. (2004). Vrednote v prehodu III. Slovensko javno mnenje 1999-2004 (Values in transition III. Slovenian public opinion surveys 1999-2004). Ljubljana, CJMMK (Public Opinion and Mass Communication Research Centre). 
Tyczynski JE et al. (2004). Breast cancer mortality patterns and time trends in 10 new EU Member States: mortality declining in young women, but still increasing in the elderly. Int $J$ Cancer, 112(6):1056-1064.

http://www.uradni-list.si/1/objava.jsp?urlid=2005101\&stevilka=4409, accessed 1 December 2007). http://www.uradni-list.si/1/ulonline.jsp?urlid=200315\&dhid=59912-, accessed 1 December 2007). http://www.uradni-list.si/1/ulonline.jsp?urlid=200593\&dhid=78487, accessed 1 December 2007. http//www.uradni-list.si/1/ulonline.jsp?urlid=200780\&dhid=91444, accessed 1 December 2007. Verdecchia A et al. (2007). Recent cancer survival in Europe: a 2000-02 period analysis of EUROCARE-4 data. Lancet Oncol, 8(9):784-796.

WHO (2006). Cancer control. Knowledge into action. WHO guide for effective programmes. Planning. Geneva, WHO.

WHO Mortality Database. Geneva, Switzerland, WHO (available at:http://www.who.int/ whosis/en/, accessed 1 December 2007).

WHO (2007). Health for all statistical database 2007. WHO Regional Office for Europe (available at: http/hfadb.who.dk/hfa, accessed 1 December 2007) 


\section{Chapter 15 \\ Researching cancer \\ Tanja Cufer and Richard Sullivan}

\section{Delivering cancer research: 50 years of European cancer research progress}

Europe and its neighbours have a long and distinguished history in cancer research. Both the ancient Egyptians and Greeks recognized this distinct and dangerous disease (Karpozilos \& Pavlidis, 2004). However, it was not until James Watson and Francis Crick wrote the immortal words: "We wish to suggest a structure for the salt of deoxyribose nucleic acid (DNA)...", that the modern era of cancer research was truly born (Watson \& Crick, 1953).

Europe's seminal contributions to the milestones of cancer research are many and can be traced back even further, to 1889. In that year Dr. Stephen Paget (a London surgeon) developed the "seed and soil" hypothesis of metastasis. The prevailing view at that time was that cancer cells spread through the blood or lymph and could take up residence in any tissue. If this had been true, metastases would have shown a random distribution to other organs. Paget thought otherwise. "When a plant goes to seed, its seeds are carried in all directions. But they can only live and grow if they fall on congenial soil..." he wrote (one of the wonderful things about research in this era was the use of the natural world as an unlimited source of metaphor and analogy, sadly lost in today's prosaic research culture). Paget examined nearly a thousand cases and found that specific tumours metastasized consistently to particular organs. Although this view was challenged by James Ewing (who gave his name to a type of soft tissue cancer, or sarcoma) claiming instead that metastases settled in the first organ they reached as they spread through the vasculature, Paget was to be proved correct in 1980 by Isaiah Fidler and Ian Hart working at the MD Anderson Cancer Center at the University of Texas (Paget, 1989).

Almost simultaneously with Paget in 1890, just a few years after the discovery of the chromosomes, David Paul Hansemann, a pathologist-in-training with 
the famous Rudolph Virchow in Berlin, produced a theory of the pathogenesis of cancer. This included the key concept: that the first change which occurs in cancer is an alteration of the hereditary material of a normal cell at the site where the cancerous process begins (Von Hansemann, 1890). In the process of linking cancer to chromosomal material, Hansemann coined the terms "anaplasia" and "dedifferentiation". These terms have remained the basis of descriptive terms concerning the microscopic appearances of tumours ever since.

The great German tradition in cancer research continued with people such as Theodor Heinrich Boveri (1862 -1915). In his work with sea urchins, this German zoologist showed that it was necessary to have all chromosomes present in order for proper embryonic development to take place. His other discovery was the centrosome (1888) which he described as the special organ of cell division. He also reasoned that cancer begins with a single cell, in which the make-up of the chromosomes is scrambled, causing the cells to divide uncontrollably (Boveri, 1914).

It was Paul Ehrlich, born into a Jewish family in Strehlen, in the Prussian Province of Silesia (now in Poland) who was to make the link between the immune system and cancer, suggesting that for the latter to survive the former had to be suppressed (Ehrlich, 1909). Paul Ehrlich, who won the 1908 Nobel Prize in physiology and medicine, also predicted autoimmunity calling it "horror autotoxicus". He coined the term "chemotherapy" and popularized the concept of a "magic bullet".

However, one should not view Europe's role in turning back the tide of cancer as an isolated one. Then, as now, research was a complex dance over distance and time. Europe's great contributions are intimately intermingled with those in other countries and continents. The recent breakthrough in controlling cervical cancer through the use of a vaccine directed against certain types of human papilloma virus (HPV) rests on the work of Harald zur Hausen, who was first to show that the papilloma virus was the most significant cause of this cancer (McIntyre, 2005). In turn, that work owed much to the groundbreaking research begun in 1910 by Peyton Rous who first discovered tumour viruses. Indeed, although Peyton Rous was born in Texas in 1879, his mother's ancestors were Huguenots who settled in Virginia after the Edict of Nantes.

Europe has also laid the foundations of many other domains of cancer research. The most important discovery in the history of cancer epidemiology was the carcinogenic effect of tobacco (Doll,1978). The pivotal studies begun by Sir Austin Bradford Hill and Sir Richard Doll, and later with Sir Richard Peto, were to provide the springboard for five decades of research on both sides of the Atlantic. 
In surgery there have been many seminal contributions by the European cancer research community. Umberto Veronesi, an Italian surgeon and oncologist, was the founder of breast-conserving surgery (Veronesi et al., 1981), inventing the technique of quadrantectomy, which challenged the idea, then dominant among surgeons, that cancers could be treated only with aggressive surgery.

Europe has also been at the forefront of treating bowel cancer through surgical advances - from 1908 when Ernst Miles (Miles et al, 1908) first described the abdominoperineal resection, to the first description of total mesorectal excision by Bill Heald and colleagues in 1982 (Heald et al., 1982). This gave rise to clinical trials in Scandinavian countries that were to change global clinical practice (Ridgway \& Darzi, 2003).

Research into the molecular and cellular biology of cancer has provided remarkable insights into the molecular basis of cancer, such as disordered cell proliferation; disturbed differentiation and altered cell survival; and disruption of normal tissue, invasion and metastasis. New discoveries in the molecular oncology of tumours in the last few decades have led to major improvements in cancer therapy. In the middle of the twentieth century an improved molecular classification of malignant lymphomas paved the way for individualized therapy in cancer. The treatment based on these molecular classifications resulted in higher response rates and improved survival of patients with malignant lymphomas. One of the most prominent scientists involved in this molecular pathology research and one of the authors of the new Kiel classification of lymphomas was Karl Lennert, a German pathologist, working with his group in Kiel (Lennert, 2005).

The field of breast cancer, the most frequent cancer in women, has also seen many new developments based on the European research, shared with other countries. Pivotal experiments performed in the late 1950s and early 1960s, primarily in the laboratories of Gerald Mueller and Elwood Jensen in Germany and in the United States of America (USA), set the stage for the development of hormonal therapy in hormone-responsive breast cancer (Malley, 1995). Acknowledgement of hormone receptors as one of the major biological determinants of breast cancer was actually one of the first discoveries that enabled the most effective strategies in the treatment of cancer, i.e. targeted therapy. Hormonal therapy with tamoxifen was the first individualized, targeted therapy in the history of cancer therapy.

Nowadays, breast cancer can be divided into hormone-receptor-positive and hormone-receptor-negative tumours, with treatment being substantially different in these two distinct diseases. Based on the largest meta-analysis in 
cancer care, undertaken at Oxford by Sir Richard Peto and his co-workers in the Early Breast Cancer Trialists' Collaborative Group, a vast amount of knowledge on the best possible adjuvant systemic therapy in hormone-positive and hormone-negative breast cancer was accumulated. Their work confirmed that adjuvant chemotherapy reduced the rate of recurrence by $33 \%$ and the rate of breast cancer death by 17\%, saving thousands of lives of women with breast cancer. The same was true for hormonal therapy in hormone-responsive breast cancer, in which adjuvant hormonal therapy with tamoxifen was found to reduce the rate of recurrence by $41 \%$ and the rate of breast cancer death by $34 \%$, according to the data from the meta-analysis (Early Breast Cancer Trialists Collaborative Group, 2005). Adjuvant systemic therapy, in addition to surgery, radiotherapy and screening programmes has been responsible for major declines in breast cancer mortality during the last decade in the United States and Europe (Berry et al., 2005).

\section{The impact of European research policies on cancer research: the good, the bad and the ugly}

European research policies - be they funding frameworks or regulatory approaches at the Member State and at European level - have never been more influential. Such policies can and do have dramatic effects on the shape and course of cancer research. One of the most striking differences between Europe and the United States is the heterogeneous funding and regulatory policies in the former. Most funding is provided through governmental and charitable organizations and the health-care/university systems of individual Member States. Layered across all this is a bewildering variety of scientific and regulatory structures in each Member State, each with a very different sociological model of engagement and dialogue between policy-makers, experts and the general public. The same can also be said for funding and regulatory policy structures at the EU level. With multiple Directorate Generals and independent regulatory bodies (e.g. the European Medicines Agency, EMEA) as well as a complex approach to EU health policy (Duncan, 2002) the picture that emerges of Europe's funding and regulatory policies can, at best, be described as Byzantine.

Turning to practicalities, there is currently little understanding between Member States on how cancer research funding is constructed in each country and how Directives have been implemented (or not); or the specific regulatory mechanisms in each country (e.g. gene therapy trials in the United Kingdom must be authorized by a separate statutory committee - the Gene Therapy Advisory Committee). What we do know comes from major studies carried 
out by consortia concerned with trans-European cancer research. The TuBaFrost tissue banking initiative has reviewed the current regulatory situation and found that, "...the regulatory regimes for research with residual tissue and accompanying data differ widely between countries in the EU: from specific consent to opt-out or even no consent at all", diplomatically suggesting that, "this seems to be a rather distressing state of affairs when researchers want to proceed with exchange programs for residual tissue" (van Veen et al., 2006). In reality this is a huge problem, particularly for many of the orphan cancers where the only realistic way of conducting research is transnational cooperation and exchange of human tissue across borders. Centralized European support to assist academic networks to overcome these regulatory hurdles will be a necessary component of any infrastructure support.

There is no doubt that a number of European policies have been well thought through. Examples abound. On the funding side, the creation of the European Research Area during the sixth Framework Programme (FP6) shone a welcome spotlight on cancer research. The Research Framework Programme has undergone significant changes since FP3 (1990-1994) but, as an assessment of FP5 made clear, there has been a disconnect between broad high-level socioeconomic goals and technical objectives. There is also a feeling that EU money is not necessarily adding European value, i.e. it is rather acting as a substitute for funding by Member States.

With the new FP7, there is promise of a less bureaucratic, more thematically focused mechanism for EU funding with, of course, the European Research Council funding to support the foundations of European science. This is to be welcomed. The key now is to implement these policies in a tangible form hypothecated monies for European cancer research networks. Already the Innovative Medicines Initiative has matured to provide public-private funding for EU translational networks, of which cancer will be one disease-specific area. However, European policy in FP7 must transcend the standard drug discovery-pharmaceutical industry paradigm and re-embrace the other essential areas of cancer research, for example clinical trials (e.g. European Clinical Research Infrastructures Network, ECRIN (www.ecrin.org); paediatric research (e.g. Innovative Therapies for Children with Cancer (www. itcc-consortium.org), and epidemiology (e.g. CONCORD study of cancer survival across five continents; CONCORD Working Group, 2007).

On the regulatory side, the new Paediatric Regulation, modelled on the American Paediatric Exclusivity Rule, has improved the incentives for industry to conduct clinical trials in the paediatric setting by making a Paediatric Investigation Plan (or deferral) a mandatory part of the drug development process (Dunne, 2007). 
Not all policies, however, have been so benign. The European Data Protection Directive (1995) sought to harmonize the widely different European approaches adopted in response to earlier guidance by the Council of Europe (Council of Europe Convention for the Protection of Individuals with regard to Automatic Processing of Personal Data). It, however, caused widespread chaos, disrupting the collection of data by cancer registries and the use of identifiable health-care data in ethically-approved research programmes (Coleman, Evans $\&$ Barrett, 2003). This situation remains a major issue even to this day, with some countries, such as Estonia, enacting legislation that goes far beyond the provisions of the Directive (Rahu \& McKee, 2003).

Turning to funding policies, Europe has seen a reversal of the hypothecated support which ran from the mid 1980s, in the Europe Against Cancer initiative, to a situation of short-term general funding for specific programmes of research. What has suffered is the badly needed long-term infrastructure support that is needed to run true trans-European research programmes e.g. in prevention (The European Prospective Investigation into Cancer, EPIC) and clinical trials (EORTC, 2003). FP7 now provides a perfect opportunity to reverse these policies and underpin these badly-needed European infrastructures, which will never receive support from individual Member States.

In some cases bad has turned ugly. The impact of regulatory policy on clinical trials over the last four years has been astonishing. The Clinical Trials Directive has probably done more damage to European cancer research that any other policy or regulation. As Markus Hartmann and colleagues have found, the differences in the application of this Directive in Member States' legislation are astonishing (Hartmann \& Hartmann-Vareilles, 2006). It has had a dramatic effect on the cost of research which has hit the non-commercial sector particularly hard. For example, costs for cancer clinical trials in the United Kingdom have doubled for the non-commercial sector since the introduction of the Clinical Trials Directive. There is little doubt that this has reduced the number of high cancer trials, to the detriment of European research and cancer patients. There is an urgent need to reconsider the regulatory paradigms that have given rise to a thriving industry around cancer research, and to reverse this trend. Good research governance is essential but bureaucracy is absorbing too much of the global investment in cancer research. Real consultation with the research community and the need for pro-research policies and a move away from the dominance of the precautionary principle, must be the way forward for European biomedical policy-makers (Graham, 2004).

Substantial progress in treatment of early breast cancer, reflected in falling mortality rates in many countries, is a direct result of more than half a century 
of sustained commitment to randomized clinical trials of multidisciplinary interventions in cancer care run by European academics. Many, if not all, of the measures ensuring the protection of clinical trial participants were previously in effect through the Guidelines for Good Clinical Practice (GCP). This is an international, ethical and scientific quality standard for designing, conducting, recording and reporting trials that involve the participation of human subjects and it is consistent with the principles of the Helsinki Declaration. The Guideline was developed to be consistent with current good clinical practice in the EU, Japan and the United States; and in Australia, Canada, the Nordic countries and WHO (www.ich.org/LOB/media/ MEDIA482.pdf). However, such a guideline had no legal basis. Although the Clinical Trials Directive briefly considers non-commercial trials, allowing some simplification of the labelling procedure for investigational medicinal products, it generally makes no distinction between commercial and non-commercial clinical trials. More importantly, it fails to distinguish clinical trials investigating new drugs from those investigating multimodality treatment strategies (most often investigated by academic cancer research groups), demonstrating how distant policy-making has become from the realities of research practice.

Despite major progress against cancer arising from clinical cancer research, participation in clinical trials appears low all over the world, although data on participation are fragmentary. According to the American National Cancer Institute (NCI) and the National Cancer Institute of Canada, only about 3\% of adult cancer patients are enrolled in clinical cancer research worldwide. There are no precise data on the number of cancer patients participating in clinical trials in Europe but certainly the percentage is not higher than in the United States (exceptions include the United Kingdom - with over 14\% of incident cases).

The importance of a healthy clinical trials community in Europe cannot be understated. Participation in clinical trials offers access to new experimental therapies and involves close monitoring and rigorous administration of care, which leads to better outcomes for participants. The way to improve enrolment into clinical trials in Europe is by promoting multinational clinical research through centres of excellence and, most of all, by disseminating knowledge and information about clinical and translational research among the scientific community and the general public.

\section{Funding cancer research in Europe}

The European Cancer Research Managers Forum, ECRM (www.ecrmforum. org), has recently completed the second survey into the global investment and 


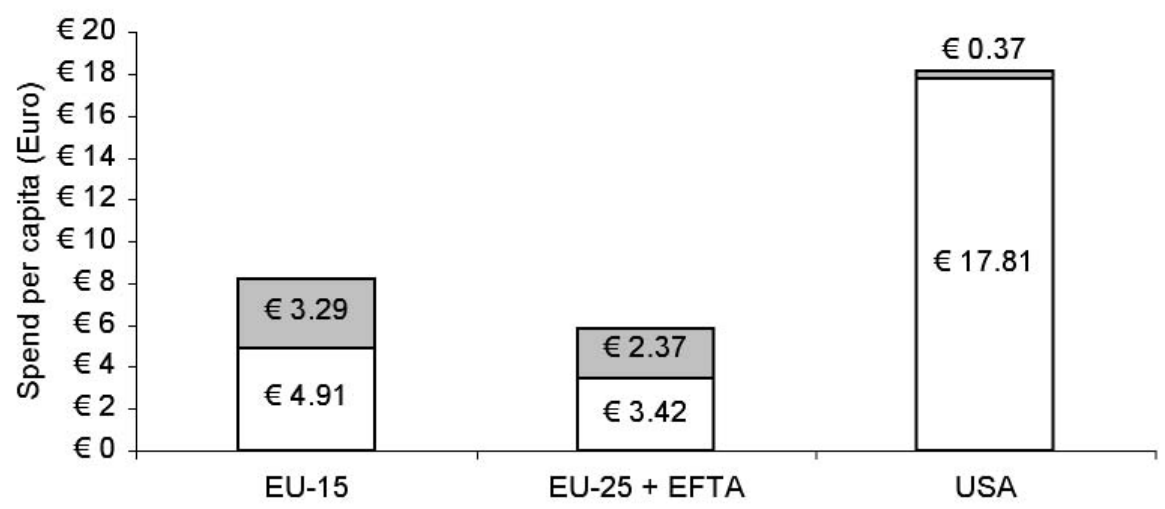

$\square$ By funding organizations $\square$ Through healthcare \& university systems

* European Free Trade Association

Source: ECRM (available at: www.ecrmforum.org, accessed 25 November 2007)

Fig. 15-1 Spend per capita for cancer research in the EU15; EU25+EFTA*; and United States - from funding organizations (governmental and philanthropic) and through healthcare/university systems

outputs of cancer research. The notable feature of this second survey has been the estimation of cancer research funding that flows through Member State health-care and university systems (Fig. 15-1). The figures for Europe are substantial and, at over $€ 1$ billion per annum, pose a major challenge to those designing policy tools to promote cancer research or create strategic frameworks. Indeed, the major policy issue is the real differences in cancer research investment between the individual Member States. To date, the overall cancer research funding gap between Europe and the United States has been the driving force behind EU policy-making (Pavitt, 1998).

A balance between philanthropic and governmental funding is also absent in a number of Member States. There are, of course, natural limits for a number of countries. The majority of cancer research funding is raised and spent within EU15 Member States. For the remainder, the priority for resources is cancer control programmes, not major cancer research funding. For example, the Warsaw Declaration signed by patient groups attending the European Cancer Patient Coalition / Slovenian Cancer summit in November 2006 strongly supports the need to prioritize investment and improvements in cancer control programmes.

Member States that aspire to become major locations of cancer research must implement specific policy actions to ensure a limited core of high-quality research within their institutions. Some of the measures outlined in the 
European Commission's Framework Programme 7 (e.g. Specific International Scientific Cooperation Activities - INCO; and ERA-NET) may facilitate this but it will still remain the responsibility of individual countries to implement action programmes to address cancer research funding deficits.

Regulatory and research policy frameworks have a major influence on the intrinsic creativity of European cancer research. Given current levels of expenditure, it is debatable whether cancer research funding at the EU level through the Framework Programmes and other streams has had a major impact on the rate and direction of European cancer research (http://cordis. europa.eu/lifescihealth/cancer/cancer-pro-calls.htm, accessed 2 December 2007). With a budget for Framework Programme 7 (2007-2013) set at $€ 5984$ million (Trias, 2006), there is now scope for the European Commission to have a major impact, in addition to its commitment to the European Research Council and Joint Technology Platforms, through which the Innovative Medicine Initiative will be funded. The EU policy of specific research programmes and thematic calls has been questioned (Laredo, 1998). However, the suggested solutions (networks, research programmes delegated to specific agencies) should be additions to ring-fenced funding for cancer, not substitutes.

EU research policy needs to recognize and fund core trans-EU infrastructure such as translational research, paediatric research networks (e.g. Innovative Therapies for Children with Cancer) and cancer registries, to name but a few. The need for such European infrastructure funding has already been recognised in a European Council report (European Strategy Forum on Research Infrastructures, 2006). In all these areas European funding to cement already cooperative groups would deliver substantial added value to existing Member State support, which mostly flows through highly competitive streams.

Philanthropy plays a remarkable and essential role in supporting cancer research. Unsurprisingly, given the fiscal dominance of the American National Cancer Institute, Europe receives a greater portion of its funding through the philanthropic sector. In Europe, philanthropy has been belatedly recognized as an underexploited source of income for research. However, charity is a complex phenomenon with different attitudes and giving patterns in each country (Wright, 2000). Furthermore, the understanding of altruism as a sociobiological phenomenon when applied to today's philanthropy, particularly around secular causes, has not been studied in any depth beyond the theoretical (Humpries, 1997). What may work at one level in one Member State may not work in another. Other health charities, overseas aid agencies, human welfare and heritage preservation groups are also increasing the pressure on charitable funds. Given these inherent uncertainties, philanthropy in cancer research should be seen in policy terms as additional to the overall 
Table 15-1 Global investment in cancer research

\begin{tabular}{|c|c|c|}
\hline Source of funding & Funding ( $€$ millions) & $\begin{array}{c}\text { As \% of estimated } \\
\text { global spend }\end{array}$ \\
\hline Pharma industry (top 24 companies) & 3095 & 22.1 \\
\hline USA (government) & 4712 & 33.5 \\
\hline USA (charitable) & 456 & 3.3 \\
\hline USA (health-care \& university systems) & 109 & 0.8 \\
\hline EU (government) & 992 & 7.1 \\
\hline EU (charitable) & 879 & 6.3 \\
\hline EU (health-care \& university systems) & 1364 & 9.7 \\
\hline Rest of world & 2423 & 17.2 \\
\hline TOTAL & 14030 & 100.0 \\
\hline
\end{tabular}

Source: ECRM (available at: www.ecrmforum.org, accessed 25 November 2007)

global effort, which is mainly funded through taxation and private enterprise (industry).

The ECRM survey has revealed some important differences in global cancer research funding, particularly the relative distribution between public and private sectors and funding through national health-care and university systems (Table 15-1).

The United States has pursued a centrist funding model (the NCI and National Institutes of Health combined are responsible for $€ 4459$ billion or $86 \%$ of total expenditure on cancer research). European funding is highly diffused - direct funding from governmental organizations (e.g. the German Federal Ministry of Education and Research) accounts for only $7.1 \%$ of funding compared to nearly $10 \%$ embedded in health-care and university systems; $6.3 \%$ is contributed by philanthropic organizations. Unlike the United States, Europe has the complicated task of networking and aligning diverse and (in many cases) hidden funding streams.

Global levels of expenditure on cancer research, as a percentage of per capita gross domestic product (GDP), continue to show substantial differences between the United States and Europe, however, this gap has substantially narrowed. A major explanation is the ECRM survey's ability to estimate cancer research funding flowing through national health-care and university systems in Europe. However, there has also been a real increase in funding in some Member States, whilst American funding has shrunk in real terms. It is important to recognize that, according to the survey, in 2002-2003 the United States spend on cancer research (\% of GDP) was four times that in the EU. In comparison, Japan, Canada and Australia spend between €7.86 and €8.66 per capita. The higher resolution of this ECRM second survey also allows comparison of spend per new case of cancer diagnosed (incidence) and per 
death from cancer (mortality). In 2004, there were an estimated 2886800 new cases of cancer with 1711000 deaths in Europe, which gives a spend of $€ 1155$ per incident case or $€ 1941$ per death (Boyle \& Ferlay, 2005). In the same year, the United States spent some $€ 3857$ per incident case and €9361 per cancer death (Jemal et al., 2004).

Whilst the gap between cancer research funding in Europe and the United States remains substantial, cancer research outputs over a ten year period have been similar. Europe has produced a steady $4-5 \%$ more cancer research publications than the United States since 1997. Indeed, Europe is now increasing its share of global cancer research outputs, with an upward trend that started in 2001, at the same time that the United States' share has stagnated. The type of research that is being conducted and then published is also changing. Europe is publishing more clinical research than the United States (Mowery, 1998), although according to the ECRM survey in 20022003, the EU spent much more on basic research than on preventive, clinical and translational research. Interestingly, a separate study has found that, globally, cancer research changed from a bipolar allegiance to either clinical or laboratory styles in the 1980s and, by 2000, to research activity structured by a common orientation to a translational research domain (Cambrosio et al., 2006). However, data from the first ECRM survey found that Europe still spends a disproportionate amount of funding on basic cancer research (Eckhouse \& Sullivan, 2006).

Although the United States is the dominant country for pivotal, commercially-sponsored phase III clinical trials (CMR International, 2006), substantial cancer research activity is conducted by the pharmaceutical industry in both Europe and the United States. Nearly all major recent policy on cancer research funding and policy initiatives have emphasized the publicprivate partnership route, such as the Innovative Medicines Initiative (IMI) Strategic Research Agenda aimed at drug development which will be funded through Framework 7 and include cancer. Whilst it is welcome that EU money is being partnered with industry, there is a real danger that if all increases in EU cancer research funding are from this source, Europe's intrinsic creativity could be distorted by encouraging subsidy-seeking behaviour. This would mean that essential areas of cancer-related public health that are not amenable to a business approach would remain orphans. Increasingly, research policy has been directed to supporting the transfer of technology from knowledge-generating organizations in the public sector (e.g. universities) to commercial firms, through the establishment of cooperative links (Faulkner \& Senker, 1995). 


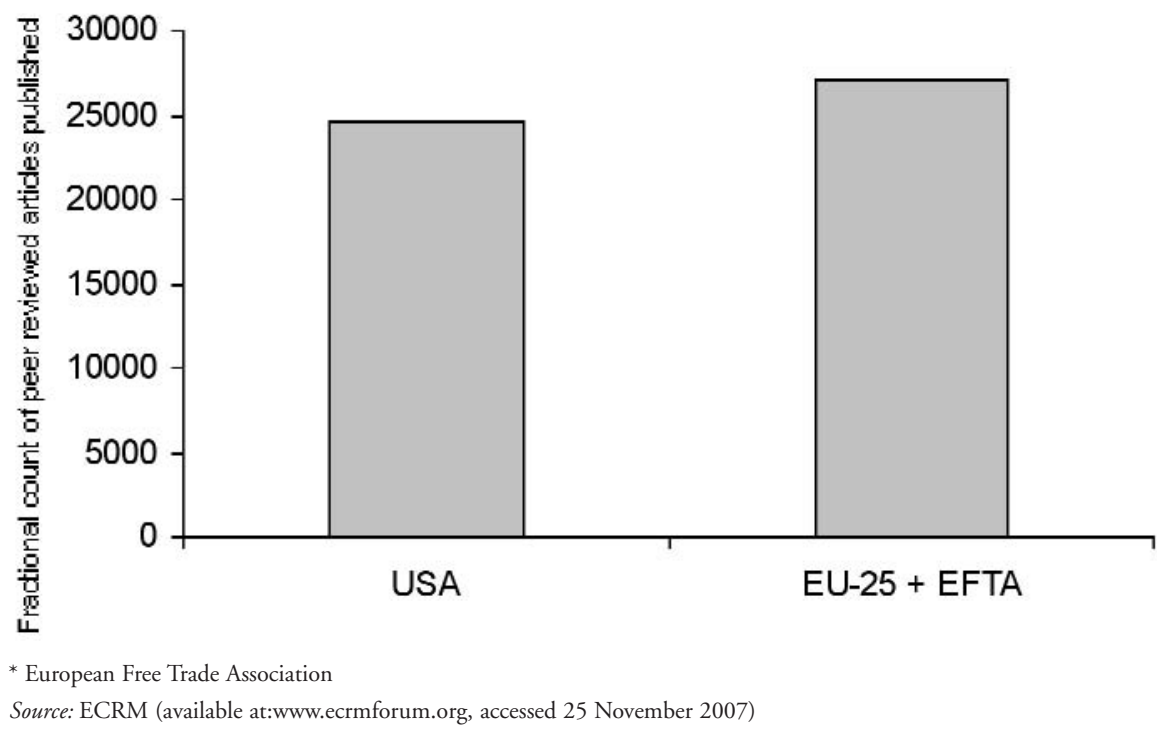

Fig. 15-2 Outputs (publications) from top 24 pharma industry in the United States of America and EU25+EFTA*, 1996-2003

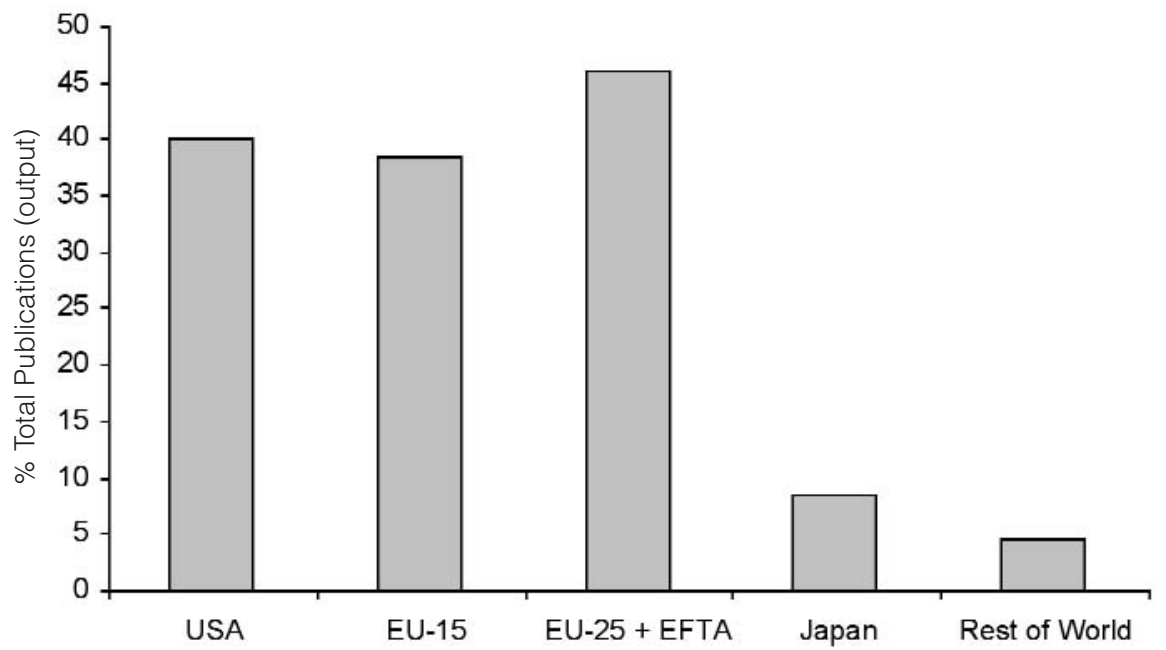

Source: ECRM (available at: www.ecrmforum.org, accessed 25 November 2007)

Fig. 15-3 Comparison of global outputs (publications) in cancer research 1999-2003.

In considering the global role of industry in cancer control it is a truism that, as the WHO have articulated, any new treatment is unlikely to be a "magic bullet". Health promotion and cancer prevention must remain a very high priority for governmental and charitable funders (Ferlay et al., 2004; Kaplan \& Laing, 2004). There are sound reasons to believe that priority-setting 
focused on predicted practical relevance, i.e. industrial utility, should be avoided by Europe. Firstly, most technological advances are derived from a broad base of scientific and technological fields and, secondly, as Pavitt describes, "our ability to understand the present and to predict successful future applications, is very limited. In detail, predictions will often be wrong, and in broad scope it will be obvious" (Pavitt, 1998). Europe must take a holistic view of cancer research funding.

\section{What will cancer research deliver for patients?}

By the end of the twentieth century Europe was witness to some 2.5 million cancer deaths a year (Ferlay et al., 2004). Although Europe comprises only one eighth of the world's population, it suffers a quarter of the global burden of cancer, in terms of incidence. In 2004, there were an estimated 2886800 new cases of cancer with 1711000 deaths (Boyle \& Ferlay, 2005). As research has unravelled the complex and complicated architecture of cancer and delivers increasingly sophisticated management (from diagnosis onwards), cost has become a dominant factor in policy making. Paradoxically, therefore, scientific research is responsible for establishing the basis for expensive new medical interventions and thus driving health care expenditure, according to Victor Fuchs of Stanford (Economist, 2004). But, of course, research provides the only realistic way to address this issue through the withdrawal of treatments that are not efficacious and/or likely to cause unacceptable toxicity.

Whilst the prevailing fashion is for new drugs, there remains tremendous scope and mileage in cancer research aimed at improving existing treatments as well as studies of the organization of care, methods to enhance quality of life and prevention. Organizational strategies should cover the broad spectrum that makes up cancer research and should fit the objectives of individual Member States as well as the broader European vision for cancer control. The plurality of strategies is one of Europe's great strengths - protean behaviour and diversification have always, in the Darwinian model, led to greater fitness and success (Beishon, 2006; Beishon, 2005).

The association between cancer research and patient outcomes for any given health-system or country is a complex one. Centres, countries and continents that are research-active should deliver higher standards of care to patients through the application of more effective medical technologies and more widespread adherence to best practice/guidelines (Coleman, et al., 2003). Delivering high-quality cancer care and being research-active are indivisible in rolling back the tide of cancer. Europe must learn from global experience in the organization of its myriad research programmes and centres of excellence. 
It is not enough to follow only American approaches (Simone, 2002); researchactive cancer centres, networks and funders across Europe must improve cooperation whilst maintaining sharpness and driving through competition a cancer research oligopoly.

The final aim of any cancer research is to improve cancer control for each patient and in society as a whole. In order to translate research findings into strategies that will ultimately improve the prevention, diagnosis, treatment and rehabilitation of cancer, it is not only scientists, but also the lay public and patients' organizations, who must be widely informed about the conduct and the results of research.

During recent years, improvements in public accessibility to clinical trial databases have been facilitated by a number of initiatives. For example, the National Library of Medicine in the United States sponsors a comprehensive web-based publicly assessed database of clinical trials (www.clinicaltrials.gov, accessed 2 December 2007). Currently, this lists more than 6000 trials on many cancer topics. Details include a brief statement on the interventions or comparisons being made; the study hypothesis; definitions of the primary and secondary study endpoints; patients' eligibility criteria; key dates (anticipated starting and closure); the targeted number of subjects; and contact information for the principal investigator. Unfortunately, this comprehensive information on ongoing clinical trials is not easy to understand and is only available in English. In addition, while clinicaltrials.gov is a very comprehensive catalogue of the clinical trials running in the United States, only a minority of those in Europe are included.

There are additional clinical trials registries worldwide that are provided or administered by the clinical research organizations and institutions that conduct research in different regions, e.g. the Southwest Oncology Group (SWOG) and the NCI in the United States; EORTC in Europe. Most of these databases are solely in English. For drug registration purposes, the European Medicines Agency (EMEA) established a comprehensive database on ongoing drug development clinical trials (EudraCT) but this database is accessible only by trial sponsors and EMEA authorities and not by the lay public or patients' organizations. There is little doubt that much more needs to be done to provide patients and their families across Europe with better information on research.

Indeed, problems around public information are deeply rooted. For example, there is a substantial difference between the number of trials completed and the number with published results. The emphasis by investigators and sponsors on trials with positive results means that many negative studies are 
never presented in the scientific media and, to an even lesser extent, to the public. One survey found a significant delay in the publication of negative trials (Krzyzanowska, Pintilie \& Tannock, 2003). For example, interferon alpha (IFN-alpha) is a drug with only modest efficacy in renal cell carcinoma. Two trials suggesting benefit from IFN-alpha were published one and three years after the presentation of preliminary results at the American Society of Clinical Oncology. Three trials showed no benefit or even a trend to harm two were published four and seven years after presentation, one remains unpublished after 13 years. In July 2005, the International Committee of Medical Journals (ICMJE) - representing 11 prestigious medical journals instituted a policy whereby a scientific report of clinical trial results cannot be published unless the trial has been recorded in a publicly accessible registry at its outset (De Angelis, 2004). They spoke only for themselves, but encouraged editors of other biomedical journals to adopt similar policies. Such practices should ensure that research is not only in the public domain but also published in a timely fashion.

\section{Conclusions}

Cancer research is a complex global activity aimed at controlling a complicated disease that will affect over 15 million people by 2020. Efforts to control and cure cancer are multifaceted and subject to many interdependencies and diverse funding streams for global cancer research as well as global outputs. Traditional research cultures, often compartmentalized until now in specific domains (laboratory or clinical), countries and institutions are evolving to fit the global research paradigm. However, the great discoveries that will help cancer patients and their families will only come from a climate of intellectual freedom and tolerance that fosters an atmosphere of creativity.

One of the most important policy messages must be the need to focus Europe on promoting creativity in cancer research (i.e. solving the problem of cancer) rather than innovation, which is too commercially orientated. This is the issue of breadth - Europe should and must pay particular attention to research domains and types of cancer that do not readily enjoy industrial support. As Europe looks forward over the next Framework and beyond, now is the time to be bold and focus on the public health needs of cancer patients rather than the economic advantage (Commission Green Paper, 2007). Hypothecated funding for clinical research is absolutely essential if Europe is to harness the research power of its social health-care systems.

The policy messages for the regulatory environment are clear - bureaucracy and overmanagement remain constant dangers to progress. There is clear 
evidence that these are damaging cancer research in Europe. Efforts must be made to simplify, harmonize and deregulate where necessary. Finally, never has there been a more urgent need for a "third culture" to drive the engagement between the research community and the public (Snow, 1964). From clinical trial registration to the public understanding of cancer, transparency and outreach must be core priorities for the European research community and policy-makers. Much has been achieved and much remains to be accomplished.

\section{REFERENCES}

European Commission, Directorate General for Research (2004). Five year assessment of the European Union Research Framework Programmes 1999-2003. Dec 15, Office for Publications of the European Communities, Luxembourg.

Anon. (2003). Protests mount at EU funding decision. Eur. J. Cancer, 39:2415-2416.

European Commission (2006). Report of the independent expert group on R\&D and the innovation appointed following the Hampton Court summit and chaired by Mr Esko Aho. Creating an Innovative Europe (EUR 22005)Office for Publication of the European Communities.

Beishon M (2005). Jose Baselga: playing to Europe's strengths. Cancer World, May - Jun:4-11.

Beishon M (2006). David Khayat: driving the French cancer plan. Cancer World, July-Aug:411 .

Berry DA et al. (2005). Effect of screening and adjuvant therapy on mortality from breast cancer. N Engl J Med, 353(17):1784-1792.

Boveri T (1914). Zur frage der entstehung maligner tumoren. Jena, Germany, Gustav Fisher.

Boyle P, Ferlay J (2005). Cancer incidence and mortality in Europe, 2004. Ann Oncol, 16(3):481-488.

Cambrosio A et al. (2006). Mapping the emergence and development of translational cancer research. Eur J Cancer, 42(18):3140-3148.

www.clinicaltrials.gov, accessed 2 December 2007.

CMR International (2006). 2006/2007 pharmaceutical R\&D factbook. Epsom, UK, Centre for Medicines Research International Ltd.

Coleman MP, Evans BG, Barrett G (2003). Confidentiality and the public interest in medical research - will we ever get it right? Clin Med, 3(3):219-228.

Coleman MP et al. (2003). EUROCARE-3 summary: cancer survival in Europe at the end of the 20th century. Ann Oncol, 14(Suppl. 5):128-149.

CONCORD Working Group (2007). www.lshtm.ac.uk/ncdeu/cancersurvival/concord/ index.htm

European Commission Green Paper (2007). The European research area: new perspectives (SEC 412) (available at: http://www.ec.europa.eu/research/era/publication_en.cfm, accessed 18 December 2007).

De Angelis C (2004). Clinical trial registration: a statement from the International

Committee of Medical Journal Editors. J Am Med Assoc, 351:1250-1251.

Doll R (1978). An epidemiological perspective of the biology of cancer. Cancer Res, 38(11 Pt 1):3573-3583.

Duncan B (2002). Health policy in the European Union: how it's made and how to influence it. Br Med J, 324(7344):1027-1030. 
Dunne J (2007). The European regulation on medicines for paediatric use. Paediatr Respir Rev, 8(2):177-183.

Early Breast Cancer Trialists Collaborative Group (2005). Effects of chemotherapy and hormonal therapy for early breast cancer on recurrence and 15-year survival: an overview of the randomised trials. Lancet, 365(9472):1687-1717.

Eckhouse S, Sullivan RA (2006). Survey of public funding of cancer research in the European Union. PLoS Medicine, 3(7):e267.

(The) Economist (2004). Survey of health care finance. The Economist Special Edition 15 July 2004:3-18 (available at: www.economist.com, accessed 18 December 2007).

www.ecrin.org, accessed 2 December 2007.

Ehrlich P (1909). Uber den jetzigen stand der karzinomforschung. Ned Tijdschr (The state of the art of cancer research). Geneeskd, 5:273-90.

European Strategy Forum on Research Infrastructures (2006). European roadmap for research infrastructures report. Luxembourg, Office for Official Publications of the European Communities, 2006.

Faulkner W, Senker J (1995). Knowledge frontiers: public sector research and industrial innovation in biotechnology. Oxford, Clarendon Press.

Ferlay J et al. (2004). GLOBOCAN 2002: Cancer incidence, mortality and prevalence Worldwide IARC CancerBase No. 5. version 2.0. Lyon, France, IARC Press.

Graham JD (2004). The perils of the precautionary principle: lessons from the American and European experience (The Heritage Foundation Lecture No. 818) (available at:http://www. heritage.org/ Research/Regulation/hl818.cfm, accessed 18 December 2007).

Hartmann M, Hartmann-Vareilles F (2006). The clinical trials directive: how is it affecting Europe's noncommercial research? PLoS Clin Trials, 1(2):e13.

http://cordis.europa.eu/lifescihealth/cancer/cancer-pro-calls.htm.

Heald RJ, Husband EM, Ryall RD (1982). The mesorectum in rectal cancer surgery: the clue to pelvic recurrence? Br J Surg, 69:613-616.

Humpries N (1997). Varieties of altruism - and the common ground between them. Social Research, 64:199-209.

www.ich.org/LOB/media/MEDIA482.pdf, accessed 2 December 2007.

www.ITCCconsortium.org, accessed 2 December 2007.

Jemal A et al. (2004). Cancer statistics, 2004. CA Cancer J Clin, 54(1):8-29.

Kaplan W, Laing R (2004). Priority medicines for Europe and the world. Geneva, World Health Organization. (WHO/EDM/PAR/2004.7, Nov).

Karpozilos A, Pavlidis N (2004). The treatment of cancer in Greek antiquity. Eur. J. Cancer, 40:2033-2040.

Krzyzanowska MK, Pintilie M, Tannock IF (2003). Factors associated with failure to publish large randomized trials presented at an oncology meeting. J Am Med Assoc, 290(4):495-501.

Laredo P (1998). The networks promoted by the framework programme and the questions they raise about its formulation and implementation. Res. Policy, 27:589-598.

Lennert K (2005). History and effect of the Kiel lymph node register. Prehistory. Pathologe, 22(3):167-174.

Malley BW (1995). Thirty years of steroid hormone action: personal recollections of an investigator. Steroids 60:490-498.

McIntyre P (2005). Finding the viral link: the story of Harald zur Hausen. Cancer World, JulyAug:32-37. 
Miles WE (1908). A method of performing abdomino-perineal resection of the rectum and of the terminal portion of the pelvic colon. Lancet, 2:1812-1813.

Mowery DC (1998). The changing structure of the US national innovation system: implications for international conflict and co-operation in R\&D policy. Res. Policy, 27:639-654.

Paget S (1989). The distribution of secondary growths in cancer of the breast, 1889. Cancer Metastasis Rev, 8(2):98-101.

Pavitt K (1998). The inevitable limits of EU R\&D funding. Res. Policy, 27:559-568.

Rahu M, McKee M (2003). Effect of Estonian law on prospects for public health research. Lancet, 362(9401):2122.

Ridgway PF, Darzi AW (2003). The role of total mesorectal excision in the management of rectal cancer. Cancer Control, 10(3):205-211.

Simone JV (2002). Understanding cancer centres. J. Clin. Oncol., 20(23):4503-4507.

Snow CP (1964). The two cultures: and a second look. An expanded version of the two cultures and the scientific revolution. Cambridge, Cambridge University Press.

Trias O (2006). Presentation at Biotechnology for Health Future Perspectives to EC-US Task Force for Biotechnology, 19-20 July (available at:http://www.ec.europa.eu/research/biotechnology/ecus/ec-us_16th_meeting_en.html, accessed 18 December 2007).

van Veen EB et al. (2006). TuBaFrost 3: regulatory and ethical issues on the exchange of residual tissue for research across Europe. Eur J Cancer, 42(17):2914-2923.

Veronesi $U$ et al.(1981). Comparing radical mastectomy with quadrantectomy, axillary dissection, and radiotherapy in patients with small cancers of the breast. $N$ Engl J Med, Jul 2;305(1):6-11.

Von Hansemann D (1890). Ueber asymmetrische Zelltheilung in epithel Krebsen und deren biologische Bedeutung. Virchow's Arch. Path. Anat, 119:299.

Watson JD, Crick FH (1953). Molecular structure of nucleic acids; a structure for deoxyribose nucleic acid. Nature, 171(4356):737-738.

Wright K (2000). Charitable change - creating a new culture of giving for Britain. LSE Magazine, Winter 2000:19-21. 


\section{Chapter 16 \\ Making progress against cancer}

Tit Albreht, Martin McKee, Delia-Marina Alexe, Michel P Coleman

and Jose M Martin-Moreno

\section{Cancer: the burden of an epidemic}

"Cancer" has been known since ancient times. Hippocrates, the father of medicine, named this kaleidoscope of diverse diseases "karkinos", the Greek word for crab, which he believed the tumours resembled. Hippocrates was not the first to document and report cases of cancer, however; ancient Egyptian writings mentioned both benign and malignant tumours.

Cancer can affect virtually any organ, but almost all types of malignancy share a common feature - the capacity of the cells to multiply without control, to spread to other organs (metastasise) and to kill the patient. The suffering of cancer patients and their families at an individual level is mirrored at the societal level, creating a huge burden for health services and the wider economy (Brown et al, 2001).

The term "epidemic" is often used to describe the growing burden of cancer. It was used in the past solely to define acute outbreaks of disease, usually infectious diseases that can spread quickly from person to person within a short period of time, and affect large numbers of people. Over time, the concept has evolved to reflect the notion of "excessive frequency", and in this sense it also applies to noninfectious diseases. But is cancer an epidemic?

In terms of "excessive frequency", cancer easily meets the definition.

First, cancer occurrence (incidence) is rising. Some 2.3 million new cases occurred in the EU25 in 2006. At current levels of incidence, one in three people in the EU will be diagnosed with cancer during their lifetime. Most people in Europe will know someone who has cancer. The EU population is growing and is expected to become significantly older. Therefore, even if the 
risks of getting cancer at each age do not increase, the number of new cancer patients diagnosed each year in the EU25 $5^{11}$ will rise by $20 \%$ in the 18 years between 2002 and 2020, simply due to population growth and ageing. Incidence rates would have to fall (by more than $1 \%$ every year over that period) in order to counterbalance the upward pressure of these demographic changes on the numbers of new patients that health systems will have to manage.

Second, total cancer mortality remains a huge and growing problem within the EU. One in four deaths is attributable to cancer. To illustrate this mortality burden, some 1.2 million deaths occurred in the EU25 in 2006 (Ferlay et al., 2007). Moreover, and in spite of the fact that survival from some cancers has improved markedly since 1990 (due to advances in medical knowledge, diagnosis and treatment), marked inequalities remain. Hence, survival for many cancers still varies widely between Member States.

This primarily human burden must be borne by millions of European families, but also by the economies and the health systems of the countries in which they live. Yet the implications for health systems go far beyond the financial. More than many other diseases, cancer conjures up deep fears and anxiety in most people; many are unaware how much can be done to reduce the risk of developing cancer and to treat successfully and care for those who develop the disease. Cancer control should thus be seen as a key priority for all countries.

\section{Cancer control}

Knowledge about the causes of cancer and the mechanisms of malignant behaviour continues to increase, ushering in an era in which prevention of many cancers is possible and many cancers can be either cured or treated more effectively, increasing both the duration and the quality of patients' lives. However, there is significant variability in the delivery of services for the prevention and treatment of cancer and in the outcomes achieved, both within and between Member States. A key component of any effective strategy for cancer control is to understand where services fall short of current standards and how to improve their quality and effectiveness. Cancer is complex, and achieving what is possible poses significant challenges. The first aim should be how best to translate existing knowledge into effective strategies at a population level.

${ }^{11}$ These calculations were made on the 25 EU Member States as of 2005. The addition of Romania and Bulgaria in 2007 is not likely to alter substantively the overall impact of these trends. 
An integrated strategy for cancer control requires simultaneous coordination of several elements, each of which must be tackled at different levels of society.

- Primary prevention (to reduce exposure to the risk factors that cause cancer, in order to avoid or minimize cancer occurrence) - through health promotion; lifestyle interventions; and government policies that both encourage individuals to make healthy choices and influence the behaviour of industry and other key stakeholders, generating commitment towards a healthier environment.

- Secondary prevention (to diagnose early those cancers which were not prevented) - through population screening to detect early disease and reduce mortality.

- Integrated care (to provide the best possible treatment for cancer patients) - by ensuring that the key prerequisites are in place, including a trained, multidisciplinary workforce; appropriate equipment and facilities; and effective diagnostics and drugs. Integrated care also includes improving the quality of life for cancer patients and their families, through support, rehabilitation and palliative care when needed.

- Advances in research - to find new solutions to all aspects of cancer management and control; and to identify new possibilities for prevention, early detection, diagnosis and treatment.

\section{Implications for patients}

First and foremost, it is important to maintain the focus on cancer patients.

For patients and their families, a diagnosis of cancer means the beginning of a long journey, often accompanied by a feeling of "betrayal" that stems from the knowledge that the cancer has arisen in one's own body. Cancer treatment is multidimensional, involving surgery, drugs, radiotherapy, chemotherapy, psycho-oncology and, when cancer is not treatable, palliative care. Effective coordination must apply to the care of the individual patient, arrangements for support for the patient's family and the overall operation of services. The outcome of this journey depends heavily on the joint efforts of cancer researchers, primary-care teams, oncologists, psychologists with training in psycho-oncology, specialist nurses, therapists, other stakeholders and policy-makers, and their collaboration with cancer patients.

In the end, what matters most for cancer patients is to regain their health and return to their everyday lives as soon as possible. For the younger patients, that means to become the productive persons that they were 
before they were diagnosed; for older patients, it means returning to the level of independence they had before diagnosis. All attempts to treat and care for cancer patients should be focused on enabling each cancer patient to reach that end-point. When the cancer is not treatable, palliative care should be provided to improve the quality of life for patients and their families.

Patients must be empowered by having access, in their own language, to the vital information that enables them and their advisers to make informed decisions about their care. This should include a Europe-wide registry of information on clinical trials, to encourage the participation of more cancer patients. National medicines regulators should set up formal links with patient groups, following the model promoted by the European Medicines Agency (EMEA).

Much can still be done to enhance equitable access to prevention and treatment within Europe. For instance, the establishment of a European cancer task force may provide a fresh European framework for tackling inequalities in cancer control and sharing best practices in cancer prevention and care.

\section{Implications for health policy}

A comprehensive approach to cancer at both national and international levels is of vital importance, starting with a detailed analysis of the current situation. At national level, such an analysis should describe the geographical and temporal patterns of incidence, mortality and survival for each of the common cancers, and establish priority areas for cancer control. A parallel analysis should consider the current status of any national cancer plan (and the creation of such a plan where it does not exist) as well as the proportion of total national expenditure on health that is allocated to cancer control.

A key requirement for successful cancer control is the development of national cancer plans. The WHO advocates the development of national cancer control programmes as the best means of reducing the incidence and impact of cancer, and improving the quality of life of cancer patients within available resources. This involves a comprehensive, planned approach that will identify and implement priorities for action and research - from cancer prevention and screening through diagnosis, treatment, rehabilitation and palliative care. Scenario planning may be used to assess the likely future burden of cancer and the potential impact on that burden of alternative policy options for prevention, screening and treatment. 
Progress in cancer control remains uneven across Europe. There is scope for the EU to facilitate shared learning and information exchange. In those areas where it has competence, such as free movement of goods and services, regulatory instruments offer a means for enhancing the quality of care in many parts of Europe.

An integrated information system is required to monitor the quality of these activities effectively and consistently. Cancer information systems, particularly cancer registries, are crucial in this respect. They require investment and protection. In some Member States, however, misguided or overzealous implementation of the 1995 EU Data Protection Directive has impeded the effective operation of cancer registries. It is time for the application of this Directive in the health domain to be reviewed. Revisions should be designed explicitly to enable population-based registration of cancer, with provisions similar to those that apply in all Member States for the notification of communicable diseases. This amendment to European law would recognize the gravity and extent of the problem posed by cancer, and the need to secure high-quality information in support of cancer control policy.

\section{Implications for reimbursement and financing agencies}

The care of cancer involves the application of many diagnostic and therapeutic approaches at various steps along the patient's care pathway. This requires deployment of some highly technical and costly resources. The economic implications are magnified because cancer can be diagnosed early in the course of what may be a very prolonged disease state, during which complex and expensive interventions may be required at any time. Payers for healthcare in Europe need to examine the cost-effectiveness of new technologies as well as the efficacy of drugs. At a national level, governments must ensure that clinically proven interventions that maximize the length and quality of life are available to all their citizens. Robust health technology assessment should be followed by equity in the distribution of treatment resources.

New systemic (drug) treatments are very attractive to investors, but developments in molecular pathology, imaging, radiotherapy and surgery are also very important in improving the management of cancer. Development of these technologies should be actively encouraged by the $\mathrm{EU}$, alongside the Innovative Medicines Initiative (IMI), which is focused solely on new drugs. Technical developments in diagnosis, investigation and treatment also require continuous training of staff and substantial long-term investment in equipment and personnel. 
Four important resource needs can be identified in relation to cancer control:

- human resources, such as highly qualified and specialized staff

- physical resources, such as appropriate equipment, facilities and drugs

- knowledge resources, such as evidence-based clinical guidelines

- social resources, such as systems of long-term support for patients and their families.

All these types of resource require sustained provision of adequate financial support and should reflect the increasing demand that will flow from the rising trend in cancer rates.

\section{Implications for research}

A thriving programme of cancer research is essential to uncover new approaches to clinical and public-health intervention. For instance, population-based research with the aid of cancer registries is an important tool in establishing the value of different interventions to reduce the burden of cancer. Research can bring new knowledge, insight and solutions. It must be stimulated appropriately.

A European research agenda should be set, embracing multiple funding bodies, covering issues such as how to broaden awareness of prevention and screening; and how to tackle the obesity epidemic. As stated in the 2007 European Commission Green Paper (European Commission, 2007), the focus should be on public health and the needs of cancer patients, rather than the economic advantage.

Consequently, research should include the following topics:

- Risk factors - research should be cast more widely than the identification of risk factors, and how to reduce or eliminate exposure to them. We should also foster behavioural research on how we can act today to prevent what it is preventable. The research question is how to improve communication, health education and social marketing in order to overcome behavioural barriers and to implement effectively (and promptly) what is already known about the prevention of cancer.

- Lifestyle challenges - research should be promoted to identify more effective ways to influence unhealthy lifestyles. This would reinforce efforts to prevent other chronic diseases and disorders that are related to the same unhealthy lifestyles. It would also provide better evidence on the relationship between lifestyles and the triggers for cancer. 
- Screening - identify new screening tests; how best to implement effective new screening programmes (i.e. for cancers other than breast, cervix and bowel); examine both the effectiveness and the costeffectiveness of existing and newly developed screening methods; examine the obstacles to the successful implementation of proven screening methods or their failure in particular circumstances.

- Treatment - the prevailing fashion is for new drugs, which should be meticulously evaluated from clinical and societal perspectives (comparing clinical and population effects, for both clinical efficacy and cost-effectiveness, in view of equity). There remains considerable scope for cancer research aimed at improving existing treatments.

- Clinical management - how to improve access, equity, effectiveness, outcome and patient satisfaction. This should also include studies of the organization of health-care systems and of methods to enhance the quality of life for cancer patients.

- Rehabilitation and palliative care - how to innovate; how to extend access to such care for all cancer patients.

- Monitoring - to quantify and explain recent trends and to predict trends in incidence, mortality and survival in the medium term (10year future).

- Development of a stable research community requires a Europe-wide commitment to the motivation and training of future researchers.

5. Implications for the pharmaceutical and medical technology industries

Technological and therapeutic advances have transformed the management of some cancers, especially those afflicting children. However, many other cancers remain stubbornly resistant to treatment. Increasing knowledge of the cellular mechanisms that underlie the development and spread of cancer is offering scope for the development of new therapeutic approaches. Yet this comes at an increasingly high cost. The ground rules for cooperation between the public and private sectors should be explicit, fair and transparent. Industry and government must find ways of working together in order to ensure not only a steady stream of innovation but also access to affordable treatment and care for all those who need it. 
Measures at the national level

1. Integrating cancer prevention into all health promotion activities

Cancer prevention is the most important lever at our disposal to stem the increasing cancer burden, which reflects the combined effects of population ageing, population growth and unhealthy lifestyles. It is an investment in the prevention of substantial future morbidity and mortality. It offers the potential for huge long-term economic benefits, but requires substantial investment in the short and medium term to provide any realistic prospect of achieving those benefits.

\section{Lifestyle interventions}

Lifestyle interventions include those in relation to tobacco and nutrition. Effective national implementation of the Framework Convention on Tobacco Control and of the Global Strategy on Diet, Physical Activity and Health would go a long way toward reducing the cancer burden.

About $40 \%$ of the total cancer burden is caused by tobacco consumption and excessive alcohol consumption. The precise figures vary from country to country. If the consequences of inappropriate diet, obesity and insufficient physical activity are added, the percentage of cancers due to an unhealthy lifestyle rises to $60 \%$. A consistent approach to promote healthy lifestyles should become an important element of cancer prevention. This would also help reduce the burden of other important chronic noncommunicable diseases - especially cardiovascular disease, the other major killer of Europeans.

\section{Implementation of the European Code Against Cancer}

The European Code Against Cancer, the third version of which was adopted in 2003 (Boyle et al., 2003), is in the process of being updated. Cancer is an extremely complex public health problem, but the Code remains highly relevant. It is an evidence-based instrument for cancer control that is periodically updated. All countries should seek to implement it as fully as possible.

\section{Development of national cancer registries}

National (and regional) cancer registries are the only source of populationbased data about the number of new cancer cases diagnosed each year, and of cancer survival. They are a crucially important resource for estimating and tracking trends in the cancer burden by age, sex and type of cancer, 
and for prioritizing the allocation of resources for cancer control. Highquality cancer registries are essential if cancer services are to be planned rationally and monitored efficiently. They are also an important resource for national and international epidemiological studies, which have provided so much evidence for the development of policies to prevent and treat cancer.

Cancer registration is statutory in many EU Member States. Where it is voluntary, experience in the last 15 years in Germany, Hungary and the United Kingdom has shown the extreme vulnerability of the complex information flows to sudden concerns about confidentiality and data security. Even in Estonia, where cancer registration is statutory, legal impediments have been placed in the way of linkage of cancer data to death records, seriously degrading the value of what was an outstanding resource.

Cancer is a public-health problem that far outweighs communicable disease in Europe on almost any conceivable measure - the number of new cases, severe morbidity, number of deaths, economic cost of treatment and the loss of economically active citizens. The crucial information systems needed to track the cancer burden should be afforded the same legislative protection as those used to collect information about communicable disease, in every EU Member State, through active removal of legal obstacles to achieving this goal.

\section{Development of national cancer screening programmes}

For three common cancers, sufficient evidence exists that mass screening (as a public-health policy) is an effective strategy for reducing risk or mortality. Thus mortality from breast cancer can be reduced by an efficient population-based mammographic screening programme and the rapid treatment of screen-detected cancers.

The incidence of invasive cervical cancer can be reduced by organized mass screening programmes using the Pap test or visual inspection. Prophylactic vaccines against the main carcinogenic strains of the human papilloma virus (HPV) may help to reduce HPV prevalence, but they will not be expected to have a substantial impact on cervical cancer incidence rates for at least a decade. In high-risk countries, continuous implementation of successful cytology screening programmes will remain a priority, alongside any vaccination programme.

Mass screening for colorectal cancer with the faecal occult blood (FOB) test, backed up by sigmoidoscopy or colonoscopy, has been shown to reduce mortality from this cancer, and should be implemented as soon as resources allow. 


\section{Development of national cancer plans}

Effective cancer plans will include all five of the elements described above. They should also cover comprehensive cancer care, the organisation and delivery of optimal treatment services to all cancer patients, rehabilitation, and research. Examples from several countries show that comprehensive national cancer plans are very effective in achieving optimal prevention and treatment of cancer.

\section{Measures at the EU level}

1. Develop coherent, Europe-wide measures to complement national policies for controlling the effects of unhealthy lifestyles.

Unhealthy lifestyles pose serious consequences for the cancer burden. Intervention through comprehensive, health-oriented policies is of great potential value. In addition to general health-promotion activities, special attention should be directed towards alcohol and tobacco. Such activities should aim in particular at reducing exposure to tobacco smoke and limiting access to alcoholic beverages. Examples include the reduction of tobacco and alcohol consumption through adequate pricing policies; policies to reduce harm related to exposure to environmental tobacco smoke and harmful drinking of alcohol; other policies to reduce demand and limit access; and by the avoidance of internal market policies that will increase consumption. Policy-makers should be aware of the tactics that have been used by the tobacco industry (Grüning et al., 2006) and, more recently, the alcohol industry (McKee, 2006) to subvert effective public health policies. European policies should also be designed to promote healthy nutrition and physical activity; the safest occupational and environmental guidelines; and a coherent approach towards preventative alternatives against infectious agents that can cause cancer, such as the hepatitis B and human papilloma viruses.

2. Develop and fund extended research programmes to broaden knowledge of cancer epidemiology and the causes, early detection, diagnosis, treatment, survival and palliation of cancer.

Research must cover a wide range. This includes epidemiology (the patterns and trends of cancer in Europe, and the factors that increase or decrease cancer risk); social sciences and economics; biomedical research (on the biological mechanisms underlying cancer); clinical research; and translational research (from the laboratory bench to the hospital and clinic, and from epidemiological insight to public health action). 
Complementary research on impact assessment should examine the effectiveness of actions designed to achieve cancer control. It addresses the question - what works? This should include testing new strategies and methods for prevention, early detection, treatment, support and rehabilitation and palliative care.

3. Reduce major inequalities in cancer incidence and survival across Europe.

This applies both to geographical inequalities between European regions and countries, and to socioeconomic and ethnic inequalities within countries. These disparities are a source of concern for European citizens in all parts of the Union. Reference centres (see below) and cross-border collaboration may offer useful approaches to removing inequities in access to optimal care. These would provide invaluable support and assistance to Member States for the successful management of cancer.

4. Develop optimal policies for evidence-based introduction of new health technology.

Rapid technological development in the management of chronic diseases has made available new methods of diagnosing and treating cancer. At the European level, this has obvious advantages but also some significant difficulties. Unifying some of the EU regulatory mechanisms (such as the evaluation and registration of new medicines through EMEA) has significantly simplified some processes, but has also brought new challenges for some Member States, which need to adapt their own systems.

The well-known provisions for the licensing of new drugs should be supplemented with a more consistent approach to the assessment of all new health technologies, including even consensus guidelines for the investigation and treatment of cancer. This will need to be carried out with full respect for the principle of subsidiarity, under which Member States are responsible for health. However, the EU has some responsibilities for public health and greater cooperation is both technically feasible and obviously desirable. Some of the newer and less wealthy Member States do not have internal access to all the resources or expertise required to assess the evidence and develop the policies independently. The experience gathered through the European Network for Health Technology Assessment (EUnetHTA) and other international health technology assessment agencies should be exploited. The potential advantages of sharing this expertise under the aegis of a European body seem clear. 
5. Develop European reference centres to improve the treatment of rare cancers.

The EU recently reviewed the definition of a rare disease on the basis of its prevalence (e.g. 1 in 5000), together with the criteria for designating centres of excellence for the diagnosis and treatment of rare diseases. By definition, few centres would be expected to have the necessary expertise and resources for optimal treatment of rare diseases. This also applies to cancer. The development of reference centres for the treatment of rare cancers should serve to ensure rapid implementation of the most up-to-date diagnostic methods and treatments. Such centres would offer particular benefits to patients living in the smaller Member States and in border areas. They should also serve as centres for research focused on their area of expertise, as well as training and knowledge transfer, while ensuring the continuing care of patients who complete their treatment in their home environment.

6. Develop common strategies for systematic assessment of the quality of cancer management programmes.

These would cover issues such as the effectiveness, misuse, overuse and sustainability of approaches to the care and treatment of cancer patients. Objective criteria for health technology assessment would also be used to monitor the appropriateness of the use of technology.

The three main pillars of public health strategy in the EU (http://ec.europa. eu/health/ph_overview/pgm2008_2013_en.htm) are to improve citizens' health and security; to promote health and reduce health inequalities; and to provide citizens with health information and knowledge. Cancer affects a large proportion of the EU population. The widespread inequalities in cancer mortality, incidence and survival across Europe are substantial and persistent. Cancer should become a key public health priority for European policymakers.

From an EU policy perspective, solidarity requires that all citizens, without discrimination or exception, benefit from whatever advances have been made in prevention, early diagnosis, treatment and palliative care, and that rapid implementation of successful strategies is facilitated by the use of policy tools such as EU Directives.

In summary, we know that at least one third of cancer can be prevented. Early detection and effective treatment of a further third is also possible. Our ability to achieve what we know is possible depends on our taking a more planned approach, involving all activities and services related to cancer. We strongly 
believe that a clear political commitment, demonstrated through coordinated, targeted actions, is needed to address the burden of cancer and improve health outcomes.

In this chapter we have outlined some of the ways in which substantial progress in cancer control in Europe could be achieved within a reasonable time frame. The details behind this summary are contained in the 14 preceding chapters covering the entire range of cancer control. The authors of those chapters have also described the current challenges for cancer control.

The résumé of research, opinion and policy proposals in this book should not be interpreted as either definitive or prescriptive. We hope new ideas will arise in discussions at the conference organised by the Slovenian Presidency of the Council of the European Union in February 2008.

\section{REFERENCES}

Boyle P et al. (2003). European Code Against Cancer and scientific justification: third version (2003). Ann Oncol, 14(7):973-1005.

Brown ML, Lipscomb J, Snyder C. The burden of illness of cancer: economic cost and quality of life. Annual Review of Public Health, 2001; 22:91-113.

http://ec.europa.eu/health/ph_overview/pgm2008_2013_en.htm, accessed 20 December 2007.

European Commission Green Paper (2007). The European research area: new perspectives. SEC 412 (available at: http://ec.europa.eu/research/era/publication_en.cfm).

Ferlay J et al. (2007). Estimates of the cancer incidence and mortality in Europe in 2006. Ann Oncol, 18(3):581-592.

Grüning T, Gilmore AB, McKee M. Tobacco industry influence on science and scientists in Germany. Am J Public Health, 2006; 96:20-32.

McKee M. A European alcohol strategy. Br Med J, 2006, 333:871-872. 


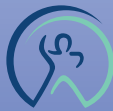

IVZ RS
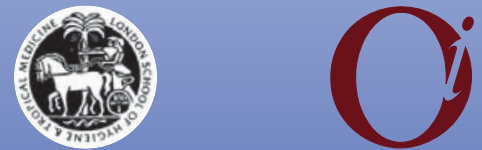

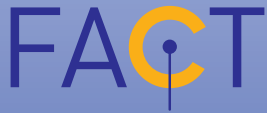

Fighting Against Cancer Today

The term "cancer" is commonly used to cover a wide range of diseases which all share a common feature, namely that cells in affected organs or tissues of the body (e.g. breast, lung, skin or bone marrow) continue to grow indefinitely, without reference to the needs of the body. Many cancers have the capacity to spread to other parts of the body and to kill the patient. With more than 3 million new cases and 1.7 million deaths each year, cancer currently represents the second most important cause of death and morbidity in Europe.

This volume, published under the Slovenian Presidency of the European Union, is a review of the current status of cancer control in the European Union. The aim was to summarize the evidence that should underpin policy for the prevention, management and palliation of cancer in Europe.

The book has been produced as a collaborative effort between internationally recognized public health institutes in the European Union, under the umbrella Fighting Against Cancer Today (FACT). FACT is co-funded by the Government of Slovenia and the European Commission's Health and Consumer Protection Directorate, with additional support from the European Observatory on Health Systems and Policies.

\section{The editors}

Michel P Coleman is Professor of Epidemiology and Vital Statistics at the London School of Hygiene and Tropical Medicine and leader of the Cancer Research UK Cancer Survival Group, London, United Kingdom. Delia-Marina Alexe is a Clinical Research Fellow at the London School of Hygiene and Tropical Medicine, London, United Kingdom. Tit Albreht is Researcher in Health Services at the Institute of Public Health, Ljubljana, the Republic of Slovenia. Martin McKee is Professor of European Public Health at the London School of Hygiene and Tropical Medicine, London, United Kingdom, co-director of the School's European Centre on Health of Societies in Transition, and a Research Director at the European Observatory on Health Systems and Policies.

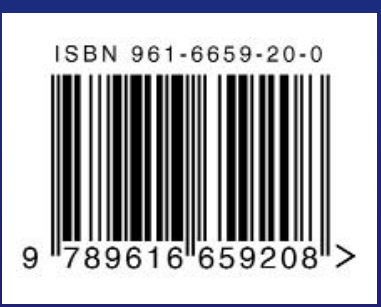

